\title{
Fabric Development, Electrical Conductivity and Graphite Formation in graphite-bearing Marbles from the Central Damara Belt, Namibia
}

\author{
Dissertation \\ zur Erlangung des Doktorgrades \\ der Mathematisch-Naturwissenschaftlichen Fakultäten \\ der Georg-August-Universität zu Göttingen
}

\author{
vorgelegt von \\ Jens Martin Walter \\ aus Windhoek/Namibia
}

Göttingen, 2004 
D 7

Referent:

Prof. K. Weber

Korreferent:

Dr. B. Leiss

Tag der mündlichen Prüfung: 29.06.2004 


\section{AbSTRACT}

Graphite-bearing marbles occur in crustal-scale dome structures of the central parts of the Damara Belt in north-western Namibia. They have been reported to show significant anomalies of high electrical conductivity in magnetotelluric profiles. This work presents conductivity measurements on a sample scale of different types of these graphite-bearing marbles. As the graphite-bearing marbles also form distinct shear zones along reactivated rims of the dome structures, these different types of graphite-bearing marbles were distinguished by their macro- and microscopic fabric characteristics. The investigation and classification of the different fabrics is the basis for understanding the conductivity potentials of the different types of graphite-bearing marbles. The classification was made using qualitative optical microscopy, cathodoluminescence microscopy (CL), scattered electron microscopy (SEM), bulk and local texture analysis and the quantification of the calcite-graphite ratios. Several studies were made to verify the tectono-metamorphic development of the different fabrics, and to characterise the modes of graphite formation within these marbles. These include Raman spectroscopic measurements, energy dispersive X-ray analysis $(\mathrm{EDX})$ and investigations of the stable isotopes.

Fabric investigations and field work show that the graphite-bearing marbles are abnormally coarsegrained. The large grain-size of the marble is according to these investigations related to the regional intrusion of granitic melts into high-grade metamorphic rocks. Calcite-graphite thermometry by carbon isotopes indicates regional peak temperatures of around $760^{\circ} \mathrm{C}$. The coarse-grained marbles were subsequently deformed in brittle-ductile shear zones along the reactivated rims of the dome structures, producing complex fabrics. The studied shear zones are composed of a mylonitic core zone and a brittle-ductile deformed boundary zone. Part of the deformation in the shear zones was by pressure solution, which resulted in the formation of graphitic stylolites. The graphitic stylolites form network structures of varying degrees of intensity. On a sample scale, the graphite networks show resistivities of 400 to $540 \Omega \mathrm{m}$. These networks are responsible for the anomalies of high electrical conductivity, measured in the magnetotelluric profiles. Many of the graphitic stylolites are cut by microveins. It is proposed that these veins were generated as tension fractures and hydrofractures during the Cretaceous break-up of Gondwana and the subsequent uplift to surface levels.

Graphite was most probably formed during metamorphism, which also lead to the abnormal graincoarsening of the marbles. The graphite is of uniform high crystallinity across all types of graphitebearing marbles. Since carbon isotopes of graphite were equilibrated with the calcite marble host rock, no isotopic indications about the origin of the graphite are preserved in the marbles. Qualitative optical microscopy and EDX investigations show, that the graphite is commonly epitaxial-intergrown with mica minerals. The fine-grained graphitic stylolites formed as a residue of pressure solution of calcite. The calcite-graphite ratios indicate, that both calcite and graphite were subject to pressure solution deformation. The calcite textures correspond to so-called 'lowtemperature' pure shear textures, both in the mylonitic core zones as well as in the brittle-ductile boundary zones. The intensity of lattice-preferred orientations is generally very high and varies strongly with the grain size of the investigated samples. The mylonitic core zones show a complex pattern of different domains of lattice preferred orientation within microscopic scales. Graphite also shows a strong lattice-preferred orientation in the mylonitic core zones with the basal plains oriented parallel to the foliation.

The abnormal grain-coarsening resulted from a combination of regional and contact metamorphism. The complex brittle-ductile deformation fabrics were formed subsequently in a seismic-aseismic transition zone with isochronous brittle, ductile and pressure solution deformation. The graphitic stylolites developed during this deformation, are responsible for crustal anomalies of high electrical conductivity in the measured magnetotelluric profiles. 


\section{CONTENTS}

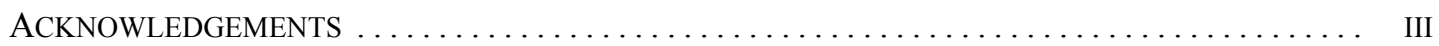

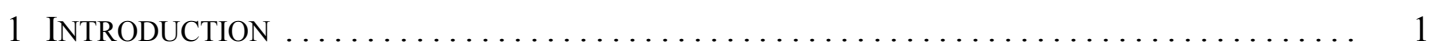

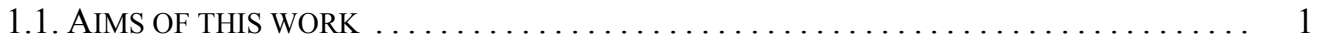

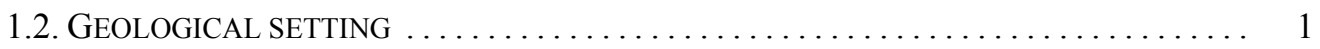

1.3. LOCATION AND DESCRIPTION OF THE FIELD AREAS $\ldots \ldots \ldots \ldots \ldots \ldots \ldots \ldots . \quad 4$

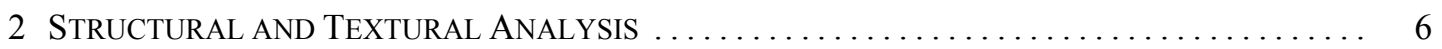

2.1. REGIONAL DEFORMATION AND STRUCTURES $\ldots \ldots \ldots \ldots \ldots \ldots \ldots \ldots \ldots \ldots \ldots$

2.2. CHARACTERISATION OF THE DIFFERENT TYPES OF MARBLES $\ldots \ldots \ldots \ldots \ldots \ldots . . \ldots$

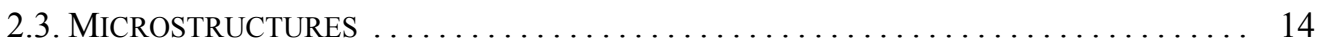

2.3.1. COARSE-GRAINED MARBLE HOST ROCK $\ldots \ldots \ldots \ldots \ldots \ldots \ldots \ldots \ldots$

2.3.2. BRITTLE-DUCTILE DEFORMED MARBLE $\ldots \ldots \ldots \ldots \ldots \ldots \ldots \ldots \ldots$

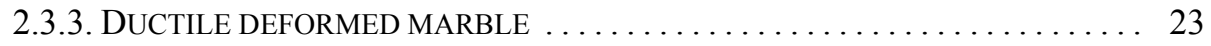

2.3.4. CATHODOLUMINESCENCE INVESTIGATIONS ................ 25

2.3.5. INVESTIGATIONS BY SCANNING ELECTRON MICROSCOPY ......... 26

2.3.6. QUANTITATIVE CALCITE-GRAPHITE RATIOS ............... 28

2.3.6.1. ANALYTICAL TECHNIQUES ...................... 30

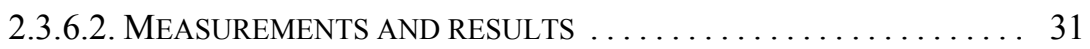

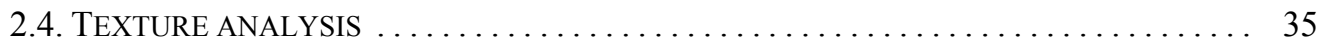

2.4.1. TEXTURE ANALYSIS BY NEUTRON DIFFRACTION $\ldots \ldots \ldots \ldots \ldots \ldots . \ldots 35$

2.4.1.1. ANALYTICAL TECHNIQUE $\ldots \ldots \ldots \ldots \ldots \ldots \ldots \ldots \ldots \ldots \ldots \ldots$

2.4.1.2. TEXTURE TYPES OF CALCITE $\ldots \ldots \ldots \ldots \ldots \ldots \ldots \ldots \ldots \ldots$

2.4.1.3. SAMPLES AND MEASUREMENTS $\ldots \ldots \ldots \ldots \ldots \ldots \ldots \ldots, 38$

2.4.2. TEXTURE ANALYSIS BY ROTATING POLARIZER STAGE $\ldots \ldots \ldots \ldots \ldots 45$

2.4.2.1. ANALYTICAL TECHNIQUE $\ldots \ldots \ldots \ldots \ldots \ldots \ldots \ldots \ldots \ldots . \ldots \ldots$

2.4.2.2. SAMPLES AND MEASUREMENTS $\ldots \ldots \ldots \ldots \ldots \ldots \ldots \ldots . \ldots . \ldots . \ldots$

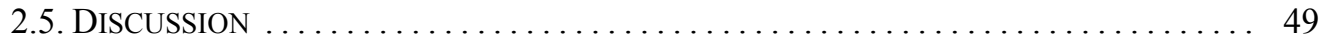

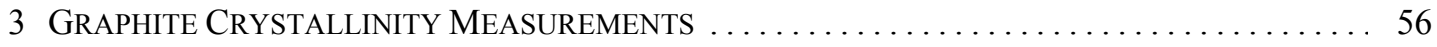

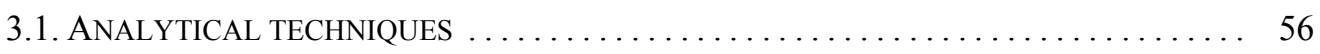

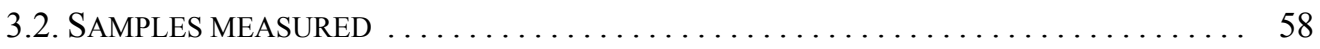

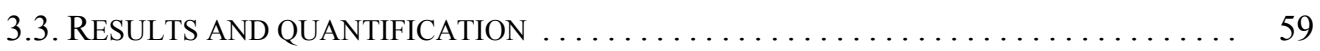

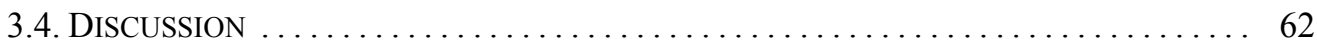

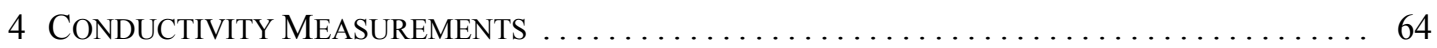

4.1. PRINCIPALS OF ELECTRICAL CONDUCTIVITY ....................... 64

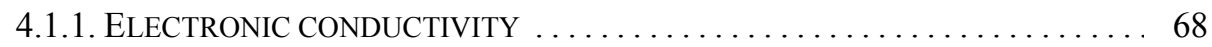

4.1.2. IONIC OR ELECTROLYTIC CONDUCTIVITY $\ldots \ldots \ldots \ldots \ldots \ldots \ldots \ldots 68$

4.1.3. ELECTRICAL CONDUCTIVITY OF ROCKS $\ldots \ldots \ldots \ldots \ldots \ldots \ldots \ldots \ldots$

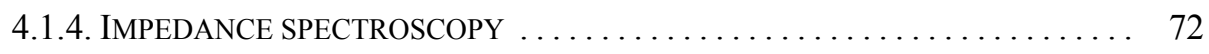

4.1.4.1 POLARISATION EFFECTS AND ELECTRICAL CONDUCTIVITY . . . 72 
4.1.4.2. THEORY OF COMPLEX IMPEDANCE $\ldots \ldots \ldots \ldots \ldots \ldots \ldots, 74$

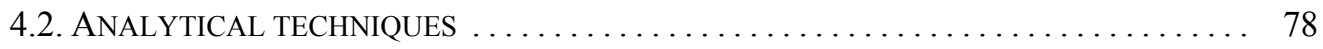

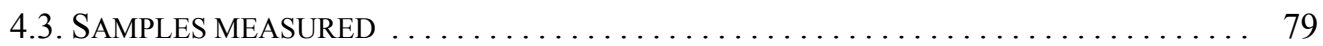

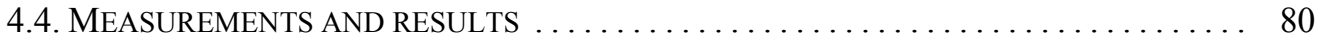

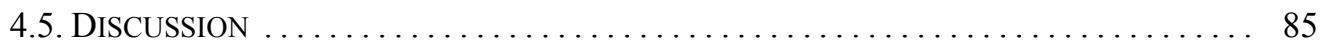

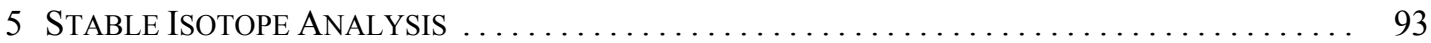

5.1. STABLE ISOTOPES OF MARBLES AND GRAPHITE $\ldots \ldots \ldots \ldots \ldots \ldots \ldots \ldots \ldots . \ldots 9 . \ldots \ldots$

5.2. CHARACTERISATION OF THE GRAPHITE-BEARING MARBLES $\ldots \ldots \ldots \ldots \ldots \ldots . . \ldots 0$

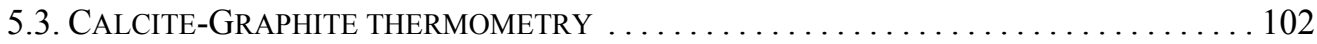

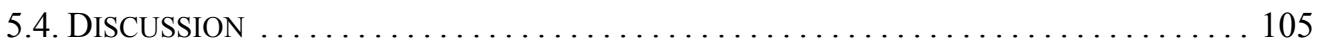

6 Mobilisation AND PRECIPITATION OF GRAPHITE $\ldots \ldots \ldots \ldots \ldots \ldots \ldots \ldots \ldots \ldots \ldots \ldots \ldots \ldots \ldots$

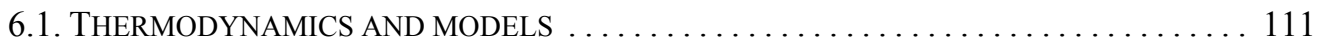

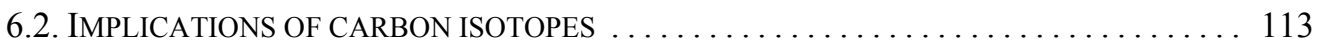

6.3. IMPLICATIONS OF GRAPHITE CRYSTALLINITIES ..................... 114

6.4. IMPLICATIONS OF REGIONAL METAMORPHISM $\ldots \ldots \ldots \ldots \ldots \ldots \ldots \ldots \ldots \ldots \ldots$

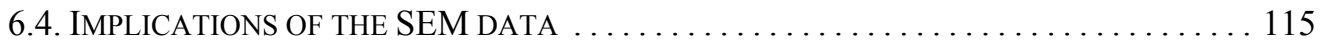

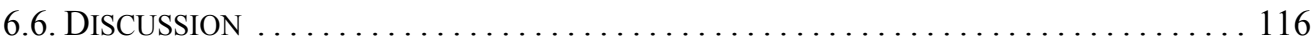

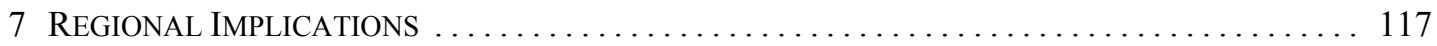

7.1. REGIONAL METAMORPHISM .................................. 117

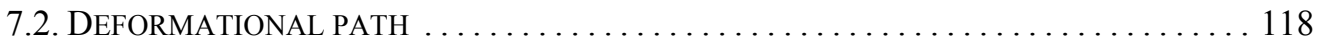

7.3. DISCUSSION ................................................... 119

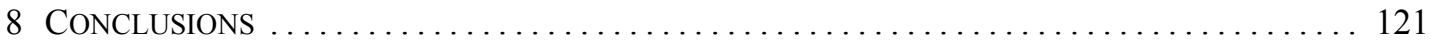

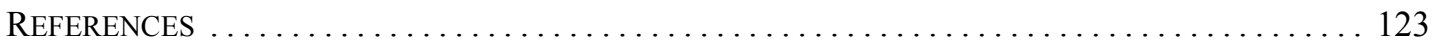

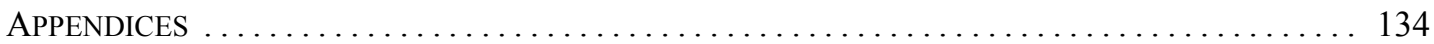

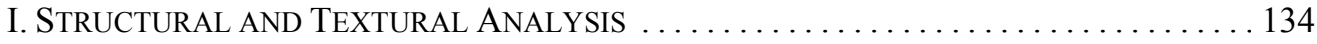

II. GRAPHITE CRYSTALLINITY MEASUREMENTS $\ldots \ldots \ldots \ldots \ldots \ldots \ldots \ldots \ldots \ldots \ldots \ldots$

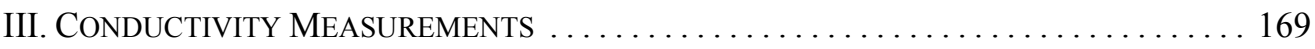

IV. STABLE ISOTOPIC ANALYSIS . . . . . . . . . . . . . . . . . . . . . . . . . . . . . . . . . . 270 


\section{ACKNOWLEDGEMENTS}

Writing a $\mathrm{PhD}$ thesis is a long and sometimes stony path of rewarding scientific progress and inevitable detours and scientific setbacks. In this process, the personal and scientific gain and progress is also based on the profound experience and knowledge, shared in discussions and laboratory setups.

First of all I want to thank Prof. Dr. Klaus Weber, my supervisor, for the initiation of the project WE 488 50-1 together with Dr. Bernd Leiss and Dr. Georg Nover. The funding of this project by the Deutsche Forschungsgemeinschaft (DFG) enabled this research. He participated in this work by fruitful discussions and observations, especially in the field. I am also very appreciative for adjuvant comments on the manuscript.

Secondly I want to express my gratitude to Dr. Bernd Leiss, my co-supervisor for his effective support and his readiness for valuable discussions. The co-operation with him was a great personal gain and a great pleasure. His constructive comments on the manuscript are also grateful acknowledged.

Special thanks also go to Dr. Klaus Ullemeyer for stimulating discussions in the field and afterwards. Furthermore, I especially want to thank him for his uncomplicated helpfulness in the organisation of the textural measurements and during the analysis of the textural data. His profound scientific attitude always had been exemplary for me.

Furthermore, I want to thank Dr. Georg Nover from the MineralogischPetrologisches Institut of the Universität Bonn for the excellent co-operation regarding the electrical conductivity measurements at his institute and afterwards.

I also want to thank Dr. Axel Vollbrecht for our prolific discussions regarding the microstructural analysis. Likewise, I want to thank Prof. Dr. Agust Gudmundsson and Dr. Sonja Brenner for their enlightening discussions with me about tensile- and hydrofractures. I also want to thank Prof. Dr. Agust Gudmundsson for his general support of this work. The stimulating discussions about the isotope geothermometry with Prof. Dr. Jochen Hoefs are also gratefully acknowledged.

I am also very grateful to Dr. Thomas Kenkmann from the Institut für Mineralogie at the Museum für Naturkunde, Humboldt-Universität Berlin for his uncomplicated and effective co-operation concerning the raman measurements at his institute.

I also want to thank Percy-Constatin von Samson-Himmelstjerna for his support in solving high-grade polynomial functions. As time becomes a very important matter during the accomplishment of a thesis, his little program helped me solving hundreds of these polynomial functions in a reasonable time. 
Additionally the readiness of Dr. Sonja Brenner for proof-reading the manuscript for spelling is very much appreciated.

The helpfulness regarding professional photos from the spherical graphite structures by Günther Schnorrer of our institute is also greatly appreciated. Likewise, I want to thank Michael Sosnitza for the many hours he spent in finding the optimal preparation of my samples.

After all I want to express my love and sincere gratefulness to my wife Mariele and my little daughter Franziska for their tolerance for late working hours and their love. They always stood beside me and without their support this work would not have been possible in this form.

Göttingen,

June 2004

Jens M. Walter 


\section{CHAPTER 1}

\section{INTRODUCTION}

This $\mathrm{PhD}$ thesis originates in the discovery of (subsurface) anomalies of high electrical conductivity in magnetotelluric profiles. These were measured by Ritter et al. (2003) and Weckmann et al. (2003) in the central Damara Belt of north-western Namibia. Questions about the correlation of potential electrical conductivities on sample scale with different fabric types of the graphite-bearing marble, led to the initiation of this work. Furthermore, precipitation and mobilisation mechanisms of graphite seem to have played an important role during the formation of the different fabrics. The work on this $\mathrm{PhD}$ thesis was accomplished at the Geowissenschaftliches Zentrum der Universität Göttingen (GZG).

\subsection{AIMS OF THIS WORK}

Previous works by Ritter et al. (2003) and Weckmann et al. (2003) show, that anomalies of high electrical conductivity correlate with graphite-bearing marbles. As these are also surface anomalies, one aim of the present work is to correlate different potentials of electrical conductivity on a sample scale with different types of marbles.

The different types of graphite-bearing marbles can be distinguished based on their fabrics and microfabrics. Consequently, another aim of this thesis is to characterise and to understand the formation of the associated fabric types. The marbles are located in the central parts of the Damara Orogen, so that their fabric development must be correlated with the regional tectono-metamorphic history of the area.

Graphite is the most important factor for the conductivity of marbles as well as to distinguish between the different types of fabrics. The third aim of this work is therefore to explore the conditions of formation, mobilisation and precipitation of graphite during the tectonic and metamorphic history of the graphite-bearing marbles. Consequently, the geological fieldwork was made primarily to understand the geological setting, as well as the structural, metamorphic and deformational history of the area.

\subsection{GeOLOGICAL SETTING}

The areas of investigation are located in the north-western Damara Belt in Namibia. The Damara Orogenesis forms a part of the neoproterozoic Pan-African orogenic belts (Fig. 1.1), which amalgamated Gondwana and eventually Pangea. The Damara Orogen is subdivided into the northern Kaoko Belt, the central Damara Belt, and the southern Gariep 


\section{Panafrican Mobile Belts}

\section{Legend}

Late Proterozoic mobile Belts

Paraguay-Araguaia Belt

(2) Sierras Pampeanas

(3) Ribeira Belt

(4) Northeastern Foldbelt

(5) Pharusian

(6) Mauritanides

(7) Rokelides

(8) Dahomeyan

(9) West Kongo Belt

(10) Damara Belt

(11) Kaoko Belt

(22) Katanga Belt

(13) Gariep Belt

(13) Malmesbury Belt

(16) Mozambique Belt

(1) Red Sea Fold Belt

(10) Transantarctic Belt

(1) Adelaidean

Fig 1.1 Panafrican Mobile Belt System with Neoproterozoic Orogenic Zones amalgamating Gondwana after Porada (1985).

Belt (Fig. 1.1 and 1.2). The Damara Belt developed during the north-westward subduction of the southern Kalahari Craton and the northern Congo Craton. Therefore, structures are trending parallel to a general strike of north-east to south-west. This is also reflected by the boundaries of the tectono-stratigraphic zones, postulated by Miller (1983) throughout the Damara Orogen (Fig. 1.2). The Kaoko Belt and Gariep Belt formed during the subsequent collision of the Kalahari and Congo Cratons with the Rio De La Plata Craton in Brazil.

The areas of investigation are situated within the Central Zone $(\mathrm{CZ})$ of the Damara Belt, which is shown in Figure 1.2. The $\mathrm{CZ}$ is characterised by the highest metamorphic grades within the Damara Belt, and the intrusion of syn- to post-tectonic granitic magmas. South of the $\mathrm{CZ}$, metamorphic grades increase up to sillimanite-in, whereas the $\mathrm{CZ}$ was dominated by partial melting during the peak metamorphism (Hartmann et al., 1983). The Okahandja Lineament (OL) in the south marks the boundary between the $\mathrm{CZ}$ and the Southern Zone (SZ).

It is postulated that the suture zone of the continental collision is located in the SZ (Kasch, 1983; Miller, 1983; Kukla, 1992). In these models, the CZ either includes a backarc, and further north a back-arc basin, or is a part of the active margin. In this case, it 


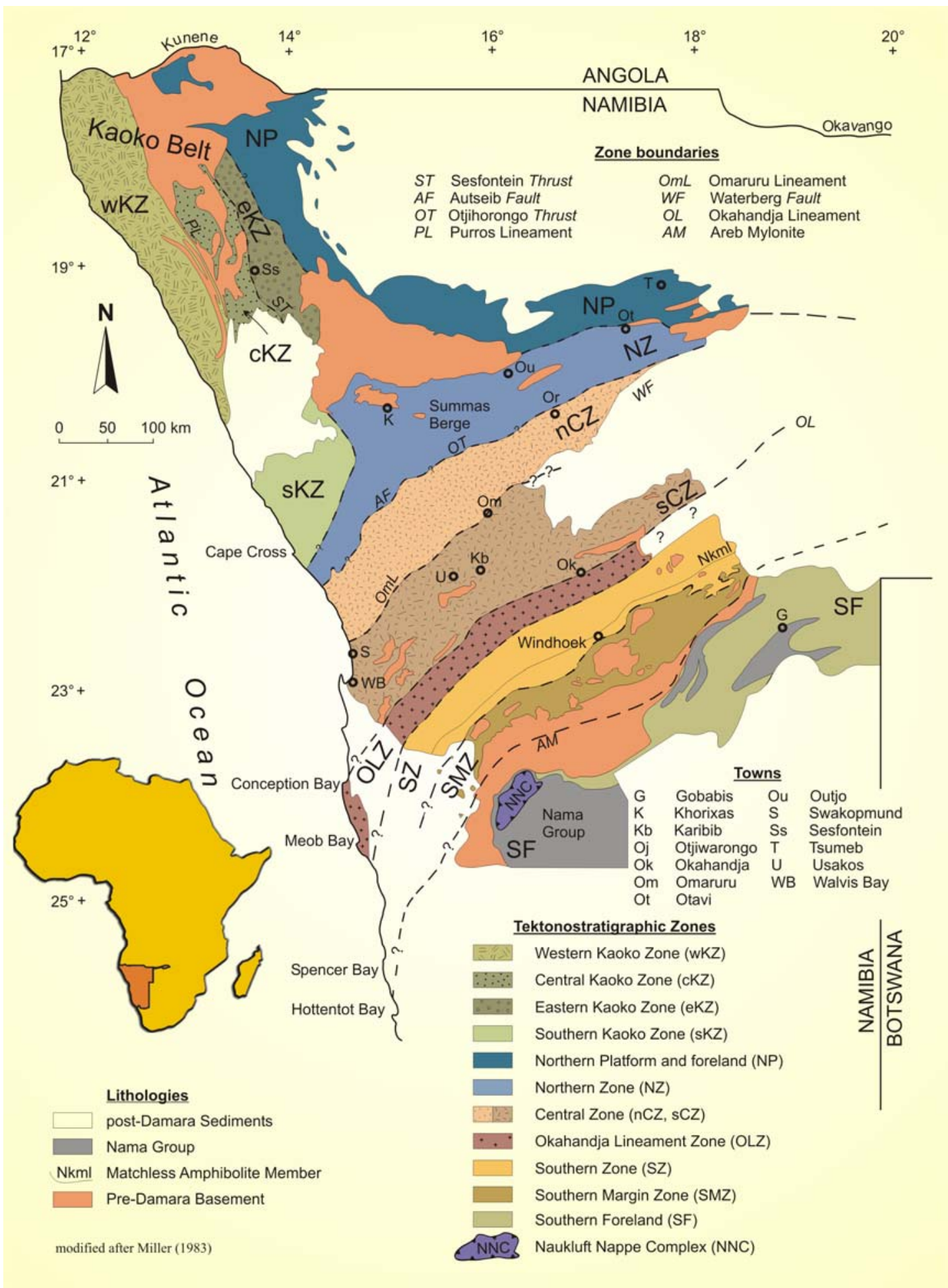

Fig 1.2 Tectonostratigraphic zones of the Kaoko Belt and Damara Belt after Miller (1983).

is assumed, that the $\mathrm{CZ}$ was subsequently stacked with the subducted Kalahari Craton. However, there is no clear geological evidence for a subduction zone in the Damara 
Orogen (Jung \& Mezger, 2003). The main reasons for this are a lack of eclogites, blueschists, a HP/LT regime and no igneous rocks with a clear subduction zone signature. Recently Jung \& Mezger (2003) argue, that the large volumes of granite in the CZ are the products of collisional orogenic processes. In general, the characteristics of the Damaran intrusives contrast with the Cordilleran-type plutonism, both in spatial distribution and the overall chemistry. However, similarities were found to the proportions and patterns of Himalayan plutons (Jung \& Mezger, 2003).

As shown in Fig. 1.2, the $\mathrm{CZ}$ itself is subdivided into the northern Central Zone $(\mathrm{nCZ})$ and the southern Central Zone $(\mathrm{sCZ})$ by the Omaruru Lineament $(\mathrm{OmL})$. The $\mathrm{nCZ}$ can be distinguished from the $\mathrm{sCZ}$, by its higher stratigraphic level (Miller, 1983). Crustalscale dome structures in the $\mathrm{nCZ}$, comprising complex refolded fold structures are mainly the effects of magmatic intrusion. Dome structures of the $\mathrm{sCZ}$, comprising mainly basement and lower stratigraphic cores, are much more elongated along the strike (Miller, 1983).

In the area of the magnetotelluric profile, the exact position of the OmL shear zone is still a matter of debate. Recent apatite fission track data of Raab et al. (2002) indicate that the $\mathrm{OmL}$ was reactivated after the Cretaceous break-up of Gondwana. According to this data, the OmL shear zone is located in the southern part of the magnetotelluric profile. This location of the OmL shear zone is also supported by (Weckmann et al., 2003).

\subsection{LOCATION AND DESCRIPTION OF THE FIELD AREAS}

The areas of investigation are situated in the $\mathrm{CZ}$ of the Damara Belt in northwestern Namibia. The magnetotelluric profile was measured in an area about 235 to 280 $\mathrm{km}$ north-west of the capital city Windhoek. The profile is located parallel to the road D 1930 west and north-west of the town of Usakos. The magnetotelluric profile was measured along this road from north to south, crosscutting the Damaran Autseib Fault and Omaruru Lineament (OmL), as postulated by Ritter et al. (2003) and Weckmann et al. (2003) (Fig. 3.1 a). The second area of investigation is situated approximately $90 \mathrm{~km}$ north-east of the first area, and locates on the road C 35 between Omaruru and Kalkfeld on the farm Epako Süd.

The vegetation and morphology of the area north-west of Usakos is mainly controlled by the climate of the Namib Desert. The Namib is one of the oldest deserts of the world, and is assumed to have developed 10 to $15 \mathrm{Ma}$ ago, because of the upwelling of the cold Benguela current. The desert extends parallel to the Namibian coast and about 80 to $150 \mathrm{~km}$ inland. The area of investigation is thus barren. A sparse bush savannah 
dominates with acacia tortilis (umbrella thorn), acacia karoo (sweet thorn), acacia reficiens (false umbrella thorn) and acacia erioloba (camelthorn tree), being the main species. After sporadic rainfalls, grass savannahs dominate the area with stipagrostis uniplumis (silky bushmen grass) as the main species. The average precipitation in this area ranges between 0 to $150 \mathrm{~mm}$ per year, with a rainfall variability of 70 to $80 \%$.

The second area of investigation, near the town of Omaruru has an annual rainfall of 100 to $350 \mathrm{~mm}$, with a rainfall variability of 40 to $50 \%$. Apart from mesozoic dykes, granite ring complexes and deeply cut river canyons, there are hardly any elevation differences in the field areas. Some graphite-bearing carbonates however, crop out in morphological elevations. The mean elevation along the southern part of the magnetotelluric profile is around $950 \mathrm{~m}$ above sea level, whereas it is around $1340 \mathrm{~m}$ above sea level at the farm Epako Süd.

The area along the magnetotelluric profile was commercially farmed after the Second World War, primarily by white famers. This farming was generally not very successful because of erratic and sparse rainfall. Since Namibia was formerly under the administration of the Apartheidsregime in the Republic of South Africa, this area became expropriated and made a part of the homeland of the Damaran people. They took over the farms for substantial farming. Traditionally the Damaran people do not own ground privately, and normally they are very friendly. Access to the outcrops is thus normally easy. The area between Omaruru and Kalkfeld however, is still farmed commercially, so that permission is needed to access that part of the field areas.

In the field areas the vegetation is normally very sparse. The fluvial and calcrete sediments are also thin or absent. Therefore, the outcrops are generally good and many large structures can easily be followed over long distances with the help of aerial photographs and satellite images. 


\section{CHAPTER 2}

\section{STRUCTURAL AND TeXTURAL ANALYSIS}

Detailed field investigations show that the graphite-bearing marbles have a complex deformational and metamorphic history. A first step to reveal the tectono-metamorphic evolution is therefore the microstructural and textural analysis regarding the field relationships and deformational structures. Textural analysis includes bulk and single-grain crystallographic orientation studies of the calcite-marbles. The structural and textural analysis forms the basis for a classification of the graphite-bearing marbles for further investigations of the graphite-bearing marbles.

\subsection{REGIONAL DEFORMATION AND STRUCTURES}

The sampling sites are located in the main magmatic province of the $\mathrm{nCZ}$ in the Damara Belt (Fig. 2.1). As shown in Figure 2.1, the plutonic rocks of the $\mathrm{CZ}$ consist mainly of monzogranite with minor granodiorite. Other intrusives are syenogranite, syenite and diorite. The sampling sites show that the graphite-bearing marbles are situated at the contact to these intrusions.

The satellite image of the southern part of the magnetotelluric profile in Figure 2.2 shows that the general geological structures are composed of a sequence of dome structures (D). Furthermore, a variety of magmatic intrusions are observable in the satellite image (I). The mesozoic dykes (MD) result from the Cretaceous break-up of Africa and South America.

It follows from Figure 2.2 that two kinds of dome structures have developed in this area. One type has developed by refolding large scale mostly isoclinal D1 folds into heteroaxial folds. This refolding event may either have developed within a transtensional deformation regime or during a second phase of deformation (D2). These dome structures are mainly developed in the north-western part of the displayed area. A compilation of models for the formation of structural dome structures is found in Soula et al. (2001).

The second type of dome structures formed during the post D1 intrusion of granitic melt into the large scale, mostly isoclinal fold structures. The dome structures comprise mica schists, quartzites, and granitic intrusions next to calcite and calc-silicate marbles which commonly include graphite. The granite intrusions also form dome structures with marble, quarzite and mica shist along their rims. 


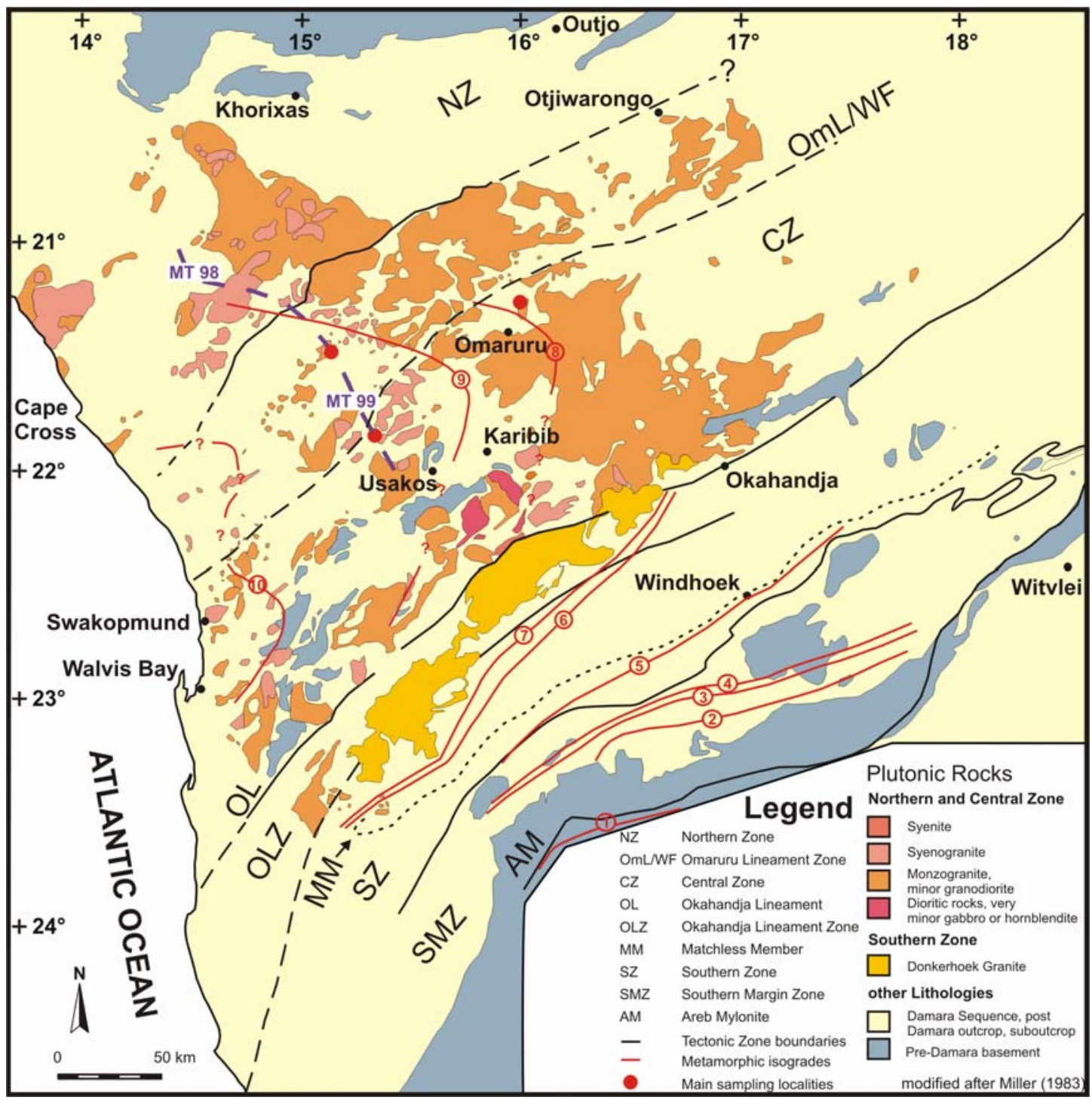

Fig 2.1 Distribution of Damaran intrusives within the Damara Belt after Miller (1983). The map also shows the course of the magnetotelluric profiles measured by Ritter et al. (2003) and Weckmann et al. (2003). The metamorphic isogrades throughout the Damara Belt increase after Hartmann et al. (1983) in metamorphic grade from south to north and within the CZ from east to west. The metamorphic isogrades are characterised as follows: 1: biotite-in; 2: garnet-in; 3: staurolite-in; 4: kyanite-in; 5: cordierite-in; 6: andalusite $\leftrightarrow$ sillimanite; 7: sillimanite-in due to the breakdown of staurolite; 8: partial melting due to: muscovite + plagioclase + quartz $+\mathrm{H}_{2} \mathrm{O} \leftrightarrow$ melt + sillimanite; 9: $\mathrm{K}$-feldspar-and cordierite-in; 10: partial melting due to: biotite $+K$-feldspar + plagioclase + quartz + cordierite $\leftrightarrow$ melt + garnet.

It is obvious from the field investigations that a regional phase of static recrystallisation postdates the formation of the dome structures. This resulted in the abnormal grain-coarsening of large parts of the calcite-marbles. This triggered a comprehensive blastesis of the calcite-marble resulting in an abnormal grain growth of 


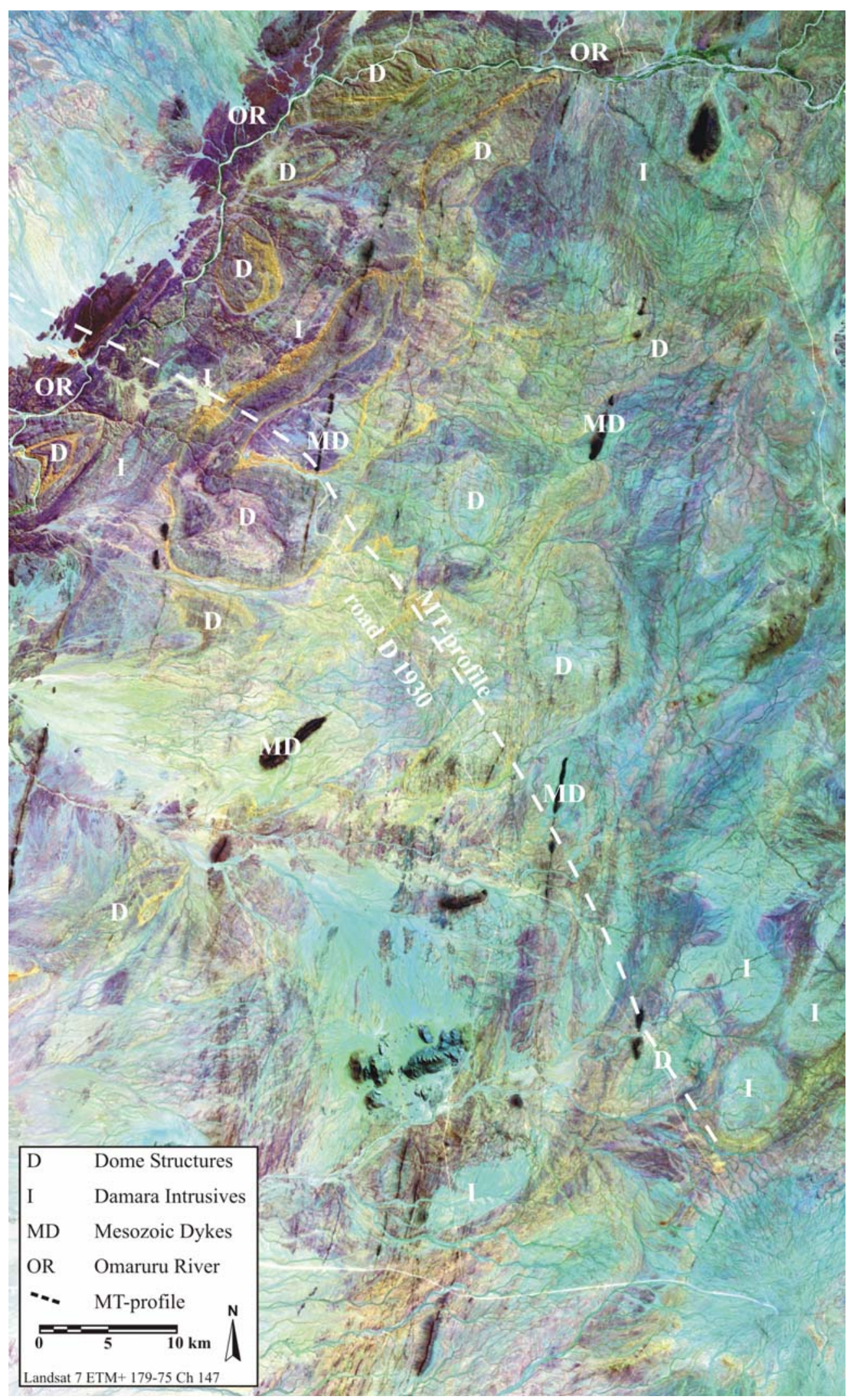

Fig 2.2 Landsat satellite image of the area of the southern part of the magnetotelluric profile. 
rhombohedral calcite crystals of up to decimeter in diameter in size (Fig. 2.3 a, b). In a regional context, this abnormal grain growth in the calcite-marble occurs only within the magmatic province of the northern Central Zone. This observation is in accordance to Covey \& Rutter (1989) who observed isolines of grain coarsening around a magmatic intrusion on Naxos.

Figure 2.1 also displays the generell pattern of the metamorphic isogrades of the Damara Belt after Hartmann et al. (1983). They increase in metamorphic grade from the SMZ and SZ to the CZ across the Damara Belt and within the CZ from east to west. As described above, the $\mathrm{CZ}$ is characterised by partial melting during peak metamorphism (Hartmann et al. 1983). Depending on the amount of crustal fluids, a amphibolite- to granulite facies metamorphic grade has dominated the central and western parts of the $\mathrm{CZ}$ (Jung et al., 2000a).

The regional peak metamorphism of the central and western $\mathrm{CZ}$ dates at about 540 to 520 Ma (Kröner, 1982; Haack \& Martin, 1983; Steven et al., 1993; Jung et al., 2000a; Jung et al., 2000b; Jung \& Mezger, 2003). Jung \& Mezger (2003) suggested that the growth of metamorphic garnet ceased at about $510 \mathrm{Ma}$ in the western $\mathrm{CZ}$ at temperatures of about 600 to $700^{\circ} \mathrm{C}$. The granitic intrusions in the Central Zone are dated between 563 Ma (Hawkesworth et al., 1981; Jacob et al. 2000) and Ar/Ar biotite and hornblende cooling ages of $461 \pm 9$ and $465 \pm 1$ Ma (Tack \& Bowden, 1999).

The main phase of intrusions started with the intrusion of the Salem granites with $\mathrm{Rb} / \mathrm{Sr}$ intrusion ages of about $550 \mathrm{Ma}$ (Haack et al., 1983; Miller, 1983) and whole rock $\mathrm{Rb} / \mathrm{Sr}$ isochron ages of $563 \pm 63 \mathrm{Ma}$ (Hawkesworth et al., 1981). Recent SHRIMP dating by Jacob et al. (2000) give intrusion ages in the area of Karibib of $563 \pm 4$ to $539 \pm 6 \mathrm{Ma}$ and a later phase of intrusions at $500 \pm 10$ to $494 \pm 8$ Ma. Marlow (1983), Miller (1983) and Haack \& Gohn (1988) published $\mathrm{Rb} / \mathrm{Sr}$ intrusion ages ranging between 530 and 460 $\mathrm{Ma}$, which coincide with $\mathrm{Rb} / \mathrm{Sr}$ datings of 512 to $468 \mathrm{Ma}$ by Steven et al. (1993). West of Omaruru $\mathrm{Rb} / \mathrm{Sr}$ ages of $507 \pm 44$ and $510 \pm 48 \mathrm{Ma}$ are reported by Jung et al. (1998), whereas $\mathrm{Pb} / \mathrm{Pb}$ ages give slightly higher intrusion ages of 521 to $528 \mathrm{Ma}$ and 496 to 490 Ma, respectively.

The extend of the abnormal grain growth and blastesis of the calcite-marble is therefore related to the combination of an amphibolite- to granulite-facies grade metamorphism and the intrusion of granitic melts. Figure $2.3 \mathrm{c}$ shows the apophysic granite intrusions, which developed during the intrusion of highly mobile melts into the graphite-bearing calcite-marble in a high-temperature metamorphic regime. The granitic 

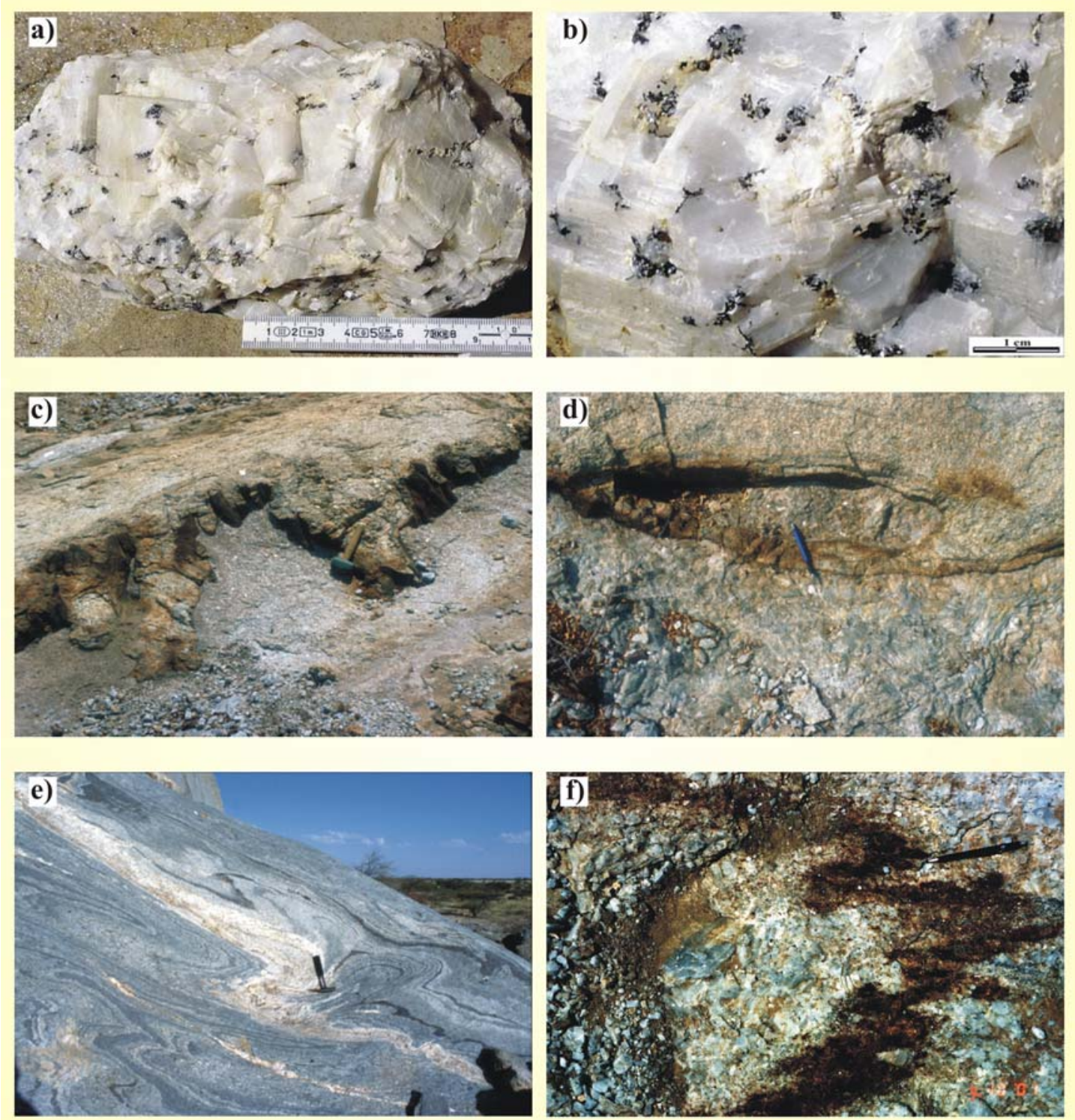

Fig 2.3 Fabrics of the graphite-bearing marble and the granitic intrusions. a) Abnormally grain-coarsened blastic calcite-marble with disseminated graphite nests. b) Rhombohedral calcite blasts with disseminated graphite nests. c) Apophysic intrusion of highly mobile granitic melts into the calcite-marble. d) Xenolith of calcite-marble in a granitic body. e) Intensively folded impure marbles which show no grain coarsening effects. The calc-silicate layer is more competent than the marble and therefore affects the development of fold structures in the marble. The fold axis mainly dip to NE. f) Folded calc-silicate layer embedded in graincoarsened calcite marble. The deformation of the calc-silicate layer predates the blastic abnormal graincoarsening by static recrystallisation.

intrusions display abnormal grain coarsening within calcite-marble xenoliths (Fig. $2.3 \mathrm{~d}$ ). They also show a blastic fabric of calcite rhombohedral crystals. Extensive parts of this marble consist of pure grain coarsened calcite-marbles with graphite occuring in disseminated nests as shown in Figure 2.3 a, b. 
Parts of this marble rich in impurities do not show any grain-coarsening fabric and often include calc-silicates. These domains of the marble are characterised by intensive folding (Fig. 2.3 e), which result from a complex deformation (Klein, 1980). This deformation is probably related to the development of the dome structures. This is also documented by folded calc-silicate layers which are imbedded in grain-coarsened calcitemarble (Fig. $2.3 \mathrm{f}$ ).

On the satellite image in Figure 2.2, the calcite-marbles appear within the dome structures in yellowish colours. Where they occur along the outer rims of the dome structures, these calcite-marbles were subsequently mylonitically and cataclastically deformed. These zones of brittle and ductile deformation developed in a preferred orientation parallel to the general north-east to south-west strike of the Damaran structures. They appear in a 3 to $10 \mathrm{~m}$ broad shear zone, including a distinct mylonitic core with adjacent zones of brittle and ductile deformation which are mutually overprinted.

The mylonite core zones are up to $1 \mathrm{~m}$ in width and are characterised by the concentration of graphite parallel to the mylonitic foliation (Fig. 2.4 a). The adjacent domains of brittleductile deformation did not overprint the distinct mylonitic core zones and are characterised by a variety of deformation fabrics (Fig. $2.4 \mathrm{~b}$ to h). Figure $2.4 \mathrm{~b}$, c show the development of graphitic stylolites from single disseminated graphite nests of the graincoarsened host rock. During deformation the graphite nests became deformed and graphite was reduced in grain size. In addition, graphite was enriched along interconnecting stylolite seams during progressive pressure solution. These graphitic stylolites commonly developed a high angle orientation between individual stylolites. The degree of network intensity of the graphitic stylolites varies within this zone as shown in Figure $2.4 \mathrm{~b}$ to $\mathrm{f}$.

According to their orientation with respect to the mylonitic core zone, some of the stylolites develop into ductile shear bands as shown in Figure $2.4 \mathrm{~d}$ to $\mathrm{f}$. With increasing stylolite network intensity, the amount of ductile shear bands developed from the graphitic stylolites increases as also shown in Figure $2.4 \mathrm{~d}$ to $\mathrm{f}$. Figure $2.4 \mathrm{f}$ shows that one set of these ductile shear bands are developed as subsidiary synthetic Riedel shear bands.

Cataclastic breccia zones often overprint the graphitic network structures (Fig. $2.4 \mathrm{~g}$ ). The calcite breccia occurs macroscopically in a very fine to ultra fine-grained matrix of calcite (Fig. $2.4 \mathrm{~h}$ ). These different fabrics of brittle-ductile deformation occur next to the mylonitic core zone in domains of different deformation characteristics. The occurrence of these different deformational domains alternate within the brittle-ductile boundary zones lateral and perpendicular to the mylonitic core zone in an irregular complex pattern. Therefore, the degree of network intensity varies extensively along the strike of the shear 

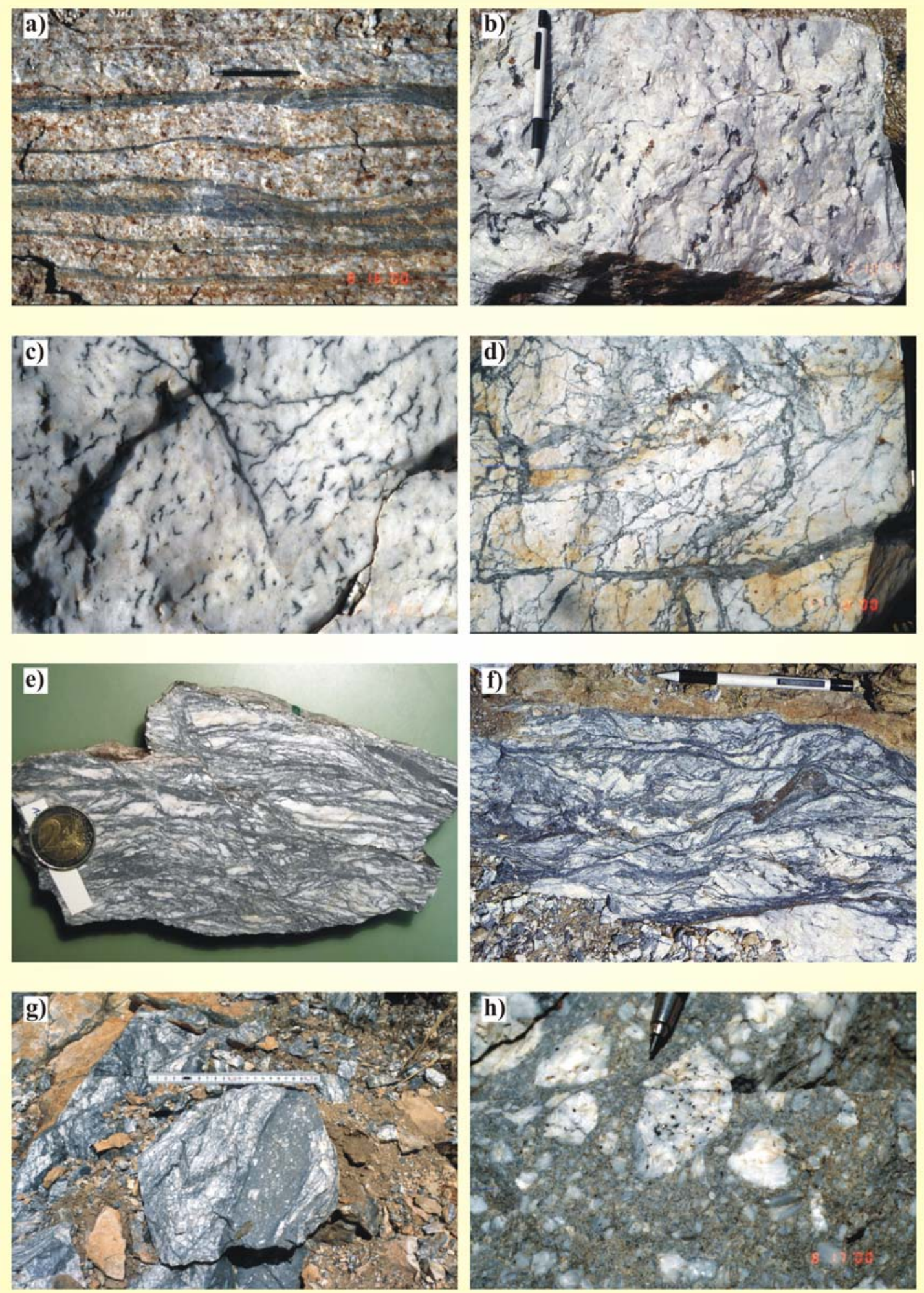

Fig 2.4 Fabrics of the mylonitically and brittle-ductile deformed graphite-bearing marble. a) Mylonite of the mylonitic core zones with graphite concentrated parallel to the foliation. b) Deformed graphite nests and short stylolitic seams developed from the graphite nests $\boldsymbol{c}$ ) Increased pressure solution and brittle-ductile 
deformation resulted in graphitic stylolites which connected to form the graphite network structures. d) Graphite network structures with higher network intensities and some graphite stylolites already developed into ductile shear bands. e) Very high graphite network intensity with a close band of ductile shear bands developed in preferred orientation. f) High network intensity with a set of conjugated ductile shear bands with the development of synthetic Riedel shears. $\mathrm{g}$ ) Cataclastic vein developed in domains within the graphite network structures. h) Cataclastic breccia in a matrix of ultra fine-grained calcite-marble.

zones. The cataclastic breccia zones also occur in irregular intervals along the shear zones. They are restricted to the brittle-ductile domains since they do not overprint the mylonitic core zones.

These shear zones are of Damaran age, as the mesozoic dykes resulting from the cretaceous break-up of Gondwana are not affected by the deformation of the investigated shear zones. Fieldwork revealed, that the mesozoic dykes intrude the graphite network structures independent of the deformational fabric of the shear zones. Therefore, these shear zones may not be related to the shear zones and structural lineaments that have been postulated as reactivated shear zones after the break-up of Gondwana as described by Raab et al. (2002).

\subsection{CHARACTERISATION OF THE DIFFERENT TYPES OF MARBLES}

Within the range of the described shear zones along the rims of the dome structures three types of graphite-bearing marbles can generally be distinguished macroscopically. The first type is characterised by the blastic, abnormally grain-coarsened marble, which is the host rock of the described shear zones. Type 2 and type 3 represent the brittle-ductile deformed marble and the mylonitically deformed marble respectively.

\section{Type 1:}

This type is the marble host rock adjacent to the shear zones. This marble host rock is composed of domains of pure calcite-marble with disseminated nests of graphite. Other domains additionally contain phases like calc silicates, phyllosilicates, dolomite and quartz. The domains of nearly pure calcite-marble are abnormally grain-coarsened to a large extent, and generally characterised by a blastic fabric of rhombohedral calcite crystals (Fig. $2.3 \mathrm{a}, \mathrm{b}$ ). The size of the calcite crystals are from 0,5 to about a decimeter in diameter. Graphite occurs in single graphite nests disseminated throughout these marbles (Fig. $2.3 \mathrm{~b}$ ). In this type of graphite-bearing marble the graphite crystals and flakes are mostly well crystallised and of up to $2 \mathrm{~mm}$ in size.

The other domains of graphite-bearing marble host rock containing calc-silicates, phyllosilicates, dolomite and quartz show grain coarsening fabrics to a much less extent. In 
some domains they are not affected by grain growth. These marbles still show prior deformation structures like extensive folding and often contain layers of calc-silicates. In this study, the marble host rock is referred to as the abnormally grain coarsened calcitemarble because this is the dominating type within the marble host rock.

\section{Type 2:}

The second type of graphite-bearing marble occurs in the boundary zone of brittleductile deformation within the investigated shear zones. In this zone network structures of graphitic stylolites, single ductile shear bands and cataclastic breccia are observable (Fig. $2.4 \mathrm{~b}$ to $\mathrm{h}$ ). The thickness of the graphitic stylolites and shear bands, as well as the graphite network intensity varies quite extensively. From the investigation of the macroscopic structures shown in Figure $2.4 \mathrm{~b}$ to $\mathrm{h}$, it is obvious that the deformational structures are mutually overprinted.

Pressure solution resulting in the formation of graphitic stylolites occurred during the brittle-ductile deformation and is restricted to this domain of the shear zone. The stylolites themselves are again overprinted by ductile shearing and cataclastic deformation. In general, a grain size reduction of the calcite-marble and the graphite is associated with the brittle-ductile deformation. Graphite is generally ultra fine-grained in the stylolites as well as in the cataclastic zones and ductile shear bands.

\section{Type 3:}

The third type also occurs in the mylonitic core zones of the shear zones and is characterised by pure ductile deformation (Fig. 2.4 a). This mylonitic core zone is up to 1 $\mathrm{m}$ wide and is unaffected by the brittle deformation of the adjacent boundary zone of brittle-ductile deformation. This mylonitic core zone is continuously existent within the investigated shear zones, whereas the boundary zone of brittle-ductile deformation varies lateraly along the shear zone. As shown in Figure 2.4 a, graphite is concentrated parallel to the mylonitic foliation.

\subsection{MicrostruCtures}

The microstructures will be investigated according to the macroscopic classification of the graphite-bearing marbles by the three different types. The classification of the microstructures has been performed by qualitative optical microscopy. The microstructural investigation will be the basis for the general interpretation of the progression of the deformational processes within the macroscopically differentiated domains of deformation. Additionally the microstructural analysis should reveal some information about the 
abnormally grain-coarsened host rock, with respect to the graphite formation within the disseminated nests.

\subsubsection{COARSE-GRAINED MARBLE HOST ROCK}

The microstructures of the calcite-marble host rock are characterised by the blastic fabric of the abnormally grain coarsened calcite marble. As shown in Figure 2.5 a to d, two sets of calcite twins are developed in the rhombohedral calcite crystals. These twin lamellae intersect each other at an angle of $80^{\circ}$. At the intersection between the twin lamellae, the elder generation of calcite twins are rotated and dislocated by the younger generation (Fig. 2.5 a). Likewise, the twin lamellae are fractured and slightly dislocated by the the development of the rhombohedral plains (Fig. 2.5 b).

These sets of twins are recrystallised in parts again by the host crystal and therefore are partially extinct (Fig. $2.5 \mathrm{c}$ ). Figure $2.5 \mathrm{~d}$ shows, that the twin lamellae are recrystallised in lobate and bulging fronts of recrystallisation. The twins are recrystallised in the orientation of the host crystal at a late stage during the abnormal grain growth and static recrystallisation.

The rhombohedral calcite crystals show inclusions of graphite, muscovite and quartz as accessory minerals. Graphite, muscovite and quartz had been overgrown by statically recrystallising calcite during abnormal grain-coarsening and are incorporated in the calcite crystals. Graphite occurs in graphite nests which are disseminated throughout the calcite crystals (Fig. 2.5 e, f). In general, the graphite flakes in the nests are oriented as shown in Figure 2.5 e. The graphite crystals in the nests are locally oriented radial forming spherical structures (Fig. 2.5 f). Some of these radial graphite nests have a single mineral grain in the centre of the radial graphite structure.

As shown in Figure $2.5 \mathrm{~g}$, $\mathrm{h}$, graphite and muscovite are partially epitaxially intergrown on their basal plains. Figure $2.5 \mathrm{~h}$ shows that the growth of graphite is also to some extent controlled by the two sets of twins developed in the calcite host crystal. Additionally, in the investigated rhombohedral calcite crystals no fluid inclusions are observable.

Nevertheless, fluid inclusions within more impure and less grain-coarsened grey marbles are reported in the same area of the dome structures (Gross et al., 1999). However, the fluid inclusions occur as secondary fluid inclusions trails and are probably of cretaceous age. These grey marbles occur in small lenses of some meters within the abnormally grain-coarsened marble. 

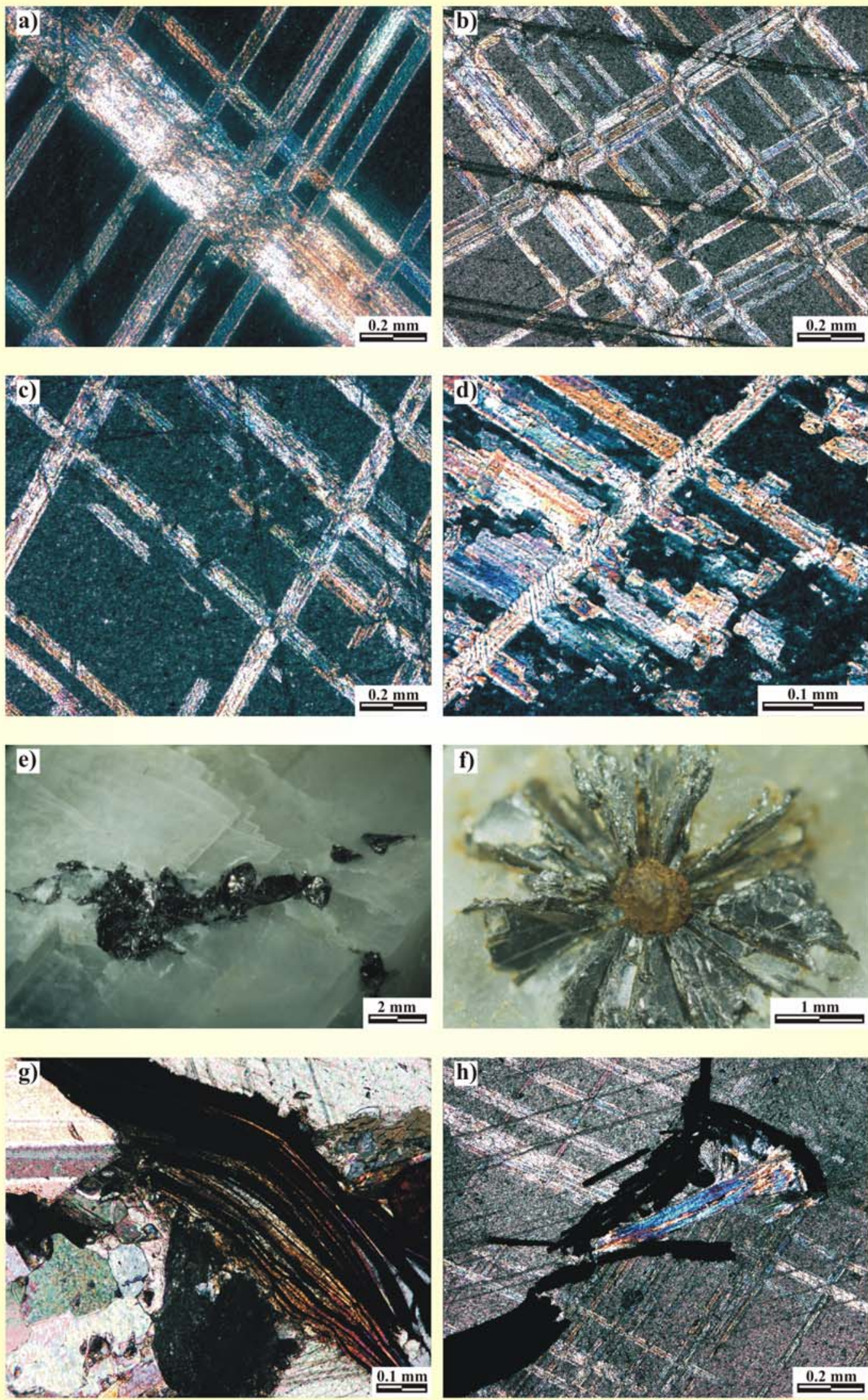
Fig 2.5 Microstructures of the undeformed grain-coarsened host rock. Figures a to $d$ and $g$ to $h$ are microphotographs in crossed polarised light, whereas Figures $e$ and $f$ are microphotographs in reflected light. a) Two sets of intersecting calcite twins. The younger set of twins rotated and dislocated the older twins at the points of intersection (sample Elim Sp). b) The rhombohedral plains fractured and slightly dislocated the twin lamellae (sample Elim Sp). c) Twin lamellae have been recrystallised by the host crystal and therefore are partially extinct (sample Elim Sp). d) Twin lamellae that have been partially recrystallised by interlobate fronts of recrystallisation (sample Elim Sp). e) Graphite nest with graphite flakes as they occur commonly throughout the grain-coarsened graphite-bearing marble (sample Elim Sp). f) Nest of radial graphite with mineral core in the center occuring in the grain-coarsened calcite-marble (sample Omaruru Sp). g) Epitaxial intergrown basal plains of graphite and muscovite (sample 13.8.97/3A from C. Gross Univ. Göttingen). h) Epitaxial intergrown graphite and muscovite. (sample Elim Sp).

\subsubsection{BRITTLE-DUCTILE DEFORMED MARBLE}

The calcite-marble of the brittle-ductile domains shows a variety of microstructures in the boundary zone of brittle-ductile deformation ranging from brittle to crystal-plastic deformation structures. The microstructural investigations revealed that despite pronounced differences in the macroscopic fabrics shown above, the microstructures of the different fabrics are very similar. The cataclastic breccia zones are exceptional in some respects, as the cataclastic microfabric dominates in these samples.

Figures 2.6 a to $\mathrm{d}$ display a general overview of the microstructures representative for the different macroscopic fabrics shown in Figure $2.4 \mathrm{~b}$ to $\mathrm{h}$. Therefore, they are arranged in an order of increasing graphite network intensity from Figure $2.6 \mathrm{a}$ to c. Figure $2.6 \mathrm{~d}$ represents the microfabrics of a cataclastic breccia zone. The fabrics and microfabrics described are identical for all investigated localities shown in Figure 2.1.

Figure 2.6 a shows an overview of the microstructures of a sample with a low degree of network intensity of graphitic stylolites. The macroscopic fabric is similar to the fabrics shown in Figure $2.4 \mathrm{~b}$ and c. The grain size of the calcite crystals ranges between 5 to $10 \mathrm{~mm}$ for the host crystals and between 50 to $200 \mu \mathrm{m}$ for the calcite grains in the fracture zones. The orientations of the host crystals are still similar, which implies that these result from one single calcite crystal as observable in the abnormally grain-coarsened and undeformed marble host rock. The fracture zones show, that this sample had already been deformed.

The microstructures of a sample with an intermediate degree of graphite network intensity are shown in Figure $2.6 \mathrm{~b}$, which applies to the macroscopic fabric shown in Figure $2.4 \mathrm{~d}$. It is obvious, that the grain size is generally reduced with an increasing degree of graphite network intensity. The calcite host crystals are reduced to grain sizes of 

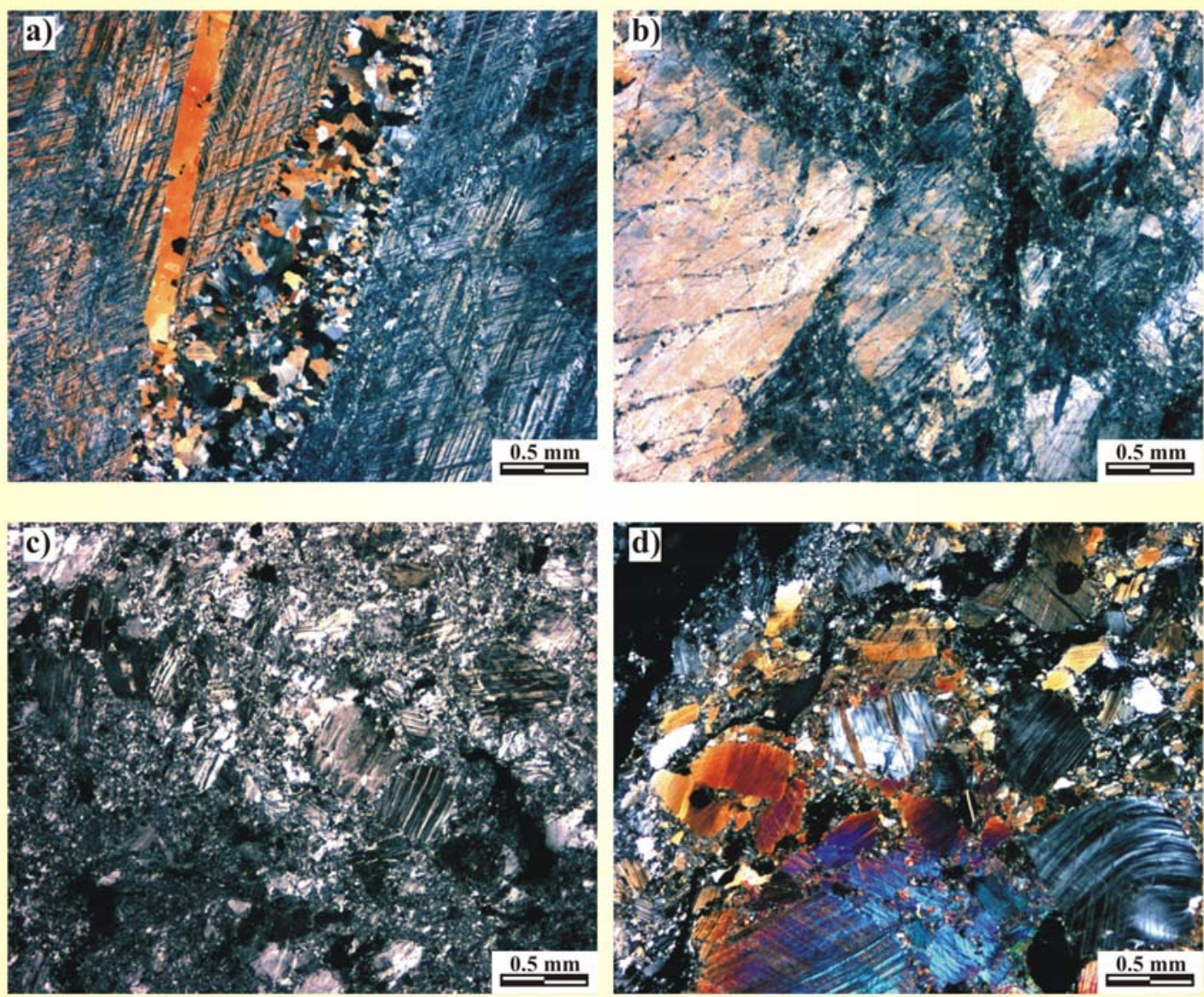

Fig 2.6 General overview of brittle-ductile deformed marbles. The samples increase in network intensity from a to c. Figure d represents purely cataclastic deformation. All figures represent microphotographs in crossed polarised light. a) Microstructures of a sample with a low degree of network intensity of graphitic stylolites. The macroscopic fabric is similar to the macroscopic fabric shown shown in Figure 2.4 b,c (sample 17/08/00/15). b) Microstructures of a sample with an intermediate degree of graphite network intensity as shown in Figure $2.4 d$ (sample 17/08/00/19). c) Overview of the microstructures of a sample with a high degree of graphite network intensity. The macroscopic fabric is similar to the fabric shown in Figure 2.4 e, $f$ (sample 17/08/00/17). d) Microstructures of a cataclastic breccia zone as shown in Figure $2.4 \mathrm{~g}, \mathrm{~h}$ (sample17/08/00/16)

about 0.5 to $3 \mathrm{~mm}$ and in the matrix to about 10 to $100 \mu \mathrm{m}$. The orientation of these calcite host crystals varies already to a greater extent than in Figure 2.6 a.

Figure $2.6 \mathrm{c}$ shows microstructures of samples with a high degree of graphite network intensity. The macroscopic fabric is equivalent to the fabric shown in Fig. 2.4 e, f. It is obvious that the grain sizes of the calcite host crystals and the matrix are reduced to a great extend compared to Figure $2.6 \mathrm{a}$, b. The grain size of the calcite host crystals are reduced to 0.1 to $0.5 \mathrm{~mm}$ and the grain size of the matrix ranges from 10 to about $100 \mu \mathrm{m}$. 
Additionally, similarities in orientation between the host crystals as obvious in Figure 2.6 a are not observable anymore.

The microstructures of the cataclastic breccia zone show a different distribution of grain sizes. The macro- and microscopic fabrics give an impression, that the distribution of grain sizes is scale invariant as it is the similar in every dimension. This excludes the ultra fine-grained matrix, which shows recrystallisation fabrics. According to the microstructures in Figure $2.6 \mathrm{c}$ there is a great variety in crystallographic orientation of the bigger calcite clasts compared to the fabric of the only slightly deformed sample shown in Figure 2.6 a.

The microstructurs of these samples show a strong cataclastic deformation, wheras the other samples of the brittle-ductile boundary zones rather show fine-grained inter- and intracrystalline fracture-zones. In these fracture zones the graphitic stylolites have developed. Locally an overprinting of the cataclastic microfabric by dynamic and static recrystallisation is observable in these samples.

From Figure $2.6 \mathrm{a}$ to $\mathrm{c}$ it is obvious that the only difference in microstructures is the distribution of grain sizes, which can be correlated directly to the macroscopic fabrics. Therefore, the microstructures will be described independently of the degree of graphite network intensity. Nevertheless, the microstructures of the graphite network fabrics are distinguished from the cataclastic breccia zones.

Nearly all calcite grains show intensive twinning, often in two sets of twin lamellae which intersect each other. Figure 2.7 a shows two sets of twins are developed, whereas the older set of twins is often overprinted by intensive twinning of the second set of twins. The twin lamellae vary quite extensive in thickness within one set of twins. The twin lamellae are often bent or kinked (Fig. 2.7 b). As shown in this Figure, the degree of bending and kinking depends on the thickness of the twins. Thick twins are folded whereas thinner twins are already kinked.

Figure $2.7 \mathrm{c}$ shows, that kink bands are sometimes strongly developed with a great variety of thickness. In some samples, higher metamorphic temperatures are indicated by a kink bands that are irrregular in shape, thickness and orientation (Fig. $2.7 \mathrm{~d}$ ). It is obvious in all samples, that the kink bands develop also from deformation bands shown in Figure 2.7 e. The deformation bands are often developed in irregular shapes. In other cases, the deformation bands are in regular shape and show a preferred orientation within distinct domains of a grain (Fig. 2.7 f). 

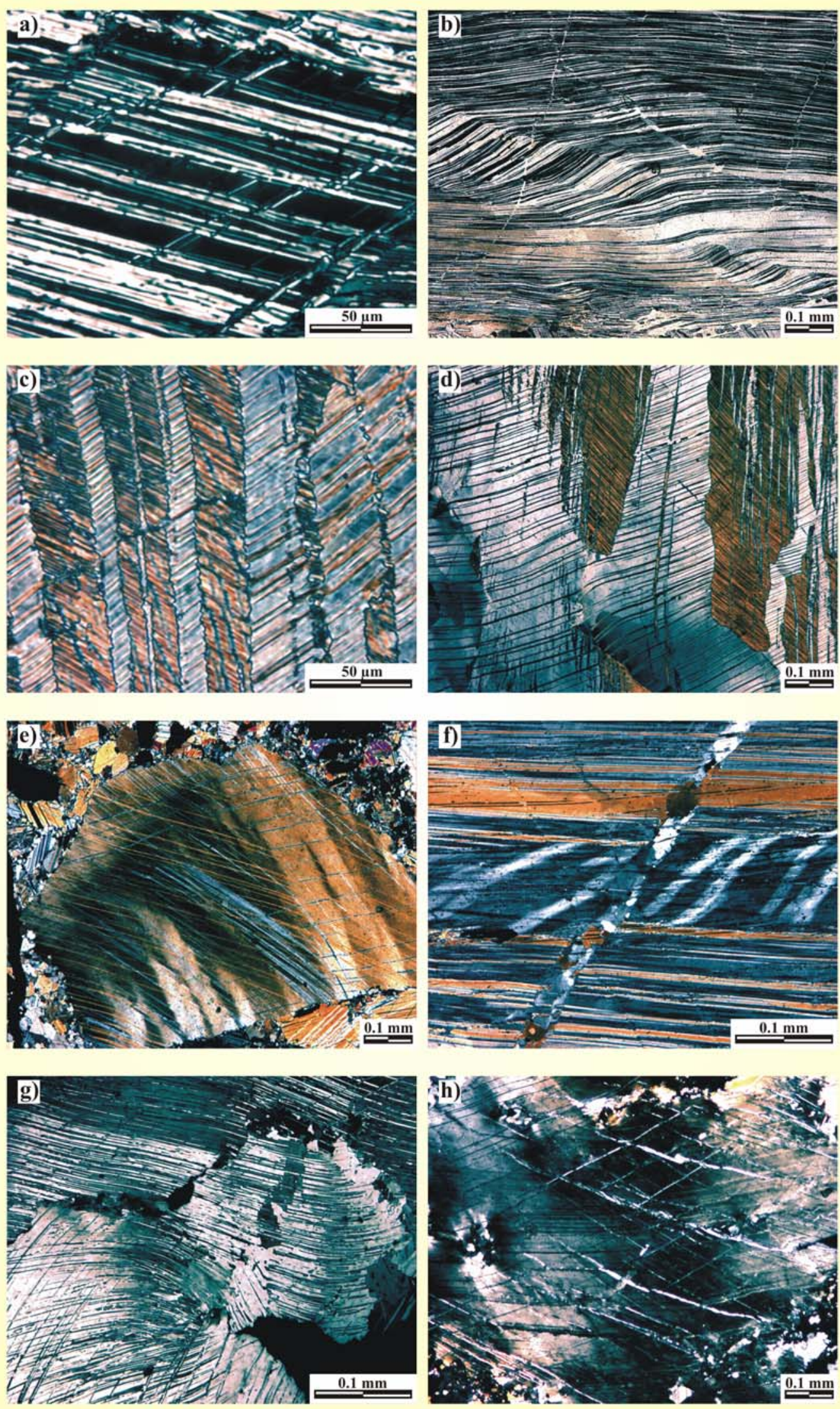

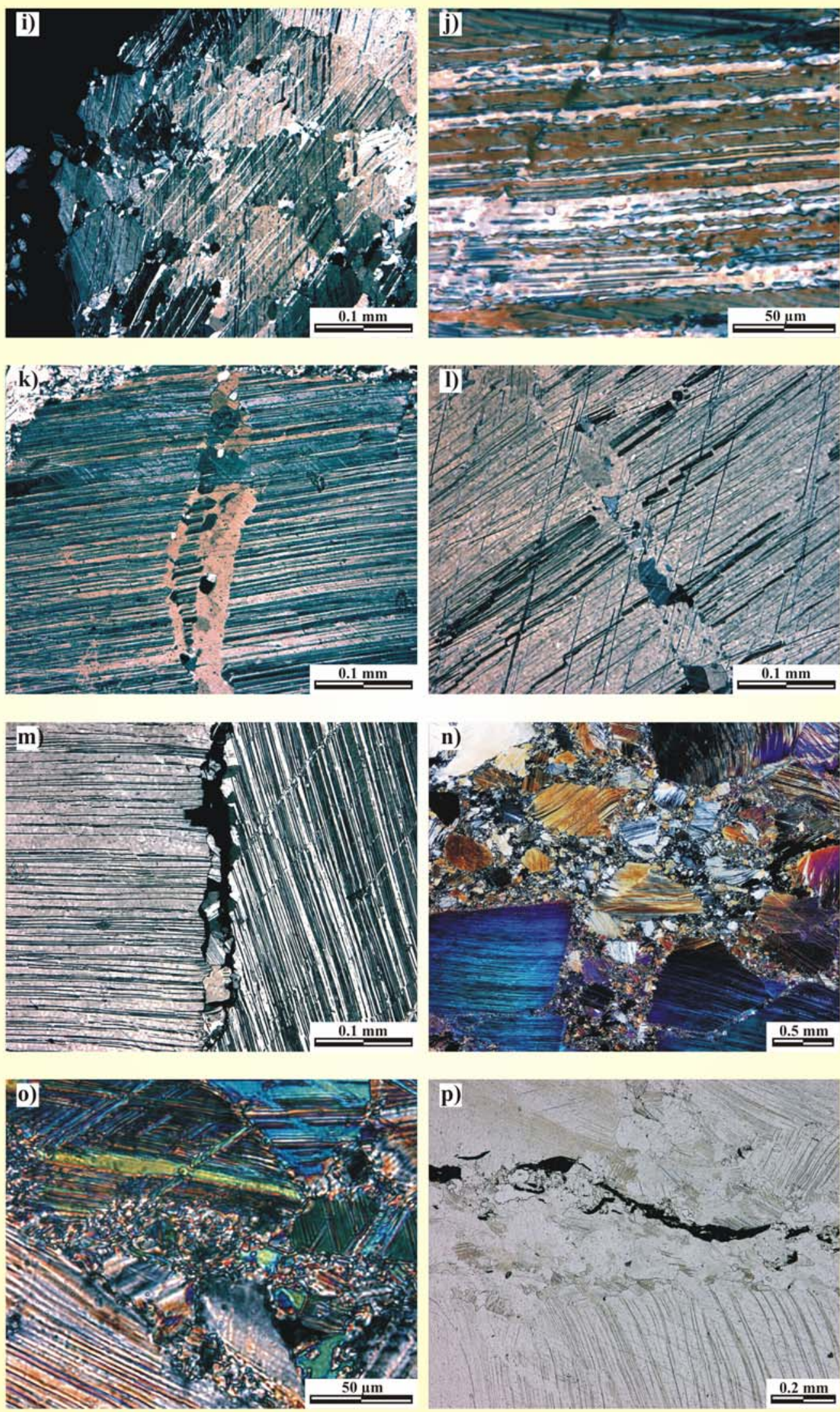
Fig 2.7 Microstructures of the brittle-ductile deformed marbles. Figures a to o represent microphotographs in crossed polarised light, whereas Figure $p$ is a microphotograph in plain polarised light. a) Intensive twinning of the calcite crystals with an extensive difference in the thickness of the twins. Often two sets of twins are developed, where the first set of twins has been overprinted by the younger genereation of twins (sample 17/08/00/19). b) Calcite twins are bent or kinked depending on their thickness (sample 3.10.01/3). c) Intensive kinking of the calcite twins (sample 17/08/00/15). d) Kink bands at higher metamorphic temperatures (sample 3.10.01/3). e) Deformation bands in varying extent are developed in the calcite host crystals (sample 17/08/00/16). f) Deformation bands developed in distinct parts of the grain which are free from intensive twinning (sample 3.10.01/3). g) Flexural slip in the calcite crystals with a thickening of the flexured twins (sample 17/08/00/16). h) Undulous extinction due to polygonisation within the calcite crystals (sample 17/08/00/16). i) Subgrain rotation recrystallisation of the calcite crystals (sample 17/08/00/16). j) Ultra fine-grained recrystallisation along the twins of a calcite crystal (sample 3.10.01/3). k) Intracrystalline multiple crack-seal vein filled mainly with breccia of the host crystal (sample 17/08/00/16). I) Intracrystalline crack-seal vein filled with breccia of different orientation (sample 17/08/00/16). m) Open fracture of a younger generation filled with fine-grained breccia (sample 17/08/00/16). n) Purely cataclastic microfabric of a breccia zone, which shows the same microstructures than the brittle-ductile deformed samples (sample 17/08/00/16). o) The purely cataclastic microstructures are scale invariant (sample 17/08/00/16). p) Graphite stylolites are developed in fine-grained fracture zones with ultra fine-grained graphite as residue of the pressure solution (sample 3.10.01/3).

Another deformation structure observed is flexural slip within the crystal lattice, which leads to a thickening of calcite twins in the area of bending and flexural slip (Fig. $2.7 \mathrm{~g})$. The deformation of the calcite crystals also lead to polygonisation as shown in Figure $2.7 \mathrm{~h}$ and eventually to subgrain rotation recrystallisation (Fig. 2.7 i). Higher metamorphic temperatures within distinct shear zones are indicated by ultra fine-grained recrystallisation along the twin lamellae (Fig. $2.7 \mathrm{j}$ ).

Intracrystalline fracturing is also observable in all investigated samples of the boundary zone of brittle-ductile deformation. Figure $2.7 \mathrm{k}$ indicates a vein of multiple crack and seal events. In this vein, breccia of the same orientation as the host rock is still observable. The fluid inclusions in the matrix of the vein shows, that the sealing was controlled by fluids percolating the fractures.

The vein in Figure 2.71 shows that the fracture had been filled with breccia, now partially in different orientation to the host crystal. The pore space between the fracture breccia has crystallised in the same orientation as the host crystal. Even the twins have developed again across the vein in the same orientation as in the host crystal. Figure $2.7 \mathrm{~m}$ shows an open fracture of a younger generation, which is also filled with breccia of the host crystal. This breccia, as well as the breccia in the sealed veins are slightly recrystallised. 
Also the ultra fine-grained matrix of the cataclastic zones shown in Figure $2.7 \mathrm{n}$ and o is recrystallised as shown in Figure 2.7 o. Both Figures show, that the cataclastic microfabric and especially the distribution in grain size is scale invariant up to the recrystallised ultra fine-grained matrix. In the brecciated cataclastic zones more brittle microstructures dominate with kinking, flexural slip within the crystal lattice, the devolopment of deformation bands and polygonisation.

Graphite in the brittle-ductile deformed graphite-bearing marble is also ultra finegrained with grain sizes of 1 to $5 \mu \mathrm{m}$. As shown in Figure $2.7 \mathrm{p}$, graphite is concentrated in the fine-grained fracture zones as residue in stylolitic seams. These graphitic stylolites occur between the grain boundaries of the fine-grained and ultra fine-grained calcite crystals. Within zones of intensive pressure solution the graphitic stylolites are interconnected around calcite grain boundaries and form the graphitic network structures. The thickness of the graphitic stylolites varies from 20 to about $100 \mu \mathrm{m}$. The graphitic stylolites develop only in the fine-grained zones between the coarser-grained calcite host crystals. Therefore, the degree of graphite network intensity is dependent on the grain size distribution in the samples. The graphitic stylolites are never developed as intracrystalline stylolites.

\subsubsection{DUCTILE DEFORMED MARBLE}

The ductile deformed marble of the mylonitic core zone commonly shows a finegrained dynamically recrystallised matrix and porphyroclasts of calcite, quartz and plagioclase. Graphite either occurs concentrated in layers or is disseminated within the matrix. The grain size of the matrix varies from 10 to $100 \mu \mathrm{m}$, whereas the grain size of the graphite ranges from 5 to $70 \mu \mathrm{m}$. The porphyroclasts show grain sizes of up to $1 \mathrm{~mm}$.

The calcite porphyroclasts are commonly twinned. As shown in Figure 2.8 a some porphyroclasts have two sets of twins developed. The twins are commonly bent or show kink bands as shown in Figure $2.8 \mathrm{~b}$. The porphyroclasts display undulose extinction (Fig. $2.8 \mathrm{a}$ ) and are recrystallised along the grain boundaries. The grain sizes of the recrystallised grains vary in domains around the clast as shown in Figure $2.8 \mathrm{~b}$.

The porphyroclast in Figure $2.8 \mathrm{c}$ shows subgrain rotation recrystallisation which caused a rotation and bending of the unrecrystallised twin lamellae within the subgrain domain. This porphyroclast also shows recrystallisation along the grain boundary. The recrystallised grains are commonly free of twins along the rim of the porphyroclast. Subgrain rotation recrystallisation and the formation of new grains also occurs along twin lamellae within the porphyroclasts as shown in Fig $2.8 \mathrm{~d}$. 

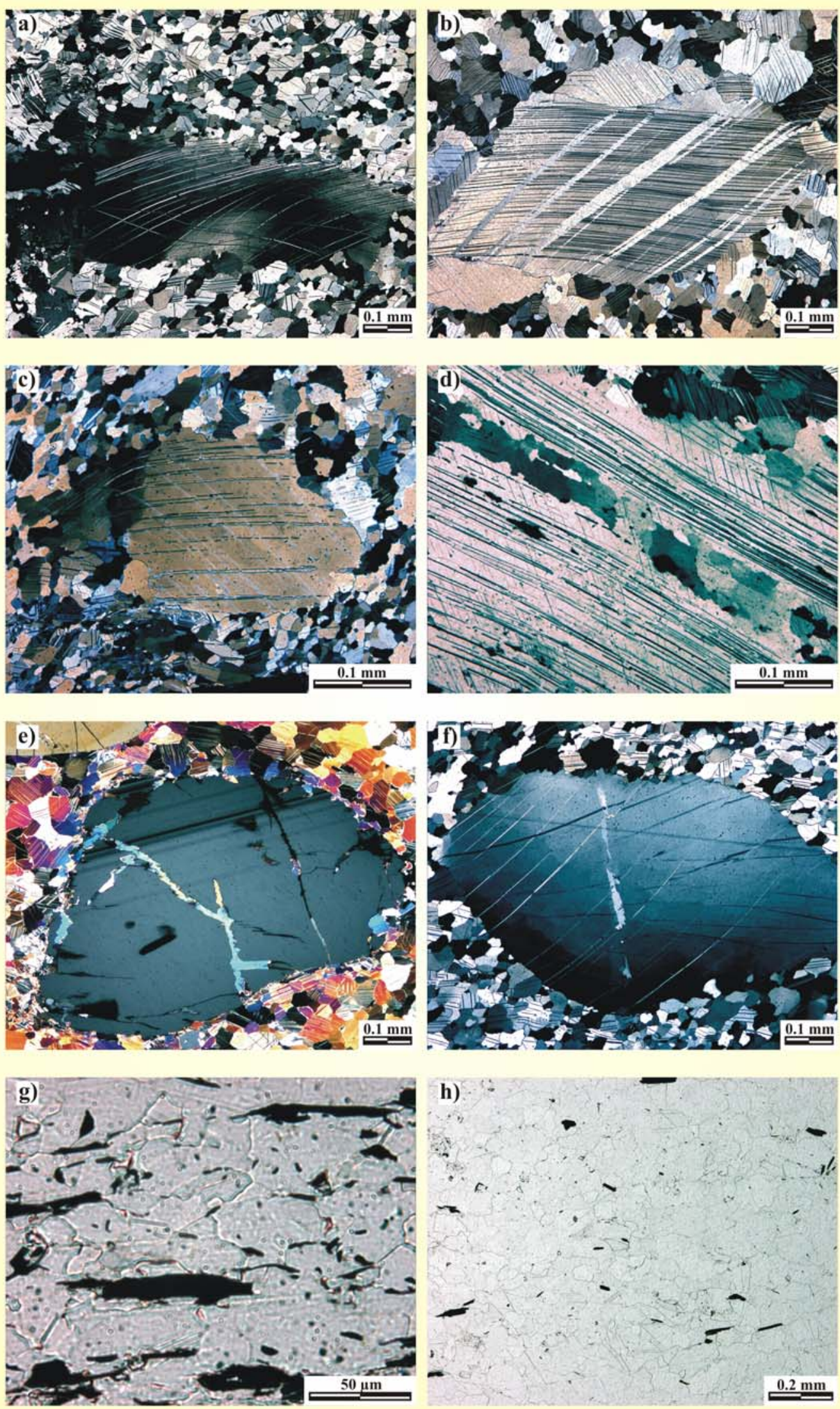
Fig 2.8 Microstructures of the mylonitic core zone. Figures a to $f$ are photomicrographs in crossed polarised light and Figures $g$ and $h$ are photomicrographs in plain polarised light. a) Twinned calcite porphyroclast with two sets of twins developed. It shows undulous extinction and is recrystallised along the grain boundary (sample 11/08/00/03). b) Calcite porphyroclast with kink bands and recrystallisation along the grain boundary (sample 16/08/00/02). c) Calcite porphyroclast with subgrain rotation recrystallisation and recrystallisation along the grain boundary (sample 12/08/00/04). d) Calcite porphyroclast with subgrain rotation recrystallisation and the formation of new grains along the twin lamellae (sample 12/08/00/04). e) Fractured plagioclase porphyroclast with healed calcite veins and an ultra fine-grained rim of recrystallised calcite around the grain boundary (sample 3.10.01/4). f) Calcite porphyroclast with a vein developed. Recrystallisation along the grain boundaries occurred in different grain size domains (sample 11/08/00/03). g) Graphite concentrated in layers in preferred orientation of the basal plains parallel to the foliation (sample 11/08/00/03). h) Graphite disseminated in the fine-grained dynamically recrystallised matrix with a preferred orientation of the basal plains parallel to the foliation (sample 11/08/00/06).

The quartz and plagioclase porphyroclasts are often fractured and sealed with calcite veins as shown in Figure 2.8 e. This plagioclase porphyroclast shows an ultra finegrained rim of recrystallised calcite along the grain boundary, which is very common in the marble mylonites. Also the calcite porphyroclasts show veins of recrystallised intracrystalline fractures as shown in Figure $2.8 \mathrm{f}$. This porphyroclast is recrystallised along domains of the grain boundary, whereas other domains do not show any recrystallisation of the grain boundaries of the porphyroclast.

Graphite occurs in the dynamically recrystallised matrix of the marble mylonites. As shown in Figure $2.8 \mathrm{~g}, \mathrm{~h}$ it either occurs concentrated in graphitic layers or disseminated throughout the matrix. The graphite is not interconnected in the graphitic layers and shows a preferred orientation with the basal planes parallel to the foliation (Fig. $2.8 \mathrm{~g}$ ). Graphite disseminated throughout the marble mylonites, also shows a preferred orientation parallel to the foliation. In both cases graphite mainly occurs on the calcite grain boundaries.

\subsubsection{CATHODOLUMINESCENCE INVESTIGATIONS}

For cathodoluminescence microscopy (CL) investigations thin sections are carbon coated. By the irradiation with electrons, the distribution of trace elements like manganese $(\mathrm{Mn})$ and iron $(\mathrm{Fe})$ is dectected. $\mathrm{Mn}$ is the most important activator element for the luminescence, whereas Fe inhibits bright luminescence colours. For calcite the luminescence colours range from dark read to bright yellow. By CL investigations, fluidcontrolled recrystallisation processes can be visualized.

The investigation by CL revealed that the brittle-ductile deformed marble shows consistent $\mathrm{CL}$ colours for the calcite host crystals, the fracture zones, the stylolites and the crack-seal 
veins (Fig. 2.9 a, b). Local differences in intensity as shown in Figure 2.9 a, are related to effects on luminescence intensities due to differences in grain geometry and lattice orientations. This is also observable in Figure $2.9 \mathrm{c}$, where the coarse-grained host crystal shows a slightly darker CL colour than the fine-grained matrix (Fig.2.9 d).

Figure $2.9 \mathrm{c}$ to $\mathrm{h}$ show additionally a younger generation of microveins, which display different CL colours than the older fabric of brittle-ductile deformation. These younger veins occur in different generations, which all show a preferred orientation. They postdate the brittle-ductile deformation as they cut the graphitic stylolites, the brittleductile fracture zones and the veins regardless of the orientation of the previous deformational structures. Figure 2.9 d shows that these younger microveins cut the coarsegrained host crystals as well as the matrix.

Graphitic stylolites are also opened and crystallised with calcite by these younger microveins (Fig. 2.9 e). It is also commonly observable that the microveins either cut the graphitic stylolites or end when a stylolite is reached by the vein (Fig. 2.9 e to h). The stylolites are not inevitably reactivated by the younger microveins as shown in Figure 2.9 g, h. A microvein has developed parallel to a graphitic stylolite cutting other stylolites of the graphite network structure and a domain of coarse-grained calcite crystals.

\subsubsection{INVESTIGATIONS BY SCANNIG ELECTRON MICROSCOPY}

Investigations by Scanning Electron Microscopy (SEM) and Energy Dispersive Xray Analysis (EDX) were conducted to characterise the different types of graphite-bearing marbles described above. EDX investigations reveal the qualitative composition of a sample by the composition of existing elements. Figure 2.10 shows the qualitative composition of the measured arrays in the left column. The according SEM phase contrast image is shown in the right column.

The measurement and composition of the calcite-marble is shown by the representative Figure $2.10 \mathrm{a}, \mathrm{b}$. The area scan of Figure $2.10 \mathrm{~b}$ shows, that the marble is mainly composed of calcite (Fig. 2.10 a). Quarz, plagioclase and muscovite occur in concentrations of about $0.5 \mathrm{Vol} \%$ and were also detected in the measurements. However, as these phases are only accessory minerals, the composition shown in Figure 2.10 a is representative for all three types of the investigated graphite-bearing marbles.

The quantitative composition of graphite within the abnormally grain coarsened marble host rock (type 1) is shown in Figure $2.10 \mathrm{c}$ to $\mathrm{f}$. According to the measurement spots shown in the phase contrast images (Fig. $2.10 \mathrm{~d}, \mathrm{f}$ ) the composition of the graphite 

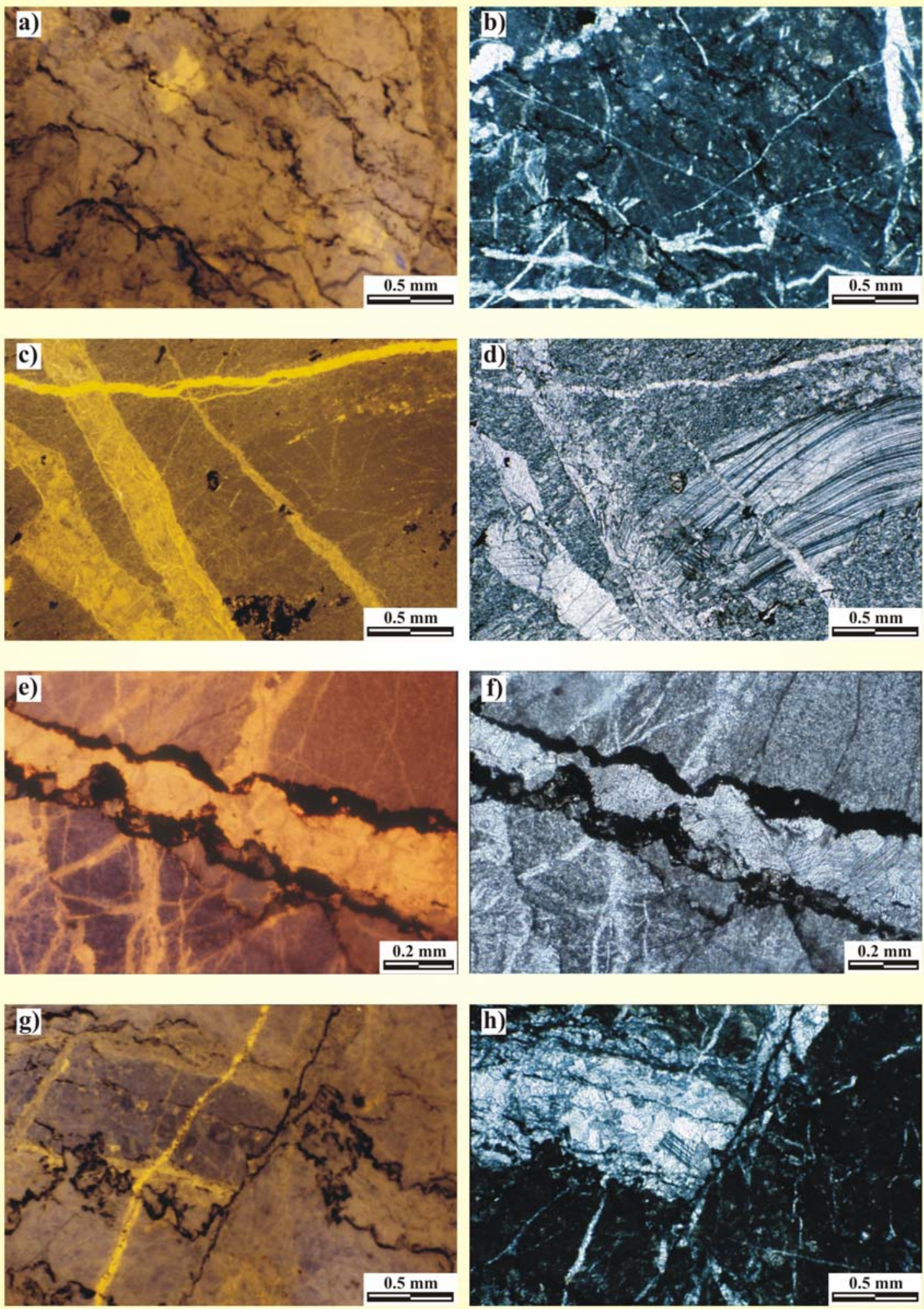

Fig 2.9 Cathodoluminescence microscopy investigations of microveins in the brittle-ductile deformed marble a) Microphotograph of uniform CL colours of the graphitic stylolites (sample 17/08/00/08a). b) Normal polarised microphotograph of the same section as in Figure a), which shows the calcite matrix with 
microveins and stylolites of the brittle-ductile deformation (sample 17/08/00/08a). c) Microphotograph in CL colours of two generations of younger microveins cutting coarse-grained host crystals and the fine-grained matrix. They are distinguished from the older deformational structures by the different CL colours (sample 12/08/00/04). d) Microphotograph in crossed polarised light of the sampe section as Figure c (sample 12/08/00/04). e) Microphotograph in CL colours of a reactivated graphitic stylolite mineralised as calcite vein. Microveins end at stylolites (sample 17/08/00/08b). f) Crossed polarised light microphotograph of the section shown in e (sample 17/08/00/08b). g) Microvein parallel to a stylolite cutting other stylolites and a domain of coarse-grained calcite crystals. Microphotograph in CL colours (sample 17/08/00/08a). h) Section of $g$ under crossed polarised light (sample 17/08/00/08a).

varies quite extensively between different graphite flakes within one radial graphite structure. The composition shown in Figure $2.10 \mathrm{c}$ results from a spot measurement of a graphite flake, that has grey colours in the phase contrast image (Fig. $2.10 \mathrm{~d}$ ). This measurement shows a strong $\mathrm{C}$ peak accompanied by $\mathrm{Ca}$ and $\mathrm{O}$ peaks which are most probaly resulting from calcite.

In contrast, the white domains in the phase contrast image of this radial graphite structure (Fig. $2.10 \mathrm{f}$ ) show a completely different quantitative composition of graphite (Fig. 2.10 e). Additional phases like Fe, Ti, K, Si, $\mathrm{Al}, \mathrm{Mg}$ and $\mathrm{Cl}$ are detected in this part of the radial graphite structure. These phases may be related to the epitaxial intergrowth of mica minerals and graphite on the basal plains.

These phases are also detected in the graphitic stylolites of the brittle-ductile deformed graphite bearing marbles (type 2) as shown in Figure $2.10 \mathrm{~g}$ to $\mathrm{j}$. The spot measurements of the phase contrast images in Figure $2.10 \mathrm{~h}, \mathrm{j}$ indicate that the graphite stylolites also display different colours in the phase contrast image.

As the EDX measurements in Figure $2.10 \mathrm{~g}$, i show, this difference in phase contrast colours is not only related to the measurement of fine-grained calcite within the stylolites. The additional phases $\mathrm{Fe}, \mathrm{Ti}, \mathrm{Al}, \mathrm{Mg}, \mathrm{P}, \mathrm{Ca}, \mathrm{O}$ are similar to the phases detected in the undeformed radial graphite structure. From their composition, they may be related to mica and calcite minerals. Therefore, the undeformed and the retrograde deformed graphite shows a similar composition in accessory minerals.

\subsubsection{QUANTITATIVE CALCITE-GRAPHITE RATIOS}

As the graphitic stylolites are formed by pressure solution, it is necessary to quantify this influence on the development of the microstructures by calculating the quantitative calcite-graphite ratios. These were approached by two methods: Firstly by the evaluation of the content of graphite in samples with a known volume. Secondly by scanning polished sample surfaces and counting the ratio between white calcite and black 

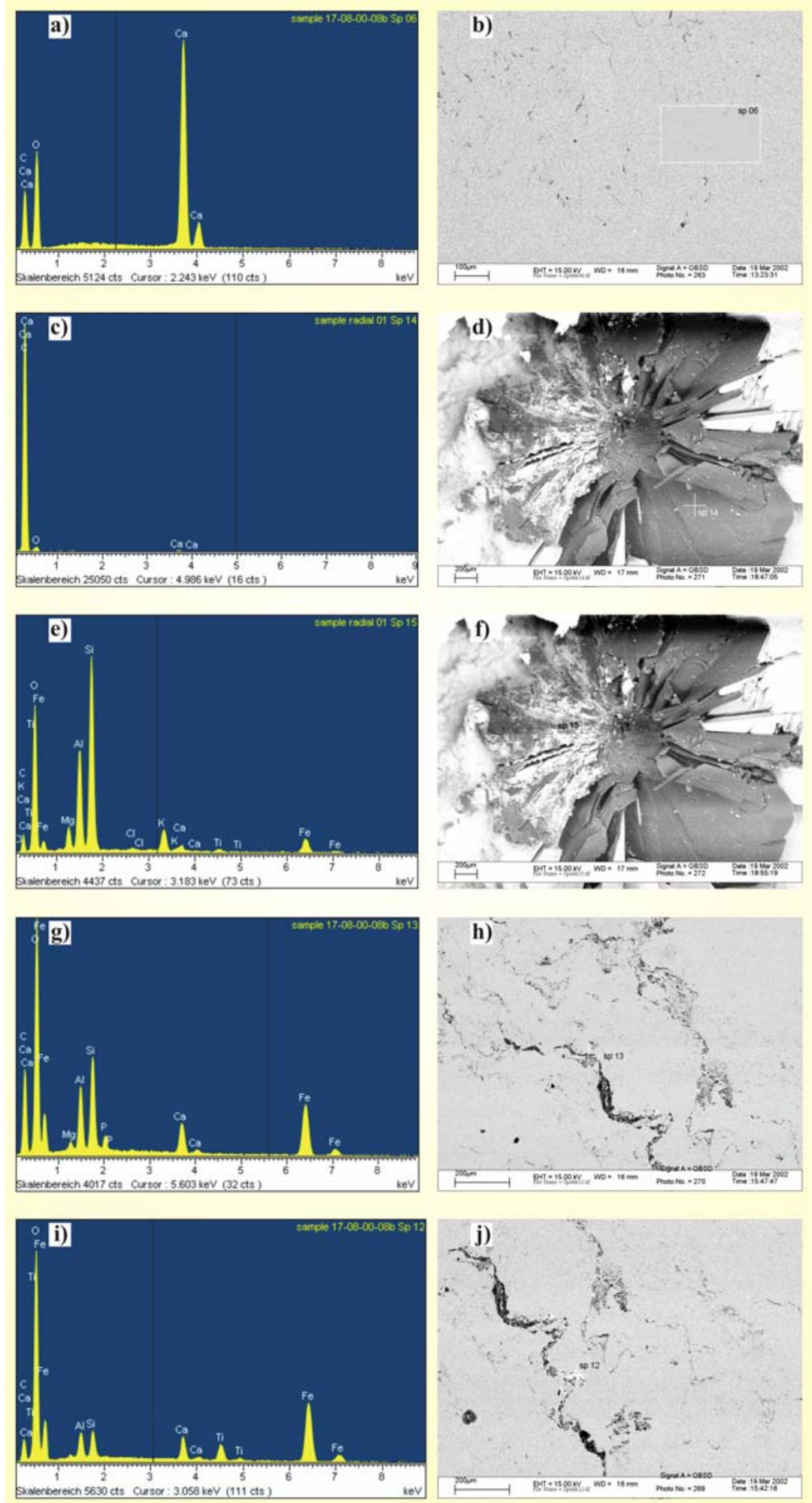

Fig 2.10 SEM and EDX investigations of the different types of graphitebearing marbles. Figures $a, c, g$ and $i$ are the EDX diagrams and Figures $b, d, h$ and $j$ represent the phase contrast images. a) Representative plot of 
the qualitative composition of a type 2 graphite-bearing marble (sample 17/08/00/08b). b) Phase contrast image with the measured area marked by the white box (sample 17/08/00/08b). c) Qualitative composition of a graphite flake (type 1 graphite-bearing marble) with grey colours in the phase contrast image (sample radial 01). d) Phase contrast image of the radial graphite structure with the locality of the spot measurement marked by a + (sample radial 01). e) Qualitative composition of a different graphite flake (type 1 graphitebearing marble) with a white colour in the phase contrast image (sample radial 01). f) Spot measurement in the white domains of the graphite flakes is marked by a + (sample radial 01).g) Qualitative composition of a graphitic stylolite from a type 2 graphite-bearing marble (sample 17/08/00/08b). h) The spot of the measurement is marked on the phase contrast image (sample 17/08/00/08b). i) Qualitative composition of a white domain of a graphitic stylolite from a type 2 graphite-bearing marble (sample 17/08/00/08b). j) Phase contrast image with the locality of the spot measurement marked (sample 17/08/00/08b).

graphite pixels in a predefined area. This area was constant for all samples. This was done to evaluate whether it is possible to estimate the amount of pressure solution by macroscopic optical methods.

\subsubsection{ANALYTICAL TECHNIQUES}

For quantifying the calcite-graphite ratio by the content of graphite, the different sample volumes were obtained of samples with varying degrees of graphite network intensity. This was done by measuring the water displacement of the samples, which then was used to calculate the sample volume by the density of the displaced water. In a second step, the samples were excavated and the volume of the permeable pore space was measured by the oven-dry mass, the saturated mass and the flotation mass. The porosity then was substracted from the calculated volume of the samples.

Afterwards the graphite bearing marbles were dissolved in concentrated hydrochloric acid. After calcite was dissolved, the graphitic residue was then treated with hydrofluoric acid to dissolve accessory silicate minerals. As the graphitic residue still contained Ca-Fluorides, it had to be boiled in hydrochloric acid. After the graphite has been rinsed with demineralised water, the volumetric content of graphite for each sample had been calculated from the graphite density.

For the second method of quantifying the calcite-graphite ratios, polished surfaces of the same samples were scanned on a flat bed scanner with a resolution of $600 \mathrm{dpi}$. An area of $50 \mathrm{~cm}^{2}$ was selected for all samples, to evaluate the content of graphite statistically. This was done by calculating the ratio between black pixels of the graphitic stylolites and the white pixels of calcite.

The evaluation of both methods is based on the assumption that the graphite in the undeformed and therefore unaltered host rock must have a statistically constant volume, 
because it is disseminated thoughout the host rock in graphite nests. Under this precondition, the amount of pressure solution of calcite may be calculated for the samples, where the volumetric content of graphite is higher than in the undeformed host rock. Likewise, for lower volumetric contents of graphite pressure solution of graphite has to be assumed. As shown in Figure $2.3 \mathrm{a}, \mathrm{b}$ this assumption is only true for a sample volume that eliminates statistically local differences in the concentration of disseminated graphite nests in the undeformed host rock. Whether this critical sample volume of the undeformed host rock has been reached in the investigated samples, has not been evaluated in detail. It is assumed however, that the sample volume is statistically appropriate.

\subsubsection{MEASUREMENTS AND RESULTS}

The results of the measurement of the concentration of graphite are shown in table 2.1. The table shows the samples sorted from the lowest to highest volumetric content of graphite. The volumetric content of graphite in the undeformed host rock is around 0.098 $\mathrm{Vol} \%$, whereas the highest concentration of graphite are observable in the brittle-ductile boundary zone with $0.442 \mathrm{Vol} \%$. This table also reveals that there are seven samples, which show a lower volumetric content of graphite than the undeformed host rock. The lowest value measured is $0.018 \mathrm{Vol} \%$ of graphite.

According to this data it can be assumed, that pressure solution has occurred for calcite as well as for graphite. From the obtained values, the calculated pressure solution of graphite amounts to a maximum of $5.44 \%$. Likewise, the pressure solution of calcite can be calculated to about $4.51 \%$. For a simultaneous pressure solution of both mineral phases, the amount of graphite pressure solution must be substracted from the field of calcite pressure solution. A maximal amount of pressure solution of calcite of $24.56 \%$ is in this case implied by the data.

The quantitative calcite-graphite ratios obtained from the same samples by macroscopic optical methods are shown in table 2.2. The proportion of graphite pixels from the measured area is given as a percentage. The samples are sorted from the lowest proportion to the maximum proportion of graphite obtained from the measured samples.

Compared to the volumetric contents of graphite (table 2.1), it becomes obvious from table 2.2 that the samples with a lower volumetric content than the undeformed host rock imply macroscopically an exceeding concentration of graphite. This is related to a high portion of dark and/or black arrays within the samples, due to fine-grained calcite, which also appears in darker colours. The macroscopic fabrics of the measured samples are shown in Figure 2.11 a to p. 


\begin{tabular}{|c|c|c|c|}
\hline sample & $\begin{array}{c}\text { sample volume } \\
{\left[\mathbf{c m}^{3}\right]}\end{array}$ & $\begin{array}{c}\text { content of graphite } \\
{\left[\mathbf{c m}^{\mathbf{3}}\right]}\end{array}$ & $\begin{array}{c}\text { volumetric content } \\
\text { of graphite } \\
{[\text { Vol \%] }}\end{array}$ \\
\hline $17 / 08 / 00 / 04$ & 2397.876 & 0.439 & 0.018 \\
\hline $17 / 08 / 00 / 17$ & 2292.347 & 0.527 & 0.023 \\
\hline $17 / 08 / 00 / 13$ & 745.192 & 0.344 & 0.046 \\
\hline $17 / 08 / 00 / 15$ & 599.671 & 0.360 & 0.060 \\
\hline $17 / 08 / 00 / 19$ & 675.834 & 0.450 & 0.067 \\
\hline $17 / 08 / 00 / 10$ & 366.988 & 0.262 & 0.071 \\
\hline $17 / 08 / 00 / 12$ & 381.800 & 0.367 & 0.096 \\
\hline $17 / 08 / 00 / 20$ & 1158.106 & 1.545 & 0.098 \\
\hline $18 / 08 / 00 / 02$ & 385.824 & 0.387 & 0.100 \\
\hline $12 / 08 / 00 / 02$ & 295.190 & 0.300 & 0.102 \\
\hline $12 / 08 / 00 / 03$ & 288.253 & 0.302 & 0.105 \\
\hline $3.10 .01 . / 4$ & 295.132 & 0.319 & 0.108 \\
\hline $15 / 08 / 00 / 01$ & 422.667 & 0.585 & 0.139 \\
\hline $17 / 08 / 00 / 11$ & 131.691 & 0.249 & 0.189 \\
\hline $3.10 .01 . / 3$ & 224.579 & 0.545 & 0.243 \\
\hline $17 / 08 / 00 / 05$ & 881.652 & 3.398 & 0.301 \\
\hline $17 / 08 / 00 / 21$ & 936.970 & 4.141 & 0.442 \\
\hline
\end{tabular}

Table 2.1 Quantitative calcite-graphite ratios calculated from the sample volume and the graphite content of the sample. The sample in orange is the host rock sample, whereas the samples in grey show pressure solution of graphite. The samples in peach colours show pressure solution of calcite as well.

Table 2.1 and Figure 2.11 a to $\mathrm{p}$ indicate, that pressure solution of graphite occurs in samples with a broad variety of graphite network intensity. The samples of Figure 2.11 b, d with short graphitic stylolites and a very low occurrence of interconnected stylolites, definitely experienced graphite pressure solution. Likewise, the samples of Figure $2.11 \mathrm{k}, 1$ with an intermediate to high degree of graphite network intensity show graphite pressure solution. Furthermore samples with a very high degree of graphite network intensity as shown in Figure $2.11 \mathrm{~m}$, o and p also clearly show graphite pressure solution.

\begin{tabular}{|c|c|}
\hline sample & $\begin{array}{c}\text { Proportion of Graphite } \\
{[\%]}\end{array}$ \\
\hline $17 / 08 / 00 / 20$ & 0.23 \\
\hline $17 / 08 / 00 / 13$ & 0.39 \\
\hline $18 / 08 / 00 / 02$ & 1.99 \\
\hline $17 / 08 / 00 / 15$ & 2.54 \\
\hline $12 / 08 / 00 / 03$ & 2.84 \\
\hline $12 / 08 / 00 / 02$ & 3 \\
\hline $15 / 08 / 00 / 01$ & 4.34 \\
\hline $17 / 08 / 00 / 05$ & 4.68 \\
\hline
\end{tabular}

\begin{tabular}{|c|c|}
\hline sample & $\begin{array}{c}\text { Proportion of Graphite } \\
{[\%]}\end{array}$ \\
\hline $17 / 08 / 00 / 111$ & 5.72 \\
\hline $17 / 08 / 00 / 112$ & 6.57 \\
\hline $17 / 08 / 00 / 17$ & 16.09 \\
\hline $17 / 08 / 00 / 19$ & 17.6 \\
\hline $17 / 08 / 00 / 04$ & 19.08 \\
\hline $17 / 08 / 00 / 21$ & 22.31 \\
\hline $17 / 08 / 00 / 12$ & 29.93 \\
\hline $17 / 08 / 00 / 10$ & 32.5 \\
\hline
\end{tabular}

Table 2.2 Quantitative calcite-graphite ratios calculated from the area percentage of graphite on defined sample surfaces. Analogue to table 2.1 the sample in orange is the host rock. Samples in grey colours show pressure solution of graphite and the samples in peach colours show pressure solution of calcite. 

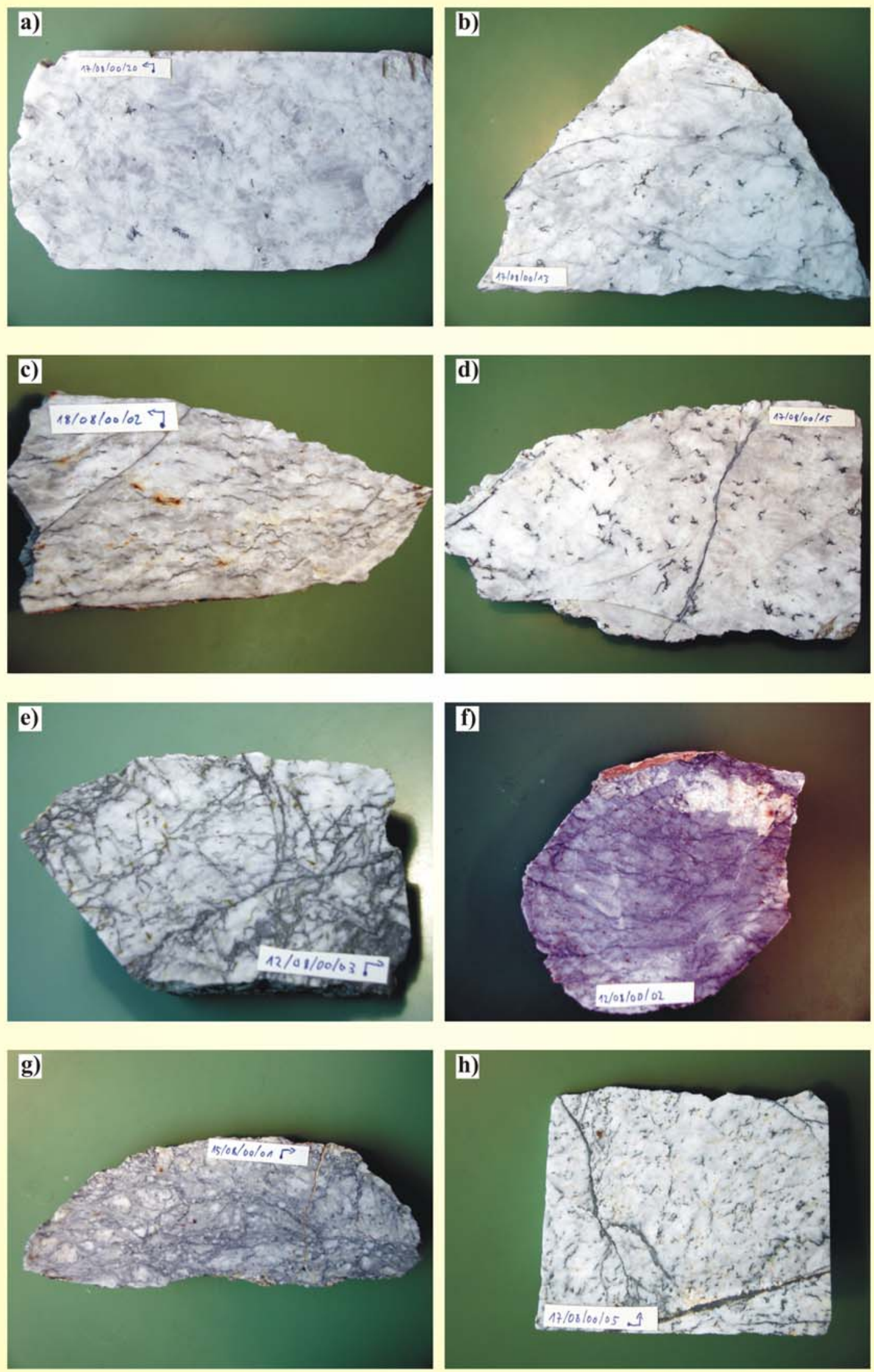

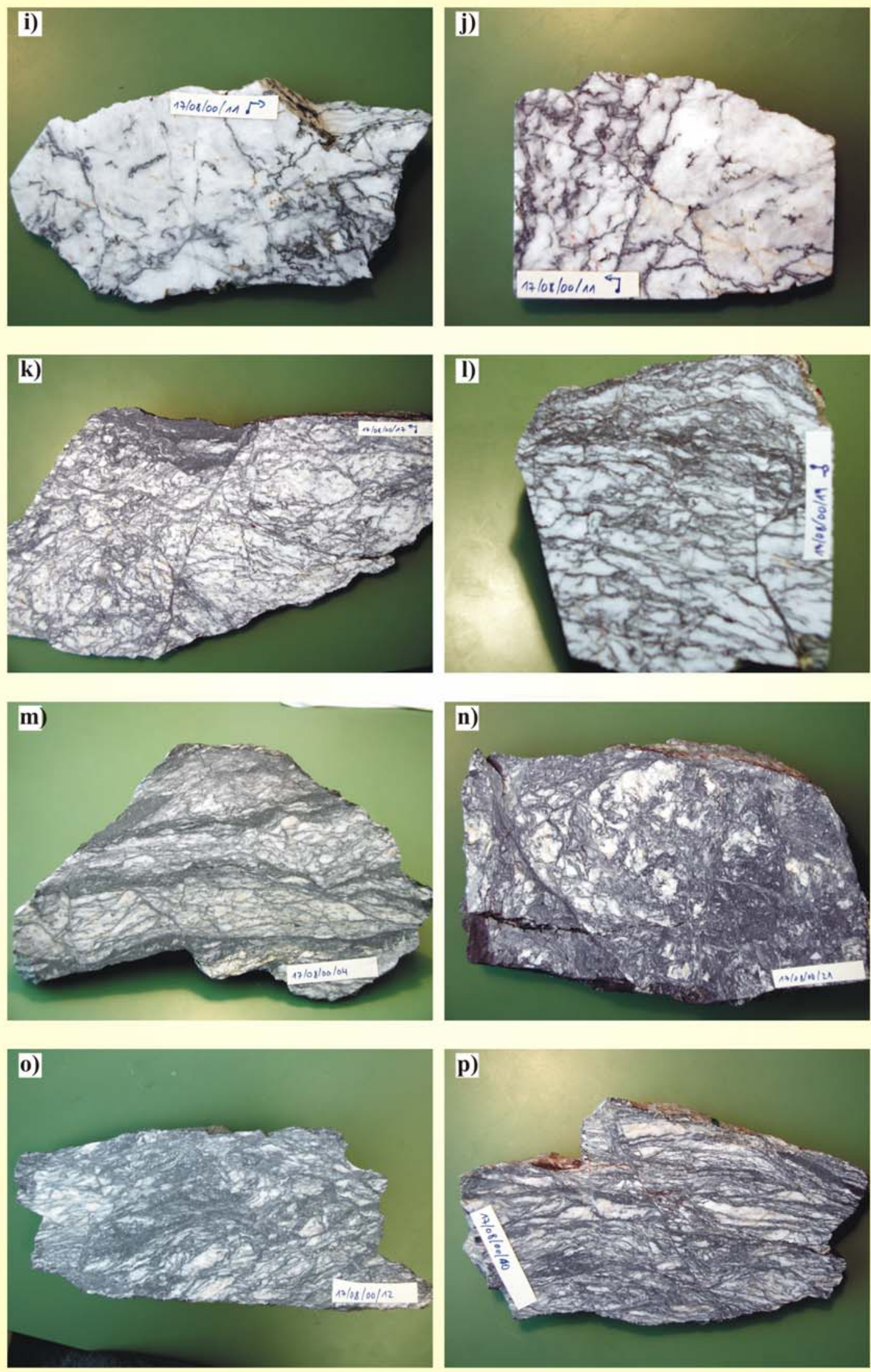
Fig 2.11 Samples with different degrees of graphite network intensity used to quantify the calcite-graphite ratios by optical methods. The calcite-graphite ratios are shown in table 2.2 a) sample 17/08/00/20 b) sample 17/08/00/13 c) sample 18/08/00/02 d) sample 17/08/00/15 e) sample 12/08/00/03 f) sample 12/08/00/02 g) sample 15/08/00/01 h) sample 17/08/00/05 I) sample 17/08/00/11 1 j) sample 17/08/00/11 2 k) sample 17/08/00/17 l) sample 17/08/00/19 m) sample 17/08/00/04 n) sample 17/08/00/21 o) sample 17/08/00/12 p) sample 17/08/00/10

\subsection{TeXture ANALYSIS}

In this study texture analysis is referred to as the quantification and analysis of crystallographic orientations of the mineral phases in the investigated samples. The texture analysis measurements were restricted to samples from the investigated shear zones, as the undeformed host rock is to coarse-grained to obtain statistically reprentative results. Texture analysis was conducted by neutron diffraction and the rotating stage polarizor.

\subsubsection{TEXTURE ANALYSIS BY NEUTRON DIFFRACTION}

For the texture analysis by neutron diffraction, the texture diffractometer SKAT (Ullemeyer et al., 1998) at the pulsed reactor IBR-2 in Dubna (Russia) was used. From the obtained time-of-flight (TOF) spectra, experimental pole figures were extracted, which represent the bulk texture of the samples.

\subsubsection{ANALYTICAL TECHNIQUE}

For the measurements with the texture diffractometer SKAT cylindrical samples of $40 \mathrm{~mm}$ in length and diameter were prepared. An exposition time of 15 min per sample position was selected. A measuring grid of $5^{\circ} \times 5^{\circ}$ and a simultaneous application of 19 detectors, resulted in measuring times of 16 hours per sample. Neutron diffraction enables texture measurements of the whole sample volume with an high $d$-resolution $(\Delta d / d \approx 0.5 \%$ at $\mathrm{d}=2 \AA$ ), where $\mathrm{d}$ is the lattice spacing. The high d-resolution is necessary to identify individual Bragg peaks of the polyphase diffraction patterns. From the obtained TOF spectra (Fig. 2.12), experimental pole figures were calculated after the background subtraction, by integrating over all intensities at a pre-defined interval.

The experimental pole figures are plotted in equal area projection and the lowest contour represents 1.0 multiples of random distribution (m.r.d.). The contour intervals for the pole figures are uniform for all plotted samples. For the calcite pole figures in Figure 2.14 the contour intervals for the c-axes are 0.5 m.r.d. The a-axes pole figures are contoured at 0.2 m.r.d exept for sample 17/08/00/15 where it is 0.4 m.r.d. For the $r$ - and f-plains the contour intervals are 0.1 m.r.d., except for samples $17 / 08 / 00 / 16 \mathrm{~b}$ and $17 / 08 / 00 / 15$ where it is 0.2 


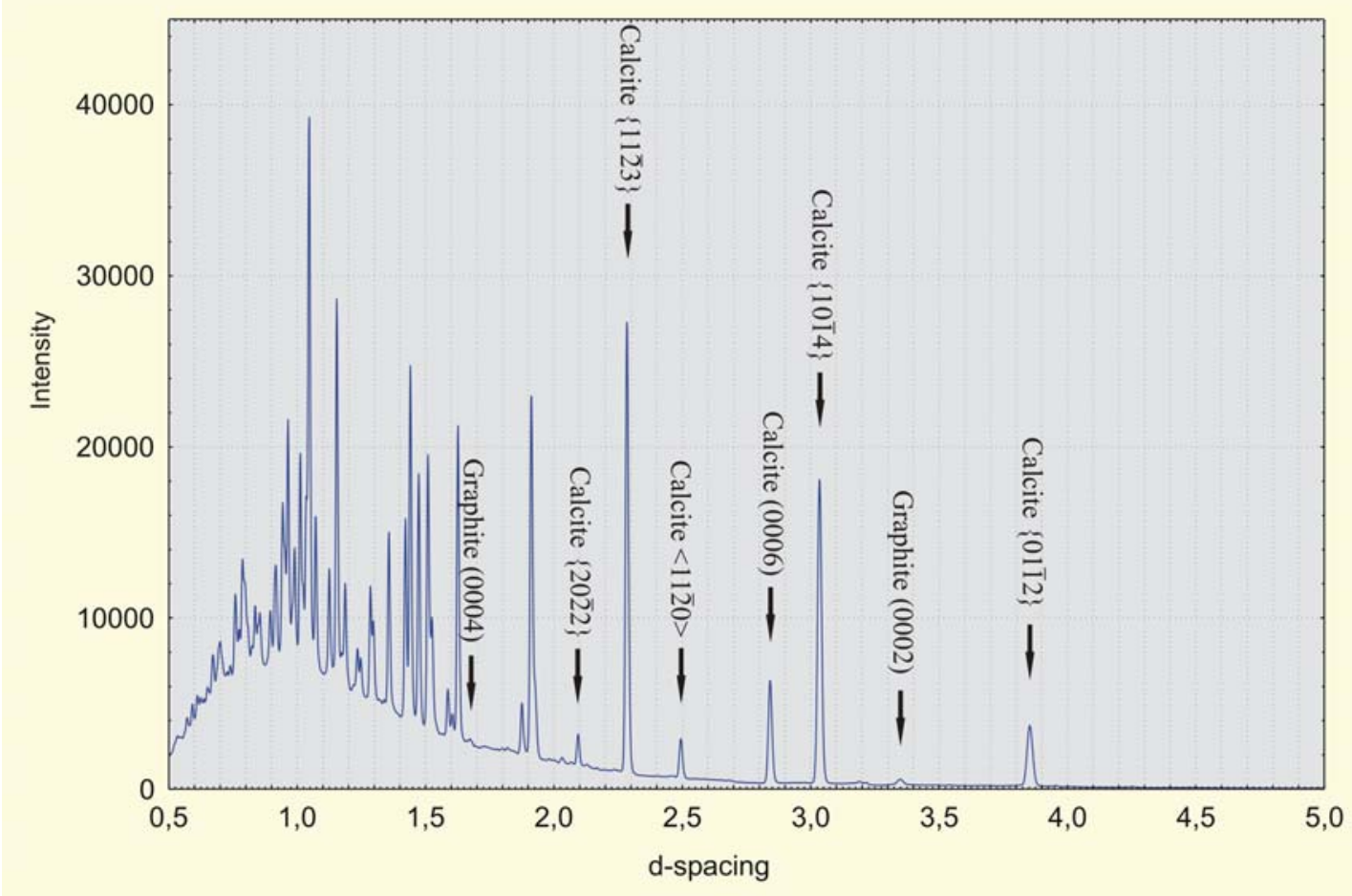

Fig 2.12 Sumarised TOF neutron diffraction pattern. From the labelled peaks of calcite and graphite the pole figures were calculated.

m.r.d. and 0.4 m.r.d. respectively. For the graphite pole figures in Figure 2.15, the contour intervals of the c-axes of the (0002) and (0004) Bragg reflections are 1.0 m.r.d. The dotted lines represent additional intermediate contours in samples of low texture intensities. The pole figures are oriented normal to the foliation and parallel to the lineation, where it was obtainable. The relative maxima are given for all pole figure in Figures 2.14 and 2.15.

\subsubsection{TEXTURE TYPES OF CALCITE}

For calcite, high-temperature and low-temperature texture types are distinguished for pure shear and simple shear deformation (Wenk et al., 1987). The different texture types of calcite are plotted in a compilation by Leiss \& Molli (2003) after Wenk et al. (1987) (Fig. 2.13). The transition between different texture types in dependence of the temperature and deformation regime, is due to different critical shear stresses for the intracrystalline slip systems at different temperatures during deformation (e.g. De Bresser $\&$ Spiers, 1997). As these critical shear stresses are not only dependent on the temperature but also on the stress and strain rate, the simple correlation for calcite textures shown in Figure 2.13 is doubted by Leiss \& Molli (2003). 


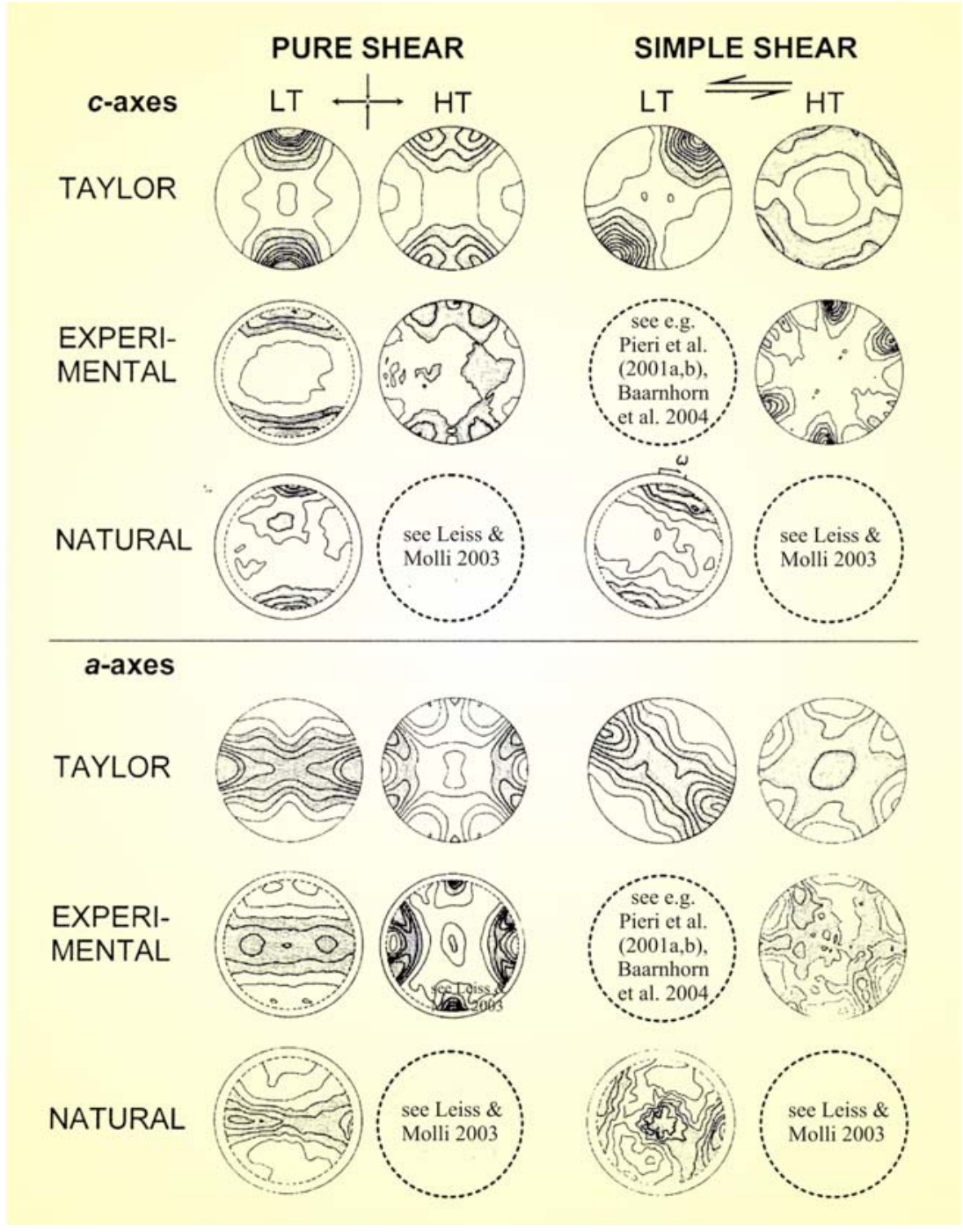

Fig 2.13 Calcite texture types for different deformation regimes at different temperatures. The textures are represented by c-axes and a-axes after Wenk et al. (1987) and Leiss \& Molli (2003).

According to the the compilation of texture types in Figure 2.13, c-axes pole figures with one maximum normal to the foliation are related to 'low-temperature' pure shear deformation. In this case the a-axes pole figures show a girdle distribution parallel to the lineation. This is the most common texture type for calcite as also described by Wenk et al. (1987) and Bestmann et al. (2000) The 'high-temperature' pure shear calcite textures show a c-axes double maximum normal to the foliation and fixed maxima of the a-axes at $45^{\circ}$ to the lineation. These types of 'high-temperature' pure shear textures have recently been reported in the literature by Leiss \& Molli (2003). However, these 'high-temperature' texture types occur in a low-temperature deformation structure. 
Simple shear low-temperature calcite textures show a maximum normal to the foliation, which is rotated against the sense of shear for both the c-axes and a-axes (Fig. 2.13). The a-axes again form a girdle distribution. These textures have recently been obtained in experimental data of Pieri et al. (2001a,b) and Barnhoorn et al. (2004).

The high-temperature variation of simple shear textures for calcite again exists in a double maximum for the c-axes, which is also rotated against the shear sense from the position normal to the foliation (Fig. 2.13). The a-axes form distinct maxima, which are rotated correspondingly to the c-axes. These pole figures were also recently reported from natural samples by Leiss \& Molli (2003).

The relationship between temperature, deformational regime and texture type as shown in Figure 2.13, is not as clearly documented in natural samples. After Leiss \& Molli (2003), high-temperature texture types also occur in low-temperature deformation structures.

After Wenk et al. (1987), the most common texture type of calcite is the single maximum texture as shown in the low-temperature pure shear type of Figure 2.13. This single maximum may be rotated against or according to the sense of shear as shown by the low-temperature simple shear texture type in Figure 2.13. Bestmann et al. (2000) also shows, that the single maximum texture type is common across different stages of mylonitisation. A spectrum of different texture types of natural samples are shown and characterised by Leiss \& Ullemeyer (1999), Weiss et al. (1999), Leiss \& Weiss (2000) with respect to their implications on the physical properties of carbonate rocks.

\subsubsection{SAMPLES AND MEASUREMENTS}

The measured samples cover a broad range of shear zones and were selected due to different macroscopic fabrics. The sample localities are shown in Fig. 2.1. Corresponding coordinates are listed in the appendix. The calcite pole figures of the measured samples are shown in Figure 2.14. They are classified between mylonitic samples of the mylonitic core zones and the samples of the brittle-ductile boundary zone. The pole figures of the samples $11 / 08 / 00 / 03$ to $16 / 08 / 00 / 02$ represent the mylonitic samples, whereas the brittle-ductile deformed marbles are the samples $17 / 08 / 00 / 15$ to $17 / 08 / 00 / 16 b$.

The samples of the brittle-ductile boundary zone were selected to cover a broad range of different macroscopic fabrics. The pole figures from sample 17/08/00/15 to sample 17/08/00/01 are sorted according to increasing degree of graphite network intensity. The pole figures of sample 17/08/00/16a represent the textures of the 


\section{Calcite}
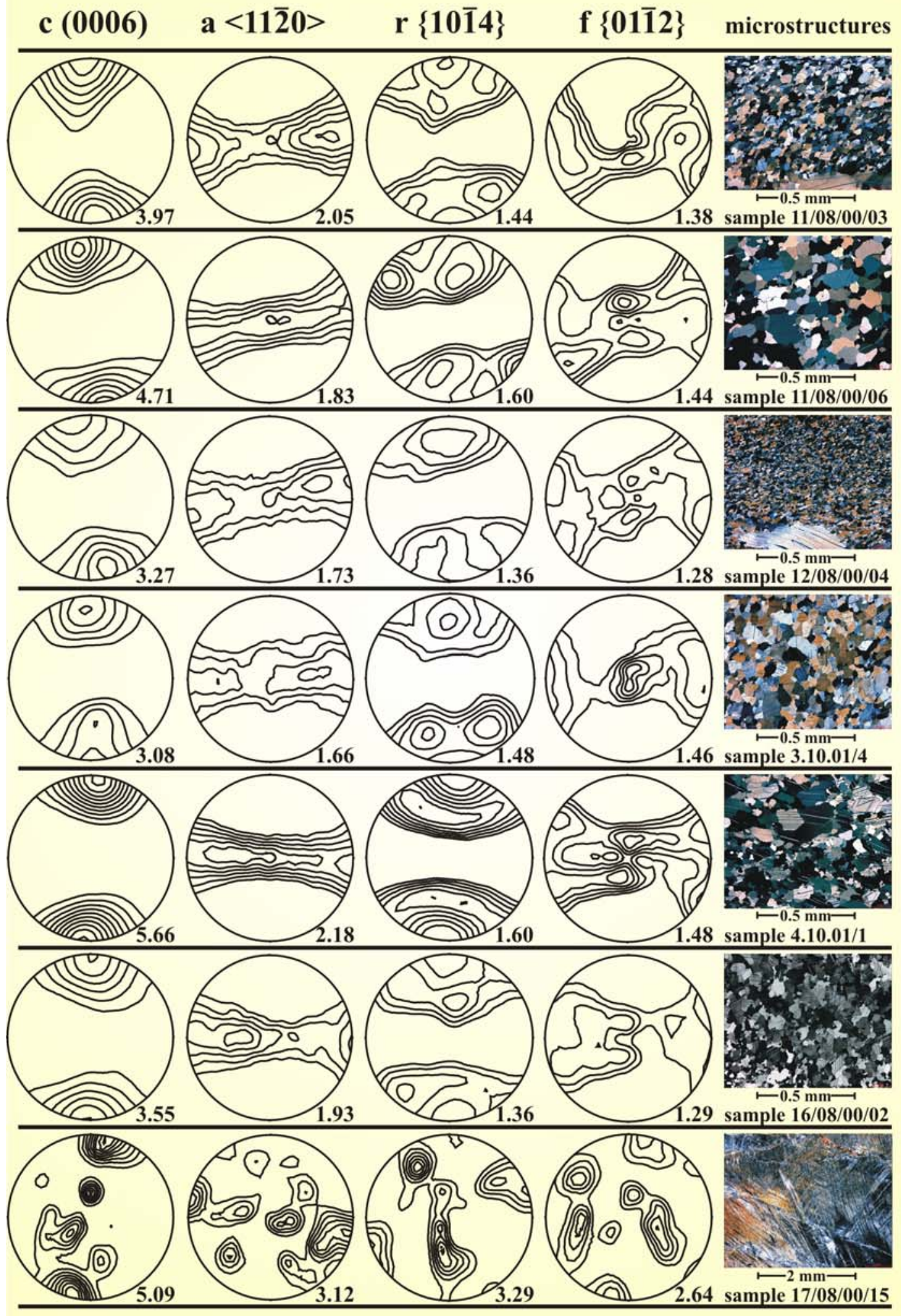


\section{Calcite}

\section{c (0006) a $<11 \overline{2} 0>\quad r\{10 \overline{1} 4\} \quad$ f $\{01 \overline{1} 2\}$ microstructures}

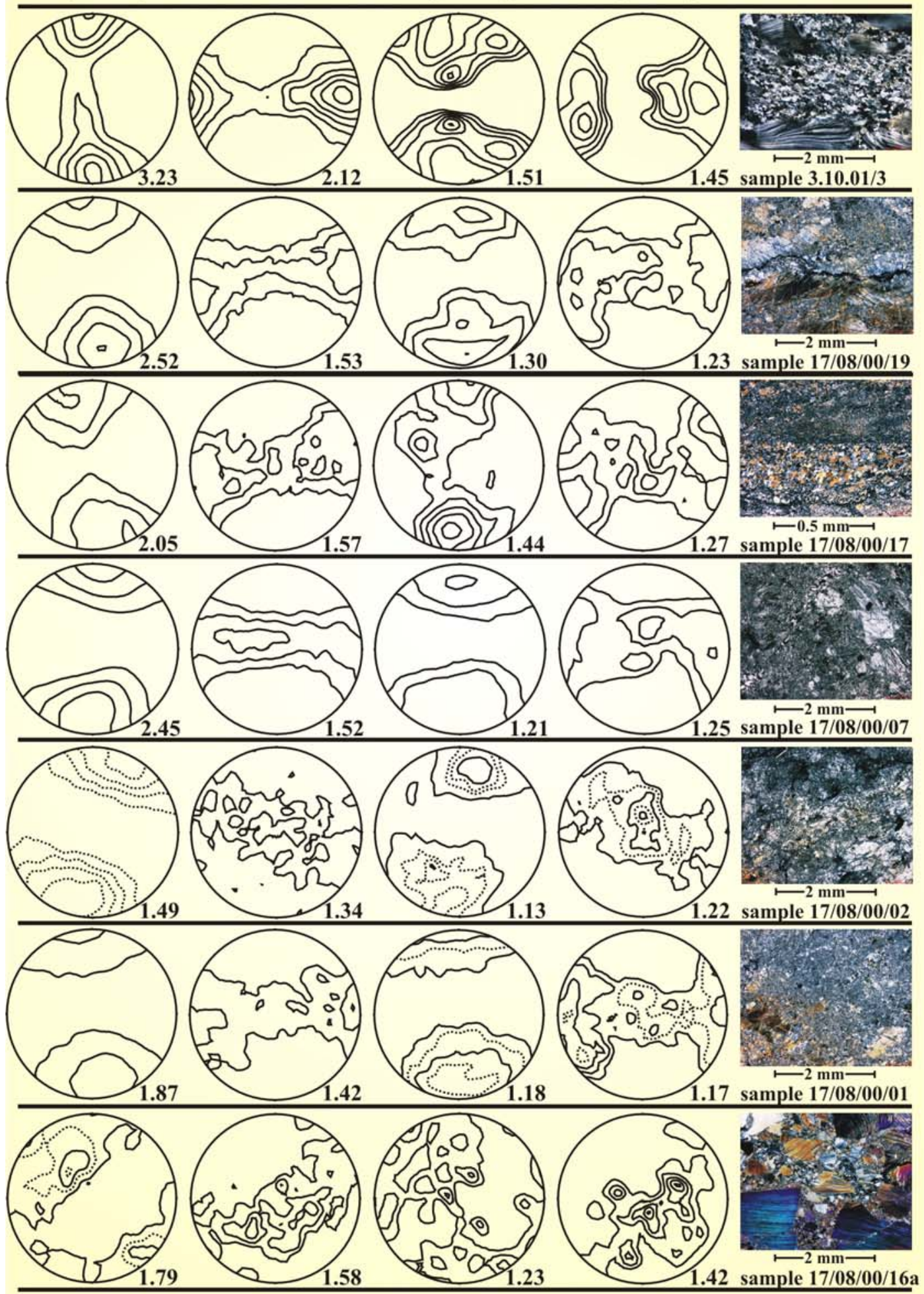




\section{Calcite}

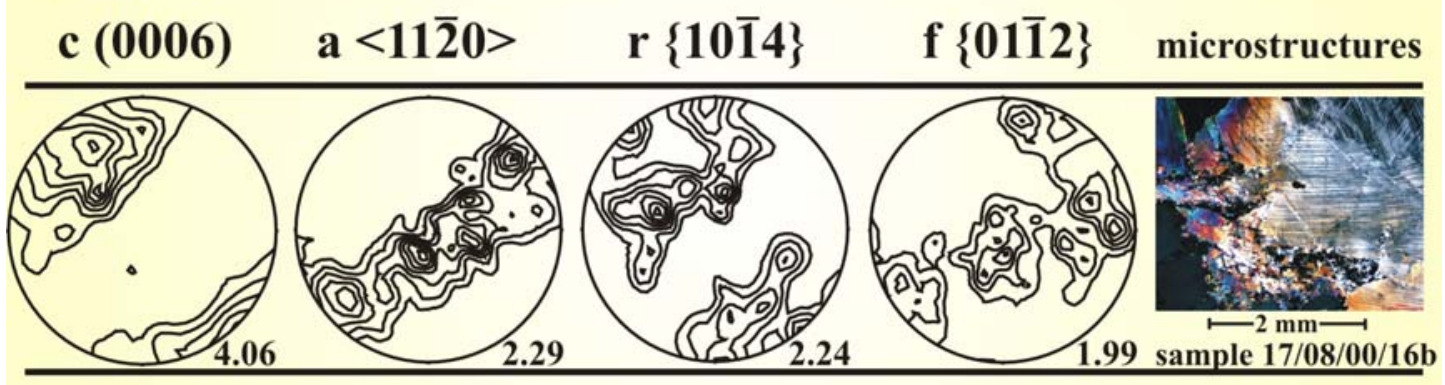

Fig 2.14 Calcite textures of mylonitically and brittle-ductile deformed graphite-bearing marble. The pole figures of the samples 11/08/00/03 to 16/08/00/02 represent the mylonitic samples, and the samples 17/08/00/15 to 17/08/00/16b represent the brittle-ductile boundary zone. The samples 17/08/00/16a and $17 / 08 / 00 / 16 b$ are from a purely cataclastic domain. Sample 17/08/00/16a represents the matrix and sample $17 / 08 / 00 / 16 b$ represents the texture of a clast.

macroscopic matrix of a cataclastic breccia zone and the pole figures of sample $17 / 08 / 00 / 16 \mathrm{~b}$ represent the textures of a macroscopic clast. The microstructures show, that the investigated clast also consists of brecciated domains. However, the general grain size distribution is clearly different between these to samples.

Sample 17/08/00/15 represents the domains of low graphite network intensity, which is characterised by short graphitic stylolites. The macroscopic fabric of this sample is shown in Figure 2.11 d. Samples 3.10.01/3, 17/08/00/19 and 17/08/00/17 show fabrics of intermediate to high to degrees of graphite network intensity. The macroscopic fabrics of the latter are shown in Figure 2.11 k, 1. Sample 17/08/00/07 to sample 17/08/00/01 are characterised by the highest degrees of graphite network intensity.

The pole figures of the c-axes of the mylonitic samples show a single maximum normal to the foliation, which shows the tendency for a girdle maximum in some samples $(11 / 08 / 00 / 03,12 / 08 / 00 / 04,3.10 .01 / 4,16 / 08 / 00 / 02)$. The pole figures of the a-axes form a girdle parallel to the lineation. The samples which show a tendency for a c-axes girdle have some distinct maxima within the a-axes girdle, whereas the other samples show a-axes girdles without distinct maxima. The latter therefore underwent a rotation in the a-axes. This is related to compression normal to the foliation, which implies pure shear deformation. The r- and f-plains show distinct maxima and small circle girdle distribution.

The intensities of the textures of the mylonitic samples are generally very high. From the microstructural overview images in Figure 2.14 it is obvious, that the intensity of the textures is directly related to the grain size of the mylonitic samples. Fine-grained 
mylonites show less high intensities than coarser-grained mylonites. This effect is also observable in the brittle-ductile deformed samples.

Sample 17/08/00/15 is the least deformed and most coarse-grained sample and it shows the highest intensities of the brittle-ductile deformed marbles. This is statistically also related to the single-crystal orientations of the single coarse grained calcite crystals, as they represent larger volume fractions compared to fine-grained samples. Nevertheless, the accordance of the crystallographic orientation between the single calcite crystals is obvious in the pole figures. Also the girdle alignment of the a-axes maxima is parallel to the mylonitic foliation, which indicates a consistent preferred lattice orientation in these samples. This relationship will have to be discussed later in this chapter.

With increasing degrees of network intensity an obvious decrease in grain size is observable in the microstructures of the brittle-ductile boundary zone. The textures all form single maxima normal to the foliation in the c-axes pole figures. Some of these maxima form girdles like in sample 3.10.01/3 and other maxima are rotated around an axis parallel to the foliation. This may either be related to the heterogeneities in deformation as shown in Figure $2.4 \mathrm{f}$ or to a rotation within or against the sense shear. Due to the heterogenous deformation it is also possible that the foliation is not perfectly parallel to an E-W axis of the pole figure. This is related to the complex deformation it was not in all cases possible to identify the exact foliation during sample preparation.

The textures of the cataclastic samples show, that the lattice preferred orientations are strongly diminished by the cataclastic deformation as obvious from the texture of the cataclastic matrix of sample 17/08/00/16a. In contrast, the texture of the coarse-grained clast of sample 17/08/00/16b again shows very high intensities. The rotation of the single maximum in the c-axes may be related to a rotation of the clast during the event of cataclastic deformation in the breccia zones.

For the c-axes textures of graphite, a strong lattice preferred orientation in the mylonitic samples is obvious (Fig. 2.15). The single maxima normal to the foliation shows that the basal plains of graphite are oriented parallel to the foliation. The highest texture intensities of graphite are rather related to the coarser-grained samples as also observed for the calcite textures. Two mylonitic samples display a slight girdle distribution of the c-axis orientation distribution. In these samples the c-axes and their equivalent basal planes are partially rotated in the direction normal to the foliation.

The samples from the brittle-ductile deformed boundary zone show no real preffered lattice orientations with the exeption of sample 3.10.01/3. This may be related to 
Graphite

\section{Calcite}

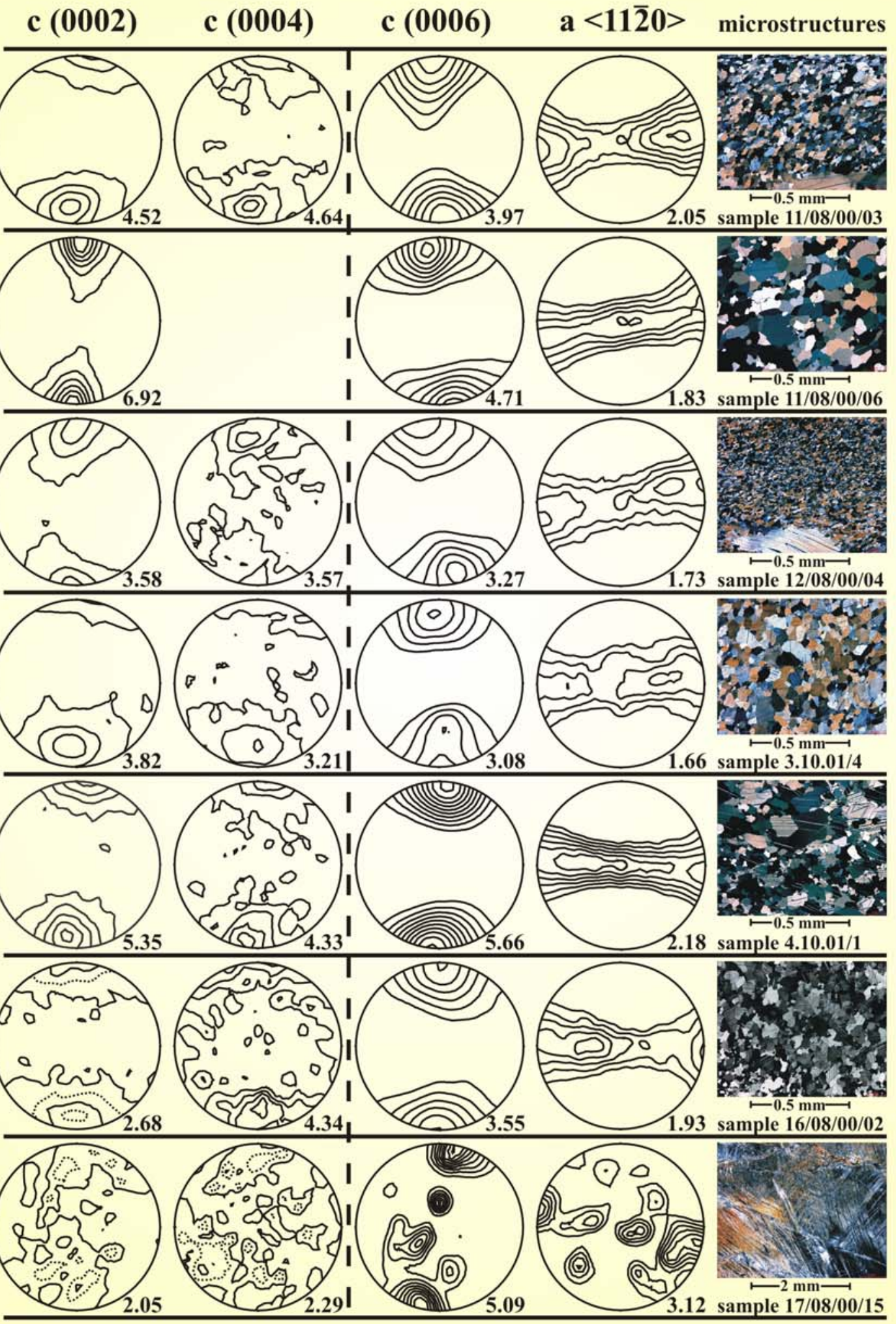




\section{Graphite}

\section{Calcite}

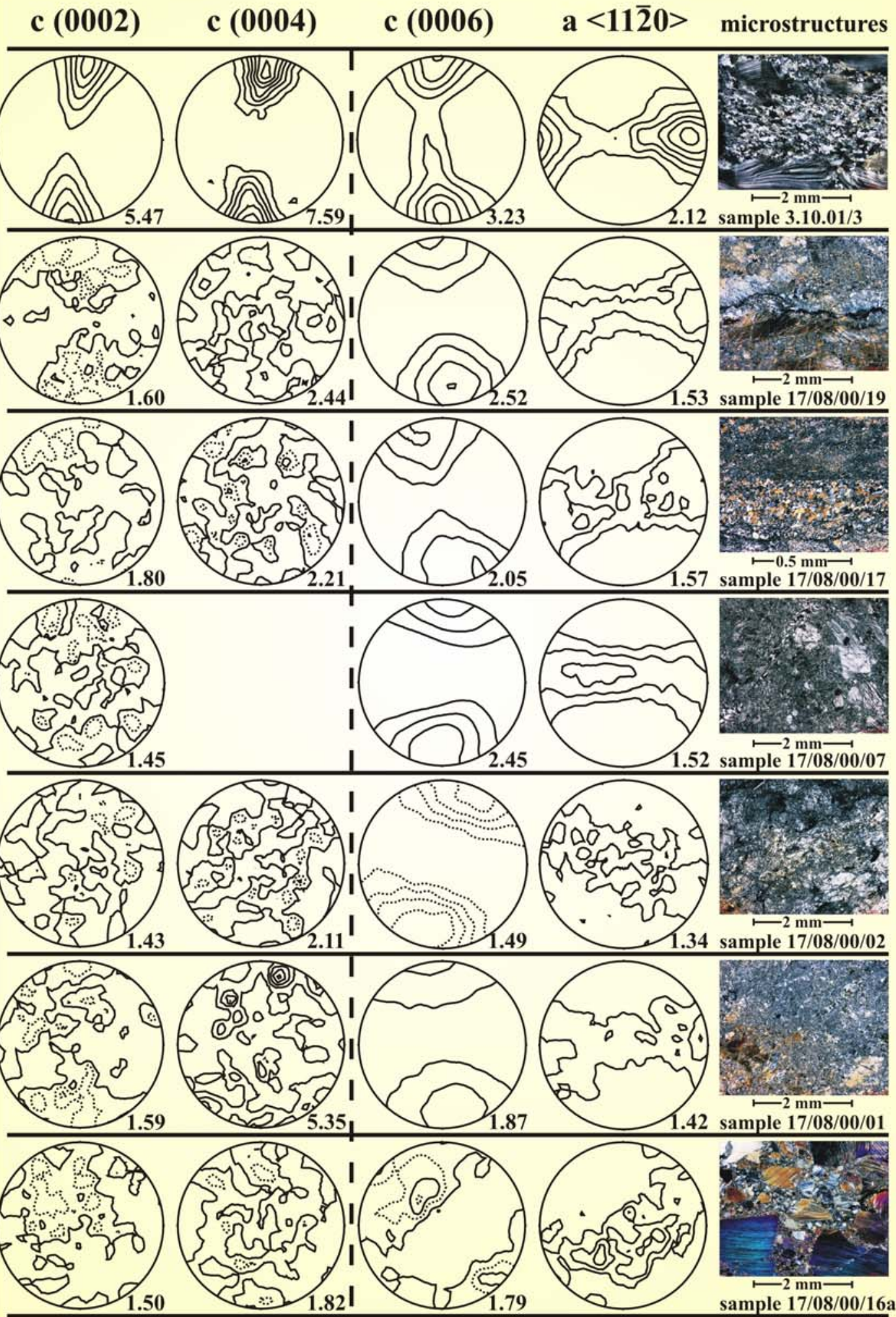




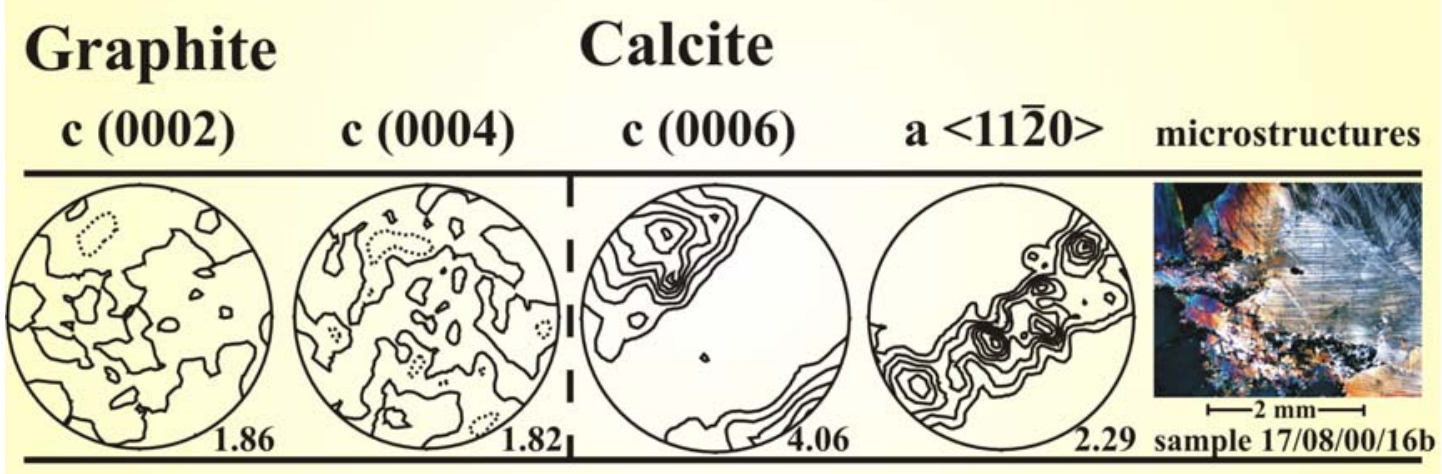

Fig 2.15 Graphite and Calcite textures of mylonitically and brittle-ductile deformed graphite-bearing marble. The graphite c(0004) pole figures are missing where the peak intensities were to low to extract the pole figures. Sample 17/08/00/16a represents the matrix of a cataclastic breccia zone, whereas sample 17/08/00/16b is derived from a macroscopic clast of the cataclastic breccia zone.

a strong influence of the cataclastic and pressure solution deformation in these samples. Sample 3.10.01/3 however is characterised by a strong influence of mylonitic deformation and therefore by dynamic recrystallisation. Therefore a strong texture is observable in this sample from the brittle-ductile boundary zone.

From the texture analysis of the investigated samples, it is not possible to evaluate whether the mutual influence between the calcite and graphite textures is existent. In the samples from the brittle-ductile boundary zone this influence is not existent as the graphite textures show no to very weak preferred orientations. In the mylonitic samples and the mylonitically overprinted sample of the brittle-ductile zone a girdle maximum is either existent in both phases or only in one of both phases. Also the texture intensity of both phases is not directly related, although a dependence of the texture intensity of both phases may is also observable in the samples.

\subsubsection{TEXTURE ANALYSIS BY ROTATING POLARIZER STAGE}

The rotating polarizer stage microscope enables the 2-dimensional measurement of crystallographic orientations in microscopic scales. These are local textures allow qualitative texture measurements on a grain scale within different domains of a sample. With these measurements the degree of homogeneity of the global textures of a sample can be evaluated. For the graphite-bearing marbles these investigations were conducted to verify, whether the texture is different between the coarse-grained and the fine-grained 
domains of the matrix. Secondly this method was used to measure the texture of the individual porphyroclasts in the samples of the mylonitic core zones.

\subsubsection{ANALYTICAL TECHNIQUE}

The applicated rotating polarizer stage was designed by Fueten \& Goodchild (2001). It is mounted on a standard petrographic transmitted light microscope. Therefore, normal polished thin sections for quartz and ultrathin sections $(<15 \mu \mathrm{m}$ thickness) for carbonates are suitable for the measurements.

The microscope of the rotating polarizer stage was modified to include two rotating polarisers, which are oriented $90^{\circ}$ to each other. The sample position is fixed between the rotating polarisers, which enables the gathering of information for every pixel in a stable position. The rotating polarisors are computer-controlled and at incremental steps a frame is captured by a video device and a frame grabber card. The different frames are then piled to form a composite picture of the thin section. This composite data set image (see upper images in Figures 2.16 and 2.17) contains the information of every pixel for every step. The maximum resolution is $0.9^{\circ}$ per step. Therefore, 200 steps are needed for a rotation of the polarisers of $180^{\circ}$ at maximum resolution.

For uniaxial minerals like quartz and calcite, the maximum intensity gathered during the rotation is a function of the angle between the light path of the microscope and the c-axis. This information is then used to calculate the trends of the c-axes, either per grain or across a grid for the composite data image as shown in the second image of Figure 2.16 and 2.17. The principles of the rotating polarizer stage are described by Fueten \& Goodchild (2001) and Heilbronner (2000).

This data is then extracted and plotted into a pole figure of equal area projection as shown in Figures 2.16 and 2.17. As it is not possible to gain the plunge of the c-axis with this method, the measurements are displayed as 2-dimensional vectors. These are plotted as linears in the equivalent pole figure. One half of a pole figure is sufficient to cover the whole range of directional data. In the pole figures of the data from the rotating polarizer stage a circular array of about 5 to $10^{\circ}$ is visible, where no data is plotted. This is due to normalisation problems. Likewise a tendency of distortion of the data along small circles is observable in the data.

The software of the rotating polarizer stage also enables the plot of an 'Achsenverteilungsanalyse (AVA)', which is shown in the third image of Figures 2.16 and 2.17. In these plots, the direction of every pixel is coloured according to a predefined 


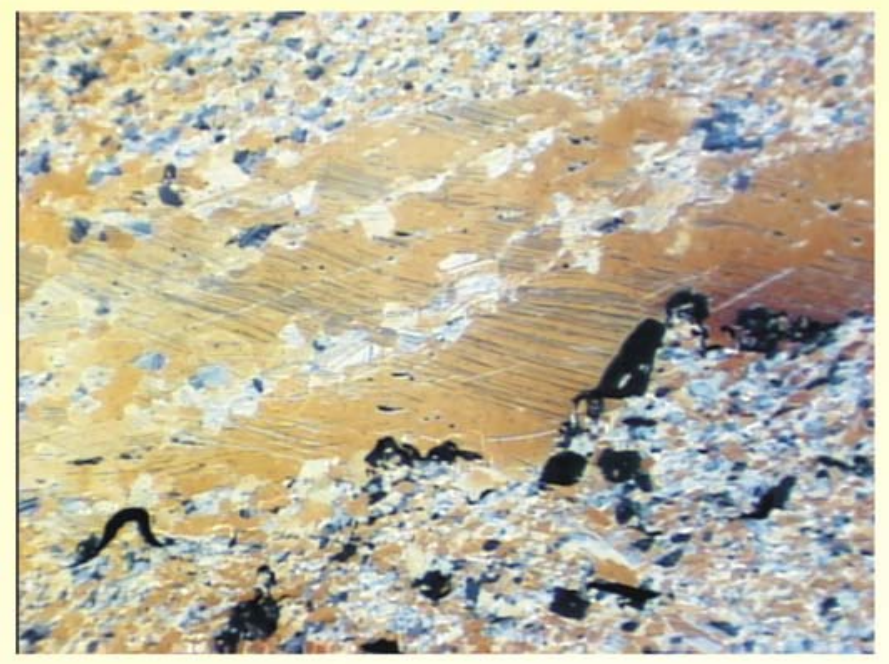

\section{sample 12/08/00/04}

bulk texture by neutron diffraction:

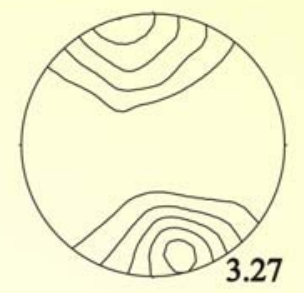

clast with surrounding matrix as shown on the left:
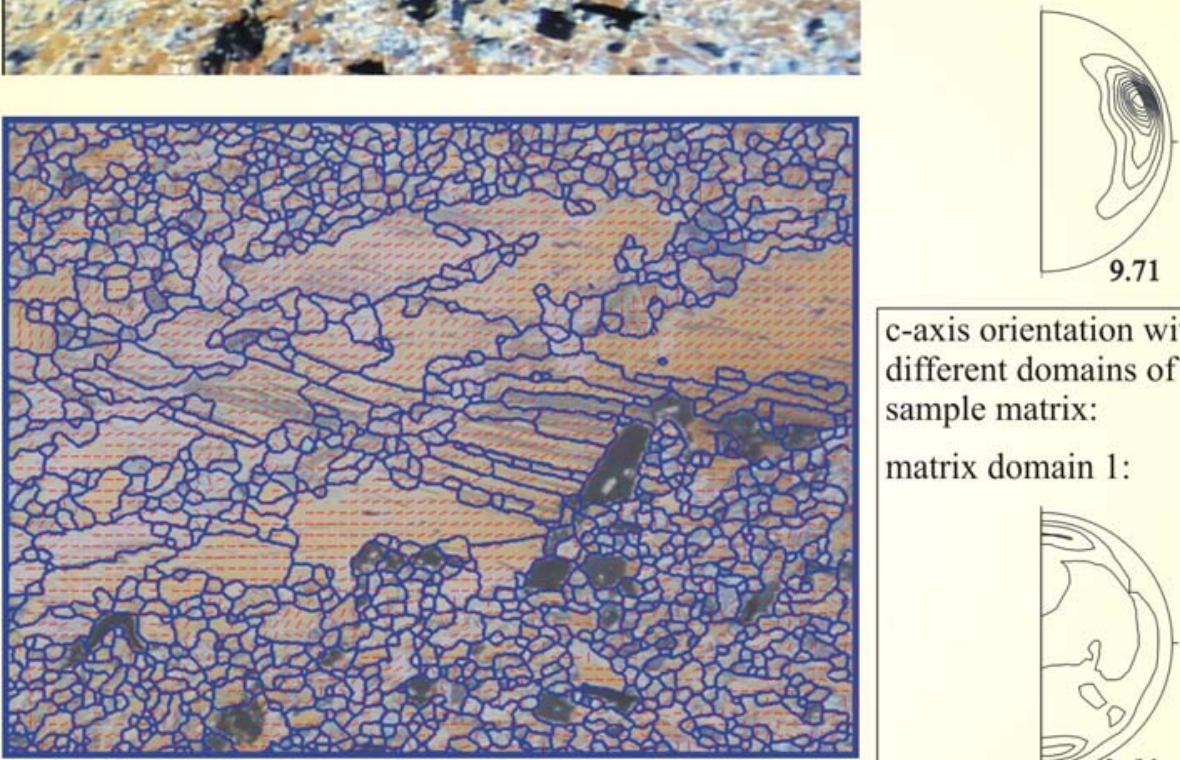

c-axis orientation within different domains of the sample matrix:

matrix domain 1:
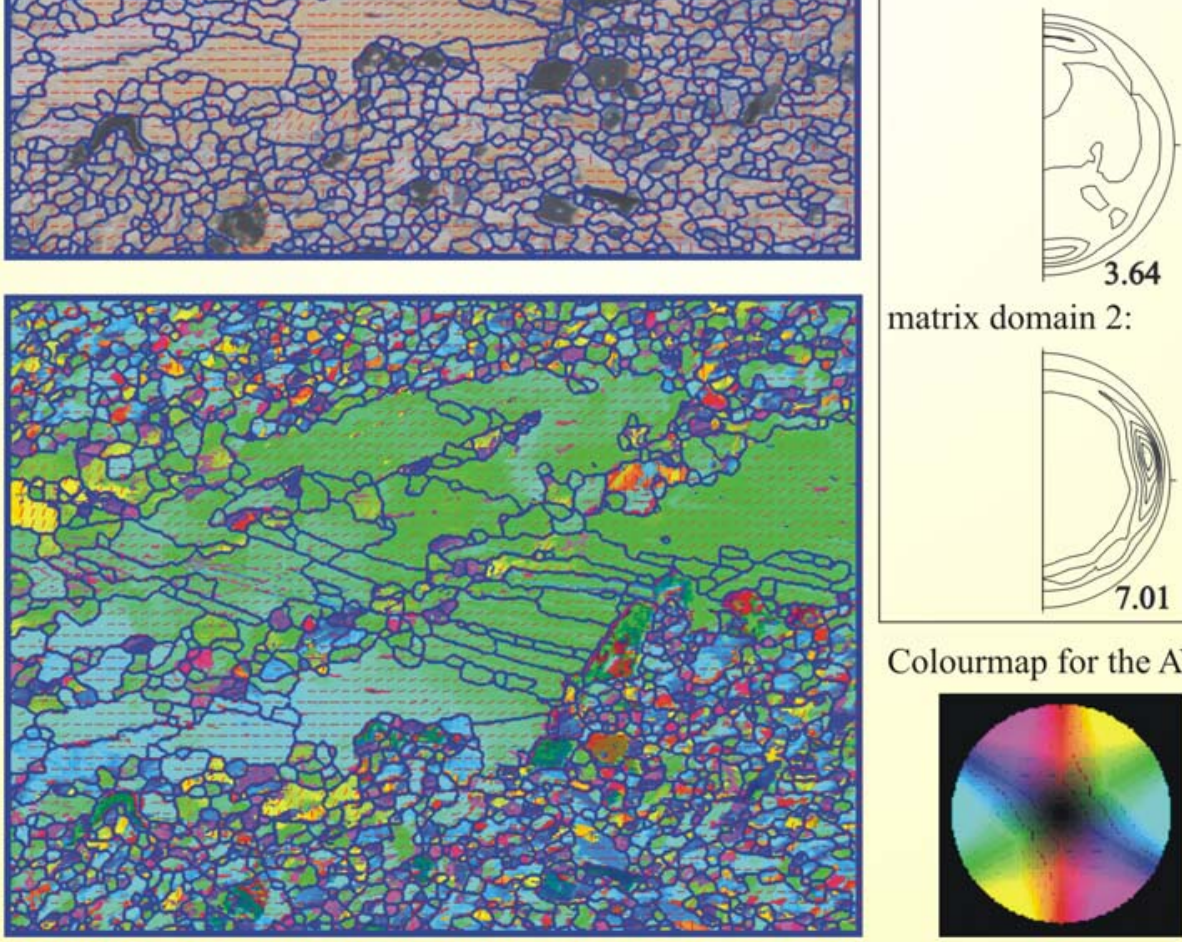

matrix domain 2:

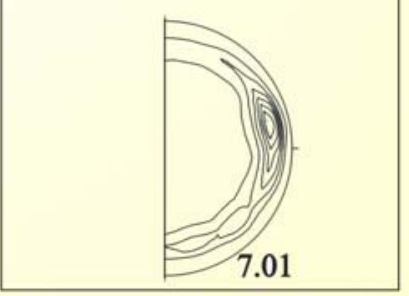

Colourmap for the AVA:

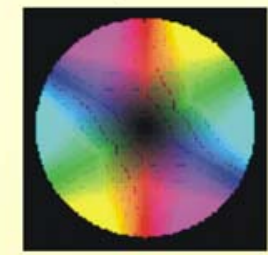

Fig 2.16 Rotating polarizer stage data shows the heterogeneities in c-axes orientation of this mylonitic sample within the matrix and the porphyroclasts. 


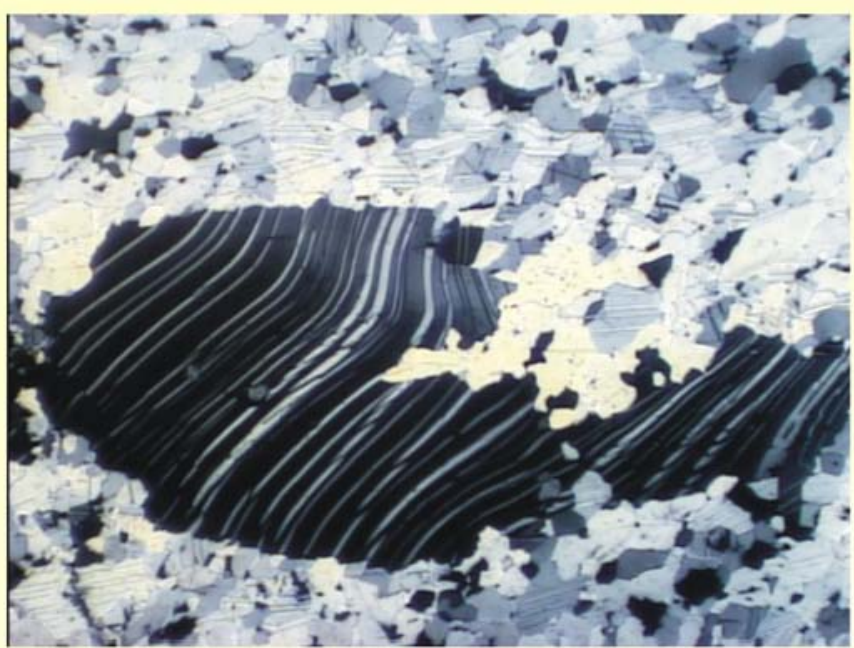

\section{sample 11/08/00/03}

bulk texture by neutron diffraction:

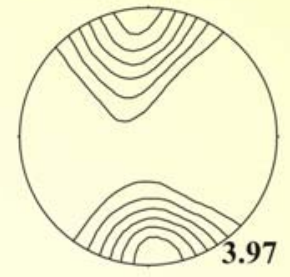

clast with surrounding matrix as shown on the left:
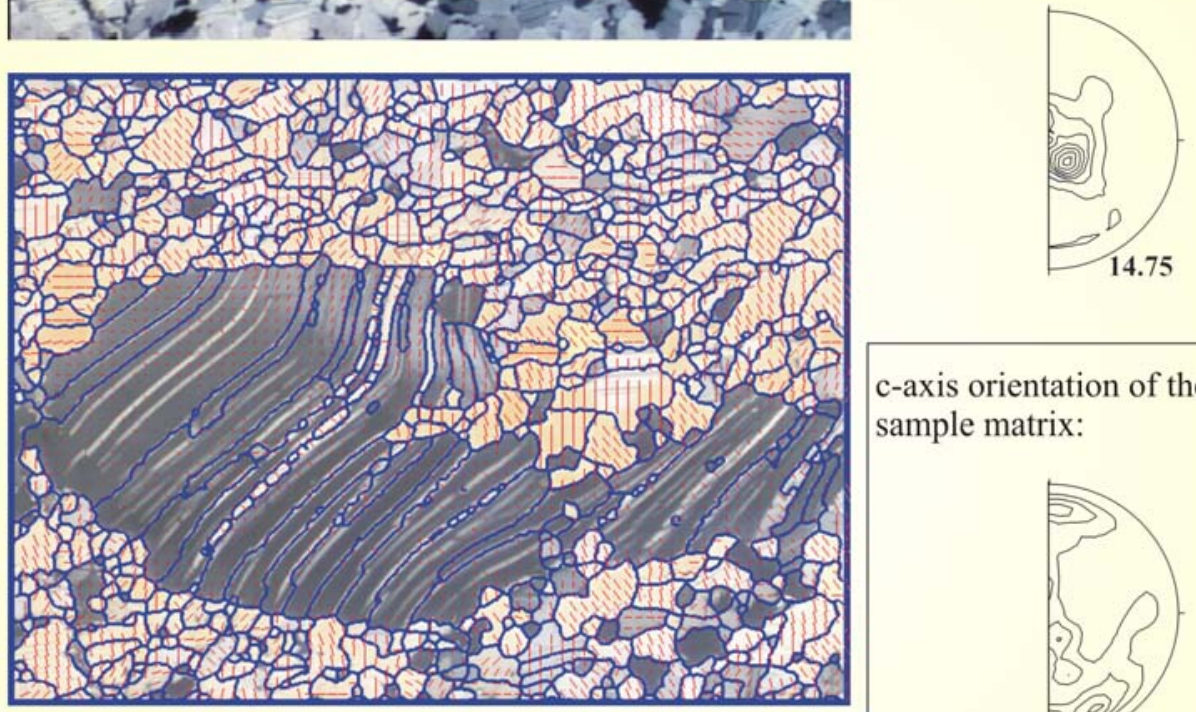

c-axis orientation of the sample matrix:
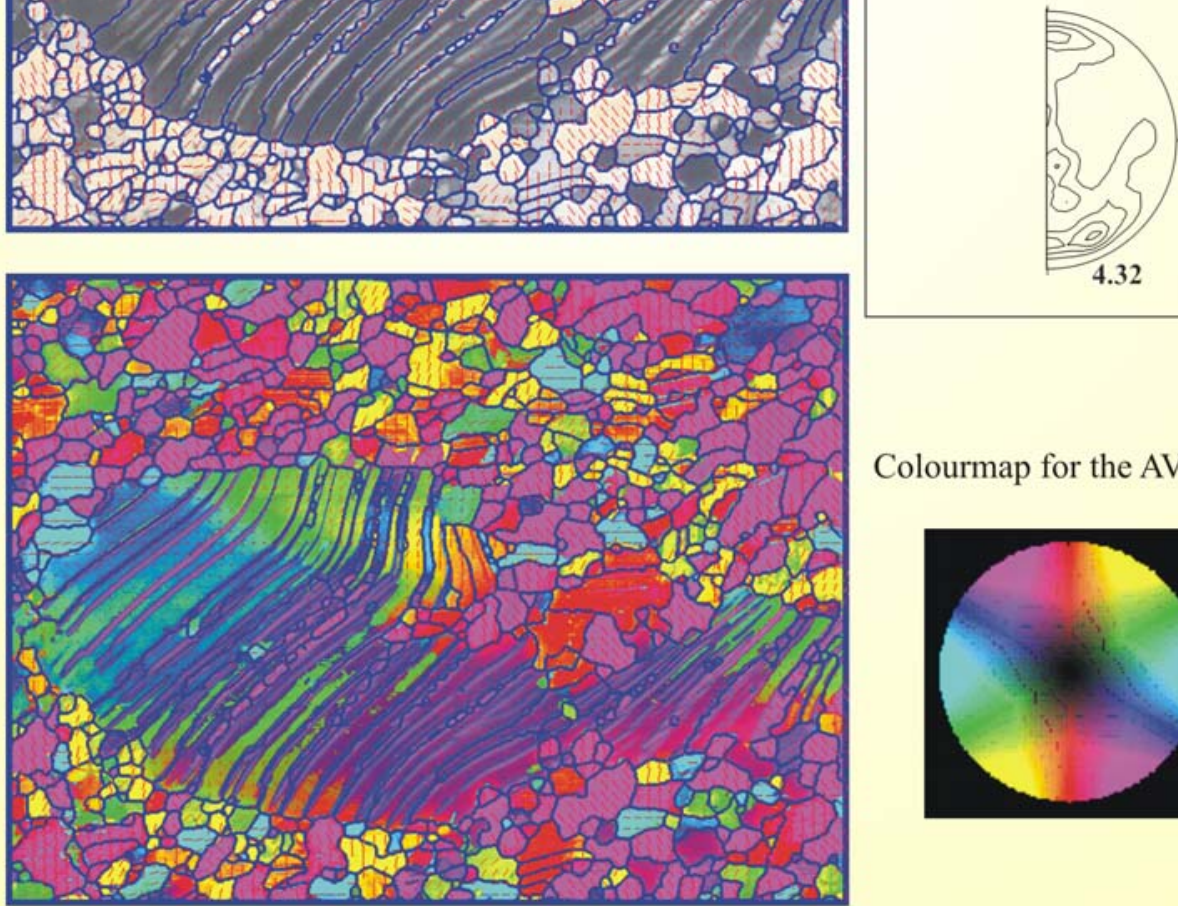

Colourmap for the AVA:

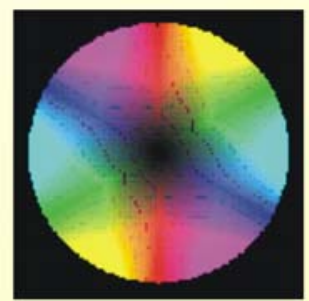

Fig 2.17 Rotating polarizer stage data showing a homogeneous texture for the mylonitic matrix of this sample. The porphyroclasts show heterogeneities due to the the development of twins, deformation bands, subgrain rotation crystallisation and crystallisation to new grains. 
colour map of orientations (Fig. 2.16, 2.17). This data can be statistically processed and gives an approach on the orientation distribution of the c-axes.

\subsubsection{SAMPLES AND MEASUREMENTS}

Complementary to the bulk textures shown above, two samples are investigated to evaluate the differences in the local textures that may occur between different domains within the mylonitic core zones. As shown in Figure 2.16 the bulk texture for this sample obtained by neutron diffraction shows one single maximum normal to the foliation. However, the investigations by the rotating polarizer stage revealed, that the c-axes orientation shows heterogeneities within the matrix and between coarse-grained domains and the fine-grained matrix.

As shown in Fig. 2.16 the matrix of domain 1 shows maxima, which coincide with the textures of the neutron diffraction data. The matrix of domain 2 however, shows a maximum nearly perpendicular to the maxima of domain 1 . For this domain the preferred orientation of the c-axes is parallel to the foliation. Other heterogeneities within the textures of the mylonitic samples are produced by porphyroclasts as shown in the images of Figure 2.16 and 2.17.

The c-axes pole figures of the porphyroclasts and their surrounding matrix show, that there is a distinct deviation to the bulk neutron diffraction textures (Fig 2.16 and 2.17). In Figure 2.16 the texture of the porphyroclast is similar to the texture in the matrix domain 2, whereas they differ completely in Figure 2.17. The AVA shows the existence of pronounced heterogeneities of the texture within a porphyroclast. These result from twinning, subgrain rotation recrystallisation, recrystallisation along twins and the development of partially bent deformation bands (Fig 2.16 and 2.17).

In contrast to the sample in Figure 2.16 the sample of Figure 2.17 shows a uniform texture in the matrix which is similar to the bulk textures measured by neutron diffraction. Even the recrystallised domains of the porphyroclast show textures corresponding to the bulk textures. As described above, the only texture heterogeneities of this sample are resulting from different orientations of $\mathrm{c}$-axes of the porphyroclasts.

\subsection{DisCUSSION}

From the structural and textural analysis of the investigated samples it is obvious, that in general three different fabric types of graphite-bearing marbles can be distinguished. These occur within crustal dome structures of the northern Central Zone of the Damara Belt. After the formation of the structural domes by the refolding of crustal 
scale isoclinal folds, another type of dome structures formed by the intrusion of mainly granitic melts. Subsequently a widespread regional phase of static recrystallisation occurred. During this phase of recrystallisation, a blastic fabric developed during the abnormal grain-coarsening of large domains of the calcite-marble.

As shown above, the peak metamorphism and the intrusion of the granitic magma coincide quite well in the geochronological data. The postulated regional peak metamorphism of upper amphibolite facies to granulite facies cannot be obtained from the investigated samples, as muscovite is the only mineral sensitive to metamorphic changes. Muscovite only occurs in the investigated samples as accessory mineral and is still stable in the marble, allthough it should not be stable in the described metamorphic conditions. This is related due to the absence of quartz in the marble host rock, which is needed for the dissociation reaction of muscovite.

In retrograde metamorphic conditions, this grain-coarsened marble was then deformed along the reactivated rims of the dome structures. Within the distinct shear zones purely ductile deformation occurred in the mylonitic core zones and brittle-ductile deformation occurred in the boundary zones of the shear zones.

Figure 2.18 shows a summarised overview of the different macroscopic and microscopic fabrics observed in the shear zones. It follows the macroscopic setup of the shear zones, showing the different macroscopic fabrics and the equivalent microfabrics. The fabrics of the brittle-ductile boundary zone vary lateral and normal to the mylonitic core zone in a complex pattern of the fabrics shown in Figure 2.18.

Therefore, the brittle-ductile boundary zones show a complex and heterogeneous setup within the shear zones. It is obvious that the different microstructures described above occur in every deformational domain of the brittle-ductile boundary zone. The only significant difference in microstructures between the different deformational domains of this zone is the grain size of the calcite crystals. The above described complex microstructural pattern comprises of brittle and crystal plastic deformational mechanisms. The observed brittle deformational mechanisms are kinking and crystal fracturing, whereas crystal-plastic deformational mechanisms caused polygonisation, the development of deformation bands, subgrain rotation recrystallisation, and ultra fine-grained dynamic recrystallisation.

It is obvious from the macroscopic and microscopic deformational fabrics that a multiple progression of deformation and recovery has occurred in the investigated shear zones. In the brittle-ductile boundary zone brittle deformation and pressure solution prevail 


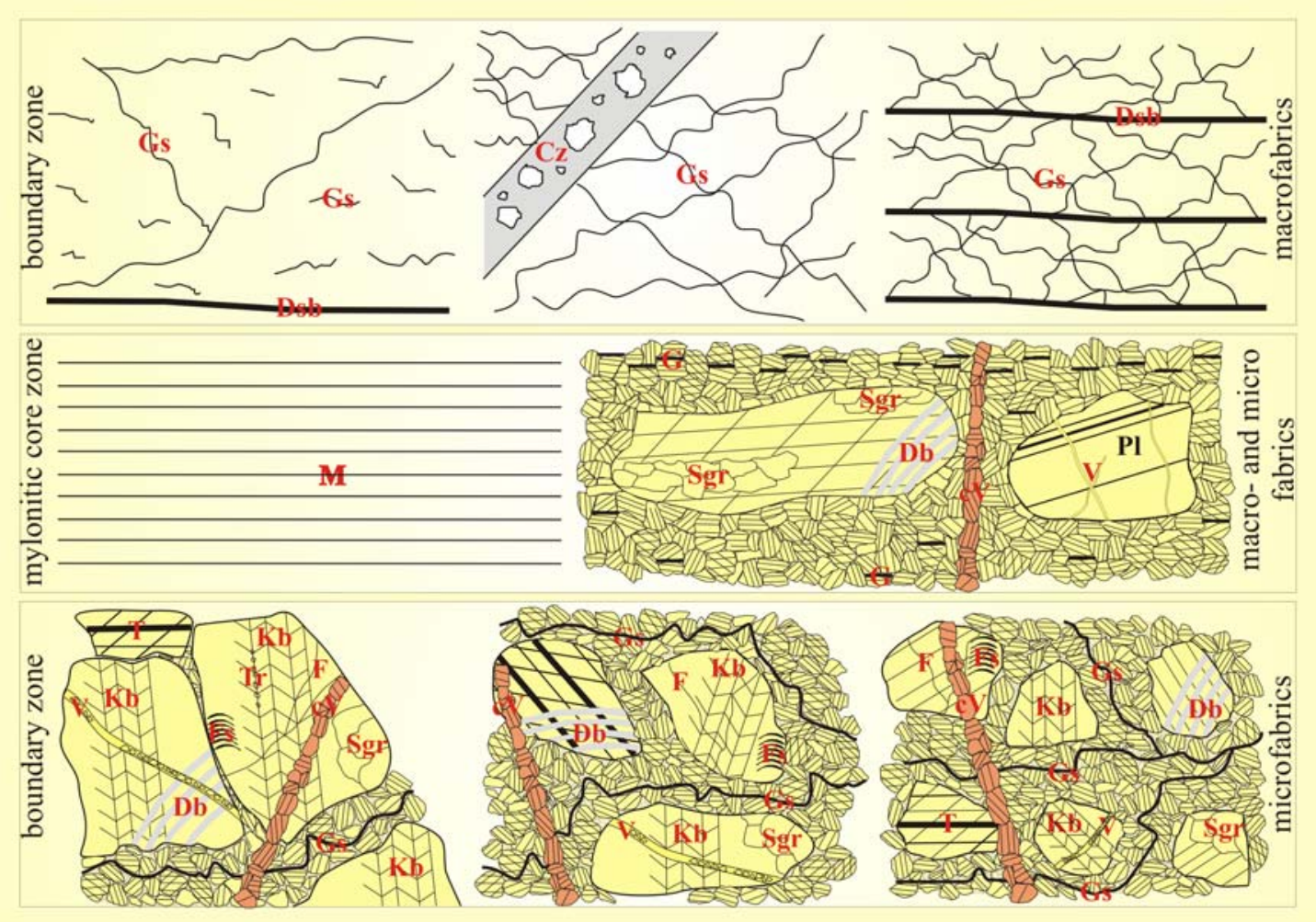

Fig. 2.18 Fabrics and microfabrics of the investigated shear zones. In the center is the mylonitic core zone, with graphite on the foliation plains. The upper part shows the fabrics of the brittle-ductile domain and in the lower parts the microfabrics of this domains are shown: $\mathrm{cV}=$ cretaceous vein; $\mathrm{Cz}=$ cataclastic zone; $\mathrm{Db}=$ deformation bands; $D s b=$ ductile shear band; $F=$ fracture; $F$ s $=$ flexural slip with thickend calcite twins; $G$ $=$ graphite with basal plane parallel to the foliation; $G s=$ graphite stylolite in fine grained matrix; Kb= kink bands; $M=$ mylonite; $S g r=$ sub grain rotation; $T=$ twins; $T r=$ finegrained recrystallisation of twins; $V=$ veins.

in different intensities. This is observable in the described macroscopic fabrics. These fabrics themselves are overprinted either by cataclastic breccia zones or by mylonitic deformation. This is observable in several cycles of mutual overprinting. In the microstructures brittle and ductile deformational fabrics are preserved next to ultra finegrained static recrystallisation of the calcite matrix.

Pressure solution is restricted to the shear zone as stylolites are not observed in the undeformed and abnormally grain-coarsened host rock. Therefore, pressure solution was enabled by the deformation of the calcite-marble along the rims of the dome structures. Pressure solution and the resulting formation of graphitic stylolites, is mutually overprinted by the brittle-ductile deformation. The graphitic stylolites are cut by cataclastic breccia zones as shown in Figure 2.18. 
Additionally the stylolites oriented parallel and subparallel to the foliation of the mylonitic core zone, develop into ductile shear bands, which themselves partly become overprinted by pressure solution again. These observations imply, that the different domains within the boundary zones do not show a successive deformation but are only related to differences in strain and strain rate within the shear zones. These occur all in the same timeframe of mutual overprinting of brittle, ductile and pressure solution deformation.

The mutual overprinting of brittle, ductile and pressure solution deformation in different domains of the brittle-ductile boundary zones, may be explained by seismicaseismic deformation within these shear zones. During seismic events, brittle deformation with high strain rates prevailed, where cataclastic breccia zones and brittle deformation fabrics developed in different deformational domains. These are distinguished by differences in deformation intensity. During aseismic deformation ductile deformation has been dominant, which is documented in the mylonitic core zones.

These mylonitic core zones have not been overprinted by the brittle deformation of the brittle-ductile boundary zones. Nevertheless, the ductile deformation in the brittleductile boundary zones is clearly related to the deformation in the mylonitic core zones. This is documented by the graphitic stylolites, which have been overprinted by ductile deformation parallel to the foliation of the mylonitic core zone. Therefore, the deformation structures of the brittle-ductile boundary zone are related to brittle seismic deformation and aseismic creep deformation, which mainly has occurred in the mylonitic core zones.

The quantitative calcite-graphite ratios imply that pressure solution has occurred for calcite and graphite. This may only be considered as a first assumption, as the amount of investigated samples, does not give a secure statistical basis for a safe interpretation of the data. Therefore, more investigations in that direction are necessary to confirm this assumption. Nevertheless, thermodynamic conditions for the solution of graphite as described by Holloway (1984) and Luque et al. (1998) may have been existent during the deformation.

These first investigations of the quantitative calcite-graphite ratios showed, that it is not possible to quantify the content of graphite and thus the graphite network intensity by macroscopic optical measurements. The obtained data revealed that the fine-grained to ultra fine-grained domains also occur in dark colours, which are in parts not differentiable anymore from purely graphitic stylolite seams. Also inside the graphitic stylolites ultra fine-grained graphite is present, which is not detectable by macroscopic optical measurements. 
The calcite textures prevailing in the mylonitic core zones as well as in the brittle-ductile boundary zones, are of the most common calcite texture type which is characterised by a single maximum normal to the foliation. The textures imply pure shear deformation during ductile deformation. The texture intensities are generally very high and decrease commonly with decreasing grain sizes. This observation applies for the brittle and ductile deformed marble. The host rock for this deformed marble is the abnormally graincoarsened and blastic graphite-bearing marble described above as type 1 . The texture of this marble may only be evaluated in the field, as it is to coarse-grained to measure a statistically relevant texture from the samples.

The grain sizes of the graphite-bearing marbles are continuously reduced by brittle and ductile deformation. This is obvious from the microstructural analysis. In the brittle-ductile boundary zone the texture intensity may be decreased by the reorientation of the grains during cataclastic deformation. This is most drastically observable in the samples $17 / 08 / 00 / 16 a$, which is the matrix of a cataclastic breccia zone, whereas a clast of several $\mathrm{cm}$ in size (sample 17/08/00/16b) still shows high calcite texture intensities (Fig. 2.14, 2.15).

As texture intensities are normally increased during dynamic recrystallisation in ductile deformed material, this implies that the host rock must have preserved strong texture intensities during the period of abnormal grain-coarsening and static recrystallisation. In this case the texture of the grain-coarsened host rock must be related to the prior formation of the dome structures and/or to the widespread intrusion of the granitic melts. From this data it is obvious that the conserved textures are similar to equal to the textures measured in the mylonitic core zones. This may imply that the pre-existing stress field was similar to the retrograde stress field, which led to the reactivation of the rims of the dome structures and thus the formation of the shear zones.

The investigation of the local textures showed that some mylonitic core zones partially show different texture domains. Whether this is related to the different generations of textures, or to differences within the ductile deformation in the shear zones is not clear and needs further investigation. The porphyroclasts often show a different crystallographic orientation than the local textures of the matrix or the overall global textures. This may be the reason for the extensive recrystallisation and subgrain rotation recrystallisation.

From the microstructures it is hard to evaluate the metamorphic temperatures of this deformation. As shown from the microstructures of the brittle-ductile deformed marble, crystal plastic and brittle deformational structures are mutually overprinted. They range from the development of kink bands to fine-grained dynamic recrystallisation along twins 
often within one sample of the investigated shear zones. In the cataclastically deformed domains, the ultra fine-grained calcite breccia is only slightly recrystallised along the grain boundaries. As the development of all the observed microstructures are depending on the temperature as well as on the applied strain rate, an estimation of the temperature becomes very difficult (De Bresser \& Spiers, 1997).

Some authors postulate temperature estimates from the thickness of the calcite twins (Burkhard, 1993; Passchier \& Trouw, 1998). As the development of calcite twins is also strongly dependent on the strain rate (De Bresser \& Spiers, 1997), this estimate may only be true at constant strain rates under varying metamorphic temperatures. In the investigated samples however, the multitude of different deformational microstructures imply a complex sequence of deformational mechanisms and different strain rates.

The granulite facies is not directly documented by HP/HT mineral assemblages. However, the regional metamorphic isogrades and the intrusion of granitic melts imply these metamorphic grades as described above. Missing fluid inclusions and no differences in cathodoluminescence colours show, that granulite facies conditions may have prevailed during peak metamorphism. This metamorphic event is also connected to a period without strain in this part of the lower crust, because the abnormaly grain-coarsened calcite crystals have been statically recrystallised on a regional scale.

The described twins in host rock are partially recrystallised in the orientation of the host crystal by crystal diffusion annealing. These twins could be either related to the seismic events and have been recrystallised while aseismic creep deformation continued, or have developed due to some post-Damara events, like the cretaceous break-up of Gondwana between Africa and South America.

As compiled by Soula et al. (2001), several models for the formation of dome structures are proposed in the literature. The mode of formation of the dome structures, which host the investigated shear zones is not clear. If their formation had been related to a crustal shear zone, as some of the models imply, this would give some implication about the source of the seismic events. Ritter et al. (2003) and Weckmann et al. (2003) postulated the anomalies of electrical conductivity, to occur at distinct crustal shear zones like the Omaruru Lineament (OmL) and the Autseib Fault further north (see Fig. 4.2).

From the satellite image in Figure 2.2 however, it is observable that there is no distinct shear zone developed which could be a crustal structural lineament of the Damara Orogen. This is also confirmed by own field investigations, which show that the investigated shear zones are only a regional set of (conjugated) shear zones along the rims 
of the dome structures. Therefore, the investigated shear zones do not correspond to one single deep crustal shear zone. They rather represent a regional set of shear zones, which are controlled by the crustal dome structures.

Since the dome structures are built up by Damara sediments of the Arandis and Karibib Formation, they have to be allocated to the $\mathrm{nCZ}$ according to the definition of the OmL by Miller (1983). Therefore, these shear zones may not be correlated with the OmL, as the $\mathrm{OmL}$ is defined to separate the basement level dome structures of the $\mathrm{SCZ}$ from the higher stratigraphic levels of the $\mathrm{nCZ}$. The basement level dome structures of the $\mathrm{sCZ}$ occur south of the area of the magnetotelluric profile measured by Ritter et al. (2003) and Weckmann et al. (2003). This implies, that the OmL has to be postulated south of the investigated area.

Due to these uncertainties, the source of the seismic events is still a matter of further research and investigation of the structural setting of the various structural domes. Furthermore, the relationship to the Autseib Fault, which is postulated north of the investigated area and the Omaruru Lineament will have to be investigated in more detail, regarding the source of the seismic events in the late Damaran stage of Orogeny. 


\section{CHAPTER 3}

\section{GrAPHite CRYSTALLinity MEASUREMENTS}

To obtain implications on the formation of different graphite fabrics, crystallinity measurements of graphite have been conducted. The crystallinity of graphite is either measured by High Resolution Transmission Electron Microscopy (HRTEM), X-ray Diffractometry (XRD) or by Raman spectroscopy. For this work, the determination of graphite crystallinities were focussed on Raman spectroscopy, as the calculation of the graphite crystallinities from the Raman spectra has been generally calibrated with HRTEM and XRD data (Tuinstra \& Koenig, 1970; Beny-Bassez \& Rouzaud, 1985; Wopenka \& Pasteris, 1993). The aim of this work is to investigate the graphite crystallinities of the whole range of microstructural types of graphite-bearing marbles.

\subsection{Analytical TeChniQues}

Raman spectroscopy is based on the Raman Effect, which originates from the scattering of electromagnetic radiation by the electron sheaths of the investigated molecules. The Raman Effect is virtually independent of the wavelength of the radiation beam. Therefore, a monochromatic laser beam is used for Raman spectroscopy. Approximately $99.99 \%$ of the laser beam radiates through the sample, while a small part is scattered elastically by the molecules (Rayleigh scattering). An even smaller part (about $10^{-6} \%$ ) is scattered inelastically, which is referred to as the Raman scattering. This Raman radiation is emitted either in longer (Stokes radiation) or in shorter wavelengths (Antistokes radiation) than the original wavelength of the laser beam. This difference in frequency is expressed by wave numbers relative to the original frequency of the laser beam. The original frequency is therefore calibrated to zero. This Raman shift is specifically characteristic for different molecules.

The Raman shift for carbonaceous material (CM) is divided into first and second order regions after Tuinstra \& Koenig (1970). The measured first-order region is shown in Figure 3.1. The first-order region lies in the range of 1100 to 1800 wave numbers $\left(\Delta \mathrm{cm}^{-1}\right)$, and the main graphite band - the $\mathrm{G}$ band - is at $\sim 1582 \Delta \mathrm{cm}^{-1}$. This band is inherent in graphite lattices. For more poorly crystalline graphite additional bands are recognisable at $\sim 1150 \Delta \mathrm{cm}^{-1}$, at $\sim 1355 \Delta \mathrm{cm}^{-1}$, at $\sim 1500 \Delta \mathrm{cm}^{-1}$ and at $\sim 1622 \Delta \mathrm{cm}^{-1}$. The band at $\sim 1355 \Delta$ $\mathrm{cm}^{-1}$ is referred to as the main defect band (D1-band) (Fig.3.1). This band occurs when defects are present in the carbon aromatic structure (Beny-Bassez \& Rouzaud, 1985). It is also sensitive to graphite intercalations (Dresselhaus \& Dresselhaus, 1982). The $1150 \Delta$ 


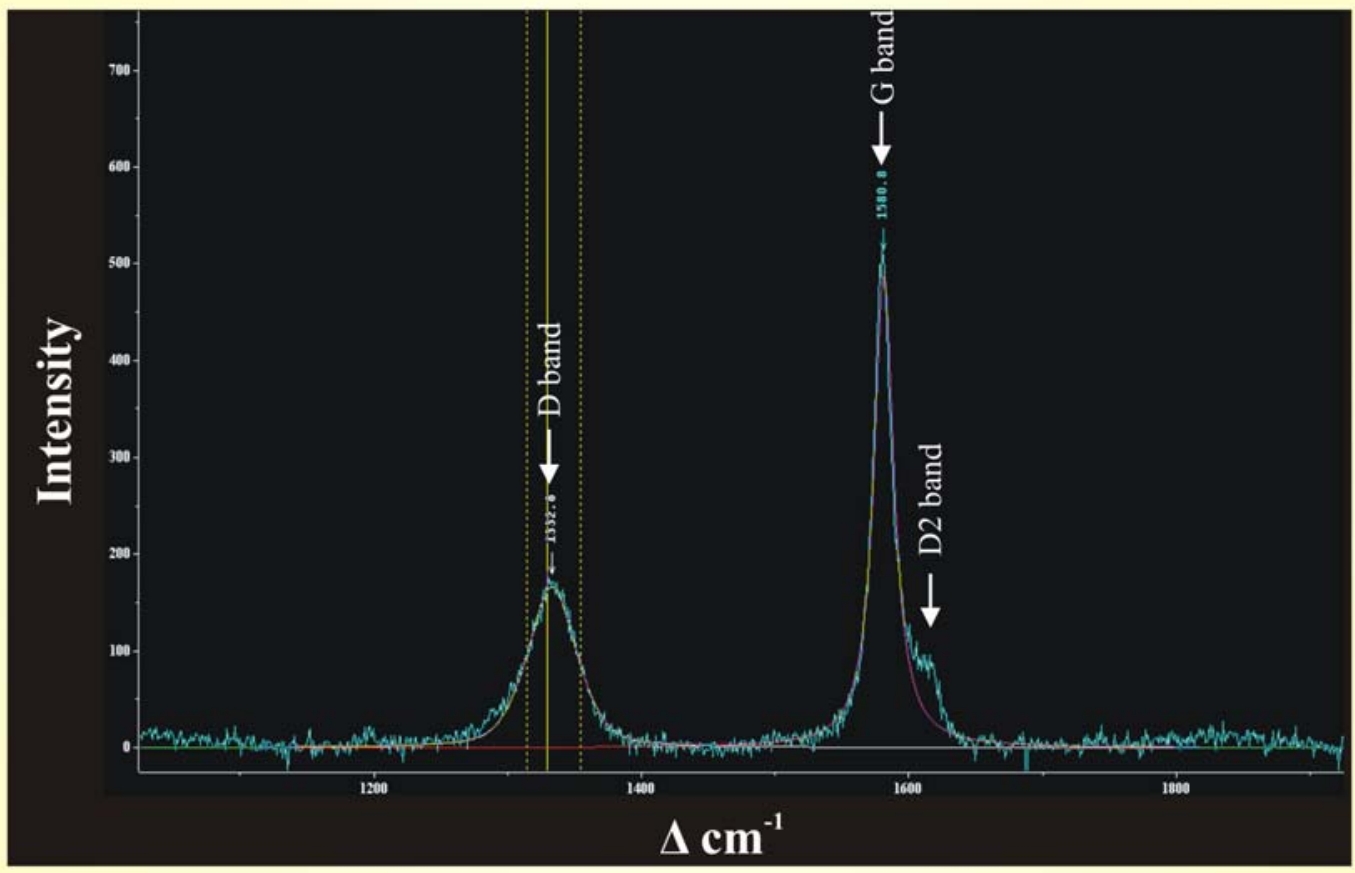

Fig. 3.1 First-order Raman spectrum measured from sample 17/08/00/20. It shows the Raman graphite band ( $G$ band), the first deffect band ( $D$ band) and the shoulder peak on the $G$ band. The D band and the shoulder peak are absent in highly crystallised graphite.

$\mathrm{cm}^{-1}$ band appears only in very poorly organised CM (Beyssac et al., 2002). Also the band at $1500 \Delta \mathrm{cm}^{-1}$ (D3 band) is attributed to defects outside the plane of aromatic layers like tetrahedral carbons (Beny-Bassez \& Rouzaud, 1985). It occurs as a wide band in poorly crystallised CM (Beyssac et al., 2002). The D2 band at $\sim 1622 \Delta \mathrm{cm}^{-1}$ appears as a shoulder peak of the $\mathrm{G}$ band and is also absent in highly crystalline graphite (Fig. 3.1).

The second order region from 2200 to $3400 \Delta \mathrm{cm}^{-1}$ includes several bands at $\sim 2400$ $\Delta \mathrm{cm}^{-1}$, at $\sim 2700 \Delta \mathrm{cm}^{-1}$, at $\sim 2900 \Delta \mathrm{cm}^{-1}$ and at $\sim 3300 \Delta \mathrm{cm}^{-1}$, depending on the degree of graphite crystallinity. The S1 band at $\sim 2700 \Delta \mathrm{cm}^{-1}$ splits up into two bands at high crystallinities. It is therefore the most important indicator band for graphite crystallinities in the second order region.

In general, Raman spectra for well crystallised graphite include the existence of D1 and D2 bands in the first order region (Wopenka \& Pasteris, 1993). To estimate the degree of graphite crystallinity, it is important to reveal some information about the variation of the $G$ band position, about the full widths at half maximum of the $G$ and D1 bands and about the intensity ratios between D1 and G. This intensity ratio can be used to quantify the crystallinity of graphite according to the linear relationship between the D1/G intensity 
ratio and $10^{3} / \mathrm{L}_{\mathrm{a}}$. $\mathrm{L}_{\mathrm{a}}$ quantifies after Tuinstra \& Koenig (1970), the crystallinity of graphite. It quantifies the mean basal plane diameter of graphite parallel to (001) and is expressed in $\AA$.

The Raman spectroscopic measurements were conducted at the laboratory of the Institut für Mineralogie at the Museum für Naturkunde, Humboldt-Universität Berlin. A Dilor LabRam spectrograph was used equipped with a Jobin Yvon detector and a notch filter to avoid the Rayleigh scattering. This Raman spectograph is equipped with an optical microscope with a 50x objective to focus the laser with an exciting line of $632.8 \mathrm{~nm}$. The grating of 1800 grooves $/ \mathrm{mm}$ produces an according spectral resolution of about $2.5 \Delta \mathrm{cm}^{-1}$. The confocal pinhole in front of the entrance slit of $100 \mu \mathrm{m}$ is $1000 \mu \mathrm{m}$ in diameter. The final laser power is about 20 to $50 \mathrm{~mW}$. Acqusition time for the spectra was generally $5 \mathrm{~s}$.

The measurements, background subtraction peak fits and calculations of the full widths at half maximum were conducted with Labspec V. 2.08 from Dilor. For peak fitting a Gauss-Lorentz function was used for all the samples. The fitted data represent the graphite crystallinities.

\subsection{SAMPLES MEASURED}

The samples for the crystallinity measurements were selected to cover all microstructurally distinguished types of graphite-bearing marbles from all the main sampling localities shown in Figure 2.1. The sample measurements plotted in Figure 3.2 are distinguished by plot numbers. Plot numbers 1 to 10 represent the type 2 brittle-ductile deformed graphite-bearing marbles with graphitic stylolites and network structures of varying intensity, thickness and orientation to the deformational framework. Samples 11 and 12 are type 3 mylonitic graphite-bearing marbles and samples 13 and 14 are type 1 coarse-grained marble host rock.

The graphite crystallinities were measured on graphite isolated from the marble host-rock as well as on graphite within the marble host-rock. This allows a control whether the processes of graphite isolation has changed the degree of graphite crystallinity. Measuring isolated graphite gives better statistics on the graphite crystallinities within a sample. As the Raman Effect varies with the crystallographic orientation, the measurements were conducted perpendicular to the basal plains of the graphite crystals where possible. This was especially difficult for the ultrafine-grained graphite of the brittle-ductile deformed boundary zone, which has been classified as type 2 of the graphitebearing marble. 


\subsection{RESULTS AND QUANTIFICATION}

The measurements in general revealed, that next to the inherent $\mathrm{G}$ band, only two of the above mentioned defect bands are recognisable in the measured samples. These are the D1 band at $\sim 1355 \Delta \mathrm{cm}^{-1}$ and the D2 shoulder band at $\sim 1622 \Delta \mathrm{cm}^{-1}$ in the first order region. Figure 3.2 shows the evaluated results of the Raman measurements on the described samples. The plots are distinguished between the measurements of the isolated graphite and of the graphite within the marble host-rock of the same samples. The density variation in the plots represent the statistical means and variations for every sample. The second order region has not been measured due to the setup of the Raman spectroscope.

The measured data shows in general, that the graphite crystallinities of all microstructural types are in the range of well-ordered graphite. In the following, a detailed description of the graphite crystallinities will be presented for the different methods of evaluating graphite crystallinities from Raman spectra.

A first approximation about the degree of graphite crystallinity is the $G$ band position and accordingly also the variation of this $\mathrm{G}$ band position within a sample (BenyBassez \& Rouzaud, 1985; Beyssac et al., 2002). For highly crystalline graphite the G band position should be at $\sim 1582 \Delta \mathrm{cm}^{-1}$ with a variation of about $20 \Delta \mathrm{cm}^{-1}$. Both authors state, that a decrease in wave numbers for the $\mathrm{G}$ band position occurs for mean wave numbers from $\sim 1590 \Delta \mathrm{cm}^{-1}$ to $\sim 1582 \Delta \mathrm{cm}^{-1}$ with increasing crystallinities. The $\mathrm{G}$ band position of poorly-ordered graphite varies about $50 \Delta \mathrm{cm}^{-1}$ (Beyssac et al., 2002).

The $G$ band positions of all measured samples are plotted into Figure $3.2 \mathrm{a}$. With the exception of sample 5, this plot shows that the mean position of the $\mathrm{G}$ band is constant at $1578 \Delta \mathrm{cm}^{-1}$ with a standard deviation of $5.5 \Delta \mathrm{cm}^{-1}$ for all samples. The variation within the samples is about $20 \Delta \mathrm{cm}^{-1}$ with the exception of sample 7, which shows a variation in $\mathrm{G}$ band position of $27 \Delta \mathrm{cm}^{-1}$. Graphite crystallinities of sample 14 are very high as no defect bands are recognisable for this sample across all measurements. The variation within the $\mathrm{G}$ band position is only $1 \Delta \mathrm{cm}^{-1}$ and the mean position is at $1580 \Delta \mathrm{cm}^{-1}$.

A second indicative parameter for the degree of graphite crystallinity is the full width at half maximum (FWHM) of the G band. Similarly to the $\mathrm{G}$ band position a decrease in the FWHM from about 60 to $120 \Delta \mathrm{cm}^{-1}$ to about $22 \Delta \mathrm{cm}^{-1}$ is observable with increasing graphite crystallinities. Also the variability in the FWHM of the G band is representative for the crystallinity of graphite. For poorly ordered CM this variation may be up to $70 \Delta \mathrm{cm}^{-1}$, whereas highly ordered graphite shows a variation of about 5 to $10 \Delta$ $\mathrm{cm}^{-1}$ (Beny-Bassez \& Rouzaud, 1985; Wopenka \& Pasteris, 1993; Beyssac et al., 2002). 

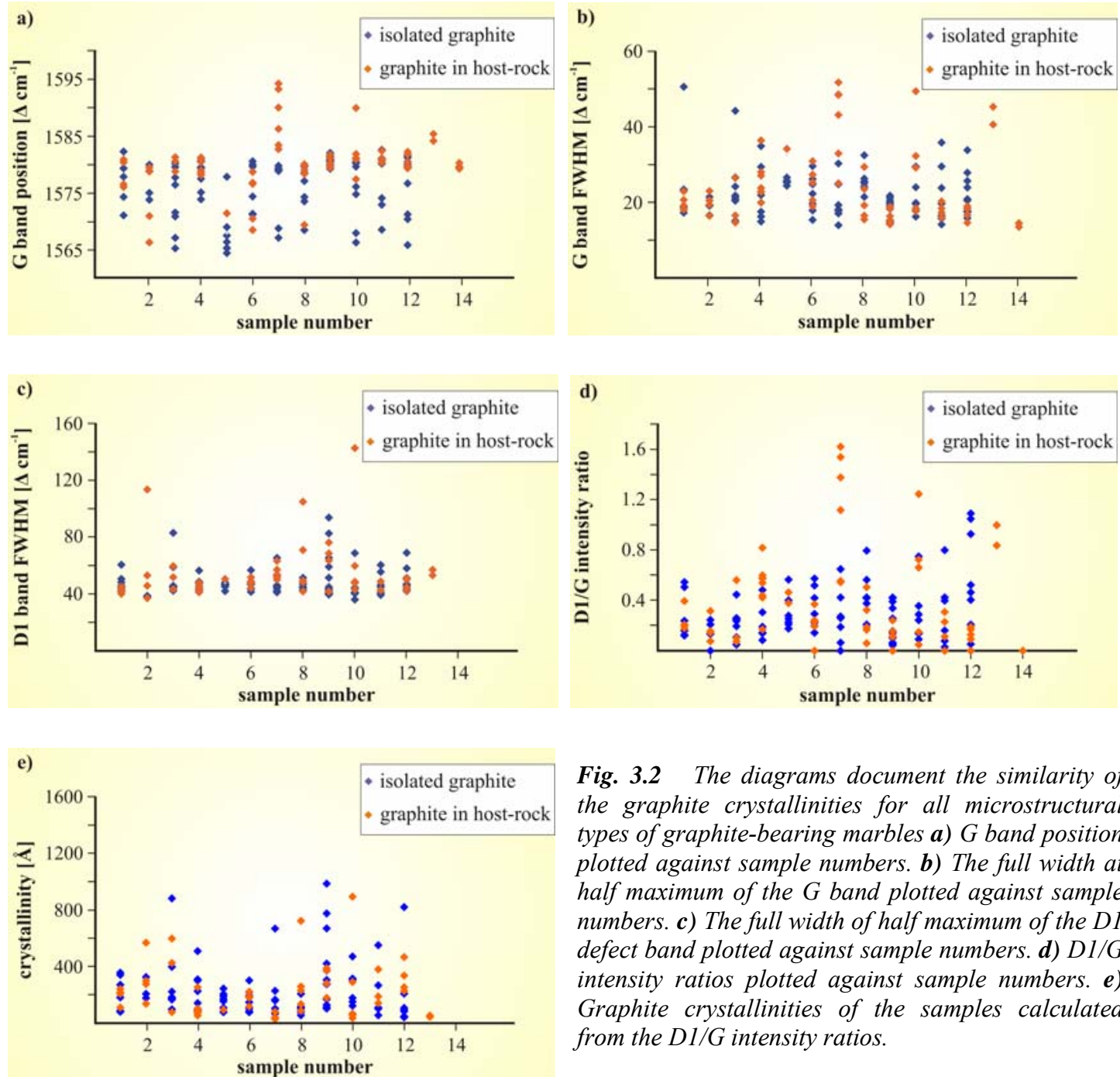

Fig. 3.2 The diagrams document the similarity of the graphite crystallinities for all microstructural types of graphite-bearing marbles a) $G$ band position plotted against sample numbers. b) The full width at half maximum of the $G$ band plotted against sample numbers. c) The full width of half maximum of the DI defect band plotted against sample numbers. d) D1/G intensity ratios plotted against sample numbers. e) Graphite crystallinities of the samples calculated from the $D 1 / G$ intensity ratios.

Figure $3.2 \mathrm{~b}$ shows the plot of the full width at half maximum of the $\mathrm{G}$ band for the measured samples. The mean FWHM is at $22 \Delta \mathrm{cm}^{-1}$ with a standard deviation of $8 \Delta \mathrm{cm}^{-1}$. In general, the samples show a variation of 10 to $20 \Delta \mathrm{cm}^{-1}$, while sample 7 again shows the highest variation of the FWHM of $30 \Delta \mathrm{cm}^{-1}$. Sample 14 with the highest crystallinities has the lowest mean full width of half maximum of $14 \Delta \mathrm{cm}^{-1}$ and a variation of only $1 \Delta$ $\mathrm{cm}^{-1}$.

Thirdly, the full width at half maximum of the D1 band gives a good indication for the degree of graphite crystallinities. After Beny-Bassez \& Rouzaud (1985) a decrease from $260 \Delta \mathrm{cm}^{-1}$ to about $50 \Delta \mathrm{cm}^{-1}$ is observable with increasing graphite crystallinities from poorly to well ordered graphite. Accordingly, the variation of the FWHM for the D1 band has to be considered evaluating the graphite crystallinities. After Beny-Bassez \& 
Rouzaud (1985) and Wopenka \& Pasteris (1993) this variation is from about $120 \Delta \mathrm{cm}^{-1}$ for poorly ordered CM to about $25 \Delta \mathrm{cm}^{-1}$ for well ordered graphite.

The full width of the half maximum for the D1 band of the measured samples is plotted in Figure $3.2 \mathrm{c}$. The mean FWHM is nearly constant over all samples with $48 \Delta \mathrm{cm}^{-}$

${ }^{1}$ and a standard deviation of $14 \Delta \mathrm{cm}^{-1}$. The biggest variation of the FWHM is shown by sample 10 with $108 \Delta \mathrm{cm}^{-1}$, whereas the other samples display a variation of 20 to $40 \Delta$ $\mathrm{cm}^{-1}$. As graphite with high crystallinities does not show the existence of the D1 band, these samples cannot be presented in this plot. Therefore, sample 14 does not show any data plot in Figure $3.2 \mathrm{c}$.

Graphite crystallinities are also characterised by the intensity ratio of the D1 band and the $\mathrm{G}$ band. This varies as much as one order of magnitude between the lowest and highest degrees of graphite crystallinity. After Wopenka \& Pasteris (1993) and Beyssac et al. (2002) poorly-ordered carbonaceous material shows mean D1/G intensity ratios between 1 and 2.6 with a standard deviation of 0.8 to 1.2. For highly-crystalline graphite mean $\mathrm{D} 1 / \mathrm{G}$ intensity ratios of 0.1 to 0.3 are measurable with a standard deviation of up to 0.2 . The variation in the measurements of a sample for highly-crystalline graphite is about 0.4 (Wopenka \& Pasteris, 1993; Beyssac et al., 2002).

The D1/G intensity ratios of the sample measurements are plotted in Figure $3.2 \mathrm{~d}$. The mean D1/G intensity ratios show a slight variation between the samples. They plot at 0.35 with a standard deviation of 0.3 . Again, sample 7 shows the highest variation of 1.5 within the measurements. Sample 14 with the highest graphite crystallinities plots at zero as no defect bands were detectable in the Raman measurements of this sample.

The $\mathrm{D} 1 / \mathrm{G}$ intensity ratio is used to calculate the crystallinities of graphite. As described above, there is a linear relationship between this ratio and a factor of the mean basal plane diameter La (Tuinstra \& Koenig, 1970; Beny-Bassez \& Rouzaud, 1985; Wopenka \& Pasteris, 1993).

The calculated crystallinities for the measured samples are plotted in Figure 3.2 e. The mean crystallinity for $L_{a}$ is $226 \AA$ with a standard deviation of $220 \AA$. The variation in crystallinities of about $890 \AA$ shows that differences may be quite substantial within a sample. As the graphite crystallinities are calculated as factors from the D1/G intensity ratios it is not possible to calculate the crystallinities of highly crystalline graphite due to the missing D1 defect band. Therefore sample 14 shows no values in this plot. 


\subsection{DisCUSSION}

The data plots of the graphite crystallinities indicate in general high graphite crystallinities across all microstructural types of graphite-bearing marbles and sampling localities. Samples 7 and 10 show a broader spectrum of graphite crystallinities but still are clearly in the range of well-ordered graphite. This general pattern of the graphite crystallinities is an important aspect considering the evolution of the complex microfabrics of the graphite-bearing marbles.

Differences in graphite crystallinity within and also between the samples may be related to measurements in different orientations. As described above the Raman Effect varies with the crystallographic orientation of graphite. As shown in Chapter 2, the graphite of the investigated shear zones is mainly ultra fine-grained. This makes a Raman investigation along distinct crystallographic orientations very difficult. Therefore, differences in the described order of magnitude may be attributed to these difficulties.

Pasteris \& Wopenka (1991) and Wopenka \& Pasteris (1993) studied the correlation of the described parameters indicative for graphite crystallinities and the metamorphic grades of the host rocks. Compared to Pasteris \& Wopenka (1991), the graphite crystallinities of the measured samples show metamorphic grades of staurolite, kyanite and and andalusite zones as well as sillimanite zones. Following Wopenka \& Pasteris (1993) similar metamorphic grades are indicated by the graphite crystallinities from staurolite and andalusite zones to sillimanite zones and granulite facies.

The obtained metamorphic grades fit very well into the metamorphic isogrades postulated by Hartmann et al. (1983) for the central parts of the Damara Belt (Fig. 2.1). South of the Central Zone (CZ), where the sampling sites are located, sillimanite-in due to staurolite breakdown is already observed. In the $\mathrm{CZ}$ upper amphibolite- to granulite facies metamorphism dominated. The $\mathrm{CZ}$ is characterised therefore by the highest metamorphic grades in the Damara Belt. The the main sampling localities shown in Figure 2.1 are located within the metamorphic isogrades of sillimanite, k-feldspar and cordierite-in accompanied with partial melting.

This implies that the measured graphite samples have preserved the crystallinities obtained during peak metamorphism. Therefore, graphite crystallinities were not clearly overprinted by the subsequent retrograde ductile and brittle-ductile deformation. However, graphite crystallinities are not only controlled by temperatures and metamorphic grades, as graphite crystallised in high-strain zones may show clearly higher crystallinities than graphite from outside these zones (Bustin, 1983; Demeny, 1989). This is in agreement with 
experimental investigations showing that strain-induced graphitisation increases the crystallinities of graphite (Ross \& Bustin 1990; Ross et al., 1991; Wilks et al., 1993; Bustin et al., 1995; Ross \& Bustin, 1997).

In addition, fluid-deposited graphite also is reported to be well crystalline (Bartels $\&$ Pasteris, 1994). This shows that particularly in high-strain zones graphite crystallinities alone do not give a secure evidence for specific deformational and metamorphic events. It is clearly necessary to discuss the graphite crystallinities together with the microstructural analysis, field relationships and the analysis of stable isotopes to determine the mode of graphite formation.

The data also implies that the method of isolating the graphite from the marble host rock as described in Chapter 2.3.6.1, does not influence the obtained degree of graphite crystallinity. This was also already observed by Hofmann (1989) and Alcántara et al. (2003), who investigated graphite crystallinities of grounded graphite and found no differences even after $40 \mathrm{~min}$ of grounding. 


\section{CHAPTER 4}

\section{CONDUCTIVITY MEASUREMENTS}

As described above, a series of follow up magnetotelluric profiles were measured by Ritter et al. (2003) and Weckmann et al. (2003) north-west of Usakos (Fig. 4.1 a). These magnetotelluric profiles revealed surface anomalies of high electrical conductivity in the southern parts of the profile (Figure $4.1 \mathrm{~b}$ ). Plotting the data on the regional geological map showed, that these anomalies coincide with the graphite-bearing carbonates, which build up the described dome structures (Fig. 4.2). The aim of the conductivity measurements on sample scale is, to understand and quantify the conductivity potentials of the different fabric types of graphite-bearing marbles.

The conductivity measurements were performed at the MineralogischPetrologisches Institut of the Universität Bonn in co-operation with Dr. Georg Nover. These measurements by complex impedance spectroscopy enabled the distinction between electrolytic and electronic conductivities. To exclude the reduction of conductivity potentials by disconnected graphite veins due to microcracks, reference samples were measured under different hydrostatic pressures. Additionally, the samples were drenched in $0.1 \mathrm{M} \mathrm{NaCl}$ under vacuum conditions, to fill up the permeable pore space and microcracks with water. Therefore, disconnected graphite veins are able to be connected by electrolytic bridges. In this way upper crustal conditions are simulated in the samples.

\subsection{PRINCIPLES OF ELECTRICAL CONDUCTIVITY}

An electrical field applied on any kind of material by a potential difference E, enforces the movement of charged material components like electrons and ions along the electric field lines. This causes an electrical current, which is directly proportional to the potential difference or electrode voltage. The degree of mobility of these charged particles characterises the intrinsic resistivity $\rho$ of the material.

In electrically conducting materials, charged components are free to move. In dielectrical or electrically isolating materials however, no free movement of charged material components is possible. In the latter case, the movability of the charged components is restricted to their position within the crystal lattice or the material fabric. An electric field applied on a dielectric material, forces the charged particles to move along the electric field lines until the field forces are balanced by the Coulomb forces within the crystal lattice. Due to these minor shifts around fixed lattice positions, polarisation effects are observable in dielectric materials. 
a)

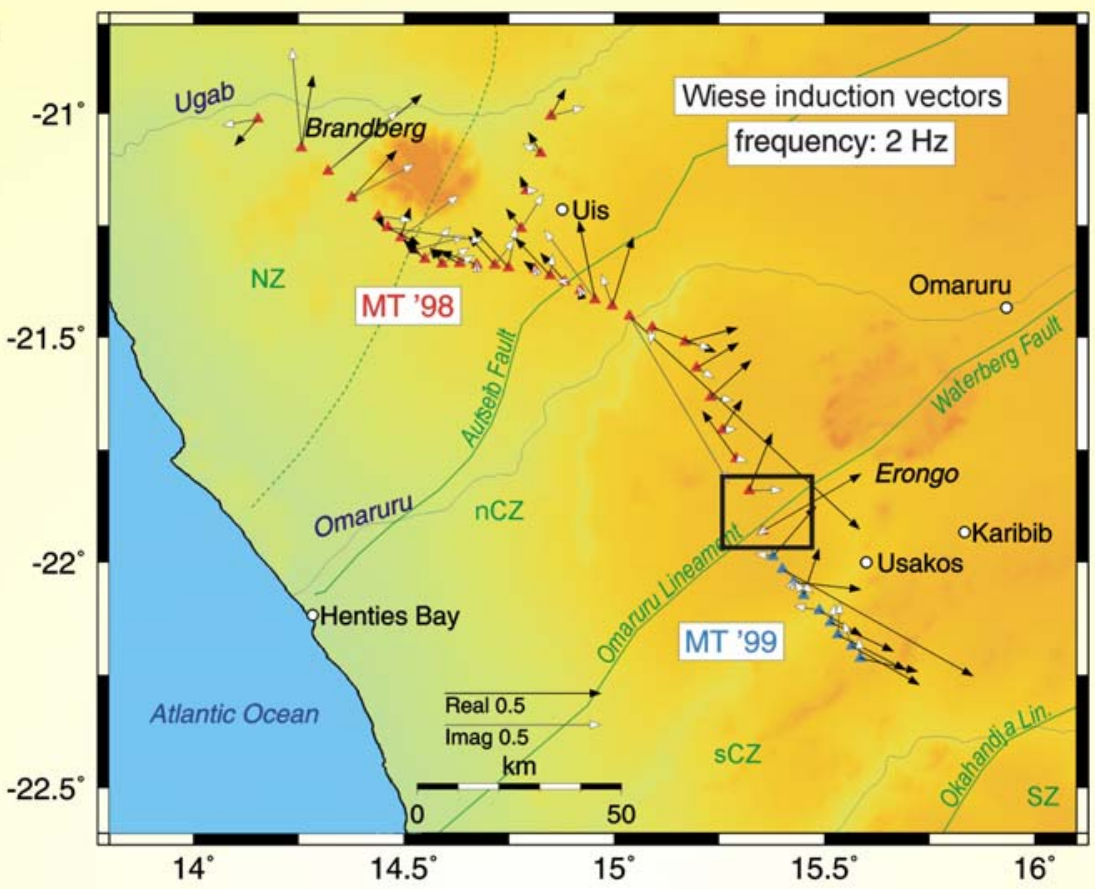

b)

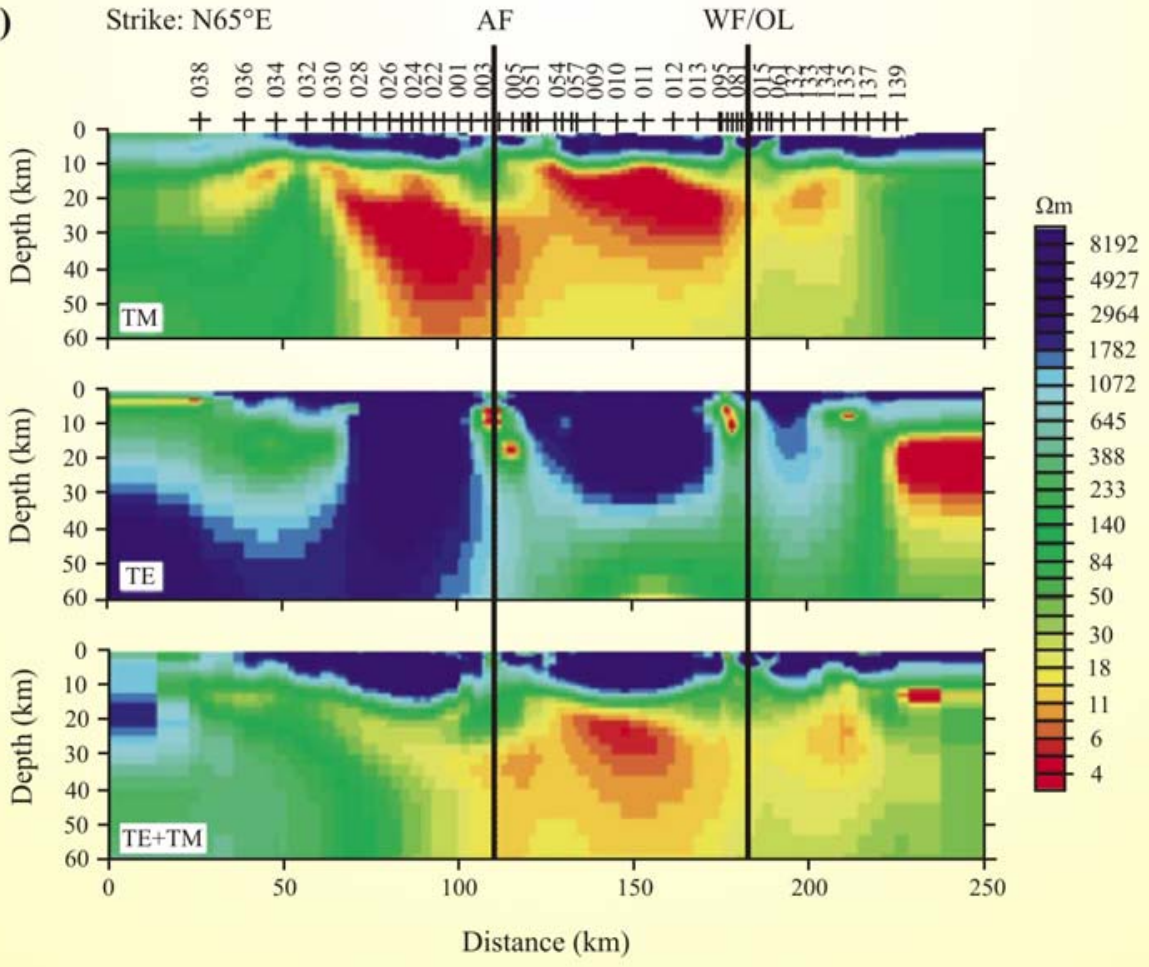

Fig 4.1 a) Map of the magnetotelluric profile across the Omaruru Lineament $(O L)$ and the Autseib Fault (AF). b) TE, TM and TE+TM calculations for the surface and upper crustal anomalies of electrical conductivity after Ritter et al. (2003) and Weckmann et al. (2003). 


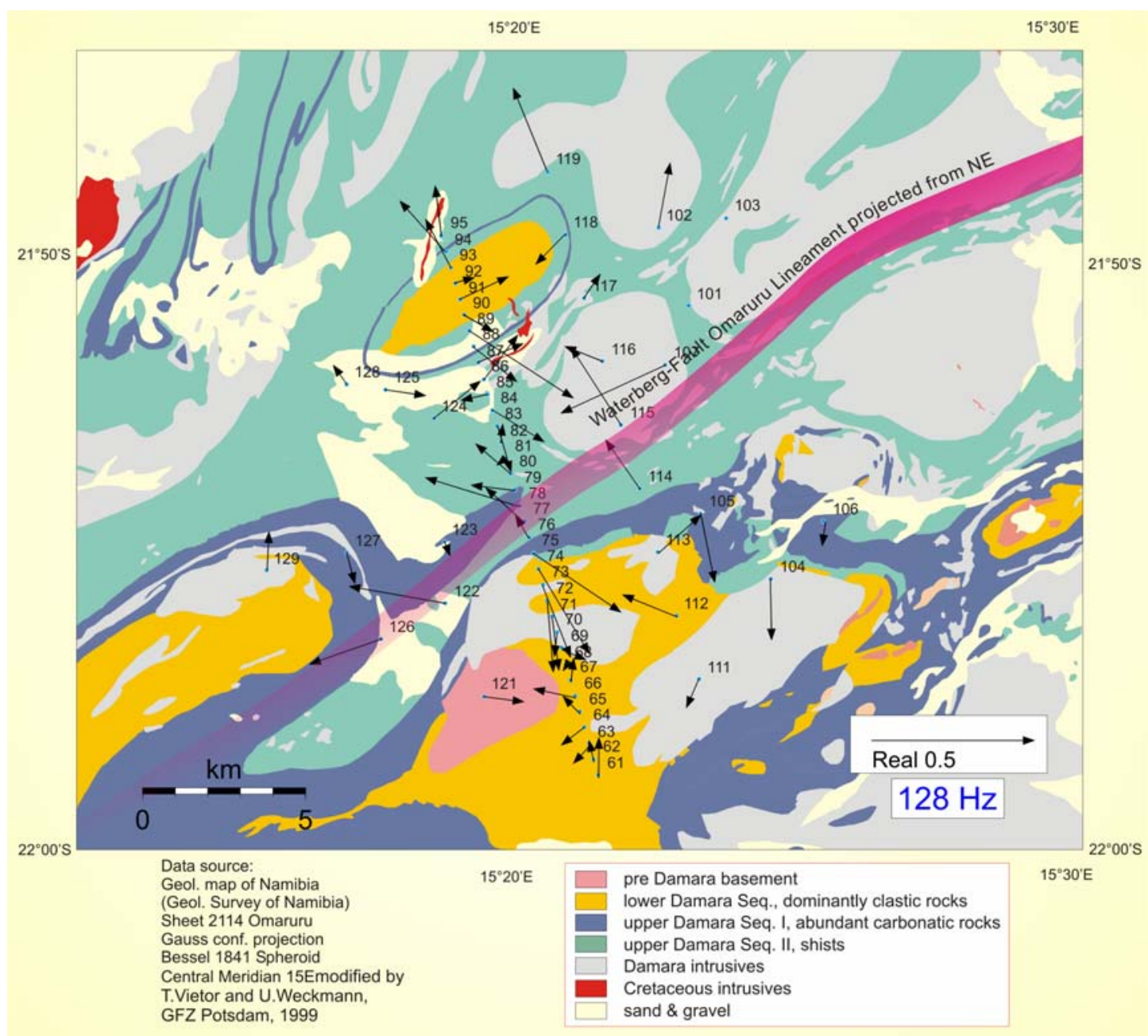

Fig 4.2 Magnetotelluric profile plotted on a simplified geological map by Weckmann et al. (2003). The anomalies of electrical conductivity coincide with the localities of Proterozoic graphite-bearing carbonates. The Omaruru Lineament is projected into the map by Weckmann et al. (2003).

In general, the correlation between voltage $\mathrm{E}$, current flow I and electrical resistivity R induced by a potential difference within a electrical conductor, is described by Ohm's Law:

$$
E=I \times R
$$

Resistivity and conductivity respectively, are material properties. This implies that the intrinsic resistivity $\rho$ for a cylindrical conductor is independent of the applied electrode voltage. The intrinsic resistivity is therefore defined by the geometric properties as:

$$
\rho=R \frac{A}{l}
$$


The geometric description of the conductor is defined by the cross sectional area A and the length of the conductor 1 . The intrinsic conductivity $\sigma$ is the reciprocal value of the intrinsic resistivity. Therefore, it is defined as:

$$
\sigma=\frac{1}{\rho}
$$

The intrinsic resistivity is expressed in $\Omega \mathrm{m}$, whereas conductivity is expressed in $\mathrm{S}$ $\mathrm{m}^{-1}$. They both quantify the transport of electrical charge in a conductor between two electrodes of a constant potential difference. This implies that Ohm's Law is only applicable for linear conductors. In linear conductors, a static electrical field strength $\vec{E}$ produces an electric current density $\vec{J}$ by the displacement either of electrons or ions. For linear conductors the electric current density is therefore defined as:

$$
\vec{J}=\sigma \vec{E}=\frac{1}{\rho} \vec{E}
$$

$\vec{J}$ is expressed in Amp $/ \mathrm{m}^{2}$ and $\vec{E}$ in V/m. When Ohm's Law is expressed in this way it is only applicable for isotropic materials. However, the conductivity $\sigma$ of crystalline materials is mostly anisotropic. Therefore, Ohm's Law has to be described by a second rank tensor, where the overall conductivity of a crystalline material is calculated according to an ellipsoid of three (positive) vectors. The magnitudes and directions of the vectors defining the ellipsoidal tensor, depend on the material properties in different directions. The tensor is then expressed by:

$$
J_{i}=\sigma_{i j} E_{j}
$$

The different components of the tensor are described by the direction-dependant conductivities. Therefore, this expression enables the determination of the conductivity $\sigma$, in dependence of distinct directions of the tensor. However, most geological materials especially silicates and germanates are anisotropic and of dielectric nature. Dielectric materials are isolators, which polarise dipoles, molecules or free electrical charge on grain and material boundaries, according to the electric field lines of a static electrical field. Because of electrical charge culmination due to polarisation effects, conductivity measurements with direct current will produce a linear decrease in the electrical current flow I. This results in a time dependant increase in the electrical resistivity R.

This implies that the linearity of Ohm's Law is not given for dielectric materials. Additionally, it is difficult to distinguish conductivities according to equation [4.5] in the different directions of the tensor ellipsoid. This is also the case for materials developing 
semiconductoral properties with increasing temperatures. In these cases, the dependency of the electrical current flow I from the voltage $\mathrm{E}$ is following a exponential law:

$$
I=I_{s} \exp \left(-\frac{e E}{k T}\right)
$$

For this expression $e$ is the elementary charge, $k$ is the Boltzmann constant and $T$ is the absolute temperature. To measure and to distinguish conductivity potentials of materials showing dielectric, semiconducting or ionic conductivity, from linear electronic conductors, impedance spectroscopic measurements were developed (MacDonald \& Kenan, 1987).

\subsubsection{ELECTRONIC CONDUCTIVITY}

Electronic conductivity varies between different materials in many orders of magnitude. The ability for electronic conductivity depends on the amount of electrons in the valence band. If a band is not completely filled with electrons, the electrons are able to move within the valence band. In this way they can react on influences of an applied electric field. If the valence band is filled up completely with electrons, the electrons have to be activated by the application of energy, to overcome the energy gap to the next free band (Fig 4.3 a). Insulators have a large energy gap between the single bands and the valence band is either filled completely by electrons, or the valence band is completely empty (Fig 4.3 b). Metals generally have partially-filled valence bands and therefore are good electronic conductors. Semiconductors have an energy gap of $\mathrm{E}_{0}<2.5 \mathrm{eV}$ and dielectric materials have energy gaps of $\mathrm{E}_{0}>2.5 \mathrm{eV}$. In Figure 4.4 the electrical conductivity/resistivity ranges of geologic materials are shown.

\subsubsection{IONIC OR ELECTROLYTIC CONDUCTIVITY}

Ionic or electrolytic conductivity is an important component in upper-crustal conductivity measurements, because most of the minerals are of dielectric nature. Electrical conduction in these materials only occurs through mobile ions as they are electrically charged. The displacement of ions occurs mainly by diffusion, which is controlled by the electrical potential $\mathrm{qV}$ between two points of a material, by the number of charge carriers $\mathrm{n}$, by the unit volume and the temperature $\mathrm{T}$. 


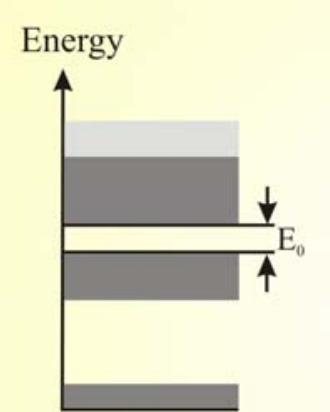

(a)

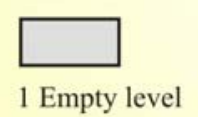

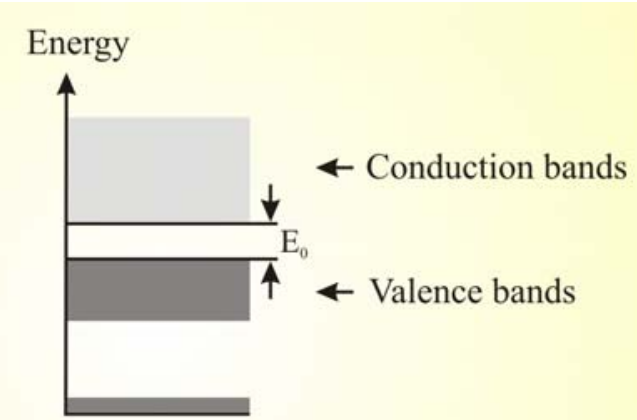

(b)

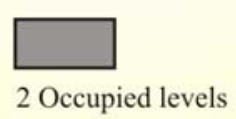

Fig 4.3 a) Partial occupation of the conduction band with electrons enables electronic conductivity. If the energy gap $E_{0}$ is $<2.5 \mathrm{eV}$ between the valence bands, the movement of electrons to higher energy states is enabled. b) The conduction band is completely empty and the valence band is completely occupied by electrons. This prohibits electronic conduction. If $E_{0}>2.5 \mathrm{eV}$ electrons are unable to move to empty electron bands. Figure after Guéguen \& Palciauskas (1994).

In a thermodynamic equilibrium the number of charge carriers $n$ follows Boltzmann's Law by applying the Boltzmann constant $\mathrm{k}$. The diffusion follows Fick's Law where $\mathrm{D}$ is the diffusion coefficient. The ionic conductivity is thus defined as:

$$
\sigma=n q^{2} \frac{D}{k T}
$$

Diffusional ionic conductivity as described by equation [4.7] depends on the material properties. Therefore, it is referred to as the intrinsic electrolytic conductivity. In the presence of impurities in the dielectric material, extrinsic ionic conductivity also occurs by a different phase of ionic material which causes the impurities. This ionic material becomes mobile in an electrical field or contributes with additional electrons to the conducting charge. At lower temperatures extrinsic electrolytic conductivity dominates because of the lower activation energy, whereas at higher temperatures intrinsic ionic conductivity is dominating. The total sum of the electrolytic conductivity of a material, should always be calculated as the sum of the extrinsic $\sigma_{\mathrm{eo}}$ and intrinsic $\sigma_{\mathrm{io}}$ ionic conductivity:

$$
\sigma=\sigma_{\text {io }} \exp \left(-\frac{E_{i}}{k T}\right)+\sigma_{e o} \exp \left(-\frac{E_{e}}{k T}\right)
$$


Ionic conduction in solutions occurs, where aquaeous pore fluids contain varying concentrations of salts such as $\mathrm{NaCl}$ and $\mathrm{KCl}$. These salts dissociate during solution and the soluted anions and cations move freely in the solution. If an electric field is applied, the anions and cations move along the electric field lines in opposite directions. This diffusion process is controlled by Stoke's Law, where the applied force is balanced by the viscosity of fluid. The conductivity is proportional to the ionic concentration and varies depending on the chemical composition of the salts, because of differences in the mobility of different ions.

\subsubsection{ELECTRICAL CONDUCTIVITY OF ROCKS}

The total amount of conductivity in rocks is strongly dependent on the composition of the rocks (Fig. 4.4), on the microstructures and on the overall macroscopic fabric. Most igneous rocks, sands and carbonates show conductivities of 1 to $10^{-2} \mathrm{mS} / \mathrm{m}$, whereas sulfides and graphite show conductivities of $10^{5}$ to $10^{2} \mathrm{mS} / \mathrm{m}$. Other conductive minerals are zeolithes and hydrated minerals, like for example some phyllosilicates.

The microstructures of rocks are of great importance, because an interconnection of conducting minerals is essential for bulk electronic conductivity. If the pore space is interconnected in a permeable network around grain boundaries, the ionic component of conductivity by melts or aqueous fluids will be relatively high.

The ionic conductivity in rocks therefore also depends on the degree of fluid saturation in the rocks, as well as on the volume of interconnected pore space. Bulk conductivities of rocks increase predominantly, when conducting minerals like sulfides and graphite are interconnected and ionic conductivity is prevailing throughout the rock.

For the electrolytic conductivity of clay-bearing rocks, a component of surface conductivity along grain boundaries of clay minerals is measurable. Extrinsic ionic conductivity occurs along charged impurities on the surface of the clay minerals. If for example $\mathrm{Al}^{3+}$ is substituted by $\mathrm{Si}^{4+}$, the charge deficit is compensated by cation absorption on the mineral surface. This results in the formation of an electrical double layer. In the combination with electrolytic conductivity, these surface ions are displaced along the grain boundaries.

Rocks with a high concentration of conducting minerals such as sulfides $\left(\mathrm{FeS}_{2} \rho=\right.$ 1,2 - $600 \times 10^{-3} \Omega \mathrm{m}, \mathrm{PbS} \rho=6,8 \times 10^{-6}-9 \times 10^{-2} \Omega \mathrm{m}$ ), graphite (parallel to basal plain $\rho$ $=33-100 \times 10^{-8} \Omega \mathrm{m}$, perpendicular to basal plain $\rho=28-200000 \times 10^{-8} \Omega \mathrm{m}$ ), amorphous carbon $\left(\mathrm{C} \rho=5 \times 10^{-5} \Omega \mathrm{m}\right)$ and magnetite $\left(\mathrm{Fe}_{3} \mathrm{O}_{4} \rho=52 \times 10^{-6} \Omega \mathrm{m}\right)$ may 


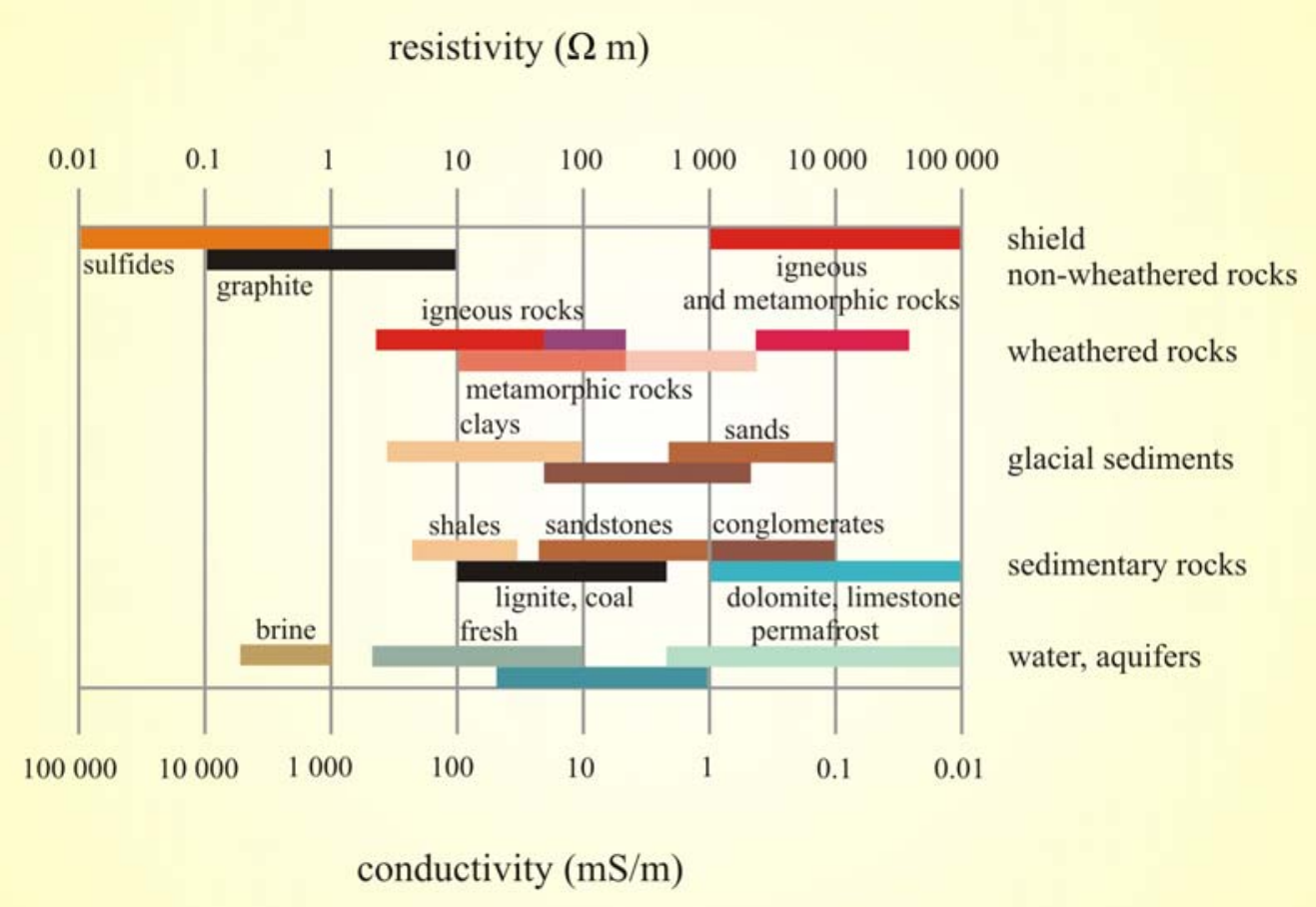

Fig 4.4 Resistivity ranges of different crustal material after Palacky (1987).

show higher conductivities, which could purely be electronic conductivity. Silicates have resistivities of about $\rho=5-1 \times 10^{5} \Omega \mathrm{m}$ depending on their structure and water content. To obtain electronic conductivities the conducting minerals have to be interconnected. Graphite is known to form films on grain boundaries, whereas dendritic patterns also could result in a strong increase in electrical conductivity. Figure 4.5 a shows, that there can be a decrease of several orders of magnitude in resistivity, when the volume fraction of conductive minerals is increasing by one order of magnitude.

Conductivity variations with increasing pressure are also measurable in crustal rocks. With increasing pressures, the energy gaps between the covalent electron bands decrease. Therefore, a lower activation energy is needed for electrons to reach the next free electron band to perform electronic conductivity. In ionic conductivity components, the effects of pressure are neglectable small, although a decrease in ionic resistivity is measurable with increasing pressure (Fig. 4.5 b). Considering the effects of temperature on the conductivity potentials, the effects of pressure are neglegible in crustal dimensions. 

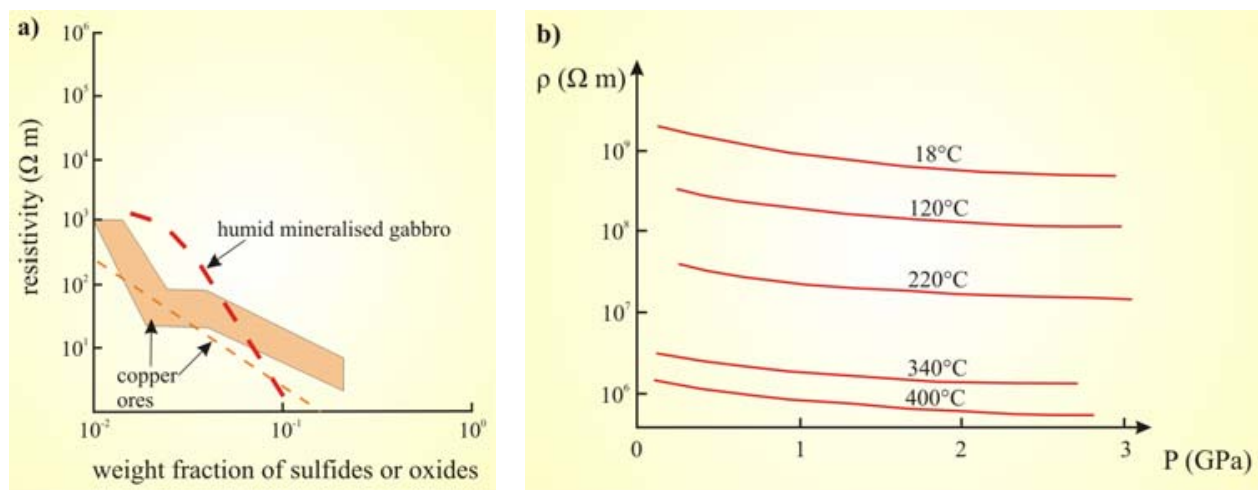

Fig 4.5 a) Resistivity variation with the increasing concentration of sulfides and oxides as conducting minerals after Keller (1987). b) Variation of resistivity with increasing pressure and temperature for basalt after Parkhomenko (1967).

\subsubsection{IMPEDANCE SPECTROSCOPY}

Impedance spectroscopy is a physical method to determine the conductivity of complex resistors, as they occur in most geological materials. Complex resistors are described by a combination of linear conductors and dielectric materials. As impedance spectroscopic investigations are based on measurements with alternating current, the above mentioned polarisation effects in measurements which occur in direct current are eliminated. Additionally, it is possible to distinguish between electronic and ionic conductivity potentials of the measured materials.

The principle of impedance spectroscopy is the theory of complex impedance. The theory of complex impedance is based on the equivalent-circuit model. This model describes complex resistors like the graphite network structures in a calcite host-rock, by a combination of elementary cells containing resistors and capacitors. Thus, the conductivity of elementary cells includes a component of linear conductivity by a fixed resistor with a constant resistivity, and a frequency-dependant component of a capacitor. Therefore, a combination of pure transport of charge and various effects of polarisation can be measured and distinguished using impedance spectroscopy.

\subsubsection{POLARISATION EFFECTS AND ELECTRICAL CONDUCTIVITY}

The polarisation effects of dielectric materials (permittivity) in alternating current depend directly on the frequency of the applied electrical current. Several different effects of polarisation are described in the measured frequency ranges from $10^{15}$ to $10^{-5} \mathrm{~Hz}$.

At high frequencies of $10^{15} \mathrm{~Hz}$ polarisation effects are induced by the distortion of the electron shells relative to the atomic nucleus by the electrical field. These shifts occur 
along electric field lines of the applied field, and produce charge concentrations and thereby charge polarisation on an atomic scale. This kind of polarisation effect is referred to as electron polarisation.

Between $10^{15}$ and $10^{12} \mathrm{~Hz}$ ionic polarisation effects are measurable. Positively and negatively charged ionic components of the molecules are displaced relative to each other around fixed lattice positions. This causes polarisation effects due to concentrations of different charges, relative to the applied field lines on a molecular scale.

Polarisation effects on the same scale are measured between $100 \mathrm{kHz}$ and $10 \mathrm{~Hz}$, either by the reorientation of pre-existing dipoles within the molecules or by the reorientation of charged components in lattice defects. These charged particles and material components are forced into a preferred orientation along the electric field lines. Thereby the materials electrical charge is polarised. These polarisation effects are referred to as dipolar and space-charge polarisation effects.

At frequencies below $10^{-1} \mathrm{~Hz}$ the effects of polarisation reach a sample-scale level by free-moving ionic components. Charged particles separate according to the electrical field lines and concentrate either on solid interfaces of the sample or on grain boundaries. These concentrations of charge on specific interfaces are regarded as electrode polarisation. These effects are referred to as the Maxwell-Wagner effect. Polarisation effects on grain-boundary scale commonly overlap with the dipolar polarisation effects at higher frequencies.

If dielectric material is polarised, the electric displacement $\vec{D}$ of two charges of opposite polarity depends on the electric field strength $\vec{E}$ and the Polarisation $\vec{P}$. This is defined as the electrical dipole moment per volume:

$$
\vec{D}=\varepsilon_{0} \vec{E}+\vec{P}
$$

$\varepsilon$ is the dielectric permittivity expressed in farads/m. Equation [4.9] shows that the electric displacement $\vec{D}$ is directly related to the electric field strength $\vec{E}$. $\varepsilon_{0}$ is the permittivity of a vacuum. The permittivity is a parameter which varies with the composition and the material properties of the dielectric material. The permittivity $\kappa$ of a material is defined as:

$$
\kappa=\frac{\varepsilon}{\varepsilon_{0}}
$$


This implies, that the linear approximation between $\vec{E}$ and $\vec{P}$ can be deduced for most dielectric materials. In comparison to a vacuum capacitor, a linear dielectric material shows a higher capacity by the factor $\varepsilon$. This higher capacity depends directly on the susceptibility $\chi$ of the polarisation:

$$
\vec{P}=\varepsilon_{0} \chi \vec{E}
$$

The susceptibility $\chi$ is here defined as $\varepsilon-1$. The parameters for the permittivity $\mathrm{K}$ and $\varepsilon$ and the susceptibility $\chi$ are material parameters and therefore second rank tensors. As complex numbers, these parameters contain imaginary parts which represent the dissipation of energy during the redistribution of charge.

Polarisation effects in an oscillating electrical field are not always static, as the redistribution of charge is not always as rapidly as the oscillation of the electric field. These described charge redistributions are additive, so that the overall polarisation is the sum of all polarisation effects. The total polarisation or permittivity $\kappa(\omega)$ decreases, with the increase of frequency $\omega$. At frequencies below $10^{-3} \mathrm{~Hz}$, all different kinds of polarisation effects are induced in dielectric materials by oscillatory electrical fields. Therefore, an electrical current is measurable.

For an ideal dielectric material, the phase difference between the electrical flow I and the electron voltage $\mathrm{E}$ is $90^{\circ}$. With increasing frequencies, the polarisation effects vary according to the frequency ranges described above. With every change in polarisation effect, a change in the phase difference is observable. This effect is used in impedance spectroscopic measurements to distinguish between different conductivity components in complex resistors.

\subsubsection{THEORY OF COMPLEX IMPEDANCE}

The theory of complex impedance describes the conductivity potentials of complex resistors with components of linear electrical currents (conductivity by charge transport) and displacement currents (conductivity by polarisation effects). It is based on the combination of elementary cells, which are either parallel or in series. The constant resistor of the elementary cell is equivalent to the electronic conductivity component by charge transport. The capacitor represents the displacement currents of the electrostatic field by the polarisation of dielectric material.

If an oscillatory electrode voltage $E$ is applied, the potential difference $E(\omega)$ is defined by the amplitude $\mathrm{E}_{0}$ and the cyclic frequency $\omega$ by: 


$$
E(\omega)=E_{0} \sin (\omega t)=E_{0} e^{(i \omega t)}
$$

An oscillatory electric potential difference $\mathrm{E}(\omega)$ always produces an alternating electrical current $\mathrm{I}(\omega)$ with the amplitude $\mathrm{I}_{0}$. The electrical current has a phase difference $\varphi$ to the electrode voltage E. This implies, that $\mathrm{I}(\omega)$ is defined analogue to equation [4.12] as:

$$
I(\omega)=I_{0} \sin (\omega t+\varphi)=I_{0} e^{(i \omega t+\varphi)}
$$

In complex resistors, either a combination or a substitution of charge transport and displacement currents is possible in oscillatory electrical fields. Therefore, the complex oscillatory resistor $\mathrm{Z}(\mathrm{w})$ (the impedance) is calculated by integrating equations [4.12] and [4.13] into Ohm's Law [4.1]:

$$
Z(w)=\frac{E(\omega)}{I(\omega)}=|Z| e^{i \varphi}=|Z| \cos \varphi+i|Z| \sin \varphi
$$

The magnitude of the impedance is described by $|Z|$, where the electrical current by charge transport is the real component of the impedance $Z^{\prime}$. This real component of the complex impedance is defined by the term $|Z| \cos \varphi$. The displacement current is defined by the imaginary component of the impedance $Z^{\prime \prime}$ with $i|Z| \sin \varphi$. The unit imaginary number $i$ is the positive square root of -1 . It thereby defines the imaginary component of the complex impedance. The complex impedance is described according to equation [4.14] by the phase difference as:

$$
\tan \varphi=\frac{Z^{\prime}}{Z^{\prime \prime}}
$$

Analogue to equations [4.5] and [4.14] the total current density $\vec{J}$ is also defined by a combination of real and imaginary components of the complex electrical conductivity:

$$
\vec{J}_{T}=\sigma \vec{E}+\frac{\delta \vec{D}}{\delta t}=\sigma \vec{E}+\varepsilon \frac{\delta \vec{E}}{\delta t}
$$

The electrical current by charge transport is defined by $\sigma \vec{E}$. The displacement currents are either defined by $\frac{\delta \vec{D}}{\delta t}$ where $\vec{D}$ is the electric displacement, or by $\varepsilon \frac{\delta \vec{E}}{\delta t}$ where $\vec{E}$ is the electric field strength and $\varepsilon$ is the permittivity.

The complex impedance is described by an elementary cell containing a constant resistor and a capacitor. The constant resistor defines the electrical conductivity by charge 
transport and is independent of the cyclic frequency $(\omega)$. Therefore, the electrical current is in phase with the electrode voltage. The capacitor represents the displacement currents which depend on the cyclic frequency $(\omega)$. This implies, that the phase difference of the electrical current is $90^{\circ}$ before the electrode voltage. The imaginary component of the complex impedance is defined by the cyclic frequency $(\omega)$ and the capacity $\mathrm{C}$ as:

$$
Z^{\prime \prime}=-\frac{i}{\omega C}
$$

If the two components of the complex impedance are connected in series, the electrode voltage of the real component $E_{R}$ and the imaginary component $E_{C}$ add to the total electrode voltage $E_{T}$ by $E_{T}=E_{R}+E_{C}$. The total complex impedance $Z_{T}$ is therefore defined as:

$$
Z_{T}=R-\frac{i}{\omega C}=R+\frac{1}{i \omega C}
$$

The complex impedance of this elementary cell plots in a Cole-Cole diagram as a straight line parallel to the imaginary axis. The resistor $\mathrm{R}$ plots at the intersection with the axis of the real component (Fig. 4.6 a). The capacity of the complex resistor cannot be differentiated in this plot. To differentiate the capacity, the complex admittance as the reciprocal value of the complex impedance, is plotted into an Argand diagram. In this case the capacity is distinguished in the maximum of the half-circle. The diameter of the halfcircle ranges from 0 to $1 / \mathrm{R}$ (Fig. $4.6 \mathrm{~b}$ ).

Due to this fact, the elementary cell is connected parallel, where the reciprocal components of the complex impedance add to the total admittance $\mathrm{Y}_{\mathrm{T}}$ :

$$
Y_{T}=\frac{1}{Z_{T}}=\frac{1}{R}+i \omega C
$$

The real component of the complex admittance is defined by $Y^{\prime}=\frac{1}{R}$ and the imaginary component is defined by $Y^{\prime \prime}=i \omega C$. The complex admittance plots in a Argand diagram as a single line parallel to axis of the imaginary component (Fig 4.6c). The total complex impedance can be calculated as the reciprocal value of the total complex admittance. It is therefore defined by: 

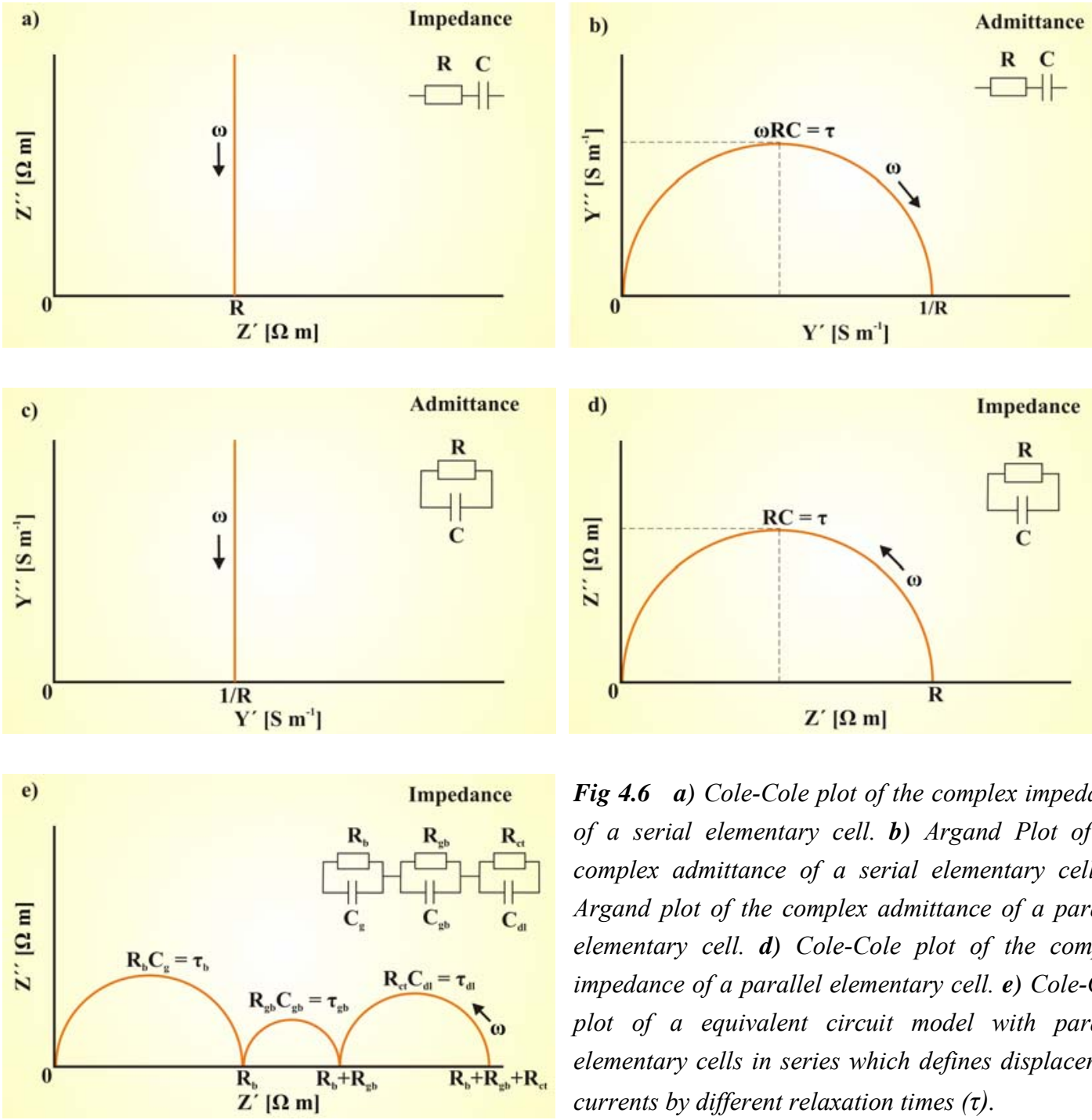

Fig 4.6 a) Cole-Cole plot of the complex impedance of a serial elementary cell. b) Argand Plot of the complex admittance of a serial elementary cell. c) Argand plot of the complex admittance of a parallel elementary cell. d) Cole-Cole plot of the complex impedance of a parallel elementary cell. e) Cole-Cole plot of a equivalent circuit model with parallel elementary cells in series which defines displacement currents by different relaxation times $(\tau)$.

$$
Z_{T}=\frac{1}{Y_{T}}=\left(\frac{1}{R}+i \omega C\right)^{-1}
$$

The real component of the complex impedance is accordingly defined as $Z^{\prime}=\frac{R}{1+(\omega R C)^{2}}$. The imaginary component is defined by $Z^{\prime \prime}=R \frac{\omega R C}{1+(\omega R C)^{2}}$. Measured in a wide frequency range, the complex impedance plots as a half circle above the axis of the real component from 0 to $\mathrm{R}$ (Fig. $4.6 \mathrm{~d}$ ). The half circle is defined by $\mathrm{R}$ and the relaxation moment $\tau=\mathrm{RC}$.

The total complex impedance of crustal materials with a fluidal component in the permeable pore space, is described by a set of parallel elementary cells. These are combined in series. The single cells represent different resistivity/conductivity 
components, which add up to the total resistivity (Fig. 4.6 e). $R_{b}$ is the electrolytic bulk resistance which is defined according to equation [4.3] by [4.21]. $\mathrm{C}_{\mathrm{g}}$ represents the electrolytic capacity, which is dependent on the permittivity constant $\varepsilon$ and is calculated by equation [4.22] as follows:

$$
\begin{gathered}
\sigma=\frac{l}{A R_{b}} \\
C_{g}=\frac{\varepsilon_{0} \varepsilon A}{L}
\end{gathered}
$$

The second elementary cell $\mathrm{R}_{\mathrm{gb}} / / \mathrm{C}_{\mathrm{gb}}$ defines the complex impedance of grain boundary effects within the measured volume. The third elementary cell $\mathrm{R}_{\mathrm{ct}} / \mathrm{C}_{\mathrm{dl}}$ represents the complex impedance on the contact between the electrolyte and the electrode. $\mathrm{R}_{\mathrm{ct}}$ defines the charge transfer whereas $\mathrm{C}_{\mathrm{dl}}$ represents charge changes in the double layer on the interface to the electrode.

Every half circle is defined by $\mathrm{R}$ and the relaxation time $\tau$. If these vary by several orders of magnitude, the three impedance components shown in Figure 4.6 e can be differentiated. If the relaxation times vary less than one order of magnitude between the different impedance components, one big half circle over $\mathrm{R}$ will be displayed as shown in Figure $4.6 \mathrm{~d}$. In this case it is very difficult to reconstruct the single components, due to the uncertainty regarding the frequency dependency of the displacement currents of the different materials. A plot of the complex admittance of the described components shows different straight lines and/or curves of different gradients. The different gradients represent at least different relaxation times for the measured conductivities. A further interpretation of these curves is doubtful up to now, due to uncertainties of the correlation of these curves to individual and overlain displacement currents.

For further information on the electrical conductivity of rocks see Guéguen \& Palciauskas (1994) and von der Gönna (1997).

\subsection{Analytical TeChNiQues}

For the measurements of the complex impedance a HP 4192A Impedance Spectroscope was used. The measurements were conducted with two different electrode voltages of 0.5 and $1 \mathrm{~V}$ in the frequency range of $785 \mathrm{~Hz}$ to $1 \mathrm{MHz}$. For every decade 15 to 20 logarithmic frequency measurements were carried out. The impedance spectroscope was operated and controlled by a graphical software HP-VEE, which was programmed and modified by the group of Dr. Georg Nover. This software shows the measured complex impedance plotted life into a Cole-Cole diagram. Additionally the quality of the 
measurements is controlled by a Bode plot, where the impedance and the phase difference is plotted as a function of the applied cyclic frequency.

Reference samples were measured under different hydrostatic pressures, to verify the loss of electronic conductivity by the existence of microcracks. These samples were measured in a autoclave press which enables pressures of up to $4 \mathrm{kbar}$. The sample and the electrodes were sleeved by a heat shrink tube and thereby separated to the pressure medium glycerine. Abnormal pore pressures are prevented by porous electrodes.

Every measured sample has been evacuated and afterwards saturated with a $0.1 \mathrm{M}$ $\mathrm{NaCl}$ pore fluid to simulate upper crustal conditions. The electrical contact between the sample interfaces and the electrodes was established by a moistened filter paper. All the sample measurements have been conducted under room temperature conditions. The analysis of the measured data was carried out by the Least Squares Program IDA by EG\&G/Parc.

\subsection{SAMPLES MEASURED}

For conductivity measurements by complex impedance graphite-bearing marbles ideally should function as complex resistors. Accordingly, the samples had to be restricted to the types where graphite occurs in interconnected graphitic stylolites and/or graphite network structures. The samples for the conductivity measurements were therefore selected according to the classification of the microstructural investigations described above.

Three main types of graphite-bearing marbles had been distinguished and classified. The microstructural investigations had revealed for the graphite-bearing marbles of type 1 that graphite occurs only in single graphite nests. These are disseminated throughout the calcite-marble host rock. As graphite is not interconnected, no electronic conductivity is measurable in this type of marble. Therefore, only a purely dielectric behaviour is measurable in these samples.

Likewise, the finer grained mylonitic graphite-bearing marble of type 3 shows no interconnection of the single graphite grains on the foliation plains. On a microscopic scale the single graphite grains are not interconnected in the graphitic layers parallel to the foliation. Therefore, they are not able to form continuous electronic conductors. Accordingly, this type of graphite bearing marble also shows no electronic component in the conductivity measurements and behaves purely dielectrically.

According to the microstructural investigations, type 2 of the graphite-bearing marbles is the only type to produce electric conductivity by interconnected graphitic 
stylolites. The samples of type 2 were selected by the degree of the graphite network intensity and the orientation of the graphite veins. The aim is to distinguish the different conductivity potentials of the samples in relation to the direction of the foliation, the degree of network intensity and the thickness of the graphitic stylolites. These relationships would reveal an estimation of the conductivity potentials of the samples. An upscaling to the results of the magnetotelluric measurements on a crustal scale, these results may give some good indication for the analysis of the magnetotelluric data.

The samples measured, are from all areas of field investigation (Fig. 2.1). Therefore, some of the discussed samples are not from the magnetotelluric profile. However, in mineral composition and in microstructural characterisation the samples of the two localities are identical. This has been investigated by optical methods, Scanning Electron Microscopy (SEM) and energy dispersive x-ray analysis (EDX) and neutron diffraction as described in chapter 2. The samples from the locality on Farm Epako Süd are considered as reference samples for a regional scale investigation. Secondly they derive from ideal outcrops with good sampling opportunities of different microstructural varieties.

\subsection{MEASUREMENTS AND RESULTS}

Out of a broad range of impedance spectroscopic measurements only the plots of several representative samples have been selected. They show the spectrum of results which will have to be discussed. Generally, measurements have been conducted in different scales and under different hydrostatic pressures. The measured samples were cut into rectangular blocks using foliation and lineation as the external reference system where possible. Due to this structural reference frame, an extrapolation of the measurement results into the crustal framework will later be possible.

The measurements presented here have been conducted in three different scales. Samples were measured either over the complete plains of the rectangular blocks, or over profiles across the blocks. These profiles have been measured either with electrodes of 30 $\mathrm{mm}$ in diameter or with point electrodes. For measurements under different hydrostatic pressures, cores have been drilled of $30 \mathrm{~mm}$ in length and $20 \mathrm{~mm}$ in diameter. These cores have been measured over their complete volume.

The representative plots in Figure 4.7 to 4.10 show, that in nearly all cases only pure ionic conductivities are measurable in the samples. This is obvious, as discussed above, by the form of a half circle in the plots of the measured complex impedance. The different sample plots show, that this result is obtained independent of the sample scale and 
sample variety also across the different localities. The sample of Figure 4.7 is from the magnetotelluric profile, whereas the other shown samples are from farm Epako Süd.

Figure 4.7 shows the plots of the complex impedance (Fig. 4.7a) and the plots of the complex admittance (Fig. 4.7 b) across a rectangular block as shown in Figure $4.7 \mathrm{c}$. The impedance spectroscopic measurements have been conducted with circular electrodes of $30 \mathrm{~mm}$ in diameter. The differences in the resistivity of the complex impedance have to be correlated to the different orientations of the measurements. Measurement 1 and 2 had been conducted in a fine-grained brittle-ductile deformed zone with fine-grained graphite parallel to the main foliation. The resistivity of measurement 2 exceeds the resistivity of measurement 1 by nearly $50000 \Omega \mathrm{m}$. This seems to be related to the orientation of the measurements relative to the lineation on the foliation planes, which is macroscopically not visible. Accordingly, the resistivity of the third measurement orthogonal to the foliation is exceeding the resistivity of the first two measurements by up to $100000 \Omega \mathrm{m}$.

The curves plotted in this diagram show, that only displacement currents are measurable in this sample due to polarisation effects and ionic conductivities as discussed above. The plots of the complex admittance show by the two gradients of the curves, that the displacement currents had two relaxation phases. These relaxation phases indicate changes in the mode of displacement currents. However, it is not possible to extrapolate from these curves which kinds of polarisation effects or ionic conductivities have been responsible for the displacement currents.

The samples measured in Figures 4.8 and 4.9 are from farm Epako Süd. They have been measured in different scales according to different graphite network densities and thickness of the graphitic stylolites. Different directions have been considered in relationship to the orientation of the foliation planes. Figure 4.8 shows the measurements of a sample with a high graphite network intensity and a distinct difference in thickness of the graphitic stylolites. The measurements were conducted in three different directions as shown in Figure $4.8 \mathrm{c}$. The circular electrodes had diameters of $30 \mathrm{~mm}$.

The plots of the complex impedance show that there is no clear structural relationship between the resistivities of the conducted measurements (Fig. 4.8 a). Measurement 1 has been conducted perpendicular, whereas measurements 2 and 3 have been performed parallel to the foliation. This implies for this sample, that there is no correlation of the displacement current resistivity with the structural framework. The different gradients in the curves of the complex admittance show the existence of two relaxation phases in the displacement currents (Figure $4.8 \mathrm{~b}$ ). 

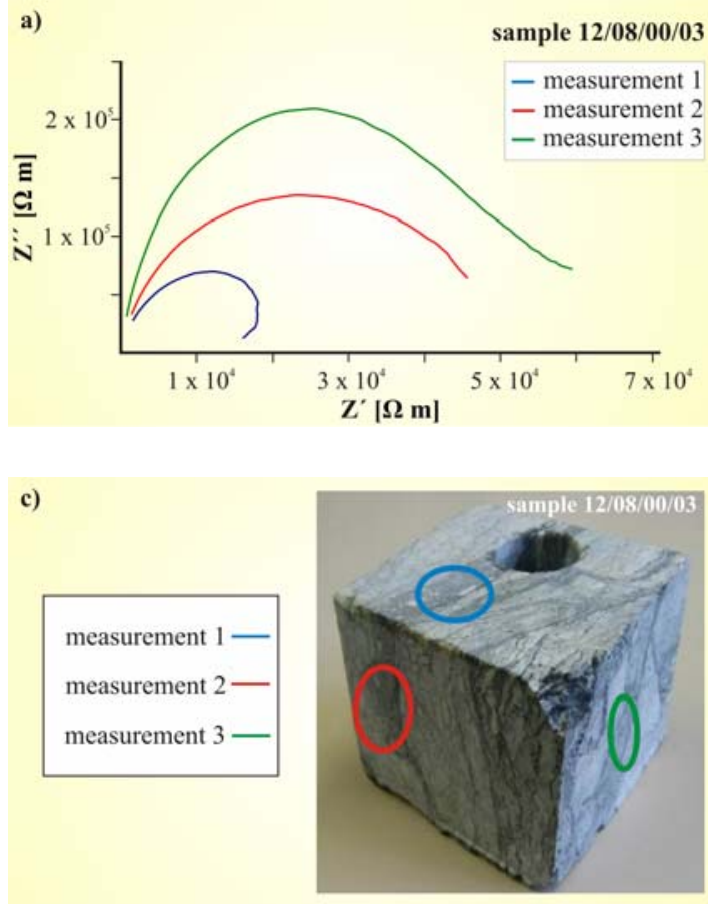

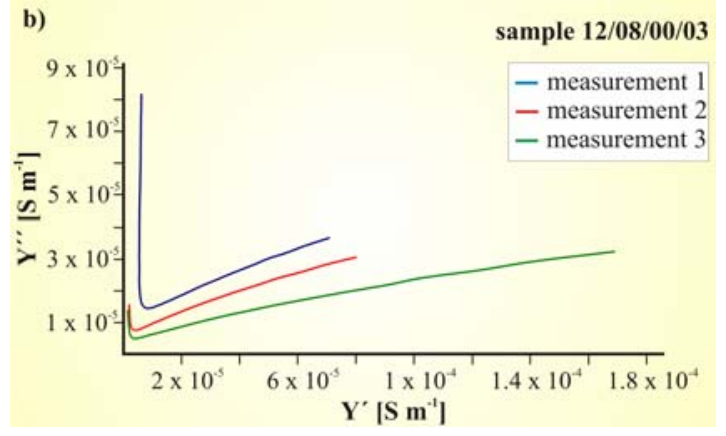

Fig 4.7 a) Cole-Cole plot of the complex impedance of sample 12/08/00/03. It shows the measurement of pure displacement currents. b) Argand plot of the complex admittance of this sample which shows two relaxation phases. c) Measurement points of the electrodes with a diameter of $30 \mathrm{~mm}$ along graphitic stylolites of varying thickness.

The measurements plotted in Figure 4.9 had been conducted across the complete plains of the rectangular sample blocks, as well as on single graphitic stylolites (Fig. 4.9 e). The plots in Figure 4.9 a, b represent the impedance spectroscopic measurements of single graphitic stylolites with point electrodes. These measurements show that the conductivity measured even in this scale is purely composed of displacement currents.

The resistivity of these small scale measurements in general is much lower than measured in Figure 4.7 a, 4.8 a and 4.9 c. This is related to geometric factors like the cross sectional area of the conductor, which is calculated into the intrinsic resistivity according to equation [4.2]. The two measurements plotted in Figure $4.9 \mathrm{c}, \mathrm{d}$ have been conducted across the plains of the rectangular sample blocks. They show, that measurements on all these sample scales show displacement currents with two relaxation phases (Fig. 4.9 a, b).

The presented representative results apply for nearly all measured samples of the magnetotelluric profile and the locality of farm Epako Süd. Furthermore, these results are obviously representative for samples of varying degrees of graphite network intensity and thickness in different directions of the samples. All the samples presented so far, showed purely displacement currents by polarisation effects and ionic conductivity. Therefore, they show no linear conductivity related to electronic conductivity along or perpendicular to the graphitic stylolites. 

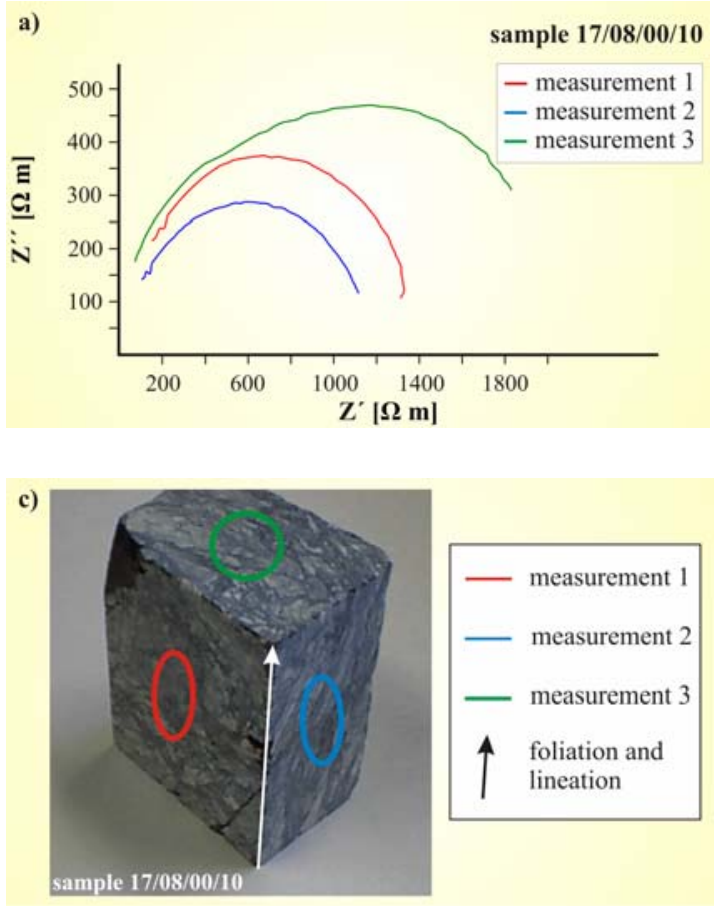

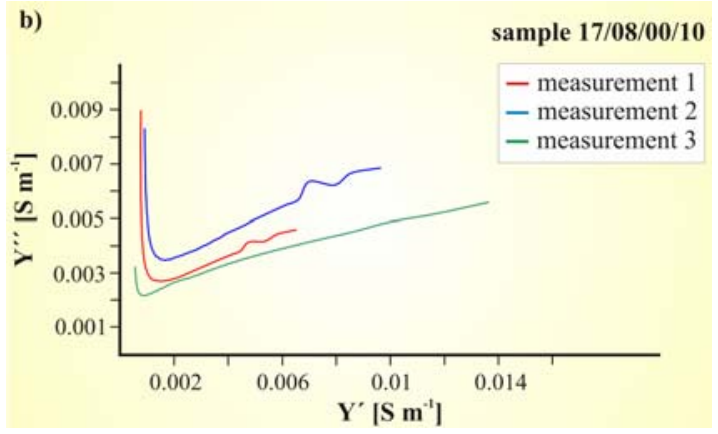

Fig 4.8 a) Cole-Cole plot of the complex impedance of sample 17/08/00/10. Pure displacement currents are observable from the plots. b) Argand plot of the complex admittance of this sample which shows two relaxation phases. c) The measurement points of the electrodes which had a diameter of $30 \mathrm{~mm}$. The foliation is indicated by the white arrow.

In contrast to the measurements presented so far, the sample of Figure 4.10 shows a definite component of electronic conductivity. The data plot of Figure 4.10 a shows, that this sample reacts as a complex resistor with linear and displacement conductivities. This sample is a core sample (Fig. $4.10 \mathrm{c}$ ), which was measured in the autoclave under different hydrostatic pressures. For all samples measured in the autoclave, this core is representative for two cores of one sample, which showed a linear intrinsic electronic conductivity component.

The results of this sample show a half circle plot from the ionic conductivity component, influenced by the linearity of the electronic conductivity component parallel to the axis of real impedance. A pure electronic conductivity would be a point plot on the axis of the real component. This implies that the results show a combination of electronic and ionic conductivity. Opposed to the other measurements presented in Figure 4.7 to 4.9, three relaxation phases are observable in this sample. This may be related to the electronic conductivity component measured in this sample.

The bulk electronic resistivity values of the two impedance measurements are shown in table 4.1. The average electronic resistivity of core 1 is about $519 \Omega \mathrm{m}$. With increasing pressure there is a continuos decrease of the bulk resistivity of about $36 \Omega \mathrm{m}$. The average electronic resistivity of core 2 is about $426 \Omega \mathrm{m}$. For this core there a slight increase measurable for the bulk resistivity up to 75 bars. This is followed by a continuous decrease of the bulk resistivities of about $20 \Omega \mathrm{m}$ with a further increase in pressure. 

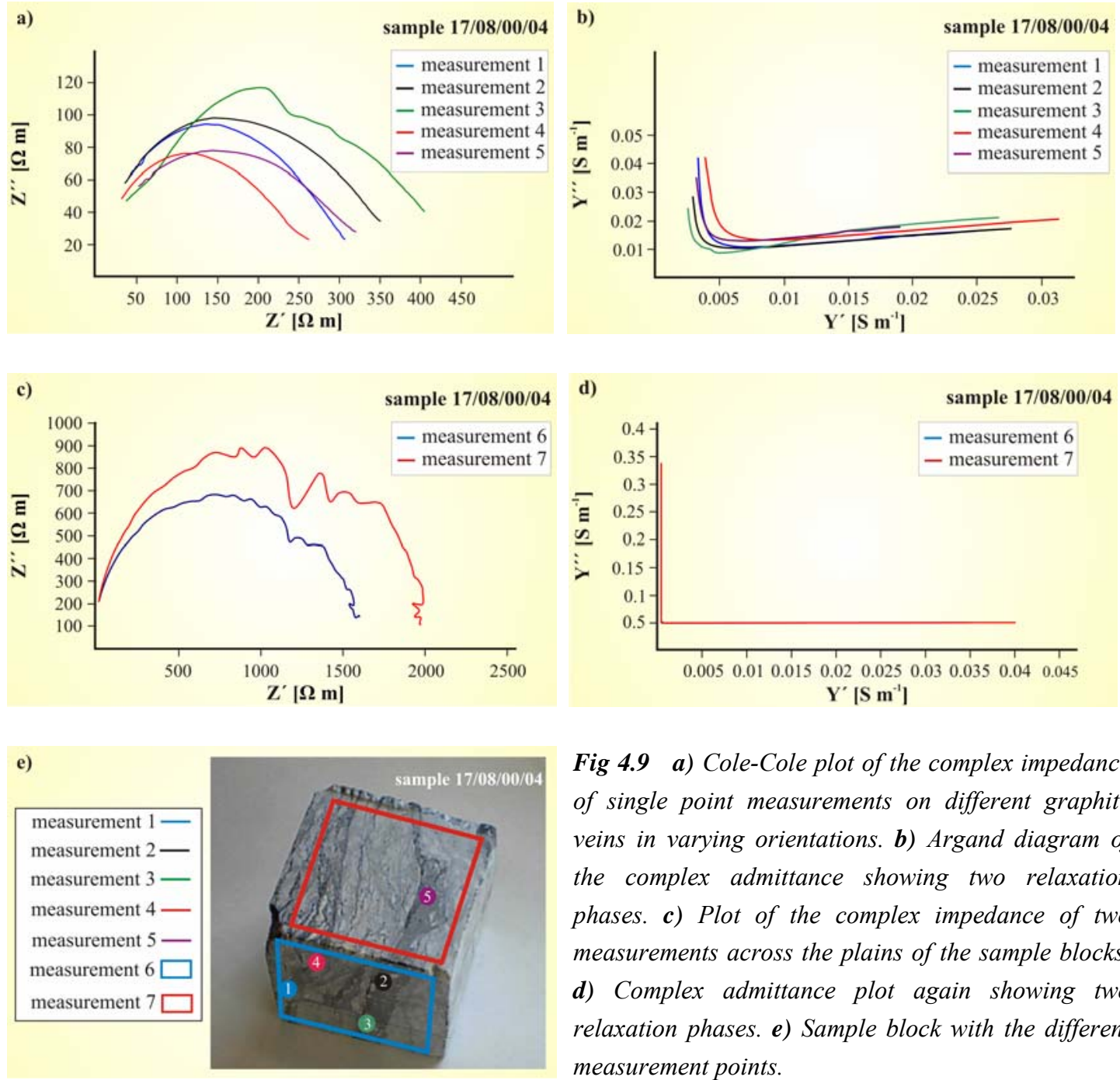

Fig 4.9 a) Cole-Cole plot of the complex impedance of single point measurements on different graphite veins in varying orientations. b) Argand diagram of the complex admittance showing two relaxation phases. c) Plot of the complex impedance of two measurements across the plains of the sample blocks. d) Complex admittance plot again showing two relaxation phases. e) Sample block with the different measurement points.

A decrease in resistivity with increasing pressure is also observable in the plots of the complex impedance in Fig. 4.10 a, b. From 0 to 150 bar, a decrease in resistivity is is obvious with an increase in pressure (Fig. 4.10 a). Accordingly, an increase in conductivity is measureable with increasing pressures accompanied by differing relaxation times $\tau$ (Fig. $4.10 \mathrm{~b})$.

All further measurements of this sample of up to 400 bar plot nearly on the same curve. This implies that the resistivity in the displacement currents decreases with increasing pressure up to an equilibrium pressure. Besides the two presented measurements of electronic conductivities, all other measurements under hydrostatic pressures also showed pure ionic conductivities, similar to the measurements shown in Figure 4.7 to 4.9. 


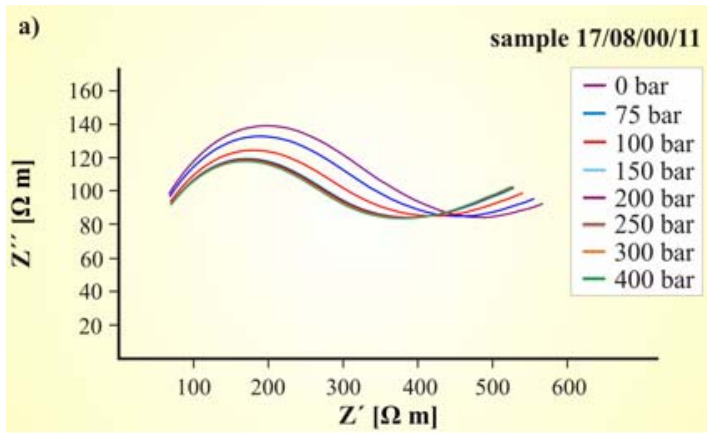

c)

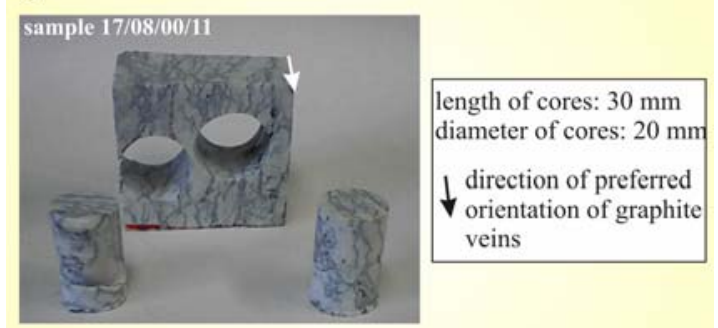

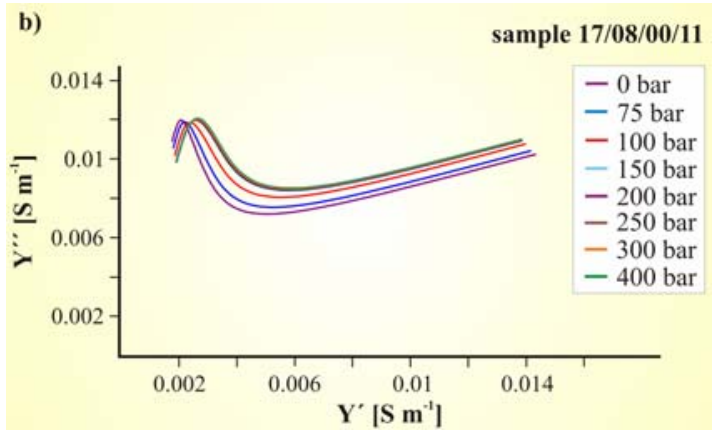

Fig 4.10 a) Cole-Cole plot of the complex impedance of sample 17/08/00/04. It shows a combination of displacement currents and linear electrical currents. b) Argand plot of the complex admittance of this sample which shows the existence of three relaxation phases. c) Sample cores drilled parallel to the foliation were measured in the autoclave.

Both cores of this sample shown in Figure $4.10 \mathrm{c}$ are drilled parallel to the foliation, which is indicated by the preferred orientation of the graphitic stylolites. This is also the direction of the potential difference between the two electrodes. Therefore, a linear conductor parallel to the graphite veins can be assumed for this sample.

In contrast to this measurements and the general microstructural characteristics of the type 2 graphite-bearing marble, hardly no electronic conductivity could be measured along the graphitic stylolites, even on different sample scales (Fig. 4.7 c, 4.8 c, 4.9 e, 4.10 e). This implies, that these interconnected network structures of graphitic stylolites do not generally show electronic conductivity. The reasons for these results have to be discussed, considering other effects on the conductivity potentials of these graphite-bearing marbles.

\subsection{Discussion}

The data presented in Figure 4.10 and table 4.1 shows the direct relationship between electronic conductivity and the orientation of the graphite fabric elements. Accordingly, interconnected graphite veins have been correlated with crustal anomalies of electrical conductivity (Alabi et al., 1975; Duba \& Shankland, 1982; Frost et al., 1989; Mareschal et al., 1992). A combination of conductivity by saline fluids and graphite, as also observed by Leger et al. (1996) is obvious in the presented data (Fig. $4.10 \mathrm{a}, \mathrm{b}$ ). The type 2 of the graphite-bearing marbles only occurs along the outer rims of dome structures. This shows, that the recognised ring structures in the magnetotelluric data of 


\begin{tabular}{|c|c|c|c|}
\hline $\begin{array}{c}\text { pressure } \\
{[\mathbf{b a r}]}\end{array}$ & $\begin{array}{c}\text { bulk resistivity } \\
\text { core } \mathbf{~} \\
\mathbf{R}[\mathbf{\Omega} \text { }]\end{array}$ & $\begin{array}{c}\text { bulk resistivity } \\
\text { core } \mathbf{2} \\
\mathbf{R}[\mathbf{\Omega} \text { }]\end{array}$ & $\begin{array}{c}\text { estimated depth } \\
{[\mathbf{m}]}\end{array}$ \\
\hline 0 & 542,6910 & & 0 \\
\hline 25 & & 400,9720 & 91 \\
\hline 50 & & 425,8540 & 182 \\
\hline 75 & 537,6420 & 433,8620 & 273 \\
\hline 100 & 523,2600 & 433,0040 & 364 \\
\hline 150 & 511,3260 & 432,7180 & 545 \\
\hline 200 & 511,0200 & 433,2900 & 727 \\
\hline 250 & 509,4900 & 432,4320 & 909 \\
\hline 300 & 508,8780 & 431,2880 & 1091 \\
\hline 400 & 506,4300 & 430,7160 & 1455 \\
\hline 500 & & 428,7140 & 1818 \\
\hline 600 & & 426,7120 & 2181 \\
\hline 700 & & 424,4240 & 2545 \\
\hline 800 & & 422,9940 & 2909 \\
\hline & & 413,8420 & \\
\hline
\end{tabular}

Table 4.1 Electronic resistivity components of the two cores of sample 17/08/00/11 with increasing pressure. The data was obtained by impedance spectroscopic measurements in an autoclave apparatus. The estimated depth was calculated after a pressure gradient of 2.75 $\mathrm{kbar} / 10 \mathrm{~km}$.

Weckmann et al. (2003) shown in Fig. 4.1 b, also confirms this correlation between crustal conductivity and the brittle-ductile deformed graphite-bearing marbles.

Other conductivity data of graphite-bearing crustal rocks, obtained by previous authors show quite a strong range. Bulk resistivities on sample scale range mostly between 0.02 and a few times $10^{3} \Omega \mathrm{m}$ and in exception to $10^{4} \Omega \mathrm{m}$ (Duba \& Shankland, 1982; Mareschal et al., 1992; Duba et al., 1994; Nover et al., 1998; Fowler et al., 2004; Jödicke et al., 2004). This confirms that the data presented above is consistent for crustal conductivity potentials. The range of the published data shows, that quite a strong variation is measurable in the conductivities of graphite-bearing rocks.

Reasons for the relative strong deviation between the above stated data can be manifold. They have to be considered and evaluated individually for every geological setting of the measured samples. Commonly conductivities of graphite-bearing rocks are also scale dependant. A variety of factors described in the following, may influence the conductivity potentials of graphite-bearing rocks to quite some extent.

A first important factor on the conductivity of graphite-bearing rocks is the composition of the conducting minerals. Even if graphite is the main interconnected conducting phase, the total conductivity varies with the volumetric content and the degree of connectivity of other conducting minerals included in the rock. Fowler et al. (2004) presents data which shows, how resistivity data varies with respect to the relative 
volumetric content of graphite, pyrite and hematite. The same dataset shows two samples from a locality with a nearly identical content of conducting minerals, but with differences in resistivity by one order of magnitude.

Another influencing factor on conductivities of interconnected graphite in rocks is the degree of the graphite crystallinity. Raab et al. (1998) describes differences in resistivity of 5 orders of magnitude between low grade metamorphic black shale and bituminous black shale. The latter had undergone burial diagenesis to a maximum temperature of $150^{\circ} \mathrm{C}$. The molecular structure of these samples lies between the beginning of oil formation and anthracite. In contrast, the low grade metamorphic black shales had been influenced by a thermal metamorphism of $250-280^{\circ} \mathrm{C}$. Raman investigations showed that the molecular structures of these samples lie between anthracite and graphite (Raab et al., 1998).

Similarly, differences in resistivity within the graphite molecular structures are to be expected with varying degrees of crystallinity. One aim of this work was, to quantify this effect by correlating graphite crystallinities (see chapter 3) with the complex impedance spectroscopic measurements. Unfortunately, this was not possible as only one sample showed electronic conductivity as discussed above. Secondly all the samples were of nearly uniform high crystallinity.

Thirdly, the conductivity of graphite-bearing rocks is also dependant on the thickness of the graphite veins. It can be calculated to the cross sectional area of the conductor. Therefore, this influence can be quantified after equation [4.2] for the intrinsic resistivity and after equation [4.3] for the intrinsic conductivity. Katsube \& Mareschal (1993) showed in model calculations, that a pore lining of connected graphite films of 50 to $200 \AA$ thickness will produce bulk resistivities of a few $100 \Omega \mathrm{m}$. Mareschal et al. (1992) shows resistivity data for graphite films of a thickness of 30 to $300 \AA$ in samples of the Kapuskasing gneisses of a few times $10^{3} \Omega \mathrm{m}$. Graphite crystallinities in these samples are of disordered graphitic carbon.

A fourth influencing factor are the microstructures of the graphite in the conducting veins. If the basal plains of the graphite are oriented randomly in the graphite veins, the interconnection of the graphite crystals is also randomly. This implies that differences in conductivity can occur due to anisotropic conductivity potentials parallel and perpendicular to the basal plains of graphite. Accordingly, the degree of interconnectivity of the graphite is then more random, which also affects the conductivity. Even for preferred oriented graphite in the graphitic stylolites, the conductivity potentials of the stylolites are still dependent on the degree of interconnectivity between the graphite crystals. Therefore, the 
conductivity depends on whether the graphite basal plains are partially or commonly interlocked between the single crystals. The local textures of the fine-grained graphite veins could be determined for example by electron backscatter diffraction (EBSD).

Another important microstructural influence in this respect is the grain-size distribution of graphite within the graphitic stylolites. The degrees of interconnectivity of the graphite crystals may vary to quite some extend within graphitic stylolites or veins, according to the grain-size of the graphite crystals. This applies also to the crustal conductivities of the graphite-bearing rocks. After Frost et al. (1989) graphite-bearing rocks with grain sizes of $1 \mathrm{~cm}$ to $0.1 \mathrm{~mm}$ are in the range of high crustal conductivities, whereas rocks of the same type with bigger grain sizes range below these conductivities. As this gradient is significantly steep, this influence is also scale dependant. Grain sizes may change for example between shear zones and host rock on a m scale within the crust.

Another influencing factor in conductivity measurements are the microfabrics of the host rock. After Katsube \& Mareschal (1993) measurements of the bulk rock resistivity showed, that the resistivity measured parallel to the foliation is lower than the resistivity measured perpendicular to the foliation. One reason may be the interconnection of conductive minerals along the foliation planes, due a shape preferred orientation. Another reason could be that a interconnected fluid network is present parallel to the foliation planes. In combination this could result in additional ionic bridging of electronic conductors.

Additionally, the graphite network intensity is an important factor influencing the conductivity of graphite-bearing carbonates. A higher graphite network intensity of interconnected graphitic stylolites and veins would also produce higher overall conductivities. As shown above, the network densities can vary to a great extend within a crustal shear zone on a $\mathrm{cm}$ to $\mathrm{m}$ scale. Therefore, this is important for the differentiation of the conductivities on sample scale. Accordingly, caution is necessary for the upscaling of these measurements to crustal dimensions.

The effects of pressure on the conductivity of rocks also have to be considered. The effects of pressure on the resistivity of basalt are shown in Figure 4.5 b. Also Shankland et al. (1997) describes an increase in conductivity with increasing pressure. As discussed below microcracks, permeability, fluid percolation and a reconnection of solid conductors by the closure of microcracks are the main factors for these observations.

For the quantification of conductivity potentials of (sub)surface crustal rocks, microcracks have to be taken into account (Glover \& Vine, 1992; Katsube \& Mareschal, 
1993). As the results shown in table 4.1 and in Figure $4.10 \mathrm{a}, \mathrm{b}$ indicate, a reduction of resistivity in linear and displacement currents with increasing hydrostatic pressures is measurable. After equations [4.2] and [4.3], this is only possible for electronic conductivity, due to the reconnection and extension of the graphitic conductors. Therefore, a closure of microcracks had been obtained by increasing the hydrostatic pressures.

Microcracks are either upper crustal tensional microcracks, or originate due to lower crustal hydrofracturing. Therefore, careful investigations on these influences are necessary for the quantification of crustal conductivity potentials. Tensional or tensile microcracks develop, as crustal segments are lifted to upper crustal levels. They develop when $\sigma_{3}$ becomes negative and tensional stresses evolve. As the development of tensile microcracks in dependent on $\sigma_{3}$, they show a preferred orientation orthogonal to $\sigma_{3}$.

Hydrofractures emerge from extensional crustal stresses in combination with fluid overpressures. Therefore, hydrofractures also show a preferred orientation relative to crustal stress field. As microcracks and open hydrofractures are reclosed at conductivity measurements under confining hydrostatic pressures, it is questionable whether these measurements are allways in accordance with deeper crustal conductivities. This matter will be discussed in more detail in the following. The effect of remineralised veins and microveins on the conductivity potentials have to be revealed by microstructural investigations.

The conductivity measurements under hydrostatic pressures of a variety of samples, showed no electronic conductivities with the exception of only one sample (Fig. 4.10 and table 4.1). The investigation of the microstructures by qualitative optical microscopy and cathodoluminescence microscopy (CL) revealed, that the graphite-bearing marbles show microcracks, which very frequently are healed as microveins by a younger generation of calcite. These microveins show a preferred orientation within the different generations.

A first generation of the microcracks originated most likely as hydrofractures, due to extensional stresses during the cretaceous break up of pangaea between Africa and South America. The crustal stresses are documented by widespread intrusions of cretaceous mafic dykes in the vicinity. This is also visible on the satellite image in Figure 2.2. Due to the fluid migration in these microcracks, the vein mineralisation of calcite formed this observed generation of microveins, which show different cathodoluminescence colours as the marble host-rock.

The latest microcracks may have originated due to tensional stresses near (sub)surface crustal levels. It is obvious from table 4.1, that open microcracks still exist up 
to estimated depths of $2900 \mathrm{~m}$. Microcracks up to this depth are generally related either to hydrofracturing or to tensional microcracks, resulting from local crustal stress concentrations due to stress inhomogeneities in the crust.

As shown in Chapter 2.3.4, most of the mineralised microcracks cut the graphite veins and network structures on a microscopic scale within $200 \mu \mathrm{m}$ and several $\mathrm{mm}$. This implies, that the electronic conductivity is cut and disturbed on a $\mu \mathrm{m}$ to $\mathrm{mm}$ scale by microcracks and microveins. The increasing hydrostatic pressures could not close the mineralised microveins to reconnect the disturbed graphite veins. Therefore, conductivity measurements even under high pressures did not show any electronic conductivity in the samples, except for the sample of Figure 4.10. In this sample the graphite veins are undisturbed by microveins.

The CL investigations of the microveins also revealed, that the graphitic stylolites themselves often opened due to hydrofracturing or tensional stresses. The remineralisation of the opened graphitic stylolites by calcite disturbed the interconnectivity of graphite along the graphitic stylolites. Therefore, they are not capable of transporting electrical charge over distances greater than on the described scale.

In contrast, the measured magnetotelluric anomalies of high electrical conductivity are crustal phenomena calculated on a kilometer scale. Therefore, the exact localisation to a sample scale of the conducting crustal material is barely possible. Differences in the bulk conductivities of graphite-bearing marbles, which occur on sample scale due to the above mentioned factors, are mostly not measurable in magnetotelluric profiles. This scale incoherence implies that only a mean and thus more general quantification for the conductivity potential of graphite-bearing marble, would be applicable for calculations of magnetotelluric data.

The sample of Figure 4.10 is from farm Epako Süd, where no magnetotelluric measurements have been conducted. This is the reason, why the above described relationship between graphitic stylolite fabrics and electrical conductivity cannot directly be related to the magnetotelluric measurements by Ritter et al. (2003) and Weckmann et al. (2003). Nevertheless, they give a first assumption for the conductivity potentials of this type of graphite-bearing marbles in a general regional context. Compared to the resistivities from the magnetotelluric data by Ritter et al. (2003), this data fits very well into their calculations of the surface anomalies of high electrical conductivity (Fig. $4.1 \mathrm{~b}$ and table 4.1). 
According to the discussed origin of the described microcracks, it is still debatable whether the conductivity potential of the graphite-bearing marble increases with increasing depth, to the large extends implied by the data in table 4.1. A decrease of resistivity should be related to a decrease of tensile microcracks with an increasing depth. The amount of tensile microcracks decreases rapidly to a depth of about $1000 \mathrm{~m}$.

This an approximate depth related to Mohr criteria, where a maximum normal stress $\left(\sigma_{\mathrm{n}}\right)$ is allowed for tensional microcracks in relationship to the shear stress. The closure of unhealed extensional cracks and microcracks, by greater confining lithostatic pressures in deeper crustal levels, is still a matter of debate. In general, the closure of microcracks in the crust is dependant on the permeability of the microcracks and on the amount of crustal fluids percolating the rocks.

After Weiss et al. (2002) ultrasonic wave velocity measurements show, that dry microcracks of different kind of marbles close completely at approximately $75 \mathrm{MPa}$ confining pressure. Most of the microcracks are already closed between 20 and $50 \mathrm{MPa}$ in these measurements. This is supported by the measurements shown in table 4.1 , where the electronic resistivity continuously reduces even to pressures of 800 bar, due to the continuous closure of the microcracks with increasing pressure.

These results only apply for dry rock material, whereas pore fluids and therefore fluid overpressure in the crust are more likely to be expected. As KTB reports by ELEKTB-Group (1997) state, ionic conduction by hydraulic conductors was still measured at depths below $3850 \mathrm{~m}$. Saline fluids were observed to depths of $6000 \mathrm{~m}$. This implies, that the fluid overpressure may still be prevailing in these crustal depths. In contrast, the impedance spectroscopic pressure measurements were conducted with porous electrodes to reduce the fluid overpressure in the sample. This was done, to prevent the sample from cracking completely during the measurement. Therefore, the decrease in resistivity of the impedance spectroscopic measurements may not always be directly applicable to crustal conditions, since the fluid overpressure in the sample was released through the porous electrodes.

Ultrasonic wave velocity measurements show, that the wave velocities are relatively stable with increasing pressure for saturated microcracks, as the lacking compressibility of the pore fluid does not allow further closure of the microcracks (Weiss et al. 2002). Investigations on microscopic scale conductivity by Losito et al. (2001), also shows this correlation between saturated and unsaturated microcracks and electrical conductivity measurements with increasing pressures. 
To quantify the conductivity potentials of these Damaran graphite-bearing marbles, a regional investigation of the intensity, distribution and orientation of the healed and unhealed microcracks has to be conducted. The investigations on the cretaceous stress field would help to understand the allocation, orientation and distribution of hydrofractures in the area of the magnetotelluric profile.

In addition, a qualitative and quantitative investigation of the preferred orientation of the microveins would give some indication about the origin of the different microcracks. Tensional microcracks are oriented perpendicular to $\sigma_{3}$ and therefore should be distinguishable from the extensional hydrofractures related to the cretaceous stress field. For further confirmation samples from boreholes can help to verify the origin of the microcracks and the conductivity potential of the graphite-bearing marbles.

A further step would be to correlate conductivity measurements with the degree of graphite network intensity. A quantification of this influence would be helpful for the interpretation of magnetotelluric profiles. Accordingly, EBSD investigations on the microstructures of graphitic stylolites, and the correlation of conductivity measurements with the degree of graphite crystallinity would further enlighten the complex matter of graphite conductivity in geological materials. 


\section{CHAPTER 5}

\section{STABLE ISOTOPE ANALYSIS}

Stable isotopes as ${ }^{13 / 12} \mathrm{C}$ and ${ }^{18 / 16} \mathrm{O}$ are a useful tool to characterise carbonate rocks. Isotopes occur when elements build atoms with different numbers of neutrons in the atomic nucleus. Most elements consist of one stable and several unstable isotopes. The natural occurrence of the different isotopes of an element varies extensively. For carbon two main stable isotopes exist with an occurrence for ${ }^{12} \mathrm{C}$ of $98.89 \%$ and for ${ }^{13} \mathrm{C}$ of $1.11 \%$ respectively (Nier, 1950). ${ }^{14} \mathrm{C}$ isotopes which are well known for radiometric dating, only occur as traces in terrestrial materials. The frequency of occurrence of oxygen isotopes also varies with $99.763 \%$ for ${ }^{16} \mathrm{O}$ isotopes, with $0.0375 \%$ for ${ }^{17} \mathrm{O}$ isotopes and with $0.1995 \%$ for ${ }^{18} \mathrm{O}$ isotopes (Garlick, 1969).

Molecules that consist of heavier isotopes are more stable, as the dissociation energy needed to break-up the molecules is higher than for lighter isotopes (Fig. 5.1). Due to the lower vibrational energies of the heavier isotopes, the bonding energy is higher between heavier isotopes than for molecules with lighter isotopes. Therefore, most inorganic molecules like crustal minerals tend to incorporate heavier isotopes, while the lighter isotopes are enriched in gaseous and fluid phases. Most biological processes are also based on the differences in dissociation energies and lead to a strong fractionation of carbon isotopes. Photosynthesis effects of $\mathrm{C}_{3}$-plants, which constitute $90 \%$ of all plants today, results in a depletion of the heavier ${ }^{13} \mathrm{C}$ isotopes relative to ${ }^{12} \mathrm{C} . \mathrm{C}_{4}$-plants show a similar depletion effects but to a less great extent.

Isotope fractionation can originate either from kinetic or equilibrated isotope exchange reactions. The described biological depletion of ${ }^{13} \mathrm{C}$ results from kinetic isotopic fractionation, as lighter ${ }^{12} \mathrm{C}$ isotopes are bound in organic molecules, whereas ${ }^{13} \mathrm{C}$ is concentrated in $\mathrm{CO}_{2}$. Kinetic fractionation is therefore a irreversible process.

After Matthews et al. (1983) and Giletti (1985) there are three main mechanisms for kinetic isotope fractionation between minerals and fluids. One mechanism is controlled by solution and precipitation processes, where mineral grains are dissolved and crystallise to bigger grains with a lower sum of total free energy. Due to the dissociation of the crystal lattices by solution processes atoms are movable and free to form new molecules and crystals. As heavier isotopes are preferably included into molecules with high rates of covalent bonds, isotope fractionation takes place during crystallisation. Lighter isotopes are enriched in the fluid phase and are mostly removed by the mobile fluids. 


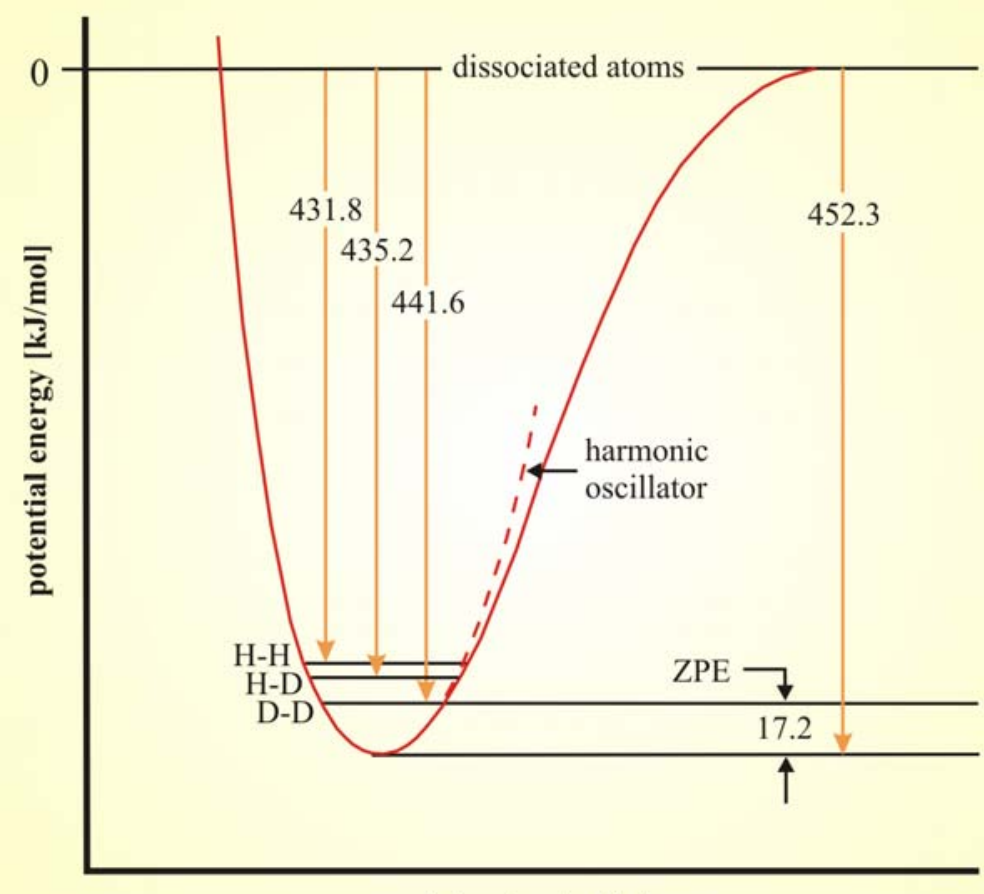

interatomic distance

Fig. 5.1 Potential energy-level diagram for different isotopic species of the hydrogen molecule. The differences of the zero-point energy levels (ZPE) for $\mathrm{H}_{2}$ is shown for different fundamental vibrational energies. The energy required for the dissociation of of the different species are indicated by the arrows. Figure after O'Neil (1986).

Further mechanisms of kinetic isotope fractionation are diffusional processes, where the exchange of isotopes occurs between interfaces without a change in phase morphology. A third mechanism is a chemical reaction between two phases with the enrichment of lighter isotopes in the fluid phase.

For kinetic isotope fractionation fluid phases play an important role, whereas equilibrated isotope exchange reactions only occur when fluid phases are absent. Equilibrated isotope fractionation is triggered by thermodynamic factors. Therefore, it is dependent on changes to PT-conditions, where diffusional processes and crystallisation are possible. In these regimes, isotopic fractionation occurs due to isotope exchange reactions between at least two mineral phases. During the isotope exchange reactions, the isotopic composition of the involved phases is approximated constantly between the phases. Equilibrated isotope exchange reactions between solid phases are mainly controlled by temperature. Therefore, several calibrations for geothermometric calculations are discussed in the literature. An overview about the geothermometric calibrations for calcite-graphite thermometry is compiled by Valley (2001). 
The isotopic composition of a mineral or a fluid phase is described by the isotopic ratio $\delta$. This ratio is obtained between the concentrations of different isotopes from one element within a phase. The isotopic ratios are calculated to an international standard as for carbon by:

$$
\delta=\left[\frac{\left({ }^{13} C /{ }^{12} C\right)_{s m p}}{\left({ }^{13} C /{ }^{12} C\right)_{s t d}}-1\right] x 10^{3}
$$

The isotopic ratio $\delta$ is expressed in \%o. Carbon and oxygen isotope ratios are nowadays calculated over the V-PDB standard (Pee Dee Belemnite), whereas older data is also calculated over the SMOW standard (standard mean ocean water). The isotope ratios used in this work are calculated over V-PDB. Cited data calculated to the SMOW standard, is here recalculated to the V-PDB standard for comparison reasons.

Some isotopic ratios are characteristic for different kind of crustal rocks. Figures $5.2 \mathrm{a}, \mathrm{b}$ show these for carbon and oxygen isotopes. They vary quite distinctively within the crustal and atmospheric material, depending on the isotopic composition of their base material and the processes of formation. In general, a organic influence in the crustal material shifts isotopic ratios to lighter values. Fluids and gaseous phases are also mostly characterised by lighter isotope ratios, whereas crustal material generally includes heavier isotope ratios.

As isotopic ratios are changed by isotope exchange reactions, the quantification of these isotope fractionations is important for the interpretation of the isotopic ratios. The fractionation factor $\alpha$ between two different phases is calculated as the overall ratio between the isotopic compositions of the two phases A and B:

$$
\alpha_{A-B}=\frac{R_{A}}{R_{B}}
$$

If all isotopes are exchanged in an equilibrated system, the fractionation factor will be identical to the equilibrium constant of the two phases for a specific temperature. Therefore, this fractionation factor $\alpha$ is very important to study the isotopic exchange reactions and rates between two phases at certain temperatures. 

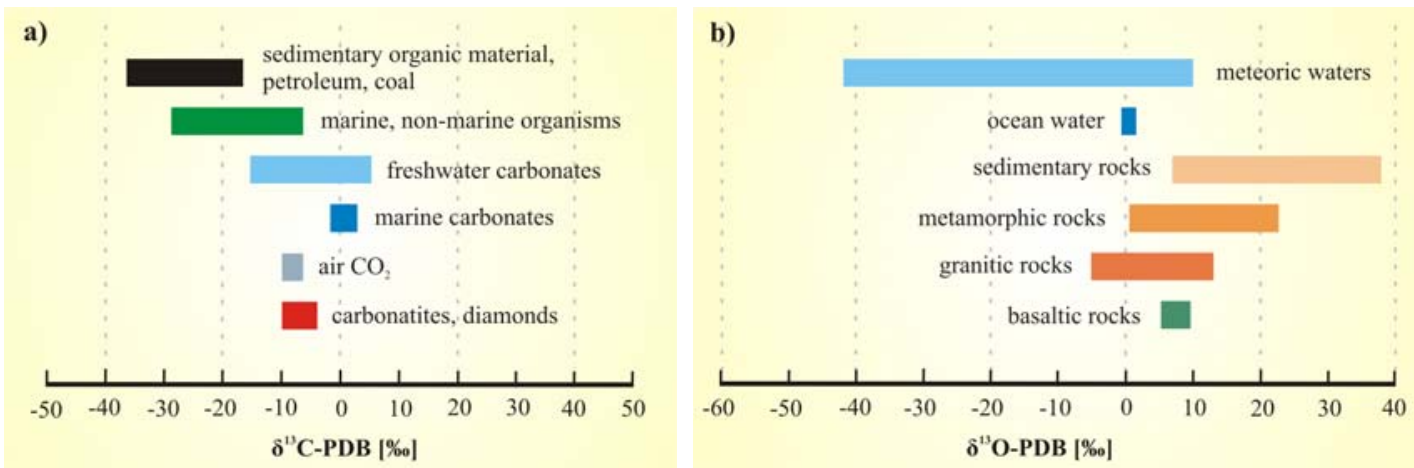

Fig. 5.2 a) Carbon isotopic composition of different crustal and atmospheric phases. Organic material generally plots to lighter carbon isotope ratios. b) Oxygen isotope composition of different geological materials. Crustal rocks generally plot to heavier oxygen isotope ratios. Both figures after Hoefs (1997).

According to equation [5.2] the fractionation factor $\alpha$ for carbon between calcite and graphite will have to be calculated as follows:

$$
\alpha_{\mathrm{CaCO}_{3}-C_{\text {graphite }}}=\frac{\left(\frac{{ }^{13} \mathrm{C}}{{ }^{12} C}\right)_{\mathrm{CaCO}_{3}}}{\left(\frac{{ }^{13} \mathrm{C}}{{ }^{12} C}\right)_{C_{\text {graphite }}}}
$$

Another dimension for the characterisation of isotope fractionation is calculated from the isotopic ratios $\delta$. The quantification of the isotopic fractionation $\Delta_{(\mathrm{A}-\mathrm{B})}$ is calculated for two isotopically equilibrated phases $\mathrm{A}$ and $\mathrm{B}$, by the subtraction of the isotopic ratios $\delta$ of the two phases. The carbon isotopic fractionation for calcite and graphite is calculated as follows:

$$
\Delta_{(\text {cal-gr })}=\delta^{13} C_{\text {calcite }}-\delta^{13} C_{\text {graphite }}
$$

The isotopic fractionation $\Delta_{(\mathrm{A}-\mathrm{B})}$ is expressed in \%o. It is generally used to describe geothermometric correlations and to correlate isotopic fractionations with metamorphic grades. It can also be calculated from the fractionation factor $\alpha$ with some neglectable differences by the following equation:

$$
\Delta_{(c a l-g r)} \approx 10^{3} \ln \alpha
$$




\subsection{STABLE ISOTOPES OF MARBLES AND GRAPHITE}

For carbonates and (in)organic carbon a range of isotope ratios are characteristic. As reported in the literature, carbonates are distinguished as heavy carbonates with carbon isotope ratios of $\delta{ }^{13} \mathrm{C}>+20 \%$ and as light methane with $\delta{ }^{13} \mathrm{C}>-80 \%$ (Hoefs, 1997). For marine carbonate sediments, the mean range of $\delta{ }^{13} \mathrm{C}$ ratios vary from -2 to $+3 \%$ (Fig. 5.2 a). For graphite the isotopic ratios reported in the literature range mainly between -35 to $-2 \%$. Variations in the carbon isotope ratios of carbonates and graphite depend mainly on the origin of the carbon material and on the metamorphic history.

Additionally carbonate rocks are characterised by oxygen isotopes. Oxygen isotope ratios are very good indicators for fluid/rock interactions (Hoefs, 1997). As described above, kinetic isotope fractionation between fluids and rocks are controlled by solutionprecipitation processes, by chemical reactions and by diffusion. By these processes, the primary isotopic compositions of the mineral and fluid phases are changed. Therefore, the $\delta{ }^{18} \mathrm{O}$ ratios vary quite strongly within different carbonate rocks.

This variation is documented in a database of oxygen isotope ratios from precambrian carbonates and other crustal rocks after Shields \& Veizer (2002) (Fig. 5.3). These $\delta{ }^{18} \mathrm{O}$ ratios generally range between -25 and $0 \%$. The data shows, that there is a slight positive trend of the mean archean to neoproterozoic oxygen isotope ratios to heavier

$\delta{ }^{18} \mathrm{O}$ ratios. This may be related to the increasing photosynthetic activity during the proterozoic. As these isotope ratios are gathered from carbonates worldwide, primary oxygen isotopic signatures for seawater are postulated throughout the precambrian (Brasier et al., 1990; Burdett et al., 1990; Kaufman et al., 1991; Veizer et al., 1992; Hall \& Veizer, 1996).

Kinetic exchange reactions of oxygen isotopes between associated mineral and fluid phases, mainly occur due to the opening of fluid pathways during deformation. Accordingly, the quantification of isotope fractionation may give some hints on the tectono-(metamorphic) history of the rocks. Kirschner \& Kennedy (2001) show the effects of fluid flow on stable isotope ratios in carbonate-hosted fault rocks. The interpretation of the oxygen isotopic ratios becomes difficult, when multiple isotope fractionations have occurred due to a complex deformational path and magmatic intrusions. In general, the interpretation of oxygen isotope fractionations becomes more significant, if several mineral phase have exchanged isotopes with the fluid phase in kinetic isotope fractionation. Additional indications on oxygen isotope exchange reactions may be revealed, by considering primary oxygen signatures for seawater at specific geological times. 


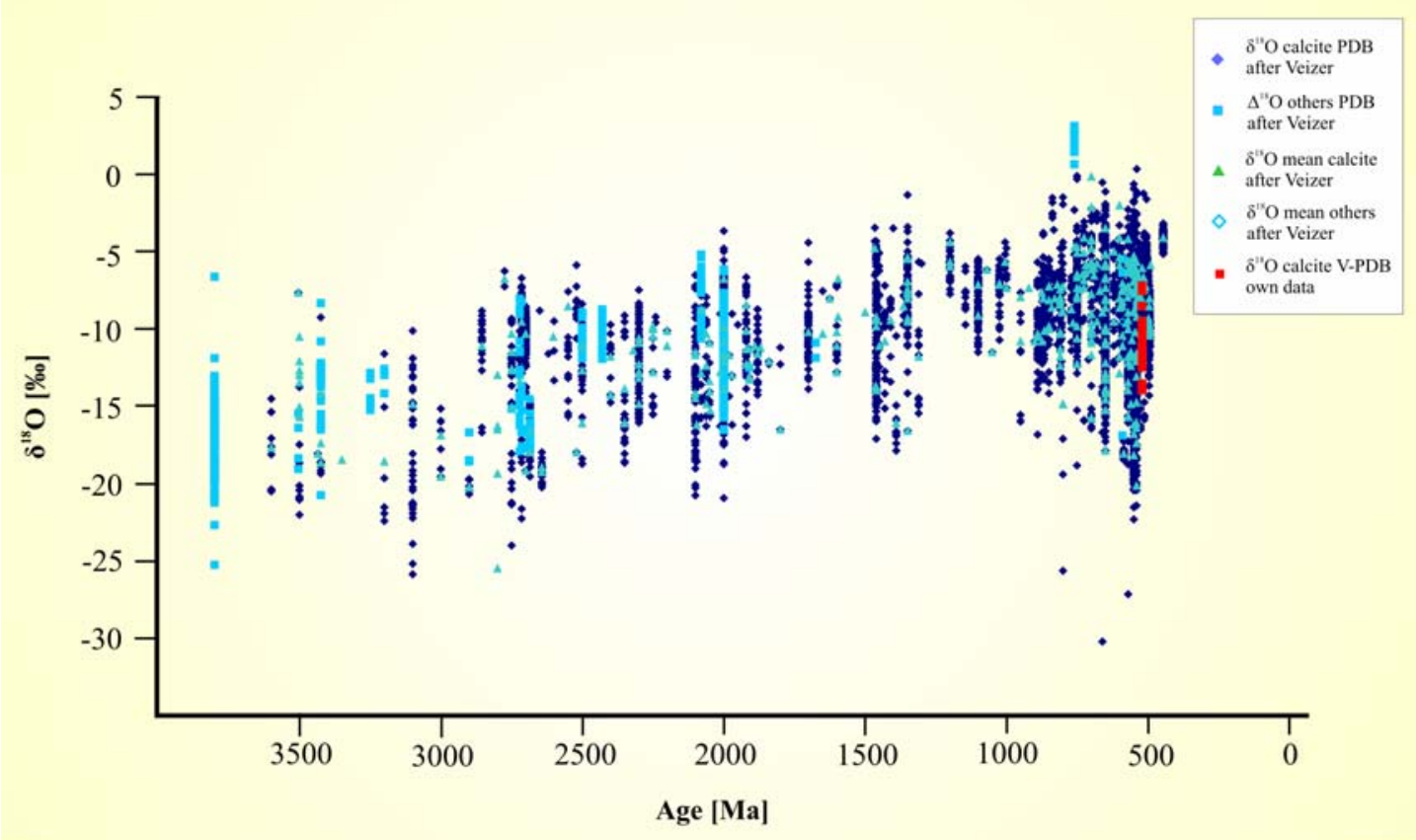

Fig. 5.3 Precambrian oxygen isotope ratios for carbonates and other crustal rocks. Own measurements are plotted in red signatures into the diagram with a neoproterozoic age of 520 Ma. Database after Shields \& Veizer (2002).

The carbon isotope composition of precambrian rocks and organic material after Shields \& Veizer (2002) is shown in Figure 5.4. The precambrian carbon isotope ratios range between -18 and $+16 \%$, while the mean precambrian $\delta{ }^{13} \mathrm{C}$ ratios for calcite plot within -5 and +6 $\%$. This is similar to the present day range for carbonates (Fig $5.2 \mathrm{a}$ ). The carbon isotope ratios of organic matter range between -46 to $-12 \%$. This shows that there is a significant difference between organic and inorganic carbon isotopes. This allows an estimation about an organic or inorganic formation of carbon bearing mineral phases. This estimate is only possible, when kinetic or equilibrated isotope exchange reactions can be excluded for the measured samples.

Reasons for the variation of carbon isotope ratios in carbonate rocks as documented in Figure 5.4 can be manifold. Differences already occur during the formation and sedimentation of the carbonates. Isotope fractionation already occurs during the precipitation of carbonate. With increasing seawater temperature, $\delta{ }^{13} \mathrm{C}$ increases in calcite within the equilibrium between dissolved bicarbonate $\left(\mathrm{HCO}_{3}^{-}\right)$and solid calcite. In contrast, the carbon isotope ratio between atmospheric $\mathrm{CO}_{2}$ and dissolved bicarbonate decreases strongly with increasing temperatures. This implies that an increase of $\delta{ }^{13} \mathrm{C}$ only occurs with decreasing seawater temperatures (Stosch, 2002). 


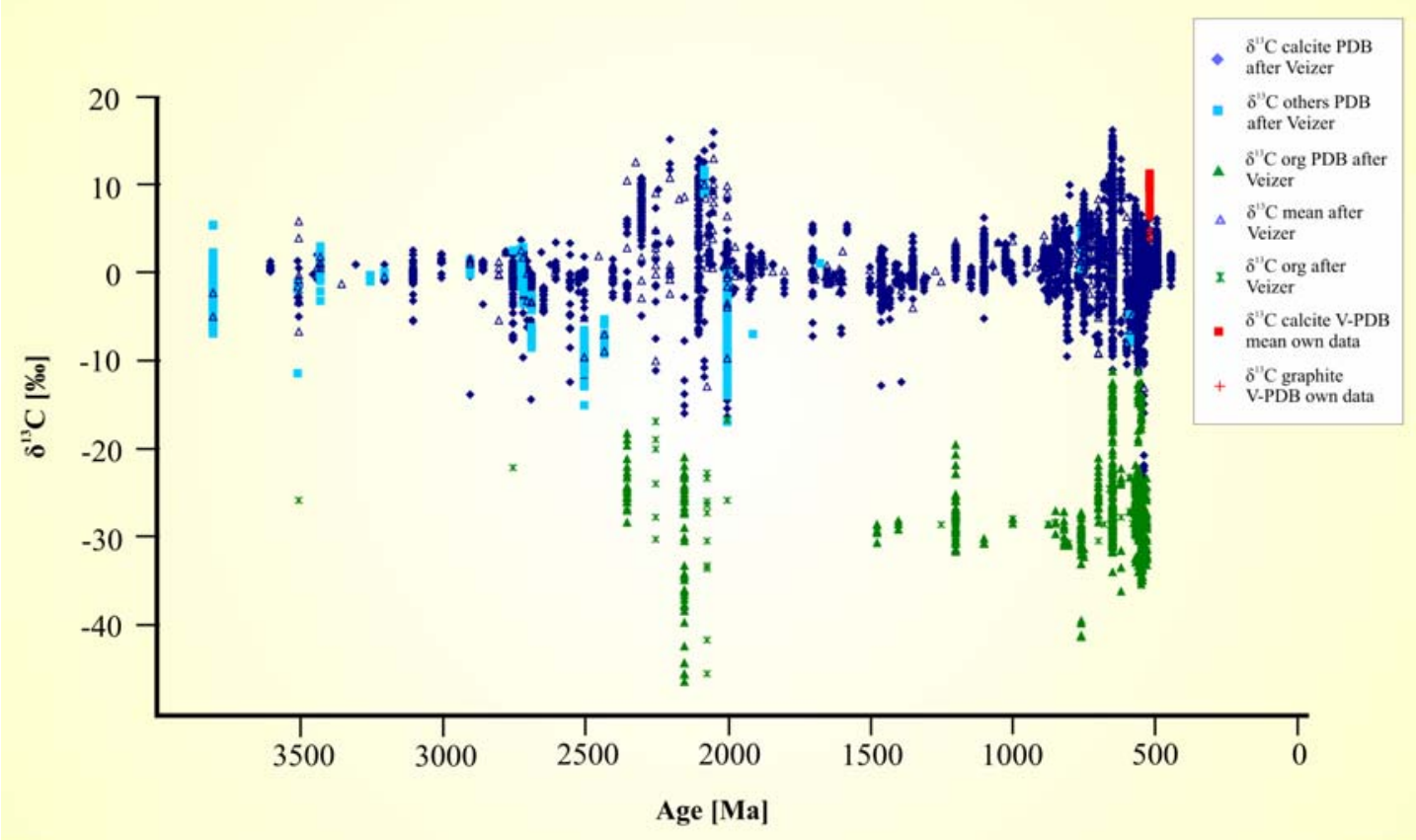

Fig. 5.4 Precambrian carbon isotope ratios for carbonates, other crustal rocks and organic material. Own measurements for calcite and graphite are plotted into the diagram in red signatures with a neoproterozoic age of 520 Ma. Database after Shields \& Veizer (2002).

Additionally, the carbon isotopic composition of the seawater is changed due to photosynthetic activity of organic material. As organic material concentrates lighter ${ }^{12} \mathrm{C}$ isotopes due to photosynthesis by diffusional processes (Park \& Epstein, 1960), dissolved bicarbonate is enriched by ${ }^{13} \mathrm{C}$ isotopes relative to $\mathrm{CO}_{2}$ in marine environments. In the equilibrium between dissolved bicarbonate and calcite, ${ }^{13} \mathrm{C}$ isotopes will therefore also be enriched in calcite. Additionally, crystallisation processes also fractionate isotopic systems, as chemical bondings are energetically more stable by incorporating heavier isotopes into molecule- and lattice structures (O'Neil, 1986) (Fig 5.1).

The primary isotopic composition of carbonate rocks can change distinctively during deformation and metamorphism. If fluids penetrate the rock pervasively or discrete along shear and fault zones, the isotopic composition of the carbonates can be changed due to isotopic exchanges with the fluid. This kinetic isotope fractionation is irreversible, due to the mobility of the fluids across the crustal segments. Secondly the isotopic composition of carbonates can change, due to the isotopic exchange reactions between carbonates and other carbon bearing mineral phases like graphite. The extent of equilibrated isotope fractionations is temperature dependent (Criss, 1991), and for isotope exchange by diffusion processes also dependent on diffusion rates (Chacko et al. 1991; Hoefs, 1997). 
According to the origin of graphite, syngenetic and epigenetic graphite is distinguished. Syngenetic graphite originates from the metamorphism and graphitisation of primarily organic carbon material. Carbon atoms are arranged in a hexagonal crystal structure during graphitisation, which forms the basal plains of the graphite crystals. Therefore, syngenetic graphite commonly has preserved the organic carbon isotope ratios, and in these cases can be related to organic origin. Epigenetic graphite originates from the precipitation of graphite from $\mathrm{CO}_{2}$ - and/or $\mathrm{CH}_{4}$-bearing fluids. Accordingly, this graphite is characterised by the lighter isotopic ratios of the magmatic or deep crustal fluids. This implies that they can be differentiated from the syngenetic graphite by their different isotopic compositions (Weis et al., 1981).

The primary carbon isotopic ratios of calcite and graphite in graphite-bearing marbles are commonly not detectable, due to isotope exchange reactions between the two phases during metamorphism. The isotope exchange is controlled either by diffusional processes mainly at lower temperatures, or by crystallisation at higher temperatures. Chacko et al. (1991) showed experimental investigations on oxygen isotope exchange rates within calcite under different temperatures. These experiments revealed that at temperatures of $500^{\circ} \mathrm{C}$, already $78 \%$ of the oxygen isotopes had been exchanged mainly by diffusion. At temperatures of 800 to $950^{\circ} \mathrm{C}$, about 80 to $86 \%$ of the oxygen isotopes had been exchanged due to crystallisation. For equilibrated isotope exchange reactions between calcite and graphite, a minimum temperature of about $600^{\circ} \mathrm{C}$ is postulated (Valley \& O’Neil, 1981; Chacko et al., 1991).

\subsection{CHARACTERISATION OF THE GRAPHITE-BEARING MARBLES}

The measured samples comprise all types of graphite-bearing calcite-marbles distinguished by the microstructural analysis. The composition of the stable isotopes of calcite and graphite have been measured for all types of graphite-bearing marbles. The main sample localities are pointed out in Figure 2.1. The samples were selected, to characterise the different microstructural types of graphite-bearing marbles in the regional context by their isotopic composition. The samples and their according carbon and oxygen isotope ratios are shown in table 5.1.

As stated above, the interpretation of oxygen isotope ratios of carbonates generally may be difficult, especially in complex deformational regimes. Also the existence of magmatic intrusions indicates the possibility of associated $\mathrm{CO}_{2}$-bearing fluids percolating the marble host rock. Therefore, this complicates the interpretation of oxygen isotopes furthermore. As field investigations and the microstructural analysis revealed, complex deformational structures, as well as related granitic intrusions have to be considered for the 
interpretation of the measured oxygen isotope ratios. Additionally, the investigated marbles are pure graphite-bearing calcite-marbles. Therefore, no additional mineral phase can be considered for the analysis of the oxygen isotope ratios.

The measured data is also plotted into the overall distribution of precambrian $\delta{ }^{18} \mathrm{O}$ ratios (Fig. 5.3). It is obvious from this plot, that the measured data fits well into the general distribution for neoproterozoic oxygen isotope ratios. However, uncertainties exist about the initial oxygen isotope ratios, because of the possible overlap of multiple fluidrock interactions.

As primary oxygen isotope signatures have been postulated for precambrian seawater, it should be possible to characterise, whether the composition of oxygen isotopes have changed after formation and sedimentation. Kumar et al. (2002) and Ray et al. (2003) show the evaluation of primary oxygen isotope ratios of the neoproterozoic formations of the Vindhyan Basin. Following these authors the primary oxygen isotope ratio for neoproterozoic seawater lies within the range of $\delta{ }^{18} \mathrm{O}=7.5 \pm 2 \%$. This implies, that the oxygen isotope ratios measured in the own samples (table 5.1), would approximately fit into this range. Following this, a fluid rock interaction by kinetic isotope fractionation can be excluded for these samples.

The same general uncertainties regarding the initial carbon isotope ratios exist for calcite and graphite in the measured samples. The own carbon isotopes are also plotted into the database of precambrian carbon isotope ratios of Figure 5.4. They are differentiated between the plots of calcite and graphite. From this plot it is obvious, that both fit quite well into the general pattern of the precambrian carbon isotope ratios. The carbon isotope ratios of graphite and calcite plot explicitly above the range of organic isotope ratios.

This implies that an organic origin of either graphite or calcite cannot be extrapolated from the isotopic data anymore. Secondly, the data shows that the carbon isotope ratios plot clearly above the mean isotopic range for carbonate rocks. Therefore, they may be considered as isotopically heavier than the average neoproterozoic carbonates. As the isotopic ratios of calcite and graphite additionally plot in the same range, it is obvious that are very similar. This indicates, that equilibrated isotope fractionation may have occurred between calcite and graphite.

Therefore, isotope fractionation values $\Delta_{\text {(cal-gr) }}$ between calcite and graphite were calculated for the measured samples. They can reveal indications on the equilibration of the isotope fractionation. Consequently, an equilibrated isotope exchange between these 
two minerals would allow geothermometric calculations. In this case, estimations on the metamorphic temperatures during isotope exchange would be possible.

\subsection{CALCITE-GRAPHITE THERMOMETRY}

Geothermometry by calcite-graphite isotope fractionation is strongly dependent on the equilibration of the isotope exchange between the two phases. After Valley (2001) there are three basic conditions to be fulfilled for stable isotope thermometry. Firstly, the isotope fractionation between the mineral phases have to be equilibrated at one single metamorphic event. Secondly, the analysis of isotope ratio is accurate at the appropriate scale. Thirdly, the isotope fractionation has to be sufficiently sensitive and is accurately calibrated.

The quantification of the equilibration of the isotope exchange $\Delta_{\text {(cal-gr) }}$ is calculated according to equation [5.4], as the difference between the carbon isotopic ratios of calcite and graphite within a sample. A range of thermometer calibrations for equilibrated isotope exchange reactions is shown in Figure 5.5. $\Delta_{\text {(cal-gr) }}$ is also empirically used, to characterise the metamorphic facies of the measured samples during isotope exchange (Fig. 5.6). As $\Delta_{\text {(cal-gr) }}$ is temperature dependent, the variation within different values of the isotope fractionation $\Delta_{\text {(cal-gr) }}$ decrease to a great extend with increasing temperatures. Figure 5.6 shows the variation within $\Delta_{\text {(cal-gr) }}$ from unmetamorphosed to granulite facies metamorphism.

Additionally, the overall value for $\Delta_{\text {(cal-gr) }}$ decreases as equilibrium isotope exchange is reached. Therefore, the histogram for $\Delta_{\text {(cal-gr) }}$ of the amphibolite facies plots mainly between 3 and $8 \%$, and for the granulite facies between 2 and $5 \%$. This implies, that an equilibration of isotope exchange is only reached in amphibolite to granulite facies metamorphism.

The $\Delta_{\text {(cal-gr) }}$ values from the measured samples (table 5.1), plot in an according histogram between 2 and $4.5 \%$, where $90 \%$ of the samples plot between 2 and $3.5 \%$ (Fig. 5.7 a). This implies, that the samples have equilibrated isotopically between calcite and graphite in granulite facies metamorphism. Therefore, the isotope fractionations are appropriate to be used to calculate calcite-graphite temperatures by different geothermometric calibrations as shown in Figure 5.5.

A first approximation of the metamorphic temperatures are shown in Figure $5.7 \mathrm{~b}$. In this figure, the isotopic ratios of calcite are plotted against the isotopic ratios of graphite. All measured samples across all microstructural types and sample localities (Fig. 2.1) are 


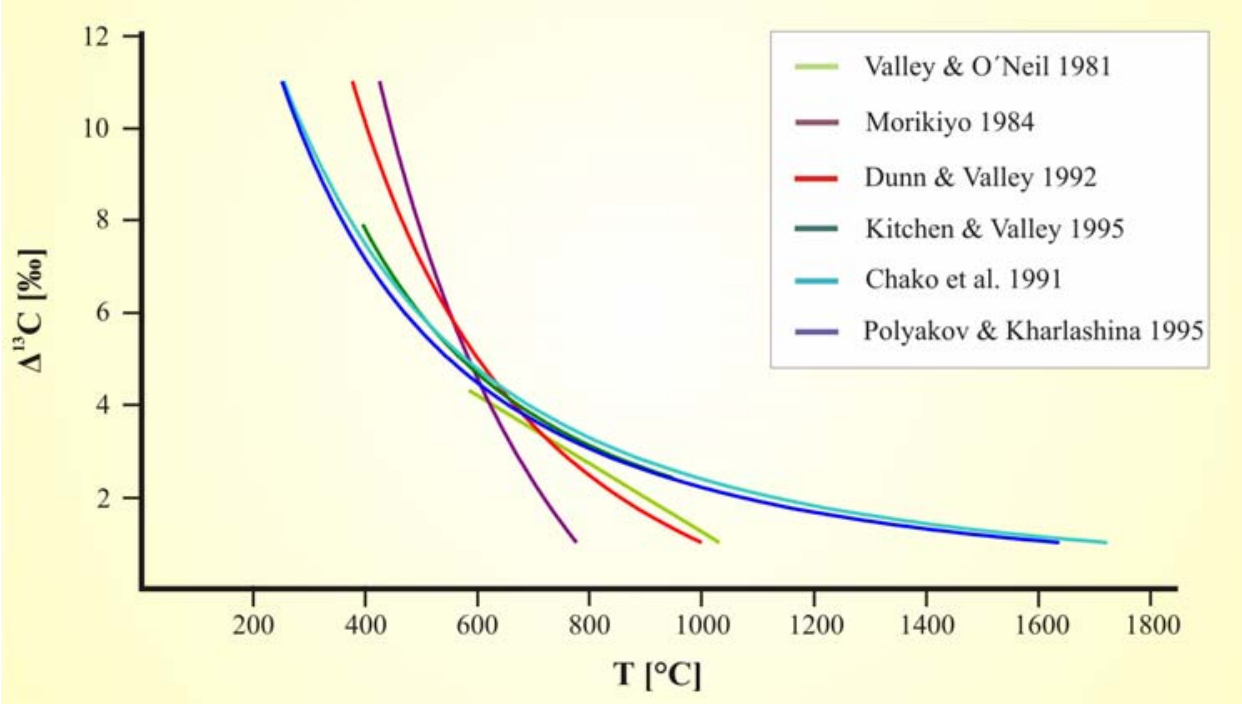

Fig. 5.5 Different geothermometer calibrations for calcite-graphite carbon isotope fractionations. The geothermometeric calibrations from Valley \& O'Neil (1981), Morikiyo (1984), Dunn \& Valley (1992), Kitchen \& Valley (1995) are empirically derived, whereas the calibration from Chacko et al. (1991) is experimentally derived. The geothermometer after Polyakov \& Kharlashina (1995) is theoretically calculated.

plotted into Figure $5.7 \mathrm{~b}$. It is obvious from this Figure, that they plot all in the same range of metamorphic temperature. The geothermometric calibration for this plot was selected after Valley \& O'Neil (1981) as this was the bottom range of temperatures obtained from this data.

The correlation between the between calcite-graphite isotope fractionations and distinct metamorphic temperatures shown in Figure 5.5, is determined either by theoretical calculations (Polyakov \& Kharlashina, 1995), experimental calibrations (Chacko et al., 1991 ) or is empirically derived by the comparison with other geothermometers (Valley \& O’Neil, 1981; Morikiyo, 1984; Dunn \& Valley, 1992; Kitchen \& Valley, 1995). There is still some debate on the correct thermometer calibrations, due to uncertainties in the theoretical calculations as well as in experimental considerations.

After Valley (2001), the geothermometric calibration of Kitchen \& Valley (1995) is in excellent agreement with independent petrologic geothermometry at temperatures above $600^{\circ} \mathrm{C}$. This applies also to the experimental calibrations of Chacko et al. (1991) and the theoretical calculations of Polyakov \& Kharlashina (1995). The empirical calibration of Dunn \& Valley (1992) fits data including lower temperatures of the amphibolite facies. For temperatures below $680^{\circ} \mathrm{C}$ the thermometer calibration of Wada \& Suzuki (1983) is more applicable. 


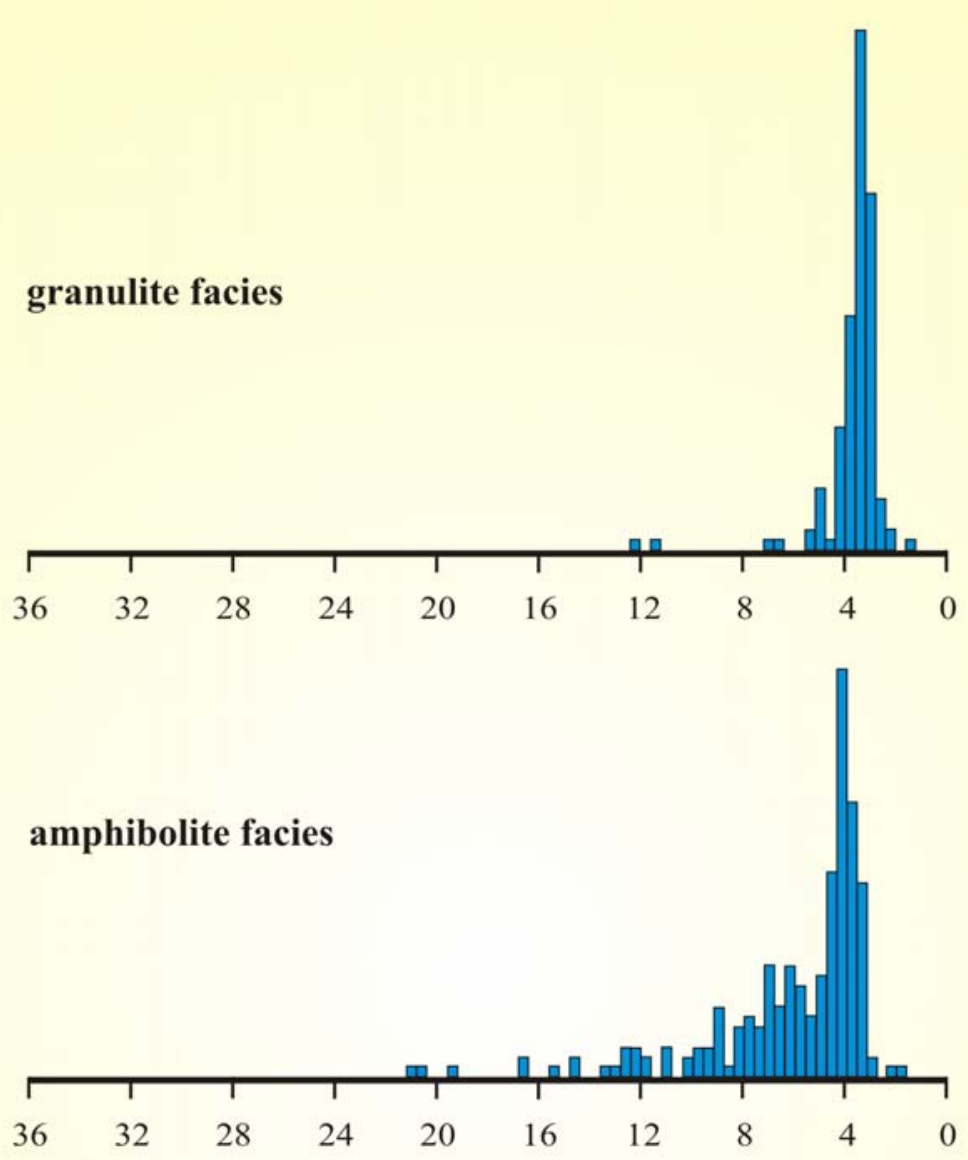

\section{greenschist facies}

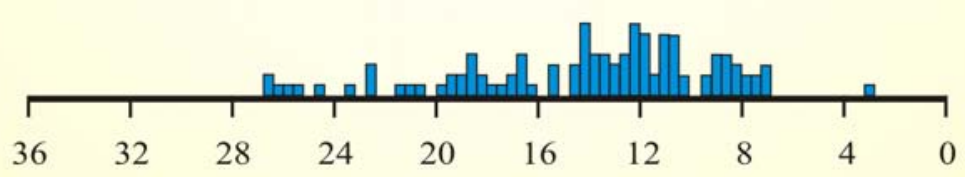

\section{unmetamorphosed}

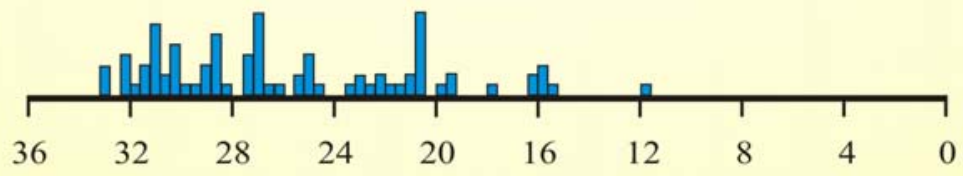

$\Delta($ cal-gr) [\%o]

Fig. 5.6 Histogram of the fractionation between calcite and graphite for several metamorphic grades after Kitchen \& Valley (1995). 

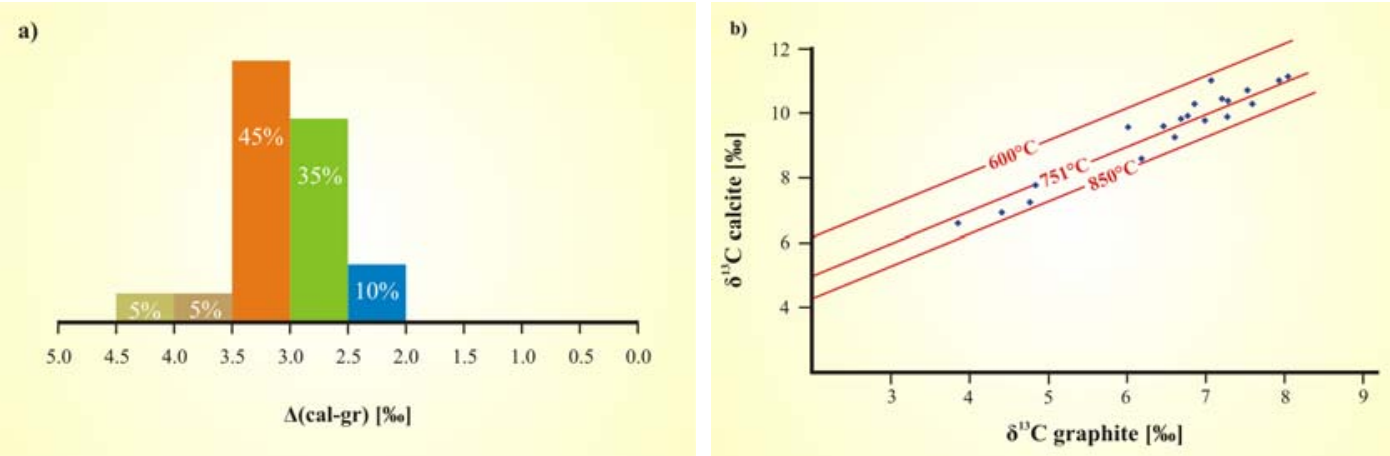

Fig. 5.7 a) Histogram of the fractionation between calcite and graphite of the measured samples. b) Plot of the isotopic ratios between calcite and graphite of all measured samples. The metamorphic temperatures were calculated according to the geothermometric calibration of Valley \& O'Neil (1981).

Figure 5.5 shows the plotted thermometer calibrations. It is obvious from this Figure, that the geothermometric calibrations after Kitchen \& Valley (1995), Chacko et al. (1991) and Polyakov \& Kharlashina (1995) plot with similar gradients. The geothermometric calibrations of Valley \& O’Neil (1981) and Morikiyo (1984) however, plot with steeper gradients. This implies that the temperatures gained by the latter are lower with higher fractionations of the carbon isotopes. Therefore the temperature calibrations for the own data were calculated for the most common calibrations used in literature as discussed above (table 5.1).

Because of the different gradients in the geothermometric calibrations, the metamorphic temperatures shown in table 5.1 vary stronger with higher fractionation and temperature values for different calibrations. As these temperatures plot all in the same range across different localities and microstructural types, mean temperatures have been calculated. The standard deviation between the samples show, that this is an appropriate assumption (table 5.1). The implications of these results have to be discussed in detail, regarding the metamorphic and deformational path of the investigated samples.

\subsection{DiscusSION}

The investigation of the isotopic composition of the graphite-bearing marbles revealed a mean peak metamorphic temperature of about $788^{\circ} \mathrm{C}$ across all differentiated microstructural types (Fig. $5.7 \mathrm{~b}$ and table 5.1). This is a mean temperature of all geothermometric callibrations calculated for the measured samples. The applicability of the different callibrations will be discussed in the following. Despite the co-existence of brittle and ductile deformational structures within the investigated samples, no differences in metamorphic temperatures are obtained across the distinguished deformational 


\begin{tabular}{|c|c|c|c|c|c|c|c|c|c|}
\hline \multirow[b]{2}{*}{ Sample } & \multicolumn{2}{|c|}{ calcite } & \multirow{2}{*}{$\begin{array}{c}\text { graphite } \\
\delta^{13} \mathrm{C} \\
\text { V-PDB } \\
{[\% 0]}\end{array}$} & \multirow[b]{2}{*}{$\begin{array}{c}\Delta \text { cal-gr } \\
{[\% 0]}\end{array}$} & \multicolumn{5}{|c|}{ Temperature $\left[{ }^{\circ} \mathrm{C}\right]$} \\
\hline & $\begin{array}{c}\delta^{13} C \\
V-P D B \\
{[\% 0]}\end{array}$ & \begin{tabular}{|c|c}
$\delta^{18} O$ \\
V-PDB \\
{$[\% 0]$} \\
\end{tabular} & & & V-O & D-V & $\mathbf{K}-\mathrm{V}$ & C et al & P-K \\
\hline $12 / 08 / 00 / 02$ & 9,64 & $-13,99$ & 6,46 & 3,19 & 733 & 727 & 782 & 811 & 769 \\
\hline $15 / 08 / 00 / 01$ & 10,34 & $-12,20$ & 6,85 & 3,49 & 693 & 702 & 735 & 760 & 721 \\
\hline $17 / 08 / 00 / 04$ & 7,80 & $-11,45$ & 4,84 & 2,96 & 759 & 745 & 816 & 849 & 805 \\
\hline $17 / 08 / 00 / 05$ & 10,43 & $-10,10$ & 7,27 & 3,16 & 733 & 727 & 782 & 811 & 769 \\
\hline $17 / 08 / 00 / 10$ & 9,87 & $-11,20$ & 6,68 & 3,19 & 733 & 727 & 782 & 811 & 769 \\
\hline $17 / 08 / 00 / 11$ & 11,20 & $-10,23$ & 8,03 & 3,16 & 733 & 727 & 782 & 811 & 769 \\
\hline $17 / 08 / 00 / 12$ & 9,97 & $-11,02$ & 6,76 & 3,20 & 733 & 727 & 782 & 811 & 769 \\
\hline $17 / 08 / 00 / 13$ & 10,50 & $-8,49$ & 7,20 & 3,31 & 719 & 718 & 705 & 793 & 752 \\
\hline $17 / 08 / 00 / 15$ & 11,07 & $-10,89$ & 7,92 & 3,16 & 733 & 727 & 782 & 811 & 769 \\
\hline $17 / 08 / 00 / 16$ & 6,61 & $-9,80$ & 3,86 & 2,75 & 786 & 763 & 854 & 891 & 845 \\
\hline $17 / 08 / 00 / 17$ & 10,34 & $-10,34$ & 7,58 & 2,76 & 786 & 763 & 854 & 891 & 845 \\
\hline $17 / 08 / 00 / 19$ & 9,82 & $-12,51$ & 6,98 & 2,84 & 786 & 763 & 854 & 891 & 845 \\
\hline $17 / 08 / 00 / 20$ & 10,77 & $-7,15$ & 7,52 & 3,25 & 719 & 718 & 705 & 793 & 752 \\
\hline $17 / 08 / 00 / 21$ & 9,94 & $-11,65$ & 7,26 & 2,67 & 799 & 773 & 875 & 913 & 866 \\
\hline $18 / 08 / 00 / 02$ & 9,61 & $-11,70$ & 6,01 & 3,60 & 679 & 694 & 721 & 745 & 706 \\
\hline $3 / 10 / 01 / 3$ & 9,30 & $-11,38$ & 6,60 & 2,70 & 799 & 773 & 875 & 913 & 866 \\
\hline $3 / 10 / 01 / 4$ & 6,95 & $-11,68$ & 4,42 & 2,53 & 826 & 793 & 920 & 962 & 913 \\
\hline $3 / 10 / 01 / 5$ & 7,26 & $-9,46$ & 4,77 & 2,49 & 826 & 793 & 920 & 962 & 913 \\
\hline Elim Am & 11,08 & $-7,46$ & 7,06 & 4,02 & 626 & 664 & 670 & 688 & 653 \\
\hline Omaruru Sp & 8,63 & \begin{tabular}{|l|}
$-7,51$ \\
\end{tabular} & \begin{tabular}{|r|}
6,18 \\
\end{tabular} & 2,45 & 826 & 793 & 920 & 962 & 913 \\
\hline \multicolumn{5}{|c|}{ mean isotopic temperatures: } & 751 & 741 & 806 & 844 & 800 \\
\hline \multicolumn{5}{|c|}{ standard deviation of temperatures } & 53 & 35 & 76 & 76 & 73 \\
\hline
\end{tabular}

Table 5.1 Stable isotopes of carbon for calcite and graphite and of oxygen for calcite. The temperatures are calculated by $\Delta_{(\text {cal-gr) }}$ after different geothermometric calibrations: V-O after Valley \& O'Neil (1981), D-V after Dunn \& Valley (1992), K-V after Kitchen \& Valley (1995), C et al. after Chacko et al. (1991) and P-K after Polyakov \& Kharlashina (1995).

structures and the undeformed grain-coarsened marble. This is also applicable on a regional scale as shown by Figure 2.1.

Furthermore, the equilibration of the carbon isotope fractionation and missing fluid inclusions imply, that fluids have not played a role during deformation, metamorphism and the intrusion of the granitic melts. As discussed above, this also may be confirmed by the oxygen isotope ratios which plot within the range of neoproterozoic seawater signatures.

As these results seem to be contradictory, the implications of the isotopic compositions on the microstructural development and the regional correlations have to be discussed in more detail. Field relationships reveal, that the intrusion of syn- to post deformational granitic melts have occurred on a regional scale within the $\mathrm{nCZ}$ of the Damara Belt (Fig. 2.1). From the apophysic form of the granitic intrusions observable along the magnetotelluric profile, it is obvious that the intruding granitic melts still had been in a mobile and liquidous phase. Figure 2.1 also includes the metamorphic isogrades 
which show, that this area of the $\mathrm{nCZ}$ was additionally overprinted by high-grade regional metamorphism and partial melting. As discussed in chapter 2, the age of the regional peak metamorphism coincides with the intrusion age of the granites at about $520 \mathrm{Ma}$.

This implies that the metamorphic regime results from the overlap of high-grade regional metamorphism and a widespread contact metamorphism. The metamorphic temperatures of the upper-amphibolite- and granulite-facies range between 650 to $900^{\circ} \mathrm{C}$. As the cotectic line of granite melts lies between 700 and $770^{\circ} \mathrm{C}$, it seems that the calcitegraphite geothermometric results shown in table 5.1 are related to this peak metamorphic event. Considering these cotectic melt temperatures, it is obvious, that the geothermometric calibrations of Valley \& O'Neil (1981) and Dunn \& Valley (1992) seem to be the most appropriate calibrations. The geothermometric calibrations of Kitchen \& Valley (1995), Chacko et al. (1991) and Polyakov \& Kharlashina (1995) therefore reveal slightly to high temperatures.

In comparison to thermobarometric calculations in the western Central Zone by Masberg (2000) and Jung \& Mezger (2003), the obtained temperatures are consistent. Nevertheless, Puhan (1983) measured slightly lower metamorphic temperatures of 580 to $645^{\circ} \mathrm{C}$ by calcite-dolomite geothermometry. After Rathmell et al. (1999) calcite-graphite geothermometry reveals the peak metamorphic temperatures, whereas calcite-dolomite geothermometry shows lower metamorphic temperatures. This may be the reason for this discrepancy.

From the microstructural analysis and field investigations it is apparent, that the abnormally grain-coarsened calcite-marble (type 1) is the host rock of the mylonitically and cataclastically deformed marble. It shows extreme grain growth of calcite due to recrystallisation processes and graphite is concentrated in disseminated nests of graphite flakes. As discussed above, regional relationships show that this abnormally graincoarsened type occurs only in conjunction with granite intrusions. The abnormal grain coarsening of calcite and graphite is therefore enhanced by the combination of isochronous regional and contact metamorphism. This interpretation seems to be in accordance with the isotopic analysis, since granulite facies metamorphic temperatures are obtained by this data. Accordingly, during this recrystallisation and abnormal grain growth equilibrated isotope fractionation occurred between calcite and graphite.

Also the mylonitically and brittle-ductile deformed types of graphite-bearing marble show the same equilibrated isotopic fractionation between calcite and graphite. Therefore, they imply the same metamorphic temperatures. In contrast, the microstructural analysis reveals, that the brittle-ductile deformed graphite-bearing marble shows partial 
static recrystallisation only within the ultrafine-grained parts. The rate of recrystallisation in the abnormally grain-coarsened graphite-bearing marbles, which is related to temperatures of about 750 to $780^{\circ} \mathrm{C}$ is exeedingly higher. This implies that this deformation may only have occurred retrograde at much lower temperatures.

The minimum temperature for static recrystallisation of calcite is dependent on several factors and can therefore not be specifically quantified. It depends mainly on the purity of the calcite grain boundaries and on the amount and presence of fluids. Furthermore, it depends on the amount of dislocations, dislocation tangles and subgrain boundaries within the calcite grains. Another main factor influencing static recrystallisation temperatures of calcite are the shape of the grain aggregates, as the decrease in total free surface energy and the total internal free energy is reduced by grain boundary area reduction (GBAR) (Passchier \& Trouw, 1998).

Additionally, the mylonitic deformation which is controlled by dynamic recrystallisation processes shows mutual overprinting effects with the cataclastic deformation in the brittle-ductile boundary zone. Temperatures for dynamic recrystallisation depend strongly the strainrate and on the according slip system activated during deformation (Barber et al., 1981). Therefore, no clear temperature for the mylonitic and cataclastic deformation can be deduced from the microstructures.

Furthermore, Rathmell et al. (1999) showed by the comparison of several geothermometers, that calcite-graphite thermometry reveals the peak metamorphic temperature. Also Dunn \& Valley (1992) found that calcite-graphite temperatures from contact aureoles are preserved, although being overprinted by a later regional metamorphism. This is mainly related to the inert diffusion rates of graphite (Thrower \& Mayer, 1978). Conclusively, this data and the microstructural analysis indicate, that the abnormal grain-coarsening and the equilibrated isotope fractionation of calcite and graphite is related to peak metamorphic granulite facies conditions. Field work and the microstructural analysis also reveal a subsequent deformation with a partial static recrystallisation in the ultra fine-grained domains.

Deduced from the experimental investigations and empirical quantification after Chacko et al. (1991) and Valley \& O'Neil (1981), it is obvious that carbon isotope fractionation is not equilibrated below $600^{\circ} \mathrm{C}$. This leaves either the possibility that the calcite and graphite isotopic ratios are preserved during the subsequent deformation, or that the deformation occurred below the temperatures where carbon isotopes are exchanged. As the strainrates of the deformation in the shear zone are not clear, it is not possible to 
estimate the temperature of deformation from the microstructures. Therefore, it may not be ruled out completely, that the deformation occurred above $600^{\circ} \mathrm{C}$.

Likewise, it can also not be precluded that the deformation occurred at temperatures where the isotope exchange rate is minimised. As the isotopic exchange rate has to be calculated over geological time spans, it is not sure whether this possibility of isotopic exchange at lower temperatures is applicable. It seems to be most likely that the equilibrated peak metamorphic isotope ratios were preserved. The already equilibrated mineral phases would in this case not change in isotope composition, as long as no further fluid phase is introduced due to the deformation. This implies that the calcite-graphite thermometry may not be applied to deduce the deformational temperature, as it would not give the retrograde deformational temperature.

Additionally, there are several hints that fluids were not present during metamorphism and retrograde metamorphic deformation. Missing fluid inclusions in the undeformed grain-coarsened host rock as well as in the deformed marble and the equilibrated isotope fractionation imply this assumption.

As the graphite network structures are most probably related to pressure solution, this implies however, that fluids would have to be considered at least for brittle-ductile domains of the boundary zone. In contrast again, the oxygen isotopes of calcite were not changed in composition by either hydrous or $\mathrm{CO}_{2}$-bearing fluids. Accordingly, the carbon isotopic ratios were not changed by the carbon isotopes of external $\mathrm{CO}_{2^{-}}$and/or $\mathrm{CH}_{4}$ bearing fluids.

For the isotopic interpretation of the graphitic stylolites this could only mean, that the fluids just caused the solution of calcite and graphite due to pressure solution effects. No recrystallisation, precipitation or overprinting of calcite and graphite therefore occurred again during pressure solution. The evacuation of calcite and graphite due to pressure solution is confirmed by the calcite-graphite ratios described in chapter 2.3.6.

The carbon isotopic ratios of calcite and graphite are heavier than the mean neoproterozoic isotopic ratios as shown in Figure 5.4. This may be related to the phase of static recrystallisation and abnormal grain growth. As described above, molecules and therefore crystals tend to incorporate heavier isotopes during recrystallisation. This isotopic fractionation must have been prior or at least simultaneously to the equilibrated isotope exchange with graphite, as graphite shows very similar isotopic composition as the the calcite-marble. 
Another observation of the stable isotope ratios measured in the samples is the missing influence of possible granitic fluids. There is no implication in the isotopic data, that carbon-bearing fluids have percolated the calcite-marbles. 


\section{CHAPTER 6}

\section{Mobilisation AND Precipitation OF GRAPHITE}

As graphite is a very important factor in the development of the macro- and microfabrics, it is necessary to know the modes of graphite formation in the investigated graphite-bearing marbles. As described above, graphite occurs in the undeformed host-rock in single nests with graphite flakes of up to $2 \mathrm{~mm}$ in size. In the brittle-ductile deformed boundary zones of the investigated shear zones, graphite occurs in graphitic stylolites and is ultra fine-grained. In the mylonitic core zones, graphite occurs in single ultra finegrained crystals, which show a preferred orientation with the basal planes parallel to the foliation.

Therefore, it is necessary to discuss the mechanisms of graphite formation in the undeformed host-rock, and possible mechanisms of mobilisation and precipitation of graphite in the deformed graphite-bearing marbles.

\subsection{THERMODYNAMICS AND MODELS}

Graphite is crystallised either from organic matter or from fluids. The origin of the graphitic carbon can be differentiated by the carbon isotopes, if these have not been significantly overprinted. As discussed above, a subsequent metamorphic or deformational event may change the isotopic composition by isotopic exchange reactions. After Weis et al. (1981) syngenetic graphite, crystallised from coal is differentiated by the mode of formation, from epigenetic graphite which originated from carbon bearing fluids.

The conversion of carbonaceous material to syngenetic graphite during metamorphism occurs by many steps of structural ordering of carbon. After Buseck \& Huang (1985) the most important factors influencing these processes are temperature, the time of heating, the total and partial pressures of various gaseous species and the presence of catalysts. Additionally, the structural characteristics of the reactants and products are also important (Buseck \& Huang, 1985).

After Franklin (1951) aliphatic carbon material must first be aromatised before the polymerisation into graphitic molecules. Otherwise this carbon material will not graphitise even at high metamorphic temperatures. After Ruland (1965), the aromatisation must result in planar hexagonal benzene-ring structures, which then are able to be stacked in graphitic structures. Other aromatic structures are unfavorable for the formation of graphite. 
Graphitisation is a complex process of stacking parallel layers of ordered carbon atoms. It includes processes of ordering, removing of foreign atoms like hydrogen and oxygen, and the removal of bonding vacancies. Many of these processes are controlled by diffusion (Buseck \& Huang, 1985). After Demeny (1989) the release of volatiles creats bubbles and pores in the carbonaceous matter, which promotes the development of a preferred orientation when shear stresses are prevailing. In general, the occurrence of a crustal stress field enhances the ordered crystallisation of graphite from carbonaceous matter (Ross \& Bustin 1990; Ross et al., 1991; Wilks et al., 1993; Bustin et al., 1995; Ross \& Bustin, 1997).

From the randomly rotated hexagonal layers, an organised stack of carbon layers develops with increasing temperatures. At temperatures of 500 to $550^{\circ} \mathrm{C}$ pure graphite develops. Catalising materials for the graphitisation of carbonaceous material are: $\mathrm{SiO}_{2}$, $\mathrm{FeS}_{2}, \mathrm{Fe}, \mathrm{Co}, \mathrm{Ni}, \mathrm{CaCO}_{3}$ (Demeny, 1989). Graphitisation is also catalysed by mica minerals, as the epitaxial intergrowth between the basal planes favours the structural ordering of the hexagonal carbon ring-structures (Kucha \& Wieczorek, 1988).

Carbon is transported in fluids either in $\mathrm{CO}-, \mathrm{CO}_{2}-$ or in $\mathrm{CH}_{4}-$ molecules. After Luque et al. (1998) three possible sources of carbon are postulated for the carbon bearing fluids. One source of carbon are the carbon bearing compounds released into fluids during the maturation of organic matter. A second source are devolatised carbonate-rich materials. Thirdly, carbon can be of igneous origin and in this case would be mantle-derived. The according source of carbon can be derived from the carbon isotope ratios, if these are not fractionated by istotopic exchange reactions during metamorphism.

As shown by Holloway (1984) and Luque et al. (1998), the stability of carbonbearing fluids is strongly dependent on the thermodynamic conditions and the oxygen fugacity of the fluids. The precipitation of epigenetic graphite from saturated $\mathrm{C}-\mathrm{O}-\mathrm{H}$ fluids (containing $\mathrm{CO}_{2}-\mathrm{CO}-\mathrm{H}_{2} \mathrm{O}-\mathrm{CH}_{4}$ ), therefore occures at according changes in temperature and pressure conditions of the fluids. Isobaric cooling and isothermal pressure increase leads to the precipitation of graphite from saturated fluids (Luque et al., 1998). This occurs also by changes and/or chemical reactions of the fluids, that influence the oxygen fugacity of the fluid. Such changes in oxygen fugacity occurs by mixing of $\mathrm{CO}_{2}$ - and $\mathrm{CH}_{4}$-bearing fluids, by fluid-rock interactions or by redox-reactions through sulfides and oxides. Carbon may also precipitate from $\mathrm{C}-\mathrm{O}-\mathrm{H}$ fluids by the hydratisation of the fluids, which leads to a oversaturation of the fluids (Luque et al., 1998).

Some processes which lead to the precipitation of thin interconnected graphite filmes are described by Glover (1996). Graphite may be precipitated by the isobaric 
cooling of C-O-H fluids. After Frost et al. (1989), this leads to a reduction of the $\mathrm{CO}_{2}$-rich fluid according to:

$$
\mathrm{C}+\mathrm{O}_{2} \Leftrightarrow \mathrm{CO}_{2}
$$

Graphite precipitation may also be triggered by titanomagnetite. As described by Fuhrmann et al. (1988) and Frost et al. (1989), oxygen fugacity is changed during interoxide equilibrium by cooling, which leads to the precipitation of graphite according to the following reaction:

$$
6 \mathrm{Fe}_{2} \mathrm{TiO}_{4}+\mathrm{CO}_{2} \Leftrightarrow 2 \mathrm{Fe}_{3} \mathrm{O}_{4}+6 \mathrm{FeTiO}_{3}+\mathrm{C}
$$

Walther \& Althaus (1993) described a third mechanism for the precipitation of graphite along strike-slip faults by the following chemical reaction:

$$
\mathrm{CH}_{4}+\mathrm{CO}_{2} \Leftrightarrow 2 \mathrm{C}+\mathrm{H}_{2} \mathrm{O}
$$

\subsection{IMPLICATIONS OF CARBON ISOTOPES}

As described above, the characterisation of graphite by carbon isotopes is a useful tool to evaluate whether it is of syngenetic or epigenetic origin. The carbon isotopes of the investigated samples show carbon isotope ratios that are clearly higher than the isotope ratios of organic carbon. Additionally, the carbon isotopes of graphite and calcite have been fractionated in equilibrium. As discussed above, this applies to all three types of graphite-bearing marble across all sampling sites shown in Figure 2.1.

This implies for all the types of the investigated graphite samples, that it is impossible to find indications about the origin of the carbon of graphite and carbonate from the carbon isotopes. The isotopic equilibration between calcite and graphite during peak metamorphism erased all isotopic signatures of the original carbon material.

The carbon isotopes nevertheless show that graphite of the abnormally graincoarsened and retrograde undeformed host rock, consists of the same isotopic composition than the deformed graphite bearing marbles in the shear zones. This strongly implies that the graphite has not been soluted by fluids and precipitated again in the interconnected graphitic stylolites. A solution of the carbon material would change the isotopic composition of the graphite, unless the fluids are purely aquaeous fluids, which do not 
carry any carbon material. However, for all other compositions of fluids, graphite in the shear zones should show a different isotopic composition, which is not observable.

Additionally, the carbon isotope ratios of graphite are consistent across all sampling localities (Fig. 2.1). This shows that they are even similar to equal on a regional scale, which would not be expected when local fluids overprint the carbon isotope ratios. The carbon isotope ratios therefore strongly imply, that the ultra fine-grained graphite of the shear zones has been grain-size reduced by deformation and concentrated by pressure solution of the calcite-marble as residue in the graphitic stylolites. Likewise, graphite in the mylonitically deformed marble is 'autochtoneous', and was concentrated as single crystals in preferred orientation along the calcite grain-boundaries during dynamic recrystallisation.

\subsection{IMPICATIONS OF GRAPHITE CRYSTALLINITIES}

As described in chapter 3, the crystallinities of graphite are equally high for all different types of the investigated graphite bearing marbles. Therefore, the graphite crystallinities of the abnormally grain-coarsened and undeformed host rock are similar to the crystallinities of graphite in the shear zones. Even within in different deformational domains of the shear zones, graphite crystallinities do not differ substantially. Again, this also applies on a regional scale across the sampling sites shown in Figure 2.1.

For the investigation of the mechanisms of graphite formation, possible mobilisation and subsequent precipitation, these results give no clear implications. It can not be clearly deduced from the measured graphite crystallinities, whether a concentration of graphite in the shear zones had occurred due to pressure solution or due to solution, fluid mobilisation and finally precipitation.

The microstructural investigation revealed, that graphite grain-sizes are reduced to about 10 to $200 \mu \mathrm{m}$ in comparison to the grain-sizes of the graphite flakes of up to $2 \mathrm{~mm}$ in the undeformed host-rock. As shown by Landis (1971) and Hofmann (1989) it is possible to grind highly crystalline graphite without changing the degree of crystallinity of the graphite. Therefore it is possible, that the deformed graphite from the shear zones remained unchanged in crystallinity and shows the same characteristics as the abnormally graincoarsened and undeformed host rock. Intensive pressure solution of calcite in the brittleductile boundary zone, must have left the deformed graphite as residue in the graphitic stylolites.

On the other hand Bustin et al. (1986), Demeny (1989), Wilks et al. (1993) and Ross \& Bustin (1997) showed that graphite formed under shear stress produces highly 
crystalline graphite even at 350 to $400^{\circ} \mathrm{C}$. This could also imply, that the highly crystalline graphite in the investigated shear zones, could have originated from crustal fluids penetrating the shear zones during deformation.

However, the isotopic composition of the graphite from the shear zones should in this case differ from the isotope ratios of the abnormally grain-coarsened undeformed host rock as discussed above. In the case of solution of graphite by purely aquaeous fluids, the carbon isotope ratios would not have been changed, as long as no mixing with other fluids or rocks had occurred. In this case a mobilisation and subsequent precipitation of graphite would have been possible, with high graphite crystallinities and according isotopic composition.

\subsection{IMPLICATIONS OF REGIONAL METAMORPHISM}

As discussed in chapter 2, amphibolite- to granulite metamorphic facies occurred in combination with contact metamorphism of granite intrusions. This occurred on a regional scale within the northern Central Zone of the Damara Belt. Calcite-graphite geothermometry revealed temperatures of $750-780^{\circ} \mathrm{C}$ for all localities. Therefore they apply on a regional scale as well.

These metamorphic conditions are well sufficient for the formation of syngenetic graphite as well as for epigenetic graphite. At the described metamorphic facies, the conversion from carbonaceous material to graphite is well possible, as this may occur already at lower metamorphic facies (Weis et al., 1981; Buseck \& Huang, 1985; Demeny, 1989).

The thermodynamic conditions for the precipitation of epigenetic graphite are also existent in these metamorphic grades (Holloway, 1984; Luque et al., 1998). Therefore, it is not possible from the evaluation of the regional metamorphism, to preclude on the mode of graphite formation in the investigated samples.

\subsection{IMPLICATIONS OF SEM DATA}

The SEM and EDX data presented in chapter 2 shows, that graphite in the undeformed host rock, as well as in the brittle-ductile and mylonitically deformed marble shows similar compositions. Additional phases like $\mathrm{Fe}, \mathrm{Ti}, \mathrm{K}, \mathrm{Si}, \mathrm{Al}, \mathrm{Mg}$, and $\mathrm{Cl}$ imply, that propably some mica minerals are epitaxial intergrown with the graphite. This was also observed by qualitative optical microscopy in the abnormally grain-coarsened undeformed host rock as described in chapter 2 . 
As these additional phases occur in the graphite of all microstructural types, they give an indication, that the graphite in the deformed graphite-bearing marbles is most likely not soluted and precipitated after being mobilized by fluids. The epitaxial intergrowth with mica minerals rather implies, that the graphite of the shear zone is either 'autochtoneous' in the case of pure mylonitic deformation. In the brittle-ductile deformed domains, graphite seems to be a residue of pressure solution in graphitic stylolites.

\subsection{DisCUSSION}

As the unfractionated carbon isotopic composition is the most reliable characterisation for the origin of graphite and calcite, it is not possible in the measured samples to pinpoint the origin of carbon in this samples. The original carbon isotope ratios have been overprinted during peak metamorphism.

The metamorphic conditions favour both mechanisms of graphite formation, which also do not give implications, whether the investigated graphite is of syngenetic or epigenetic origin. However, the carbon isotopes of graphite and calcite are equilibrated equally within the deformed and undeformed graphite-bearing marble. This gives an first indication, that the graphite of all the investigated types of graphite-bearing marble are genetically related.

From the graphite crystallinities it is obvious, that graphite either had been deformed in the shear zones while keeping the high crystallinities, or it was precipitated under shear stress, obtaining high crystallinities. Regarding the carbon isotope ratios, the latter may only have been possible, when the original graphite had been soluted and mobilised by purely aquaeous fluids.

The investigations by EDX revealed, that it is most likely, that the graphite in all microstructural types is the same, as graphite is epitaxial intergrown with mica minerals. This would not be expected in epigenetic graphite. Considering these implications together with the indications of the carbon isotope ratios, the graphite crystallinities and the thermodynamic crustal conditions, it seems to be the most likely that the graphite in the shear zones is the same as in the undeformed host rock. 


\section{CHAPTER 7}

\section{REGIONAL IMPLICATIONS}

The data gathered in this work implies some new regional proxies for the deformational and metamorphic history. They will be related to the tectono-metamorphic history postulated so far for the northern Central Zone of the Damara Belt.

\subsection{REGIONAL METAMORPHISM}

The regional metamorphic isogrades of the Damara Belt postulated by Hartmann et al. (1983) are in good accordance with the obtained peak metamorphic temperatures of the calcite-graphite geothermometry. The metamorphic isogrades increase from east to west in the area of investigation (Fig. 2.1). In the east they are described by partial melting due to the reaction of muscovite, plagioclase, quartz and water to melt and sillimanite. To the west, increasing metamorphic grades are characterised by k-feldspar- and cordierite-in. This already shows, that upper-amphibolite- to granulite-facies metamorphism was prevailing in the region. The metamorphic temperatures implied by these facies are around $650^{\circ} \mathrm{C}$ for the upper amphibolite-facies and 650 to $900^{\circ} \mathrm{C}$ for the granulite-facies.

Fieldwork and the geochronological data discussed in chapter 2 shows that the intrusion of the granitic melts occurred during this metamorphic event. The cotectic temperatures of granitic melts are between 700 to $770^{\circ} \mathrm{C}$. In the combination of regional and contact metamorphism the obtained calcite-graphite geothermometric temperatures possibly represent peak metamorphism. Thermobarometric calculations of the western part of the Central Zone by Masberg (2000) and Jung \& Mezger (2003) show, that the obtained metamorphic temperatures are consistent with this data. Nevertheless, calcite-dolomite geothermometric calculations of the Central Zone by Puhan (1983) show lower temperatures of 580 to $645^{\circ} \mathrm{C}$. These temperatures are generally lower as the obtained data. After Rathmell et al. (1999) calcite-graphite geothermometry rather represents peak metamorphic temperatures, while calcite-dolomite may also represent lower metamorphic temperatures. This could be the reason for the discrepancy between these temperatures.

The combination of regional and contact metamorphism as also postulated by Jung et al. (2003) is in accordance with fieldwork, geochronological data, geothermometric data. The geochronological data of the regional widespread intrusions and the metamorphic events in the northern Central Zone are discussed in chapter 2. In general, peak metamorphism is dated to about 520 to $504 \mathrm{Ma}$ and the intrusion ages range mainly from 540 to $510 \mathrm{Ma}$ and a later stage of intrusions at $469 \mathrm{Ma}$ (Miller, 1983; Jacob et al., 2000; 
McDermott et al., 2000, Jung et al., 2003). The geothermometric data also shows, that in general temperatures of 700 to $760^{\circ} \mathrm{C}$ have been present during metamorphism. These temperatures do not directly imply a combination of regional and contact metamorphism. They show however, that the metamorphic temperatures do not exclude the possibility of a combined regional and contact metamorphism in these parts of the northern Central Zone.

Likewise, the apophysic granitic intrusions imply, that mobile melts have intruded the marble in a high-temperature regime. Therefore, this correlation of metamorphic events may also be verified by these field investigations.

\subsection{DEFORMATIONAL PATH}

As shown on the satellite image in Figure 2.2, the regional structures are dominated by crustal scale dome structures. It is obvious from this image, that two kinds of dome structures are developed in the region. The dominating type of dome structures has developed by by refolding crustal scale isoclinal folds. The second type of dome structures emerged from the widespread intrusion of the granitic melts.

After the emplacement of the granites, a regional phase of static recrystallisation dominated. This is documented by regionally widespread abnormally grain-coarsened calcite-marbles as described in chapter 2. During retrograde metamorphism the rims of the crustal-scale dome structures became reactivated and shear zones developed along the rims of the dome-structures. Based on the investigations of the microstructures, the carbon isotopes and the graphite crystallinities, the reactivation of the dome structures occurred most probably at temperatures below $600^{\circ} \mathrm{C}$. The microstructural analysis implies even lower temperatures. As these estimates also depend strongly on the strain rate on geological time scales, it is not possible to estimate an exact temperature.

The shear zones are built up by mylonitic core zones and brittle-ductile boundary zones. The mylonitic core zones are consistent in the shear zones, whereas the boundary zones consist of different domains of brittle-ductile deformation, which vary lateral and perpendicular to the shear zones. The analysis of the macro- and microstructures shows, that the mylonitic core zones are characterised by aseismic creep deformation, whereas the brittle-ductile boundary zones show a mutual overprinting of brittle and ductile deformation, related to seismic events and aseismic creep deformation. The transition between brittle and ductile deformation, is controlled by the differences in strain rates between short-termed seismic deformation with high strain rates and aseismic ductile deformation. 
As revealed by fieldwork, this deformation is of Damaran age. The Cretaceous dykes, which occur widespread in the region (Fig. 2.2) are not overprinted by these shear zones. Consequently, the Cretaceous dykes cut the shear zones across the crustal dome structures regardless of the general Damaran strike, whereas the shear zones are oriented parallel to the Damaran strike from north-east to south-west.

\subsection{DisCUSSION}

The deformational path described above, implies for this part of the Central Zone of the Damara Belt at least two deformational stages during the Damara Orogenesis. This is documented by a regional widespread phase of static recrystallisation. Fieldwork revealed that the abnormally grain-coarsened marbles occur at least from the western sampling locality (Fig. 2.1) to the coast near Swakopmund. This phase of static recrystallisation postdates either a transtensional or a multistage deformation, which led to the formation of the crustal dome structures. After the phase of static recrystallisation a second regional stress field built up, which induced the formation of the investigated shear zones along the rims of the dome structures.

Therefore, a wide range of this crustal segment was free of crustal stresses during the late stage of the Damara Orogenesis. Whether this is purely related to the structural setting of this crustal segment during this stage of orogenesis, or to the widespread magmatic intrusions is not clear. More detailed investigations on a regional scale will reveal more information on this matter.

The models for the formation of dome structures discussed in the literature are related to large-scale buckling, thrust-related antiformal stacking, syn-contractional gravitational collapse or post-contractional extension. A compilation of models is shown by Soula et al. (2001). Which of these models are applicable for the investigated structural domes, is not clear at present. Further investigation and detailed mapping of these dome structures will be necessary on this matter. A thrust-related formation of the dome structures, would reveal some indications about the source of the seismic activity observed in the fabrics of the brittle-ductile boundary zone.

The location of the Omaruru Lineament (OmL) as postulated by Raab (2001), Ritter et al. (2003) and Weckmann et al. (2003) is still a matter of debate. After Miller (1983), the OmL devides the northern and the southern Central Zone of the Damara Belt. Main differences between the two parts of the Central Zone are the stratigraphic levels of the dome structures. In the northern Central Zone, the dome structures are built up by Damaran lithologies, whereas the dome structures of the southern Central zone comprise 
mainly basement rocks. After this definition, the OmL is located further south, as the investigated shear zones all are built up by Damaran sediments. Furthermore, there is no distinct shear zone in this area, which strictly divides the crustal segments of different straticgraphic levels. Whether the existing shear zones along the dome structures may be regarded as a conjugated shear zone system, will have to be revealed by further investigations. Nevertheless, the investigated shear zone system would not fulfil the original definition of the OmL by Miller (1983) and may thereby not be defined as such. 


\section{CHAPTER 8}

\section{COnClusions}

1. The graphite-bearing marbles, that produce high anomalies of electrical conductivity in magnetotelluric profiles occur within crustal scale domestructures in the northern Central Zone of the Damara Belt in north-western Namibia.

2. The undeformed graphite-bearing marbles are abnormally grain-coarsened due to a combination of amphibolite- to granulite facies metamorphism and contact metamorphism of granitic intrusions. Calcite-graphite geothermometry revealed regional peak-metamorphic temperatures of about 750 to $780^{\circ} \mathrm{C}$.

3. The granitic melts intruded after the formation of the structural domes and themselves formed widespread granitic domes in the Central Zone of the Damara Belt.

4. After the intrusion of the granitic melts, a regional phase of static recrystallisation occurred with the abnormal grain-coarsening of the graphite-bearing marbles.

5. These coarse-grained marbles were subsequently deformed in complex shear zones along the rims of the retrograde reactivated dome structures. The shear zones comprise a mylonitic core zone and a brittle-ductile boundary zone. The brittleductile boundary is characterised by brittle and ductile deformation fabrics, which show a mutual overprinting of both deformation mechanisms and pressure solution. This was revealed by detailed investigations of the macro- and microfabrics of the graphite-bearing marbles. The brittle-ductile boundary zone is attributed to a combination of seismic deformation with high strain rates and cataclastic deformation and aseismic creep deformation. Aseismic creep deformation has also dominated the mylonitic core zones of the shear zones.

6. Texture measurements revealed high intensities in preferred-crystallographic orientation, which possibly is related to recrystallisation. The intensities decrease with the decrease in grain size in the mylonitic core zones as well as in the brittleductile boundary zones. The mylonitic core zones show a complex pattern of different texture domains in microscopic scales.

7. The graphitic stylolites of the brittle-ductile boundary zone produce the anomalies of high electrical conductivity in magnetotelluric profiles. Electrical conductivity 
measurements on sample scale however, showed mainly ionic conductivity as most of the graphitic stylolites are cut by younger microveins. These microveins are either related to hydrofracturing during the Cretaceous break-up of Gondwana, or to tensile fracturing during the exhumation of this crustal segment.

8. The investigations of this work show, that it is not possible to reveal the origin of the graphite. As graphite and calcite are isotopically equilibrated, it is impossible to find any implications on the origin of graphite and on the mode of formation. It is most likely that the graphite in the shear zone is of the same origin as in the undeformed host rock. Several findings imply that graphite from the coarse-grained marble had been deformed in the shear zone, and then formed graphitic stylolites as a residue during pressure solution.

9. Graphite is of uniformly high crystallinity in the abnormally grain-coarsened undeformed marble as well as in the deformed marble. Graphite is epitaxial intergrown with mica minerals, which is observable by qualitative optical microscopy in the undeformed host rock. Within the deformed graphite-bearing marbles it is not possible to observe this directly. EDX data implies this assumption however. 


\section{REFERENCES}

Alabi, A. O., Camfield, P. A. \& Gough, D. I. 1975. The North American Central Plains conductivity anomaly. The Geophysical Journal of the Royal Astronomical Society 43(3), 815-833.

Alcántara, R., Lavela, P., Ortiz, G. F., Tirado, J. L., Menéndez, R., Santamaría, R. \& JiménezMateos, J. M. 2003. Electrochemical, textural and microstructural effects of mechanical grinding on graphitized petroleum coke for lithium and sodium batteries. Carbon 41(15), 3003-3013.

Barber, D. J., Heard, H. C. \& Wenk, H. R. 1981. Deformation of dolomite single crystals from 20800 degrees C. Physics and Chemistry of Minerals 7(6), 271-286.

Barnhoorn, A., Bystricky, M., Burlini, L. \& Kunze, K. 2004. The role of recrystallisation on the deformation behaviour of calcite rocks: large strain torsion experiments on Carrara marble. Journal of Structural Geology 26, 885-903.

Bartels, K. S. \& Pasteris, J. D. 1994. Multiple generations of fluid-deposited graphite in rocks from the KTB. In: Geological Society of America, 1994 annual meeting. (edited by Anonymous). Abstracts with Programs - Geological Society of America 26; 7. Geological Society of America (GSA), Boulder, CO, United States, 227.

Beny-Bassez, C. \& Rouzaud, J. N. 1985. Characterization of carbonaceous materials by correlated electron and optical microscopy and Raman microspectroscopy. Scanning Electron Microscopy 1985(1), 119-132.

Bestmann, M., Kunze, K. \& Matthews, A. 2000. Evolution of a calcite marble shear zone complex on Thassos Island, Greece: microstructural and textural fabrics and their kinematic significance. Journal of Structural Geology 22(11-12), 1789-1807.

Beyssac, O., Rouzaud, J. N., Goffe, B., Brunet, F. \& Chopin, C. 2002. Graphitization in a highpressure, low-temperature metamorphic gradient; a Raman microspectroscopy and HRTEM study. Contributions to Mineralogy and Petrology 143(1), 19-31.

Brasier, M. D., Magaritz, M., Corfield, R., Luo, H., Wu, X., Ouyang, L., Jiang, Z., Hamdi, B., He, T. \& Fraser, A. G. 1990. The carbon- and oxygen-isotope record of the PrecambrianCambrian boundary interval in China and Iran and their correlation. Geological Magazine 127(4), 319-332.

Burdett, J. W., Grotzinger, J. P. \& Arthur, M. A. 1990. Did major changes in the stable-isotope composition of Proterozoic seawater occur? Geology (Boulder) 18(3), 227-230. 
Burkhard, M. 1993. Calcite twins, their geometry, appearance and significance as stress-strain markers and indicators of tectonic regime: a review. In: The geometry of naturally deformed rocks. (edited by Casey, M., Dietrich, D., Ford, M., Watkinson, J. \& Hudleston Peter, J.). Journal of Structural Geology 15; 3-5. Pergamon, Oxford-New York, International, 351-368.

Buseck, P. R. \& Huang, B. 1985. Conversion of carbonaceous material to graphite during metamorphism. Geochimica et Cosmochimica Acta 49(10), 2003-2016.

Bustin, R. M. 1983. Heating during thrust faulting in the Rocky Mountains: friction or fiction? Tectonophysics 95(3-4), 309-328.

Bustin, R. M., Ross, J. V. \& Moffat, I. 1986. Vitrinite anisotropy under differential stress and high confining pressure and temperature: preliminary observations. International Journal of Coal Geology 6(4), 343-351.

Bustin, R. M., Ross, J. V. \& Rouzaud, J. N. 1995. The role of strain in the formation of natural graphite: experimental considerations. In: Geological Association of Canada; Mineralogical Association of Canada; annual meeting; program with abstracts-Association Geologique du Canada; Association Mineralogique du Canada; reunion annuelle; programme et resumes. (edited by Anonymous). Program with Abstracts Geological Association of Canada; Mineralogical Association of Canada; Canadian Geophysical Union, Joint Annual Meeting 20. Geological Association of Canada, Waterloo, ON, Canada, 13.

Chacko, T., Mayeda, T. K., Clayton, R. N. \& Goldsmith, J. R. 1991. Oxygen and carbon isotope fractionations between $\mathrm{CO}_{2}$ and calcite. Geochimica et Cosmochimica Acta 55(10), 28672882.

Covey, C. S. J. \& Rutter, E. H. 1989. Thermally-induced grain growth of calcite marbles on Naxos Islands, Greece. Contributions to Mineralogy and Petrology 101(1), 69-86.

Criss, R. E. 1991. Temperature dependence of isotopic fractionation factors. In: Stable isotope geochemistry; a tribute to Samuel Epstein. (edited by Taylor Hugh, P. J., O’Neil, J. R. \& Kaplan Isaac, R.). Special Publication - Geochemical Society 3. Geochemical Society, University Park, PA, United States, 11-16.

De Bresser, J. H. P. \& Spiers, C. J. 1997. Strength characteristics of the r, f, and c slip systems in calcite. Tectonophysics 272(1-2), 1-23.

Demeny, A. 1989. Structural ordering of carbonaceous matter in Penninic terranes. Acta Mineralogica-Petrographica (Szeged) 30, 103-113. 
Dresselhaus, M. S. \& Dresselhaus, G. 1982. Light scattering in graphite intercalation compounds. In: Light scattering in solids (edited by Cardona, M. \& Guntherodth, G.). Springer, New York, 3-57.

Duba, A., Heikamp, S., Meurer, W., Nover, G. \& Will, G. 1994. Evidence from borehole samples for the role of accessory minerals in lower-crustal conductivity. Nature (London) 367(6458), 59-61.

Duba, A. G. \& Shankland, T. J. 1982. Free carbon and electrical conductivity in the Earth's mantle. Geophysical Research Letters 9(11), 1171-1174.

Dunn, S. R. \& Valley, J. W. 1992. Calcite-graphite isotope thermometry: a test for polymetamorphsim in marble, Tudor gabbro aureole, Ontario, Canada. Journal of Metamorphic Geology 10(4), 487-501.

ELEKTB-Group. 1997. KTB and the electrical conductivity of the crust. The KTB deep drill hole. 102(8), 18,289-18,305.

Fowler, C. M. R., Stead, D., Pandit, B. I., Janser, B. W., Nisbet, E. G. \& Nover, G. 2004. A data base for physical properties of rocks from the Trans-Hudson Orogen, Canada. Canadian Journal of Earth Sciences = Journal Canadien des Sciences de la Terre in print.

Franklin, R. E. 1951. The structure of graphitic carbons. Acta Crystallographica 4, 253-261.

Frost, B. R., Fyfe, W. S., Tazaki, K. \& Chan, T. 1989. Grain-boundary graphite in rocks and implications for high electrical conductivity in the lower crust. Nature (London) 340(6229), 134-136.

Fueten, F. \& Goodchild, J. S. 2001. Quartz c-axes orientation determination using the rotating stage polarizer microscope. Journal of Structural Geology 23(6-7), 895-902.

Fuhrmann, M. L., Frost, B. R. \& Lindsley, D. H. 1988. Crystallisation conditions of the Sybille Monzosyenite, Laramie anorthosite complex, Wyoming. Journal of Petrology 29, 699-729.

Garlick, G. D. 1969. The stable isotopes of oxygen. In: Handbook of Geochemistry (edited by Wedepohl, K. H.) 8B. Springer, Berlin Heidelberg New York.

Giletti, B. J. 1985. The nature of oxygen transport within minerals in the presence of hydrothermal water and the role of diffusion. Chemical Geology 53(3-4), 197-206.

Glover, P. W. J. 1996. Graphite and electrical conductivity in the lower continental crust: a review. In: Geophysical studies of the continental lithosphere; density inhomogeneities in the crust and mantle and their relation to tectonics. (edited by Goetze, H. J. \& Jacoby, W. R.). Physics and Chemistry of the Earth 21; 4. Pergamon, Oxford-New York-Toronto, International, 279-287. 
Glover, P. W. J. \& Vine, F. J. 1992. Electrical conductivity of carbon-bearing granulite at raised temperatures and pressures. Nature (London) 360(6406), 723-726.

Gross, C. J., Weber, K., Siegesmund, S. \& Vollbrecht, A. 1999. Fabric Development and Spathization Processes in Metacarbonates: Two Case Studies from the Damara Orogen, Namibia. In: Sonderforschungsbereich 486 Wechselwirkungen an geologischen Grenzflächen Arbeits- und Ergebnisbericht für die Jahre 1997-1999. Georg-August Universität Göttingen, Göttingen, B3-1 - B3-33.

Guéguen, Y. \& Palciauskas, V. 1994. Introduction to the Physics of Rocks. Princeton University Press, Princeton, New Jersey 08540.

Haack, U. \& Gohn, E. 1988. Rb-Sr data on some pegmatites in the Damara Orogen (Namibia). Communications of the Geological Survey of Namibia 4, 13-17.

Haack, U., Hoefs, J. \& Gohn, E. 1983. Genesis of Damaran granites in the light of Rb/Sr and delta ${ }^{18} \mathrm{O}$ data. In: Intercontinental fold belts (edited by Martin, H. \& Eder, F. W.). Springer, Berlin, 847-872.

Haack, U. \& Martin, H. 1983. Geochronology of the Damara Orogen - a review. In: Intercontinental fold belts (edited by Martin, H. \& Eder, F. W.). Springer, Berlin, 839-845.

Hall, S. M. \& Veizer, J. 1996. Geochemistry of Precambrian carbonates: VII, Belt Supergroup, Montana and Idaho, USA. Geochimica et Cosmochimica Acta 60(4), 667-677.

Hartmann, O., Hoffer, E. \& Haak, U. 1983. Regional metamorphism in the Damara Orogen: Interaction of crustal motion and heat transfer. In: Evolution of the Damara Orogen of South-West Africa/Namibia (edited by Miller, R. M. G.). Special Publication - Geological Society of South Africa 11, 233-241.

Hawkesworth, C. J., Kramers, K. D. \& Miller, R. M. G. 1981. Old model Nd ages in Namibian Pan-African rocks. Nature 289, 278-282.

Heilbronner, R. 2000. Automatic grain boundary detection and grain size analysis using polarization micrographs or orientation images. Journal of Structural Geology 22(7), 969981.

Hoefs, J. 1997. Stable isotope geochemistry. 4 ed. Springer, Berlin, Federal Republic of Germany.

Hofmann, K. H. 1989. Das Auftreten von Graphit in metamorphen Gesteinen in Bayern Translated Title: The occurrence of graphite in metamorphic rocks in Bavaria. Geologische Blaetter fuer Nordost-Bayern und Angrenzende Gebiete 39(1-2), 59-98.

Holloway, J. R. 1984. Graphite- $\mathrm{CH}_{4}-\mathrm{H}_{2} \mathrm{O}-\mathrm{CO}_{2}$ equilibria at low-grade metamorphic conditions. Geology (Boulder) 12(8), 455-458. 
Jacob, R. E., Moore, J. M. \& Armstrong, R. A. 2000. Zircon and titanite age determinations from igneous rocks in the Karibib District, Namibia: implications for Navachab vein-style gold mineralization. In: Henno Martin commemorative volume. (edited by Miller, R. M.). Communications of the Geological Survey of South West Africa/Namibia 12. Geological Survey of South West Africa/Namibia, Windhoek, Namibia, 157-166.

Jödicke, H., Kruhl, J. H., Ballhaus, C., Giese, P. \& Untiedt, J. 2004. Syngenetic, thin graphite-rich horizons in lower crustal rocks from the Serre San Bruno, Calabria (Italy), and implications for the nature of high-conducting deep crustal layers. Physics of the Earth and Planetary Interiors 141(1), 37-89.

Jung, S., Hoernes, S. \& Mezger, K. 2000a. Geochronology and petrogenesis of Pan-African, syntectonic, S-type and post-tectonic A-type granite (Namibia): products of melting of crustal sources, fractional crystallization and wall rock entrainment. Lithos 50(4), 259-287.

Jung, S., Hoernes, S. \& Mezger, K. 2000b. Geochronology and petrology of migmatites from the Proterozoic Damara Belt - importance of episodic fluid-present disequilibrium melting and consequences for granite petrology. Lithos 51(3), 153-179.

Jung, S. \& Mezger, K. 2003. Petrology of basement-dominated terranes: I. Regional metamorphic $\mathrm{T}-\mathrm{t}$ path form $\mathrm{U}-\mathrm{Pb}$ monazite and $\mathrm{Sm}-\mathrm{Nd}$ garnet geochronology (Central Damara orogen, Namiba). Chemical Geology 198(3-4), 223-247.

Jung, S., Mezger, K. \& Hoernes, S. 1998. Petrology and geochemistry of syn- to post-collisional metaluminous A-type granites - a major and trace element and $\mathrm{Nd}-\mathrm{Sr}-\mathrm{Pb}-\mathrm{O}-$-isotope study from the Proterozoic Damara Belt, Namibia. Lithos 45, 147-175.

Jung, S., Mezger, K. \& Hoernes, S. 2003. Petrology of basement-dominated terranes: II. Contrasting isotopic ( $\mathrm{Sr}, \mathrm{Nd}, \mathrm{Pb}$ and $\mathrm{O}$ ) signatures of basement-derived granites and constraints on the source region of granite (Damara orogen, Namibia). Chemical Geology 199(1-2), 1-28.

Kasch, K. W. 1983. Continental collision, suture propagation and thermal relaxation: a plate tectonic model for the Damara Orogen in central Namibia. In: Evolution of the Damara Orogen of South-West Africa/Namibia (edited by Miller, R. M. G.). Special Publication Geological Society of South Africa 11, 423-429.

Katsube, T. J. \& Mareschal, M. 1993. Petrophysical model of deep electrical conductors; graphite lining as a source and its disconnection due to uplift. Journal of Geophysical Research, B, Solid Earth and Planets 98(5), 8019-8030.

Kaufman, A. J., Hayes, J. M., Knoll, A. H. \& Germs, G. J. B. 1991. Isotopic compositions of carbonates and organic carbon from upper Proterozoic successions in Namibia: 
stratigraphic variation and the effects of diagenesis and metamorphism. Precambrian Research 49(3-4), 301-327.

Keller, G. V. 1987. Resistivity Characteristics of Geologic Targets. In: Electromagnetic Methods in Applied Geophysics (edited by Nabighian, M. N.). Investigations in Geophysics, Vol 31. Soc. of Epl. Geophys., Tusla, Okla.

Kirschner, D. L. \& Kennedy, L. A. 2001. Limited syntectonic fluid flow in carbonate-hosted thrust faults of the Front Ranges, Canadian Rockies, inferred from stable isotope data and structures. Journal of Geophysical Research, B, Solid Earth and Planets 106(5), 88278840 .

Kitchen, N. E. \& Valley, J. W. 1995. Carbon isotope thermometry in marbles of the Adirondack Mountains, New York. Journal of Metamorphic Geology 13(5), 577-594.

Klein, J. 1980. Evolution of the first generation folds in a marble unit (Damara Orogenic Belt, Namibia). Geologische Rundschau 69(3), 770-800.

Kröner, A. 1982. Rb-Sr geochronology and tectonic evolution of the Pan-African Damara Belt of Namibia, South-western Africa. American Journal of Science 282, 1471-1507.

Kucha, H. \& Wieczorek, A. 1988. Graphite in Kupferschiefer (Poland) and its genetic meaning. Mineralium Deposita 23(3), 174-178.

Kukla, P. A. 1992. Tectonics and sedimentation of a late Proterozoic Damaran convergent continental margin, Khomas Hochland, central Namibia. Ministry of Mines and Energy, Geological Survey of Namibia, Windhoek, Namibia.

Kumar, B., Das Sharma, S., Sreenivas, B., Dayal, A. M., Rao, M. N., Dubey, N. \& Chawla, B. R. 2002. Carbon, oxygen and strontium isotope geochemistry of Proterozoic carbonate rocks of the Vindhyan Basin, central India. Precambrian Research 113(1-2), 43-63.

Landis, C. A. 1971. Graphitization of dispersed carbonaceous material in metamorphic rocks. Contributions to Mineralogy and Petrology 30, 34-45.

Leger, A., Mathez, E. A., Duba, A., Pineau, F. \& Ginsberg, S. 1996. Carbonaceous material in metamorphosed carbonate rocks from the Waits River Formation, NE Vermont, and its effect on electrical conductivity. Journal of Geophysical Research, B, Solid Earth and Planets 101(10), 22,203-22,214.

Leiss, B. \& Molli, G. 2003. 'High-temperature' texture in naturally deformed Carrara Marble from the Alpi Apuane, Italy. Journal of Structural Geology 25(4), 649-658. 
Leiss, B. \& Ullemeyer, K. 1999. Texture characterisation of carbonate rocks and some implications for the modeling of physical anisotropies, derived from idealized texture types. Zeitschrift der Deutschen Geologischen Gesellschaft 150(2), 259-274.

Leiss, B. \& Weiss, T. 2000. Fabric anisotropy and its influence on physical weathering of different types of Carrara marbles. Journal of Structural Geology 22, 1737-1745.

Losito, G., Schnegg, P. A., Lambelet, C., Viti, C. \& Trova, A. 2001. Microscopic scale conductivity as explanation of magnetotelluric results from the Alps of western Switzerland. Geophysical Journal International 147(3), 602-609.

Luque, F. J., Pasteris, J. D., Wopenka, B., Rodas, M. \& Barrenechea, J. F. 1998. Natural fluiddeposited graphite; mineralogical characteristics and mechanisms of formation. American Journal of Science 298(6), 471-498.

MacDonald, J. R. \& Kenan, W. R. 1987. Impedance Spectroscopy : Emphasizing Solid Materials and Systems. John Wiley \& Sons, New York.

Mareschal, M., Fyfe, W. S., Percival, J. \& Chan, T. 1992. Grain-boundary graphite in Kapuskasing gneisses and implications for lower-crustal conductivity. Nature (London) 357(6380), 674676.

Marlow, A. G. 1983. Geology and Rb-Sr geochronology of mineralized and radioactive granites and alaskites, Namibia. In: Evolution of the Damara Orogen of South-West Africa/Namibia (edited by Miller, R. M. G.). Special Publication - Geological Society of South Africa 11, 289-298.

Masberg, P. 2000. Garnet growth in medium-pressure granulite facies metapelites from the central Damara Orogen:

igneous versus metamorphic history. In: Henno Martin commemorative volume. (edited by Miller, R. M.). Communications of the Geological Survey of South West Africa/Namibia 12. Geological Survey of South West Africa/Namibia, Windhoek, Namibia, 115-124.

Matthews, A., Goldsmith, J. R. \& Clayton, R. N. 1983. Oxygen isotope fractionation between zoisite and water. Geochimica et Cosmochimica Acta 47(3), 645-654.

McDermott, F., Harris, N. B. W. \& Hawkesworth, C. J. 2000. Geochemical constraints on the petrogenesis of Pan-Africa A-type granites in the Damara Belt, Namibia. In: Henno Martin commemorative volume. (edited by Miller, R. M.). Communications of the Geological Survey of South West Africa/Namibia 12. Geological Survey of South West Africa/Namibia, Windhoek, Namibia, 139-148. 
Miller, R. M. G. 1983. The Pan-African Damara Orogen of South West Africa/Namibia. In: Evolution of the Damara Orogen of South-West Africa/Namibia (edited by Miller, R. M. G.). Special Publication - Geological Society of South Africa 11, 431-515.

Morikiyo, T. 1984. Carbon isotopic study on coexisting calcite and graphite in the Ryoke metamorphic rocks, northern Kiso District, central Japan. Contributions to Mineralogy and Petrology 87(3), 251-259.

Nier, A. O. 1950. A redetermination of the relative abundances of the isotopes of carbon, nitrogen, oxygen, argon and potassium. Physical Reviews 77, 789.

Nover, G., Heikamp, S., Meurer, H. J. \& Freund, D. 1998. In-situ electrical conductivity and permeability of mid-crustal rocks from the KTB drilling; consequences for high conductive layers in the Earth crust. Surveys in Geophysics 19(1), 73-85.

O'Neil, J. R. 1986. Theoretical and experimental aspects of isotopic fractionation. In: Stable isotopes in high temperature geological processes. (edited by Valley John, W., Taylor Hugh, P., Jr. \& O’Neil, J. R.). Reviews in Mineralogy 16. Mineralogical Society of America, Washington, DC, United States, 1-40.

Palacky, G. J. 1987. Resistivity Characteristics of Geologic Targets. In: Electromagnetic Methods in Applied Geophysics (edited by Nabighian, M. N.). Investigations in Geophysics, Vol 31. Soc. of Epl. Geophys., Tusla, Okla.

Park, R. \& Epstein, S. 1960. Carbon isotope fractionation during photosynthesis. Geochimica et Cosmochimica Acta 21, 110-126.

Parkhomenko, E. I. 1967. Electrical Properties of Rocks. Plenum Press, New York.

Passchier, C. W. \& Trouw, R. A. J. 1998. Microtectonics. Springer Berlin Heidelberg.

Pasteris, J. D. \& Wopenka, B. 1991. Raman spectra of graphite as indicators of degree of metamorphism. The Canadian Mineralogist 29 Part 1, 1-9.

Polyakov, V. B. \& Kharlashina, N. N. 1995. The use of heat capacity data to calculate carbon isotope fractionation between graphite, diamond, and carbon dioxide: a new approach. Geochimica et Cosmochimica Acta 59(12), 2561-2572.

Porada, H. 1985. Stratigraphy and facies in the upper Proterozoic Damara Orogen, Namibia, based on a geodynamic model. In: Stratigraphic methods as applied to the Proterozoic record. (edited by Young, G. M., Chen, J. B. \& Zhang, H.). Precambrian Research 29; 1-3. Elsevier, Amsterdam, International, 235-264. 
Pieri, M., Burlini, L., Kunze, K., Stretton, I. \& Olgaard, D. L. 2001a. Rheological and microstructural evolution of Carrara Marble with high shear strain: results from high temperature torsion experiments. Journal of Structural Geology 23(9), 1393-1413.

Pieri, M., Kunze, K., Burlini, L., Stretton, I., Olgaard, D. L., Burg, J. P. \& Wenk, H. R. 2001 b. Texture development of calcite by deformation and dynamic recrystallization at $1000 \mathrm{~K}$ during torsion experiments of marble to large strains. Tectonophysics 330(1-2), 119-140.

Puhan, D. 1983. Temperature and pressure of metamorphism in the Central Damara orogen. In: Evolution of the Damara Orogen of South-West Africa/Namibia (edited by Miller, R. M. G.). Special Publication - Geological Society of South Africa 11, 219-223.

Raab, M. J. 2001. The Geomorphic Response of the Passive Continental Margin of Northern Namibia to Gondwana Break-Up and Global Scale Tectonics. Doctoral thesis GeorgAugust-Universität Göttingen, 253.

Raab, M. J., Brown, R. W., Gallagher, K., Carter, A. \& Weber, K. 2002. Late Cretaceous reactivation of major crustal shear zones in northern Namibia: constraints from apatite fission track analysis. Tectonophysics 349(1-4), 75-92.

Raab, S., Hoth, P., Huenges, E. \& Mueller, H. J. 1998. Role of sulfur and carbon in the electrical conductivity of the middle crust. Journal of Geophysical Research, B, Solid Earth and Planets 103(5), 9681-9689.

Rathmell, M. A., Streepey, M. M., Essene, E. J. \& van, d. P. B. A. 1999. Comparison of garnetbiotite, calcite-graphite, and calcite-dolomite thermometry in the Grenville Orogen; Ontario, Canada. Contributions to Mineralogy and Petrology 134(2-3), 217-231.

Ray, J. S., Veizer, J. \& Davis, W. J. 2003. C, O, Sr and Pb isotope systematics of carbonate sequences of the Vindhyan Supergroup, India: age, diagenesis, correlations and implications for global events. Precambrian Research 121(1-2), 103-140.

Ritter, O., Weckmann, U., Vietor, T. \& Haak, V. 2003. A magnetotelluric study of the Damara Belt in Namibia : 1. Regional scale conductivity anomalies. Physics of the Earth and Planetary Interiors 138(2), 71-91.

Ross, J. V. \& Bustin, R. M. 1990. The role of strain energy in creep graphitization of anthracite. Nature (London) 343(6253), 58-60.

Ross, J. V. \& Bustin, R. M. 1997. Vitrinite anisotropy resulting from simple shear experiments at high temperature and high confining pressure. International Journal of Coal Geology 33(2), 153-168.

Ross, J. V., Bustin, R. M. \& Rouzaud, J. N. 1991. Graphitization of high rank coals-the role of shear strain: experimental considerations. Organic Geochemistry 17(5), 585-596. 
Ruland, W. 1965. X-ray studies on the carbonization and graphitization of acenaphthylene and bifluorenyl. Carbon 2, 365-378.

Shankland, T. J., Duba, A. G., Mathez, E. A. \& Peach, C. L. 1997. Increase of electrical conductivity with pressure as an indicator of conduction through a solid phase in midcrustal rocks. Journal of Geophysical Research, B, Solid Earth and Planets 102(7), 14,741-14,750.

Shields, G. \& Veizer, J. 2002. Precambrian marine carbonate isotope database: version 1.1. Geochemics Geophysics Geosystems 3 3(5), 1030.

Soula, J. C., Debat, P., Brusset, S., Bessiere, G., Christophoul, F. \& Deramond, J. 2001. Thrustrelated, diapiric, and extensional doming in a frontal orogenic wedge: example of the Montagne Noire, southern French Hercynian Belt. Journal of Structural Geology 23(11), 1677-1699.

Steven, N. M., Armstrong, R. A. \& Moore, J. M. 1993. New Rb-Sr data from the Central Zone of the Damara Orogen, Namibia. Communications of the Geological Survey of Namibia 8, 514.

Stosch, H.-G. 2002. Einführung in die Isotopengeochemie. Online in Internet http://129.13.109.66/WWW_only/html/ftp-Inhalte.html.

Tack, L. \& Bowden, P. 1999. Post-collisional granite magmatism in the central Damaran (PanAfrican) orogenic belt, western Namibia. Journal of African Earth Sciences (1994) 28(3), 653-674.

Thrower, P. A. \& Mayer, R. M. 1978. Point defects and self-diffusion in graphite. Physica Status Solidi 47, 11-37.

Tuinstra, F. \& Koenig, J. L. 1970. Raman Spectrum of Graphite. Journal of Chemical Physics 53(3), 1126-1130.

Ullemeyer, K., Spalthoff, P., Heinitz, J., Isakov, N. N., Nikitin, A. \& Weber, K. 1998. The SKAT texture diffractometer at the pulsed reactor IBR-2 at Dubna: experimental layout and first measurements. Nuclear Instruments and Methods in Physics Research A 412(1), 80-88.

Valley, J. W. 2001. Stable Isotope Thermometry at High Temperatures. In: Stable Isotope Geochemistry (edited by Valley, J. W. \& Cole, D. R.). Reviews in Mineralogy and Geochemistry 43. Geochemical Society, 365-413.

Valley, J. W. \& O'Neil, J. R. $1981 .{ }^{13} \mathrm{C} /{ }^{12} \mathrm{C}$ exchange between calcite and graphite: a possible thermometer in Grenville marbles. Geochimica et Cosmochimica Acta 45(3), 411-419. 
Veizer, J., Clayton, R. N. \& Hinton, R. W. 1992. Geochemistry of Precambrian carbonates: IV, Early Paleoproterozoic $(2.25$ + or - $0.25 \mathrm{Ga})$ seawater. In: The Taylor Colloquium; Origin and evolution of planetary crusts. (edited by McLennan Scott, M. \& Rudnick Roberta, L.). Geochimica et Cosmochimica Acta 56; 3. Pergamon, Oxford, International, 875-885.

von der Gönna, J. 1997. In-situ Untersuchungen zur Phasentransformation Olivin-Spinell im Modellsystem $\mathrm{Mg}_{2} \mathrm{GeO}_{4}$ mitttels Impedanzspektroskopie und energiedispersiver Röntgenbeugung. Doctoral thesis Rheinische-Friedrich-Wilhelms-Universität Bonn, 4-26.

Wada, H. \& Suzuki, K. 1983. Carbon isotopic thermometry calibrated by dolomite-calcite solvus temperatures. Geochimica et Cosmochimica Acta 47(4), 697-706.

Walther, J. \& Althaus, E. 1993. Graphite deposition in tectonically mobilized fault planes if the KTB-pilot drill hole. KTB Report 93-2, 493-497.

Weckmann, U., Ritter, O. \& Haak, V. 2003. A magnetotelluric study of the Damara Belt in Namibia : 2. MT phases over $90^{\circ}$ reveal the internal structure of the Waterberg Fault/Omaruru Lineament. Physics of the Earth and Planetary Interiors 138(2), 91-112.

Weis, P. L., Friedman, I. \& Gleason, J. P. 1981. The origin of epigenetic graphite: evidence from isotopes. Geochimica et Cosmochimica Acta 45(12), 2325-2332.

Weiss, T., Leiss, B., Oppermann, H. \& Siegesmund, S. 1999. Microfabric of fresh and weathered marbles: Implications and consequences for the reconstruction of the Marmorpalais Potsdam. Zeitschrift der Deutschen Geologischen Gesellschaft 150(2), 313-332.

Weiss, T., Rasolofosaon, P. N. J. \& Siegesmund, S. 2002. Ultrasonic wave velocities as a diagnostic tool for the quality assessment of marble. In: Natural Stone, Weathering Phenomena, Conservation strategies and Case Studies (edited by Siegesmund, S., Weiss, T. \& Vollbrecht, A.) 205. Geological Society of London, Special Publications, London, $149-164$.

Wenk, H. R., Takeshita, T., Bechler, E., Erskine, B. G. \& Matthies, S. 1987. Pure shear and simple shear on calcite textures. Comparison of experimental, theoretical and natural data. Journal of Structural Geology 9(5-6), 731-745.

Wilks, K. R., Mastalerz, M., Ross, J. V. \& Bustin, R. M. 1993. The effect of experimental deformation on the graphitization of Pennsylvania anthracite. In: Advances in organic petrology and geochemistry. (edited by Goodarzi, F. \& Bustin, R. M.). International Journal of Coal Geology 24; 1-4. Elsevier, Amsterdam, International, 347-369.

Wopenka, B. \& Pasteris, J. D. 1993. Structural characterization of kerogens to granulite-facies graphite; applicability of Raman microprobe spectroscopy. American Mineralogist 78(5-6), 533-557. 


\section{APPENDICES}

\section{Structural and TeXtural Analysis}

\section{I.1 SAMPLE LOCALITIES:}

\begin{tabular}{|c|c|}
\hline sample & geographical coordinates \\
\hline Elim Sp & $\mathrm{S} 21^{\circ} 29^{\prime} 06.3^{\prime \prime} \mathrm{E} 015^{\circ} 08^{\prime} 14.6^{\prime \prime}$ \\
\hline Radial 01 & $\mathrm{~S} \mathrm{21} 1^{\circ} 30^{\prime} 42.69^{\prime \prime}$ E $015^{\circ} 02^{\prime} 19.97^{\prime \prime}$ \\
\hline Omaruru $\mathrm{Sp}$ & 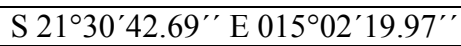 \\
\hline $13.8 .97 / 3 \mathrm{~A}$ & 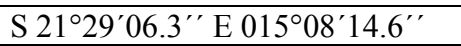 \\
\hline $11 / 08 / 00 / 03$ & 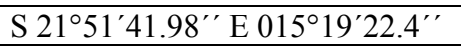 \\
\hline $11 / 08 / 00 / 06$ & 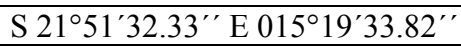 \\
\hline $12 / 08 / 00 / 02$ & $\mathrm{~S} 21^{\circ} 49^{\prime} 58.55^{\prime \prime}$ E $015^{\circ} 18^{\prime} 45.25^{\prime \prime}$ \\
\hline $12 / 08 / 00 / 03$ & 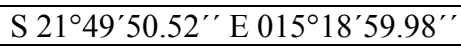 \\
\hline $12 / 08 / 00 / 04$ & 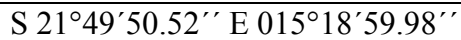 \\
\hline $15 / 08 / 00 / 01$ & 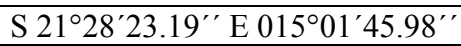 \\
\hline $16 / 08 / 00 / 02$ & $\mathrm{~S} 21^{\circ} 25^{\prime} 50.88^{\prime \prime}$ E $015^{\circ} 09^{\prime} 09.50^{\prime \prime}$ \\
\hline $17 / 08 / 00 / 01$ & $\mathrm{~S} 21^{\circ} 16^{\prime} 31.62^{\prime \prime}$ E $015^{\circ} 59^{\prime} 59.24^{\prime \prime}$ \\
\hline $17 / 08 / 00 / 02$ & $\mathrm{~S} 21^{\circ} 16^{\prime} 31.62^{\prime \prime}$ E $015^{\circ} 59^{\prime} 59.24^{\prime \prime}$ \\
\hline $17 / 08 / 00 / 04$ & 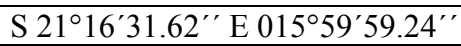 \\
\hline $17 / 08 / 00 / 05$ & 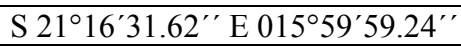 \\
\hline $17 / 08 / 00 / 07$ & 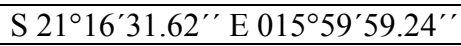 \\
\hline $17 / 08 / 00 / 08 \mathrm{a}$ & $\mathrm{S} 21^{\circ} 16^{\prime} 31.62^{\prime \prime}$ E $015^{\circ} 59^{\prime} 59.24^{\prime \prime}$ \\
\hline $17 / 08 / 00 / 08 b$ & $\mathrm{~S} 21^{\circ} 16^{\prime} 31.62^{\prime \prime}$ E $015^{\circ} 59^{\prime} 59.24^{\prime \prime}$ \\
\hline $17 / 08 / 00 / 10$ & 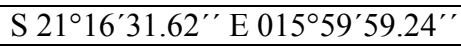 \\
\hline $17 / 08 / 00 / 11$ & 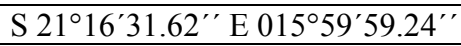 \\
\hline $17 / 08 / 00 / 12$ & 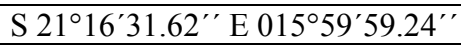 \\
\hline $17 / 08 / 00 / 13$ & 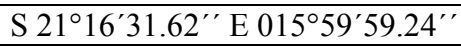 \\
\hline $17 / 08 / 00 / 15$ & $\mathrm{~S} 21^{\circ} 16^{\prime} 31.62^{\prime \prime}$ E $015^{\circ} 59^{\prime} 59.24^{\prime \prime}$ \\
\hline $17 / 08 / 00 / 16 a$ & $\mathrm{~S} 21^{\circ} 16^{\prime} 31.62^{\prime \prime}$ E $015^{\circ} 59^{\prime} 59.24^{\prime \prime}$ \\
\hline $17 / 08 / 00 / 16 b$ & 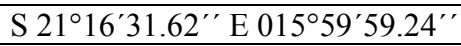 \\
\hline $17 / 08 / 00 / 17$ & 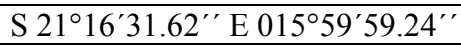 \\
\hline $17 / 08 / 00 / 19$ & 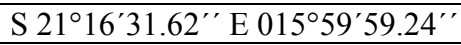 \\
\hline $17 / 08 / 00 / 20$ & $\mathrm{~S} 21^{\circ} 16^{\prime} 31.62^{\prime \prime}$ E $015^{\circ} 59^{\prime} 59.24^{\prime \prime}$ \\
\hline $17 / 08 / 00 / 21$ & $\mathrm{~S} 21^{\circ} 16^{\prime} 31.62^{\prime \prime}$ E $015^{\circ} 59^{\prime} 59.24^{\prime \prime}$ \\
\hline $18 / 08 / 00 / 02$ & 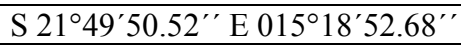 \\
\hline $3.10 .01 / 3$ & 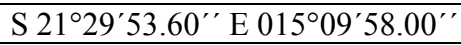 \\
\hline $3.10 .01 / 4$ & 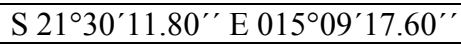 \\
\hline $4.10 .01 / 1$ & $\mathrm{~S} 21^{\circ} 28^{\prime} 48.10^{\prime \prime}$ E $015^{\circ} 08^{\prime} 43.60^{\prime \prime}$ \\
\hline
\end{tabular}




\section{Graphite Crystallinity Measurements}

\section{II.1 RAMAN MEASUREMENTS AND CALCULATIONS:}

\begin{tabular}{|c|c|c|c|c|}
\hline sample & lab number & $\begin{array}{c}G \text { band }(G \\
\text { (ordered peak }(O) \\
\text { measurement } \\
\text { wave number }(F\end{array}$ & $\begin{array}{c}\text { G band }(G \\
\text { (ordered peak }(O) \\
\text { measurement } \\
\text { intensity }(I\end{array}$ & $\begin{array}{c}\text { G band }(G) \\
\text { (ordered peak }(O) \\
\text { measurement } \\
\text { f. width at half } \\
\text { max. }(W) \\
\end{array}$ \\
\hline \multirow[t]{10}{*}{$12 / 08 / 00 / 02$} & $12-02-2 \mathrm{i}$ & 1582,49 & 397,11 & 22,77 \\
\hline & $12-02-3 \mathrm{i}$ & 1579,86 & 528 & 17,53 \\
\hline & $12-02-4 i$ & 1580,74 & 449,5 & 21,91 \\
\hline & $12-02-5 i$ & 1570,22 & 633,67 & 28,09 \\
\hline & $12-02-6 i$ & 1573,73 & 629,1 & 22,8 \\
\hline & $12-02-7 \mathrm{i}$ & 1578,99 & 517,54 & 18,41 \\
\hline & $12-02-1 \mathrm{~g}$ & 1580,74 & 697,32 & 17,52 \\
\hline & $12-02-2 \mathrm{~g}$ & 1577,24 & 555,52 & 18,41 \\
\hline & $12-02-3 g$ & 1576,36 & 702,4 & 19,29 \\
\hline & $12-02-4 g$ & 1581,62 & 479,52 & 21,9 \\
\hline \multirow[t]{8}{*}{$12 / 08 / 00 / 03$} & $12-03-2 \mathrm{i}$ & 1580,74 & 449,53 & 15,77 \\
\hline & $12-03-3 i$ & 1579,86 & 306,45 & 20,16 \\
\hline & $12-03-4 \mathrm{i}$ & 1575,48 & 652,63 & 21,05 \\
\hline & $12-03-5 i$ & 1574,6 & 618,06 & 22,8 \\
\hline & $12-03-1 \mathrm{~g}$ & 1571,97 & 413,22 & 19,31 \\
\hline & $12-03-2 \mathrm{~g}$ & 1564,95 & 418,01 & 21,08 \\
\hline & $12-03-3 g$ & 1578,11 & 524,78 & 17,53 \\
\hline & $12-03-4 g$ & 1579,86 & 505,1 & 20,15 \\
\hline \multirow[t]{11}{*}{$15 / 08 / 00 / 01$} & $15-01-1 \mathrm{i}$ & 1579,86 & 446,55 & 19,28 \\
\hline & $15-01-2 \mathrm{i}$ & 1562,66 & 556,11 & 25,43 \\
\hline & $15-01-3 i$ & 1570,55 & 643,54 & 26,28 \\
\hline & $15-01-4 i$ & 1573,18 & 908,48 & 30,66 \\
\hline & $15-01-5 i$ & 1580,18 & 481,25 & 15,74 \\
\hline & $15-01-6 \mathrm{i}$ & 1580,18 & 739,49 & 24,5 \\
\hline & $15-01-7 i$ & 1578,43 & 924,37 & 36,77 \\
\hline & $15-01-8 \mathrm{i}$ & 1568,79 & 687,86 & 26,3 \\
\hline & $15-01-1 \mathrm{~g}$ & 1577,55 & 470,64 & 17,49 \\
\hline & $15-01-2 \mathrm{~g}$ & 1579,3 & 469,41 & 24,49 \\
\hline & $15-01-3 g$ & 1581,05 & 507,22 & 14,86 \\
\hline \multirow[t]{10}{*}{$17 / 08 / 00 / 11$} & $17-11-1 \mathrm{i}$ & 1578,43 & 152,7 & 18,36 \\
\hline & $17-11-2 \mathrm{i}$ & 1574,05 & 821,56 & 32,4 \\
\hline & $17-11-3 i$ & 1579,3 & 499,62 & 16,62 \\
\hline & $17-11-4 i$ & 1579,3 & 556,36 & 14,87 \\
\hline & $17-11-5 i$ & 1580,18 & 567,05 & 16,62 \\
\hline & $17-11-6 i$ & 1574,93 & 504,51 & 22,76 \\
\hline & $17-11-7 i$ & 1577,55 & 449,47 & 26,25 \\
\hline & $17-11-1 \mathrm{~g}$ & 1576,67 & 717,06 & 33,24 \\
\hline & $17-11-2 \mathrm{~g}$ & 1576,67 & 777,14 & 27,11 \\
\hline & $17-11-3 g$ & 1579,3 & 675 & 21,86 \\
\hline
\end{tabular}




\begin{tabular}{|c|c|c|c|c|}
\hline sample & lab number & $\begin{array}{c}G \text { band }(G \\
\text { (ordered peak }(O) \\
\text { measurement } \\
\text { wave number }(F\end{array}$ & $\begin{array}{c}\text { G band }(G \\
\text { (ordered peak }(O) \\
\text { measurement } \\
\text { intensity (I }\end{array}$ & $\begin{array}{c}\text { G band }(G \\
\text { (ordered peak }(O) \\
\text { measurement } \\
\text { f. width at half } \\
\text { max. }(W) \\
\end{array}$ \\
\hline & $17-11-4 \mathrm{~g}$ & 1578,43 & 596,57 & 21 \\
\hline & $17-11-5 \mathrm{~g}$ & 1579,3 & 539,54 & 26,61 \\
\hline & $17-11-6 \mathrm{~g}$ & 1580,18 & 296,35 & 20,98 \\
\hline & $17-11-7 \mathrm{~g}$ & 1578,43 & 827,44 & 23,62 \\
\hline \multirow[t]{9}{*}{$17 / 08 / 00 / 12$} & $17-12-1 \mathrm{i}$ & 1564,41 & 731,54 & 27,18 \\
\hline & $17-12-2 \mathrm{i}$ & 1574,93 & 437,36 & 22,75 \\
\hline & $17-12-3 \mathrm{i}$ & 1566,16 & 643,55 & 25,43 \\
\hline & $17-12-4 \mathrm{i}$ & 1566,16 & 605,89 & 31,56 \\
\hline & $17-12-5 i$ & 1562,66 & 840,53 & 23,68 \\
\hline & $17-12-6 \mathrm{i}$ & 1568,79 & 399,86 & 27,17 \\
\hline & $17-12-7 \mathrm{i}$ & 1564,41 & 621,73 & 25,43 \\
\hline & $17-12-2 \mathrm{~g}$ & 1568,79 & 522,08 & 30,68 \\
\hline & $17-12-3 g$ & 1568,79 & 427,22 & 32,41 \\
\hline \multirow[t]{12}{*}{$17 / 08 / 00 / 15$} & $17-15-1 \mathrm{i}$ & 1580,18 & 510 & 20,11 \\
\hline & $17-15-2 \mathrm{i}$ & 1580,18 & 563,5 & 20,12 \\
\hline & $17-15-3 \mathrm{i}$ & 1579,3 & 479,78 & 26,23 \\
\hline & $17-15-4 \mathrm{i}$ & 1580,18 & 504,99 & 18,36 \\
\hline & $17-15-5 i$ & 1573,18 & 323,5 & 22,78 \\
\hline & $17-15-6 \mathrm{i}$ & 1574,93 & 289,27 & 22,76 \\
\hline & $17-15-7 \mathrm{i}$ & 1573,18 & 518,72 & 31,54 \\
\hline & $17-15-1 \mathrm{~g}$ & 1578,43 & 663,36 & 19,25 \\
\hline & $17-15-2 \mathrm{~g}$ & 1578,43 & 335,45 & 17,49 \\
\hline & $17-15-3 g$ & 1566,16 & 405,01 & 25,42 \\
\hline & $17-15-4 \mathrm{~g}$ & 1577,55 & 222 & 23,62 \\
\hline & $17-15-5 \mathrm{~g}$ & 1568,79 & 366,95 & 26,28 \\
\hline \multirow[t]{13}{*}{$17 / 08 / 00 / 16$} & $17-16-1 \mathrm{i}$ & 1567,92 & 897,73 & 19,29 \\
\hline & $17-16-2 \mathrm{i}$ & 1579,3 & 359,09 & 16,62 \\
\hline & $17-16-3 \mathrm{i}$ & 1580,18 & 507,71 & 16,62 \\
\hline & $17-16-4 \mathrm{i}$ & 1563,53 & 516,27 & 32,42 \\
\hline & $17-16-5 i$ & 1579,3 & 509 & 22,74 \\
\hline & $17-16-6 i$ & 1580,18 & 403,51 & 16,62 \\
\hline & $17-16-7 \mathrm{i}$ & 1579,3 & 549,93 & 16,62 \\
\hline & $17-16-1 \mathrm{~g}$ & 1587,17 & 344 & 50,57 \\
\hline & $17-16-2 \mathrm{~g}$ & 1581,62 & 122,66 & 51,52 \\
\hline & $17-16-3 g$ & 1579,86 & 442,42 & 48,1 \\
\hline & $17-16-4 \mathrm{~g}$ & 1586 & 501,35 & 54,18 \\
\hline & $17-16-5 \mathrm{~g}$ & 1581,62 & 169,53 & 19,27 \\
\hline & $17-16-6 \mathrm{~g}$ & 1582,49 & 168,96 & 20,15 \\
\hline \multirow[t]{8}{*}{$17 / 08 / 00 / 19$} & $17-19-1 \mathrm{i}$ & 1572,3 & 476,66 & 27,15 \\
\hline & $17-19-2 \mathrm{i}$ & 1570,55 & 583,4 & 27,17 \\
\hline & $17-19-3 i$ & 1576,67 & 579,43 & 22,74 \\
\hline & $17-19-4 \mathrm{i}$ & 1575,8 & 658,61 & 20,12 \\
\hline & $17-19-5 i$ & 1575,8 & 596,03 & 33,27 \\
\hline & $17-19-6 \mathrm{i}$ & 1576,67 & 627,39 & 28 \\
\hline & $17-19-1 \mathrm{~g}$ & 1581,05 & 627,95 & 16,62 \\
\hline & $17-19-2 \mathrm{~g}$ & 1579,3 & 533,18 & 19,25 \\
\hline
\end{tabular}




\begin{tabular}{|c|c|c|c|c|}
\hline sample & lab number & $\begin{array}{c}G \text { band }(G \\
\text { (ordered peak }(O) \\
\text { measurement } \\
\text { wave number }(F\end{array}$ & $\begin{array}{c}\text { G band }(G \\
\text { (ordered peak }(O) \\
\text { measurement } \\
\text { intensity (I }\end{array}$ & $\begin{array}{c}\text { G band }(G \\
\text { (ordered peak }(O) \\
\text { measurement } \\
\text { f. width at half } \\
\text { max. }(W) \\
\end{array}$ \\
\hline & $17-19-3 g$ & 1579,3 & 377,45 & 28,86 \\
\hline & $17-19-4 \mathrm{~g}$ & 1579,3 & 499 & 19,24 \\
\hline & $17-19-5 \mathrm{~g}$ & 1567,92 & 398,14 & 28,05 \\
\hline \multirow[t]{21}{*}{$17 / 08 / 00 / 20$} & $17-2010 \mathrm{i}$ & 1580,18 & 550,69 & 14,87 \\
\hline & $17-2011 \mathrm{i}$ & 1581,92 & 483,49 & 19,23 \\
\hline & $17-2012 \mathrm{i}$ & 1581,92 & 418,37 & 14,86 \\
\hline & $17-2013 i$ & 1580,18 & 822,29 & 23,61 \\
\hline & $17-2014 i$ & 1580,18 & 606,06 & 17,49 \\
\hline & $17-2015 \mathrm{i}$ & 1578,43 & 573,36 & 19,24 \\
\hline & $17-20-1 \mathrm{i}$ & 1582,8 & 775,5 & 14,86 \\
\hline & $17-20-2 \mathrm{i}$ & 1579,3 & 775,25 & 14,87 \\
\hline & $17-20-3 i$ & 1581,05 & 903,48 & 14,87 \\
\hline & $17-20-4 \mathrm{i}$ & 1582,8 & 533,7 & 15,73 \\
\hline & $17-20-5 i$ & 1580,18 & 513,22 & 21,86 \\
\hline & $17-20-6 \mathrm{i}$ & 1579,3 & 510,96 & 17,48 \\
\hline & $17-20-7 \mathrm{i}$ & 1581,05 & 599 & 18,36 \\
\hline & $17-20-8 \mathrm{i}$ & 1581,05 & 514,26 & 14,87 \\
\hline & $17-20-9 \mathrm{i}$ & 1580,18 & 514,68 & 15,74 \\
\hline & $17-20-1 \mathrm{~g}$ & 1581,05 & 457,9 & 18,36 \\
\hline & $17-20-2 \mathrm{~g}$ & 1581,05 & 516,81 & 14,87 \\
\hline & $17-20-3 \mathrm{~g}$ & 1581,05 & 476,97 & 14,87 \\
\hline & $17-20-4 \mathrm{~g}$ & 1579,3 & 487,48 & 14,87 \\
\hline & $17-20-5 \mathrm{~g}$ & 1582,8 & 224,38 & 17,48 \\
\hline & $17-20-6 \mathrm{~g}$ & 1581,05 & 252,85 & 23,6 \\
\hline \multirow[t]{12}{*}{$18 / 08 / 00 / 02$} & $18-02-1 \mathrm{i}$ & 1579,86 & 554,29 & 22,78 \\
\hline & $18-02-2 \mathrm{i}$ & 1571,1 & 630,7 & 25,47 \\
\hline & $18-02-3 \mathrm{i}$ & 1565,83 & 755,07 & 17,57 \\
\hline & $18-02-4 \mathrm{i}$ & 1576,36 & 610,36 & 28,94 \\
\hline & $18-02-5 i$ & 1579,86 & 442,42 & 15,77 \\
\hline & $18-02-6 \mathrm{i}$ & 1578,99 & 745,52 & 28,92 \\
\hline & $18-02-7 \mathrm{i}$ & 1575,48 & 649,66 & 19,29 \\
\hline & $18-02-1 \mathrm{~g}$ & 1579,86 & 498,65 & 29,79 \\
\hline & $18-02-2 \mathrm{~g}$ & 1581,62 & 546,91 & 20,15 \\
\hline & $18-02-3 \mathrm{~g}$ & 1581,62 & 419,25 & 17,52 \\
\hline & $18-02-5 \mathrm{~g}$ & 1586,87 & 191,15 & 42,83 \\
\hline & $18-02-6 g$ & 1574,6 & 430,54 & 23,67 \\
\hline \multirow[t]{10}{*}{$3.10 .01 / 3$} & 3-013-1i & 1581,62 & 712,84 & 19,27 \\
\hline & $3-013-2 i$ & 1579,86 & 488,13 & 16,65 \\
\hline & $3-013-3 i$ & 1574,6 & 318,55 & 14,91 \\
\hline & $3-013-4 i$ & 1581,62 & 404,48 & 40,26 \\
\hline & $3-013-5 i$ & 1571,97 & 375,72 & 17,54 \\
\hline & $3-013-6 i$ & 1564,95 & 377,88 & 34,25 \\
\hline & $3-013-7 i$ & 1579,86 & 422,76 & 26,28 \\
\hline & $3-013-1 g$ & 1579,86 & 476,51 & 28,04 \\
\hline & $3-013-2 \mathrm{~g}$ & 1580,74 & 707,54 & 19,27 \\
\hline & $3-013-3 g$ & 1580,74 & 731,55 & 21,02 \\
\hline
\end{tabular}




\begin{tabular}{|c|c|c|c|c|}
\hline sample & lab number & $\begin{array}{c}\text { G band }(G \\
\text { (ordered peak }(O) \\
\text { measurement } \\
\text { wave number }(F\end{array}$ & $\begin{array}{c}\text { G band (G } \\
\text { (ordered peak }(O) \\
\text { measurement } \\
\text { intensity (I }\end{array}$ & $\begin{array}{c}\text { G band }(G \\
\text { (ordered peak }(O) \\
\text { measurement } \\
\text { f. width at half } \\
\text { max. }(W)\end{array}$ \\
\hline & $3-013-4 g$ & 1580,74 & 480,65 & 18,4 \\
\hline \multirow[t]{15}{*}{$3.10 .01 / 4$} & $3-01410 \mathrm{i}$ & 1569,67 & 520,42 & 21,02 \\
\hline & 3-014-1i & 1580,18 & 186,79 & 22,74 \\
\hline & $3-014-2 i$ & 1580,18 & 265,44 & 17,49 \\
\hline & $3-014-3 i$ & 1572,3 & 202,25 & 20,15 \\
\hline & $3-014-4 i$ & 1565,29 & 267,7 & 21,92 \\
\hline & $3-014-5 i$ & 1580,18 & 311,78 & 20,11 \\
\hline & $3-014-6 i$ & 1579,3 & 198,15 & 21,85 \\
\hline & $3-014-7 i$ & 1581,05 & 445 & 27,11 \\
\hline & $3-014-8 \mathrm{i}$ & 1577,55 & 169,98 & 22,74 \\
\hline & 3-014-9i & 1576,67 & 448,55 & 18,38 \\
\hline & $3-014-1 g$ & 1580,74 & 512 & 21,02 \\
\hline & $3-014-2 \mathrm{~g}$ & 1581,62 & 428,43 & 17,51 \\
\hline & $3-014-3 g$ & 1580,18 & 467,73 & 22,74 \\
\hline & $3-014-4 g$ & 1579,86 & 390,9 & 21,02 \\
\hline & $3-014-5 \mathrm{~g}$ & 1580,74 & 427,83 & 22,78 \\
\hline \multirow[t]{2}{*}{ Elim Spatkristall } & Elim 01 & 1580,18 & 118,97 & 46,26 \\
\hline & Elim 02 & 1581,92 & 108,65 & 24,49 \\
\hline \multirow[t]{4}{*}{ Omaruru Spatkristall } & Rad 01 & 1579,3 & 464,73 & 14,87 \\
\hline & $\operatorname{Rad} 02$ & 1580,18 & 459,53 & 15,74 \\
\hline & $\operatorname{Rad} 03$ & 1579,3 & 477,09 & 13,99 \\
\hline & $\operatorname{Rad} 04$ & 1580,18 & 514,62 & 16,61 \\
\hline
\end{tabular}




\begin{tabular}{|c|c|c|c|c|}
\hline sample & lab number & $\begin{array}{c}\text { G band }(G \\
\text { (ordered peak }(O) \\
\text { function of meas. } \\
\text { wave number }(F\end{array}$ & $\begin{array}{c}\text { G band (G } \\
\text { (ordered peak }(O) \\
\text { function of meas. } \\
\text { Intensity (I }\end{array}$ & $\begin{array}{c}\text { G band }(G \\
\text { (ordered peak }(O) \\
\text { function of meas. } \\
\text { f. width at half } \\
\text { max. }(W)\end{array}$ \\
\hline \multirow[t]{10}{*}{$12 / 08 / 00 / 02$} & $12-02-2 \mathrm{i}$ & 1582,51 & 376,82 & 22,7186 \\
\hline & $12-02-3 i$ & 1579,53 & 505,003 & 17,6463 \\
\hline & $12-02-4 i$ & 1579,53 & 444,274 & 16,7984 \\
\hline & $12-02-5 \mathrm{i}$ & 1571,18 & 524,488 & 50,643 \\
\hline & $12-02-6 \mathrm{i}$ & 1574,46 & 618,807 & 23,0683 \\
\hline & $12-02-7 i$ & 1578,04 & 515,438 & 18,25 \\
\hline & $12-02-1 \mathrm{~g}$ & 1580,61 & 682,731 & 17,6251 \\
\hline & $12-02-2 \mathrm{~g}$ & 1576,7 & 543,339 & 18,6876 \\
\hline & $12-02-3 g$ & 1576,21 & 654,683 & 20,2408 \\
\hline & $12-02-4 g$ & 1581,09 & 463,953 & 22,5514 \\
\hline \multirow[t]{8}{*}{$12 / 08 / 00 / 03$} & $12-03-2 \mathrm{i}$ & 1580,17 & 443,64 & 16,0985 \\
\hline & $12-03-3 i$ & 1579,05 & 287,076 & 18,6463 \\
\hline & $12-03-4 i$ & 1575,18 & 649,911 & 20,0762 \\
\hline & $12-03-5 \mathrm{i}$ & 1573,94 & 605,779 & 21,0218 \\
\hline & $12-03-1 \mathrm{~g}$ & 1571,07 & 413,225 & 18,9978 \\
\hline & $12-03-2 \mathrm{~g}$ & 1566,39 & 388,051 & 22,6218 \\
\hline & $12-03-3 g$ & 1578,97 & 518,289 & 15,9374 \\
\hline & $12-03-4 g$ & 1579,68 & 489,659 & 20,1115 \\
\hline \multirow[t]{11}{*}{$15 / 08 / 00 / 01$} & $15-01-1 \mathrm{i}$ & 1579,76 & 440,623 & 20,0054 \\
\hline & $15-01-2 \mathrm{i}$ & 1565,32 & 540,429 & 23,8225 \\
\hline & $15-01-3 i$ & 1570,98 & 636,002 & 21,4134 \\
\hline & $15-01-4 i$ & 1571,69 & 896,341 & 26,2946 \\
\hline & $15-01-5 i$ & 1580,39 & 474,05 & 14,6029 \\
\hline & $15-01-6 i$ & 1577,89 & 717,593 & 20,3826 \\
\hline & $15-01-7 i$ & 1576,61 & 699,493 & 44,161 \\
\hline & $15-01-8 \mathrm{i}$ & 1567,19 & 665,791 & 20,9289 \\
\hline & $15-01-1 \mathrm{~g}$ & 1578,96 & 470,398 & 16,0457 \\
\hline & $15-01-2 \mathrm{~g}$ & 1580,79 & 448,116 & 26,1242 \\
\hline & $15-01-3 g$ & 1581,5 & 499,717 & 14,1854 \\
\hline \multirow[t]{14}{*}{$17 / 08 / 00 / 11$} & $17-11-1 \mathrm{i}$ & 1580,87 & 121,579 & 29,1035 \\
\hline & $17-11-2 \mathrm{i}$ & 1574,04 & 789,533 & 34,6974 \\
\hline & $17-11-3 i$ & 1579,6 & 476,836 & 17,0527 \\
\hline & $17-11-4 \mathrm{i}$ & 1579,72 & 540,656 & 14,4205 \\
\hline & $17-11-5 i$ & 1579,64 & 553,606 & 15,7714 \\
\hline & $17-11-6 \mathrm{i}$ & 1575,26 & 495,817 & 19,5114 \\
\hline & $17-11-7 i$ & 1577,69 & 438,791 & 21,5306 \\
\hline & $17-11-1 \mathrm{~g}$ & 1579,11 & 650,848 & 36,2335 \\
\hline & $17-11-2 \mathrm{~g}$ & 1578,36 & 728,443 & 27,7066 \\
\hline & $17-11-3 g$ & 1581,46 & 621,383 & 26,7658 \\
\hline & $17-11-4 \mathrm{~g}$ & 1578,41 & 584,374 & 19,5215 \\
\hline & $17-11-5 \mathrm{~g}$ & 1580,64 & 516,118 & 22,4875 \\
\hline & $17-11-6 \mathrm{~g}$ & 1581,26 & 279,409 & 22,357 \\
\hline & $17-11-7 \mathrm{~g}$ & 1578,77 & 790,566 & 23,4237 \\
\hline \multirow[t]{3}{*}{$17 / 08 / 00 / 12$} & $17-12-1 \mathrm{i}$ & 1566,46 & 731,538 & 25,4083 \\
\hline & $17-12-2 \mathrm{i}$ & 1578,03 & 402,193 & 25,0189 \\
\hline & $17-12-3 i$ & 1565,34 & 629,65 & 24,9894 \\
\hline
\end{tabular}




\begin{tabular}{|c|c|c|c|c|}
\hline sample & lab number & $\begin{array}{c}\text { G band }(G \\
\text { (ordered peak }(O) \\
\text { function of meas. } \\
\text { wave number }(F\end{array}$ & $\begin{array}{c}\text { G band (G } \\
\text { (ordered peak }(O) \\
\text { function of meas. } \\
\text { Intensity (I }\end{array}$ & $\begin{array}{c}\text { G band }(G \\
\text { (ordered peak }(O) \\
\text { function of meas. } \\
\text { f. width at half } \\
\text { max. }(W)\end{array}$ \\
\hline & $17-12-4 i$ & 1567,59 & 605,888 & 25,0486 \\
\hline & $17-12-5 i$ & 1564,47 & 839,506 & 24,0268 \\
\hline & $17-12-6 i$ & 1569,1 & 388,083 & 26,2521 \\
\hline & $17-12-7 \mathrm{i}$ & 1565,38 & 598,649 & 24,9041 \\
\hline & $17-12-2 \mathrm{~g}$ & 1568,01 & 511,148 & 32,2915 \\
\hline & $17-12-3 \mathrm{~g}$ & 1571,55 & 411,942 & 33,9613 \\
\hline \multirow[t]{12}{*}{$17 / 08 / 00 / 15$} & $17-15-1 \mathrm{i}$ & 1580,21 & 503,688 & 17,3981 \\
\hline & $17-15-2 \mathrm{i}$ & 1578,93 & 541,596 & 19,2132 \\
\hline & $17-15-3 i$ & 1580,78 & 465,561 & 21,885 \\
\hline & $17-15-4 i$ & 1579,83 & 495,903 & 14,8532 \\
\hline & $17-15-5 i$ & 1571,46 & 305,607 & 25,8086 \\
\hline & $17-15-6 \mathrm{i}$ & 1574,53 & 270,41 & 24,4327 \\
\hline & $17-15-7 i$ & 1571,34 & 503,172 & 29,3287 \\
\hline & $17-15-1 \mathrm{~g}$ & 1576,76 & 645,69 & 20,1826 \\
\hline & $17-15-2 \mathrm{~g}$ & 1578,89 & 315,39 & 18,5458 \\
\hline & $17-15-3 g$ & 1568,59 & 405,011 & 24,8774 \\
\hline & $17-15-4 \mathrm{~g}$ & 1576,98 & 199,76 & 27,0177 \\
\hline & $17-15-5 \mathrm{~g}$ & 1570,59 & 301,114 & 30,6547 \\
\hline \multirow[t]{13}{*}{$17 / 08 / 00 / 16$} & $17-16-1 \mathrm{i}$ & 1567,19 & 879,676 & 16,5687 \\
\hline & $17-16-2 \mathrm{i}$ & 1579,33 & 337,19 & 18,8498 \\
\hline & $17-16-3 i$ & 1579,99 & 488,244 & 17,6551 \\
\hline & $17-16-4 i$ & 1568,87 & 495,667 & 30,0261 \\
\hline & $17-16-5 i$ & 1579,05 & 478,145 & 24,43 \\
\hline & $17-16-6 \mathrm{i}$ & 1579,52 & 379,945 & 17,3173 \\
\hline & $17-16-7 i$ & 1579,47 & 541,672 & 13,435 \\
\hline & $17-16-1 \mathrm{~g}$ & 1593,6 & 322,803 & 43,0641 \\
\hline & $17-16-2 \mathrm{~g}$ & 1594,57 & 115,486 & 48,4573 \\
\hline & $17-16-3 g$ & 1586,54 & 395,684 & 48,6091 \\
\hline & $17-16-4 \mathrm{~g}$ & 1590,32 & 480,845 & 51,7922 \\
\hline & $17-16-5 \mathrm{~g}$ & 1582,94 & 147,219 & 24,6986 \\
\hline & $17-16-6 \mathrm{~g}$ & 1583,66 & 136,845 & 32,7022 \\
\hline \multirow[t]{11}{*}{$17 / 08 / 00 / 19$} & 17-19-1i & 1573,66 & 465,982 & 24,7576 \\
\hline & $17-19-2 \mathrm{i}$ & 1568,56 & 573,285 & 25,1858 \\
\hline & $17-19-3 i$ & 1579,84 & 556,082 & 23,7654 \\
\hline & $17-19-4 \mathrm{i}$ & 1577,28 & 634,145 & 21,0438 \\
\hline & $17-19-5 i$ & 1574,44 & 584,054 & 25,9746 \\
\hline & $17-19-6 i$ & 1578,7 & 564,548 & 32,2254 \\
\hline & 17-19-1g & 1580,07 & 620,789 & 16,027 \\
\hline & $17-19-2 \mathrm{~g}$ & 1578,55 & 517,039 & 18,7833 \\
\hline & $17-19-3 \mathrm{~g}$ & 1579,22 & 363,881 & 23,1723 \\
\hline & $17-19-4 \mathrm{~g}$ & 1580,25 & 483,038 & 14,9974 \\
\hline & $17-19-5 \mathrm{~g}$ & 1569,49 & 379,902 & 29,1222 \\
\hline \multirow[t]{4}{*}{$17 / 08 / 00 / 20$} & $17-2010 \mathrm{i}$ & 1580,17 & 536,754 & 14,7645 \\
\hline & $17-2011 \mathrm{i}$ & 1581,42 & 474,423 & 14,668 \\
\hline & $17-2012 \mathrm{i}$ & 1580,93 & 422,76 & 20,8158 \\
\hline & $17-2013 \mathrm{i}$ & 1580,53 & 807,982 & 18,8048 \\
\hline
\end{tabular}




\begin{tabular}{|c|c|c|c|c|}
\hline \multirow[t]{18}{*}{ sample } & lab number & $\begin{array}{c}\text { G band }(G \\
\text { (ordered peak }(O) \\
\text { function of meas. } \\
\text { wave number }(F\end{array}$ & $\begin{array}{c}\text { G band (G } \\
\text { (ordered peak }(O) \\
\text { function of meas. } \\
\text { Intensity (I }\end{array}$ & $\begin{array}{c}\text { G band }(G \\
\text { (ordered peak }(O) \\
\text { function of meas. } \\
\text { f. width at half } \\
\text { max. }(W)\end{array}$ \\
\hline & $17-2014 \mathrm{i}$ & 1579,99 & 572,242 & 19,4036 \\
\hline & $17-2015 \mathrm{i}$ & 1580,01 & 559,823 & 19,5359 \\
\hline & $17-20-1 \mathrm{i}$ & 1581,65 & 768,895 & 14,6654 \\
\hline & $17-20-2 \mathrm{i}$ & 1580,09 & 770,899 & 13,9147 \\
\hline & $17-20-3 i$ & 1580,91 & 900,805 & 14,4525 \\
\hline & $17-20-4 \mathrm{i}$ & 1581,93 & 533,7 & 13,9119 \\
\hline & $17-20-5 i$ & 1580,75 & 491,547 & 17,8184 \\
\hline & $17-20-6 \mathrm{i}$ & 1581,1 & 472,94 & 20,0949 \\
\hline & $17-20-7 i$ & 1582,26 & 533,853 & 21,3611 \\
\hline & $17-20-8 \mathrm{i}$ & 1580,84 & 514,256 & 13,8199 \\
\hline & $17-20-9 \mathrm{i}$ & 1579,45 & 514,679 & 14,4007 \\
\hline & $17-20-1 \mathrm{~g}$ & 1580,62 & 452,776 & 13,9929 \\
\hline & $17-20-2 \mathrm{~g}$ & 1579,62 & 521,401 & 13,6727 \\
\hline & $17-20-3 \mathrm{~g}$ & 1580,09 & 480,675 & 15,8578 \\
\hline & $17-20-4 \mathrm{~g}$ & 1580,15 & 487,484 & 14,6909 \\
\hline & $17-20-5 \mathrm{~g}$ & 1581,95 & 211,757 & 17,9347 \\
\hline & $17-20-6 \mathrm{~g}$ & 1581,41 & 234,852 & 18,3847 \\
\hline \multirow[t]{12}{*}{$18 / 08 / 00 / 02$} & $18-02-1 \mathrm{i}$ & 1580,45 & 538,406 & 19,4269 \\
\hline & $18-02-2 \mathrm{i}$ & 1568,03 & 624,69 & 23,6295 \\
\hline & $18-02-3 \mathrm{i}$ & 1566,36 & 733,16 & 17,4281 \\
\hline & $18-02-4 i$ & 1576,25 & 590,288 & 29,2048 \\
\hline & $18-02-5 i$ & 1580,63 & 436,15 & 15,7241 \\
\hline & $18-02-6 \mathrm{i}$ & 1579,83 & 727,495 & 29,173 \\
\hline & $18-02-7 i$ & 1574,94 & 628,937 & 19,1773 \\
\hline & $18-02-1 \mathrm{~g}$ & 1582,08 & 466,898 & 32,0512 \\
\hline & $18-02-2 \mathrm{~g}$ & 1581,11 & 546,913 & 17,1895 \\
\hline & $18-02-3 g$ & 1581,37 & 419,245 & 18,0022 \\
\hline & $18-02-5 \mathrm{~g}$ & 1590,25 & 161,268 & 49,4372 \\
\hline & $18-02-6 \mathrm{~g}$ & 1577,59 & 381,023 & 28,9114 \\
\hline \multirow[t]{11}{*}{$3.10 .01 / 3$} & 3-013-1i & 1581,29 & 712,836 & 19,2287 \\
\hline & $3-013-2 \mathrm{i}$ & 1580,34 & 485,443 & 15,2394 \\
\hline & $3-013-3 i$ & 1574,25 & 307,747 & 16,0103 \\
\hline & $3-013-4 i$ & 1582,81 & 382,145 & 29,2093 \\
\hline & $3-013-5 i$ & 1573,06 & 370,921 & 13,64 \\
\hline & $3-013-6 \mathrm{i}$ & 1568,63 & 360,802 & 35,6522 \\
\hline & $3-013-7 i$ & 1581,13 & 390,469 & 23,4603 \\
\hline & $3-013-1 \mathrm{~g}$ & 1580,54 & 466,589 & 16,8247 \\
\hline & $3-013-2 \mathrm{~g}$ & 1581,23 & 699,92 & 17,9541 \\
\hline & $3-013-3 g$ & 1582,63 & 715,139 & 19,839 \\
\hline & $3-013-4 \mathrm{~g}$ & 1580,9 & 467,438 & 15,4609 \\
\hline \multirow[t]{6}{*}{$3.10 .01 / 4$} & $3-01410 \mathrm{i}$ & 1570,51 & 514,422 & 20,4356 \\
\hline & 3-014-1i & 1579,82 & 171,891 & 17,205 \\
\hline & $3-014-2 \mathrm{i}$ & 1580,26 & 243,459 & 20,0774 \\
\hline & $3-014-3 i$ & 1571,35 & 198,997 & 17,0248 \\
\hline & 3-014-4i & 1565,91 & 247,854 & 17,9389 \\
\hline & $3-014-5 i$ & 1579,76 & 282,444 & 23,5544 \\
\hline
\end{tabular}




\begin{tabular}{|c|c|c|c|c|}
\hline \multirow{2}{*}{ sample } & lab number & $\begin{array}{c}\text { G band (G } \\
\text { (ordered peak (O) } \\
\text { function of meas. } \\
\text { wave number (F }\end{array}$ & $\begin{array}{c}\text { G band (G } \\
\text { ordered peak (O) } \\
\text { function of meas. } \\
\text { Intensity (I }\end{array}$ & $\begin{array}{c}\text { G band (G } \\
\text { (ordered peak (O) } \\
\text { function of meas. } \\
\text { f. width at half } \\
\text { max. (W) }\end{array}$ \\
\hline & $3-014-6 \mathrm{i}$ & 1581,77 & 161,818 & 33,6083 \\
\hline & $3-014-7 \mathrm{i}$ & 1580,75 & 415,185 & 25,3214 \\
\hline & $3-014-8 \mathrm{i}$ & 1581,45 & 156,678 & 27,5599 \\
\hline & $3-014-9 \mathrm{i}$ & 1576,85 & 443,49 & 15,3604 \\
\hline & $3-014-1 \mathrm{~g}$ & 1582,02 & 511,654 & 16,2126 \\
\hline & $3-014-2 \mathrm{~g}$ & 1582,46 & 425,59 & 14,1091 \\
\hline & $3-014-3 \mathrm{~g}$ & 1579,55 & 447,737 & 18,5711 \\
\hline & $3-014-4 \mathrm{~g}$ & 1580,66 & 388,84 & 16,2443 \\
\hline & $3-014-5 \mathrm{~g}$ & 1579,91 & 421,448 & 17,8496 \\
\hline & Elim 01 & 1585,64 & 92,033 & 40,4928 \\
\hline Elim Spatkristall & Elim 02 & 1584,4 & 86,7425 & 45,2778 \\
\hline & Rad 01 & 1579,85 & 457,607 & 13,9269 \\
\hline & Rad 02 & 1579,65 & 417,175 & 13,9287 \\
\hline & Rad 03 & 1579,46 & 451,104 & 13,9798 \\
\hline & Rad 04 & 1580,48 & 497,532 & 12,9713 \\
\hline
\end{tabular}




\begin{tabular}{|c|c|c|c|}
\hline sample & lab number & $\begin{array}{l}\text { shoulder peak (D2) } \\
\text { wave number (F }\end{array}$ & $\begin{array}{c}\text { shoulder peak (D2) } \\
\text { intensitiy (I }\end{array}$ \\
\hline \multirow[t]{10}{*}{$12 / 08 / 00 / 02$} & $12-02-2 \mathrm{i}$ & 1620,89 & 86,35 \\
\hline & $12-02-3 \mathrm{i}$ & 1611,32 & 56 \\
\hline & $12-02-4 \mathrm{i}$ & & \\
\hline & $12-02-5 i$ & & \\
\hline & $12-02-6 \mathrm{i}$ & 1613,06 & 112,5 \\
\hline & $12-02-7 i$ & 1612,19 & 62,76 \\
\hline & $12-02-1 \mathrm{~g}$ & 1619,5 & 83,5 \\
\hline & $12-02-2 \mathrm{~g}$ & 1616,54 & 69,27 \\
\hline & $12-02-3 \mathrm{~g}$ & 1610,44 & 99,7 \\
\hline & $12-02-4 \mathrm{~g}$ & 1615,67 & 99,62 \\
\hline \multirow[t]{8}{*}{$12 / 08 / 00 / 03$} & $12-03-2 \mathrm{i}$ & 1620,02 & 50,36 \\
\hline & $12-03-3 i$ & 1619,15 & 41,96 \\
\hline & $12-03-4 i$ & 1611,32 & 83,78 \\
\hline & $12-03-5 i$ & & \\
\hline & $12-03-1 \mathrm{~g}$ & 1606,96 & 61,51 \\
\hline & $12-03-2 \mathrm{~g}$ & 1601,73 & 80,66 \\
\hline & $12-03-3 \mathrm{~g}$ & 1616,54 & 44,64 \\
\hline & $12-03-4 \mathrm{~g}$ & 1619,15 & 111,53 \\
\hline \multirow[t]{11}{*}{$15 / 08 / 00 / 01$} & $15-01-1 \mathrm{i}$ & & \\
\hline & $15-01-2 \mathrm{i}$ & & \\
\hline & $15-01-3 i$ & & \\
\hline & $15-01-4 \mathrm{i}$ & & \\
\hline & $15-01-5 i$ & & \\
\hline & $15-01-6 \mathrm{i}$ & & \\
\hline & $15-01-7 i$ & & \\
\hline & $15-01-8 \mathrm{i}$ & 1603,73 & 124,73 \\
\hline & $15-01-1 \mathrm{~g}$ & 1617,64 & 39,81 \\
\hline & $15-01-2 \mathrm{~g}$ & 1615,04 & 116,18 \\
\hline & $15-01-3 \mathrm{~g}$ & & \\
\hline \multirow[t]{14}{*}{$17 / 08 / 00 / 11$} & $17-11-1 \mathrm{i}$ & 1616,78 & 44,64 \\
\hline & $17-11-2 \mathrm{i}$ & & \\
\hline & $17-11-3 i$ & & \\
\hline & $17-11-4 \mathrm{i}$ & & \\
\hline & $17-11-5 \mathrm{i}$ & & \\
\hline & 17-11-6i & & \\
\hline & $17-11-7 \mathrm{i}$ & & \\
\hline & $17-11-1 \mathrm{~g}$ & & \\
\hline & $17-11-2 \mathrm{~g}$ & & \\
\hline & $17-11-3 \mathrm{~g}$ & 1611,56 & 221 \\
\hline & $17-11-4 \mathrm{~g}$ & & \\
\hline & $17-11-5 \mathrm{~g}$ & & \\
\hline & $17-11-6 \mathrm{~g}$ & 1612,43 & 67,66 \\
\hline & $17-11-7 \mathrm{~g}$ & 1614,17 & 212,05 \\
\hline \multirow[t]{3}{*}{$17 / 08 / 00 / 12$} & $17-12-1 \mathrm{i}$ & 1596,76 & 174,5 \\
\hline & $17-12-2 \mathrm{i}$ & 1608,96 & 125,71 \\
\hline & $17-12-3 \mathrm{i}$ & 1595,89 & 147,8 \\
\hline
\end{tabular}




\begin{tabular}{|c|c|c|c|}
\hline sample & lab number & $\begin{array}{l}\text { shoulder peak (D2) } \\
\text { wave number ( } F\end{array}$ & $\begin{array}{c}\text { shoulder peak (D2) } \\
\text { intensitiy (I }\end{array}$ \\
\hline & $17-12-4 \mathrm{i}$ & 1601,99 & 133,38 \\
\hline & $17-12-5 \mathrm{i}$ & & \\
\hline & $17-12-6 i$ & 1600,25 & 106,85 \\
\hline & $17-12-7 i$ & & \\
\hline & $17-12-2 \mathrm{~g}$ & & \\
\hline & $17-12-3 \mathrm{~g}$ & & \\
\hline \multirow[t]{12}{*}{$17 / 08 / 00 / 15$} & $17-15-1 \mathrm{i}$ & 1604,6 & 97,63 \\
\hline & $17-15-2 \mathrm{i}$ & 1615,04 & 64,13 \\
\hline & $17-15-3 \mathrm{i}$ & & \\
\hline & $17-15-4 \mathrm{i}$ & & \\
\hline & $17-15-5 \mathrm{i}$ & 1606,34 & 78,74 \\
\hline & $17-15-6 \mathrm{i}$ & 1608,09 & 76,49 \\
\hline & $17-15-7 \mathrm{i}$ & 1608,09 & 84,89 \\
\hline & $17-15-1 \mathrm{~g}$ & 1619,38 & 82,99 \\
\hline & $17-15-2 \mathrm{~g}$ & 1613,3 & 58,91 \\
\hline & $17-15-3 \mathrm{~g}$ & 1604,6 & 89,12 \\
\hline & $17-15-4 \mathrm{~g}$ & 1616,78 & 79 \\
\hline & $17-15-5 \mathrm{~g}$ & 1609,82 & 183,57 \\
\hline \multirow[t]{13}{*}{$17 / 08 / 00 / 16$} & $17-16-1 \mathrm{i}$ & & \\
\hline & $17-16-2 \mathrm{i}$ & 1615,04 & 57,93 \\
\hline & $17-16-3 \mathrm{i}$ & & \\
\hline & $17-16-4 \mathrm{i}$ & 1609,82 & 82,96 \\
\hline & $17-16-5 \mathrm{i}$ & 1613,3 & 111,68 \\
\hline & $17-16-6 i$ & 1612,43 & 67,74 \\
\hline & $17-16-7 \mathrm{i}$ & & \\
\hline & $17-16-1 \mathrm{~g}$ & & \\
\hline & $17-16-2 \mathrm{~g}$ & 1604,34 & 111,52 \\
\hline & $17-16-3 \mathrm{~g}$ & 1610,44 & 278,31 \\
\hline & $17-16-4 \mathrm{~g}$ & 1602,6 & 443,46 \\
\hline & $17-16-5 \mathrm{~g}$ & 1620,02 & 47,03 \\
\hline & $17-16-6 \mathrm{~g}$ & 1611,32 & 63,8 \\
\hline \multirow[t]{11}{*}{$17 / 08 / 00 / 19$} & $17-19-1 \mathrm{i}$ & & \\
\hline & $17-19-2 \mathrm{i}$ & & \\
\hline & $17-19-3 i$ & 1615,91 & 163,49 \\
\hline & $17-19-4 \mathrm{i}$ & 1612,43 & 126,48 \\
\hline & $17-19-5 \mathrm{i}$ & & \\
\hline & $17-19-6 \mathrm{i}$ & & \\
\hline & $17-19-1 \mathrm{~g}$ & 1619,38 & 65,53 \\
\hline & $17-19-2 \mathrm{~g}$ & 1615,91 & 71,31 \\
\hline & $17-19-3 \mathrm{~g}$ & 1610,69 & 95,59 \\
\hline & $17-19-4 \mathrm{~g}$ & & \\
\hline & $17-19-5 \mathrm{~g}$ & 1600,25 & 112 \\
\hline \multirow[t]{4}{*}{$17 / 08 / 00 / 20$} & $17-2010 \mathrm{i}$ & 1612,43 & 61,34 \\
\hline & $17-2011 \mathrm{i}$ & 1608,96 & 44,36 \\
\hline & $17-2012 \mathrm{i}$ & 1620,25 & 80,64 \\
\hline & $17-2013 \mathrm{i}$ & 1617,64 & 127,76 \\
\hline
\end{tabular}




\begin{tabular}{|c|c|c|c|}
\hline \multirow[t]{18}{*}{ sample } & lab number & $\begin{array}{l}\text { shoulder peak (D2) } \\
\text { wave number ( } F\end{array}$ & $\begin{array}{c}\text { shoulder peak (D2) } \\
\text { intensitiy (I }\end{array}$ \\
\hline & $17-2014 \mathrm{i}$ & 1617,64 & 82,26 \\
\hline & $17-2015 i$ & & \\
\hline & $17-20-1 \mathrm{i}$ & 1615,04 & 75,05 \\
\hline & $17-20-2 \mathrm{i}$ & & \\
\hline & $17-20-3 \mathrm{i}$ & & \\
\hline & $17-20-4 \mathrm{i}$ & & \\
\hline & $17-20-5 \mathrm{i}$ & 1614,17 & 88,57 \\
\hline & $17-20-6 \mathrm{i}$ & 1615,91 & 104,85 \\
\hline & $17-20-7 \mathrm{i}$ & 1612,43 & 137 \\
\hline & $17-20-8 \mathrm{i}$ & & \\
\hline & $17-20-9 \mathrm{i}$ & & \\
\hline & $17-20-1 \mathrm{~g}$ & 1617,64 & 36,69 \\
\hline & $17-20-2 \mathrm{~g}$ & & \\
\hline & $17-20-3 \mathrm{~g}$ & 1617,64 & 45,82 \\
\hline & $17-20-4 \mathrm{~g}$ & & \\
\hline & $17-20-5 \mathrm{~g}$ & & \\
\hline & $17-20-6 \mathrm{~g}$ & 1619,38 & 37,8 \\
\hline \multirow[t]{12}{*}{$18 / 08 / 00 / 02$} & $18-02-1 \mathrm{i}$ & 1617,41 & 99,11 \\
\hline & $18-02-2 \mathrm{i}$ & 1603,47 & 77,18 \\
\hline & $18-02-3 i$ & & \\
\hline & $18-02-4 \mathrm{i}$ & & \\
\hline & $18-02-5 \mathrm{i}$ & & \\
\hline & $18-02-6 \mathrm{i}$ & 1613,93 & 206,17 \\
\hline & $18-02-7 \mathrm{i}$ & 1612,19 & 81,55 \\
\hline & $18-02-1 \mathrm{~g}$ & 1610,44 & 177,1 \\
\hline & $18-02-2 \mathrm{~g}$ & 1618,28 & 70,43 \\
\hline & $18-02-3 \mathrm{~g}$ & & \\
\hline & $18-02-5 \mathrm{~g}$ & 1613,06 & 135,99 \\
\hline & $18-02-6 \mathrm{~g}$ & 1606,08 & 157,11 \\
\hline \multirow[t]{11}{*}{$3.10 .01 / 3$} & $3-013-1 \mathrm{i}$ & 1619,15 & 89,97 \\
\hline & $3-013-2 \mathrm{i}$ & 1615,67 & 45,58 \\
\hline & $3-013-3 i$ & 1613,06 & 33,14 \\
\hline & $3-013-4 i$ & 1618,28 & 153,61 \\
\hline & $3-013-5 i$ & & \\
\hline & $3-013-6 i$ & & \\
\hline & $3-013-7 i$ & 1617,41 & 87,42 \\
\hline & $3-013-1 \mathrm{~g}$ & & \\
\hline & $3-013-2 \mathrm{~g}$ & 1611,32 & 102,02 \\
\hline & $3-013-3 g$ & 1614,8 & 123,31 \\
\hline & $3-013-4 \mathrm{~g}$ & & \\
\hline \multirow[t]{6}{*}{$3.10 .01 / 4$} & $3-01410 \mathrm{i}$ & 1601,99 & 80,8 \\
\hline & 3-014-1i & 1601,12 & 48,45 \\
\hline & 3-014-2i & 1618,51 & 47,97 \\
\hline & $3-014-3 i$ & & \\
\hline & $3-014-4 i$ & & \\
\hline & $3-014-5 \mathrm{i}$ & 1610,69 & 74,55 \\
\hline
\end{tabular}




\begin{tabular}{|c|c|c|c|}
\hline sample & lab number & $\begin{array}{c}\text { shoulder peak (D2) } \\
\text { wave number (F }\end{array}$ & $\begin{array}{c}\text { shoulder peak (D2) } \\
\text { intensitiy (I }\end{array}$ \\
\hline & $3-014-6 \mathrm{i}$ & 1610,69 & 75,59 \\
\hline & $3-014-7 \mathrm{i}$ & 1615,91 & 59,94 \\
\hline & $3-014-8 \mathrm{i}$ & 1618,51 & \\
\hline & $3-014-9 \mathrm{i}$ & & \\
\hline & $3-014-1 \mathrm{~g}$ & & \\
\hline & $3-014-2 \mathrm{~g}$ & & \\
\hline & $3-014-3 \mathrm{~g}$ & & 45,49 \\
\hline & $3-014-4 \mathrm{~g}$ & & \\
\hline & $3-014-5 \mathrm{~g}$ & & \\
\hline Elim Spatkristall & Elim 01 & 1603,73 & \\
\hline & Elim 02 & 1620,25 & \\
\hline & Rad 01 & & \\
\hline & Rad 02 & & \\
\hline & Rad 03 & & \\
\hline & Rad 04 & & \\
\hline
\end{tabular}




\begin{tabular}{|c|c|c|c|c|}
\hline sample & lab number & $\begin{array}{c}\text { dissorderd peak } \\
\text { (D1) } \\
\text { measurement } \\
\text { wave number (F }\end{array}$ & $\begin{array}{c}\text { dissorderd peak } \\
\text { (D1) } \\
\text { measurement } \\
\text { intensity (I }\end{array}$ & $\begin{array}{c}\text { dissorderd peak } \\
\text { (D1) } \\
\text { measurement } \\
\text { f. width at half } \\
\text { max. (W) }\end{array}$ \\
\hline \multirow[t]{10}{*}{$12 / 08 / 00 / 02$} & $12-02-2 \mathrm{i}$ & 1339,81 & 224,18 & 41,19 \\
\hline & $12-02-3 \mathrm{i}$ & 1332,47 & 74,01 & 43,98 \\
\hline & $12-02-4 \mathrm{i}$ & 1339,81 & 59,52 & 30,2 \\
\hline & $12-02-5 i$ & 1324,22 & 312,13 & 45,87 \\
\hline & $12-02-6 \mathrm{i}$ & 1329,73 & 161,51 & 38,54 \\
\hline & $12-02-7 \mathrm{i}$ & 1335,23 & 90,36 & 44 \\
\hline & $12-02-1 \mathrm{~g}$ & 1340,72 & 155,92 & 41,21 \\
\hline & $12-02-2 \mathrm{~g}$ & 1338,89 & 115,33 & 41,23 \\
\hline & $12-02-3 g$ & 1334,31 & 158,47 & 33,94 \\
\hline & $12-02-4 g$ & 1339,81 & 190,98 & 48,55 \\
\hline \multirow[t]{8}{*}{$12 / 08 / 00 / 03$} & $12-03-2 \mathrm{i}$ & 1335,23 & 71 & 32,06 \\
\hline & $12-03-3 i$ & 1342,55 & 43,67 & 47,62 \\
\hline & $12-03-4 \mathrm{i}$ & 1334,31 & 171,82 & 41,25 \\
\hline & $12-03-5 i$ & 1333,39 & 137 & 43,11 \\
\hline & $12-03-1 \mathrm{~g}$ & 1337,97 & 80,63 & 40,33 \\
\hline & $12-03-2 \mathrm{~g}$ & 1328,81 & 77,9 & 44,04 \\
\hline & $12-03-3 g$ & 1341,64 & 43,59 & 32,98 \\
\hline & $12-03-4 g$ & 1336,14 & 168,87 & 45,79 \\
\hline \multirow[t]{11}{*}{$15 / 08 / 00 / 01$} & $15-01-1 \mathrm{i}$ & 1331,56 & 128,34 & 45,83 \\
\hline & $15-01-2 \mathrm{i}$ & 1330,46 & 127,37 & 36,67 \\
\hline & $15-01-3 i$ & 1329,54 & 179,72 & 45,81 \\
\hline & $15-01-4 i$ & 1327,71 & 233 & 45,82 \\
\hline & $15-01-5 i$ & & & \\
\hline & $15-01-6 \mathrm{i}$ & 1335,04 & 186,04 & 46,7 \\
\hline & $15-01-7 i$ & 1336,87 & 359,99 & 75,91 \\
\hline & $15-01-8 \mathrm{i}$ & 1323,13 & 79,16 & 36,69 \\
\hline & $15-01-1 \mathrm{~g}$ & 1338,7 & 57,34 & 33,84 \\
\hline & $15-01-2 \mathrm{~g}$ & 1335,04 & 263,26 & 45,77 \\
\hline & $15-01-3 g$ & 1337,78 & 46,85 & \\
\hline \multirow[t]{14}{*}{$17 / 08 / 00 / 11$} & $17-11-1 \mathrm{i}$ & 1339,61 & 69,44 & 31,09 \\
\hline & $17-11-2 \mathrm{i}$ & 1333,21 & 472,09 & 51,32 \\
\hline & $17-11-3 i$ & 1337,78 & 100,44 & 43,04 \\
\hline & $17-11-4 \mathrm{i}$ & 1321,38 & 56,78 & 22,89 \\
\hline & $17-11-5 i$ & 1328,63 & 89,07 & 36,61 \\
\hline & $17-11-6 \mathrm{i}$ & 1326,8 & 164,51 & 43,97 \\
\hline & $17-11-7 \mathrm{i}$ & 1331,38 & 76,13 & 37,54 \\
\hline & $17-11-1 \mathrm{~g}$ & 1328,63 & 565,09 & 49,49 \\
\hline & $17-11-2 \mathrm{~g}$ & 1331,38 & 420,72 & 43,07 \\
\hline & $17-11-3 g$ & 1330,46 & 376,3 & 44,85 \\
\hline & $17-11-4 \mathrm{~g}$ & 1331,38 & 114,5 & 37,54 \\
\hline & $17-11-5 \mathrm{~g}$ & 1331,38 & 228,53 & 46,7 \\
\hline & $17-11-6 \mathrm{~g}$ & 1330,46 & 132,32 & 43,94 \\
\hline & $17-11-7 \mathrm{~g}$ & 1327,71 & 511 & 43,04 \\
\hline $17 / 08 / 00 / 12$ & $17-12-1 \mathrm{i}$ & 1323,13 & 188,37 & 43,09 \\
\hline
\end{tabular}




\begin{tabular}{|c|c|c|c|c|}
\hline \multirow[t]{9}{*}{ sample } & lab number & $\begin{array}{c}\text { dissorderd peak } \\
\text { (D1) } \\
\text { measurement } \\
\text { wave number (F }\end{array}$ & $\begin{array}{c}\text { dissorderd peak } \\
\text { (D1) } \\
\text { measurement } \\
\text { intensity (I }\end{array}$ & $\begin{array}{c}\text { dissorderd peak } \\
\text { (D1) } \\
\text { measurement } \\
\text { f. width at half } \\
\text { max. (W) } \\
\end{array}$ \\
\hline & $17-12-2 \mathrm{i}$ & 1334,12 & 243,06 & 50,37 \\
\hline & $17-12-3 i$ & 1327,71 & 152,53 & 43,99 \\
\hline & $17-12-4 i$ & 1324,05 & 180,78 & 47,67 \\
\hline & $17-12-5 i$ & 1316,71 & 167,92 & 42,17 \\
\hline & $17-12-6 i$ & 1323,13 & 172,89 & 46,76 \\
\hline & $17-12-7 i$ & 1329,54 & 160,42 & 43,09 \\
\hline & $17-12-2 \mathrm{~g}$ & 1325,88 & 202,5 & 48,58 \\
\hline & $17-12-3 \mathrm{~g}$ & 1329,54 & 210,74 & 41,22 \\
\hline \multirow[t]{12}{*}{$17 / 08 / 00 / 15$} & $17-15-1 \mathrm{i}$ & 1326,8 & 124,51 & 42,11 \\
\hline & $17-15-2 \mathrm{i}$ & 1333,21 & 113,72 & 41,22 \\
\hline & $17-15-3 i$ & 1330,46 & 252,93 & 43,94 \\
\hline & $17-15-4 i$ & 1333,21 & 76,92 & 48,52 \\
\hline & $17-15-5 i$ & 1326,8 & 138,79 & 43,97 \\
\hline & $17-15-6 \mathrm{i}$ & 1331,38 & 173,24 & 40,3 \\
\hline & $17-15-7 i$ & 1334,12 & 157,06 & 48,57 \\
\hline & $17-15-1 \mathrm{~g}$ & 1333,21 & 141,78 & 36,62 \\
\hline & $17-15-2 \mathrm{~g}$ & 1335,04 & 83,25 & 39,34 \\
\hline & $17-15-3 \mathrm{~g}$ & 1330,46 & 117,06 & 31,14 \\
\hline & $17-15-4 \mathrm{~g}$ & 1341,44 & 102,87 & 15,56 \\
\hline & $17-15-5 \mathrm{~g}$ & 1327,71 & 149,23 & \\
\hline \multirow[t]{13}{*}{$17 / 08 / 00 / 16$} & $17-16-1 \mathrm{i}$ & 1322,21 & 72,55 & 33,93 \\
\hline & $17-16-2 \mathrm{i}$ & 1335,95 & 80,96 & 42,13 \\
\hline & $17-16-3 i$ & 1333,21 & 144,05 & 43,95 \\
\hline & $17-16-4 \mathrm{i}$ & 1329,54 & 149,49 & 42,16 \\
\hline & $17-16-5 i$ & 1330,46 & 213,06 & 46,71 \\
\hline & $17-16-6 \mathrm{i}$ & 1341,44 & 252,2 & 43,88 \\
\hline & $17-16-7 i$ & & & \\
\hline & $17-16-1 \mathrm{~g}$ & 1332,29 & 536,38 & 54,04 \\
\hline & $17-16-2 \mathrm{~g}$ & 1331,56 & 170,9 & 47,67 \\
\hline & $17-16-3 \mathrm{~g}$ & 1330,64 & 458,2 & 53,19 \\
\hline & $17-16-4 \mathrm{~g}$ & 1331,56 & 768,59 & 54,12 \\
\hline & $17-16-5 \mathrm{~g}$ & 1331,56 & 94,46 & 44,93 \\
\hline & $17-16-6 \mathrm{~g}$ & 1333,39 & 90,88 & 39,42 \\
\hline \multirow[t]{11}{*}{$17 / 08 / 00 / 19$} & $17-19-1 \mathrm{i}$ & 1324,96 & 218,39 & 44,9 \\
\hline & $17-19-2 i$ & 1325,88 & 135,98 & 34,83 \\
\hline & $17-19-3 i$ & 1335,95 & 327,45 & 49,46 \\
\hline & $17-19-4 i$ & 1331,38 & 269,31 & 45,79 \\
\hline & $17-19-5 \mathrm{i}$ & 1333,21 & 262,7 & 46,73 \\
\hline & $17-19-6 i$ & 1323,13 & 461,78 & 54,99 \\
\hline & $17-19-1 \mathrm{~g}$ & 1332,29 & 115,2 & 43,02 \\
\hline & $17-19-2 \mathrm{~g}$ & 1337,78 & 113,59 & 40,28 \\
\hline & $17-19-3 \mathrm{~g}$ & 1335,95 & 195,92 & 47,61 \\
\hline & $17-19-4 \mathrm{~g}$ & 1332,29 & 43,7 & 35,68 \\
\hline & $17-19-5 \mathrm{~g}$ & 1328,63 & 143,6 & 40,33 \\
\hline
\end{tabular}




\begin{tabular}{|c|c|c|c|c|}
\hline sample & lab number & $\begin{array}{c}\text { dissorderd peak } \\
\text { (D1) } \\
\text { measurement } \\
\text { wave number (F }\end{array}$ & $\begin{array}{c}\text { dissorderd peak } \\
\text { (D1) } \\
\text { measurement } \\
\text { intensity (I }\end{array}$ & $\begin{array}{c}\text { dissorderd peak } \\
\text { (D1) } \\
\text { measurement } \\
\text { f. width at half } \\
\text { max. (W) } \\
\end{array}$ \\
\hline \multirow[t]{21}{*}{$17 / 08 / 00 / 20$} & $17-2010 \mathrm{i}$ & 1337,78 & 73,11 & 38,44 \\
\hline & $17-2011 i$ & 1329,54 & 20,33 & \\
\hline & $17-2012 \mathrm{i}$ & 1337,78 & 186,86 & 42,08 \\
\hline & $17-2013 i$ & 1335,04 & 219,79 & 40,25 \\
\hline & $17-2014 i$ & 1338,7 & 105,81 & 35,68 \\
\hline & $17-2015 \mathrm{i}$ & 1337,78 & 115,82 & 36,62 \\
\hline & $17-20-1 i$ & 1331,38 & 144,6 & 37,52 \\
\hline & $17-20-2 \mathrm{i}$ & 1346,92 & 75,01 & 19,18 \\
\hline & $17-20-3 \mathrm{i}$ & 1336,87 & 102,39 & 40,25 \\
\hline & $17-20-4 \mathrm{i}$ & 1346,01 & 39,21 & 13,7 \\
\hline & $17-20-5 i$ & 1329,54 & 180,27 & 39,35 \\
\hline & $17-20-6 \mathrm{i}$ & 1334,12 & 200,1 & 40,27 \\
\hline & $17-20-7 \mathrm{i}$ & 1335,95 & 216,49 & 41,18 \\
\hline & $17-20-8 \mathrm{i}$ & & & \\
\hline & $17-20-9 \mathrm{i}$ & 1346,92 & 46,01 & \\
\hline & $17-20-1 \mathrm{~g}$ & 1346,01 & 40,75 & \\
\hline & $17-20-2 \mathrm{~g}$ & & & \\
\hline & $17-20-3 \mathrm{~g}$ & 1345,09 & 62,44 & 34,75 \\
\hline & $17-20-4 \mathrm{~g}$ & & & \\
\hline & $17-20-5 \mathrm{~g}$ & 1348,74 & 55,76 & 14,6 \\
\hline & $17-20-6 \mathrm{~g}$ & 1344,18 & 49 & 26,49 \\
\hline \multirow[t]{12}{*}{$18 / 08 / 00 / 02$} & $18-02-1 \mathrm{i}$ & 1337,97 & 169 & 40,3 \\
\hline & $18-02-2 i$ & 1326,98 & 96,88 & 36,7 \\
\hline & $18-02-3 \mathrm{i}$ & 1321,47 & 71,94 & 36,71 \\
\hline & $18-02-4 \mathrm{i}$ & 1333,39 & 228,89 & 44,92 \\
\hline & $18-02-5 \mathrm{i}$ & 1332,47 & 74,01 & 45,83 \\
\hline & $18-02-6 i$ & 1334,31 & 572,55 & 46,74 \\
\hline & $18-02-7 \mathrm{i}$ & 1332,47 & 174,71 & 39,42 \\
\hline & $18-02-1 \mathrm{~g}$ & 1336,14 & 359,12 & 50,39 \\
\hline & $18-02-2 \mathrm{~g}$ & 1344,38 & 89,04 & 41,21 \\
\hline & $18-02-3 g$ & 1340,72 & 36,58 & \\
\hline & $18-02-5 \mathrm{~g}$ & 1334,31 & 230,09 & 44,9 \\
\hline & $18-02-6 \mathrm{~g}$ & 1328,81 & 283,93 & 44,04 \\
\hline \multirow[t]{11}{*}{$3.10 .01 / 3$} & 3-013-1i & 1338,89 & 132 & 37,55 \\
\hline & $3-013-2 i$ & 1338,89 & 23 & \\
\hline & $3-013-3 i$ & 1320,55 & 34,71 & \\
\hline & $3-013-4 i$ & 1333,39 & 313,83 & 48,57 \\
\hline & $3-013-5 i$ & & & \\
\hline & $3-013-6 i$ & 1326,06 & 161,03 & 50,49 \\
\hline & $3-013-7 i$ & 1337,97 & 170,04 & 46,74 \\
\hline & $3-013-1 \mathrm{~g}$ & 1336,14 & 70,56 & \\
\hline & $3-013-2 \mathrm{~g}$ & 1337,06 & 169,77 & 42,15 \\
\hline & $3-013-3 \mathrm{~g}$ & 1333,39 & 236,9 & 43,97 \\
\hline & $3-013-4 \mathrm{~g}$ & & & \\
\hline
\end{tabular}




\begin{tabular}{|c|c|c|c|c|}
\hline sample & lab number & $\begin{array}{c}\text { dissorderd peak } \\
\text { (D1) } \\
\text { measurement } \\
\text { wave number (F }\end{array}$ & $\begin{array}{c}\text { dissorderd peak } \\
\text { (D1) } \\
\text { measurement } \\
\text { intensity (I }\end{array}$ & $\begin{array}{c}\text { dissorderd peak } \\
\text { (D1) } \\
\text { measurement } \\
\text { f. width at half } \\
\text { max. (W) }\end{array}$ \\
\hline \multirow[t]{15}{*}{$3.10 .01 / 4$} & 3-01410i & 1328,63 & 127,56 & 38,48 \\
\hline & $3-014-1 i$ & 1335,04 & 87,09 & 41,18 \\
\hline & $3-014-2 i$ & 1339,61 & 116,53 & 36,61 \\
\hline & $3-014-3 i$ & & & \\
\hline & $3-014-4 \mathrm{i}$ & & & \\
\hline & $3-014-5 i$ & 1336,87 & 157,6 & 50,34 \\
\hline & $3-014-6 i$ & 1330,46 & 175,57 & 50,34 \\
\hline & $3-014-7 i$ & 1335,04 & 392,39 & 46,69 \\
\hline & $3-014-8 \mathrm{i}$ & 1330,46 & 183,17 & 46,68 \\
\hline & 3-014-9i & 1330,46 & 38,48 & \\
\hline & $3-014-1 \mathrm{~g}$ & 1337,97 & 61,68 & 29,31 \\
\hline & $3-014-2 g$ & & & \\
\hline & $3-014-3 g$ & 1337,78 & 90,98 & 41,2 \\
\hline & $3-014-4 g$ & 1332,47 & 60,54 & 35,76 \\
\hline & $3-014-5 g$ & 1341,64 & 77 & 42,15 \\
\hline \multirow[t]{2}{*}{ Elim Spatkristall } & Elim 01 & 1336,87 & 105,46 & 44,87 \\
\hline & Elim 02 & 1329,54 & 96,4 & 28,36 \\
\hline \multirow[t]{4}{*}{ Omaruru Spatkristall } & $\operatorname{Rad} 01$ & & & \\
\hline & $\operatorname{Rad} 02$ & 1332,29 & 53,74 & \\
\hline & $\operatorname{Rad} 03$ & 1324,96 & 178,1 & 2,75 \\
\hline & $\operatorname{Rad} 04$ & & & \\
\hline
\end{tabular}




\begin{tabular}{|c|c|c|c|c|}
\hline sample & lab number & $\begin{array}{l}\text { dissorderd peak } \\
\text { (D1) } \\
\text { function of meas. } \\
\text { wave number ( } F\end{array}$ & $\begin{array}{c}\text { dissorderd peak } \\
\text { (D1) } \\
\text { function of meas. } \\
\text { Intensity (I }\end{array}$ & $\begin{array}{l}\text { dissorderd peak } \\
\text { (D1) } \\
\text { function of meas. } \\
\text { F. width at half } \\
\text { max. (W) }\end{array}$ \\
\hline \multirow[t]{10}{*}{$12 / 08 / 00 / 02$} & $12-02-2 \mathrm{i}$ & 1339,87 & 205,35 & 41,0948 \\
\hline & $12-02-3 i$ & 1332,7 & 63,6505 & 49,9216 \\
\hline & $12-02-4 i$ & 1335,48 & 53,6588 & 43,3199 \\
\hline & $12-02-5 i$ & 1326,53 & 265,075 & 60,0061 \\
\hline & $12-02-6 \mathrm{i}$ & 1329,78 & 147,265 & 47,6603 \\
\hline & $12-02-7 \mathrm{i}$ & 1331,91 & 81,9594 & 45,1889 \\
\hline & $12-02-1 \mathrm{~g}$ & 1337,24 & 141,97 & 40,794 \\
\hline & $12-02-2 \mathrm{~g}$ & 1335,01 & 97,35 & 39,3494 \\
\hline & $12-02-3 \mathrm{~g}$ & 1331,53 & 130,263 & 41,9071 \\
\hline & $12-02-4 \mathrm{~g}$ & 1336,42 & 183,071 & 44,438 \\
\hline \multirow[t]{8}{*}{$12 / 08 / 00 / 03$} & $12-03-2 \mathrm{i}$ & 1335,69 & 58,6312 & 37,8724 \\
\hline & $12-03-3 i$ & & & \\
\hline & $12-03-4 \mathrm{i}$ & 1330,94 & 159,339 & 37,7999 \\
\hline & $12-03-5 i$ & 1328,26 & 126,468 & 37,251 \\
\hline & $12-03-1 \mathrm{~g}$ & 1331,56 & 64,3954 & 52,3581 \\
\hline & $12-03-2 \mathrm{~g}$ & 1323,22 & 55,8303 & 113,451 \\
\hline & $12-03-3 \mathrm{~g}$ & 1334,04 & 39,1355 & 36,2262 \\
\hline & $12-03-4 \mathrm{~g}$ & 1337,33 & 154,6 & 45,0413 \\
\hline \multirow[t]{11}{*}{$15 / 08 / 00 / 01$} & $15-01-1 \mathrm{i}$ & 1332,01 & 113,009 & 45,0875 \\
\hline & $15-01-2 \mathrm{i}$ & 1324,93 & 104,916 & 44,7885 \\
\hline & $15-01-3 \mathrm{i}$ & 1326,6 & 164,809 & 43,437 \\
\hline & $15-01-4 \mathrm{i}$ & 1327,35 & 211,992 & 41,4662 \\
\hline & $15-01-5 i$ & 133,23 & 23,0564 & 58,2942 \\
\hline & $15-01-6 \mathrm{i}$ & 1332,01 & 179,115 & 44,2934 \\
\hline & $15-01-7 \mathrm{i}$ & 1334,95 & 312,083 & 82,658 \\
\hline & $15-01-8 \mathrm{i}$ & 1322,32 & 71,9885 & 44,57 \\
\hline & $15-01-1 \mathrm{~g}$ & 1335,36 & 47,5938 & 51,1475 \\
\hline & $15-01-2 \mathrm{~g}$ & 1333,58 & 251,2 & 42,6133 \\
\hline & $15-01-3 \mathrm{~g}$ & 1332,39 & 35,889 & 58,9871 \\
\hline \multirow[t]{14}{*}{$17 / 08 / 00 / 11$} & $17-11-1 \mathrm{i}$ & 1333,66 & 58,6302 & 55,8544 \\
\hline & $17-11-2 \mathrm{i}$ & 1326,47 & 453,967 & 46,6628 \\
\hline & $17-11-3 \mathrm{i}$ & 1332,05 & 90,8531 & 46,933 \\
\hline & $17-11-4 \mathrm{i}$ & 1329,87 & 45,6286 & 42,25 \\
\hline & $17-11-5 \mathrm{i}$ & 1329,14 & 77,0558 & 47,9653 \\
\hline & $17-11-6 \mathrm{i}$ & 1327,42 & 151,572 & 44,6187 \\
\hline & $17-11-7 \mathrm{i}$ & 1329,2 & 62,6733 & 40,7432 \\
\hline & $17-11-1 \mathrm{~g}$ & 1325,65 & 532,274 & 47,3183 \\
\hline & $17-11-2 \mathrm{~g}$ & 1327,21 & 392,237 & 42,7238 \\
\hline & $17-11-3 \mathrm{~g}$ & 1330,8 & 356,305 & 44,8143 \\
\hline & $17-11-4 \mathrm{~g}$ & 1329,52 & 99,4069 & 40,404 \\
\hline & $17-11-5 \mathrm{~g}$ & 1331,17 & 217,712 & 43,144 \\
\hline & $17-11-6 \mathrm{~g}$ & 1331,4 & 122,866 & 43,42 \\
\hline & $17-11-7 \mathrm{~g}$ & 1328,08 & 473,295 & 43,1196 \\
\hline $17 / 08 / 00 / 12$ & $17-12-1 \mathrm{i}$ & 1322,71 & 166,924 & 45,7517 \\
\hline
\end{tabular}




\begin{tabular}{|c|c|c|c|c|}
\hline \multirow[t]{9}{*}{ sample } & lab number & $\begin{array}{l}\text { dissorderd peak } \\
\text { (D1) } \\
\text { function of meas. } \\
\text { wave number (F }\end{array}$ & $\begin{array}{l}\text { dissorderd peak } \\
\text { (D1) } \\
\text { function of meas. } \\
\text { Intensity (I }\end{array}$ & $\begin{array}{l}\text { dissorderd peak } \\
\text { (D1) } \\
\text { function of meas. } \\
\text { F. width at half } \\
\text { max. (W) }\end{array}$ \\
\hline & $17-12-2 i$ & 1329,69 & 227,06 & 47,317 \\
\hline & $17-12-3 i$ & 1322,21 & 132,304 & 44,3789 \\
\hline & $17-12-4 i$ & 1324,07 & 168,714 & 41,2751 \\
\hline & $17-12-5 i$ & 1321,83 & 147,911 & 45,6466 \\
\hline & $17-12-6 \mathrm{i}$ & 1324,89 & 155,087 & 44,5394 \\
\hline & $17-12-7 \mathrm{i}$ & 1324,23 & 151,9 & 46,1856 \\
\hline & $17-12-2 \mathrm{~g}$ & 1324,35 & 193,224 & 47,1725 \\
\hline & $17-12-3 \mathrm{~g}$ & 1326,62 & 190,801 & 49,87 \\
\hline \multirow[t]{12}{*}{$17 / 08 / 00 / 15$} & $17-15-1 \mathrm{i}$ & 1331,08 & 112,607 & 40,791 \\
\hline & $17-15-2 \mathrm{i}$ & 1330,13 & 106,684 & 47,434 \\
\hline & $17-15-3 \mathrm{i}$ & 1331,75 & 241,326 & 42,6803 \\
\hline & $17-15-4 \mathrm{i}$ & 1332,02 & 70,7673 & 56,1229 \\
\hline & $17-15-5 i$ & 1325,97 & 128,099 & 48,124 \\
\hline & $17-15-6 \mathrm{i}$ & 1326,78 & 155,17 & 46,6375 \\
\hline & $17-15-7 \mathrm{i}$ & 1325,37 & 147,449 & 45,9974 \\
\hline & $17-15-1 \mathrm{~g}$ & 1333,14 & 125,548 & 46,9232 \\
\hline & $17-15-2 \mathrm{~g}$ & 1335,96 & 72,7349 & 51,0356 \\
\hline & $17-15-3 g$ & 1325,68 & 97,593 & 43,5395 \\
\hline & $17-15-4 \mathrm{~g}$ & 1335,69 & 73,8345 & 47,8316 \\
\hline & $17-15-5 \mathrm{~g}$ & & & \\
\hline \multirow[t]{13}{*}{$17 / 08 / 00 / 16$} & $17-16-1 \mathrm{i}$ & 1321,58 & 56,5026 & 41,6044 \\
\hline & $17-16-2 \mathrm{i}$ & 1329,34 & 63,6368 & 64,8709 \\
\hline & $17-16-3 \mathrm{i}$ & 1330,36 & 126,936 & 44,5031 \\
\hline & $17-16-4 i$ & 1325,7 & 134,896 & 43,5138 \\
\hline & $17-16-5 i$ & 1329,41 & 202,204 & 45,2838 \\
\hline & $17-16-6 i$ & 1339,55 & 246,109 & 40,4072 \\
\hline & $17-16-7 \mathrm{i}$ & & & \\
\hline & $17-16-1 \mathrm{~g}$ & 1329,87 & 522,875 & 49,4426 \\
\hline & $17-16-2 \mathrm{~g}$ & 1331,26 & 158,95 & 56,4573 \\
\hline & $17-16-3 \mathrm{~g}$ & 1328,73 & 441,88 & 53,0644 \\
\hline & $17-16-4 \mathrm{~g}$ & 1327,88 & 739,196 & 51,187 \\
\hline & $17-16-5 \mathrm{~g}$ & 1330,07 & 81,4696 & 52,1528 \\
\hline & $17-16-6 \mathrm{~g}$ & 1330,79 & 74,4376 & 62,7218 \\
\hline \multirow[t]{11}{*}{$17 / 08 / 00 / 19$} & $17-19-1 \mathrm{i}$ & 1325,94 & 194,989 & 44,1855 \\
\hline & $17-19-2 \mathrm{i}$ & 1325,21 & 119,789 & 42,0041 \\
\hline & $17-19-3 i$ & 1329,83 & 313,258 & 46,1914 \\
\hline & $17-19-4 i$ & 1329,4 & 238,52 & 43,6325 \\
\hline & $17-19-5 \mathrm{i}$ & 1326,98 & 247,321 & 43,7256 \\
\hline & $17-19-6 \mathrm{i}$ & 1325,39 & 448,75 & 50,7063 \\
\hline & $17-19-1 \mathrm{~g}$ & 1333,01 & 103,198 & 47,5681 \\
\hline & $17-19-2 \mathrm{~g}$ & 1332,68 & 96,7854 & 41,0647 \\
\hline & $17-19-3 \mathrm{~g}$ & 1332,01 & 183,506 & 48,7009 \\
\hline & $17-19-4 \mathrm{~g}$ & 1324,61 & 28,6701 & 104,759 \\
\hline & $17-19-5 \mathrm{~g}$ & 1321,9 & 122,856 & 70,4486 \\
\hline
\end{tabular}




\begin{tabular}{|c|c|c|c|c|}
\hline sample & lab number & $\begin{array}{l}\text { dissorderd peak } \\
\text { (D1) } \\
\text { function of meas. } \\
\text { wave number ( } F\end{array}$ & $\begin{array}{c}\text { dissorderd peak } \\
\text { (D1) } \\
\text { function of meas. } \\
\text { Intensity (I }\end{array}$ & $\begin{array}{l}\text { dissorderd peak } \\
\text { (D1) } \\
\text { function of meas. } \\
\text { F. width at half } \\
\text { max. (W) }\end{array}$ \\
\hline \multirow[t]{21}{*}{$17 / 08 / 00 / 20$} & $17-2010 \mathrm{i}$ & 1334,02 & 62,153 & 50,7895 \\
\hline & $17-2011 \mathrm{i}$ & & & \\
\hline & $17-2012 \mathrm{i}$ & 1336,62 & 178,59 & 42,8265 \\
\hline & $17-2013 \mathrm{i}$ & 1336,42 & 207,194 & 41,7477 \\
\hline & $17-2014 \mathrm{i}$ & 1335,22 & 80,6838 & 64,8443 \\
\hline & $17-2015 \mathrm{i}$ & 1332,84 & 87,7097 & 82,2106 \\
\hline & $17-20-1 \mathrm{i}$ & 1333,47 & 117,696 & 40,2888 \\
\hline & $17-20-2 \mathrm{i}$ & 1342,83 & 49,3757 & 44,1207 \\
\hline & $17-20-3 \mathrm{i}$ & 1333,39 & 91,9725 & 58,521 \\
\hline & $17-20-4 \mathrm{i}$ & 1340,74 & 23,2153 & 39,5124 \\
\hline & $17-20-5 \mathrm{i}$ & 1332,76 & 166,114 & 39,4883 \\
\hline & $17-20-6 \mathrm{i}$ & 1334,39 & 197,989 & 40,0849 \\
\hline & $17-20-7 \mathrm{i}$ & 1333,5 & 208,294 & 38,7959 \\
\hline & $17-20-8 \mathrm{i}$ & & & \\
\hline & $17-20-9 \mathrm{i}$ & 1338,75 & 28,4746 & 93,5214 \\
\hline & $17-20-1 \mathrm{~g}$ & 1331,54 & 50,2055 & 62,9814 \\
\hline & $17-20-2 \mathrm{~g}$ & & & \\
\hline & $17-20-3 \mathrm{~g}$ & 1337,78 & 55,3621 & 40,9091 \\
\hline & $17-20-4 \mathrm{~g}$ & & & \\
\hline & $17-20-5 \mathrm{~g}$ & 1330,03 & 51,1333 & 68,082 \\
\hline & $17-20-6 \mathrm{~g}$ & 1338,1 & 36,0354 & 75,7599 \\
\hline \multirow[t]{12}{*}{$18 / 08 / 00 / 02$} & $18-02-1 \mathrm{i}$ & 1335,64 & 154,881 & 39,4901 \\
\hline & $18-02-2 i$ & 1324,86 & 85,3399 & 35,2597 \\
\hline & $18-02-3 i$ & 1323,91 & 66,8553 & 40,1324 \\
\hline & $18-02-4 \mathrm{i}$ & 1330,54 & 210,327 & 43,5993 \\
\hline & $18-02-5 i$ & 1332,37 & 61,2759 & 68,2714 \\
\hline & $18-02-6 i$ & 1332,32 & 544,528 & 43,5378 \\
\hline & $18-02-7 \mathrm{i}$ & 1329,8 & 152,677 & 40,3124 \\
\hline & $18-02-1 \mathrm{~g}$ & 1334,29 & 338,249 & 47,8929 \\
\hline & $18-02-2 \mathrm{~g}$ & 1335,85 & 81,1213 & 42,3511 \\
\hline & $18-02-3 \mathrm{~g}$ & 1329,74 & 20,1215 & 142,871 \\
\hline & $18-02-5 \mathrm{~g}$ & 1331,87 & 200,768 & 59,1313 \\
\hline & $18-02-6 \mathrm{~g}$ & 1327,93 & 251,836 & 47,0394 \\
\hline \multirow[t]{11}{*}{$3.10 .01 / 3$} & $3-013-1 i$ & 1336,14 & 115,056 & 38,5804 \\
\hline & $3-013-2 \mathrm{i}$ & 1339,53 & 14,0829 & 59,809 \\
\hline & $3-013-3 i$ & 1327,14 & 23,998 & 40,0794 \\
\hline & $3-013-4 i$ & 1332,93 & 305,292 & 45,646 \\
\hline & $3-013-5 i$ & & & \\
\hline & 3-013-6i & 1322,35 & 143,295 & 54,902 \\
\hline & $3-013-7 i$ & 1335,12 & 165,442 & 44,1698 \\
\hline & $3-013-1 \mathrm{~g}$ & 1333,63 & 52,7411 & 48,0881 \\
\hline & $3-013-2 \mathrm{~g}$ & 1333,46 & 160,311 & 41,9454 \\
\hline & $3-013-3 \mathrm{~g}$ & 1335,34 & 220,21 & 41,7939 \\
\hline & $3-013-4 \mathrm{~g}$ & & & \\
\hline
\end{tabular}




\begin{tabular}{|c|c|c|c|c|}
\hline sample & lab number & $\begin{array}{l}\text { dissorderd peak } \\
\text { (D1) } \\
\text { function of meas. } \\
\text { wave number (F }\end{array}$ & $\begin{array}{l}\text { dissorderd peak } \\
\text { (D1) } \\
\text { function of meas. } \\
\text { Intensity (I }\end{array}$ & $\begin{array}{l}\text { dissorderd peak } \\
\text { (D1) } \\
\text { function of meas. } \\
\text { F. width at half } \\
\text { max. (W) }\end{array}$ \\
\hline \multirow[t]{15}{*}{$3.10 .01 / 4$} & 3-01410i & 1326,24 & 106,783 & 44,4356 \\
\hline & $3-014-1 i$ & 1332,94 & 79,6503 & 46,088 \\
\hline & $3-014-2 i$ & 1333,89 & 98,358 & 41,2725 \\
\hline & $3-014-3 i$ & & & \\
\hline & $3-014-4 \mathrm{i}$ & & & \\
\hline & $3-014-5 i$ & 1332,61 & 147,585 & 57,4559 \\
\hline & $3-014-6 i$ & 1331,56 & 169,465 & 50,6634 \\
\hline & $3-014-7 i$ & 1333,15 & 384,729 & 42,577 \\
\hline & $3-014-8 \mathrm{i}$ & 1332,2 & 170,752 & 45,7596 \\
\hline & 3-014-9i & 1326,31 & 23,1992 & 68,4843 \\
\hline & $3-014-1 \mathrm{~g}$ & 1333,31 & 47,0691 & 49,487 \\
\hline & $3-014-2 g$ & & & \\
\hline & $3-014-3 g$ & 1332,98 & 84,7304 & 44,0079 \\
\hline & $3-014-4 g$ & 1330,75 & 49,7358 & 41,5414 \\
\hline & $3-014-5 g$ & 1334,24 & 71,6761 & 50,2227 \\
\hline \multirow[t]{2}{*}{ Elim Spatkristall } & Elim 01 & 1331,06 & 91,7932 & 56,3895 \\
\hline & Elim 02 & 1332,39 & 72,5575 & 52,4464 \\
\hline \multirow[t]{4}{*}{ Omaruru Spatkristall } & $\operatorname{Rad} 01$ & & & \\
\hline & Rad 02 & & & \\
\hline & $\operatorname{Rad} 03$ & & & \\
\hline & $\operatorname{Rad} 04$ & & & \\
\hline
\end{tabular}




\begin{tabular}{|c|c|c|c|c|}
\hline sample & lab number & D1/G intensity ratio & $\begin{array}{c}\text { graphite } \\
\text { crystallinity } \\
\text { own calculation } \\
\text { based on Tuinstra } \\
\text { \& Koenig } \\
\mathbf{L}_{\mathrm{a}}[\AA ̊] \\
\end{array}$ & $\begin{array}{c}\text { graphite } \\
\text { crystallinity } \\
\text { after Zheng \& Chen } \\
\mathrm{L}_{\mathrm{a}}[\AA]\end{array}$ \\
\hline \multirow[t]{10}{*}{$12 / 08 / 00 / 02$} & $12-02-2 \mathrm{i}$ & 0,544955151 & 79,18072888 & 80,74058924 \\
\hline & $12-02-3 i$ & 0,126039845 & 342,3516266 & 349,0959537 \\
\hline & $12-02-4 \mathrm{i}$ & 0,120778619 & 357,2647755 & 364,3028916 \\
\hline & $12-02-5 \mathrm{i}$ & 0,505397645 & 85,3782096 & 87,06016033 \\
\hline & $12-02-6 \mathrm{i}$ & 0,237982117 & 181,3159181 & 184,8878416 \\
\hline & $12-02-7 \mathrm{i}$ & 0,159009231 & 271,3675539 & 276,7134947 \\
\hline & $12-02-1 \mathrm{~g}$ & 0,207944271 & 207,5072609 & 211,5951539 \\
\hline & $12-02-2 \mathrm{~g}$ & 0,17916991 & 240,832548 & 245,5769492 \\
\hline & $12-02-3 g$ & 0,198971105 & 216,8653888 & 221,1376369 \\
\hline & $12-02-4 g$ & 0,394589538 & 109,3540043 & 111,5082782 \\
\hline \multirow[t]{8}{*}{$12 / 08 / 00 / 03$} & $12-03-2 \mathrm{i}$ & 0,132159409 & 326,4992371 & 332,9312721 \\
\hline & $12-03-3 i$ & 0 & & \\
\hline & $12-03-4 i$ & 0,245170493 & 175,9997527 & 179,4669478 \\
\hline & $12-03-5 i$ & 0,208769205 & 206,6873136 & 210,7590537 \\
\hline & $12-03-1 g$ & 0,155836167 & 276,8930151 & 282,3478075 \\
\hline & $12-03-2 \mathrm{~g}$ & 0,143873615 & 299,9156322 & 305,8239701 \\
\hline & $12-03-3 g$ & 0,075509031 & 571,4541119 & 582,7117579 \\
\hline & $12-03-4 g$ & 0,315729926 & 136,6672667 & 139,3596119 \\
\hline \multirow[t]{11}{*}{$15 / 08 / 00 / 01$} & $15-01-1 \mathrm{i}$ & 0,25647549 & 168,2419868 & 171,5563539 \\
\hline & $15-01-2 \mathrm{i}$ & 0,19413466 & 222,2681212 & 226,6468032 \\
\hline & $15-01-3 i$ & 0,259132833 & 166,5167072 & 169,7970863 \\
\hline & $15-01-4 i$ & 0,236508204 & 182,4458744 & 186,0400581 \\
\hline & $15-01-5 i$ & 0,048637064 & 887,1823845 & 904,6598775 \\
\hline & $15-01-6 i$ & 0,249605278 & 172,8727312 & 176,278324 \\
\hline & $15-01-7 i$ & 0,446156002 & 96,71492911 & 98,62021321 \\
\hline & $15-01-8 \mathrm{i}$ & 0,108124772 & 399,0754876 & 406,9372747 \\
\hline & $15-01-1 \mathrm{~g}$ & 0,101177726 & 426,4767329 & 434,8783245 \\
\hline & $15-01-2 \mathrm{~g}$ & 0,560569138 & 76,97524375 & 78,49165605 \\
\hline & $15-01-3 g$ & 0,071818649 & 600,8181224 & 612,6542395 \\
\hline \multirow[t]{14}{*}{$17 / 08 / 00 / 11$} & $17-11-1 \mathrm{i}$ & 0,482239531 & 89,47824316 & 91,24096455 \\
\hline & $17-11-2 \mathrm{i}$ & 0,574981666 & 75,04577726 & 76,52417907 \\
\hline & $17-11-3 i$ & 0,190533223 & 226,469407 & 230,9308543 \\
\hline & $17-11-4 i$ & 0,084394883 & 511,2862818 & 521,3586216 \\
\hline & $17-11-5 i$ & 0,139188882 & 310,0100063 & 316,1172034 \\
\hline & $17-11-6 i$ & 0,305701499 & 141,1505872 & 143,9312538 \\
\hline & $17-11-7 i$ & 0,142831781 & 302,1032558 & 308,05469 \\
\hline & $17-11-1 \mathrm{~g}$ & 0,817816141 & 52,7624045 & 53,80182387 \\
\hline & $17-11-2 \mathrm{~g}$ & 0,538459426 & 80,13592843 & 81,71460622 \\
\hline & $17-11-3 g$ & 0,573406418 & 75,25194127 & 76,73440451 \\
\hline & $17-11-4 \mathrm{~g}$ & 0,170108355 & 253,6615324 & 258,6586645 \\
\hline & $17-11-5 \mathrm{~g}$ & 0,421826017 & 102,2932308 & 104,3084074 \\
\hline & $17-11-6 \mathrm{~g}$ & 0,439735298 & 98,12709195 & 100,0601957 \\
\hline & $17-11-7 \mathrm{~g}$ & 0,598678668 & 72,07530242 & 73,49518588 \\
\hline
\end{tabular}




\begin{tabular}{|c|c|c|c|c|}
\hline sample & lab number & D1/G intensity ratio & $\begin{array}{c}\text { graphite } \\
\text { crystallinity } \\
\text { own calculation } \\
\text { based on Tuinstra } \\
\& \text { Koenig } \\
\left.\mathrm{L}_{\mathrm{a}}[\AA]\right] \\
\end{array}$ & $\begin{array}{c}\text { graphite } \\
\text { crystallinity } \\
\text { after Zheng \& Chen } \\
\mathrm{L}_{\mathrm{a}}[\AA]\end{array}$ \\
\hline \multirow[t]{9}{*}{$17 / 08 / 00 / 12$} & $17-12-1 \mathrm{i}$ & 0,228182268 & 189,1029765 & 192,8283051 \\
\hline & $17-12-2 \mathrm{i}$ & 0,564554828 & 76,4318077 & 77,93751431 \\
\hline & $17-12-3 i$ & 0,210123084 & 205,3555715 & 209,4010763 \\
\hline & $17-12-4 i$ & 0,278457405 & 154,9606702 & 158,0133955 \\
\hline & $17-12-5 i$ & 0,176188139 & 244,9083477 & 249,7330422 \\
\hline & $17-12-6 i$ & 0,399623276 & 107,9765584 & 110,1036966 \\
\hline & $17-12-7 i$ & 0,253738 & 170,0570906 & 173,4072153 \\
\hline & $17-12-2 \mathrm{~g}$ & 0,378019673 & 114,1473556 & 116,3960585 \\
\hline & $17-12-3 g$ & 0,463174427 & 93,16133082 & 94,99660903 \\
\hline \multirow[t]{12}{*}{$17 / 08 / 00 / 15$} & $17-15-1 \mathrm{i}$ & 0,223564985 & 193,0085166 & 196,8107844 \\
\hline & $17-15-2 \mathrm{i}$ & 0,196980775 & 219,0566363 & 223,372052 \\
\hline & $17-15-3 i$ & 0,518355275 & 83,24396061 & 84,88386664 \\
\hline & $17-15-4 i$ & 0,142703916 & 302,3739453 & 308,3307121 \\
\hline & $17-15-5 i$ & 0,419162519 & 102,9432358 & 104,9712176 \\
\hline & $17-15-6 i$ & 0,573832329 & 75,19608761 & 76,67745054 \\
\hline & $17-15-7 i$ & 0,293038961 & 147,2498604 & 150,1506826 \\
\hline & $17-15-1 \mathrm{~g}$ & 0,194440056 & 221,9190164 & 226,290821 \\
\hline & $17-15-2 \mathrm{~g}$ & 0,230618916 & 187,1049728 & 190,7909408 \\
\hline & $17-15-3 g$ & 0,240963826 & 179,0722983 & 182,6000225 \\
\hline & $17-15-4 \mathrm{~g}$ & 0,369616039 & 116,7426234 & 119,0424531 \\
\hline & $17-15-5 \mathrm{~g}$ & 0 & & \\
\hline \multirow[t]{13}{*}{$17 / 08 / 00 / 16$} & $17-16-1 \mathrm{i}$ & 0,064231149 & 671,7915981 & 685,0258926 \\
\hline & $17-16-2 \mathrm{i}$ & 0,188726831 & 228,6370514 & 233,1412013 \\
\hline & $17-16-3 i$ & 0,259984762 & 165,9710584 & 169,2406882 \\
\hline & $17-16-4 i$ & 0,272150456 & 158,5518052 & 161,6752758 \\
\hline & $17-16-5 i$ & 0,422892637 & 102,0352266 & 104,0453206 \\
\hline & $17-16-6 \mathrm{i}$ & 0,647749016 & 66,61522438 & 67,9275443 \\
\hline & $17-16-7 i$ & 0 & & \\
\hline & $17-16-1 \mathrm{~g}$ & 1,619795975 & 26,63912415 & 27,16391489 \\
\hline & $17-16-2 \mathrm{~g}$ & 1,376357307 & 31,35083153 & 31,96844291 \\
\hline & $17-16-3 g$ & 1,11674973 & 38,6388686 & 39,40005431 \\
\hline & $17-16-4 \mathrm{~g}$ & 1,537285404 & 28,06892328 & 28,62188107 \\
\hline & $17-16-5 \mathrm{~g}$ & 0,553390527 & 77,9737707 & 79,50985398 \\
\hline & $17-16-6 \mathrm{~g}$ & 0,54395557 & 79,32623256 & 80,88895934 \\
\hline \multirow[t]{9}{*}{$17 / 08 / 00 / 19$} & $17-19-1 \mathrm{i}$ & 0,418447494 & 103,1191409 & 105,150588 \\
\hline & $17-19-2 \mathrm{i}$ & 0,208951917 & 206,506581 & 210,5747606 \\
\hline & $17-19-3 i$ & 0,563330588 & 76,59791069 & 78,10688953 \\
\hline & $17-19-4 i$ & 0,376128488 & 114,7212919 & 116,9813014 \\
\hline & $17-19-5 i$ & 0,423455708 & 101,89955 & 103,9069711 \\
\hline & $17-19-6 i$ & 0,794883695 & 54,28460334 & 55,35401003 \\
\hline & $17-19-1 \mathrm{~g}$ & 0,166236837 & 259,5690989 & 264,6826101 \\
\hline & $17-19-2 \mathrm{~g}$ & 0,187191682 & 230,5120913 & 235,0531795 \\
\hline & $17-19-3 \mathrm{~g}$ & 0,504302231 & 85,56366289 & 87,24926705 \\
\hline
\end{tabular}




\begin{tabular}{|c|c|c|c|c|}
\hline sample & lab number & D1/G intensity ratio & $\begin{array}{c}\text { graphite } \\
\text { crystallinity } \\
\text { own calculation } \\
\text { based on Tuinstra } \\
\text { \& Koenig } \\
\mathbf{L}_{\mathrm{a}}[\AA ̊] \\
\end{array}$ & $\begin{array}{c}\text { graphite } \\
\text { crystallinity } \\
\text { after Zheng \& Chen } \\
\mathrm{L}_{\mathrm{a}}[\AA]\end{array}$ \\
\hline & $17-19-4 \mathrm{~g}$ & 0,059353715 & 726,996545 & 741,318377 \\
\hline & $17-19-5 \mathrm{~g}$ & 0,323388663 & 133,4306083 & 136,0591912 \\
\hline \multirow[t]{21}{*}{$17 / 08 / 00 / 20$} & $17-2010 \mathrm{i}$ & 0,1157942 & 372,6434146 & 379,9844899 \\
\hline & $17-2011 \mathrm{i}$ & 0 & & \\
\hline & $17-2012 \mathrm{i}$ & 0,422438263 & 102,1449756 & 104,1572316 \\
\hline & $17-2013 \mathrm{i}$ & 0,25643393 & 168,2692535 & 171,5841578 \\
\hline & $17-2014 \mathrm{i}$ & 0,140995942 & 306,0367934 & 312,0657183 \\
\hline & $17-2015 \mathrm{i}$ & 0,156673984 & 275,4123233 & 280,8379461 \\
\hline & $17-20-1 \mathrm{i}$ & 0,153071616 & 281,8938433 & 287,447152 \\
\hline & $17-20-2 \mathrm{i}$ & 0,064049506 & 673,6967834 & 686,9686101 \\
\hline & $17-20-3 i$ & 0,102100344 & 422,6229271 & 430,9485988 \\
\hline & $17-20-4 i$ & 0,043498782 & 991,9805565 & 1011,522573 \\
\hline & $17-20-5 i$ & 0,337941235 & 127,6847619 & 130,2001517 \\
\hline & $17-20-6 \mathrm{i}$ & 0,418634499 & 103,0730772 & 105,1036169 \\
\hline & $17-20-7 \mathrm{i}$ & 0,390171077 & 110,592375 & 112,7710448 \\
\hline & $17-20-8 \mathrm{i}$ & 0 & & \\
\hline & $17-20-9 \mathrm{i}$ & 0,05532497 & 779,9361919 & 795,3009349 \\
\hline & $17-20-1 \mathrm{~g}$ & 0,110883748 & 389,1458103 & 396,8119828 \\
\hline & $17-20-2 \mathrm{~g}$ & 0 & & \\
\hline & $17-20-3 g$ & 0,115175742 & 374,6443925 & 382,0248871 \\
\hline & $17-20-4 \mathrm{~g}$ & 0 & & \\
\hline & $17-20-5 \mathrm{~g}$ & 0,241471592 & 178,6957448 & 182,216051 \\
\hline & $17-20-6 \mathrm{~g}$ & 0,153438761 & 281,2193325 & 286,7593533 \\
\hline \multirow[t]{12}{*}{$18 / 08 / 00 / 02$} & $18-02-1 \mathrm{i}$ & 0,287665814 & 150,0002574 & 152,9552624 \\
\hline & $18-02-2 \mathrm{i}$ & 0,136611599 & 315,858582 & 322,0809961 \\
\hline & $18-02-3 i$ & 0,091187872 & 473,1983022 & 482,5203088 \\
\hline & $18-02-4 \mathrm{i}$ & 0,356312512 & 121,1014057 & 123,4871034 \\
\hline & $18-02-5 \mathrm{i}$ & 0,14049272 & 307,132967 & 313,1834865 \\
\hline & $18-02-6 i$ & 0,748497241 & 57,64877107 & 58,78445186 \\
\hline & $18-02-7 i$ & 0,242754044 & 177,7517087 & 181,2534173 \\
\hline & $18-02-1 \mathrm{~g}$ & 0,72446016 & 59,56151686 & 60,73487874 \\
\hline & $18-02-2 \mathrm{~g}$ & 0,148325785 & 290,9133169 & 296,6443092 \\
\hline & $18-02-3 g$ & 0,047994609 & 899,0581784 & 916,7696245 \\
\hline & $18-02-5 \mathrm{~g}$ & 1,244933899 & 34,66043145 & 35,34324195 \\
\hline & $18-02-6 \mathrm{~g}$ & 0,660946977 & 65,2850343 & 66,57114948 \\
\hline \multirow[t]{8}{*}{$3.10 .01 / 3$} & $3-013-1 i$ & 0,16140599 & 267,3379481 & 272,6045056 \\
\hline & $3-013-2 \mathrm{i}$ & 0,029010409 & 1487,3953 & 1516,696987 \\
\hline & $3-013-3 i$ & 0,077979639 & 553,3488812 & 564,2498542 \\
\hline & $3-013-4 i$ & 0,798890474 & 54,01234273 & 55,07638589 \\
\hline & $3-013-5 i$ & 0 & & \\
\hline & $3-013-6 i$ & 0,397156889 & 108,6471045 & 110,7874525 \\
\hline & $3-013-7 i$ & 0,423700729 & 101,8406226 & 103,8468829 \\
\hline & $3-013-1 g$ & 0,113035455 & 381,7381546 & 389,2583962 \\
\hline
\end{tabular}




\begin{tabular}{|c|c|c|c|c|}
\hline sample & lab number & D1/G intensity ratio & $\begin{array}{c}\text { graphite } \\
\text { crystallinity } \\
\text { own calculation } \\
\text { based on Tuinstra } \\
\& \text { Koenig } \\
\left.\mathrm{L}_{\mathrm{a}}[\AA]\right] \\
\end{array}$ & $\begin{array}{c}\text { graphite } \\
\text { crystallinity } \\
\text { after Zheng \& Chen } \\
\mathrm{L}_{\mathrm{a}}[\AA]\end{array}$ \\
\hline & $3-013-2 g$ & 0,229041891 & 188,3932497 & 192,1045967 \\
\hline & $3-013-3 g$ & 0,307926151 & 140,1308264 & 142,8914037 \\
\hline & $3-013-4 g$ & 0 & & \\
\hline \multirow[t]{15}{*}{$3.10 .01 / 4$} & 3-01410i & 0,207578603 & 207,8728033 & 211,9678975 \\
\hline & 3-014-1i & 0,463376791 & 93,12064586 & 94,95512258 \\
\hline & $3-014-2 i$ & 0,404002317 & 106,8061847 & 108,9102666 \\
\hline & $3-014-3 i$ & 0 & & \\
\hline & $3-014-4 i$ & 0 & & \\
\hline & $3-014-5 i$ & 0,52252836 & 82,5791467 & 84,20595589 \\
\hline & $3-014-6 i$ & 1,047256795 & 41,20283228 & 42,01452807 \\
\hline & $3-014-7 i$ & 0,926644749 & 46,56579139 & 47,48313748 \\
\hline & $3-014-8 \mathrm{i}$ & 1,089827544 & 39,59337079 & 40,37336019 \\
\hline & $3-014-9 i$ & 0,052310537 & 824,8805812 & 841,1307286 \\
\hline & $3-014-1 g$ & 0,091994004 & 469,051724 & 478,292043 \\
\hline & $3-014-2 \mathrm{~g}$ & 0 & & \\
\hline & $3-014-3 g$ & 0,189241452 & 228,0152979 & 232,5071993 \\
\hline & $3-014-4 g$ & 0,127908137 & 337,3510636 & 343,9968795 \\
\hline & $3-014-5 g$ & 0,170071041 & 253,717187 & 258,7154156 \\
\hline \multirow[t]{2}{*}{ Elim Spatkristall } & Elim 01 & 0,997394413 & 43,26267072 & 44,11494533 \\
\hline & Elim 02 & 0,836470012 & 51,58576572 & 52,60200531 \\
\hline \multirow[t]{4}{*}{ Omaruru Spatkristall } & Rad 01 & 0 & & \\
\hline & Rad 02 & 0 & & \\
\hline & Rad 03 & 0 & & \\
\hline & Rad 04 & 0 & & \\
\hline
\end{tabular}




\section{II..2 STATISTICAL ANALYSIS OF SAMPLE MEASUREMENTS:}

\begin{tabular}{|c|c|c|c|c|}
\hline sample & \multicolumn{2}{|c|}{$\begin{array}{c}\text { G band postion of sample } \\
{\left[\Delta \mathbf{c m}^{-1}\right]}\end{array}$} & \multicolumn{2}{|c|}{$\begin{array}{c}\text { G band FWHM of sample } \\
{\left[\mathrm{cm}^{-1}\right]}\end{array}$} \\
\hline $12 / 08 / 00 / 02$ & G band position 1: & & G band FWHM 1: & \\
\hline & sum of raman shift: & 15779,86 & sum of wavenumbers: & 228,2295 \\
\hline & mean raman shift: & 1577,986 & mean wavenumbers: & 22,82295 \\
\hline & standard deviation: & 3,420848498 & standard deviation: & 10,04927333 \\
\hline & maximum raman shift: & 1582,51 & maximum wavenumbers: & 50,643 \\
\hline & minimum raman shift: & 1571,18 & minimum wavenumbers: & 16,7984 \\
\hline & variation of raman shift: & 11,33 & variation of wavenumbers: & 33,8446 \\
\hline \multirow[t]{7}{*}{$12 / 08 / 00 / 03$} & G band position 2: & & G band FWHM 2: & \\
\hline & sum of raman shift: & 12604,45 & sum of wavenumbers: & 153,5113 \\
\hline & mean raman shift: & 1575,55625 & mean wavenumbers: & 19,1889125 \\
\hline & standard deviation: & 4,914299652 & standard deviation: & 2,306406458 \\
\hline & maximum raman shift: & 1580,17 & maximum wavenumbers: & 22,6218 \\
\hline & minimum raman shift: & 1566,39 & minimum wavenumbers: & 15,9374 \\
\hline & variation of raman shift: & 13,78 & variation of wavenumbers: & 6,6844 \\
\hline \multirow[t]{7}{*}{ 15/08/00/01 } & G band position 3: & & G band FWHM 3: & \\
\hline & sum of raman shift: & 17331,08 & sum of wavenumbers: & 247,9666 \\
\hline & mean raman shift: & 1575,552727 & mean wavenumbers: & 22,54241818 \\
\hline & standard deviation: & 5,766836379 & standard deviation: & 8,282343669 \\
\hline & maximum raman shift: & 1581,5 & maximum wavenumbers: & 44,161 \\
\hline & minimum raman shift: & 1565,32 & minimum wavenumbers: & 14,1854 \\
\hline & variation of raman shift: & 16,18 & variation of wavenumbers: & 29,9756 \\
\hline \multirow[t]{7}{*}{ 17/08/00/11 } & G band position 4: & & G band FWHM 4: & \\
\hline & sum of raman shift: & 22104,83 & sum of wavenumbers: & 330,5831 \\
\hline & mean raman shift: & 1578,916429 & mean wavenumbers: & 23,61307857 \\
\hline & standard deviation: & 2,142319401 & standard deviation: & 6,614637212 \\
\hline & maximum raman shift: & 1581,46 & maximum wavenumbers: & 36,2335 \\
\hline & minimum raman shift: & 1574,04 & minimum wavenumbers: & 14,4205 \\
\hline & variation of raman shift: & 7,42 & variation of wavenumbers: & 21,813 \\
\hline \multirow[t]{7}{*}{ 17/08/00/12 } & G band position 5: & & G band FWHM 5: & \\
\hline & sum of raman shift: & 14115,93 & sum of wavenumbers: & 241,901 \\
\hline & mean raman shift: & 1568,436667 & mean wavenumbers: & 26,87788889 \\
\hline & standard deviation: & 4,207701273 & standard deviation: & 3,612933014 \\
\hline & maximum raman shift: & 1571,55 & maximum wavenumbers: & 33,9613 \\
\hline & minimum raman shift: & 1564,47 & minimum wavenumbers: & 24,0268 \\
\hline & variation of raman shift: & 7,08 & variation of wavenumbers: & 9,9345 \\
\hline \multirow[t]{7}{*}{ 17/08/00/15 } & G band position 6: & & G band FWHM 6: & \\
\hline & sum of raman shift: & 18908,89 & sum of wavenumbers: & 274,1977 \\
\hline & mean raman shift: & 1575,740833 & mean wavenumbers: & 22,84980833 \\
\hline & standard deviation: & 4,280767685 & standard deviation: & 4,946035567 \\
\hline & maximum raman shift: & 1580,78 & maximum wavenumbers: & 30,6547 \\
\hline & minimum raman shift: & 1570,59 & minimum wavenumbers: & 14,8532 \\
\hline & variation of raman shift: & 10,19 & variation of wavenumbers: & 15,8015 \\
\hline \multirow[t]{2}{*}{ 17/08/00/16 } & G band position 7: & & G band FWHM 7: & \\
\hline & sum of raman shift: & 20565,05 & sum of wavenumbers: & 387,6055 \\
\hline
\end{tabular}




\begin{tabular}{|c|c|c|c|c|}
\hline \multirow[t]{2}{*}{ sample } & \multicolumn{2}{|c|}{$\begin{array}{c}G \text { band postion of sample } \\
{\left[\Delta \mathrm{cm}^{-1}\right]}\end{array}$} & \multicolumn{2}{|c|}{$\begin{array}{c}\text { G band FWHM of sample } \\
{\left[\mathrm{cm}^{-1}\right]}\end{array}$} \\
\hline & mean raman shift: & 1581,926923 & mean wavenumbers: & 29,81580769 \\
\hline & standard deviation: & 8,216469624 & standard deviation: & 13,81697602 \\
\hline & maximum raman shift: & 1594,57 & maximum wavenumbers: & 51,7922 \\
\hline & minimum raman shift: & 1567,19 & minimum wavenumbers: & 13,435 \\
\hline & variation of raman shift: & 27,38 & variation of wavenumbers: & 38,3572 \\
\hline \multirow[t]{7}{*}{ 17/08/00/19 } & G band position 8: & & G band FWHM 8: & \\
\hline & sum of raman shift: & 17340,06 & sum of wavenumbers: & 255,0548 \\
\hline & mean raman shift: & 1576,369091 & mean wavenumbers: & 23,1868 \\
\hline & standard deviation: & 4,234580155 & standard deviation: & 5,232664364 \\
\hline & maximum raman shift: & 1580,25 & maximum wavenumbers: & 32,2254 \\
\hline & minimum raman shift: & 1568,56 & minimum wavenumbers: & 14,9974 \\
\hline & variation of raman shift: & 11,69 & variation of wavenumbers: & 17,228 \\
\hline \multirow[t]{7}{*}{$17 / 08 / 00 / 20$} & G band position 9: & & G band FWHM 9: & \\
\hline & sum of raman shift: & 33195,87 & sum of wavenumbers: & 346,9658 \\
\hline & mean raman shift: & 1580,755714 & mean wavenumbers: & 16,52218095 \\
\hline & standard deviation: & 0,797493395 & standard deviation: & 2,667774257 \\
\hline & maximum raman shift: & 1582,26 & maximum wavenumbers: & 21,3611 \\
\hline & minimum raman shift: & 1579,45 & minimum wavenumbers: & 13,6727 \\
\hline & variation of raman shift: & 2,81 & variation of wavenumbers: & 7,6884 \\
\hline \multirow[t]{7}{*}{$18 / 08 / 00 / 02$} & G band position 10: & & G band FWHM 10: & \\
\hline & sum of raman shift: & 18938,89 & sum of wavenumbers: & 299,3552 \\
\hline & mean raman shift: & 1578,240833 & mean wavenumbers: & 24,94626667 \\
\hline & standard deviation: & 6,401668047 & standard deviation: & 9,617434428 \\
\hline & maximum raman shift: & 1590,25 & maximum wavenumbers: & 49,4372 \\
\hline & minimum raman shift: & 1566,36 & minimum wavenumbers: & 15,7241 \\
\hline & variation of raman shift: & 23,89 & variation of wavenumbers: & 33,7131 \\
\hline \multirow[t]{7}{*}{$3.10 .01 / 3$} & G band position 11: & & G band FWHM 11: & \\
\hline & sum of raman shift: & 17366,81 & sum of wavenumbers: & 222,5189 \\
\hline & mean raman shift: & 1578,800909 & mean wavenumbers: & 20,22899091 \\
\hline & standard deviation: & 4,638653801 & standard deviation: & 6,750722562 \\
\hline & maximum raman shift: & 1582,81 & maximum wavenumbers: & 35,6522 \\
\hline & minimum raman shift: & 1573,06 & minimum wavenumbers: & 13,64 \\
\hline & variation of raman shift: & 9,75 & variation of wavenumbers: & 22,0122 \\
\hline \multirow[t]{7}{*}{$3.10 .01 / 4$} & G band position 12: & & G band FWHM 12: & \\
\hline & sum of raman shift: & 23673,03 & sum of wavenumbers: & 301,0728 \\
\hline & mean raman shift: & 1578,202 & mean wavenumbers: & 20,07152 \\
\hline & standard deviation: & 4,9394537 & standard deviation: & 5,316176167 \\
\hline & maximum raman shift: & 1582,46 & maximum wavenumbers: & 33,6083 \\
\hline & minimum raman shift: & 1565,91 & minimum wavenumbers: & 14,1091 \\
\hline & variation of raman shift: & 16,55 & variation of wavenumbers: & 19,4992 \\
\hline \multirow[t]{6}{*}{ Elim Spatkristall } & G band position 13: & & G band FWHM 13: & \\
\hline & sum of raman shift: & 3170,04 & sum of wavenumbers: & 85,7706 \\
\hline & mean raman shift: & 1585,02 & mean wavenumbers: & 42,8853 \\
\hline & standard deviation: & 0,876812409 & standard deviation: & 3,383505948 \\
\hline & maximum raman shift: & 1585,64 & maximum wavenumbers: & 45,2778 \\
\hline & minimum raman shift: & 1584,4 & minimum wavenumbers: & 40,4928 \\
\hline
\end{tabular}




\begin{tabular}{|l|l|c|l|c|}
\hline \multirow{2}{*}{ sample } & \multicolumn{2}{|c|}{$\begin{array}{c}\text { G band postion of sample } \\
{\left[\mathbf{\Delta} \mathbf{~ c m}^{-1}\right]}\end{array}$} & \multicolumn{2}{c|}{ G band FWHM of sample } \\
& \multicolumn{2}{|c|}{$\left[\mathbf{c m}^{-1}\right]$} \\
\hline & variation of raman shift: & 1,24 & variation of wavenumbers: & 4,785 \\
\hline Omaruru Spatkristall & G band position 14: & & G band FWHM 14: & \\
\hline & sum of raman shift: & 6319,44 & sum of wavenumbers: & 54,8067 \\
\hline & mean raman shift: & 1579,86 & mean wavenumbers: & 13,701675 \\
\hline & standard deviation: & 0,442944691 & standard deviation: & 0,487533865 \\
\hline & maximum raman shift: & 1580,48 & maximum wavenumbers: & 13,9798 \\
\hline & minimum raman shift: & 1579,46 & minimum wavenumbers: & 12,9713 \\
\hline & variation of raman shift: & 1,02 & variation of wavenumbers: & 1,0085 \\
\hline
\end{tabular}




\begin{tabular}{|c|c|c|c|c|}
\hline \multirow{2}{*}{$\begin{array}{c}\text { sample } \\
\text { 12/08/00/02 } \\
\end{array}$} & \multicolumn{2}{|c|}{$\begin{array}{l}\text { D1 band postion of sample } \\
{\left[\Delta \mathrm{cm}^{-1}\right]}\end{array}$} & \multicolumn{2}{|c|}{$\begin{array}{l}\text { D1 band FWHM of sample } \\
\qquad\left[\mathrm{cm}^{-1}\right]\end{array}$} \\
\hline & D1 band FWHM 1: & & D1/G intensity ratio 1: & \\
\hline & sum of wavenumbers: & 453,6801 & sum of intensity ratios: & 2,67483743 \\
\hline & mean wavenumbers: & 45,36801 & mean intensity ratios: & 0,26748374 \\
\hline & standard deviation: & 6,080375201 & standard deviation: & 0,15633062 \\
\hline & maximum wavenumbers: & 60,0061 & maximum intensity ratios: & 0,54495515 \\
\hline & minimum wavenumbers: & 41,0948 & minimum intensity ratios: & 0,12077861 \\
\hline & variation of wavenumbers: & 18,9113 & variation of intensity ratios: & 0,42417653 \\
\hline \multirow[t]{7}{*}{ 12/08/00/03 } & D1 band FWHM 2: & & D1/G intensity ratio 2: & \\
\hline & sum of wavenumbers: & 359,9999 & sum of intensity ratios: & 1,27704784 \\
\hline & mean wavenumbers: & 44,9999875 & mean intensity ratios: & 0,15963098 \\
\hline & standard deviation: & 27,95850796 & standard deviation: & 0,09832481 \\
\hline & maximum wavenumbers: & 113,451 & maximum intensity ratios: & 0,31572992 \\
\hline & minimum wavenumbers: & 36,2262 & minimum intensity ratios: & 0,07550903 \\
\hline & variation of wavenumbers: & 77,2248 & variation of intensity ratios: & 0,24022089 \\
\hline \multirow[t]{7}{*}{ 15/08/00/01 } & D1 band FWHM 3: & & D1/G intensity ratio 3: & \\
\hline & sum of wavenumbers: & 557,3427 & sum of intensity ratios: & 2,53233981 \\
\hline & mean wavenumbers: & 50,66751818 & mean intensity ratios: & 0,23021271 \\
\hline & standard deviation: & 12,23244323 & standard deviation: & 0,15767821 \\
\hline & maximum wavenumbers: & 82,658 & maximum intensity ratios: & 0,56056913 \\
\hline & minimum wavenumbers: & 41,4662 & minimum intensity ratios: & 0,07181864 \\
\hline & variation of wavenumbers: & 41,1918 & variation of intensity ratios: & 0,48875048 \\
\hline \multirow[t]{7}{*}{ 17/08/00/11 } & D1 band FWHM 4: & & D1/G intensity ratio 4: & \\
\hline & sum of wavenumbers: & 629,9714 & sum of intensity ratios: & 5,47990179 \\
\hline & mean wavenumbers: & 44,99795714 & mean intensity ratios: & 0,39142155 \\
\hline & standard deviation: & 3,923606228 & standard deviation: & 0,22206625 \\
\hline & maximum wavenumbers: & 55,8544 & maximum intensity ratios: & 0,81781614 \\
\hline & minimum wavenumbers: & 40,404 & minimum intensity ratios: & 0,08439488 \\
\hline & variation of wavenumbers: & 15,4504 & variation of intensity ratios: & 0,73342125 \\
\hline \multirow[t]{7}{*}{ 17/08/00/12 } & D1 band FWHM 5: & & D1/G intensity ratio 5: & \\
\hline & sum of wavenumbers: & 412,1368 & sum of intensity ratios: & 2,95206110 \\
\hline & mean wavenumbers: & 45,79297778 & mean intensity ratios: & 0,32800678 \\
\hline & standard deviation: & 2,37190353 & standard deviation: & 0,13073679 \\
\hline & maximum wavenumbers: & 49,87 & maximum intensity ratios: & 0,56455482 \\
\hline & minimum wavenumbers: & 41,2751 & minimum intensity ratios: & 0,17618813 \\
\hline & variation of wavenumbers: & 8,5949 & variation of intensity ratios: & 0,38836668 \\
\hline \multirow[t]{7}{*}{ 17/08/00/15 } & D1 band FWHM 6: & & D1/G intensity ratio 6: & \\
\hline & sum of wavenumbers: & 517,117 & sum of intensity ratios: & 3,40327759 \\
\hline & mean wavenumbers: & 43,09308333 & mean intensity ratios: & 0,28360646 \\
\hline & standard deviation: & 4,147101138 & standard deviation: & 0,16213686 \\
\hline & maximum wavenumbers: & 56,1229 & maximum intensity ratios: & 0,57383232 \\
\hline & minimum wavenumbers: & 40,791 & minimum intensity ratios: & 0,14270391 \\
\hline & variation of wavenumbers: & 15,3319 & variation of intensity ratios: & 0,43112841 \\
\hline \multirow[t]{6}{*}{ 17/08/00/16 } & D1 band FWHM 7: & & D1/G intensity ratio 7: & \\
\hline & sum of wavenumbers: & 605,2091 & sum of intensity ratios: & 8,60326936 \\
\hline & mean wavenumbers: & 46,55454615 & mean intensity ratios: & 0,66178995 \\
\hline & standard deviation: & 7,949872904 & standard deviation: & 0,56397877 \\
\hline & maximum wavenumbers: & 64,8709 & maximum intensity ratios: & 1,61979597 \\
\hline & minimum wavenumbers: & 40,4072 & minimum intensity ratios: & 0,06423114 \\
\hline
\end{tabular}




\begin{tabular}{|c|c|c|c|c|}
\hline \multirow[t]{2}{*}{ sample } & \multicolumn{2}{|c|}{$\begin{array}{l}\text { D1 band postion of sample } \\
{\left[\Delta \mathrm{cm}^{-1}\right]}\end{array}$} & \multicolumn{2}{|c|}{$\begin{array}{l}\text { D1 band FWHM of sample } \\
\qquad\left[\mathrm{cm}^{-1}\right]\end{array}$} \\
\hline & variation of wavenumbers: & 24,4637 & variation of intensity ratios: & 1,55556482 \\
\hline \multirow[t]{7}{*}{ 17/08/00/19 } & D1 band FWHM 8: & & D1/G intensity ratio 8: & \\
\hline & sum of wavenumbers: & 582,9867 & sum of intensity ratios: & 4,02567101 \\
\hline & mean wavenumbers: & 52,99879091 & mean intensity ratios: & 0,36597009 \\
\hline & standard deviation: & 18,96806464 & standard deviation: & 0,20989332 \\
\hline & maximum wavenumbers: & 104,759 & maximum intensity ratios: & 0,79488369 \\
\hline & minimum wavenumbers: & 41,0647 & minimum intensity ratios: & 0,05935371 \\
\hline & variation of wavenumbers: & 63,6943 & variation of intensity ratios: & 0,73552997 \\
\hline \multirow[t]{7}{*}{$17 / 08 / 00 / 20$} & D1 band FWHM 9: & & D1/G intensity ratio 9: & \\
\hline & sum of wavenumbers: & 924,4844 & sum of intensity ratios: & 3,27809819 \\
\hline & mean wavenumbers: & 44,02306667 & mean intensity ratios: & 0,15609991 \\
\hline & standard deviation: & 17,38052537 & standard deviation: & 0,13826649 \\
\hline & maximum wavenumbers: & 93,5214 & maximum intensity ratios: & 0,42243826 \\
\hline & minimum wavenumbers: & 38,7959 & minimum intensity ratios: & 0,04349878 \\
\hline & variation of wavenumbers: & 54,7255 & variation of intensity ratios: & 0,37893948 \\
\hline \multirow[t]{7}{*}{ 18/08/00/02 } & D1 band FWHM 10: & & D1/G intensity ratio 10: & \\
\hline & sum of wavenumbers: & 649,8888 & sum of intensity ratios: & 4,83018323 \\
\hline & mean wavenumbers: & 54,1574 & mean intensity ratios: & 0,40251526 \\
\hline & standard deviation: & 29,40042191 & standard deviation: & 0,36521965 \\
\hline & maximum wavenumbers: & 142,871 & maximum intensity ratios: & 1,24493389 \\
\hline & minimum wavenumbers: & 35,2597 & minimum intensity ratios: & 0,04799460 \\
\hline & variation of wavenumbers: & 107,6113 & variation of intensity ratios: & 1,19693928 \\
\hline \multirow[t]{7}{*}{$3.10 .01 / 3$} & D1 band FWHM 11: & & D1/G intensity ratio 11: & \\
\hline & sum of wavenumbers: & 415,014 & sum of intensity ratios: & 2,53814762 \\
\hline & mean wavenumbers: & 37,72854545 & mean intensity ratios: & 0,23074069 \\
\hline & standard deviation: & 7,087753325 & standard deviation: & 0,24142451 \\
\hline & maximum wavenumbers: & 59,809 & maximum intensity ratios: & 0,79889047 \\
\hline & minimum wavenumbers: & 40,0794 & minimum intensity ratios: & 0,02901040 \\
\hline & variation of wavenumbers: & 19,7296 & variation of intensity ratios: & 0,76988006 \\
\hline \multirow[t]{7}{*}{$3.10 .01 / 4$} & D1 band FWHM 12: & & D1/G intensity ratio 12: & \\
\hline & sum of wavenumbers: & 581,9953 & sum of intensity ratios: & 5,29274032 \\
\hline & mean wavenumbers: & 38,79968667 & mean intensity ratios: & 0,35284935 \\
\hline & standard deviation: & 7,827510915 & standard deviation: & 0,38377432 \\
\hline & maximum wavenumbers: & 68,4843 & maximum intensity ratios: & 1,08982754 \\
\hline & minimum wavenumbers: & 41,2725 & minimum intensity ratios: & 0,05231053 \\
\hline & variation of wavenumbers: & 27,2118 & variation of intensity ratios: & 1,03751700 \\
\hline \multirow[t]{7}{*}{ Elim Spatkristall } & D1 band FWHM 13: & & D1/G intensity ratio 13: & \\
\hline & sum of wavenumbers: & 108,8359 & sum of intensity ratios: & 1,83386442 \\
\hline & mean wavenumbers: & 54,41795 & mean intensity ratios: & 0,91693221 \\
\hline & standard deviation: & 2,788192749 & standard deviation: & 0,11379073 \\
\hline & maximum wavenumbers: & 56,3895 & maximum intensity ratios: & 0,99739441 \\
\hline & minimum wavenumbers: & 52,4464 & minimum intensity ratios: & 0,83647001 \\
\hline & variation of wavenumbers: & 3,9431 & variation of intensity ratios: & 0,16092440 \\
\hline \multirow{5}{*}{$\begin{array}{l}\text { Omaruru Spatkristall } \\
\\
\end{array}$} & D1 band FWHM 14: & & D1/G intensity ratio 14: & \\
\hline & sum of wavenumbers: & 0 & sum of intensity ratios: & 0 \\
\hline & mean wavenumbers: & 0 & mean intensity ratios: & 0 \\
\hline & standard deviation: & \#DIV/0! & standard deviation: & 0 \\
\hline & maximum wavenumbers: & & maximum intensity ratios: & 0 \\
\hline
\end{tabular}




\begin{tabular}{|l|l|c|l|c|}
\hline \multirow{2}{*}{ sample } & \multicolumn{2}{|c|}{$\begin{array}{c}\text { D1 band postion of sample } \\
{\left[\Delta \mathbf{c m}^{-1}\right]}\end{array}$} & \multicolumn{2}{c|}{$\begin{array}{c}\text { D1 band FWHM of sample } \\
{\left[\mathbf{c m}^{-1}\right]}\end{array}$} \\
\hline & minimum wavenumbers: & & minimum intensity ratios: & 0 \\
\hline & variation of wavenumbers: & 0 & variation of intensity ratios: & 0 \\
\hline
\end{tabular}




\begin{tabular}{|c|c|c|}
\hline sample & \multicolumn{2}{|c|}{$\begin{array}{c}\text { Crystallinities of samples } \\
\left.\qquad \mathbf{L}_{\mathrm{a}}[\AA]\right]\end{array}$} \\
\hline \multirow[t]{7}{*}{$12 / 08 / 00 / 02$} & Crystallinities 1: & \\
\hline & sum of crystallinities: & 2091,418015 \\
\hline & mean crystallinities: & 209,1418015 \\
\hline & standard deviation: & 98,63689724 \\
\hline & maximum crystallinity: & 357,2647755 \\
\hline & minimum crystallinity: & 79,18072888 \\
\hline & variation of crystallinity: & 278,0840466 \\
\hline \multirow[t]{7}{*}{$12 / 08 / 00 / 03$} & Crystallinities 2: & \\
\hline & sum of crystallinities: & 1994,116329 \\
\hline & mean crystallinities: & 249,2645412 \\
\hline & standard deviation: & 143,8070253 \\
\hline & maximum crystallinity: & 571,4541119 \\
\hline & minimum crystallinity: & 136,6672667 \\
\hline & variation of crystallinity: & 434,7868451 \\
\hline \multirow[t]{7}{*}{$15 / 08 / 00 / 01$} & Crystallinities 3: & \\
\hline & sum of crystallinities: & 3399,588321 \\
\hline & mean crystallinities: & 309,0534837 \\
\hline & standard deviation: & 249,4896342 \\
\hline & maximum crystallinity: & 600,8181224 \\
\hline & minimum crystallinity: & 76,97524375 \\
\hline & variation of crystallinity: & 523,8428787 \\
\hline \multirow[t]{7}{*}{ 17/08/00/11 } & Crystallinities 4: & \\
\hline & sum of crystallinities: & 2389,85099 \\
\hline & mean crystallinities: & 170,7036422 \\
\hline & standard deviation: & 133,0645958 \\
\hline & maximum crystallinity: & 511,2862818 \\
\hline & minimum crystallinity: & 52,7624045 \\
\hline & variation of crystallinity: & 458,5238773 \\
\hline \multirow[t]{7}{*}{$17 / 08 / 00 / 12$} & Crystallinities 5: & \\
\hline & sum of crystallinities: & 1356,101709 \\
\hline & mean crystallinities: & 150,6779677 \\
\hline & standard deviation: & 56,69868814 \\
\hline & maximum crystallinity: & 244,9083477 \\
\hline & minimum crystallinity: & 76,4318077 \\
\hline & variation of crystallinity: & 168,47654 \\
\hline \multirow[t]{7}{*}{ 17/08/00/15 } & Crystallinities 6: & \\
\hline & sum of crystallinities: & 1827,911154 \\
\hline & mean crystallinities: & 152,3259295 \\
\hline & standard deviation: & 69,08918422 \\
\hline & maximum crystallinity: & 302,3739453 \\
\hline & minimum crystallinity: & 75,19608761 \\
\hline & variation of crystallinity: & 227,1778577 \\
\hline \multirow[t]{4}{*}{$17 / 08 / 00 / 16$} & Crystallinities 7: & \\
\hline & sum of crystallinities: & 1675,599715 \\
\hline & mean crystallinities: & 128,8922858 \\
\hline & standard deviation: & 179,2634085 \\
\hline
\end{tabular}




\begin{tabular}{|c|c|c|}
\hline \multirow[t]{2}{*}{ sample } & \multicolumn{2}{|c|}{$\begin{array}{c}\text { Crystallinities of samples } \\
\qquad \mathbf{L}_{\mathbf{a}}[\AA]\end{array}$} \\
\hline & maximum crystallinity: & 671,7915981 \\
\hline & minimum crystallinity: & 26,63912415 \\
\hline & variation of crystallinity: & 645,152474 \\
\hline \multirow[t]{7}{*}{ 17/08/00/19 } & Crystallinities 8: & \\
\hline & sum of crystallinities: & 2093,201084 \\
\hline & mean crystallinities: & 190,2910077 \\
\hline & standard deviation: & 190,1264101 \\
\hline & maximum crystallinity: & 726,996545 \\
\hline & minimum crystallinity: & 54,28460334 \\
\hline & variation of crystallinity: & 672,7119417 \\
\hline \multirow[t]{7}{*}{$17 / 08 / 00 / 20$} & Crystallinities 9: & \\
\hline & sum of crystallinities: & 5939,692557 \\
\hline & mean crystallinities: & 282,8425027 \\
\hline & standard deviation: & 252,2481041 \\
\hline & maximum crystallinity: & 991,9805565 \\
\hline & minimum crystallinity: & 102,1449756 \\
\hline & variation of crystallinity: & 889,8355809 \\
\hline \multirow[t]{7}{*}{ 18/08/00/02 } & Crystallinities 10: & \\
\hline & sum of crystallinities: & 2952,170472 \\
\hline & mean crystallinities: & 246,014206 \\
\hline & standard deviation: & 246,0173612 \\
\hline & maximum crystallinity: & 899,0581784 \\
\hline & minimum crystallinity: & 34,66043145 \\
\hline & variation of crystallinity: & 864,397747 \\
\hline \multirow[t]{7}{*}{$3.10 .01 / 3$} & Crystallinities 11: & \\
\hline & sum of crystallinities: & 3282,844429 \\
\hline & mean crystallinities: & 298,4404027 \\
\hline & standard deviation: & 449,792051 \\
\hline & maximum crystallinity: & 1487,3953 \\
\hline & minimum crystallinity: & 54,01234273 \\
\hline & variation of crystallinity: & 1433,382957 \\
\hline \multirow[t]{7}{*}{$3.10 .01 / 4$} & Crystallinities 12: & \\
\hline & sum of crystallinities: & 2730,756629 \\
\hline & mean crystallinities: & 182,0504419 \\
\hline & standard deviation: & 230,5586932 \\
\hline & maximum crystallinity: & 824,8805812 \\
\hline & minimum crystallinity: & 39,59337079 \\
\hline & variation of crystallinity: & 785,2872104 \\
\hline \multirow[t]{7}{*}{ Elim Spatkristall } & Crystallinities 13: & \\
\hline & sum of crystallinities: & 94,84843644 \\
\hline & mean crystallinities: & 47,42421822 \\
\hline & standard deviation: & 5,885316916 \\
\hline & maximum crystallinity: & 51,58576572 \\
\hline & minimum crystallinity: & 43,26267072 \\
\hline & variation of crystallinity: & 8,323095001 \\
\hline Omaruru Spatkristall & Crystallinities 14: & \\
\hline
\end{tabular}




\begin{tabular}{|l|l|c|}
\hline \multirow{2}{*}{ sample } & \multicolumn{2}{c|}{$\begin{array}{c}\text { Crystallinities of samples } \\
\mathbf{L}_{\mathbf{a}}[\mathbf{\AA} \mathbf{\mathbf { A }}\end{array}$} \\
\hline & \multicolumn{1}{|c|}{0} \\
\hline & sum of crystallinities: & 0 \\
\hline & mean crystallinities: & 0 \\
\hline & standard deviation: & \#DIV/0! \\
\hline & maximum crystallinity: & 0 \\
\hline & minimum crystallinity: & 0 \\
\hline & variation of crystallinity: & 0 \\
\hline
\end{tabular}




\section{II.3 SAMPLES AND ACCORDING PLOT NUMBERS:}

\begin{tabular}{|c|c|c|c|}
\hline sample & type of marble & sample locality & plot number for sample \\
\hline $12 / 08 / 00 / 02$ & $\begin{array}{c}\text { cataclasite with graphite } \\
\text { network structures }\end{array}$ & MT-Station 94 & 1 \\
\hline $12 / 08 / 00 / 03$ & $\begin{array}{c}\text { cataclasite with graphite } \\
\text { network structures }\end{array}$ & MT-Station 94 & 3 \\
\hline $15 / 08 / 00 / 01$ & $\begin{array}{c}\text { cataclasite with graphite } \\
\text { network structures }\end{array}$ & $\begin{array}{c}\text { Omaruru River near Farm } \\
\text { Elim }\end{array}$ & 4 \\
\hline $17 / 08 / 00 / 11$ & $\begin{array}{c}\text { cataclasite with graphite } \\
\text { network structures }\end{array}$ & Farm Epako Süd & 5 \\
\hline $17 / 08 / 00 / 12$ & $\begin{array}{c}\text { cataclasite with graphite } \\
\text { network structures }\end{array}$ & Farm Epako Süd & 6 \\
\hline $17 / 08 / 00 / 15$ & $\begin{array}{c}\text { cataclasite with graphite } \\
\text { network structures }\end{array}$ & Farm Epako Süd & 8 \\
\hline $17 / 08 / 00 / 16$ & $\begin{array}{c}\text { cataclasite with graphite } \\
\text { network structures }\end{array}$ & Farm Epako Süd & 9 \\
\hline $17 / 08 / 00 / 19$ & $\begin{array}{c}\text { cataclasite with graphite } \\
\text { network structures }\end{array}$ & Farm Epako Süd & 10 \\
\hline $17 / 08 / 00 / 20$ & $\begin{array}{c}\text { cataclasite with graphite } \\
\text { network structures }\end{array}$ & Farm Epako Süd & 11 \\
\hline $18 / 08 / 00 / 02$ & $\begin{array}{c}\text { cataclasite with graphite } \\
\text { network structures }\end{array}$ & MT-Station 94 & 12 \\
\hline $3.10 .01 / 3$ & mylonite & Farm Elim & 13 \\
\hline $3.10 .01 / 4$ & mylonite & Farm Elim & 14 \\
\hline Elim Spatkristall & grain coarsened marble & Farm Elim & Elim \\
\hline Omaruru Spatkristall & grain coarsened marble & Omaruru River near Farm & \\
\hline
\end{tabular}




\section{Conductivity Measurements}

\section{III.1 COMPLEX IMPEDANCE DATA FOR SAMPLE 12/08/00/03:}

\begin{tabular}{|c|c|c|c|c|}
\hline $\begin{array}{l}\text { frequency } \\
\qquad[\mathrm{Hz}]\end{array}$ & $\begin{array}{c}\text { measurement } \\
1\end{array}$ & $\begin{array}{c}\text { phase } \\
\text { difference } \\
{\left[{ }^{\circ}\right]} \\
\end{array}$ & $\begin{array}{c}\text { real impedance } \\
\qquad Z^{\prime}[\Omega \mathrm{m}]\end{array}$ & $\begin{array}{c}\text { imaginary } \\
\text { impedance } \\
Z^{\prime \prime}[\Omega \mathrm{m}]\end{array}$ \\
\hline 785,412918011400 & 159120,000000000000 & 4,4000000000 & 158651,033934348000 & 12207,53175553940 \\
\hline 881,247788255900 & 160004,000000000000 & 4,6000000000 & 159488,607668513000 & 12832,14868831470 \\
\hline 988,776281235900 & 161772,000000000000 & 4,8000000000 & 161204,642826511000 & 13536,73207155530 \\
\hline 1109,425234722800 & 163540,000000000000 & 5,0000000000 & 162917,680925924000 & 14253,45016895200 \\
\hline 1244,795587027200 & 164424,000000000000 & 5,3000000000 & 163721,037774302000 & 15187,94147031340 \\
\hline 1396,683620478200 & 166192,000000000000 & 5,5000000000 & 165426,884999038000 & 15928,79730 \\
\hline 1567,104796997800 & 167076,000000000000 & 5,8000000000 & 166220,689308279000 & 16884,0819 \\
\hline 1758,320502056700 & 168844,000000000000 & 6,1000000000 & 167887,995839227000 & 17942,05086 \\
\hline 1972,868051885100 & 172380,000000000000 & 6,5000000000 & 171271,916481530000 & 19513,9699 \\
\hline 2213,594362117900 & 175916,000000000000 & 7,0000000000 & 174604,748812135000 & 21438,7 \\
\hline 2483,693724635000 & 177684,000000000000 & 7,5000000000 & 176163,888748344000 & 23192,41593842 \\
\hline 2786,750193874500 & 178568,000000000000 & 8,0000000000 & 176830,188499045000 & 24851,86229223700 \\
\hline 3126,785145056700 & 179452,000000000000 & 8,5000000000 & 177480,874712023000 & 26524,69444603090 \\
\hline 3508,310635390900 & 180336,000000000000 & 9,0000000000 & 178115,764589565000 & 28210,76568749510 \\
\hline 3936,389276332400 & 180336,000000000000 & 9,6000000000 & 177810,581341147000 & 30074,40870779500 \\
\hline 4416,701411361400 & 181220,000000000000 & 10,300000000 & 178299,646574316000 & 32402,53742338490 \\
\hline 4955,620490689000 & 181220,000000000000 & 11,000000000 & 177890,478184386000 & 34578,40614213740 \\
\hline 5560,297643070000 & 181220,000000000000 & 11,700000000 & 177454,757740876000 & 36749,11366450720 \\
\hline 6238,756566936200 & 182104,000000000000 & 12,400000000 & 177855,928573765000 & 39104,162 \\
\hline 7000,000000000000 & 183872,000000000000 & 13,500000000 & 178791,602003362000 & 42924,0659429 \\
\hline 7854,129180113700 & 182988,000000000000 & 14,300000000 & 177318,250658680000 & 45197,8553401 \\
\hline 8812,477882559200 & 182104,000000000000 & 15,300000000 & 175649,764130839000 & 48052,33789089430 \\
\hline 9887,762812359300 & 180336,000000000000 & 16,200000000 & 173175,522100237000 & 50312,14009869040 \\
\hline 11094,252347227800 & 179452,000000000 & 0000000 & 171426,612639579000 & 53065,40099650430 \\
\hline 12447,955870272400 & 176800,000000000000 & 18,100000000 & 168051,181351792000 & 54927,59275871340 \\
\hline 13966,836204782100 & 175032,000000000000 & 19,100000000 & 165396,297992757000 & 57273,60329411050 \\
\hline 15671,047969978300 & 173264,000000000000 & 20,100000000 & 162711,226494938000 & 59543,85332268220 \\
\hline 17583,205020567000 & 170612,000000000000 & 21,200000000 & 159065,628372981000 & 61697,49115084590 \\
\hline 19728,680518851200 & 167076,000000000000 & 22,200000000 & 154690,753827715000 & 63128,12729848220 \\
\hline 22135,943621178600 & 163540,000000000000 & 23,200000000 & 150315,393381801000 & 64425,25989450410 \\
\hline 24836,937246350300 & 160004,000000000000 & 24,200000000 & 145942,867068028000 & 65589,32510678700 \\
\hline 27867,501938744800 & 156468,000000000000 & 25,300000000 & 141459,988380323000 & 66867,83016846720 \\
\hline 31267,851450567400 & 152932,000000000000 & 26,400000000 & 136982,990916934000 & 67998,94722310930 \\
\hline 35083,106353909100 & 148512,000000000000 & 27,500000000 & 131731,752856964000 & 68575,21004876130 \\
\hline 39363,892763324500 & 144976,000000000000 & 28,500000000 & 127407,389725281000 & 69176,56842739700 \\
\hline 44167,014113613600 & 140556,000000000000 & 29,700000000 & 122091,371143374000 & 69639,68860018550 \\
\hline 49556,204906889700 & 136136,000000000000 & 30,800000000 & 116935,364528553000 & 69707,46745202010 \\
\hline 55602,976430699800 & 130832,000000000000 & 32,000000000 & 110951,828516338000 & 69330,39717815870 \\
\hline 62387,565669362300 & 126412,000000000000 & 33,100000000 & 105897,698405423000 & 69033,84109575600 \\
\hline 70000,000000000100 & 121992,000000000000 & 34,200000000 & 100897,213416902000 & 68569,67543093710 \\
\hline
\end{tabular}




\begin{tabular}{|c|c|c|c|c|}
\hline $\begin{array}{l}\text { frequency } \\
\qquad[\mathrm{Hz}]\end{array}$ & $\begin{array}{c}\text { measurement } \\
1\end{array}$ & $\begin{array}{c}\text { phase } \\
\text { difference } \\
{\left[{ }^{\circ}\right]} \\
\end{array}$ & $\begin{array}{l}\text { real impedance } \\
\quad Z^{\prime}[\Omega \mathrm{m}]\end{array}$ & $\begin{array}{c}\text { imaginary } \\
\text { impedance } \\
\mathbf{Z}^{\prime \prime}[\Omega \mathrm{m}]\end{array}$ \\
\hline 78541,291801137600 & 117572,000000000000 & 35,500000000 & 95717,189724189200 & 68274,40790884660 \\
\hline 88124,778825591900 & 112268,000000000000 & 36,600000000 & 90130,712304756000 & 66936,97426263980 \\
\hline 98877,628123593000 & 107848,000000000000 & 37,700000000 & 85331,875583477900 & 65951,96822996150 \\
\hline 110942,523472278000 & 103428,000000000000 & 38,900000000 & 80492,132365749300 & 64948,96312655570 \\
\hline 124479,558702724000 & 98124,000000000000 & 40,200000000 & 74946,721513745600 & 63334,89015022490 \\
\hline 139668,362047821000 & 93942,680000000000 & 41,430000000 & 70434,905732656800 & 62162,29709409190 \\
\hline 156710,479699 & 89337,040000000 & 42,760000000 & 65591,614364967200 & 60653,49817577740 \\
\hline 175832,050205670 & 3,720000000000 & 44,040000000 & 60896,852561569800 & 58889,62306156180 \\
\hline 197286,805188511000 & 80134,600000000000 & 45,300000000 & 56366,253151838600 & 56959,63151902270 \\
\hline 221359,436211786000 & 75767,640000000000 & 46,560000000 & 52097,419219758500 & 55014,49065301200 \\
\hline 248369,372463502000 & 71542,120000000000 & 47,880000000 & 47982,267998859200 & 53065,77891428760 \\
\hline 278675,019387447000 & 67325,440000000000 & 49,090000000 & 44089,594358757300 & 50880,47307635650 \\
\hline 312678,514505673000 & 63259,040000000000 & 50,330000000 & 40382,349055193700 & 48692,62805092880 \\
\hline 350831,063539090000 & 59325,240000000000 & 51,560000000 & 36882,190257027200 & 46467,06514190500 \\
\hline 393638,927633243000 & 55603,600000000000 & 52,860000000 & 33571,488611337400 & 44325,11122804810 \\
\hline 441670,141136134000 & 51996,880000000000 & 54,130000000 & 30467,475099205100 & 42135,59648104820 \\
\hline 495562,049068895000 & 48513,920000000000 & 55,370000000 & 27569,231412155200 & 39919,14218904790 \\
\hline 556029,764306995000 & 45154,720000000000 & 56,620000000 & 24843,643322244900 & 37705,99587274520 \\
\hline 623875,656693619000 & 41945,800000000000 & 57,840000000 & 22327,136595155200 & 35509,84524186610 \\
\hline 699999,999999997000 & 38878,320000000000 & 58,790000000 & 20145,823646590000 & 33251,60982002070 \\
\hline 785412,918011371000 & 36058,360000000000 & 60,290000000 & 17870,893379747600 & 31318,30927268100 \\
\hline 881247,788255913000 & 33291,440000000000 & 61,300000000 & 15987,331751720100 & 29201,45888023480 \\
\hline 988776,281235923000 & 30736,680000000000 & 62,590000000 & 14149,776046779200 & 27286,02820581250 \\
\hline
\end{tabular}




\begin{tabular}{|c|c|c|c|c|}
\hline $\begin{array}{l}\text { frequency } \\
\quad[\mathrm{Hz}]\end{array}$ & $\begin{array}{c}\text { measurement } \\
1\end{array}$ & $\mid \begin{array}{c}\text { phase difference } \\
{\left[{ }^{\circ}\right]}\end{array}$ & $\begin{array}{c}\text { real admittance } \\
\mathbf{Y}^{\prime}[\mathrm{S} \mathbf{m}-1]\end{array}$ & $\begin{array}{l}\text { imaginary } \\
\text { admittance } \\
Y^{\prime \prime}[S \text { m-1] }\end{array}$ \\
\hline 785,412918011400 & 159120,000000000000 & 4,4000000000 & 0,000006303142 & 0,000081916641 \\
\hline 881,247788255900 & 160004,000000000000 & 4,6000000000 & 0,000006270040 & 0,000077929272 \\
\hline 988,776281235900 & 161772,000000000000 & 4,8000000000 & 0,000006203295 & 0,000073873073 \\
\hline 1109,425234722800 & 163540,000000000000 & 5,0000000000 & 0,000006138069 & 0,000070158452 \\
\hline 1244,795587027200 & 164424,000000000000 & 5,3000000000 & 0,000006107951 & 0,000065841708 \\
\hline 1396,683620478200 & 166192,000000000000 & 5,5000000000 & 0,000006044967 & 0,000062779379 \\
\hline 1567,104796997800 & 167076,000000000000 & 5,8000000000 & 0,000006016098 & 0,000059227384 \\
\hline 1758,320502056700 & 168844,000000000000 & 6,1000000000 & 0,000005956352 & 0,000055734989 \\
\hline 1972,868051885100 & 172380,000000000000 & 6,5000000000 & 0,000005838669 & 0,000051245339 \\
\hline 2213,594362117900 & 175916,000000000000 & 7,0000000000 & 0,000005727221 & 0,000046644473 \\
\hline 2483,693724635000 & 177684,000000000000 & 7,5000000000 & 0,000005676532 & 0,000043117543 \\
\hline 2786,750193874500 & 178568,000000000000 & 8,0000000000 & 0,000005655143 & 0,000040238433 \\
\hline 3126,785145056700 & 179452,000000000000 & 8,5000000000 & 0,000005634410 & 0,000037700717 \\
\hline 3508,310635390900 & 180336,000000000000 & 9,0000000000 & 0,000005614326 & 0,000035447460 \\
\hline 3936,389276332400 & 180336,000000000000 & 9,6000000000 & 0,000005623962 & 0,000033250862 \\
\hline 4416,701411361400 & 181220,000000000000 & 10,3000000000 & 0,000005608536 & 0,000030861781 \\
\hline 4955,620490689000 & 181220,000000000000 & 11,0000000000 & 0,000005621436 & 0,000028919783 \\
\hline 5560,297643070000 & 181220,000000000000 & 11,7000000000 & 0,000005635239 & 0,000027211541 \\
\hline 6238,756566936200 & 182104,000000000000 & 12,4000000000 & 0,000005622528 & 0,000025572725 \\
\hline 7000,000000000000 & 183872,000000000000 & 13,5000000000 & 0,000005593104 & 0,000023296954 \\
\hline 7854,129180113700 & 182988,000000000000 & 14,3000000000 & 0,000005639577 & 0,000022124944 \\
\hline 8812,477882559200 & 182104,000000000000 & 15,3000000000 & 0,000005693147 & 0,000020810642 \\
\hline 9887,762812359300 & 180336,000000000000 & 16,2000000000 & 0,000005774488 & 0,000019875919 \\
\hline 11094,252347227800 & 179452,000000000000 & 17,2000000000 & 0,000005833400 & 0,000018844671 \\
\hline 12447,955870272400 & 176800,000000000000 & 18,1000000000 & 0,000005950568 & 0,000018205786 \\
\hline 13966,836204782100 & 175032,000000000000 & 19,1000000000 & 0,000006046085 & 0,000017460050 \\
\hline 15671,047969978300 & 173264,000000000000 & 20,1000000000 & 0,000006145857 & 0,000016794345 \\
\hline 17583,205020567000 & 170612,000000000000 & 21,2000000000 & 0,000006286713 & 0,000016208114 \\
\hline 19728,680518851200 & 167076,000000000000 & 22,2000000000 & 0,000006464510 & 0,000015840799 \\
\hline 22135,943621178600 & 163540,000000000000 & 23,2000000000 & 0,000006652679 & 0,000015521862 \\
\hline 24836,937246350300 & 160004,000000000000 & 24,2000000000 & 0,000006851996 & 0,000015246383 \\
\hline 27867,501938744800 & 156468,000000000000 & 25,3000000000 & 0,000007069137 & 0,000014954874 \\
\hline 31267,851450567400 & 152932,000000000000 & 26,4000000000 & 0,000007300176 & 0,000014706110 \\
\hline 35083,106353909100 & 148512,000000000000 & 27,5000000000 & 0,000007591184 & 0,000014582529 \\
\hline 39363,892763324500 & 144976,000000000000 & 28,5000000000 & 0,000007848838 & 0,000014455762 \\
\hline 44167,014113613600 & 140556,000000000000 & 29,7000000000 & 0,000008190587 & 0,000014359628 \\
\hline 49556,204906889700 & 136136,000000000000 & 30,8000000000 & 0,000008551733 & 0,000014345665 \\
\hline 55602,976430699800 & 130832,000000000000 & 32,0000000000 & 0,000009012920 & 0,000014423688 \\
\hline 62387,565669362300 & 126412,000000000000 & 33,1000000000 & 0,000009443076 & 0,000014485649 \\
\hline 70000,000000000100 & 121992,000000000000 & 34,2000000000 & 0,000009911076 & 0,000014583706 \\
\hline 78541,291801137600 & 117572,000000000000 & 35,5000000000 & 0,000010447444 & 0,000014646777 \\
\hline 88124,778825591900 & 112268,000000000000 & 36,6000000000 & 0,000011094997 & 0,000014939426 \\
\hline 98877,628123593000 & 107848,000000000000 & 37,7000000000 & 0,000011718950 & 0,000015162550 \\
\hline 110942,523472278000 & 103428,000000000000 & 38,9000000000 & 0,000012423574 & 0,000015396705 \\
\hline 124479,558702724000 & 98124,000000000000 & 40,2000000000 & 0,000013342812 & 0,000015789086 \\
\hline 139668,362047821000 & 93942,680000000000 & 41,4300000000 & 0,000014197506 & 0,000016086922 \\
\hline 156710,479699784000 & 89337,040000000000 & 42,7600000000 & 0,000015245851 & 0,000016487095 \\
\hline
\end{tabular}




\begin{tabular}{|c|c|c|c|c|}
\hline $\begin{array}{c}\text { frequency } \\
{[\mathbf{H z}]}\end{array}$ & $\begin{array}{c}\text { measurement } \\
\mathbf{1}\end{array}$ & $\begin{array}{c}\text { phase difference } \\
{\left[{ }^{\circ} \text { ] }\right.}\end{array}$ & $\begin{array}{c}\text { real admittance } \\
\mathbf{Y}^{\prime} \text { [S m-1] }\end{array}$ & $\begin{array}{c}\text { imaginary } \\
\text { admittance } \\
\mathbf{Y}^{\prime \prime} \text { [S m-1] }\end{array}$ \\
\hline 175832,050205670000 & 84713,720000000000 & 44,0400000000 & 0,000016421210 & 0,000016980920 \\
\hline 197286,805188511000 & 80134,600000000000 & 45,3000000000 & 0,000017741112 & 0,000017556293 \\
\hline 221359,436211786000 & 75767,640000000000 & 46,5600000000 & 0,000019194809 & 0,000018177029 \\
\hline 248369,372463502000 & 71542,120000000000 & 47,8800000000 & 0,000020841032 & 0,000018844536 \\
\hline 278675,019387447000 & 67325,440000000000 & 49,0900000000 & 0,000022681089 & 0,000019653905 \\
\hline 312678,514505673000 & 63259,040000000000 & 50,3300000000 & 0,000024763294 & 0,000020536990 \\
\hline 350831,063539090000 & 59325,240000000000 & 51,5600000000 & 0,000027113357 & 0,000021520619 \\
\hline 393638,927633243000 & 55603,600000000000 & 52,8600000000 & 0,000029787181 & 0,000022560575 \\
\hline 441670,141136134000 & 51996,880000000000 & 54,1300000000 & 0,000032821886 & 0,000023732902 \\
\hline 495562,049068895000 & 48513,920000000000 & 55,3700000000 & 0,000036272321 & 0,000025050638 \\
\hline 556029,764306995000 & 45154,720000000000 & 56,6200000000 & 0,000040251745 & 0,000026520981 \\
\hline 623875,656693619000 & 41945,800000000000 & 57,8400000000 & 0,000044788547 & 0,000028161204 \\
\hline 699999,999999997000 & 38878,320000000000 & 58,7900000000 & 0,000049638080 & 0,000030073732 \\
\hline 785412,918011371000 & 36058,360000000000 & 60,2900000000 & 0,000055956912 & 0,000031930204 \\
\hline 881247,788255913000 & 33291,440000000000 & 61,3000000000 & 0,000062549525 & 0,000034244864 \\
\hline 988776,281235923000 & 30736,680000000000 & 62,5900000000 & 0,000070672497 & 0,000036648793 \\
\hline
\end{tabular}




\begin{tabular}{|c|c|c|c|c|}
\hline $\begin{array}{l}\text { frequency } \\
\qquad[\mathrm{Hz}]\end{array}$ & $\begin{array}{c}\text { measurement } \\
2\end{array}$ & $\begin{array}{c}\text { phase } \\
\text { difference } \\
{\left[{ }^{\circ}\right]}\end{array}$ & $\begin{array}{l}\text { real impedance } \\
\quad Z^{\prime}[\Omega \mathbf{~ m}]\end{array}$ & $\begin{array}{c}\text { imaginary impedance } \\
Z^{\prime \prime}[\Omega \mathrm{m}]\end{array}$ \\
\hline 785,412918011400 & 10156,6664206088 & 8,0000000000 & 454794,474322529000 & 63917,19503932350 \\
\hline 881,247788255900 & 10676,3477086778 & 8,1900000000 & 452932,987605396000 & 65187,94933766660 \\
\hline 988,776281235900 & 11262,5610835340 & 8,6000000000 & 449164,338730985000 & 67929,71953686080 \\
\hline 1109,425234722800 & 11858,8705405681 & 8,9000000000 & 446336,556317832000 & 69894,43948082850 \\
\hline 1244,795587027200 & 12636,3673033007 & 9,3000000000 & 442553,472311200000 & 72470,91036616010 \\
\hline 1396,683620478200 & 13252,7593559638 & 9,6900000000 & 439589,540837530000 & 75061,45409096340 \\
\hline 1567,104796997800 & 14047,5561475707 & 10,200000000 & 435628,596781778000 & 78381,95609921520 \\
\hline 1758,320502056700 & 14927,7863160982 & 10,700000000 & 431657,871070424000 & 81562,60147242250 \\
\hline 1972,868051885100 & 16235,6230311074 & 11,200000000 & 428481,211856527000 & 84841,56461288380 \\
\hline 2213,594362117900 & 17837,0544888306 & 11,700000000 & 423650,956787401000 & 87733,89546304160 \\
\hline 2483,693724635000 & 19296,0900607718 & 12,300000000 & 417831,585816092000 & 91102,01862972120 \\
\hline 2786,750193874500 & 20676,7494271412 & 12,900000000 & 411988,667292085000 & 94358,00103272470 \\
\hline 3126,785145056700 & 22068,5457790977 & 13,400000000 & 405484,114865779000 & 96599,94210927660 \\
\hline 3508,310635390900 & 23471,3570519959 & 14,100000000 & 397818,223741436000 & 99925,03107434360 \\
\hline 3936,389276332400 & 25021,9080448854 & 14,800000000 & 390936,970828990000 & 103290,0031128530 \\
\hline 4416,701411361400 & 26958,9111362562 & 15,500000000 & 383231,976719256000 & 106279,6332128180 \\
\hline 4955,620490689000 & 28769,2339102583 & 16,200000000 & 376312,207193595000 & 109328,8027058050 \\
\hline 5560,297643070000 & 30575,2625688700 & 17,000000000 & 367588,247316095000 & 112383,0053481440 \\
\hline 6238,756566936200 & 32534,6627993345 & 17,800000000 & 359645,931261703000 & 115469,6761531040 \\
\hline 7000,000000000000 & 35712,8228645036 & 18,600000000 & 350113,232804821000 & 117826,1205291810 \\
\hline 7854,129180113700 & 37604,6156429709 & 19,500000000 & 341160,808456081000 & 120811,3784938860 \\
\hline 8812,477882559200 & 39979,5451252241 & 20,300000000 & 331637,527278767000 & 122676,4463963030 \\
\hline 9887,762812359300 & 41859,7005621104 & 21,300000000 & 321694,347060738000 & 125423,3848577100 \\
\hline 11094,252347227800 & 44150,4136290915 & 22,200000000 & 311211,027931014000 & 127002,8679917180 \\
\hline 12447,955870272400 & 45699,7571752495 & 23,200000000 & 301299,917290580000 & 129137,3094991900 \\
\hline 13966,836204782100 & 47651,6379406999 & 24,200000000 & 290652,547738992000 & 130624,4342718200 \\
\hline 15671,047969978300 & 49540,4859644716 & 25,300000000 & 279817,165450210000 & 132268,9681497900 \\
\hline 17583,205020567000 & 51332,3126375038 & 26,300000000 & 269261,492338527000 & 133077,3183064240 \\
\hline 19728,680518851200 & 52522,6019123372 & 27,400000000 & 258531,840151720000 & 134010,1773290580 \\
\hline 22135,943621178600 & 53601,8162322274 & 28,500000000 & 247137,433154347000 & 134184,6779251660 \\
\hline 24836,937246350300 & 54570,3184888468 & 29,700000000 & 236323,364344112000 & 134796,4671683950 \\
\hline 27867,501938744800 & 55634,0347001647 & 30,900000000 & 224881,650492053000 & 134588,8913394050 \\
\hline 31267,851450567400 & 56575,1240896269 & 32,000000000 & 213789,532848650000 & 133590,5268361340 \\
\hline 35083,106353909100 & 57054,5747605694 & 33,200000000 & 202590,681460176000 & 132571,6271662280 \\
\hline 39363,892763324500 & 57554,9049315943 & 34,400000000 & 191531,946128344000 & 131144,6605710240 \\
\hline 44167,014113613600 & 57940,2209153543 & 35,700000000 & 181075,136492227000 & 130115,6851433420 \\
\hline 49556,204906889700 & 57996,6129200807 & 36,900000000 & 170326,434780482000 & 127884,7046326110 \\
\hline 55602,976430699800 & 57682,8904522280 & 38,200000000 & 159535,236169752000 & 125541,8515247460 \\
\hline 62387,565669362300 & 57436,1557916690 & 39,500000000 & 149584,055237210000 & 123307,5794701660 \\
\hline 70000,000000000100 & 57049,9699585397 & 40,700000000 & 139399,676665335000 & 119902,6377091020 \\
\hline 78541,291801137600 & 56804,3073801604 & 42,000000000 & 129842,263907410000 & 116910,4995430200 \\
\hline 88124,778825591900 & 55691,5625865163 & 43,300000000 & 120495,879939789000 & 113549,5730574800 \\
\hline 98877,628123593000 & 54872,0375673279 & 44,700000000 & 111771,795667580000 & 110607,4102275460 \\
\hline 110942,523472278000 & 54037,5373212943 & 46,000000000 & 102876,126031496000 & 106531,3470709530 \\
\hline 124479,558702724000 & 52694,6286049871 & 47,400000000 & 94611,015538363600 & 102888,7064492480 \\
\hline 139668,362047821000 & 51719,0311822845 & 48,800000000 & 86588,681669361300 & 98909,45426379630 \\
\hline 156710,479699784000 & 50463,7104822468 & 50,200000000 & 79353,119225751000 & 95242,57185284190 \\
\hline
\end{tabular}




\begin{tabular}{|c|c|c|c|c|}
\hline $\begin{array}{c}\text { frequency } \\
{[\mathbf{H z}]}\end{array}$ & $\begin{array}{c}\text { measurement } \\
\mathbf{2}\end{array}$ & $\begin{array}{c}\text { phase } \\
\text { difference } \\
{\left[{ }^{\circ}\right]}\end{array}$ & $\begin{array}{c}\text { real impedance } \\
\mathbf{Z}^{\prime}[\mathbf{\Omega} \mathbf{~ m}]\end{array}$ & $\begin{array}{c}\text { imaginary impedance } \\
\mathbf{Z}^{\prime \prime}[\mathbf{\Omega} \text { m] }\end{array}$ \\
\hline 175832,050205670000 & 48996,1663872194 & 51,600000000 & 71834,498493626000 & 90632,58095281870 \\
\hline 197286,805188511000 & 47390,4134238269 & 53,000000000 & 65092,312904125600 & 86380,41676671520 \\
\hline 221359,436211786000 & 45772,0562233060 & 54,200000000 & 58888,859056313000 & 81651,41678529350 \\
\hline 248369,372463502000 & 44150,7280566873 & 55,500000000 & 53251,248770725100 & 77481,04774948740 \\
\hline 278675,019387447000 & 42332,5535995286 & 56,780000000 & 47664,636205540600 & 72783,73596079740 \\
\hline 312678,514505673000 & 40512,2665383728 & 58,020000000 & 42760,337184282400 & 68484,02693829320 \\
\hline 350831,063539090000 & 38660,5981980650 & 59,250000000 & 38255,848576116500 & 64302,30026382290 \\
\hline 393638,927633243000 & 36878,4925417360 & 60,450000000 & 34118,787646768700 & 60182,15199006020 \\
\hline 441670,141136134000 & 35056,8162722321 & 61,630000000 & 30357,857793125200 & 56216,01701588960 \\
\hline 495562,049068895000 & 33212,7263012879 & 62,760000000 & 26954,580888031400 & 52358,00014355180 \\
\hline 556029,764306995000 & 31371,3885661240 & 63,880000000 & 23856,479077356000 & 48654,13744192030 \\
\hline 623875,656693619000 & 29544,1912412326 & 64,970000000 & 21075,028140770300 & 45133,82980704540 \\
\hline 699999,999999997000 & 27665,3393702572 & 65,740000000 & 18801,776312341400 & 41718,95022050140 \\
\hline 785412,918011371000 & 26056,8333148706 & 67,060000000 & 16334,291564378200 & 38593,49650595920 \\
\hline 881247,788255913000 & 24295,6137883554 & 67,890000000 & 14430,095985142700 & 35519,25552053660 \\
\hline 988776,281235923000 & 22701,9754672360 & 69,060000000 & 12533,242292735200 & 32752,68799765290 \\
\hline
\end{tabular}




\begin{tabular}{|c|c|c|c|c|}
\hline $\begin{array}{l}\text { frequency } \\
\text { [Hz] }\end{array}$ & $\begin{array}{c}\text { measurement } \\
2\end{array}$ & $\begin{array}{c}\text { phase difference } \\
{\left[{ }^{\circ}\right]}\end{array}$ & $\begin{array}{l}\text { real admittance } \\
\mathbf{Y}^{\prime}[\mathbf{S} \mathrm{m}-1]\end{array}$ & $\begin{array}{l}\text { imaginary } \\
\text { admittance } \\
Y^{\prime \prime}[\text { S m-1] }\end{array}$ \\
\hline 785,412918011400 & 10156,6664206088 & 8,0000000000 & 0,000002198795 & 0,000015645242 \\
\hline 881,247788255900 & 10676,3477086778 & & & \\
\hline 988,776281235900 & 11262,5610835340 & & & \\
\hline 1109,425234722800 & 881 & & & \\
\hline 1244,795587027200 & 007 & & & \\
\hline 1396,683620478200 & 13252,7593559638 & & & \\
\hline 1567,104796997800 & 14047,5561475707 & & & \\
\hline 1758,320502056700 & 14927,7863160982 & & & \\
\hline 1972,868051885100 & 16235,623 & & & \\
\hline & & & & \\
\hline & & & & \\
\hline & & & & \\
\hline & & & & \\
\hline 3508,310635390900 & 23471 & & & \\
\hline 3936,389276332400 & 25021, & & & \\
\hline 4416,701411361400 & & & & \\
\hline & & & & \\
\hline 5560,2 & 305 & & & \\
\hline & & & & \\
\hline & & & & \\
\hline 7854 & & & & \\
\hline 8812,4 & & & & \\
\hline 9887,76 & & & & \\
\hline & & & & \\
\hline & & & & \\
\hline & & & & \\
\hline & & & & \\
\hline 17583,2 & & & & \\
\hline 19728,680518851200 & & & & \\
\hline 22135,943621178600 & 53601 & & & \\
\hline 24836,937246350300 & 54570,3184 & & & \\
\hline 27867,501938744800 & & & & \\
\hline & & & & \\
\hline & & & & \\
\hline 1500 & & & & \\
\hline 44167,014113613600 & & & 0,000005522569 & 0,000007685469 \\
\hline 49556,204906889700 & 57996,6129200807 & & 0,000005871079 & 0,000007819543 \\
\hline 55602,976430699800 & 57682,890452 & & 0,000006268208 & 0,000007965471 \\
\hline 62387,565669362300 & 57436,1557916690 & & & \\
\hline & & & & \\
\hline 78541,291801137600 & 56804 & & & \\
\hline 88124,7788 & 55691, & & & 6726 \\
\hline 98877,628123593000 & 54872,0375673279 & & 0,000008946801 & 0,000009040986 \\
\hline 110942,523472278000 & 54037,5373212943 & 46,0000000000 & 0,000009720428 & 0,000009386908 \\
\hline 124479,558702724000 & 52694,6286049871 & 47,4000000000 & 0,000010569594 & 0,000009719240 \\
\hline 139668,36204782100 & 51719,0311822845 & & & 0,000010110257 \\
\hline 156710,479699784000 & 50463,7104822468 & 50,2000000000 & 0,000012601899 & 0,000010499506 \\
\hline
\end{tabular}




\begin{tabular}{|c|c|c|c|c|}
\hline $\begin{array}{c}\text { frequency } \\
{[\mathbf{H z}]}\end{array}$ & $\begin{array}{c}\text { measurement } \\
\mathbf{2}\end{array}$ & $\begin{array}{c}\text { phase difference } \\
\text { ['] }\end{array}$ & $\begin{array}{c}\text { real admittance } \\
\mathbf{Y}^{\prime} \text { [S m-1] }\end{array}$ & $\begin{array}{c}\text { imaginary } \\
\text { admittance } \\
\mathbf{Y}^{\prime \prime}[\mathbf{S} \text { m-1] }\end{array}$ \\
\hline 175832,050205670000 & 48996,1663872194 & 51,6000000000 & 0,000013920888 & 0,000011033560 \\
\hline 197286,805188511000 & 47390,4134238269 & 53,0000000000 & 0,000015362797 & 0,000011576698 \\
\hline 221359,436211786000 & 45772,0562233060 & 54,2000000000 & 0,000016981141 & 0,000012247185 \\
\hline 248369,372463502000 & 44150,7280566873 & 55,5000000000 & 0,000018778902 & 0,000012906382 \\
\hline 278675,019387447000 & 42332,5535995286 & 56,7800000000 & 0,000020979915 & 0,000013739333 \\
\hline 312678,514505673000 & 40512,2665383728 & 58,0200000000 & 0,000023386158 & 0,000014601945 \\
\hline 350831,063539090000 & 38660,5981980650 & 59,2500000000 & 0,000026139794 & 0,000015551543 \\
\hline 393638,927633243000 & 36878,4925417360 & 60,4500000000 & 0,000029309365 & 0,000016616222 \\
\hline 441670,141136134000 & 35056,8162722321 & 61,6300000000 & 0,000032940401 & 0,000017788525 \\
\hline 495562,049068895000 & 33212,7263012879 & 62,7600000000 & 0,000037099445 & 0,000019099278 \\
\hline 556029,764306995000 & 31371,3885661240 & 63,8800000000 & 0,000041917334 & 0,000020553237 \\
\hline 623875,656693619000 & 29544,1912412326 & 64,9700000000 & 0,000047449521 & 0,000022156329 \\
\hline 699999,999999997000 & 27665,3393702572 & 65,7400000000 & 0,000053186464 & 0,000023969922 \\
\hline 785412,918011371000 & 26056,8333148706 & 67,0600000000 & 0,000061220898 & 0,000025911101 \\
\hline 881247,788255913000 & 24295,6137883554 & 67,8900000000 & 0,000069299608 & 0,000028153743 \\
\hline 988776,281235923000 & 22701,9754672360 & 69,0600000000 & 0,000079787814 & 0,000030531845 \\
\hline
\end{tabular}




\begin{tabular}{|c|c|c|c|c|}
\hline $\begin{array}{c}\text { frequency } \\
{[\text { [Hz] }}\end{array}$ & $\begin{array}{c}\text { measurement } \\
3\end{array}$ & $\begin{array}{c}\text { phase } \\
\text { difference } \\
{\left[{ }^{\circ}\right]} \\
\end{array}$ & $\begin{array}{l}\text { real impedance } \\
\quad Z^{\prime}[\Omega \mathrm{m}]\end{array}$ & $\begin{array}{c}\text { imaginary } \\
\text { impedance } \\
\mathrm{Z}^{\prime \prime}[\Omega \mathrm{m}]\end{array}$ \\
\hline 785,412918011400 & 596980,000000000000 & 6,9000000000 & 592656,277985040000 & 71719,2900475076 \\
\hline 881,247788255900 & 590696,000000000000 & 7,1000000000 & 586166,506381207000 & \\
\hline 988,776281235900 & 584412,000000000000 & & & \\
\hline 1109,425234722800 & 579699,000000000000 & 7,5000000000 & 574739,5946 & \\
\hline 1244,795587027200 & 574986,000000000000 & 7,8000000000 & 569666,137801313000 & \\
\hline 1396,683620478200 & 568702,000000000000 & 8,0000000000 & 563167,431229469000 & 79148,0208621911 \\
\hline 1567,104796997800 & 563989,000000000000 & 8,3000000000 & 558081,660251219000 & \\
\hline 1758,320502056700 & 559276,000000000000 & 8,6000000000 & & \\
\hline 1972,868051885100 & & & & \\
\hline & 548279,00000 & & & \\
\hline & 5435 & & & \\
\hline & 5388 & & & \\
\hline 3126,7 & 53256 & & & \\
\hline 3508,3 & 526285,00000 & & & \\
\hline 3936,3 & 521 & & & \\
\hline 4416,70 & 5152 & & & \\
\hline 4955,62 & & & & \\
\hline & & & & \\
\hline & & & & \\
\hline 7000 , & & & & \\
\hline 7854,1 & 4822 & & & \\
\hline 8812,4 & 474 & & & \\
\hline 9887,76 & 4681 & & & \\
\hline 2347227800 & 4603 & & & \\
\hline & & & & \\
\hline & & & & \\
\hline & & & & \\
\hline & & & & \\
\hline & 4163 & & & \\
\hline & 40688 & & & \\
\hline 246350300 & 39589 & 28,4 & & \\
\hline & & & & \\
\hline & 372 & & & \\
\hline & & & & \\
\hline & 344049 & & 275130,707067825000 & 206573,978103145 \\
\hline 44167,014113613600 & 329910,000000000000 & 39,30 & 255297,623590872000 & 208958,683688555 \\
\hline 889700 & 312629,000000000000 & 41,70 & 18289700000 & 207970,300545238 \\
\hline 55602 & 29691 & 44,10 & & 206629,731651393 \\
\hline & & & & \\
\hline & & & & \\
\hline & 24507 & & & \\
\hline 88124,778825591900 & 227795,000000000000 & & 136136,020355289000 & \\
\hline 98877,628123593000 & 212085,000000000000 & 55,4000000000 & 120431,140669587000 & 174574,876720627 \\
\hline 110942,523472278000 & 196375,0000000000 & 57,6000000000 & 105222,986864000000 & 165804,896370458 \\
\hline 124479,55870272400 & 260000000000 & 59,8000000000 & 91668,342994107500 & 157501,982808835 \\
\hline 139668,36204782100 & & 61,9100000000 & 78469,262386722200 & 147021,621904080 \\
\hline & & & & \\
\hline
\end{tabular}




\begin{tabular}{|c|c|c|c|c|}
\hline $\begin{array}{c}\text { frequency } \\
{[\mathbf{H z}]}\end{array}$ & $\begin{array}{c}\text { measurement } \\
\mathbf{3}\end{array}$ & $\begin{array}{c}\text { phase } \\
\text { difference } \\
{\left[{ }^{\circ}\right.}\end{array}$ & $\begin{array}{c}\text { real impedance } \\
\mathbf{Z}^{\prime}[\mathbf{\Omega} \mathbf{~ m ]}\end{array}$ & $\begin{array}{c}\text { imaginary } \\
\text { impedance } \\
\mathbf{Z}^{\prime \prime}\end{array}$ \\
{$[\mathbf{\Omega} \mathbf{~ m ]}$}
\end{tabular}




\begin{tabular}{|c|c|c|c|c|}
\hline $\begin{array}{c}\text { frequency } \\
{[\mathrm{Hz}]}\end{array}$ & $\begin{array}{c}\text { measurement } \\
3\end{array}$ & $\begin{array}{c}\text { phase difference } \\
{\left[{ }^{\circ}\right]}\end{array}$ & $\begin{array}{l}\text { real admittance } \\
\mathbf{Y}^{\prime}[\mathrm{S} \text { m-1] }\end{array}$ & $\begin{array}{l}\text { imaginary } \\
\text { admittance } \\
Y^{\prime \prime}[S \text { m-1] }\end{array}$ \\
\hline 785,412918011400 & 596980,000000000000 & 6,9000000000 & $1,68732 \mathrm{E}-06$ & $1,39433 \mathrm{E}-05$ \\
\hline 881,247788255900 & 590696,000000000000 & 7,1000000000 & $1,706 \mathrm{E}-06$ & $1,36966 \mathrm{E}-05$ \\
\hline 988,776281235900 & 584412,000000000000 & 7,3000000000 & $1,7251 \mathrm{E}-06$ & $1,34665 \mathrm{E}-05$ \\
\hline 1109,425234722800 & 579699,00000000000 & 7,5000000000 & $1,73992 \mathrm{E}-06$ & $1,3216 \mathrm{E}-05$ \\
\hline 1244,795587027200 & 574986,000000000000 & 7,8000000000 & $1,75541 \mathrm{E}-06$ & $1,28148 \mathrm{E}-05$ \\
\hline 1396,683620478200 & 568702,00000000000 & 8,0000000000 & $1,77567 \mathrm{E}-06$ & $1,26346 \mathrm{E}-05$ \\
\hline 1567,104796997800 & 563989,000000000000 & 8,3000000000 & $1,79185 \mathrm{E}-06$ & $1,22827 \mathrm{E}-05$ \\
\hline 1758,320502056700 & 559276,000000000000 & 8,6000000000 & $1,80836 \mathrm{E}-06$ & 1,19572E-05 \\
\hline 1972,868051885100 & 554563,000000000000 & 9,0000000000 & $1,8257 \mathrm{E}-06$ & $1,1527 \mathrm{E}-05$ \\
\hline 2213,594362117900 & 548279,000000000000 & 9,3000000000 & & $1,12862 \mathrm{E}-05$ \\
\hline 2483,693724635000 & 543566,000000000000 & 9,6900000000 & $1,86633 \mathrm{E}-06$ & $1,093 \mathrm{E}-05$ \\
\hline 2786,750193874500 & 538853,000000000000 & 10,1000000000 & $1,88501 \mathrm{E}-06$ & $1,05824 \mathrm{E}-05$ \\
\hline 3126,785145056700 & 532569,000000000000 & 10,6000000000 & $1,91029 \mathrm{E}-06$ & $1,02075 \mathrm{E}-05$ \\
\hline 3508,310635390900 & 526285,000000000000 & 11,1000000000 & $1,93633 \mathrm{E}-06$ & $9,86958 \mathrm{E}-06$ \\
\hline 3936,389276332400 & 521572,000000000000 & 11,6000000000 & $1,95726 \mathrm{E}-06$ & $9,53501 \mathrm{E}-06$ \\
\hline 4416,701411361400 & 515288,000000000000 & 12,1000000000 & & $9,25807 \mathrm{E}-06$ \\
\hline 4955,620490689000 & 509004,000000000000 & 12,7000000000 & $2,01389 \mathrm{E}-06$ & $8,93634 \mathrm{E}-06$ \\
\hline 5560,297643070000 & 502720,000000000000 & 13,3000000000 & $2,044 \mathrm{E}-06$ & $8,64673 \mathrm{E}-06$ \\
\hline 6238,756566936200 & 494865,000000000000 & 14,0000000000 & $2,08262 \mathrm{E}-06$ & $8,35292 \mathrm{E}-06$ \\
\hline 7000,000000000000 & 488581,000000000000 & 14,7000000000 & $2,11601 \mathrm{E}-06$ & $8,06573 \mathrm{E}-06$ \\
\hline 7854,129180113700 & 482297,000000000000 & 15,5000000000 & $2,15167 \mathrm{E}-06$ & $7,75866 \mathrm{E}-06$ \\
\hline 8812,477882559200 & 474442,000000000000 & 16,4000000000 & $2,19713 \mathrm{E}-06$ & $7,46521 \mathrm{E}-06$ \\
\hline 9887,762812359300 & 468158,000000000000 & 17,3000000000 & $2,23724 \mathrm{E}-06$ & $7,18296 \mathrm{E}-06$ \\
\hline 11094,252347227800 & 460303,000000000000 & 18,4000000000 & $2,28953 \mathrm{E}-06$ & $6,88259 \mathrm{E}-06$ \\
\hline 12447,955870272400 & 452448,000000000000 & 19,5000000000 & $2,34469 \mathrm{E}-06$ & 6,62119E-06 \\
\hline 13966,836204782100 & 444593,000000000000 & 20,7000000000 & $2,40447 \mathrm{E}-06$ & $6,36325 \mathrm{E}-06$ \\
\hline 15671,047969978300 & 435167,000000000000 & 22,0000000000 & $2,47844 \mathrm{E}-06$ & $6,13435 \mathrm{E}-06$ \\
\hline 17583,205020567000 & 425741,000000000000 & 23,4000000000 & $2,55934 \mathrm{E}-06$ & $5,91429 \mathrm{E}-06$ \\
\hline 19728,680518851200 & 416315,000000000000 & 25,0000000000 & $2,65034 \mathrm{E}-06$ & $5,68368 \mathrm{E}-06$ \\
\hline 22135,943621178600 & 406889,000000000000 & 26,6000000000 & $2,7486 \mathrm{E}-06$ & $5,48883 \mathrm{E}-06$ \\
\hline 24836,937246350300 & 395892,000000000000 & 28,4000000000 & $2,87153 \mathrm{E}-06$ & $5,31079 \mathrm{E}-06$ \\
\hline 27867,501938744800 & 383324,000000000000 & 30,3000000000 & $3,02151 \mathrm{E}-06$ & $5,1707 \mathrm{E}-06$ \\
\hline 31267,851450567400 & 372327,000000000000 & 32,4000000000 & & $5,01246 \mathrm{E}-06$ \\
\hline 35083,106353909100 & 358188,000000000000 & 34,6000000000 & 3,3917E-06 & $4,91655 \mathrm{E}-06$ \\
\hline 39363,892763324500 & 344049,000000000000 & 36,9000000000 & $3,63464 \mathrm{E}-06$ & $4,84088 \mathrm{E}-06$ \\
\hline 44167,014113613600 & 329910,000000000000 & 39,3000000000 & $3,917 \mathrm{E}-06$ & $4,78564 \mathrm{E}-06$ \\
\hline 49556,204906889700 & 312629,000000000000 & 41,7000000000 & $4,28411 \mathrm{E}-06$ & $4,80838 \mathrm{E}-06$ \\
\hline 55602,976430699800 & 296919,000000000000 & 44,1000000000 & $4,68987 \mathrm{E}-06$ & $4,83957 \mathrm{E}-06$ \\
\hline 62387,565669362300 & 279638,000000000000 & 46,5000000000 & $5,19507 \mathrm{E}-06$ & $4,92994 \mathrm{E}-06$ \\
\hline 70000,000000000100 & 262357,000000000000 & 48,8000000000 & $5,78664 \mathrm{E}-06$ & $5,06582 \mathrm{E}-06$ \\
\hline 78541,291801137600 & 245076,000000000000 & 51,1000000000 & $6,49778 \mathrm{E}-06$ & $5,24305 \mathrm{E}-06$ \\
\hline 88124,778825591900 & 227795,000000000000 & 53,3000000000 & $7,34559 \mathrm{E}-06$ & $5,47524 \mathrm{E}-06$ \\
\hline 98877,628123593000 & 212085,000000000000 & 55,4000000000 & $8,3035 \mathrm{E}-06$ & $5,7282 \mathrm{E}-06$ \\
\hline 110942,523472278000 & 196375,000000000000 & 57,6000000000 & $9,50363 \mathrm{E}-06$ & $6,03118 \mathrm{E}-06$ \\
\hline 124479,558702724000 & 182236,000000000000 & 59,8000000000 & $1,09089 \mathrm{E}-05$ & $6,34913 \mathrm{E}-06$ \\
\hline 139668,362047821000 & 166651,680000000000 & 61,9100000000 & $1,27438 \mathrm{E}-05$ & $6,80172 \mathrm{E}-06$ \\
\hline 156710,479699784000 & 152088,510000000000 & 63,9500000000 & $1,49722 \mathrm{E}-05$ & $7,31861 \mathrm{E}-06$ \\
\hline
\end{tabular}




\begin{tabular}{|c|c|c|c|c|}
\hline $\begin{array}{c}\text { frequency } \\
\text { [Hz] }\end{array}$ & $\begin{array}{c}\text { measurement } \\
\mathbf{3}\end{array}$ & $\begin{array}{c}\text { phase difference } \\
{\left[{ }^{\circ} \text { ] }\right.}\end{array}$ & $\begin{array}{c}\text { real admittance } \\
\mathbf{Y}^{\prime} \text { [S m-1] }\end{array}$ & $\begin{array}{c}\text { imaginary } \\
\text { admittance } \\
\mathbf{Y}^{\prime \prime} \text { [S m-1] }\end{array}$ \\
\hline 175832,050205670000 & 138248,000000000000 & 65,5300000000 & $1,74628 \mathrm{E}-05$ & $7,94721 \mathrm{E}-06$ \\
\hline 197286,805188511000 & 125507,190000000000 & 67,0100000000 & $2,04001 \mathrm{E}-05$ & $8,65512 \mathrm{E}-06$ \\
\hline 221359,436211786000 & 114038,890000000000 & 68,1700000000 & $2,35817 \mathrm{E}-05$ & $9,44632 \mathrm{E}-06$ \\
\hline 248369,372463502000 & 103670,290000000000 & 69,2800000000 & $2,72638 \mathrm{E}-05$ & $1,0313 \mathrm{E}-05$ \\
\hline 278675,019387447000 & 94244,290000000000 & 70,3400000000 & $3,15384 \mathrm{E}-05$ & $1,12676 \mathrm{E}-05$ \\
\hline 312678,514505673000 & 85603,790000000000 & 71,3400000000 & $3,65109 \mathrm{E}-05$ & $1,23299 \mathrm{E}-05$ \\
\hline 350831,063539090000 & 77717,370000000000 & 72,3300000000 & $4,23911 \mathrm{E}-05$ & $1,35043 \mathrm{E}-05$ \\
\hline 393638,927633243000 & 70475,060000000000 & 73,2400000000 & $4,92067 \mathrm{E}-05$ & $1,48189 \mathrm{E}-05$ \\
\hline 441670,141136134000 & 63876,860000000000 & 74,0800000000 & $5,7074 \mathrm{E}-05$ & $1,62795 \mathrm{E}-05$ \\
\hline 495562,049068895000 & 57844,220000000000 & 74,9100000000 & $6,64058 \mathrm{E}-05$ & $1,79052 \mathrm{E}-05$ \\
\hline 556029,764306995000 & 52345,720000000000 & 75,7000000000 & $7,73435 \mathrm{E}-05$ & $1,97146 \mathrm{E}-05$ \\
\hline 623875,656693619000 & 47318,520000000000 & 76,4500000000 & $9,02003 \mathrm{E}-05$ & $2,17384 \mathrm{E}-05$ \\
\hline 699999,999999997000 & 42731,200000000000 & 76,8100000000 & 0,000102559 & $2,40362 \mathrm{E}-05$ \\
\hline 785412,918011371000 & 38662,310000000000 & 77,7700000000 & 0,000122099 & $2,64656 \mathrm{E}-05$ \\
\hline 881247,788255913000 & 34939,040000000000 & 78,2600000000 & 0,000140665 & $2,92328 \mathrm{E}-05$ \\
\hline 988776,281235923000 & 31482,840000000000 & 79,1100000000 & 0,000168128 & $3,23458 \mathrm{E}-05$ \\
\hline
\end{tabular}




\section{III.2 COMPLEX IMPEDANCE DATA FOR SAMPLE 17/08/00/04:}

\begin{tabular}{|c|c|c|c|c|}
\hline $\begin{array}{l}\text { frequency } \\
\qquad[\mathrm{Hz}]\end{array}$ & $\begin{array}{c}\text { measurement } \\
1\end{array}$ & $\begin{array}{c}\text { phase } \\
\text { difference } \\
{\left[{ }^{\circ}\right]} \\
\end{array}$ & $\begin{array}{c}\text { real impedance } \\
\qquad Z^{\prime}[\Omega \mathrm{m}]\end{array}$ & $\begin{array}{c}\text { imaginary } \\
\text { impedance } \\
Z^{\prime \prime}[\Omega \mathrm{m}]\end{array}$ \\
\hline 785,412918011374 & 306,987179487180 & 4,42000000000 & 306,074172444177 & 23,658599538134 \\
\hline 881,247788255917 & 306,282051282051 & 4,47000000000 & 305,350426478213 & 23,870734951281 \\
\hline 988,7762812 & 384615 & 4,54000000000 & & 24,1727 \\
\hline 1109,4252 & & 4,65000000000 & 303 & 24,68 \\
\hline 1244,79 & 303,782051282051 & 4,75000000000 & 302,7 & 25,15 \\
\hline 1396,6 & & 4,86000000000 & 301,8 & 25,66 \\
\hline 1567,10 & & 5,00000000000 & & 26,34 \\
\hline 1758,3 & 6666666667 & 5,10000000000 & 300,4723 & 26,81 \\
\hline 1972,868051885120 & 301,217948717949 & 5,26000000000 & 299,949502574478 & 27,6142 \\
\hline 2213,594362117870 & 300,256410256410 & 5,41000000000 & 298,918925415394 & 28,308 \\
\hline 2483,693724635030 & 299,102564102564 & 5,58000000000 & 297,68523 & 29,083 \\
\hline 2786,750193874480 & 297,5000000 & 5,75000000000 & 296,00313 & 29,80 \\
\hline 3126,78 & 54 & 5,96000000000 & 294,7 & 30,77 \\
\hline 3508,310635390910 & 397 & 6,17000000000 & 293,22 & 31,69 \\
\hline 3936,389276332440 & & 6,43000000000 & 291,55 & 32,85 \\
\hline 4416, & & 6,71000000000 & 289 , & 881 \\
\hline 4955, & & 6,99000000000 & & 35,3 \\
\hline 5560 & & 000 & & 36,58 \\
\hline 6238,75656 & & 7,61000 & & 37,9 \\
\hline & & & & \\
\hline 7854,129180113750 & & 3000000000 & & 41,02 \\
\hline 8812,477882559180 & 281,08974 & 8,73000000000 & 277,8331 & 42,66 \\
\hline 9887,762812359290 & 278,782051282051 & 9,16000000000 & 275,2269229 & 44,37 \\
\hline 11094,252347227800 & 276,474358974359 & 9,61000000000 & 272,594570909531 & 46,15 \\
\hline 12447,955870272500 & 274,102564102564 & 10,0700000000 & & 47,92 \\
\hline 13966 , & 359 & 10,6200000000 & 266 , & 50,0 \\
\hline 15671,047969978400 & 268,717948717 & 1700000000 & 263,6 & 52,0 \\
\hline & & & & 53,87 \\
\hline 19728,680518851200 & & & & 56,33 \\
\hline & & 13,0500000000 & & 343024 \\
\hline 24836,937246350300 & & & & 61,11 \\
\hline 27867,501938744900 & & 5300000000 & & 63,39 \\
\hline 31267,851450567500 & 249,230769230769 & 15,3600000000 & & 66,0169 \\
\hline 35083,106353909100 & 245,384615384615 & 16,2100000000 & & 68,501251373062 \\
\hline 39363,892763324500 & 241,282051282051 & 17,0900000000 & 230,628078225908 & 70,906401718737 \\
\hline 44167,014113613600 & 237,179487179487 & 18,0500000000 & 225,507051165268 & 73,489312239738 \\
\hline 49556,204906889700 & 232,692307692308 & 19,0100000000 & 220,001673844574 & 75,795603861684 \\
\hline 55602,976430699800 & 227,948717948718 & 20,0300000000 & 214,160877454386 & 78,075198254195 \\
\hline 62387,565669362300 & 223,076923076923 & 21,0800000000 & 208,148423666982 & 80,234327656048 \\
\hline 70000,000000000200 & 218,076923076923 & 22,1900000000 & 201,925386453581 & 82,363114828606 \\
\hline 78541,291801137600 & 212,756410256410 & 23,3300000000 & 195,361264951993 & 84,257143682605 \\
\hline 88124,778825591900 & 207,500000000000 & 24,5900000000 & 188,681565604794 & 86,345334563739 \\
\hline 98877,628123593100 & 201,987179487179 & 25,8100000000 & 181,837503123370 & 87,942840157937 \\
\hline 110942,523472278000 & 196,410256410256 & 27,1300000000 & 174,800052932269 & 89,565229403037 \\
\hline 124479,558702725000 & 190,641025641026 & 28,5200000000 & 167,506832365850 & 91,024511908722 \\
\hline
\end{tabular}




\begin{tabular}{|c|c|c|c|c|}
\hline $\begin{array}{c}\text { frequency } \\
{[\mathbf{H z}]}\end{array}$ & $\begin{array}{c}\text { measurement } \\
\mathbf{1}\end{array}$ & $\begin{array}{c}\text { phase } \\
\text { difference } \\
{\left[{ }^{\circ}\right]}\end{array}$ & $\begin{array}{c}\text { real impedance } \\
\mathbf{Z}^{\prime} \mathbf{[} \mathbf{~ m ]}\end{array}$ & $\begin{array}{c}\text { imaginary } \\
\text { impedance } \\
\mathbf{Z}^{\prime \prime}[\mathbf{\Omega} \text { m] }\end{array}$ \\
\hline 139668,362047822000 & 184,743589743590 & 30,0000000000 & 159,992641904279 & 92,371794871795 \\
\hline 156710,479699784000 & 178,461538461538 & 31,6000000000 & 152,000499016298 & 93,511330911583 \\
\hline 175832,050205671000 & 171,474358974359 & 33,1400000000 & 143,581870146241 & 93,742745591194 \\
\hline 197286,805188512000 & 164,743589743590 & 34,8000000000 & 135,279068427796 & 94,021401855704 \\
\hline 221359,436211787000 & 157,628205128205 & 36,3300000000 & 126,988150003823 & 93,384478424126 \\
\hline 248369,372463503000 & 150,705128205128 & 37,9100000000 & 118,902858810643 & 92,596683709410 \\
\hline 278675,019387448000 & 143,782051282051 & 39,4800000000 & 110,977683046069 & 91,417898338349 \\
\hline 312678,514505674000 & 136,858974358974 & 41,0700000000 & 103,179005824182 & 89,913689834884 \\
\hline 350831,063539090000 & 129,871794871795 & 42,6600000000 & 95,506141406560 & 88,007159122720 \\
\hline 393638,927633244000 & 123,012820512821 & 44,2400000000 & 88,129302470171 & 85,821792434326 \\
\hline 441670,141136134000 & 116,282051282051 & 45,8400000000 & 81,009569554549 & 83,420411716488 \\
\hline 495562,049068895000 & 110,576923076923 & 47,2100000000 & 75,116367209447 & 81,146702301544 \\
\hline 556029,764306995000 & 103,141025641026 & 49,0400000000 & 67,612241012745 & 77,888741391277 \\
\hline 623875,656693620000 & 96,730769230769 & 50,6100000000 & 61,384924028940 & 74,757827803766 \\
\hline 699999,999999997000 & 89,743589743590 & 50,9000000000 & 56,599110923321 & 69,645190377767 \\
\hline 785412,918011371000 & 84,807692307692 & 53,6100000000 & 50,314571753820 & 68,269968095685 \\
\hline 881247,788255913000 & 79,807692307692 & 54,4000000000 & 46,457890887553 & 64,891695352792 \\
\hline 988776,281235923000 & 73,333333333333 & 56,6400000000 & 40,325836629822 & 61,250344308279 \\
\hline
\end{tabular}




\begin{tabular}{|c|c|c|c|c|}
\hline $\begin{array}{c}\text { frequency } \\
{[\mathrm{Hz}]}\end{array}$ & $\begin{array}{c}\text { measurement } \\
1\end{array}$ & $\begin{array}{c}\text { phase } \\
\text { difference } \\
{\left[^{\circ}\right]} \\
\end{array}$ & $\begin{array}{c}\text { real admittance } \\
\mathbf{Y}^{\prime}\left[\mathbf{S ~ m}^{-1}\right]\end{array}$ & $\begin{array}{c}\text { imaginary } \\
\text { admittance } \\
\mathbf{Y}^{\prime \prime}\left[\mathrm{S} \mathrm{m}^{-1}\right]\end{array}$ \\
\hline 785,412918011374 & 306,987179487180 & 4,42000000000 & 0,003267181912 & 0,042267928767 \\
\hline 881,247788255917 & 306,282051282051 & 4,47000000000 & 0,003274925834 & 0,041892300427 \\
\hline 988,776281235928 & 305,384615384615 & 4,54000000000 & 0,003284866066 & 0,041368920110 \\
\hline 1109,425234722780 & 304,487179487180 & 4,65000000000 & & \\
\hline 1244,795587027250 & 303,782051282051 & 4,75000000000 & 0,003303178478 & 0,039752505447 \\
\hline 1396,683620478220 & 302,948717948718 & 4,86000000000 & 0,003312799256 & 0,038961723181 \\
\hline 1567,104796997840 & 302,243589743590 & 5,00000000000 & 0,003321227882 & 0,037961808406 \\
\hline 1758,320502056710 & 301,666666666667 & 5,10000000000 & 0,003328092829 & 0,037290548932 \\
\hline 1972,868051885120 & 301,217948717949 & 5,26000000000 & 0,0033333894510 & 0,036213145903 \\
\hline 2213,594362117870 & 300,256410256410 & 5,41000000000 & 0,0033453388716 & 0,035324709730 \\
\hline 2483,693724635030 & 299,102564102564 & 5,58000000000 & & \\
\hline 2786,750193874480 & 297,500000000000 & 5,75000000000 & & \\
\hline 3126,785145056740 & 6153846154 & 5,96000000000 & & \\
\hline 3508,310635390910 & 294,935897435897 & 6,17000000000 & & 0,031546380493 \\
\hline 3936,389276332440 & 293,397435897436 & 6,43000000000 & 0,003429922286 & 0,030434581891 \\
\hline 4416,701411361350 & 291,858974358974 & 6,71000000000 & 0,0034499943498 & 0,029323799974 \\
\hline 4955,620490688970 & 290,384615384615 & 6,99000000000 & 0,003469495962 & 0,028297606403 \\
\hline 5560,297643069970 & 288,717948717949 & 7,28000000000 & & \\
\hline 6238,756566936220 & 286,923076923077 & 7,61000000000 & & \\
\hline 7000,000000000000 & 285,1 & 7,9600 & & \\
\hline 7854 & 283,2 & 8,3300 & & 9226 \\
\hline 8812,477882559180 & 281,089743589744 & 8,73000000000 & 0,003599281 & 0,023439325261 \\
\hline 9887,762812359290 & 278,782051282051 & 9,16000000000 & 0,003633365477 & 0,022532737538 \\
\hline 11094,252347227800 & 276,474358974359 & 9,61000000000 & 0,003668451637 & 0,021666190838 \\
\hline 12447,955870272500 & 274,102564102564 & 10,0700000000 & 0,003705350834 & 0,020864992904 \\
\hline 13966,836204782200 & & 10,6200000000 & & \\
\hline 15671,0 & & 11,17 & & \\
\hline 17583, & 265,8 & 11,69 & 0,0 & 0,0185 \\
\hline 19728,680518851200 & 262,5641025 & 12,3900000000 & 0,00389 & 0,017750314319 \\
\hline 22135,943621178700 & 258,974358974359 & 13,0500000000 & 0,003963756545 & 0,017100816980 \\
\hline 24836,937246350300 & 256,217948717949 & 13,8000000000 & 0,004018936697 & 0,016362179273 \\
\hline 27867,501938744900 & 252,692307692308 & 14,5300000000 & 0,004088135286 & 0,015773570431 \\
\hline 31267,851450567500 & 249,230769230769 & 15,3600000000 & 0,004160973305 & 0,015147615634 \\
\hline 35083,106353909100 & & 16,2100000000 & & \\
\hline 39363,8 & 241,282051 & 17,0900000000 & 0,0043335985487 & 0,014103098955 \\
\hline 44167,014113613600 & 237,179487179487 & 18,0500000000 & 0,004434451139 & 0,013607420855 \\
\hline 49556,204906889700 & 232,692307692308 & 19,0100000000 & 0,004545419962 & 0,013193377308 \\
\hline 55602,976430699800 & 227,948717948718 & 20,0300000000 & 0,0046693869920 & 0,012808164723 \\
\hline 62387,5656693362300 & 223,076923076923 & 21,0800000000 & 0,004804264103 & 0,012463493236 \\
\hline 70000,000000000200 & 218,076923076923 & 22,1900000000 & 0,004952324309 & 0,012141357234 \\
\hline 78541,291801137600 & & 23,3300000000 & & 0,011868429860 \\
\hline 88124,778825591900 & 207,500000000000 & 24,5900000000 & 0,005299934823 & 0,011581401648 \\
\hline 98877,628123593100 & 201,987179487179 & 25,8100000000 & 0,005499415593 & 0,011371022339 \\
\hline 110942,523472278000 & 196,410256410256 & 27,1300000000 & 0,005720822066 & 0,011165047046 \\
\hline 124479,558702725000 & 190,641025641026 & 28,5200000000 & 0,005969905740 & 0,010986051768 \\
\hline 139668,362047822000 & 184,743589743590 & 30,0000000000 & 0,006250287439 & 0,010825815406 \\
\hline 156710,479699784000 & 178,461538461538 & 31,6000000000 & 0,006578925770 & 0,010693891214 \\
\hline
\end{tabular}




\begin{tabular}{|c|c|c|c|c|}
\hline $\begin{array}{l}\text { frequency } \\
\qquad[\mathrm{Hz}]\end{array}$ & $\begin{array}{c}\text { measurement } \\
1\end{array}$ & $\begin{array}{c}\text { phase } \\
\text { difference } \\
{\left[{ }^{\circ}\right]}\end{array}$ & $\begin{array}{l}\text { real admittance } \\
\qquad \mathbf{Y}^{\prime}\left[\mathrm{S} \mathrm{m}^{-1}\right]\end{array}$ & $\begin{array}{c}\text { imaginary } \\
\text { admittance } \\
\mathbf{Y}^{\prime \prime}\left[\mathrm{S} \mathrm{m}^{-1}\right]\end{array}$ \\
\hline 175832,050205671000 & 171,474358974359 & 33,1400000000 & 0,006964667607 & 0,010667492121 \\
\hline 197286,805188512000 & 164,743589743590 & 34,8000000000 & 0,007392126599 & 0,010635876303 \\
\hline 221359,436211787000 & 157,628205128205 & 36,3300000000 & 0,007874750518 & 0,010708417682 \\
\hline 248369,372463503000 & 150,705128205128 & 37,9100000000 & & 0,01079 \\
\hline 278675,019387448000 & 143,782051282051 & 39,4800000000 & 0,009010820667 & 0,010938776959 \\
\hline 312678,514505674000 & 136,8 & 41,0700000000 & 0,009 & 0,01 \\
\hline 350831,063539090000 & 129,871794871795 & 42,6600000000 & 0,0104705 & 0,011 \\
\hline 393638,927633244000 & 123,012820512821 & 44,2400000000 & 0,011346963745 & 0,011 \\
\hline 441670,141136134000 & 116,282051282051 & 45,8400000000 & 0,012344220633 & .73802 \\
\hline 495562,049068895000 & 110,576923076923 & 47,2100000000 & 0,013312677878 & 0,0123 \\
\hline 556029,764306995000 & 103,141025641026 & 49,0400000000 & 0,014790221194 & 0,012838826025 \\
\hline 623875,656693620000 & 96,730769230769 & 50,6100000000 & 0,016290644907 & 0,013376525635 \\
\hline 699999,999999997000 & 89,743589743590 & 50,9000000000 & 0,017668122055 & 0,014358493308 \\
\hline 785412,918011371000 & 84,807692307692 & 53,6100000000 & 0,019874957992 & 0,014647729124 \\
\hline 881247,788255913000 & 79,807692307692 & 54,4000000000 & 0,021524868669 & 0,015410292404 \\
\hline 988776,281235923000 & 73,333333333333 & 56,6400000000 & 0,024797997601 & 0,016326438835 \\
\hline
\end{tabular}




\begin{tabular}{|c|c|c|c|c|}
\hline $\begin{array}{l}\text { frequency } \\
\qquad[\mathrm{Hz}]\end{array}$ & $\begin{array}{c}\text { measurement } \\
2\end{array}$ & $\begin{array}{c}\text { phase difference } \\
{\left[{ }^{\circ}\right]}\end{array}$ & $\begin{array}{c}\text { real impedance } \\
\mathbf{Z}^{\prime}[\mathbf{\Omega} \mathrm{m}]\end{array}$ & $\begin{array}{c}\text { imaginary } \\
\text { impedance } \\
\mathbf{Z}^{\prime \prime}[\Omega \mathbf{~ m}]\end{array}$ \\
\hline 785,412918011374 & 351,217948717949 & 5,700000000000 & 349,481376142151 & 34,882878772141 \\
\hline 881,247788255917 & 349,871794871795 & 5,780000000000 & 348,093019908048 & 35,235242841604 \\
\hline 988,776281235928 & 348,333333333333 & 5,910000000000 & 346,481893347588 & 35,866540025237 \\
\hline 1109,425234722780 & 346,923076923077 & 6,060000000000 & 344,984431958917 & 36,624622970869 \\
\hline 1244,795587027250 & 345,576923076923 & 6,210000000000 & 343,549112038215 & 37,382046239708 \\
\hline 1396,683620478220 & 343,910256410256 & 6,370000000000 & 341,787005362097 & 38,156355037900 \\
\hline 1567,104796997840 & 342,435897435897 & 6,560000000000 & 340,193889068752 & 39,121115692244 \\
\hline 1758,320502056710 & 341,025641025641 & 6,710000000000 & 338,689707982184 & 39,846826020305 \\
\hline 1972,868051885120 & 339,551282051282 & 6,930000000000 & 337,070623046443 & 40,969113021303 \\
\hline 2213,594362117870 & 338,012820512821 & 7,130000000000 & 335,398995859464 & 41,95450 \\
\hline 2483,693724635030 & 336,153846153846 & 7,380000000000 & 333,369167898705 & 43,178769998102 \\
\hline 2786,750193874480 & 334,679487179487 & 7,650000000000 & 331,700755192164 & 44,552981310714 \\
\hline 3126,785145056740 & 332,371794871795 & 7,920000000000 & 329,201441810073 & 45,797606 \\
\hline 3508,310635390910 & 330,384615384615 & 8,190000000000 & 327,015058795921 & 47,065331227353 \\
\hline 3936,389276332440 & 328,269230769231 & 8,560000000000 & 324,612487309646 & 48,861241822810 \\
\hline 4416,701411361350 & 326,089743589744 & 8,900000000000 & 322,163579305698 & 50,449470199064 \\
\hline 4955,620490688970 & 323,397435897436 & 9,250000000000 & 319,192094185665 & 51,983733555853 \\
\hline 5560,297643069970 & 321,153846153846 & 9,630000000000 & 316,628333066895 & 53,724217990471 \\
\hline 6238,756566936220 & 318,397435897436 & 10,04000000000 & 313,521587903890 & 55,508027386002 \\
\hline 7000,000000000000 & 315,512820512821 & 10,47000000000 & 310,259592337944 & 57,335200969806 \\
\hline 7854,129180113750 & 312,371794871795 & 10,92000000000 & 306,715568237367 & 59,175150462469 \\
\hline 8812,477882559180 & 308,974358974359 & 11,39000000000 & 302,889312095637 & 61,018186812194 \\
\hline 9887,762812359290 & 305,576923076923 & 11,90000000000 & 299,009764870524 & 63,011240499086 \\
\hline 11094,252347227800 & 302,179487179487 & 12,44000000000 & 295,084955495262 & 65,094635050955 \\
\hline 12447,955870272500 & 298,269230769231 & 13,00000000000 & 290,624609708058 & 67,095977939872 \\
\hline 13966,836204782200 & 294,487179487180 & 13,58000000000 & 286,254207725035 & 69,146420312457 \\
\hline 15671,047969978400 & 290,512820512821 & 14,20000000000 & 281,636302931075 & 71,264940564278 \\
\hline 17583,205020567100 & 286,217948717949 & 14,86000000000 & 276,645491419986 & 73,402903520941 \\
\hline 19728,680518851200 & 281,602564102564 & 15,54000000000 & 271,308202436679 & 75,444439157016 \\
\hline 22135,943621178700 & 276,858974358974 & 16,26000000000 & 265,784892350847 & 77,519563215694 \\
\hline 24836,937246350300 & 272,115384615385 & 17,00000000000 & 260,225236478403 & 79,558838881283 \\
\hline 27867,501938744900 & 267,115384615385 & 17,77000000000 & 254,371129020376 & 81,522741729681 \\
\hline 31267,851450567500 & 261,858974358974 & 18,58000000000 & 248,210803457044 & 83,435720764807 \\
\hline 35083,106353909100 & 256,410256410256 & 19,43000000000 & 241,807335768890 & 85,296142707186 \\
\hline 39363,892763324500 & 250,897435897436 & 20,33000000000 & 235,268319802076 & 87,168463548557 \\
\hline 44167,014113613600 & 245,064102564103 & 21,23000000000 & 228,432662374537 & 88,740819953558 \\
\hline 49556,204906889700 & 239,038461538462 & 22,18000000000 & 221,350193789715 & 90,241220093316 \\
\hline 55602,976430699800 & 232,948717948718 & 23,16000000000 & 214,175413051393 & 91,618762479181 \\
\hline 62387,565669362300 & 226,794871794872 & 24,19000000000 & 206,880387712128 & 92,932335882254 \\
\hline 70000,000000000200 & 220,448717948718 & 25,25000000000 & 199,385977257537 & 94,036531828277 \\
\hline 78541,291801137600 & 214,102564102564 & 26,36000000000 & 191,840598239371 & 95,063625127872 \\
\hline 88124,778825591900 & 207,628205128205 & 27,52000000000 & 184,134990348862 & 95,936316866892 \\
\hline 98877,628123593100 & 201,025641025641 & 28,67000000000 & 176,379392437792 & 96,444897599849 \\
\hline 110942,523472278000 & 194,551282051282 & 29,92000000000 & 168,622010732158 & 97,040294952363 \\
\hline 124479,558702725000 & 187,948717948718 & 31,23000000000 & 160,713615149168 & 97,446675080537 \\
\hline 139668,362047822000 & 181,217948717949 & 32,62000000000 & 152,633404955677 & 97,688221547828 \\
\hline 156710,479699784000 & 174,358974358974 & 34,14000000000 & 144,311471395954 & 97,853212328713 \\
\hline
\end{tabular}




\begin{tabular}{|c|c|c|c|c|}
\hline $\begin{array}{c}\text { frequency } \\
{[\mathbf{H z}]}\end{array}$ & $\begin{array}{c}\text { measurement } \\
\mathbf{2}\end{array}$ & $\begin{array}{c}\text { phase difference } \\
{\left[{ }^{\circ}\right]}\end{array}$ & $\begin{array}{c}\text { real impedance } \\
\mathbf{Z}^{\prime}[\mathbf{\Omega} \mathbf{~ m}]\end{array}$ & $\begin{array}{c}\text { imaginary } \\
\text { impedance } \\
\mathbf{Z}^{\prime \prime}[\mathbf{\Omega} \text { }]\end{array}$ \\
\hline 175832,050205671000 & 166,923076923077 & 35,57000000000 & 135,776140241174 & 97,098678418795 \\
\hline 197286,805188512000 & 159,679487179487 & 37,12000000000 & 127,324162976500 & 96,364392532915 \\
\hline 221359,436211787000 & 152,307692307692 & 38,57000000000 & 119,081316552160 & 94,959323840785 \\
\hline 248369,372463503000 & 145,000000000000 & 40,06000000000 & 110,978780152054 & 93,320471258787 \\
\hline 278675,019387448000 & 137,820512820513 & 41,54000000000 & 103,157681143343 & 91,394674764110 \\
\hline 312678,514505674000 & 130,705128205128 & 43,05000000000 & 95,513853023823 & 89,224068611908 \\
\hline 350831,063539090000 & 123,717948717949 & 44,58000000000 & 88,120719455178 & 86,838180759841 \\
\hline 393638,927633244000 & 116,858974358974 & 46,11000000000 & 81,015529064719 & 84,217005044083 \\
\hline 441670,141136134000 & 110,128205128205 & 47,64000000000 & 74,202918172355 & 81,376584466705 \\
\hline 495562,049068895000 & 103,525641025641 & 49,19000000000 & 67,659463722878 & 78,356590778967 \\
\hline 556029,764306995000 & 97,051282051282 & 50,72000000000 & 61,444206362919 & 75,123637107295 \\
\hline 623875,656693620000 & 90,833333333333 & 52,24000000000 & 55,622270546665 & 71,811262791278 \\
\hline 699999,999999997000 & 84,487179487180 & 53,10000000000 & 50,727811344841 & 67,563101274743 \\
\hline 785412,918011371000 & 79,038461538462 & 55,23000000000 & 45,074333385544 & 64,925979948058 \\
\hline 881247,788255913000 & 73,589743589744 & 56,42000000000 & 40,702543929948 & 61,308672137267 \\
\hline 988776,281235923000 & 68,358974358974 & 58,17000000000 & 36,052572706620 & 58,078923687038 \\
\hline
\end{tabular}




\begin{tabular}{|c|c|c|c|c|}
\hline $\begin{array}{l}\text { frequency } \\
\qquad[\mathrm{Hz}]\end{array}$ & $\begin{array}{c}\text { measurement } \\
2\end{array}$ & $\begin{array}{c}\text { phase difference } \\
{\left[{ }^{\circ}\right]}\end{array}$ & $\begin{array}{l}\text { real admittance } \\
\qquad \mathbf{Y}^{\prime}\left[\mathrm{S} \mathrm{m}^{-1}\right]\end{array}$ & $\begin{array}{c}\text { imaginary } \\
\text { admittance } \\
\mathbf{Y}^{\prime \prime}\left[\mathrm{S} \mathrm{m}^{-1}\right]\end{array}$ \\
\hline 785,412918011374 & 351,217948717949 & 5,700000000000 & 0,002861382804 & 0,028667358750 \\
\hline 881,247788255917 & 349,871794871795 & 5,780000000000 & 0,002872795324 & 0,028380675692 \\
\hline 988,776281235928 & 348,333333333333 & 5,910000000000 & 0,002886153704 & 0,027881139338 \\
\hline 1109,425234722780 & 346,923076923077 & 6,060000000000 & 0,002898681527 & 0,027304035342 \\
\hline 1244,795587027250 & 345,576923076923 & 6,210000000000 & 0,002910791980 & 0,026750809562 \\
\hline 1396,683620478220 & 343,910256410256 & 6,370000000000 & 0,002925798770 & 0,026207954062 \\
\hline 1567,104796997840 & 342,435897435897 & 6,560000000000 & 0,002939500185 & 0,025561643177 \\
\hline 1758,320502056710 & 341,025641025641 & 6,710000000000 & 0,002952555027 & 0,025096101744 \\
\hline 1972,868051885120 & 339,551282051282 & 6,930000000000 & 0,002966737329 & 0,024408631924 \\
\hline 2213,594362117870 & 338,012820512821 & 7,130000000000 & 0,002981523536 & 0,023835343150 \\
\hline 2483,693724635030 & 336,153846153846 & 7,380000000000 & 0,002999677524 & 0,023159529557 \\
\hline 2786,750193874480 & 334,679487179487 & 7,650000000000 & 0,003014765521 & 0,022445187069 \\
\hline 3126,785145056740 & 332,371794871795 & 7,920000000000 & 0,003037653768 & 0,021835202368 \\
\hline 3508,310635390910 & 330,384615384615 & 8,190000000000 & 0,003057963152 & 0,021247061774 \\
\hline 3936,389276332440 & 328,269230769231 & 8,560000000000 & 0,003080596216 & 0,020466119212 \\
\hline 4416,701411361350 & 326,089743589744 & 8,900000000000 & 0,003104013192 & 0,019821813709 \\
\hline 4955,620490688970 & 323,397435897436 & 9,250000000000 & 0,003132909675 & 0,019236786810 \\
\hline 5560,297643069970 & 321,153846153846 & 9,630000000000 & 0,003158277057 & 0,018613579451 \\
\hline 6238,756566936220 & 318,397435897436 & 10,040000000000 & 0,003189573026 & 0,018015412312 \\
\hline 7000,000000000000 & 315,512820512821 & 10,470000000000 & 0,003223107439 & 0,017441292314 \\
\hline 7854,129180113750 & 312,371794871795 & 10,920000000000 & 0,003260349665 & 0,016898985337 \\
\hline 8812,477882559180 & 308,974358974359 & 11,390000000000 & 0,003301536106 & 0,016388556466 \\
\hline 9887,762812359290 & 305,576923076923 & 11,900000000000 & 0,003344372383 & 0,015870184305 \\
\hline 11094,252347227800 & 302,179487179487 & 12,440000000000 & 0,003388854570 & 0,015362249120 \\
\hline 12447,955870272500 & 298,269230769231 & 13,000000000000 & 0,003440864836 & 0,014904023023 \\
\hline 13966,836204782200 & 294,487179487180 & 13,580000000000 & 0,003493398431 & 0,014462064637 \\
\hline 15671,047969978400 & 290,512820512821 & 14,200000000000 & 0,003550678622 & 0,014032145289 \\
\hline 17583,205020567100 & 286,217948717949 & 14,860000000000 & 0,003614734492 & 0,013623439292 \\
\hline 19728,680518851200 & 281,602564102564 & 15,540000000000 & 0,003685845069 & 0,013254787380 \\
\hline 22135,943621178700 & 276,858974358974 & 16,260000000000 & 0,003762441090 & 0,012899969485 \\
\hline 24836,937246350300 & 272,115384615385 & 17,000000000000 & 0,003842824829 & 0,012569313656 \\
\hline 27867,501938744900 & 267,115384615385 & 17,770000000000 & 0,003931263756 & 0,012266515806 \\
\hline 31267,851450567500 & 261,858974358974 & 18,580000000000 & 0,004028833500 & 0,011985274303 \\
\hline 35083,106353909100 & 256,410256410256 & 19,430000000000 & 0,004135523833 & 0,011723859582 \\
\hline 39363,892763324500 & 250,897435897436 & 20,330000000000 & 0,004250466025 & 0,011472038846 \\
\hline 44167,014113613600 & 245,064102564103 & 21,230000000000 & 0,004377657685 & 0,011268771243 \\
\hline 49556,204906889700 & 239,038461538462 & 22,180000000000 & 0,004517728143 & 0,011081410457 \\
\hline 55602,976430699800 & 232,948717948718 & 23,160000000000 & 0,004669070020 & 0,010914794884 \\
\hline 62387,565669362300 & 226,794871794872 & 24,190000000000 & 0,004833710972 & 0,010760517214 \\
\hline 70000,000000000200 & 220,448717948718 & 25,250000000000 & 0,005015397842 & 0,010634165048 \\
\hline 78541,291801137600 & 214,102564102564 & 26,360000000000 & 0,005212660976 & 0,010519270632 \\
\hline 88124,778825591900 & 207,628205128205 & 27,520000000000 & 0,005430798340 & 0,010423581316 \\
\hline 98877,628123593100 & 201,025641025641 & 28,670000000000 & 0,005669596579 & 0,010368614876 \\
\hline 110942,523472278000 & 194,551282051282 & 29,920000000000 & 0,005930423885 & 0,010304997532 \\
\hline 124479,558702725000 & 187,948717948718 & 31,230000000000 & 0,006222248184 & 0,010262022785 \\
\hline 139668,362047822000 & 181,217948717949 & 32,620000000000 & 0,006551645757 & 0,010236648637 \\
\hline 156710,479699784000 & 174,358974358974 & $34,140000000000 \mid$ & 0,006929456060 & 0,010219388574 \\
\hline
\end{tabular}




\begin{tabular}{|c|c|c|c|c|}
\hline $\begin{array}{l}\text { frequency } \\
\qquad[\mathrm{Hz}]\end{array}$ & $\begin{array}{c}\text { measurement } \\
2\end{array}$ & $\begin{array}{c}\text { phase difference } \\
{\left[{ }^{\circ}\right]}\end{array}$ & $\begin{array}{l}\text { real admittance } \\
\mathbf{Y}^{\prime}\left[\mathrm{S} \mathrm{m}^{-1}\right]\end{array}$ & $\begin{array}{c}\text { imaginary } \\
\text { admittance } \\
\mathbf{Y}^{\prime \prime}\left[\mathrm{S} \mathrm{m}^{-1}\right]\end{array}$ \\
\hline 175832,050205671000 & 166,923076923077 & 35,570000000000 & & 0,010298801346 \\
\hline 197286,8051 & 159,679487179487 & 37,120000000000 & & \\
\hline 221359,436 & 152,307692307692 & 38,570000000000 & & \\
\hline 248369 , & 145 , & 0000 & & 431 \\
\hline & & & & \\
\hline 312 & & & & \\
\hline 350831, & & 000 & & \\
\hline 393638 & & 46,11 & & 468 \\
\hline 441670, & & 47,640000000000 & & 210 \\
\hline 495562,0490 & & 49,190000000000 & & 314 \\
\hline 556029,764306995000 & 97,051282 & 50,720000000000 & 0,0162749 & 89577 \\
\hline 623875,656693620000 & 90,833333 & 52,240000000000 & 0,017978410269 & 0,013 \\
\hline 699999,999999997000 & 84,487179487180 & 53,100000000000 & 0,019713052337 & 0,014800978361 \\
\hline 785412,918011371000 & 79,038461538462 & 55,230000000000 & 0,022185574913 & 0,015402154897 \\
\hline 881247,788255913000 & 73,589743589744 & 56,420000000000 & 0,024568488931 & 0,016310906192 \\
\hline 988776,281235923000 & 68,358974358974 & 58,170000000000 & 0,027737271571 & 0,017217949929 \\
\hline
\end{tabular}




\begin{tabular}{|c|c|c|c|c|}
\hline $\begin{array}{l}\text { frequency } \\
\qquad[\mathrm{Hz}]\end{array}$ & $\begin{array}{c}\text { measurement } \\
3\end{array}$ & $\begin{array}{c}\text { phase } \\
\text { difference } \\
\left.{ }^{\circ}\right]\end{array}$ & $\begin{array}{c}\text { real impedance } \\
Z^{\prime}[\Omega \mathrm{m}]\end{array}$ & $\begin{array}{c}\text { imaginary } \\
\text { impedance } \\
Z^{\prime \prime}[\Omega \mathrm{m}]\end{array}$ \\
\hline 785,412918011374 & 405,256410256410 & 5,770000000000 & 403,203172738621 & 40,742601137157 \\
\hline 881,247788255917 & 403,397435897436 & 5,950000000000 & 401,224226995073 & 41,816395837422 \\
\hline 988,776281235928 & 401,474358974359 & 6,130000000000 & 399,178796076143 & 42,871315317747 \\
\hline 1109,425234722780 & 399,423076923077 & 6,370000000000 & 396,957097933127 & 44,315423716897 \\
\hline 1244,795587027250 & 397,179487179487 & 6,620000000000 & 394,531326489454 & 45,788398690410 \\
\hline 1396,683620478220 & 395,192307692308 & 6,850000000000 & 392,371347175458 & 47,134763974035 \\
\hline 1567,104796997840 & 392,435897435897 & 7,160000000000 & 389,375666075978 & 48,913436438333 \\
\hline 1758,320502056710 & 389,807692307692 & 7,390000000000 & 386,569803964202 & 50,138045886601 \\
\hline 1972,868051885120 & 386,987179487180 & 7,720000000000 & 383,479670622107 & 51,984798806984 \\
\hline 2213,594362117870 & 383,717948717949 & 8,020000000000 & 379,964967629560 & 53,535852870557 \\
\hline 2483,693724635030 & 380,641025641026 & 8,410000000000 & 376,547924784937 & 55,670914679117 \\
\hline 2786,750193874480 & 377,179487179487 & 8,770000000000 & 372,769636317125 & 57,507945442166 \\
\hline 3126,785145056740 & 373,525641025641 & 9,190000000000 & 368,731123633023 & 59,655368307844 \\
\hline 3508,310635390910 & 369,807692307692 & 9,610000000000 & 364,618149681680 & 61,73600 \\
\hline 3936,389276332440 & 365,961538461538 & 10,09000000000 & 360,301493958901 & 64,114593378733 \\
\hline 4416,701411361350 & 361,858974358974 & 10,59000000000 & 355,695589574663 & 66,502367486142 \\
\hline 4955,620490688970 & 357,564102564103 & 11,09000000000 & 350,887040071227 & 68,777703891049 \\
\hline 5560,297643069970 & 353,076923076923 & 11,64000000000 & 345,815766229704 & 71,237415986476 \\
\hline 6238,756566936220 & 348,141025641026 & 12,17000000000 & 340,317046865422 & 73,392651860954 \\
\hline 7000,000000000000 & 343,076923076923 & 12,73000000000 & 334,643851818843 & 75,599388805580 \\
\hline 7854,129180113750 & 337,820512820513 & 13,31000000000 & 328,746216943343 & 77,772898414259 \\
\hline 8812,477882559180 & 332,307692307692 & 13,89000000000 & 322,590481863316 & 79,773324978076 \\
\hline 9887,762812359290 & 326,602564102564 & 14,48000000000 & 316,228027285894 & 81,664371896446 \\
\hline 11094,252347227800 & 320,641025641026 & 15,06000000000 & 309,628372995236 & 83,312291772894 \\
\hline 12447,955870272500 & 314,615384615385 & 15,65000000000 & 302,951813089597 & 84,870720407054 \\
\hline 13966,836204782200 & 308,269230769231 & 16,54000000000 & 295,513426430672 & 87,759520499242 \\
\hline 15671,047969978400 & 301,858974358974 & 17,67000000000 & 287,617431555069 & 91,624524373758 \\
\hline 17583,205020567100 & 294,871794871795 & 18,45000000000 & 279,715442004956 & 93,320131348416 \\
\hline 19728,680518851200 & 287,435897435897 & 19,24000000000 & 271,381610302595 & 94,717562913909 \\
\hline 22135,943621178700 & 280,000000000000 & 20,13000000000 & 262,895971402012 & 96,362379695565 \\
\hline 24836,937246350300 & 273,012820512821 & 21,07000000000 & 254,759702280901 & 98,150365552617 \\
\hline 27867,501938744900 & 265,192307692308 & 21,90000000000 & 246,055037332041 & 98,913490802316 \\
\hline 31267,851450567500 & 257,179487179487 & 23,15000000000 & 236,471078129545 & 101,107456867209 \\
\hline 35083,106353909100 & 249,230769230769 & 25,98000000000 & 224,045255282396 & 109,177378228308 \\
\hline 39363,892763324500 & 242,307692307692 & 28,37000000000 & 213,205929912658 & 115,135785929302 \\
\hline 44167,014113613600 & 233,589743589744 & 29,90000000000 & 202,498189302650 & 116,441623311728 \\
\hline 49556,204906889700 & 224,294871794872 & 31,13000000000 & 191,995627695841 & 115,956321341952 \\
\hline 55602,976430699800 & 214,743589743590 & 32,41000000000 & 181,293924186076 & 115,097012946368 \\
\hline 62387,565669362300 & 205,064102564103 & 33,51000000000 & 170,980291072481 & 113,212305979476 \\
\hline 70000,000000000200 & 195,384615384615 & 34,48000000000 & 161,060198674591 & 110,610850877730 \\
\hline 78541,291801137600 & 185,833333333333 & 35,50000000000 & 151,289800494549 & 107,913965936283 \\
\hline 88124,778825591900 & 176,538461538462 & 36,40000000000 & 142,094712687063 & 104,761257288884 \\
\hline 98877,628123593100 & 167,564102564103 & 37,16000000000 & 133,540515447592 & 101,215903899064 \\
\hline 110942,523472278000 & 158,910256410256 & 37,84000000000 & 125,495708942081 & 97,484853333726 \\
\hline 124479,558702725000 & 150,769230769231 & 38,47000000000 & 118,042356540247 & 93,794258935026 \\
\hline 139668,362047822000 & 143,076923076923 & 38,96000000000 & 111,254486478761 & 89,963576826996 \\
\hline 156710,479699784000 & 135,641025641026 & 39,45000000000 & 104,739202060217 & 86,186932818951 \\
\hline
\end{tabular}




\begin{tabular}{|c|c|c|c|c|}
\hline $\begin{array}{c}\text { frequency } \\
{[\mathbf{H z}]}\end{array}$ & $\begin{array}{c}\text { measurement } \\
\mathbf{3}\end{array}$ & $\begin{array}{c}\text { phase } \\
\text { difference } \\
{\left[{ }^{\circ} \mathbf{]}\right.}\end{array}$ & $\begin{array}{c}\text { real impedance } \\
\mathbf{Z}^{\prime}[\boldsymbol{\Omega} \mathbf{~ m}]\end{array}$ & $\begin{array}{c}\text { imaginary } \\
\text { impedance } \\
\mathbf{Z}^{\prime \prime}[\boldsymbol{\Omega} \mathbf{~ m}]\end{array}$ \\
\hline 175832,050205671000 & 128,333333333333 & 39,73000000000 & 98,696673915251 & 82,026891947160 \\
\hline 197286,805188512000 & 121,730769230769 & 40,07000000000 & 93,155512973625 & 78,360899561807 \\
\hline 221359,436211787000 & 115,512820512821 & 40,22000000000 & 88,202202315047 & 74,589430951058 \\
\hline 248369,372463503000 & 110,000000000000 & 40,48000000000 & 83,669588134454 & 71,410083471530 \\
\hline 278675,019387448000 & 104,935897435897 & 40,72000000000 & 79,531616036113 & 68,456297163676 \\
\hline 312678,514505674000 & 100,128205128205 & 41,06000000000 & 75,498883817923 & 65,769111324722 \\
\hline 350831,063539090000 & 95,641025641026 & 41,53000000000 & 71,597701107166 & 63,411158180846 \\
\hline 393638,927633244000 & 91,410256410256 & 42,13000000000 & 67,792104419221 & 61,319373410059 \\
\hline 441670,141136134000 & 87,307692307692 & 42,88000000000 & 63,977371852956 & 59,409839478686 \\
\hline 495562,049068895000 & 83,333333333333 & 43,78000000000 & 60,166815638908 & 57,657599154995 \\
\hline 556029,764306995000 & 79,423076923077 & 44,82000000000 & 56,336752560259 & 55,983885707345 \\
\hline 623875,656693620000 & 75,576923076923 & 45,98000000000 & 52,519116165609 & 54,347159437808 \\
\hline 699999,999999997000 & 71,679487179487 & 47,13000000000 & 48,766222830835 & 52,533840456678 \\
\hline 785412,918011371000 & 67,846153846154 & 48,61000000000 & 44,858583515721 & 50,899981106864 \\
\hline 881247,788255913000 & 63,910256410256 & 49,93000000000 & 41,140503877368 & 48,907870687048 \\
\hline 988776,281235923000 & 60,275641025641 & 51,67000000000 & 37,382341053567 & 47,283331930044 \\
\hline
\end{tabular}




\begin{tabular}{|c|c|c|c|c|}
\hline $\begin{array}{l}\text { frequency } \\
\qquad[\mathrm{Hz}]\end{array}$ & $\begin{array}{c}\text { measurement } \\
3\end{array}$ & $\begin{array}{c}\text { phase difference } \\
{\left[{ }^{\circ}\right]}\end{array}$ & $\begin{array}{l}\text { real admittance } \\
\quad \mathbf{Y}^{\prime}\left[\mathrm{S} \mathrm{m}^{-1}\right]\end{array}$ & $\begin{array}{c}\text { imaginary } \\
\text { admittance } \\
\mathbf{Y}^{\prime \prime}\left[\mathrm{S} \mathrm{m}^{-1}\right]\end{array}$ \\
\hline 785,412918011374 & 405,256410256410 & 5,770000000000 & 0,002480139214 & 0,024544333746 \\
\hline 881,247788255917 & 403,397435897436 & 5,950000000000 & 0,002492371928 & 0,023914064806 \\
\hline 988,776281235928 & 401,474358974359 & 6,130000000000 & 0,002505143083 & 0,023325619767 \\
\hline 1109,425234722780 & 399,423076923077 & 6,370000000000 & 0,002519163923 & 0,022565506908 \\
\hline 1244,795587027250 & 397,179487179487 & 6,620000000000 & 0,002534652974 & 0,021839593185 \\
\hline 1396,683620478220 & 395,192307692308 & 6,850000000000 & 0,002548606077 & 0,021215763392 \\
\hline 1567,104796997840 & 392,435897435897 & 7,160000000000 & 0,002568213905 & 0,020444280198 \\
\hline 1758,320502056710 & 389,807692307692 & 7,390000000000 & 0,002586854922 & 0,019944933679 \\
\hline 1972,868051885120 & 386,987179487180 & 7,720000000000 & 0,002607700164 & 0,019236392618 \\
\hline 2213,594362117870 & 383,717948717949 & 8,020000000000 & 0,002631821576 & 0,018679071059 \\
\hline 2483,693724635030 & 380,641025641026 & 8,410000000000 & 0,002655704451 & 0,017962701094 \\
\hline 2786,750193874480 & 377,179487179487 & 8,770000000000 & 0,002682621927 & 0,017388901522 \\
\hline 3126,785145056740 & 373,525641025641 & 9,190000000000 & 0,002712003235 & 0,016762950735 \\
\hline 3508,310635390910 & 369,807692307692 & 9,610000000000 & 0,002742595235 & 0,016198003308 \\
\hline 3936,389276332440 & 365,961538461538 & 10,09000000000 & 0,002775453382 & 0,015597073105 \\
\hline 4416,701411361350 & 361,858974358974 & 10,59000000000 & 0,002811392745 & 0,015037058646 \\
\hline 4955,620490688970 & 357,564102564103 & 11,09000000000 & 0,002849920019 & 0,014539595587 \\
\hline 5560,297643069970 & 353,076923076923 & 11,64000000000 & 0,002891713154 & 0,014037567003 \\
\hline 6238,756566936220 & 348,141025641026 & 12,17000000000 & 0,002938436406 & 0,013625342247 \\
\hline 7000,000000000000 & 343,076923076923 & 12,73000000000 & 0,002988251523 & 0,013227620167 \\
\hline 7854,129180113750 & 337,820512820513 & 13,31000000000 & 0,003041860099 & 0,012857949496 \\
\hline 8812,477882559180 & 332,307692307692 & 13,89000000000 & 0,003099905472 & 0,012535518612 \\
\hline 9887,762812359290 & 326,602564102564 & 14,48000000000 & 0,003162275047 & 0,012245242041 \\
\hline 11094,252347227800 & 320,641025641026 & 15,06000000000 & 0,003229678179 & 0,012003030750 \\
\hline 12447,955870272500 & 314,615384615385 & 15,65000000000 & 0,003300854977 & 0,011782626508 \\
\hline 13966,836204782200 & 308,269230769231 & 16,54000000000 & 0,003383941001 & 0,011394775112 \\
\hline 15671,047969978400 & 301,858974358974 & 17,67000000000 & 0,003476840728 & 0,010914108497 \\
\hline 17583,205020567100 & 294,871794871795 & 18,45000000000 & 0,003575061830 & 0,010715801463 \\
\hline 19728,680518851200 & 287,435897435897 & 19,24000000000 & 0,003684848059 & 0,010557704076 \\
\hline 22135,943621178700 & 280,000000000000 & 20,13000000000 & 0,003803785941 & 0,010377493822 \\
\hline 24836,937246350300 & 273,012820512821 & 21,07000000000 & 0,003925267580 & 0,010188449064 \\
\hline 27867,501938744900 & 265,192307692308 & 21,90000000000 & 0,004064131386 & 0,010109844389 \\
\hline 31267,851450567500 & 257,179487179487 & 23,15000000000 & 0,004228846961 & 0,009890467340 \\
\hline 35083,106353909100 & 249,230769230769 & 25,98000000000 & 0,004463383966 & 0,009159406612 \\
\hline 39363,892763324500 & 242,307692307692 & 28,37000000000 & 0,004690301064 & 0,008685396916 \\
\hline 44167,014113613600 & 233,589743589744 & 29,90000000000 & 0,004938315762 & 0,008587994323 \\
\hline 49556,204906889700 & 224,294871794872 & 31,13000000000 & 0,005208451942 & 0,008623936914 \\
\hline 55602,976430699800 & 214,743589743590 & 32,41000000000 & 0,005515904653 & 0,008688322784 \\
\hline 62387,565669362300 & 205,064102564103 & 33,51000000000 & 0,005848627311 & 0,008832962030 \\
\hline 70000,000000000200 & 195,384615384615 & 34,48000000000 & 0,006208858602 & 0,009040704344 \\
\hline 78541,291801137600 & 185,833333333333 & 35,50000000000 & 0,006609830912 & 0,009266641174 \\
\hline 88124,778825591900 & 176,538461538462 & 36,40000000000 & 0,007037559534 & 0,009545513541 \\
\hline 98877,628123593100 & 167,564102564103 & 37,16000000000 & 0,007488364087 & 0,009879870272 \\
\hline 110942,523472278000 & 158,910256410256 & 37,84000000000 & 0,007968399943 & 0,010258003842 \\
\hline 124479,558702725000 & 150,769230769231 & 38,47000000000 & 0,008471535382 & 0,010661633360 \\
\hline 139668,362047822000 & 143,076923076923 & 38,96000000000 & 0,008988401562 & 0,011115609620 \\
\hline 156710,479699784000 & 135,641025641026 & 39,45000000000 & 0,009547523566 & 0,011602686942 \\
\hline
\end{tabular}




\begin{tabular}{|c|c|c|c|c|}
\hline $\begin{array}{l}\text { frequency } \\
\qquad[\mathrm{Hz}]\end{array}$ & $\begin{array}{c}\text { measurement } \\
3\end{array}$ & $\begin{array}{c}\text { phase difference } \\
{\left[{ }^{\circ}\right]}\end{array}$ & $\begin{array}{c}\text { real admittance } \\
\mathbf{Y}^{\prime}\left[\mathrm{S} \mathrm{m}^{-1}\right]\end{array}$ & $\begin{array}{c}\text { imaginary } \\
\text { admittance } \\
\mathbf{Y}^{\prime \prime}\left[\mathrm{S} \mathrm{m}^{-1}\right]\end{array}$ \\
\hline 175832,050205671000 & 128,333333333333 & 39,73000000000 & 0,010132053699 & 0,012191123865 \\
\hline 197286,805188512000 & 121,730769230769 & 40,07000000000 & 0,010734737731 & 0,012761466568 \\
\hline 221359,436211787000 & 115,512820512821 & 40,22000000000 & 0,011337585386 & 0,013406725152 \\
\hline 248369,372463503000 & 110,000000000000 & 40,48000000000 & 0,011951 & 0,014003624578 \\
\hline 278675,019387448000 & & 0000 & & \\
\hline 312678,514505674000 & 100,1 & 000 & 0,01 & 0,015 \\
\hline 350831,063539090000 & 95,641025 & 000000000 & 0,013966 & 0,015 \\
\hline 393638,927633244000 & & 42,13000000000 & 0,012 & 9662 \\
\hline 441670,141136134000 & 87,307692307692 & 42,88000000000 & 0,01563 & 8614 \\
\hline 495562,049068895000 & 83,333333333333 & 43,78000000000 & 0,016620 & 67598 \\
\hline 556029,764306995000 & 79,423076923077 & 44,82000000000 & 0,017750401906 & 0,017862282822 \\
\hline 623875,656693620000 & 75,576923076923 & 45,98000000000 & 0,019040686002 & 0,018400225704 \\
\hline 699999,999999997000 & 71,679487179487 & 47,13000000000 & 0,020505996609 & 0,019035349240 \\
\hline 785412,918011371000 & 67,846153846154 & 48,61000000000 & 0,022292277679 & 0,019646372715 \\
\hline 881247,788255913000 & 63,910256410256 & 49,93000000000 & 0,024306945850 & 0,020446606772 \\
\hline 988776,281235923000 & 60,275641025641 & 51,67000000000 & 0,026750598593 & 0,021149101791 \\
\hline
\end{tabular}




\begin{tabular}{|c|c|c|c|c|}
\hline $\begin{array}{l}\text { frequency } \\
\qquad[\mathrm{Hz}]\end{array}$ & $\begin{array}{c}\text { measurement } \\
4\end{array}$ & $\begin{array}{c}\text { phase difference } \\
{\left[{ }^{\circ}\right]}\end{array}$ & $\begin{array}{l}\text { real impedance } \\
\quad Z^{\prime}[\Omega \mathbf{m}]\end{array}$ & $\begin{array}{c}\text { imaginary } \\
\text { impedance } \\
Z^{\prime \prime}[\Omega \mathrm{m}]\end{array}$ \\
\hline 785,412918011374 & 262,820512820513 & 5,150000000000 & 261,759535648509 & 23,591681930588 \\
\hline 881,247788255917 & 261,602564102564 & 5,230000000000 & 260,513462137979 & 23,846123163315 \\
\hline 988,776281235928 & 260,705128205128 & 5,290000000000 & 259,594734155580 & 24,036178380642 \\
\hline 1109,425234722780 & 259,679487179487 & 5,380000000000 & 258,535535143734 & 24,347754100628 \\
\hline 1244,795587027250 & 258,589743589744 & 5,510000000000 & 257,394918034404 & 24,829652834296 \\
\hline 1396,683620478220 & 257,371794871795 & 5,630000000000 & 256,130276899613 & 25,249199014163 \\
\hline 1567,104796997840 & 256,025641025641 & 5,750000000000 & 254,737452685522 & 25,650712697348 \\
\hline 1758,320502056710 & 254,679487179487 & 5,870000000000 & 253,344076003960 & 26,046503483313 \\
\hline 1972,868051885120 & 253,205128205128 & 6,010000000000 & 251,813420796267 & 26,511093079271 \\
\hline 2213,594362117870 & 251,602564102564 & 6,170000000000 & 250,145125057341 & 27,041942848694 \\
\hline 2483,693724635030 & 250,256410256410 & 6,300000000000 & 248,745100647245 & 27,461714775605 \\
\hline 2786,750193874480 & 248,653846153846 & 6,490000000000 & 247,060372406425 & 28,105294759156 \\
\hline 3126,785145056740 & 247,179487179487 & 6,660000000000 & 245,511485158158 & 28,667220614318 \\
\hline 3508,310635390910 & 245,641025641026 & 6,860000000000 & 243,882474495767 & 29,340281385778 \\
\hline 3936,389276332440 & 243,974358974359 & 7,090000000000 & 242,108807891439 & 30,113335556455 \\
\hline 4416,701411361350 & 242,307692307692 & 7,340000000000 & 240,322100238377 & 30,956516091038 \\
\hline 4955,620490688970 & 240,641025641026 & 7,600000000000 & 238,527124237454 & 31,826313399054 \\
\hline 5560,297643069970 & 239,038461538462 & 7,870000000000 & 236,787028368250 & 32,730555925739 \\
\hline 6238,756566936220 & 237,243589743590 & 8,160000000000 & 234,841632078817 & 33,673858065007 \\
\hline 7000,000000000000 & 235,705128205128 & 8,480000000000 & 233,128257925558 & 34,758061205859 \\
\hline 7854,129180113750 & 234,743589743590 & 8,820000000000 & 231,967727806529 & 35,993418592612 \\
\hline 8812,477882559180 & 232,948717948718 & 9,210000000000 & 229,945623562292 & 37,284251346916 \\
\hline 9887,762812359290 & 231,025641025641 & 9,610000000000 & 227,783638664027 & 38,567612945115 \\
\hline 11094,252347227800 & 228,974358974359 & 10,08000000000 & 225,439987367841 & 40,075792734595 \\
\hline 12447,955870272500 & 226,730769230769 & 10,56000000000 & 222,890750784286 & 41,551834265094 \\
\hline 13966,836204782200 & 224,358974358974 & 11,06000000000 & 220,191917107031 & 43,040318494885 \\
\hline 15671,047969978400 & 221,794871794872 & 11,61000000000 & 217,256979760795 & 44,635970917198 \\
\hline 17583,205020567100 & 219,294871794872 & 12,20000000000 & 214,342293227739 & 46,342444145762 \\
\hline 19728,680518851200 & 216,410256410256 & 12,79000000000 & 211,040686634498 & 47,908534358637 \\
\hline 22135,943621178700 & 213,333333333333 & 13,44000000000 & 207,490954795721 & 49,584420830247 \\
\hline 24836,937246350300 & 210,833333333333 & 14,18000000000 & 204,409435477880 & 51,647624650692 \\
\hline 27867,501938744900 & 207,692307692308 & 14,91000000000 & 200,699554111807 & 53,439532687686 \\
\hline 31267,851450567500 & 204,358974358974 & 15,70000000000 & 196,734697923565 & 55,299629600709 \\
\hline 35083,106353909100 & 200,897435897436 & 16,53000000000 & 192,594524344587 & 57,158804594276 \\
\hline 39363,892763324500 & 197,371794871795 & 17,41000000000 & 188,329822189434 & 59,055088561530 \\
\hline 44167,014113613600 & 193,653846153846 & 18,34000000000 & 183,817400090498 & 60,934190354409 \\
\hline 49556,204906889700 & 189,807692307692 & 19,29000000000 & 179,151627068218 & 62,702907253027 \\
\hline 55602,976430699800 & 185,833333333333 & 20,33000000000 & 174,257245556009 & 64,563458310492 \\
\hline 62387,565669362300 & 181,666666666667 & 21,37000000000 & 169,176490602997 & 66,197377629569 \\
\hline 70000,000000000200 & 177,307692307692 & 22,47000000000 & 163,846453022576 & 67,766935768117 \\
\hline 78541,291801137600 & 172,820512820513 & 23,62000000000 & 158,342115853460 & 69,243801156454 \\
\hline 88124,778825591900 & 168,333333333333 & 24,86000000000 & 152,735184831951 & 70,767749896835 \\
\hline 98877,628123593100 & 163,589743589744 & 26,10000000000 & 146,908100855198 & 71,969535991814 \\
\hline 110942,523472278000 & 158,846153846154 & 27,39000000000 & 141,038815631743 & 73,076351009825 \\
\hline 124479,558702725000 & 154,102564102564 & 28,79000000000 & 135,054061511094 & 74,215906194982 \\
\hline 139668,362047822000 & 149,102564102564 & 30,27000000000 & 128,773861213000 & 75,158946841040 \\
\hline 156710,479699784000 & 143,910256410256 & 31,87000000000 & 122,215534982995 & 75,983714761033 \\
\hline
\end{tabular}




\begin{tabular}{|c|c|c|c|c|}
\hline $\begin{array}{c}\text { frequency } \\
{[\mathbf{H z}]}\end{array}$ & $\begin{array}{c}\text { measurement } \\
\mathbf{4}\end{array}$ & $\begin{array}{c}\text { phase difference } \\
{\left[{ }^{\circ}\right]}\end{array}$ & $\begin{array}{c}\text { real impedance } \\
\mathbf{Z}^{\prime}[\mathbf{\Omega} \mathbf{~ m}]\end{array}$ & $\begin{array}{c}\text { imaginary } \\
\text { impedance } \\
\mathbf{Z}^{\prime \prime}[\mathbf{\Omega} \text { }]\end{array}$ \\
\hline 175832,050205671000 & 138,012820512821 & 33,39000000000 & 115,232966412786 & 75,953288787345 \\
\hline 197286,805188512000 & 132,435897435897 & 35,03000000000 & 108,445347584934 & 76,018902365362 \\
\hline 221359,436211787000 & 126,538461538462 & 36,54000000000 & 101,666238706496 & 75,338955101557 \\
\hline 248369,372463503000 & 120,897435897436 & 38,15000000000 & 95,073291071348 & 74,681050685146 \\
\hline 278675,019387448000 & 115,192307692308 & 39,69000000000 & 88,641751264751 & 73,565669195605 \\
\hline 312678,514505674000 & 109,423076923077 & 41,28000000000 & 82,230837392051 & 72,190713704158 \\
\hline 350831,063539090000 & 103,782051282051 & 42,87000000000 & 76,061784742766 & 70,606791954142 \\
\hline 393638,927633244000 & 98,205128205128 & 44,43000000000 & 70,128894737634 & 68,747256881009 \\
\hline 441670,141136134000 & 92,756410256410 & 46,02000000000 & 64,410722020717 & 66,745865283367 \\
\hline 495562,049068895000 & 87,371794871795 & 47,64000000000 & 58,869951960955 & 64,561283252689 \\
\hline 556029,764306995000 & 82,051282051282 & 49,21000000000 & 53,603156798128 & 62,121771284585 \\
\hline 623875,656693620000 & 76,923076923077 & 50,75000000000 & 48,669640658655 & 59,568664929399 \\
\hline 699999,999999997000 & 71,884615384615 & 52,24000000000 & 44,018923211736 & 56,830734011390 \\
\hline 785412,918011371000 & 67,083333333333 & 53,76000000000 & 39,657579584915 & 54,105914580360 \\
\hline 881247,788255913000 & 62,371794871795 & 55,10000000000 & 35,685765055288 & 51,154344565717 \\
\hline 988776,281235923000 & 58,051282051282 & 56,78000000000 & 31,803701148839 & 48,564142543988 \\
\hline
\end{tabular}




\begin{tabular}{|c|c|c|c|c|}
\hline $\begin{array}{c}\text { frequency } \\
{[\text { [Hz] }}\end{array}$ & $\begin{array}{c}\text { measurement } \\
4\end{array}$ & $\begin{array}{c}\text { phase difference } \\
{\left[^{\circ}\right]}\end{array}$ & $\begin{array}{l}\text { real admittance } \\
\quad \mathbf{Y}^{\prime}\left[\mathbf{S ~ m}^{-1}\right]\end{array}$ & $\begin{array}{c}\text { imaginary } \\
\text { admittance } \\
Y^{\prime \prime}\left[\mathrm{S} \mathrm{m}^{-1}\right]\end{array}$ \\
\hline 785,412918011374 & 262,820512820513 & 5,150000000000 & 0,003820300176 & 0,042387821392 \\
\hline 881,247788255917 & 261,602564102564 & 5,230000000000 & 0,003838573223 & 0,041935537829 \\
\hline 988,776281235928 & & 5,290000000000 & & \\
\hline 1109,425234722780 & 259,679487179487 & 5,380000000000 & 0,003867940241 & 0,041071550003 \\
\hline 1244,795587027250 & 258,589743589744 & 5,510000000000 & 0,003885080590 & 0,040274425368 \\
\hline 1396,683620478220 & 257,371794871795 & 5,630000000000 & 0,003904263143 & 0,039605216761 \\
\hline 1567,104796997840 & 256,025641025641 & 5,750000000000 & 0,003925610425 & 0,038985271552 \\
\hline 1758,320502056710 & 254,679487179487 & 5,870000000000 & 0,003947201039 & \\
\hline 1972,868051885120 & & & & \\
\hline 2213,594362117870 & 251,602564102564 & 6,170000000000 & & \\
\hline 2483,6 & & 0000 & & \\
\hline 2786,750193874480 & & 6,490000000000 & & \\
\hline 3126,785145056740 & & 6,660000000000 & 0,004073129204 & 0,034883046859 \\
\hline 3508,310635390910 & 245,641025641026 & 6,860000000000 & 0,004100335631 & 0,034082836045 \\
\hline 3936,389276332440 & 243,974358974359 & 7,090000000000 & & \\
\hline 4416,701411361350 & 242,3076923076 & & & \\
\hline 4955,620490688970 & & 7,600000000000 & & \\
\hline 5560,2 & & 000 & & \\
\hline 6238,7 & 237,2 & 0000 & & \\
\hline 7000,000000000000 & 235,705128205128 & 8,480000000000 & 0,004289484 & \\
\hline 7854,129180113750 & 234,743589743590 & 8,820000000000 & 0,004310944 & 0,027782856953 \\
\hline 8812,477882559180 & 232,948717948718 & 9,210000000000 & 0,004348854240 & 0,026820975717 \\
\hline 9887,762812359290 & 231,025641025641 & 9,610000000000 & 0,004390130 & 0,025 \\
\hline 11094,252347227800 & & & & \\
\hline 12447,955870272500 & & & & \\
\hline 13966,83 & 224,3 & 000 & & \\
\hline 15671, & 221,7 & 11,610 & 0,00 & 0,02 \\
\hline 17583,205020567100 & 219,294871794872 & 12,20000000000 & 0,00466 & 0,02157 \\
\hline 19728,680518851200 & 216,410256410256 & 12,79000000000 & 0,004738422794 & 0,020873107754 \\
\hline 22135,943621178700 & 213,333333333333 & 13,44000000000 & 0,004819487196 & 0,020167624896 \\
\hline 24836,937246350300 & 210,833333333333 & 14,18000000000 & 0,004892142076 & 0,019361974665 \\
\hline 27867,501938744900 & 207,692307692308 & & & \\
\hline 31267,8 & 204,3589743589 & 15,70000000000 & & 0,0 \\
\hline 35083 & 200,8 & 16,53000000000 & 0,005192255613 & 0,017495117456 \\
\hline 39363,892763324500 & 197,371794871795 & 17,41000000000 & 0,005309833506 & 0,016933341806 \\
\hline 44167,014113613600 & 193,653846153846 & 18,34000000000 & 0,005440181395 & 0,016411147735 \\
\hline 49556,204906889700 & 189,807692307692 & 19,29000000000 & 0,005581863901 & 0,015948223835 \\
\hline 55602,976430699800 & 185,833333333333 & 20,33000000000 & 0,005738642298 & 0,015488637476 \\
\hline 62387,565669362300 & & & & 0,015106338586 \\
\hline 70000,000000000200 & & 22,47000000000 & & 0,014756458864 \\
\hline 78541,291801137600 & 172,820512820513 & 23,62000000000 & 0,006315439165 & 0,014441725949 \\
\hline 88124,778825591900 & 168,333333333333 & 24,86000000000 & 0,006547279863 & 0,014130730473 \\
\hline 98877,628123593100 & 163,589743589744 & 26,10000000000 & 0,006806976567 & 0,013894767921 \\
\hline 110942,523472278000 & 158,846153846154 & 27,39000000000 & 0,007090246721 & 0,013684317651 \\
\hline 124479,558702725000 & 154,102564102564 & 28,79000000000 & 0,007404442257 & 0,013474200495 \\
\hline 139668,362047822000 & 149,102564102564 & 30,27000000000 & 0,007765551103 & 0,013305135876 \\
\hline 156710,479699784000 & 143,910256410256 & 31,87000000000 & 0,008182265864 & 0,013160714808 \\
\hline
\end{tabular}




\begin{tabular}{|c|c|c|c|c|}
\hline $\begin{array}{l}\text { frequency } \\
\qquad[\mathrm{Hz}]\end{array}$ & $\begin{array}{c}\text { measurement } \\
4\end{array}$ & $\begin{array}{c}\text { phase difference } \\
{\left[{ }^{\circ}\right]}\end{array}$ & $\begin{array}{l}\text { real admittance } \\
\mathbf{Y}^{\prime}\left[\mathbf{S ~ m}^{-1}\right]\end{array}$ & $\begin{array}{c}\text { imaginary } \\
\text { admittance } \\
\mathbf{Y}^{\prime \prime}\left[\mathrm{S} \mathrm{m}^{-1}\right]\end{array}$ \\
\hline 175832,050205671000 & 138,012820512821 & 33,39000000000 & 0,008678072180 & 0,013165986832 \\
\hline 197286,805188512000 & 132,435897435897 & 35,03000000000 & 0,009221234680 & 0,013154622980 \\
\hline 221359,436211787000 & 126,538461538462 & 36,54000000000 & 0,009836106978 & 0,013273345757 \\
\hline 248369,372463503000 & 120,897435897436 & 38,15000000000 & & \\
\hline 278675,019387448000 & 115,192307692308 & 39,69000000000 & 0,01128 & 0,013593297131 \\
\hline 312678,514505674000 & 109,4 & 41,28000000000 & 0,012 & 0,0 \\
\hline 350831,063539090000 & 103,78205128 & 42,87000000000 & & 0,01 \\
\hline 393638,927633244000 & 98,2051282051 & 44,43000000000 & 0,0142594 & 0,01 \\
\hline 441670,141136134000 & 92,756410256410 & 46,02000000000 & 0,015525365477 & 201456 \\
\hline 495562,049068895000 & 87,371794871795 & 47,64000000000 & 0,016986594463 & 159286 \\
\hline 556029,764306995000 & 82,051282051282 & 49,21000000000 & 0,018655617686 & 0,0160 \\
\hline 623875,656693620000 & 76,923076923077 & 50,75000000000 & 0,020546689609 & 0,016787349543 \\
\hline 699999,999999997000 & 71,884615384615 & 52,24000000000 & 0,022717502543 & 0,017596112691 \\
\hline 785412,918011371000 & 67,083333333333 & 53,76000000000 & 0,025215860637 & 0,018482267748 \\
\hline 881247,788255913000 & 62,371794871795 & 55,10000000000 & 0,028022378067 & 0,019548681710 \\
\hline 988776,281235923000 & 58,051282051282 & 56,78000000000 & 0,031442881296 & 0,020591324125 \\
\hline
\end{tabular}




\begin{tabular}{|c|c|c|c|c|}
\hline $\begin{array}{l}\text { frequency } \\
\qquad[\mathrm{Hz}]\end{array}$ & $\begin{array}{c}\text { measurement } \\
5\end{array}$ & $\begin{array}{c}\text { phase difference } \\
{\left[{ }^{\circ}\right]}\end{array}$ & $\begin{array}{l}\text { real impedance } \\
\quad Z^{\prime}[\Omega \mathbf{m}]\end{array}$ & $\begin{array}{c}\text { imaginary } \\
\text { impedance } \\
\mathbf{Z}^{\prime \prime}[\Omega \mathrm{m}]\end{array}$ \\
\hline 785,412918011374 & 320,576923076923 & 5,050000000000 & 319,332527146526 & 28,218800748451 \\
\hline 881,247788255917 & 318,910256410256 & 5,160000000000 & 317,617850080201 & 28,681927307741 \\
\hline 988,776281235928 & 317,307692307692 & 5,270000000000 & 315,966409470802 & 29,144462317264 \\
\hline 1109,425234722780 & 315,576923076923 & 5,390000000000 & 314,181560087362 & 29,643577378066 \\
\hline 1244,795587027250 & 314,1666666666667 & 5,580000000000 & 312,677957193149 & 30,548151007287 \\
\hline 1396,683620478220 & 312,435897435897 & 5,730000000000 & 310,874789561411 & 31,193833071987 \\
\hline 1567,104796997840 & 310,641025641026 & 5,890000000000 & 309,001071722896 & 31,877648680694 \\
\hline 1758,320502056710 & 308,846153846154 & 6,050000000000 & 307,125972929450 & 32,551244180541 \\
\hline 1972,868051885120 & 307,115384615385 & 6,250000000000 & 305,289994642509 & 33,434692142036 \\
\hline 2213,594362117870 & 304,871794871795 & 6,480000000000 & 302,924063643151 & 34,406728619120 \\
\hline 2483,693724635030 & 302,884615384615 & 6,690000000000 & 300,822273219631 & 35,285268479353 \\
\hline 2786,750193874480 & 300,897435897436 & 6,920000000000 & 298,705502121314 & 36,253136858836 \\
\hline 3126,785145056740 & 298,782051282051 & 7,180000000000 & 296,439119087062 & 37,343846122178 \\
\hline 3508,310635390910 & 296,538461538462 & 7,460000000000 & 294,028484154325 & 38,500775024761 \\
\hline 3936,389276332440 & 294,294871794872 & 7,700000000000 & 291,641273710950 & 39,431447259960 \\
\hline 4416,701411361350 & 291,987179487180 & 8,020000000000 & 289,131377806990 & 40,737689579918 \\
\hline 4955,620490688970 & 289,679487179487 & 8,350000000000 & 286,608721743480 & 42,067159556349 \\
\hline 5560,297643069970 & 287,179487179487 & 8,690000000000 & 283,882744706645 & 43,389458564209 \\
\hline 6238,756566936220 & 284,615384615385 & 9,020000000000 & 281,095738125005 & 44,621779073921 \\
\hline 7000,000000000000 & 282,051282051282 & 9,380000000000 & 278,280006235367 & 45,969161798057 \\
\hline 7854,129180113750 & 279,358974358974 & 9,770000000000 & 275,307398996051 & 47,405407000967 \\
\hline 8812,477882559180 & 276,474358974359 & 10,200000000000 & 272,104849818676 & 48,959390063998 \\
\hline 9887,762812359290 & 273,461538461538 & 10,650000000000 & 268,751012253533 & 50,538168055966 \\
\hline 11094,25234722780 & 270,384615384615 & 11,120000000000 & 265,308252753927 & 52,147591098195 \\
\hline 12447,95587027250 & 267,307692307692 & 11,620000000000 & 261,829221283782 & 53,841073993672 \\
\hline 13966,83620478220 & 263,910256410256 & 12,120000000000 & 258,027698512456 & 55,410560716280 \\
\hline 15671,04796997840 & 260,384615384615 & 12,680000000000 & 254,034153668199 & 57,155898200238 \\
\hline 17583,20502056710 & 256,858974358974 & 13,280000000000 & 249,990338319524 & 59,003080052096 \\
\hline 19728,68051885120 & 252,948717948718 & 13,900000000000 & 245,541489506894 & 60,765375359954 \\
\hline 22135,94362117870 & 248,717948717949 & 14,550000000000 & 240,741259171865 & 62,484111154829 \\
\hline 24836,93724635030 & 244,871794871795 & 15,210000000000 & 236,294111992138 & 64,243976850606 \\
\hline 27867,50193874490 & 240,448717948718 & 15,870000000000 & 231,283924275647 & 65,752051943944 \\
\hline 31267,85145056750 & 236,153846153846 & 16,590000000000 & 226,323333478340 & 67,426906917836 \\
\hline 35083,10635390910 & 231,538461538462 & 17,320000000000 & 221,039798703998 & 68,930882487414 \\
\hline 39363,89276332450 & 226,666666666667 & 18,070000000000 & 215,487074788078 & 70,307171590493 \\
\hline 44167,01411361360 & 221,6666666666667 & 18,830000000000 & 209,803153656499 & 71,545424919407 \\
\hline 49556,20490688970 & 216,538461538462 & 19,610000000000 & 203,978990648758 & 72,673768991003 \\
\hline 55602,97643069980 & 211,346153846154 & 20,420000000000 & 198,065216022180 & 73,738503833813 \\
\hline 62387,56566936230 & 206,025641025641 & 21,220000000000 & 192,056590308043 & 74,570978800568 \\
\hline 70000,00000000020 & 200,769230769231 & 22,060000000000 & 186,071128028505 & 75,404372140271 \\
\hline 78541,29180113760 & 195,256410256410 & 22,890000000000 & 179,880613559392 & 75,947551716407 \\
\hline 88124,77882559190 & 189,935897435897 & 23,770000000000 & 173,823794468761 & 76,556734591163 \\
\hline 98877,62812359310 & 184,423076923077 & 24,630000000000 & 167,643900283684 & 76,859573245298 \\
\hline 110942,5234722780 & 179,038461538462 & 25,540000000000 & 161,543630715036 & 77,190841979235 \\
\hline 124479,5587027250 & 173,589743589744 & 26,500000000000 & 155,351426360146 & 77,455363968036 \\
\hline 139668,3620478220 & 168,141025641026 & 27,510000000000 & 149,129358431998 & 77,664914583592 \\
\hline 156710,4796997840 & 162,628205128205 & 28,610000000000 & 142,771206073926 & 77,873717128555 \\
\hline
\end{tabular}




\begin{tabular}{|c|c|c|c|c|}
\hline $\begin{array}{c}\text { frequency } \\
{[\mathbf{H z}]}\end{array}$ & $\begin{array}{c}\text { measurement } \\
\mathbf{5}\end{array}$ & $\begin{array}{c}\text { phase difference } \\
\mathbf{[}^{\circ} \mathbf{]}\end{array}$ & $\begin{array}{c}\text { real impedance } \\
\mathbf{Z}^{\prime}[\boldsymbol{\Omega} \mathbf{~ m}]\end{array}$ & $\begin{array}{c}\text { imaginary } \\
\text { impedance } \\
\mathbf{Z}^{\prime \prime}[\mathbf{\Omega} \text { m] }\end{array}$ \\
\hline 175832,0502056710 & 156,602564102564 & 29,640000000000 & 136,111099980220 & 77,447605164216 \\
\hline 197286,8051885120 & 150,961538461538 & 30,770000000000 & 129,710363286549 & 77,230873041460 \\
\hline 221359,4362117870 & 145,064102564103 & 31,810000000000 & 123,275577195829 & 76,463886376274 \\
\hline 248369,3724635030 & 139,423076923077 & 32,900000000000 & 117,062384959125 & 75,731053118122 \\
\hline 278675,0193874480 & 133,782051282051 & 33,980000000000 & 110,936453895815 & 74,771254117175 \\
\hline 312678,5145056740 & 128,205128205128 & 35,080000000000 & 104,916715676116 & 73,682003703960 \\
\hline 350831,0635390900 & 122,692307692308 & 36,210000000000 & 98,995174265500 & 72,480051317637 \\
\hline 393638,9276332440 & 117,243589743590 & 37,330000000000 & 93,226952878976 & 71,097078652111 \\
\hline 441670,1411361340 & 111,858974358974 & 38,490000000000 & 87,553897816400 & 69,618568800264 \\
\hline 495562,0490688950 & 106,538461538462 & 39,670000000000 & 82,006266310878 & 68,010411502443 \\
\hline 556029,7643069950 & 101,346153846154 & 40,870000000000 & 76,637575108578 & 66,315344988059 \\
\hline 623875,6566936200 & 96,217948717949 & 42,060000000000 & 71,436410448956 & 64,457217731284 \\
\hline 699999,9999999970 & 90,448717948718 & 42,410000000000 & 66,781693078904 & 61,001442999996 \\
\hline 785412,9180113710 & 86,282051282051 & 44,470000000000 & 61,572368495032 & 60,443658156589 \\
\hline 881247,7882559130 & 81,346153846154 & 45,350000000000 & 57,167973758891 & 57,870713853078 \\
\hline 988776,2812359230 & 76,923076923077 & 46,990000000000 & 52,471230382717 & 56,248819947066 \\
\hline
\end{tabular}




\begin{tabular}{|c|c|c|c|c|}
\hline $\begin{array}{l}\text { frequency } \\
{[\mathrm{Hz}]}\end{array}$ & $\begin{array}{c}\text { measurement } \\
5\end{array}$ & $\begin{array}{c}\text { phase difference } \\
{\left[\left[^{\circ}\right]\right.}\end{array}$ & $\begin{array}{l}\text { real admittance } \\
\mathbf{Y}^{\prime}\left[\mathbf{S ~ m}^{-1}\right]\end{array}$ & $\begin{array}{c}\text { imaginary } \\
\text { admittance } \\
\mathrm{Y}^{\prime \prime}\left[\mathrm{S} \mathrm{m}^{-1}\right]\end{array}$ \\
\hline 785,412918011374 & 320,576923076923 & 5,050000000000 & 0,003131532 & 0,035437367 \\
\hline 881,247788255917 & 318,910256410256 & 5,160000000000 & 0,003148438 & 0,034865161 \\
\hline 988,776281235928 & 317,307692307692 & 5,270000000000 & 0,003164893 & 0,034311836 \\
\hline 1109,425234722780 & 315,576923076923 & 5,390000000000 & 0,003182873 & 0,03373412 \\
\hline 1244,795587027250 & 314,166666666667 & 5,580000000000 & 0,003198179 & 0,032735205 \\
\hline 1396,683620478220 & 312,435897435897 & 5,730000000000 & 0,003216729 & 0,032057618 \\
\hline 1567,104796997840 & 310,641025641026 & 5,890000000000 & 0,003236235 & 0,031369942 \\
\hline 1758,320502056710 & 308,846153846154 & 6,050000000000 & & 0,030720792 \\
\hline 1972,868051885120 & 307,115384615385 & 6,250000000000 & 0,003275574 & 0,029909054 \\
\hline 2213,594362117870 & 304,871794871795 & 6,480000000000 & & 0,029064083 \\
\hline 2483,693724635030 & 302,884615384615 & 6,690000000000 & 0,003324222 & 0,028340439 \\
\hline 2786,750193874480 & 300,897435897436 & 6,920000000000 & 0,003347779 & 0,02758382 \\
\hline 3126,785145056740 & 298,782051282051 & 7,180000000000 & 0,003373374 & 0,026778174 \\
\hline 3508,310635390910 & 296,538461538462 & 7,460000000000 & 0,003401031 & 0,025973503 \\
\hline 3936,389276332440 & 294,294871794872 & 7,700000000000 & & 0,025360469 \\
\hline 4416,701411361350 & 291,987179487180 & 8,020000000000 & 0,00345 & 293 \\
\hline 4955,620490688970 & 289,679487179487 & 8,350000000000 & & 1512 \\
\hline 5560,297643069970 & 287,179487179487 & 8,690000000000 & 0,003522581 & 0,023047073 \\
\hline 6238,756566936220 & 284,615384615385 & 9,020000000000 & 0,003557507 & 0,022410581 \\
\hline 7000,000000000000 & 282,051282051282 & 9,380000000000 & 0,003593503 & 0,021753714 \\
\hline 7854,129180113750 & 279,358974358974 & 9,770000000000 & 0,003632303 & 0,02109464 \\
\hline 8812,477882559180 & 276,474358974359 & 10,200000000000 & 0,003675054 & 0,020425091 \\
\hline 9887,762812359290 & 273,461538461538 & 10,650000000000 & 0,003720916 & 0,019787025 \\
\hline 11094,252347227800 & 270,384615384615 & 11,120000000000 & 0,003769201 & 0,019176341 \\
\hline 12447,955870272500 & 267,307692307692 & 11,620000000000 & 0,003819283 & 0,018573181 \\
\hline 13966,836204782200 & 263,910256410256 & 12,120000000000 & 0,003875553 & 0,018047101 \\
\hline 15671,047969978400 & 260,384615384615 & 12,680000000000 & 0,003936479 & 0,017496007 \\
\hline 17583,205020567100 & 256,858974358974 & 13,280000000000 & 0,004000155 & 0,016948268 \\
\hline 19728,680518851200 & 252,948717948718 & 13,900000000000 & 0,004072631 & 0,01645674 \\
\hline 22135,943621178700 & 248,717948717949 & 14,550000000000 & 0,004153837 & 0,016004069 \\
\hline 24836,937246350300 & 244,871794871795 & 15,210000000000 & & 0,0155656662 \\
\hline 27867,501938744900 & 240,448717948718 & 15,870000000000 & 0,00432369 & 0,015208651 \\
\hline 31267,851450567500 & 236,153846153846 & 16,590000000000 & 0,004418457 & 0,014830875 \\
\hline 35083,106353909100 & 231,538461538462 & 17,320000000000 & 0,004524072 & 0,014507286 \\
\hline 39363,892763324500 & 226,666666666667 & 18,070000000000 & 0,00464065 & 0,0142233 \\
\hline 44167,014113613600 & 221,666666666667 & 18,830000000000 & 0,004766373 & 0,013977134 \\
\hline 49556,204906889700 & 216,538461538462 & 19,610000000000 & 0,004902466 & 0,013760123 \\
\hline 55602,976430699800 & 211,346153846154 & 20,420000000000 & & 0,013561436 \\
\hline 62387,5656699362300 & 206,025641025641 & 21,220000000000 & 0,005206799 & 0,013410043 \\
\hline 70000,000000000200 & 200,769230769231 & 22,060000000000 & 0,005374289 & 0,01326183 \\
\hline 78541,291801137600 & 195,256410256410 & 22,890000000000 & 0,005559243 & 0,013166981 \\
\hline 88124,7788255591900 & 189,935897435897 & 23,770000000000 & 0,005752952 & 0,013062208 \\
\hline 98877,628123593100 & 184,423076923077 & 24,630000000000 & 0,005965025 & 0,013010741 \\
\hline 110942,523472278000 & 179,038461538462 & 25,540000000000 & 0,006190278 & 0,012954905 \\
\hline 124479,558702725000 & 173,589743589744 & 26,500000000000 & 0,006437018 & 0,012910662 \\
\hline 139668,362047822000 & 168,141025641026 & 27,510000000000 & 0,006705588 & 0,012875827 \\
\hline
\end{tabular}




\begin{tabular}{|c|c|c|c|c|}
\hline $\begin{array}{l}\text { frequency } \\
\qquad[\mathrm{Hz}]\end{array}$ & $\begin{array}{c}\text { measurement } \\
5\end{array}$ & $\begin{array}{c}\text { phase difference } \\
{\left[^{\circ}\right]}\end{array}$ & $\begin{array}{l}\text { real admittance } \\
\qquad \mathbf{Y}^{\prime}\left[\mathrm{S} \mathrm{m}^{-1}\right]\end{array}$ & $\begin{array}{c}\text { imaginary } \\
\text { admittance } \\
\mathbf{Y}^{\prime \prime}\left[\mathrm{S} \mathrm{m}^{-1}\right]\end{array}$ \\
\hline 156710,479699784000 & 162,628205128205 & 28,610000000000 & 0,007004213 & 0,012841303 \\
\hline 175832,050205671000 & 156,602564102564 & 29,640000000000 & 0,007346939 & 0,012911955 \\
\hline 197286,805188512000 & 150,961538461538 & 30,770000000000 & 0,007709484 & 0,01294819 \\
\hline 221359,436211787000 & 145,064102564103 & 31,810000000000 & 0,008111907 & 0,013078069 \\
\hline 248369,372463503000 & 139,423076923077 & 32,900000000000 & 0,008542454 & 0,013204623 \\
\hline 278675,019387448000 & 133,782051282051 & 33,980000000000 & 0,00901417 & 0,013374124 \\
\hline 312678,514505674000 & 128,205128205128 & 35,080000000000 & 0,00953137 & 0,013571835 \\
\hline 350831,063539090000 & 122,692307692308 & 36,210000000000 & 0,010101502 & 0,0137969 \\
\hline 393638,927633244000 & 117,243589743590 & 37,330000000000 & 0,010726512 & 0,014065276 \\
\hline 441670,141136134000 & 111,858974358974 & 38,490000000000 & 0,011421536 & 0,014363984 \\
\hline 495562,049068895000 & 106,538461538462 & 39,670000000000 & 0,01219419 & 0,014703631 \\
\hline 556029,764306995000 & 101,346153846154 & 40,870000000000 & 0,01304843 & 0,015079466 \\
\hline 623875,656693620000 & 96,217948717949 & 42,060000000000 & 0,013998464 & 0,015514166 \\
\hline 699999,999999997000 & 90,448717948718 & 42,410000000000 & 0,014974164 & 0,016393055 \\
\hline 785412,918011371000 & 86,282051282051 & 44,470000000000 & 0,016241051 & 0,016544333 \\
\hline 881247,788255913000 & 81,346153846154 & 45,350000000000 & 0,017492311 & 0,017279897 \\
\hline 988776,281235923000 & 76,923076923077 & 46,990000000000 & 0,019058063 & 0,017778151 \\
\hline
\end{tabular}




\begin{tabular}{|c|c|c|c|c|}
\hline $\begin{array}{l}\text { frequency } \\
\qquad[\mathrm{Hz}]\end{array}$ & $\begin{array}{c}\text { measurement } \\
6\end{array}$ & $\begin{array}{c}\text { phase difference } \\
{\left[{ }^{\circ}\right]}\end{array}$ & $\begin{array}{c}\text { real impedance } \\
Z^{\prime}[\Omega \mathrm{m}]\end{array}$ & $\begin{array}{c}\text { imaginary } \\
\text { impedance } \\
\mathrm{Z}^{\prime \prime}[\Omega \mathrm{m}] \\
\end{array}$ \\
\hline 785,4129180114 & 1598,3000000000 & 1,2800000000 & 1597,9011726658 & 35,7033947042 \\
\hline 881,2477882559 & 1597,2000000000 & 1,5200000000 & 1596,6379877966 & 42,3671562042 \\
\hline 988,7762812359 & 1577,4000000000 & 1,2000000000 & 1577,0540501132 & 33,0345731240 \\
\hline 1109,4252347228 & 1567,5000000000 & 1,8100000000 & 1566,7179161439 & 49,5098094685 \\
\hline 1244,7955870272 & 1562,0000000000 & 2,5600000000 & 1560,4411172602 & 69,7676111371 \\
\hline 1396,6836204782 & 1548,8000000000 & 2,8700000000 & 1546,8573567045 & 77,5484236400 \\
\hline 1567,1047969978 & 1542,2000000000 & 3,5800000000 & 1539,1905287905 & 96,2982662450 \\
\hline 1758,3205020567 & 1566,4000000000 & 3,1900000000 & 1563,9728501446 & 87,1658419938 \\
\hline 1972,8680518851 & 1568,6000000000 & 3,9400000000 & 1564,8926955916 & 107,7813123136 \\
\hline 2213,5943621179 & 1562,0000000000 & 4,9700000000 & 1556,1271864232 & 135,3225024693 \\
\hline 2483,6937246350 & 1549,9000000000 & 5,8600000000 & 1541,8007455934 & 158,2418114397 \\
\hline 2786,7501938745 & 1532,3000000000 & 5,9800000000 & 1523,9617169457 & 159,6370110088 \\
\hline 3126,7851450567 & 1523,5000000000 & 6,8000000000 & 1512,7829516003 & 180,3884457150 \\
\hline 3508,3106353909 & 1518,0000000000 & 7,6500000000 & 1504,4894165016 & 202,0781918833 \\
\hline 3936,3892763324 & 1503,7000000000 & 8,2300000000 & 1488,2140175848 & 215,2503841209 \\
\hline 4416,7014113614 & 1497,1000000000 & 8,6000000000 & 1480,2671780655 & 223,8693626696 \\
\hline 4955,6204906890 & 1480,6000000000 & 9,2600000000 & 1461,3052600309 & 238,2504921421 \\
\hline 5560,2976430700 & 1469,6000000000 & 9,8900000000 & 1447,7607423785 & 252,4143276990 \\
\hline 6238,7565669362 & 1459,7000000000 & 10,7800000000 & 1433,9400891996 & 273,0199820276 \\
\hline 7000,0000000000 & 1445,4000000000 & 11,7500000000 & 1415,1123259292 & 294,3437870980 \\
\hline 7854,1291801137 & 1436,6000000000 & 13,1200000000 & 1399,1001309724 & 326,0956661978 \\
\hline 8812,4778825592 & 1425,6000000000 & 14,2100000000 & 1381,9802338153 & 349,9514156904 \\
\hline 9887,7628123593 & 1397,0000000000 & 14,7500000000 & 1350,9631766628 & 355,6789216431 \\
\hline 11094,2523472278 & 1369,5000000000 & 15,1600000000 & 1321,8404439055 & 358,1453487842 \\
\hline 12447,9558702724 & 1331,0000000000 & 15,5500000000 & 1282,2812430707 & 356,8134157640 \\
\hline 13966,8362047821 & 1310,1000000000 & 16,9800000000 & 1252,9884893027 & 382,5988181828 \\
\hline 15671,0479699783 & 1272,7000000000 & 17,7100000000 & 1212,3847144529 & 387,1544835862 \\
\hline 17583,2050205670 & 1234,2000000000 & 17,4500000000 & 1177,4008853551 & 370,1037627004 \\
\hline 19728,6805188512 & 1236,4000000000 & 20,5000000000 & 1158,1014947867 & 432,9964061892 \\
\hline 22135,9436211786 & 1223,9700000000 & 23,0100000000 & 1126,5868391394 & 478,4398131196 \\
\hline 24836,9372463503 & 1190,0900000000 & 24,4900000000 & 1083,0219282504 & 493,3332656824 \\
\hline 27867,5019387448 & 1166,1100000000 & 26,7600000000 & 1041,2200537411 & 525,0460282559 \\
\hline 31267,8514505674 & 1126,0700000000 & 27,8400000000 & 995,7332084447 & 525,8792851030 \\
\hline 35083,1063539091 & 1101,3200000000 & 30,5200000000 & 948,7342545711 & 559,2933546926 \\
\hline 39363,8927633245 & 1055,0100000000 & 31,6200000000 & 898,3874108274 & 553,1240007149 \\
\hline 44167,0141136136 & 1022,2300000000 & 34,3000000000 & 844,4624595824 & 576,0532330055 \\
\hline 49556,2049068897 & 978,8900000000 & 35,7000000000 & 794,9404436391 & 571,2226563844 \\
\hline 55602,9764306998 & 938,3000000000 & 38,1900000000 & 737,4707848184 & 580,1238932671 \\
\hline 62387,5656693623 & 891,7700000000 & 40,1500000000 & 681,6324306226 & 575,0051846927 \\
\hline 70000,0000000001 & 842,6000000000 & 41,0500000000 & 635,4356452805 & 553,3500706668 \\
\hline 78541,2918011376 & 800,2500000000 & 43,4000000000 & 581,4413804445 & 549,8417805212 \\
\hline 88124,7788255919 & 754,6000000000 & 45,7500000000 & 526,5526809965 & 540,5214465082 \\
\hline 98877,6281235930 & 709,7200000000 & 47,6100000000 & 478,4744111279 & 524,1800418709 \\
\hline 110942,5234722780 & 667,0400000000 & 50,4900000000 & 424,3794424667 & 514,6304017561 \\
\hline 124479,5587027240 & 623,5900000000 & 52,6800000000 & 378,0614373694 & 495,9173698049 \\
\hline 139668,3620478210 & 579,7000000000 & 54,6800000000 & 335,1491927196 & 472,9980006506 \\
\hline 156710,4796997840 & 536,6900000000 & 56,5800000000 & 295,5938909189 & 447,9513452948 \\
\hline
\end{tabular}




\begin{tabular}{|c|c|c|c|c|}
\hline $\begin{array}{l}\text { frequency } \\
\qquad[\mathrm{Hz}]\end{array}$ & $\begin{array}{c}\text { measurement } \\
6\end{array}$ & $\begin{array}{c}\text { phase difference } \\
{\left[{ }^{\circ}\right]}\end{array}$ & $\begin{array}{l}\text { real impedance } \\
Z^{\prime}[\Omega \mathrm{m}]\end{array}$ & $\begin{array}{c}\text { imaginary } \\
\text { impedance } \\
\mathbf{Z}^{\prime \prime}[\Omega \mathrm{m}]\end{array}$ \\
\hline 175832,0502056700 & 492,6900000000 & 57,7000000000 & 263,2700590209 & 416,4520526101 \\
\hline 197286,8051885110 & 454,7400000000 & 59,7500000000 & 229,0861783217 & 392,8205576366 \\
\hline 221359,4362117860 & 417,4500000000 & 61,3600000000 & 200,0857424165 & 366,3743961873 \\
\hline 248369,3724635020 & 385,0000000000 & 63,3900000000 & 172,4473290435 & 344,2192886893 \\
\hline 278675,0193874470 & & & & 318,9683939979 \\
\hline 312678,5145056730 & 321,3100000000 & 66,3700000000 & 128,7902966061 & 294,3691145486 \\
\hline 350831,0635390900 & 292,7100000000 & 67,7000000000 & 111,0706124557 & 270,8181366687 \\
\hline 393638,9276332430 & 265,9800000000 & 68,9300000000 & 95,6220084487 & 248,1970827795 \\
\hline 441670,1411361340 & 241,2300000000 & 70,0800000000 & 82,1889310014 & 226,7970293475 \\
\hline 495562,0490688950 & 218,4600000000 & 71,1400000000 & 70,6186908250 & 206,7311590113 \\
\hline 556029,7643069950 & 197,4500000000 & 72,1100000000 & 60,6547698650 & 187,9029041623 \\
\hline 623875,6566936190 & 177,8700000000 & 72,9900000000 & 52,0338420156 & 170,0888479151 \\
\hline 785412,9180113710 & 143,5500000000 & 74,5100000000 & 38,3379253104 & 138,3358448953 \\
\hline 988776,2812359230 & 114,6310000000 & 75,5900000000 & 28,5269483520 & 111,0246791426 \\
\hline
\end{tabular}




\begin{tabular}{|c|c|c|c|c|}
\hline $\begin{array}{c}\text { frequency } \\
{[\mathrm{Hz}]}\end{array}$ & $\begin{array}{c}\text { measurement } \\
6\end{array}$ & $\begin{array}{c}\text { phase difference } \\
{\left[^{\circ}\right]}\end{array}$ & $\begin{array}{l}\text { real admittance } \\
\quad \mathbf{Y}^{\prime}\left[\mathbf{S ~ m}^{-1}\right]\end{array}$ & $\begin{array}{c}\text { imaginary } \\
\text { admittance } \\
\mathbf{Y}^{\prime \prime}\left[\mathrm{S} \mathrm{m}^{-1}\right]\end{array}$ \\
\hline 785,4129180114 & 1598,3000000000 & 1,2800000000 & 0,0006258209 & 0,0280085412 \\
\hline 881,2477882559 & 1597,2000000000 & 1,5200000000 & 0,0006263161 & 0,0236031891 \\
\hline 988,7762812359 & 1577,4000000000 & 1,2000000000 & 0,0006340937 & 0,0302713159 \\
\hline 1109,4252347228 & 1567,5000000000 & 1,8100000000 & 0,0006382770 & 0,0201980175 \\
\hline 1244,7955870272 & 1562,0000000000 & 2,5600000000 & 0,0006408444 & 0,0143332986 \\
\hline 1396,6836204782 & 1548,8000000000 & 2,8700000000 & 0,0006464720 & 0,0128951686 \\
\hline 1567,1047969978 & 1542,2000000000 & 3,5800000000 & 0,0006496921 & 0,0103844029 \\
\hline 1758,3205020567 & 1566,4000000000 & 3,1900000000 & 0,0006393973 & 0,0114723839 \\
\hline 1972,8680518851 & 1568,6000000000 & 3,9400000000 & 0,0006390215 & 0,0092780462 \\
\hline 2213,5943621179 & 1562,0000000000 & 4,9700000000 & 0,0006426210 & 0,0073897540 \\
\hline 2483,6937246350 & 1549,9000000000 & 5,8600000000 & 0,0006485922 & 0,0063194423 \\
\hline 2786,7501938745 & 1532,3000000000 & 5,9800000000 & 0,0006561845 & 0,0062642115 \\
\hline 3126,7851450567 & 1523,5000000000 & 6,8000000000 & 0,0006610334 & 0,0055435923 \\
\hline 3508,3106353909 & 1518,0000000000 & 7,6500000000 & 0,0006646773 & 0,0049485795 \\
\hline 3936,3892763324 & 1503,7000000000 & 8,2300000000 & 0,0006719464 & 0,0046457525 \\
\hline 4416,7014113614 & 1497,1000000000 & 8,6000000000 & 0,0006755537 & 0,0044668908 \\
\hline 4955,6204906890 & 1480,6000000000 & 9,2600000000 & 0,0006843197 & 0,0041972631 \\
\hline 5560,2976430700 & 1469,6000000000 & 9,8900000000 & 0,0006907219 & 0,0039617402 \\
\hline 6238,7565669362 & 1459,7000000000 & 10,7800000000 & 0,0006973792 & 0,0036627356 \\
\hline 7000,0000000000 & 1445,4000000000 & 11,7500000000 & 0,0007066577 & 0,0033973878 \\
\hline 7854,1291801137 & 1436,6000000000 & 13,1200000000 & 0,0007147451 & 0,0030665848 \\
\hline 8812,4778825592 & 1425,6000000000 & 14,2100000000 & 0,0007235994 & 0,0028575395 \\
\hline 9887,7628123593 & 1397,0000000000 & 14,7500000000 & 0,0007402126 & 0,0028115245 \\
\hline 11094,2523472278 & 1369,5000000000 & 15,1600000000 & 0,0007565210 & 0,0027921625 \\
\hline 12447,9558702724 & 1331,0000000000 & 15,5500000000 & 0,0007798601 & 0,0028025852 \\
\hline 13966,8362047821 & 1310,1000000000 & 16,9800000000 & 0,0007980919 & 0,0026137038 \\
\hline 15671,0479699783 & 1272,7000000000 & 17,7100000000 & 0,0008248207 & 0,0025829483 \\
\hline 17583,2050205670 & 1234,2000000000 & 17,4500000000 & 0,0008493284 & 0,0027019450 \\
\hline 19728,6805188512 & 1236,4000000000 & 20,5000000000 & 0,0008634822 & 0,0023094880 \\
\hline 22135,9436211786 & 1223,9700000000 & 23,0100000000 & 0,0008876369 & 0,0020901271 \\
\hline 24836,9372463503 & 1190,0900000000 & 24,4900000000 & 0,0009233423 & 0,0020270273 \\
\hline 27867,5019387448 & 1166,1100000000 & 26,7600000000 & 0,0009604118 & 0,0019045949 \\
\hline 31267,8514505674 & 1126,0700000000 & 27,8400000000 & 0,0010042851 & 0,0019015771 \\
\hline 35083,1063539091 & 1101,3200000000 & 30,5200000000 & 0,0010540359 & 0,0017879705 \\
\hline 39363,8927633245 & 1055,0100000000 & 31,6200000000 & 0,0011131055 & 0,0018079129 \\
\hline 44167,0141136136 & 1022,2300000000 & 34,3000000000 & 0,0011841853 & 0,0017359507 \\
\hline 49556,2049068897 & 978,8900000000 & 35,7000000000 & 0,0012579559 & 0,0017506308 \\
\hline 55602,9764306998 & 938,3000000000 & 38,1900000000 & 0,0013559859 & 0,0017237697 \\
\hline 62387,5656693623 & 891,7700000000 & 40,1500000000 & 0,0014670663 & 0,0017391148 \\
\hline 70000,0000000001 & 842,6000000000 & 41,0500000000 & 0,0015737235 & 0,0018071743 \\
\hline 78541,2918011376 & 800,2500000000 & 43,4000000000 & 0,0017198638 & 0,0018187050 \\
\hline 88124,7788255919 & 754,6000000000 & 45,7500000000 & 0,0018991452 & 0,0018500654 \\
\hline 98877,6281235930 & 709,7200000000 & 47,6100000000 & 0,0020899759 & 0,0019077415 \\
\hline 110942,5234722780 & 667,0400000000 & 50,4900000000 & 0,0023563818 & 0,0019431421 \\
\hline 124479,5587027240 & 623,5900000000 & 52,6800000000 & 0,0026450727 & 0,0020164650 \\
\hline 139668,3620478210 & 579,7000000000 & 54,6800000000 & 0,0029837458 & 0,0021141738 \\
\hline 156710,4796997840 & 536,6900000000 & 56,5800000000 & 0,0033830198 & 0,0022323853 \\
\hline
\end{tabular}




\begin{tabular}{|c|c|c|c|c|}
\hline $\begin{array}{l}\text { frequency } \\
\qquad[\mathrm{Hz}]\end{array}$ & $\begin{array}{c}\text { measurement } \\
6\end{array}$ & $\begin{array}{c}\text { phase difference } \\
{\left[{ }^{\circ}\right]}\end{array}$ & $\begin{array}{l}\text { real admittance } \\
\qquad \mathbf{Y}^{\prime}\left[\mathrm{S} \mathrm{m}^{-1}\right]\end{array}$ & $\begin{array}{c}\text { imaginary } \\
\text { admittance } \\
Y^{\prime \prime}\left[\mathrm{S} \mathrm{m}^{-1}\right]\end{array}$ \\
\hline 175832,0502056700 & 492,6900000000 & 57,7000000000 & 0,0037983810 & 0,0024012368 \\
\hline 197286,8051885110 & 454,7400000000 & 59,7500000000 & 0,0043651695 & 0,0025456916 \\
\hline 221359,4362117860 & 417,4500000000 & 61,3600000000 & 0,0049978574 & 0,0027294484 \\
\hline 248369,3724635020 & 385,0000000000 & 63,3900000000 & 0,0057988721 & 0,0029051248 \\
\hline 278675,0193874470 & 352,0000000000 & 64,9800000000 & 0,0067171357 & 0,0031351069 \\
\hline 312678,5145056730 & 321,3100000000 & 66,3700000000 & 0,0077645601 & 0,0033970955 \\
\hline 350831,0635390900 & 292,7100000000 & 67,7000000000 & 0,0090032816 & 0,0036925149 \\
\hline 393638,9276332430 & 265,9800000000 & 68,9300000000 & 0,0104578435 & 0,0040290562 \\
\hline 441670,1411361340 & 241,2300000000 & 70,0800000000 & 0,0121670885 & 0,0044092288 \\
\hline 495562,0490688950 & 218,4600000000 & 71,1400000000 & 0,0141605570 & 0,0048372002 \\
\hline 556029,7643069950 & 197,4500000000 & 72,1100000000 & 0,0164867496 & 0,0053218975 \\
\hline 623875,6566936190 & 177,8700000000 & 72,9900000000 & 0,0192182618 & 0,0058792802 \\
\hline 785412,9180113710 & 143,5500000000 & 74,5100000000 & 0,0260838319 & 0,0072287844 \\
\hline 988776,2812359230 & 114,6310000000 & 75,5900000000 & 0,0350545732 & 0,0090070064 \\
\hline
\end{tabular}




\begin{tabular}{|c|c|c|c|c|}
\hline $\begin{array}{l}\text { frequency } \\
\quad[\mathrm{Hz}]\end{array}$ & $\begin{array}{c}\text { measurement } \\
7\end{array}$ & $\begin{array}{c}\text { phase difference } \\
{\left[{ }^{\circ}\right]}\end{array}$ & $\begin{array}{l}\text { real impedance } \\
\quad \mathbf{Z}^{\prime}[\mathbf{\Omega} \mathrm{m}]\end{array}$ & $\begin{array}{c}\text { imaginary } \\
\text { impedance } \\
\mathbf{Z}^{\prime \prime}[\Omega \mathbf{m}]\end{array}$ \\
\hline 785,4129180114 & 1962,0000000000 & 0,200000 & 1961,9880468200 & 6,8486580800 \\
\hline 881,2477882559 & 1969,0000000000 & 0,010000 & 1968,9999700100 & 0,3436553300 \\
\hline 988,7762812359 & 1964,0000000000 & 0,330000 & 1963,9674243600 & 11,3117654100 \\
\hline 1109,4252347228 & 1969,0000000000 & 1,010000 & 1968,6940841700 & 34,7073907400 \\
\hline 1244,7955870272 & 1933,0000000000 & 1,130000 & 1932,6240765600 & 38,1205809400 \\
\hline 1396,6836204782 & 1962,0000000000 & 1,920000 & 1960,8984973200 & 65,7349466900 \\
\hline 1567,1047969978 & 1923,0000000000 & 2,860000 & 1920,6047772300 & 95,9494121800 \\
\hline 1758,3205020567 & 1983,0000000000 & 2,690000 & 1980,8148953700 & 93,0663757600 \\
\hline 1972,8680518851 & 1987,0000000000 & 4,200000 & 1981,6638662500 & 145,5242976900 \\
\hline 2213,5943621179 & 1984,0000000000 & 5,010000 & 1976,4200712000 & 173,2619466200 \\
\hline 2483,6937246350 & 1962,0000000000 & 5,970000 & 1951,3590734100 & 204,0631437300 \\
\hline 2786,7501938745 & 1943,0000000000 & 6,420000 & 1930,8153471700 & 217,2581302000 \\
\hline 3126,7851450567 & 1936,0000000000 & 7,470000 & 1919,5693012400 & 251,6936585500 \\
\hline 3508,3106353909 & 1931,0000000000 & 8,000000 & 1912,2076407400 & 268,7432579500 \\
\hline 3936,3892763324 & 1921,0000000000 & 8,930000 & 1897,7150291400 & 298,1923341800 \\
\hline 4416,7014113614 & 1912,0000000000 & 9,830000 & 1883,9292433600 & 326,4270301500 \\
\hline 4955,6204906890 & 1894,0000000000 & 10,540000 & 1862,0433781100 & 346,4541211200 \\
\hline 5560,2976430700 & 1883,0000000000 & 11,470000 & 1845,3945299800 & 374,4436255600 \\
\hline 6238,7565669362 & 1869,0000000000 & 12,640000 & 1823,7033485100 & 408,9830028500 \\
\hline 7000,0000000000 & 1849,0000000000 & 13,970000 & 1794,3107647900 & 446,3741472800 \\
\hline 7854,1291801137 & 1834,0000000000 & 15,650000 & 1766,0090776700 & 494,7402728500 \\
\hline 8812,4778825592 & 1817,0000000000 & 17,120000 & 1736,4893062300 & 534,8774526400 \\
\hline 9887,7628123593 & 1781,0000000000 & 17,850000 & 1695,2666864000 & 545,9229450900 \\
\hline 11094,2523472278 & 1738,0000000000 & 18,200000 & 1651,0514255500 & 542,8380883700 \\
\hline 12447,9558702724 & 1679,0000000000 & 18,930000 & 1588,1923367000 & 544,6889953400 \\
\hline 13966,8362047821 & 1647,0000000000 & 20,890000 & 1538,7372921500 & 587,2789335000 \\
\hline 15671,0479699783 & 1589,0000000000 & 21,680000 & 1476,5966520400 & 587,0122036200 \\
\hline 17583,2050205670 & 1523,0000000000 & 21,170000 & 1420,2173288300 & 550,0106716100 \\
\hline 19728,6805188512 & 1533,0000000000 & 25,660000 & 1381,8148545400 & 663,8348497700 \\
\hline 22135,9436211786 & 1509,0000000000 & 26,450000 & 1351,0430133700 & 672,1337486200 \\
\hline 24836,9372463503 & 1312,0000000000 & 23,290000 & 1205,0922088200 & 518,7453790000 \\
\hline 27867,5019387448 & 1337,0000000000 & 30,060000 & 1157,1752785500 & 669,7121581100 \\
\hline 31267,8514505674 & 1333,0000000000 & 33,450000 & 1112,2114247700 & 734,7616937500 \\
\hline 35083,1063539091 & 1300,0000000000 & 37,380000 & 1033,0145621900 & 789,2280496200 \\
\hline 39363,8927633245 & 1218,0000000000 & 37,830000 & 962,0177957900 & 747,0246050700 \\
\hline 44167,0141136136 & 1185,0000000000 & 41,680000 & 885,0413600000 & 787,9890805600 \\
\hline 49556,2049068897 & 1132,0000000000 & 41,400000 & 849,1257308200 & 748,6050315500 \\
\hline 55602,9764306998 & 1061,0000000000 & 46,360000 & 732,2225644900 & 767,8353443600 \\
\hline 62387,5656693623 & 994,6000000000 & 48,350000 & 660,9898126100 & 743,1834414400 \\
\hline 70000,0000000001 & 928,9000000000 & 49,210000 & 606,8396630100 & 703,2786314100 \\
\hline 78541,2918011376 & 868,0000000000 & 51,800000 & 536,7784871700 & 682,1237832800 \\
\hline 88124,7788255919 & 807,5000000000 & 54,040000 & 474,1803992700 & 653,6124225800 \\
\hline 98877,6281235930 & 749,9000000000 & 55,720000 & 422,3719151700 & 619,6385844000 \\
\hline 110942,5234722780 & 694,2000000000 & 58,490000 & 362,8218046300 & 591,8394867600 \\
\hline 124479,5587027240 & 640,8000000000 & 60,520000 & 315,3503157200 & 557,8340419700 \\
\hline 139668,3620478210 & 588,9000000000 & 62,250000 & 274,2003910200 & 521,1692197000 \\
\hline 156710,4796997840 & 539,1000000000 & 63,850000 & 237,5935963200 & 483,9195108500 \\
\hline
\end{tabular}




\begin{tabular}{|c|c|c|c|c|}
\hline $\begin{array}{c}\text { frequency } \\
{[\mathbf{H z}]}\end{array}$ & $\begin{array}{c}\text { measurement } \\
\mathbf{7}\end{array}$ & $\begin{array}{c}\text { phase difference } \\
{\left[{ }^{\circ}\right]}\end{array}$ & $\begin{array}{c}\text { real impedance } \\
\mathbf{Z}^{\prime}[\mathbf{\Omega} \mathbf{~ m}]\end{array}$ & $\begin{array}{c}\text { imaginary } \\
\text { impedance } \\
\mathbf{Z}^{\prime \prime}[\mathbf{\Omega} \mathbf{~ m}]\end{array}$ \\
\hline 175832,0502056700 & 490,8000000000 & 64,620000 & 210,3665898800 & 443,4304205400 \\
\hline 197286,8051885110 & 447,8000000000 & 66,340000 & 179,7059130200 & 410,1592676400 \\
\hline 221359,4362117860 & 407,0000000000 & 67,590000 & 155,1613158500 & 376,2631606500 \\
\hline 248369,3724635020 & 371,7000000000 & 69,240000 & 131,7506426700 & 347,5667679100 \\
\hline 278675,0193874470 & 337,3000000000 & 70,410000 & 113,0923538700 & 317,7757220000 \\
\hline 312678,5145056730 & 305,6000000000 & 71,420000 & 97,3728562400 & 289,6720332800 \\
\hline 350831,0635390900 & 276,6000000000 & 72,330000 & 83,9575616200 & 263,5501619200 \\
\hline 393638,9276332430 & 249,8000000000 & 73,160000 & 72,3670753700 & 239,0879470000 \\
\hline 441670,1411361340 & 225,5000000000 & 73,910000 & 62,4966398100 & 216,6666102800 \\
\hline 495562,0490688950 & 203,2000000000 & 74,580000 & 54,0293832500 & 195,8853382600 \\
\hline 556029,7643069950 & 182,9000000000 & 75,170000 & 46,8136117400 & 176,8075104600 \\
\hline 623875,6566936190 & 164,2000000000 & 75,630000 & 40,7516000200 & 159,0627143500 \\
\hline 785412,9180113710 & 131,8000000000 & 76,200000 & 31,4387097100 & 127,9954980900 \\
\hline 881247,7882559130 & 118,0000000000 & 76,220000 & 28,1069454800 & 114,6036631900 \\
\hline 988776,2812359230 & 105,1000000000 & 76,250000 & 24,9807872800 & 102,0880515400 \\
\hline
\end{tabular}




\begin{tabular}{|c|c|c|c|c|}
\hline $\begin{array}{l}\text { frequency } \\
\qquad[\mathrm{Hz}]\end{array}$ & $\begin{array}{c}\text { measurement } \\
7\end{array}$ & $\begin{array}{c}\text { phase difference } \\
{\left[{ }^{\circ}\right]}\end{array}$ & $\begin{array}{l}\text { real admittance } \\
\qquad \mathbf{Y}^{\prime}\left[\mathrm{S} \mathrm{m}^{-1}\right]\end{array}$ & $\begin{array}{c}\text { imaginary } \\
\text { admittance } \\
\mathbf{Y}^{\prime \prime}\left[\mathrm{S} \mathrm{m}^{-1}\right]\end{array}$ \\
\hline 785,4129180114 & 1962,0000000000 & 0,200000 & 0,0005096871 & 0,1460140057 \\
\hline 881,2477882559 & 1969,0000000000 & 0,010000 & 0,0005078720 & 2,9098923040 \\
\hline 988,7762812359 & 1964,0000000000 & 0,330000 & 0,0005091734 & 0,0884035306 \\
\hline 1109,4252347228 & 1969,0000000000 & 1,010000 & 0,0005079509 & 0,0288123071 \\
\hline 1244,7955870272 & 1933,0000000000 & 1,130000 & 0,0005174312 & 0,0262325488 \\
\hline 1396,6836204782 & 1962,0000000000 & 1,920000 & 0,0005099703 & 0,0152126084 \\
\hline 1567,1047969978 & 1923,0000000000 & 2,860000 & 0,0005206693 & 0,0104221587 \\
\hline 1758,3205020567 & 1983,0000000000 & 2,690000 & 0,0005048427 & 0,0107450193 \\
\hline 1972,8680518851 & 1987,0000000000 & 4,200000 & 0,0005046264 & 0,0068717047 \\
\hline 2213,5943621179 & 1984,0000000000 & 5,010000 & 0,0005059653 & 0,0057716078 \\
\hline 2483,6937246350 & 1962,0000000000 & 5,970000 & 0,0005124633 & 0,0049004440 \\
\hline 2786,7501938745 & 1943,0000000000 & 6,420000 & 0,0005179159 & 0,0046028197 \\
\hline 3126,7851450567 & 1936,0000000000 & 7,470000 & 0,0005209502 & 0,0039730838 \\
\hline 3508,3106353909 & 1931,0000000000 & 8,000000 & 0,0005229558 & 0,0037210236 \\
\hline 3936,3892763324 & 1921,0000000000 & 8,930000 & 0,0005269495 & 0,0033535403 \\
\hline 4416,7014113614 & 1912,0000000000 & 9,830000 & 0,0005308055 & 0,0030634718 \\
\hline 4955,6204906890 & 1894,0000000000 & 10,540000 & 0,0005370444 & 0,0028863851 \\
\hline 5560,2976430700 & 1883,0000000000 & 11,470000 & 0,0005418895 & 0,0026706290 \\
\hline 6238,7565669362 & 1869,0000000000 & 12,640000 & 0,0005483348 & 0,0024450894 \\
\hline 7000,0000000000 & 1849,0000000000 & 13,970000 & 0,0005573171 & 0,0022402731 \\
\hline 7854,1291801137 & 1834,0000000000 & 15,650000 & 0,0005662485 & 0,0020212626 \\
\hline 8812,4778825592 & 1817,0000000000 & 17,120000 & 0,0005758746 & 0,0018695871 \\
\hline 9887,7628123593 & 1781,0000000000 & 17,850000 & 0,0005898777 & 0,0018317603 \\
\hline 11094,2523472278 & 1738,0000000000 & 18,200000 & 0,0006056747 & 0,0018421699 \\
\hline 12447,9558702724 & 1679,0000000000 & 18,930000 & 0,0006296467 & 0,0018359100 \\
\hline 13966,8362047821 & 1647,0000000000 & 20,890000 & 0,0006498835 & 0,0017027684 \\
\hline 15671,0479699783 & 1589,0000000000 & 21,680000 & 0,0006772330 & 0,0017035421 \\
\hline 17583,2050205670 & 1523,0000000000 & 21,170000 & 0,0007041176 & 0,0018181465 \\
\hline 19728,6805188512 & 1533,0000000000 & 25,660000 & 0,0007236860 & 0,0015063988 \\
\hline 22135,9436211786 & 1509,0000000000 & 26,450000 & 0,0007401689 & 0,0014877991 \\
\hline 24836,9372463503 & 1312,0000000000 & 23,290000 & 0,0008298120 & 0,0019277280 \\
\hline 27867,5019387448 & 1337,0000000000 & 30,060000 & 0,0008641733 & 0,0014931788 \\
\hline 31267,8514505674 & 1333,0000000000 & 33,450000 & 0,0008991096 & 0,0013609855 \\
\hline 35083,1063539091 & 1300,0000000000 & 37,380000 & 0,0009680406 & 0,0012670609 \\
\hline 39363,8927633245 & 1218,0000000000 & 37,830000 & 0,0010394818 & 0,0013386440 \\
\hline 44167,0141136136 & 1185,0000000000 & 41,680000 & 0,0011298907 & 0,0012690531 \\
\hline 49556,2049068897 & 1132,0000000000 & 41,400000 & 0,0011776819 & 0,0013358179 \\
\hline 55602,9764306998 & 1061,0000000000 & 46,360000 & 0,0013657050 & 0,0013023626 \\
\hline 62387,5656693623 & 994,6000000000 & 48,350000 & 0,0015128826 & 0,0013455628 \\
\hline 70000,0000000001 & 928,9000000000 & 49,210000 & 0,0016478817 & 0,0014219115 \\
\hline 78541,2918011376 & 868,0000000000 & 51,800000 & 0,0018629659 & 0,0014660096 \\
\hline 88124,7788255919 & 807,5000000000 & 54,040000 & 0,0021089020 & 0,0015299587 \\
\hline 98877,6281235930 & 749,9000000000 & 55,720000 & 0,0023675817 & 0,0016138440 \\
\hline 110942,5234722780 & 694,2000000000 & 58,490000 & 0,0027561739 & 0,0016896473 \\
\hline 124479,5587027240 & 640,8000000000 & 60,520000 & 0,0031710766 & 0,0017926479 \\
\hline 139668,3620478210 & 588,9000000000 & 62,250000 & 0,0036469678 & 0,0019187626 \\
\hline 156710,4796997840 & 539,1000000000 & 63,850000 & 0,0042088676 & 0,0020664594 \\
\hline
\end{tabular}




\begin{tabular}{|c|c|c|c|c|}
\hline $\begin{array}{l}\text { frequency } \\
\qquad[\mathrm{Hz}]\end{array}$ & $\begin{array}{c}\text { measurement } \\
7\end{array}$ & $\begin{array}{c}\text { phase difference } \\
{\left[{ }^{\circ}\right]}\end{array}$ & $\begin{array}{l}\text { real admittance } \\
\qquad \mathbf{Y}^{\prime}\left[\mathrm{S} \mathrm{m}^{-1}\right]\end{array}$ & $\begin{array}{c}\text { imaginary } \\
\text { admittance } \\
\mathbf{Y}^{\prime \prime}\left[\mathrm{S} \mathrm{m}^{-1}\right]\end{array}$ \\
\hline 175832,0502056700 & 490,8000000000 & 64,620000 & 0,0047536066 & 0,0022551452 \\
\hline 197286,8051885110 & 447,8000000000 & 66,340000 & 0,0055646472 & 0,0024380773 \\
\hline 221359,4362117860 & 407,0000000000 & 67,590000 & 0,0064449054 & 0,0026577143 \\
\hline 248369,3724635020 & 371,7000000000 & 69,240000 & 0,0075900958 & 0,0028771450 \\
\hline 278675,0193874470 & 337,3000000000 & 70,410000 & 0,0088423308 & 0,0031468735 \\
\hline 312678,5145056730 & 305,6000000000 & 71,420000 & 0,0102698025 & 0,0034521800 \\
\hline 350831,0635390900 & 276,6000000000 & 72,330000 & 0,0119107795 & 0,0037943441 \\
\hline 393638,9276332430 & 249,8000000000 & 73,160000 & 0,0138184388 & 0,0041825613 \\
\hline 441670,1411361340 & 225,5000000000 & 73,910000 & 0,0160008603 & 0,0046153858 \\
\hline 495562,0490688950 & 203,2000000000 & 74,580000 & 0,0185084474 & 0,0051050273 \\
\hline 556029,7643069950 & 182,9000000000 & 75,170000 & 0,0213613084 & 0,0056558683 \\
\hline 623875,6566936190 & 164,2000000000 & 75,630000 & 0,0245389138 & 0,0062868285 \\
\hline 785412,9180113710 & 131,8000000000 & 76,200000 & 0,0318079212 & 0,0078127748 \\
\hline 881247,7882559130 & 118,0000000000 & 76,220000 & 0,0355783947 & 0,0087257246 \\
\hline 988776,2812359230 & 105,1000000000 & 76,250000 & 0,0400307640 & 0,0097954656 \\
\hline
\end{tabular}




\section{III.3 COMPLEX IMPEDANCE DATA FOR SAMPLE 17/08/00/10:}

\begin{tabular}{|c|c|c|c|c|}
\hline $\begin{array}{l}\text { frequency } \\
\qquad[\mathrm{Hz}]\end{array}$ & $\begin{array}{c}\text { measurement } \\
1\end{array}$ & $\begin{array}{c}\text { phase } \\
\text { difference } \\
\left.{ }^{\circ}\right] \\
\end{array}$ & $\begin{array}{l}\text { real impedance } \\
\qquad \mathbf{Z}^{\prime}[\mathbf{\Omega} \mathrm{m}]\end{array}$ & $\begin{array}{c}\text { imaginary } \\
\text { impedance } \\
\mathbf{Z}^{\prime \prime}[\Omega \mathrm{m}]\end{array}$ \\
\hline 785,412918011374 & 1313,496143958870 & 4,85000000000 & 1308,793114703990 & 111,052704146510 \\
\hline 881,247788255917 & 1319,151670951160 & 5,02000000000 & 1314,091687845160 & 115,430355234186 \\
\hline 988,776281235928 & 1329,177377892030 & 5,28000000000 & 1323,537520023870 & 122,314900927837 \\
\hline 1109,425234722780 & 1332,005141388170 & 5,53000000000 & 1325,805825895310 & 128,361243008038 \\
\hline 1244,795587027250 & 1329,434447300770 & 5,77000000000 & 1322,698848268760 & 133,655177447041 \\
\hline 1396,683620478220 & 1327,892030848330 & 6,03000000000 & 1320,544841513560 & 139,493968122016 \\
\hline 1567,104796997840 & 1326,221079691520 & 6,36000000000 & 1318,058848043810 & 146,912304833781 \\
\hline 1758,320502056710 & 1323,264781491000 & 6,59000000000 & 1314,521720701390 & 151,862858325157 \\
\hline 1972,868051885120 & 1321,593830334190 & 6,96000000000 & 1311,854992987280 & 160,145963894662 \\
\hline 2213,594362117870 & 1323,007712082260 & 7,29000000000 & 1312,313317417000 & 167,878417788331 \\
\hline 2483,693724635030 & 1317,737789203090 & 7,63000000000 & 1306,070743194690 & 174,963124299702 \\
\hline 2786,750193874480 & 1311,182519280210 & 8,00000000000 & 1298,422181135320 & 182,481337132858 \\
\hline 3126,785145056740 & 1303,727506426740 & 8,30000000000 & 1290,071989527840 & 188,201149968236 \\
\hline 3508,310635390910 & 1296,015424164520 & 8,80000000000 & 1280,759224951870 & 198,272003502821 \\
\hline 3936,389276332440 & 1285,861182519280 & 9,26000000000 & 1269,104221048900 & 206,914129110959 \\
\hline 4416,701411361350 & 1278,663239074550 & 9,72000000000 & 1260,307510609540 & 215,881119280498 \\
\hline 4955,620490688970 & 1271,593830334190 & 10,2200000000 & 1251,418384091280 & 225,616704395646 \\
\hline 5560,297643069970 & 1258,997429305910 & 10,7100000000 & 1237,066168276490 & 233,969703817852 \\
\hline 6238,756566936220 & 1245,758354755780 & 11,2000000000 & 1222,033080417010 & 241,969065812849 \\
\hline 7000,000000000000 & 1233,933161953730 & 11,7600000000 & 1208,032800472020 & 251,491155217956 \\
\hline 7854,129180113750 & 1220,565552699230 & 12,3300000000 & 1192,411863235790 & 260,641932256750 \\
\hline 8812,477882559180 & 1206,683804627250 & 12,8900000000 & 1176,275546320480 & 269,187004660625 \\
\hline 9887,762812359290 & 1191,259640102830 & 13,4900000000 & 1158,393560343210 & 277,891866547603 \\
\hline 11094,252347227800 & 1174,935732647820 & 14,1300000000 & 1139,387259939250 & 286,828603421639 \\
\hline 12447,955870272500 & 1158,097686375320 & 14,7700000000 & 1119,830672809890 & 295,244839788984 \\
\hline 13966,836204782200 & 1139,588688946020 & 15,4100000000 & 1098,619383014100 & 302,816497634172 \\
\hline 15671,047969978400 & 1120,694087403600 & 16,0900000000 & 1076,793743161450 & 310,596961076140 \\
\hline 17583,205020567100 & 1102,185089974290 & 16,8100000000 & 1055,087660101460 & 318,750689510248 \\
\hline 19728,680518851200 & 1079,948586118250 & 17,5000000000 & 1029,965272517560 & 324,746803012198 \\
\hline 22135,943621178700 & 1058,354755784060 & 18,2500000000 & 1005,118586779720 & 331,438403931515 \\
\hline 24836,937246350300 & 1039,845758354760 & 19,1000000000 & 982,601118168953 & 340,256144311178 \\
\hline 27867,501938744900 & 1016,580976863750 & 19,8600000000 & 956,120359615868 & 345,355961942572 \\
\hline 31267,851450567500 & 994,087403598972 & 20,6800000000 & 930,035727629119 & 351,060267372315 \\
\hline 35083,106353909100 & 971,336760925450 & 21,5600000000 & 903,375493064975 & 356,942322588339 \\
\hline 39363,892763324500 & 949,485861182519 & 22,4700000000 & 877,400684229042 & 362,893152181077 \\
\hline 44167,014113613600 & 924,550128534704 & 23,3300000000 & 848,958122598449 & 366,146208839659 \\
\hline 49556,204906889700 & 898,843187660668 & 24,1800000000 & 819,981518389530 & 368,170321323694 \\
\hline 55602,976430699800 & 874,935732647815 & 24,9600000000 & 793,219018238046 & 369,210137143389 \\
\hline 62387,565669362300 & 849,228791773779 & 26,0100000000 & 763,216795703499 & 372,410611467551 \\
\hline 70000,000000000200 & 824,293059125964 & 27,0000000000 & 734,450493524422 & 374,221217844436 \\
\hline 78541,291801137600 & 799,357326478149 & 27,7700000000 & 707,291389103750 & 372,439292091989 \\
\hline 88124,778825591900 & 773,778920308483 & 29,0400000000 & 676,500233809390 & 375,607842263181 \\
\hline 98877,628123593100 & 748,200514138818 & 30,0800000000 & 647,437678424819 & 375,004615856221 \\
\hline 110942,523472278000 & 722,750642673522 & 31,1300000000 & 618,672028465200 & 373,648782521425 \\
\hline
\end{tabular}




\begin{tabular}{|c|c|c|c|c|}
\hline $\begin{array}{l}\text { frequency } \\
\qquad[\mathrm{Hz}]\end{array}$ & $\begin{array}{c}\text { measurement } \\
1\end{array}$ & $\begin{array}{c}\text { phase } \\
\text { difference } \\
{\left[{ }^{\circ}\right]} \\
\end{array}$ & $\begin{array}{c}\text { real impedance } \\
Z^{\prime}[\Omega \mathrm{m}]\end{array}$ & $\begin{array}{c}\text { imaginary } \\
\text { impedance } \\
\mathbf{Z}^{\prime \prime}[\Omega \mathrm{m}]\end{array}$ \\
\hline 124479,558702725000 & 997,172236503856 & 32,2200000000 & 589,812665956604 & 371,712451269731 \\
\hline 139668,362047822000 & 671,593830334190 & 33,4000000000 & 560,6786 & 369,69 \\
\hline 156710,4796 & & 34,6800000000 & & \\
\hline 175832,0502 & & 00000000 & & \\
\hline & & & & \\
\hline 221359 & & & & \\
\hline 248369, & & & & \\
\hline 278675 & & & & \\
\hline & & 400000000 & & 325 \\
\hline & & 00000000 & & 738 \\
\hline & & & & \\
\hline & & 000 & & 296 , \\
\hline & & 300000000 & & \\
\hline & & & & \\
\hline 623875,656693620000 & & & & \\
\hline 699999,999999997000 & & 49,0300000000 & & 242,52923 \\
\hline 785412,918011371000 & & 52,1200000000 & & 240,949080850009 \\
\hline & & & & 226,492867808710 \\
\hline 988776,281235923000 & 266,838046272494 & 54,8600000000 & 153,585652204136 & 218,206302327755 \\
\hline
\end{tabular}




\begin{tabular}{|c|c|c|c|c|}
\hline $\begin{array}{l}\text { frequency } \\
\quad[\mathrm{Hz}]\end{array}$ & $\begin{array}{c}\text { measurement } \\
1\end{array}$ & $\begin{array}{c}\text { phase difference } \\
{\left[{ }^{\circ}\right]}\end{array}$ & $\begin{array}{c}\text { real admittance } \\
\mathbf{Y}^{\prime}[\mathrm{S} \mathrm{m}-1]\end{array}$ & $\begin{array}{l}\text { imaginary } \\
\text { admittance } \\
Y^{\prime \prime}[S \text { m-1] }\end{array}$ \\
\hline 785,412918011374 & 1313,496143958870 & 4,850000000000 & 0,000764062699 & 0,009004733452 \\
\hline 881,247788255917 & 1319,151670951160 & 5,020000000000 & 0,000760981908 & 0,008663232457 \\
\hline 988,776281235928 & 1329,177377892030 & 5,280000000000 & 0,000755550927 & 0,008175618771 \\
\hline 1109,425234722780 & 1332,005141388170 & 5,530000000000 & 0,000754258264 & 0,007790513527 \\
\hline 1244,795587027250 & 1329,434447300770 & 5,770000000000 & 0,000756029992 & 0,007481939863 \\
\hline 1396,683620478220 & 1327,892030848330 & 6,030000000000 & 0,000757263191 & 0,007168768754 \\
\hline 1567,104796997840 & 1326,221079691520 & 6,360000000000 & 0,000758691466 & 0,006806781781 \\
\hline 1758,320502056710 & 1323,264781491000 & 6,590000000000 & 0,000760732960 & 0,006584888570 \\
\hline 1972,868051885120 & 1321,593830334190 & 6,960000000000 & 0,000762279372 & 0,006244303482 \\
\hline 2213,594362117870 & 1323,007712082260 & 7,290000000000 & 0,000762013146 & 0,005956691832 \\
\hline 2483,693724635030 & 1317,737789203090 & 7,630000000000 & 0,000765655310 & 0,005715490073 \\
\hline 2786,750193874480 & 1311,182519280210 & 8,000000000000 & 0,000770165524 & 0,005480012453 \\
\hline 3126,785145056740 & 1303,727506426740 & 8,300000000000 & 0,000775150541 & 0,005313463813 \\
\hline 3508,310635390910 & 1296,015424164520 & 8,800000000000 & 0,000780786881 & 0,005043576412 \\
\hline 3936,389276332440 & 1285,861182519280 & 9,260000000000 & 0,000787957351 & 0,004832922741 \\
\hline 4416,701411361350 & 1278,663239074550 & 9,720000000000 & 0,000793457146 & 0,004632179059 \\
\hline 4955,620490688970 & 1271,593830334190 & 10,220000000000 & 0,000799093263 & 0,004432295927 \\
\hline 5560,297643069970 & 1258,997429305910 & 10,710000000000 & 0,000808364197 & 0,004274057639 \\
\hline 6238,756566936220 & 1245,758354755780 & 11,200000000000 & 0,000818308453 & 0,004132759684 \\
\hline 7000,000000000000 & 1233,933161953730 & 11,760000000000 & 0,000827792093 & 0,003976282980 \\
\hline 7854,129180113750 & 1220,565552699230 & 12,330000000000 & 0,000838636406 & 0,003836681195 \\
\hline 8812,477882559180 & 1206,683804627250 & 12,890000000000 & 0,000850140941 & 0,003714889585 \\
\hline 9887,762812359290 & 1191,259640102830 & 13,490000000000 & 0,000863264467 & 0,003598522017 \\
\hline 11094,252347227800 & 1174,935732647820 & 14,130000000000 & 0,000877664720 & 0,003486402639 \\
\hline 12447,955870272500 & 1158,097686375320 & 14,770000000000 & 0,000892992150 & 0,003387019400 \\
\hline 13966,836204782200 & 1139,588688946020 & 15,410000000000 & 0,000910233349 & 0,003302329985 \\
\hline 15671,047969978400 & 1120,694087403600 & 16,090000000000 & 0,000928682959 & 0,003219606517 \\
\hline 17583,205020567100 & 1102,185089974290 & 16,810000000000 & 0,000947788547 & 0,003137248116 \\
\hline 19728,680518851200 & 1079,948586118250 & 17,500000000000 & 0,000970906521 & 0,003079322077 \\
\hline 22135,943621178700 & 1058,354755784060 & 18,250000000000 & 0,000994907480 & 0,003017151869 \\
\hline 24836,937246350300 & 1039,845758354760 & 19,100000000000 & 0,001017706963 & 0,002938962357 \\
\hline 27867,501938744900 & 1016,580976863750 & 19,860000000000 & 0,001045893427 & 0,002895563159 \\
\hline 31267,851450567500 & 994,087403598972 & 20,680000000000 & 0,001075227510 & 0,002848513754 \\
\hline 35083,106353909100 & 971,336760925450 & 21,560000000000 & 0,001106959407 & 0,002801573074 \\
\hline 39363,892763324500 & 949,485861182519 & 22,470000000000 & 0,001139730135 & 0,002755632048 \\
\hline 44167,014113613600 & 924,550128534704 & 23,330000000000 & 0,001177914403 & 0,002731149404 \\
\hline 49556,204906889700 & 898,843187660668 & 24,180000000000 & 0,001219539682 & 0,002716134197 \\
\hline 55602,976430699800 & 874,935732647815 & 24,960000000000 & 0,001260685860 & 0,002708484680 \\
\hline 62387,565669362300 & 849,228791773779 & 26,010000000000 & 0,001310243702 & 0,002685208126 \\
\hline 70000,000000000200 & 824,293059125964 & 27,000000000000 & 0,001361562159 & 0,002672216198 \\
\hline 78541,291801137600 & 799,357326478149 & 27,770000000000 & 0,001413844443 & 0,002685001344 \\
\hline 88124,778825591900 & 773,778920308483 & 29,040000000000 & 0,001478196089 & 0,002662351228 \\
\hline 98877,628123593100 & 748,200514138818 & 30,080000000000 & 0,001544550207 & 0,002666633843 \\
\hline 110942,523472278000 & 722,750642673522 & 31,130000000000 & 0,001616365302 & 0,002676310072 \\
\hline 124479,558702725000 & 697,172236503856 & 32,220000000000 & 0,001695453587 & 0,002690251555 \\
\hline 139668,362047822000 & 671,593830334190 & 33,400000000000 & 0,001783552766 & 0,002704899748 \\
\hline 156710,479699784000 & 645,886889460154 & $34,680000000000 \mid$ & 0,001882741457 & 0,002721052895 \\
\hline
\end{tabular}




\begin{tabular}{|c|c|c|c|c|}
\hline $\begin{array}{l}\text { frequency } \\
\qquad[\mathrm{Hz}]\end{array}$ & $\begin{array}{c}\text { measurement } \\
1\end{array}$ & $\begin{array}{c}\text { phase difference } \\
{\left[{ }^{\circ}\right]}\end{array}$ & $\begin{array}{c}\text { real admittance } \\
Y^{\prime}[S \text { m-1] }\end{array}$ & $\begin{array}{l}\text { imaginary } \\
\text { admittance } \\
\mathbf{Y}^{\prime \prime}[\mathrm{S} \text { m-1] }\end{array}$ \\
\hline 175832,050205671000 & & 35,830000000000 & & 0,002763089539 \\
\hline 197286,80 & & & & \\
\hline 221359,4 & & & & \\
\hline 248369 , & & & & \\
\hline & & & & \\
\hline 312 & & & & \\
\hline 350 & & & & \\
\hline 393638 & & & & \\
\hline 441670 & & 0000000000 & & 793 \\
\hline 49556 & & 0000000000 & & 647 \\
\hline 556029, & 368 , & 0000 & 0,00407 & 278 \\
\hline 623875,656693620000 & 346,658097686375 & 49,490000000000 & 0,0044408 & 9601 \\
\hline 699999,999999997000 & 321,208226221080 & 49,030000000000 & 0,00474823 & 4325 \\
\hline 785412,918011371000 & 305,269922879177 & 52,120000000000 & & 0,004150254471 \\
\hline 881247,788255913000 & 284,575835475578 & 52,740000000000 & & 0,004415150065 \\
\hline 988776,281235923000 & 266,838046272494 & 54,860000000000 & 0,006511024862 & 0,004582819054 \\
\hline
\end{tabular}




\begin{tabular}{|c|c|c|c|c|}
\hline $\begin{array}{l}\text { frequency } \\
\qquad[\mathrm{Hz}]\end{array}$ & $\begin{array}{c}\text { measurement } \\
2\end{array}$ & $\begin{array}{c}\text { phase difference } \\
{\left[{ }^{\circ}\right]}\end{array}$ & $\begin{array}{l}\text { real impedance } \\
\qquad \mathbf{Z}^{\prime}[\Omega \mathbf{m}]\end{array}$ & $\begin{array}{c}\text { imaginary } \\
\text { impedance } \\
Z^{\prime \prime}[\Omega \mathbf{m}]\end{array}$ \\
\hline 785,412918011374 & 1120,000000000000 & 6,150000000000 & 1113,554212549080 & 119,987564831539 \\
\hline 881,247788255917 & 1115,566037735850 & 6,460000000000 & 1108,482912149740 & 125,511824230595 \\
\hline 988,776281235928 & 1109,905660377360 & 6,730000000000 & 1102,257771898030 & 130,070670130143 \\
\hline 1109,425234722780 & 1104,056603773580 & 7,110000000000 & 1095,566795180010 & 136,654241189414 \\
\hline 1244,795587027250 & 1096,132075471700 & 7,490000000000 & 1086,779468175390 & 142,884269359171 \\
\hline 1396,683620478220 & 1090,943396226420 & 7,850000000000 & 1080,720197361480 & 149,001170414865 \\
\hline 1567,104796997840 & 1085,566037735850 & 8,200000000000 & 1074,467461231690 & 154,833126429181 \\
\hline 1758,320502056710 & 1078,207547169810 & 8,600000000000 & 1066,084592357190 & 161,230135869637 \\
\hline 1972,868051885120 & 1070,849056603770 & 9,000000000000 & 1057,665127744850 & 167,517699308647 \\
\hline 2213,594362117870 & 1061,037735849060 & 9,440000000000 & 1046,669054777060 & 174,025764379269 \\
\hline 2483,693724635030 & 1050,377358490570 & 9,900000000000 & 1034,73653 & 180,590 \\
\hline 2786,750193874480 & 1041,226415094340 & 10,360000000000 & 1024,25156870 & 187,24628 \\
\hline 3126,785145056740 & 1030,849056603770 & 10,830000000000 & 1012,488607 & 193,692 \\
\hline 3508,310635390910 & 1018,679245283020 & 11,320000000000 & 998,862063 & 199,95 \\
\hline 3936,389276332440 & 1006,981132075470 & 11,870000000000 & 985,44867245 & 207,12777 \\
\hline 4416,701411361350 & & 12,400000000000 & 971,973194730862 & 213,702166630693 \\
\hline 4955,620490688970 & 984,433962264151 & 12,990000000000 & 959,241619200057 & 221,282041868626 \\
\hline 5560,297643069970 & 972,169811320755 & 13,640000000000 & 944,751320732405 & 229,257680390010 \\
\hline 6238,756566936220 & 957,830188679245 & 14,160000000000 & 928,727831098483 & 234,314502514092 \\
\hline 7000,000000000000 & 943,018867924528 & 14,780000000000 & 911,816728388951 & 240,571900045971 \\
\hline 7854,129180113750 & 927,547169811321 & 15,370000000000 & 894,372811703016 & 245,847566413486 \\
\hline 8812,477882559180 & 912,264150943396 & 16,010000000000 & 876,880684507625 & 251,607126755014 \\
\hline 9887,762812359290 & 895,660377358491 & 16,610000000000 & 858,286886162258 & 256,029554176565 \\
\hline 11094,252347227800 & 879,339622641509 & 17,300000000000 & 839,5590011 & 261,49350 \\
\hline 12447,955870272500 & 862,169811320755 & 17,970000000000 & 820,111604671752 & 265,99575 \\
\hline 13966,836204782200 & 843,867924528302 & 18,620000000000 & 799,697357963757 & 269,43869 \\
\hline 15671,047969978400 & 827,641509433962 & 19,310000000000 & 781,081089243232 & 273,683759409895 \\
\hline 17583,205020567100 & & 20,100000000000 & 761,020719386184 & 278,494035517966 \\
\hline 19728,680518851200 & 789,433962264151 & 20,720000000000 & 738,373837649655 & 279,302804587778 \\
\hline 22135,943621178700 & 768,113207547170 & 21,380000000000 & 715,254057081823 & 280,017023476062 \\
\hline 24836,937246350300 & 752,075471698113 & 22,290000000000 & 695,877332924980 & 285,258221005383 \\
\hline 27867,501938744900 & 732,830188679245 & 23,060000000000 & 674,273521521137 & 287,045821455638 \\
\hline 31267,851450567500 & 711,792452830189 & 23,830000000000 & 651,110898180718 & 287,581456593285 \\
\hline 35083,106353909100 & 692,735849056604 & 24,670000000000 & 629,507664380235 & 289,141932370099 \\
\hline 39363,892763324500 & 672,735849056604 & 25,500000000000 & 607,201477613100 & 289,620248239618 \\
\hline 44167,014113613600 & 653,113207547170 & 26,320000000000 & 585,406081244990 & 289,580009520576 \\
\hline 49556,204906889700 & 632,924528301887 & 26,980000000000 & 564,040150859467 & 287,144853244135 \\
\hline 55602,976430699800 & 611,886792452830 & 28,060000000000 & 539,962852740507 & 287,829054194580 \\
\hline 62387,565669362300 & 591,415094339623 & 28,830000000000 & 518,111744937934 & 285,187716373118 \\
\hline 70000,000000000200 & 570,660377358491 & 29,540000000000 & 496,481209234013 & 281,353292435806 \\
\hline 78541,291801137600 & 550,377358490566 & 30,500000000000 & 474,221181322251 & 279,337623538938 \\
\hline 88124,778825591900 & 530,849056603774 & 31,500000000000 & 452,623226869857 & 277,367870156287 \\
\hline 98877,628123593100 & 511,415094339623 & 32,400000000000 & 431,802045674191 & 274,029910903881 \\
\hline 110942,523472278000 & 492,075471698113 & 33,320000000000 & 411,185972678059 & 270,304209585642 \\
\hline 124479,558702725000 & 473,113207547170 & 34,350000000000 & 390,605202540951 & 266,952585496965 \\
\hline 139668,362047822000 & 454,150943396226 & 35,410000000000 & 370,145135468149 & 263,145697431951 \\
\hline 156710,479699784000 & 434,716981132075 & 36,550000000000 & 349,224441437292 & 258,884420518880 \\
\hline
\end{tabular}




\begin{tabular}{|c|c|c|c|c|}
\hline $\begin{array}{l}\text { frequency } \\
\qquad[\mathrm{Hz}]\end{array}$ & $\begin{array}{c}\text { measurement } \\
2\end{array}$ & $\begin{array}{c}\text { phase difference } \\
{\left[{ }^{\circ}\right]}\end{array}$ & $\begin{array}{c}\text { real impedance } \\
\qquad Z^{\prime}[\Omega \mathrm{m}]\end{array}$ & $\begin{array}{c}\text { imaginary } \\
\text { impedance } \\
Z^{\prime \prime}[\Omega \mathrm{m}]\end{array}$ \\
\hline 175832,050205671000 & 413,962264150943 & 37,530000000000 & 328,286350884117 & 252,176184371494 \\
\hline 197286,805188512000 & 394,811320754717 & 38,700000000000 & 308,122759878388 & 246,852878940087 \\
\hline 221359,436211787000 & 376,320754716981 & 39,550000000000 & 290,169345798018 & 239,622747647022 \\
\hline 248369,372463503000 & 358,018867924528 & 40,680000000000 & 271,507874388745 & 233,368772407248 \\
\hline 278675,019387448000 & 343,396226415094 & 41,250000000000 & 258,178952756932 & 226,416864807948 \\
\hline 312678,514505674000 & 324,150943396226 & 42,560000000000 & 238,759684127059 & 219,243351872321 \\
\hline 350831,063539090000 & 306,226415094340 & 43,830000000000 & 220,911038834624 & 212,068220680371 \\
\hline 393638,927633244000 & 290,754716981132 & 44,780000000000 & 206,382541226660 & 204,803691674745 \\
\hline 441670,141136134000 & 275,188679245283 & 45,860000000000 & 191,645241629227 & 197,486481931393 \\
\hline 495562,049068895000 & 261,037735849057 & 46,930000000000 & 178,260416523810 & 190,693270562860 \\
\hline 556029,764306995000 & 246,037735849057 & 48,070000000000 & 164,407873324019 & 183,042668934879 \\
\hline 623875,656693620000 & 232,169811320755 & 49,180000000000 & 151,765877909497 & 175,698433661415 \\
\hline 699999,999999997000 & 212,924528301887 & 47,680000000000 & 143,355836094424 & 157,435570981432 \\
\hline 785412,918011371000 & 204,433962264151 & 51,850000000000 & 126,283431772419 & 160,766102729403 \\
\hline 881247,788255913000 & 190,283018867925 & 52,010000000000 & 117,123751990654 & 149,965509331722 \\
\hline 988776,281235923000 & 179,056603773585 & 54,550000000000 & 103,851448743970 & 145,863442811107 \\
\hline
\end{tabular}




\begin{tabular}{|c|c|c|c|c|}
\hline $\begin{array}{c}\text { frequency } \\
{[\mathrm{Hz}]}\end{array}$ & $\begin{array}{c}\text { measurement } \\
2\end{array}$ & $\begin{array}{c}\text { phase difference } \\
{\left[{ }^{\circ}\right]}\end{array}$ & $\begin{array}{l}\text { real admittance } \\
\mathbf{Y}^{\prime}[\mathrm{S} \mathrm{m} \text {-1] }\end{array}$ & $\begin{array}{l}\text { imaginary } \\
\text { admittance } \\
\mathrm{Y}^{\prime \prime}[\mathrm{S} \mathrm{m}-1]\end{array}$ \\
\hline 785,412918011374 & 1120,000000000000 & 6,150000000000 & 0,000898025430 & 0,008334196976 \\
\hline 881,247788255917 & & & & \\
\hline & & & & \\
\hline 1109,425234722780 & & & & \\
\hline 1244,795587027250 & 1096,132075471700 & & & 0,006998671054 \\
\hline 1396,683620478220 & 1090,943396226420 & & & \\
\hline 1567,104796997840 & 1085,566037735850 & 8,200000000000 & & \\
\hline 1758,320502056710 & 1078,207547169810 & & & \\
\hline 1972,868051885120 & & & & \\
\hline 2213,5 & & & & \\
\hline & & & & \\
\hline & & & & \\
\hline 3126,78 & & & & \\
\hline 3508,310635390910 & 1018,679245 & & & \\
\hline 3936,389276332440 & 1006,98 & & & \\
\hline 4416,701411361350 & & & & \\
\hline & & & & \\
\hline 5560 & & & & \\
\hline 6238,7 & & & & \\
\hline 7000,0 & & & & \\
\hline 7854,129180113750 & & & & \\
\hline 8812,477882559180 & 912,26 & & 0,0011 & \\
\hline 9887,762812359290 & 895,66 & & & \\
\hline & & & & \\
\hline & & & & \\
\hline & & & & \\
\hline 1567 & & & & \\
\hline 17583,205020567100 & 810,37735 & & & \\
\hline 19728,680518851200 & 789,433962264151 & 20,720000000000 & 0,001354327509 & 0,0035 \\
\hline 22135,943621178700 & 768,113207547170 & 21,380000000000 & 0,001398104618 & 11448 \\
\hline 24836,937246350300 & 752,075471698113 & & & 725 \\
\hline & & & & \\
\hline 31267 & & & & \\
\hline & 692,7 & & & \\
\hline 39363,892763324500 & 672,735849056604 & 25,500000000000 & 0,0016468998 & 0,003452797262 \\
\hline 44167,014113613600 & 653,113207547170 & 26,320000000000 & 0,001708215941 & 0,003453277046 \\
\hline 49556,204906889700 & 632,924528301887 & 26,980000000000 & 0,001772923432 & 0,003482562855 \\
\hline 55602,976430699800 & 611,886792452830 & 28,060000000000 & 0,001851979252 & 0,003474284425 \\
\hline 62387,565669362300 & & & & \\
\hline & & & & \\
\hline & 550,3773584905 & & & 0,003579897285 \\
\hline 88124,778825591900 & 530,849056603774 & 31,500000000000 & 0,002209343093 & 0,003605320254 \\
\hline 98877,628123593100 & 511,415094339623 & 32,400000000000 & 0,002315876013 & 0,003649236672 \\
\hline 110942,523472278000 & 492,075471698113 & 33,320000000000 & 0,002431989578 & 0,003699535429 \\
\hline 124479,55870272500 & 473,113207547170 & 34,3500000000 & 0,002560129751 & 0,0037459983573 \\
\hline 139668,362047822000 & 454,150943396226 & & 0,002701642962 & 0,003800176137 \\
\hline 156710,479699784000 & 434,716981132075 & 36,550000000000 & 0,002863488008 & 0,003862727614 \\
\hline
\end{tabular}




\begin{tabular}{|c|c|c|c|c|}
\hline $\begin{array}{c}\text { frequency } \\
\text { [Hz] }\end{array}$ & $\begin{array}{c}\text { measurement } \\
\mathbf{2}\end{array}$ & $\begin{array}{c}\text { phase difference } \\
\text { ['] }\end{array}$ & $\begin{array}{c}{ }^{\circ} \\
\text { real admittance } \\
\mathbf{Y}^{\prime} \text { [S m-1] }\end{array}$ & $\begin{array}{c}\text { imaginary } \\
\text { admittance } \\
\mathbf{Y}^{\prime \prime} \text { [S m-1] }\end{array}$ \\
\hline 175832,050205671000 & 413,962264150943 & 37,530000000000 & 0,003046121160 & 0,003965481524 \\
\hline 197286,805188512000 & 394,811320754717 & 38,700000000000 & 0,003245459701 & 0,004050995898 \\
\hline 221359,436211787000 & 376,320754716981 & 39,550000000000 & 0,003446263413 & 0,004173226498 \\
\hline 248369,372463503000 & 358,018867924528 & 40,680000000000 & 0,003683134429 & 0,004285063463 \\
\hline 278675,019387448000 & 343,396226415094 & 41,250000000000 & 0,003873282424 & 0,004416632131 \\
\hline 312678,514505674000 & 324,150943396226 & 42,560000000000 & 0,004188311790 & 0,004561141724 \\
\hline 350831,063539090000 & 306,226415094340 & 43,830000000000 & 0,004526709056 & 0,004715463716 \\
\hline 393638,927633244000 & 290,754716981132 & 44,780000000000 & 0,004845371096 & 0,004882724485 \\
\hline 441670,141136134000 & 275,188679245283 & 45,860000000000 & 0,005217974584 & 0,005063637725 \\
\hline 495562,049068895000 & 261,037735849057 & 46,930000000000 & 0,005609770355 & 0,005244023541 \\
\hline 556029,764306995000 & 246,037735849057 & 48,070000000000 & 0,006082433765 & 0,005463207053 \\
\hline 623875,656693620000 & 232,169811320755 & 49,180000000000 & 0,006589096401 & 0,005691570375 \\
\hline 699999,999999997000 & 212,924528301887 & 47,680000000000 & 0,006975649037 & 0,006351804702 \\
\hline 785412,918011371000 & 204,433962264151 & 51,850000000000 & 0,007918695160 & 0,006220216719 \\
\hline 881247,788255913000 & 190,283018867925 & 52,010000000000 & 0,008537977848 & 0,006668199938 \\
\hline 988776,281235923000 & 179,056603773585 & 54,550000000000 & 0,009629138660 & 0,006855727389 \\
\hline
\end{tabular}




\begin{tabular}{|c|c|c|c|c|}
\hline $\begin{array}{l}\text { frequency } \\
\qquad[\mathrm{Hz}]\end{array}$ & $\begin{array}{c}\text { measurement } \\
3\end{array}$ & $\begin{array}{c}\text { phase difference } \\
{\left[{ }^{\circ}\right]}\end{array}$ & $\begin{array}{c}\text { real impedance } \\
\qquad Z^{\prime}[\Omega \mathrm{m}]\end{array}$ & $\begin{array}{c}\text { imaginary } \\
\text { impedance } \\
\mathrm{Z}^{\prime \prime}[\Omega \mathrm{m}] \\
\end{array}$ \\
\hline 785,412918011374 & 1851,851851851850 & 9,690000000000 & 1825,431224110850 & 311,698776462586 \\
\hline 881,247788255917 & 1838,383838383840 & 10,100000000000 & 1809,894734902500 & 322,391355037795 \\
\hline 988,776281235928 & 1818,181818181820 & 10,500000000000 & 1787,736195570830 & 331,337319076632 \\
\hline 1109,425234722780 & 1804,713804713800 & 11,000000000000 & 1771,556129050330 & 344,355628019622 \\
\hline 1244,795587027250 & 1784,511784511780 & 11,400000000000 & 1749,305463129670 & 352,721853201807 \\
\hline 1396,683620478220 & 1764,309764309760 & 11,900000000000 & 1726,392956879900 & 363,808057736814 \\
\hline 1567,104796997840 & 1750,841750841750 & 12,500000000000 & 1709,339810445670 & 378,951512618900 \\
\hline 1758,320502056710 & 1723,905723905720 & 13,000000000000 & 1679,722131885650 & 387,794410182016 \\
\hline 1972,868051885120 & 1696,969696969700 & 13,500000000000 & 1650,082289159700 & 396,149708361537 \\
\hline 2213,594362117870 & 1676,767676767680 & 14,000000000000 & 410 & 405,646814843888 \\
\hline 2483,693724635030 & 1649,831649831650 & 14,600000000000 & 490 & 415,872005182241 \\
\hline 2786,750193874480 & 1616,161616161620 & 15,200000000000 & 990 & 423,740086692308 \\
\hline 3126,785145056740 & 1589,225589225590 & 15,700000000 & 230 & 430,04515 \\
\hline 3508,310635390910 & 1562,289562289560 & 16,300000000000 & 120 & 438,4 \\
\hline 3936,389276332440 & 1528,619528619530 & 16,900000000000 & 900 & 444,373050 \\
\hline 4416,701411361350 & 1494,949494949490 & 17,500000000000 & 1425,7586738 & 449,539983097296 \\
\hline 4955,620490688970 & 1468,013468013470 & 18,100000000000 & 1395,36989558 & 456,077182892253 \\
\hline 5560,297643069970 & 1434,343434343430 & 18,700000000000 & 1358,624842827630 & 459,869138011777 \\
\hline 6238,756566936220 & 1400,673400673400 & 19,300000000000 & 870 & 462,942718413134 \\
\hline 7000,000000000000 & 1367,003367003370 & 19,900000000000 & 6540 & 465,299991200332 \\
\hline 7854,129180113750 & 1333,333333333330 & 20,500000000000 & 1248,896252331190 & 466,943175012622 \\
\hline 8812,477882559180 & 1299,663299663300 & 21,100000000000 & 1212,525469 & 467,874639509562 \\
\hline 9887,762812359290 & 1265,993265993270 & 21,800000000000 & 1175,45680440 & 470,149179013093 \\
\hline 11094,252347227800 & 1232,323232323230 & 22,400000000000 & 750 & 469,601877926600 \\
\hline 12447,955870272500 & 1198,653198653200 & 23,000000000000 & 1103,366086966560 & 468,351116977042 \\
\hline 13966,836204782200 & 1164,983164983160 & 23,600000000000 & 1067,547152958030 & 466,399883046078 \\
\hline 15671,047969978400 & 1131,313131313130 & 24,300000000000 & 1031,082494779480 & 465,551597613860 \\
\hline 17583,205020567100 & 1097,643097643100 & 25,000000000000 & 994,802486780971 & 463,884017937604 \\
\hline 19728,680518851200 & 1063,973063973060 & 25,600000000000 & 959,525516309815 & 459,727598051939 \\
\hline 22135,943621178700 & 1030,303030303030 & 26,300000000000 & 923,652685849605 & 456,497590546124 \\
\hline 24836,937246350300 & 1003,367003367000 & 27,000000000000 & 894,006546155329 & 455,519087280756 \\
\hline 27867,501938744900 & 969,696969696970 & 27,700000000000 & 858,563515883070 & 450,755923126904 \\
\hline 31267,851450567500 & 942,760942760943 & 28,100000000000 & 831,634755841572 & 444,051605190016 \\
\hline 35083,106353909100 & 902,356902356902 & 29,200000000000 & 787,687261644138 & 440,223530805321 \\
\hline 39363,892763324500 & 875,420875420875 & 30,000000000000 & 758,136717117690 & 437,710437710437 \\
\hline 44167,014113613600 & 841,750841750842 & 30,800000000000 & 723,030216271603 & 431,012512517316 \\
\hline 49556,204906889700 & 808,080808080808 & 31,500000000000 & 689,002153013408 & 422,221062396726 \\
\hline 55602,976430699800 & 781,144781144781 & 32,400000000000 & 659,542352580698 & 418,558304495378 \\
\hline 62387,565669362300 & 754,208754208754 & 33,200000000000 & 631,094970420227 & 412,976976640846 \\
\hline 70000,000000000200 & 722,020202020202 & 34,120000000000 & 597,734954366093 & 405,001353644970 \\
\hline 78541,291801137600 & 693,265993265993 & 35,030000000000 & 567,681973423671 & 397,938329982034 \\
\hline 88124,778825591900 & 665,185185185185 & 35,970000000000 & 538,350765256814 & 390,704215663527 \\
\hline 98877,628123593100 & 638,249158249158 & 36,930000000000 & 510,197337868765 & 383,484894666510 \\
\hline 110942,523472278000 & 612,659932659933 & 38,010000000000 & 482,716775617946 & 377,275373731029 \\
\hline 124479,558702725000 & 587,609427609428 & 39,240000000000 & 455,105299885128 & 371,704190764574 \\
\hline 139668,362047822000 & 562,356902356902 & 40,630000000000 & 426,789781851992 & 366,190889749021 \\
\hline 156710,479699784000 & 536,026936026936 & 42,250000000000 & 396,776854868363 & 360,406719675418 \\
\hline
\end{tabular}




\begin{tabular}{|c|c|c|c|c|}
\hline $\begin{array}{l}\text { frequency } \\
\quad[\mathrm{Hz}]\end{array}$ & $\begin{array}{c}\text { measurement } \\
3\end{array}$ & $\begin{array}{c}\text { phase difference } \\
{\left[{ }^{\circ}\right]}\end{array}$ & $\begin{array}{c}\text { real impedance } \\
\qquad Z^{\prime}[\Omega \mathrm{m}]\end{array}$ & $\begin{array}{c}\text { imaginary } \\
\text { impedance } \\
Z^{\prime \prime}[\Omega \mathrm{m}]\end{array}$ \\
\hline 175832,050205671000 & 508,148148148148 & 43,680000000000 & 367,496940745425 & 350,942358527929 \\
\hline 197286,805188512000 & 480,740740740741 & 45,220000000000 & 338,627278224155 & 341,237785496356 \\
\hline 221359,436211787000 & 453,670033670034 & 46,620000000000 & 311,595934287521 & 329,733943029311 \\
\hline 248369,372463503000 & 428,215488215488 & 48,110000000000 & 285,920611297477 & 318,775639569440 \\
\hline 278675,019387448000 & 403,973063973064 & 49,590000000000 & 261,876671743586 & 307,595262012092 \\
\hline 312678,514505674000 & 380,606060606061 & 51,170000000000 & 238,644485768427 & 296,495839401479 \\
\hline 350831,063539090000 & 357,845117845118 & 52,850000000000 & 216,104019769710 & 285,223037297059 \\
\hline 393638,927633244000 & 335,286195286195 & 54,610000000000 & 194,177277277758 & 273,334991793018 \\
\hline 441670,141136134000 & 313,400673400673 & 56,480000000000 & 173,068637332648 & 261,279981743370 \\
\hline 495562,049068895000 & 291,784511784512 & 58,460000000000 & 152,630637673743 & 248,680698407883 \\
\hline 556029,764306995000 & 270,976430976431 & 60,440000000000 & 133,682082864558 & 235,706017881848 \\
\hline 623875,656693620000 & 250,505050505050 & 62,380000000000 & 116,135481689805 & 221,957946965669 \\
\hline 699999,999999997000 & 229,629629629630 & 63,920000000000 & 100,951080305981 & 206,248990758492 \\
\hline 785412,918011371000 & 211,986531986532 & 66,160000000000 & 85,681556030284 & 193,899357141550 \\
\hline 881247,788255913000 & 193,535353535354 & 67,700000000000 & 73,438181985614 & 179,060789942138 \\
\hline 988776,281235923000 & 175,892255892256 & 69,700000000000 & & \\
\hline
\end{tabular}




\begin{tabular}{|c|c|c|c|c|}
\hline $\begin{array}{l}\text { frequency } \\
\qquad[\mathrm{Hz}]\end{array}$ & $\begin{array}{c}\text { measurement } \\
3\end{array}$ & $\begin{array}{c}\text { phase difference } \\
{\left[{ }^{\circ}\right]}\end{array}$ & $\begin{array}{l}\text { real admittance } \\
\quad Y^{\prime}[S \text { m-1] }\end{array}$ & $\begin{array}{l}\text { imaginary } \\
\text { admittance } \\
Y^{\prime \prime}[S \text { m-1] }\end{array}$ \\
\hline 785,412918011374 & 1851,851851851850 & 9,690000000000 & 0,000547816 & 0,003208226 \\
\hline 881,247788255917 & 1838,383838383840 & 10,100000000000 & 0,000552518 & 0,00310182 \\
\hline 988,776281235928 & 1818,181818181820 & 10,500000000000 & 0,000559367 & 0,003018072 \\
\hline 1109,425234722780 & 1804,713804713800 & 11,000000000000 & 0,000564475 & 0,002903975 \\
\hline 1244,795587027250 & 1784,511784511780 & 11,400000000000 & 0,000571655 & 0,002835095 \\
\hline 1396,683620478220 & 1764,309764309760 & 11,900000000000 & 0,000579242 & 0,002748702 \\
\hline 1567,104796997840 & 1750,841750841750 & 12,500000000000 & 0,000585021 & 0,00263886 \\
\hline 1758,320502056710 & 1723,905723905720 & 13,000000000000 & 0,000595337 & 0,002578686 \\
\hline 1972,868051885120 & 1696,969696969700 & 13,500000000000 & 0,00060603 & 0,002524298 \\
\hline 2213,594362117870 & 1676,767676767680 & 14,000000000000 & 0,000614643 & 0,002465199 \\
\hline 2483,693724635030 & 1649,831649831650 & 14,600000000000 & 0,000626348 & 0,002404586 \\
\hline 2786,750193874480 & 1616,161616161620 & 15,200000000000 & 0,000641181 & 0,002359937 \\
\hline 3126,785145056740 & 1589,225589225590 & 15,700000000000 & 0,000653623 & 0,002325337 \\
\hline 3508,310635390910 & 1562,289562289560 & 16,300000000000 & 0,000666892 & 0,002280592 \\
\hline 3936,389276332440 & 1528,619528619530 & 16,900000000000 & 0,000683712 & 0,002250361 \\
\hline 4416,701411361350 & 1494,949494949490 & 17,500000000000 & 0,000701381 & 0,002224496 \\
\hline 4955,620490688970 & 1468,013468013470 & 18,100000000000 & 0,000716656 & 0,002192611 \\
\hline 5560,297643069970 & 1434,343434343430 & 18,700000000000 & 0,000736038 & 0,002174532 \\
\hline 6238,756566936220 & 1400,673400673400 & 19,300000000000 & 0,000756454 & 0,002160094 \\
\hline 7000,000000000000 & 1367,003367003370 & 19,900000000000 & 0,000777982 & 0,002149151 \\
\hline 7854,129180113750 & 1333,333333333330 & 20,500000000000 & 0,000800707 & 0,002141588 \\
\hline 8812,477882559180 & 1299,663299663300 & 21,100000000000 & 0,000824725 & 0,002137325 \\
\hline 9887,762812359290 & 1265,993265993270 & 21,800000000000 & 0,000850733 & 0,002126984 \\
\hline 11094,252347227800 & 1232,323232323230 & 22,400000000000 & 0,000877701 & 0,002129463 \\
\hline 12447,955870272500 & 1198,653198653200 & 23,000000000000 & 0,000906318 & 0,00213515 \\
\hline 13966,836204782200 & 1164,983164983160 & 23,600000000000 & 0,000936727 & 0,002144083 \\
\hline 15671,047969978400 & 1131,313131313130 & 24,300000000000 & 0,000969855 & 0,00214799 \\
\hline 17583,205020567100 & 1097,643097643100 & 25,000000000000 & 0,001005225 & 0,002155711 \\
\hline 19728,680518851200 & 1063,973063973060 & 25,600000000000 & 0,001042182 & 0,002175201 \\
\hline 22135,943621178700 & 1030,303030303030 & 26,300000000000 & 0,001082658 & 0,002190592 \\
\hline 24836,937246350300 & 1003,367003367000 & 27,000000000000 & 0,00111856 & 0,002195298 \\
\hline 27867,501938744900 & 969,696969696970 & 27,700000000000 & 0,001164736 & 0,002218496 \\
\hline 31267,851450567500 & 942,760942760943 & 28,100000000000 & 0,001202451 & 0,002251991 \\
\hline 35083,106353909100 & 902,356902356902 & 29,200000000000 & 0,001269539 & 0,002271573 \\
\hline 39363,892763324500 & 875,420875420875 & 30,000000000000 & 0,001319023 & 0,002284615 \\
\hline 44167,014113613600 & 841,750841750842 & 30,800000000000 & 0,001383068 & 0,002320118 \\
\hline 49556,204906889700 & 808,080808080808 & 31,500000000000 & 0,001451374 & 0,002368428 \\
\hline 55602,976430699800 & 781,144781144781 & 32,400000000000 & 0,001516203 & 0,002389153 \\
\hline 62387,565669362300 & 754,208754208754 & 33,200000000000 & 0,001584548 & 0,002421442 \\
\hline 70000,000000000200 & 722,020202020202 & 34,120000000000 & 0,001672982 & 0,002469128 \\
\hline 78541,291801137600 & 693,265993265993 & 35,030000000000 & 0,00176155 & 0,002512952 \\
\hline 88124,778825591900 & 665,185185185185 & 35,970000000000 & 0,001857525 & 0,002559481 \\
\hline 98877,628123593100 & 638,249158249158 & 36,930000000000 & 0,001960026 & 0,002607665 \\
\hline 110942,523472278000 & 612,659932659933 & 38,010000000000 & 0,002071608 & 0,002650584 \\
\hline 124479,558702725000 & 587,609427609428 & 39,240000000000 & 0,002197294 & 0,002690311 \\
\hline 139668,362047822000 & 562,356902356902 & 40,630000000000 & 0,002343074 & 0,002730816 \\
\hline 156710,479699784000 & 536,026936026936 & 42,250000000000 & 0,002520308 & 0,002774643 \\
\hline
\end{tabular}




\begin{tabular}{|c|c|c|c|c|}
\hline $\begin{array}{c}\text { frequency } \\
{[\mathbf{H z}]}\end{array}$ & $\begin{array}{c}\text { measurement } \\
\mathbf{3}\end{array}$ & $\begin{array}{c}\text { phase difference } \\
\text { [ }^{\circ} \text { ] }\end{array}$ & $\begin{array}{c}\text { real admittance } \\
\mathbf{Y}^{\prime} \text { [S m-1] }\end{array}$ & $\begin{array}{c}\text { imaginary } \\
\text { admittance } \\
\mathbf{Y}^{\prime \prime} \text { [S m-1] }\end{array}$ \\
\hline 175832,050205671000 & 508,148148148148 & 43,680000000000 & 0,002721111 & 0,002849471 \\
\hline 197286,805188512000 & 480,740740740741 & 45,220000000000 & 0,002953099 & 0,002930508 \\
\hline 221359,436211787000 & 453,670033670034 & 46,620000000000 & 0,003209284 & 0,003032748 \\
\hline 248369,372463503000 & 428,215488215488 & 48,110000000000 & 0,003497474 & 0,003137003 \\
\hline 278675,019387448000 & 403,973063973064 & 49,590000000000 & 0,003818591 & 0,003251025 \\
\hline 312678,514505674000 & 380,606060606061 & 51,170000000000 & 0,004190334 & 0,003372729 \\
\hline 350831,063539090000 & 357,845117845118 & 52,850000000000 & 0,004627401 & 0,003506028 \\
\hline 393638,927633244000 & 335,286195286195 & 54,610000000000 & 0,005149933 & 0,003658514 \\
\hline 441670,141136134000 & 313,400673400673 & 56,480000000000 & 0,005778054 & 0,003827312 \\
\hline 495562,049068895000 & 291,784511784512 & 58,460000000000 & 0,006551765 & 0,004021221 \\
\hline 556029,764306995000 & 270,976430976431 & 60,440000000000 & 0,007480434 & 0,004242573 \\
\hline 623875,656693620000 & 250,505050505050 & 62,380000000000 & 0,008610633 & 0,004505358 \\
\hline 699999,999999997000 & 229,629629629630 & 63,920000000000 & 0,009905788 & 0,004848509 \\
\hline 785412,918011371000 & 211,986531986532 & 66,160000000000 & 0,011671123 & 0,005157315 \\
\hline 881247,788255913000 & 193,535353535354 & 67,700000000000 & 0,013616895 & 0,005584696 \\
\hline 988776,281235923000 & 175,892255892256 & 69,700000000000 & & \\
\hline
\end{tabular}




\section{III.4 COMPLEX IMPEDANCE DATA FOR SAMPLE 17/08/00/11 CORE 1:}

\begin{tabular}{|c|c|c|c|c|}
\hline $\begin{array}{c}\text { frequency } \\
{[\mathrm{Hz}]}\end{array}$ & $\begin{array}{c}\text { real impedance } \\
\text { at } 0 \text { bar } \\
Z^{\prime}[\Omega \mathrm{m}]\end{array}$ & $\begin{array}{c}\text { imaginary } \\
\text { impedance } \\
\text { at } 0 \text { bar } \\
Z^{\prime \prime}[\Omega \mathrm{m}]\end{array}$ & $\begin{array}{c}\text { real admittance } \\
\text { at } 0 \text { bar } \\
Y^{\prime}[S \text { m-1] }\end{array}$ & $\begin{array}{c}\text { imaginary } \\
\text { admittance } \\
\text { at } 0 \text { bar } \\
Y^{\prime \prime}[S \text { m-1] }\end{array}$ \\
\hline 785,412900 & 562,322400 & 91,834880 & 0,001778 & 0,010889 \\
\hline 881,247800 & 555,725800 & 90,459010 & 0,001799 & 0,011055 \\
\hline 988,776300 & 549,518700 & 89,547050 & 0,001820 & 0,011167 \\
\hline 1109,425000 & 543,312000 & 88,632920 & 0,001841 & 0,011282 \\
\hline 1244,796000 & 537,074800 & 87,904000 & 0,001862 & 0,011376 \\
\hline 1396,684000 & 530,989700 & 87,193380 & 0,001883 & 0,011469 \\
\hline 1567,105000 & 524,920400 & 86,384850 & 0,001905 & 0,011576 \\
\hline 1758,320000 & 518,987800 & 85,687520 & 0,001927 & 0,011670 \\
\hline 1972,868000 & 513,192300 & 85,098530 & 0,001949 & 0,011751 \\
\hline 2213,594000 & 507,383000 & 84,590010 & 0,001971 & 0,011822 \\
\hline 2483,694000 & 501,560300 & 84,158920 & 0,001994 & 0,011882 \\
\hline 2786,750000 & 495,890000 & 83,741220 & 0,002017 & 0,011942 \\
\hline 3126,785000 & 490,192000 & 83,482750 & 0,002040 & 0,011979 \\
\hline 3508,311000 & 484,481200 & 83,293070 & 0,002064 & 0,012006 \\
\hline 3936,389000 & 478,864800 & 83,445990 & 0,002088 & 0,011984 \\
\hline 4416,701000 & 473,114200 & 83,464740 & 0,002114 & 0,011981 \\
\hline 4955,620000 & 467,802800 & 83,622060 & 0,002138 & 0,011959 \\
\hline 5560,297000 & 462,162900 & 83,862450 & 0,002164 & 0,011924 \\
\hline 6238,757000 & 456,495600 & 84,233180 & 0,002191 & 0,011872 \\
\hline 7000,000000 & 450,800700 & 84,728320 & 0,002218 & 0,011802 \\
\hline 7854,129000 & 444,942500 & 85,235510 & 0,002247 & 0,011732 \\
\hline 8812,479000 & 439,206500 & 85,885410 & 0,002277 & 0,011643 \\
\hline 9887,763000 & 433,441900 & 86,643810 & 0,002307 & 0,011542 \\
\hline 11094,250000 & 427,498400 & 87,473970 & 0,002339 & 0,011432 \\
\hline 12447,960000 & 421,510100 & 88,472630 & 0,002372 & 0,011303 \\
\hline 13966,840000 & 415,641200 & 89,590190 & 0,002406 & 0,011162 \\
\hline 15671,050000 & 409,458000 & 90,652210 & 0,002442 & 0,011031 \\
\hline 17583,200000 & 403,212100 & 91,929430 & 0,002480 & 0,010878 \\
\hline 19728,680000 & 396,785100 & 93,235310 & 0,002520 & 0,010726 \\
\hline 22135,940000 & 390,309200 & 94,664350 & 0,002562 & 0,010564 \\
\hline 24836,940000 & 383,783200 & 96,206460 & 0,002606 & 0,010394 \\
\hline 27867,500000 & 377,205700 & 97,851480 & 0,002651 & 0,010220 \\
\hline 31267,850000 & 370,410200 & 99,614070 & 0,002700 & 0,010039 \\
\hline 35083,110000 & 363,560700 & 101,453900 & 0,002751 & 0,009857 \\
\hline 39363,890000 & 356,454700 & 103,504300 & 0,002805 & 0,009661 \\
\hline 44167,020000 & 348,998200 & 105,511500 & 0,002865 & 0,009478 \\
\hline 49556,210000 & 341,574800 & 107,771000 & 0,002928 & 0,009279 \\
\hline 55602,980000 & 333,887900 & 110,169100 & 0,002995 & 0,009077 \\
\hline 62387,570000 & 326,060300 & 112,783100 & 0,003067 & 0,008867 \\
\hline 70000,000000 & 317,839300 & 115,369300 & 0,003146 & 0,008668 \\
\hline 78541,290000 & 309,451800 & 118,164400 & 0,003232 & 0,008463 \\
\hline 88124,780000 & 300,466200 & 120,962600 & 0,003328 & 0,008267 \\
\hline 98877,620000 & 291,211900 & 123,722000 & 0,003434 & 0,008083 \\
\hline 110942,500000 & 281,508300 & 126,442400 & 0,003552 & 0,007909 \\
\hline
\end{tabular}




\begin{tabular}{|c|c|c|c|c|}
\hline $\begin{array}{c}\text { frequency } \\
{[\mathbf{H z}]}\end{array}$ & $\begin{array}{c}\text { real impedance } \\
\text { at 0 bar } \\
\mathbf{Z}^{\prime}[\mathbf{\Omega} \mathbf{~ m}]\end{array}$ & $\begin{array}{c}\text { imaginary } \\
\text { impedance } \\
\text { at 0 bar } \\
\mathbf{Z}^{\prime \prime}[\mathbf{\Omega} \text { m] }\end{array}$ & $\begin{array}{c}\text { real admittance } \\
\text { at 0 bar } \\
\mathbf{Y}^{\prime} \text { [S m-1] }\end{array}$ & $\begin{array}{c}\text { imaginary } \\
\text { admittance } \\
\text { at 0 bar } \\
\mathbf{Y}^{\prime \prime} \text { [S m-1] }\end{array}$ \\
\hline 124479,600000 & 271,477100 & 129,180400 & 0,003684 & 0,007741 \\
\hline 139668,400000 & 260,917500 & 131,607100 & 0,003833 & 0,007598 \\
\hline 156710,500000 & 249,914200 & 133,866500 & 0,004001 & 0,007470 \\
\hline 175832,000000 & 238,506900 & 135,848600 & 0,004193 & 0,007361 \\
\hline 197286,800000 & 226,812900 & 137,331300 & 0,004409 & 0,007282 \\
\hline 221359,400000 & 214,636400 & 138,341600 & 0,004659 & 0,007228 \\
\hline 248369,400000 & 202,365600 & 138,835300 & 0,004942 & 0,007203 \\
\hline 278675,000000 & 189,882700 & 138,727300 & 0,005266 & 0,007208 \\
\hline 312678,500000 & 177,301800 & 137,880500 & 0,005640 & 0,007253 \\
\hline 350831,100000 & 164,737000 & 136,427200 & 0,006070 & 0,007330 \\
\hline 393638,900000 & 152,345000 & 134,211000 & 0,006564 & 0,007451 \\
\hline 441670,100000 & 140,249300 & 131,372200 & 0,007130 & 0,007612 \\
\hline 495562,000000 & 128,521000 & 127,882200 & 0,007781 & 0,007820 \\
\hline 556029,800000 & 117,181200 & 123,770100 & 0,008534 & 0,008079 \\
\hline 623875,700000 & 106,495400 & 119,173700 & 0,009390 & 0,008391 \\
\hline 700000,000000 & 96,365100 & 114,173000 & 0,010377 & 0,008759 \\
\hline 785412,900000 & 86,921900 & 108,822600 & 0,011505 & 0,009189 \\
\hline 881247,800000 & 78,163280 & 103,249900 & 0,012794 & 0,009685 \\
\hline 988776,300000 & 70,098200 & 97,489000 & 0,014266 & 0,010258 \\
\hline
\end{tabular}




\begin{tabular}{|c|c|c|c|c|}
\hline $\begin{array}{c}\text { frequency } \\
\text { [Hz] }\end{array}$ & $\begin{array}{c}\text { real impedance } \\
\text { at } 75 \text { bar } \\
Z^{\prime}[\Omega \mathrm{m}]\end{array}$ & $\begin{array}{c}\text { imaginary } \\
\text { impedance } \\
\text { at } 75 \mathrm{bar} \\
\mathrm{Z}^{\prime \prime}[\Omega \mathrm{m}]\end{array}$ & $\begin{array}{c}\text { real admittance } \\
\text { at } 75 \text { bar } \\
Y^{\prime}[S \text { m-1] }\end{array}$ & $\begin{array}{c}\text { imaginary } \\
\text { admittance } \\
\text { at } 75 \text { bar } \\
Y^{\prime \prime}[S \text { m-1] }\end{array}$ \\
\hline 785,412900 & 550,811300 & 94,795620 & 0,001816 & 0,010549 \\
\hline 881,247800 & 544,209500 & 93,463950 & 0,001838 & 0,010699 \\
\hline 988,776300 & 537,574600 & 92,324460 & 0,001860 & 0,010831 \\
\hline 1109,425000 & 531,058700 & 91,396160 & 0,001883 & 0,010941 \\
\hline 1244,796000 & 524,694200 & 90,489320 & 0,001906 & 0,011051 \\
\hline 1396,684000 & 518,346100 & 89,487630 & 0,001929 & 0,011175 \\
\hline 1567,105000 & 512,133700 & 88,599130 & 0,001953 & 0,011287 \\
\hline 1758,320000 & 506,072600 & 87,732430 & 0,001976 & 0,011398 \\
\hline 1972,868000 & 499,997200 & 86,948740 & 0,002000 & 0,011501 \\
\hline 2213,594000 & 494,058300 & 86,271190 & 0,002024 & 0,011591 \\
\hline 2483,694000 & 488,090800 & 85,755710 & 0,002049 & 0,011661 \\
\hline 2786,750000 & 482,441100 & 85,196930 & 0,002073 & 0,011738 \\
\hline 3126,785000 & 476,461900 & 84,741110 & 0,002099 & 0,011801 \\
\hline 3508,311000 & 470,469800 & 84,352890 & 0,002126 & 0,011855 \\
\hline 3936,389000 & 464,887400 & 84,273060 & 0,002151 & 0,011866 \\
\hline 4416,701000 & 459,020700 & 84,036880 & 0,002179 & 0,011900 \\
\hline 4955,620000 & 453,413300 & 84,073430 & 0,002205 & 0,011894 \\
\hline 5560,297000 & 447,657700 & 84,056740 & 0,002234 & 0,011897 \\
\hline 6238,757000 & 442,025500 & 84,197180 & 0,002262 & 0,011877 \\
\hline 7000,000000 & 436,230700 & 84,355820 & 0,002292 & 0,011855 \\
\hline 7854,129000 & 430,394300 & 84,708020 & 0,002323 & 0,011805 \\
\hline 8812,479000 & 424,545500 & 85,096530 & 0,002355 & 0,011751 \\
\hline 9887,763000 & 418,819500 & 85,621990 & 0,002388 & 0,011679 \\
\hline 11094,250000 & 412,915800 & 86,217620 & 0,002422 & 0,011599 \\
\hline 12447,960000 & 406,999500 & 86,836330 & 0,002457 & 0,011516 \\
\hline 13966,840000 & 401,024700 & 87,684960 & 0,002494 & 0,011404 \\
\hline 15671,050000 & 395,036300 & 88,544300 & 0,002531 & 0,011294 \\
\hline 17583,200000 & 389,003400 & 89,546980 & 0,002571 & 0,011167 \\
\hline 19728,680000 & 382,791600 & 90,581560 & 0,002612 & 0,011040 \\
\hline 22135,940000 & 376,550000 & 91,675060 & 0,002656 & 0,010908 \\
\hline 24836,940000 & 370,245700 & 92,950190 & 0,002701 & 0,010758 \\
\hline 27867,500000 & 363,745300 & 94,291960 & 0,002749 & 0,010605 \\
\hline 31267,850000 & 357,344100 & 95,766050 & 0,002798 & 0,010442 \\
\hline 35083,110000 & 350,728400 & 97,345570 & 0,002851 & 0,010273 \\
\hline 39363,890000 & 344,026900 & 99,115170 & 0,002907 & 0,010089 \\
\hline 44167,020000 & 336,978900 & 100,852100 & 0,002968 & 0,009916 \\
\hline 49556,210000 & 329,953000 & 102,903000 & 0,003031 & 0,009718 \\
\hline 55602,980000 & 322,706600 & 104,983700 & 0,003099 & 0,009525 \\
\hline 62387,570000 & 315,183900 & 107,238800 & 0,003173 & 0,009325 \\
\hline 70000,000000 & 307,545300 & 109,633200 & 0,003252 & 0,009121 \\
\hline 78541,290000 & 299,481400 & 112,088200 & 0,003339 & 0,008922 \\
\hline 88124,780000 & 291,115500 & 114,668100 & 0,003435 & 0,008721 \\
\hline 98877,620000 & 282,486900 & 117,232700 & 0,003540 & 0,008530 \\
\hline 110942,500000 & 273,395000 & 119,829900 & 0,003658 & 0,008345 \\
\hline 124479,600000 & 264,025000 & 122,373700 & 0,003788 & 0,008172 \\
\hline 139668,400000 & 254,085000 & 124,732100 & 0,003936 & 0,008017 \\
\hline
\end{tabular}




\begin{tabular}{|c|c|c|c|c|}
\hline $\begin{array}{c}\text { frequency } \\
{[\mathrm{Hz}]}\end{array}$ & $\begin{array}{c}\text { real impedance } \\
\text { at } 75 \text { bar } \\
Z^{\prime}[\Omega \mathrm{m}]\end{array}$ & $\begin{array}{c}\text { imaginary } \\
\text { impedance } \\
\text { at } 75 \mathrm{bar} \\
\mathrm{Z}^{\prime \prime}[\Omega \mathrm{m}]\end{array}$ & $\begin{array}{c}\text { real admittance } \\
\text { at } 75 \text { bar } \\
Y^{\prime}[S \text { m-1] }\end{array}$ & $\begin{array}{c}\text { imaginary } \\
\text { admittance } \\
\text { at } 75 \text { bar } \\
\mathrm{Y}^{\prime \prime}[\mathrm{S} \text { m-1] }\end{array}$ \\
\hline 156710,500000 & 243,884400 & 126,936800 & 0,004100 & 0,007878 \\
\hline 175832,000000 & 233,254600 & 128,951200 & 0,004287 & 0,007755 \\
\hline 197286,800000 & 222,172500 & 130,475900 & 0,004501 & 0,007664 \\
\hline 221359,400000 & 210,753500 & 131,610400 & 0,004745 & 0,007598 \\
\hline 248369,400000 & 199,200200 & 132,313000 & 0,005020 & 0,007558 \\
\hline 278675,000000 & 187,391200 & 132,494400 & 0,005336 & 0,007547 \\
\hline 312678,500000 & 175,433700 & 132,012900 & 0,005700 & 0,007575 \\
\hline 350831,100000 & 163,465800 & 130,962500 & 0,006117 & 0,007636 \\
\hline 393638,900000 & 151,636500 & 129,187400 & 0,006595 & 0,007741 \\
\hline 441670,100000 & 139,934800 & 126,745600 & 0,007146 & 0,007890 \\
\hline 495562,000000 & 128,528200 & 123,718800 & 0,007780 & 0,008083 \\
\hline 556029,800000 & 117,535600 & 120,052100 & 0,008508 & 0,008330 \\
\hline 623875,700000 & 107,044700 & 115,946500 & 0,009342 & 0,008625 \\
\hline 700000,000000 & 97,079630 & 111,383300 & 0,010301 & 0,008978 \\
\hline 785412,900000 & 87,726700 & 106,475400 & 0,011399 & 0,009392 \\
\hline 881247,800000 & 79,027740 & 101,273300 & 0,012654 & 0,009874 \\
\hline 988776,300000 & 70,960200 & 95,837270 & 0,014092 & 0,010434 \\
\hline
\end{tabular}




\begin{tabular}{|c|c|c|c|c|}
\hline $\begin{array}{c}\text { frequency } \\
{\left[\begin{array}{l}\mathrm{Hz}]\end{array}\right.}\end{array}$ & $\begin{array}{c}\text { real impedance } \\
\text { at } 100 \text { bar } \\
Z^{\prime}[\Omega \mathrm{m}]\end{array}$ & $\begin{array}{c}\text { imaginary } \\
\text { impedance } \\
\text { at } 100 \mathrm{bar} \\
\mathrm{Z}^{\prime \prime}[\Omega \mathrm{m}]\end{array}$ & $\begin{array}{c}\text { real admittance } \\
\text { at } 100 \text { bar } \\
Y^{\prime}[S \text { m-1] }\end{array}$ & $\begin{array}{c}\text { imaginary } \\
\text { admittance } \\
\text { at } 100 \text { bar } \\
Y^{\prime \prime}[S \mathrm{~m}-1]\end{array}$ \\
\hline 785,412900 & 536,058900 & 98,237580 & 0,001865 & 0,010179 \\
\hline 881,247800 & 529,002600 & 96,849060 & 0,001890 & 0,010325 \\
\hline 988,776300 & 522,230100 & 95,609170 & 0,001915 & 0,010459 \\
\hline 1109,425000 & 515,608200 & 94,396840 & 0,001939 & 0,010594 \\
\hline 1244,796000 & 508,803300 & 93,334470 & 0,001965 & 0,010714 \\
\hline 1396,684000 & 502,149500 & 92,295030 & 0,001991 & 0,010835 \\
\hline 1567,105000 & 495,813500 & 91,219890 & 0,002017 & 0,010963 \\
\hline 1758,320000 & 489,295900 & 90,285530 & 0,002044 & 0,011076 \\
\hline 1972,868000 & 482,945500 & 89,287980 & 0,002071 & 0,011200 \\
\hline 2213,594000 & 476,730800 & 88,397040 & 0,002098 & 0,011313 \\
\hline 2483,694000 & 470,637100 & 87,691810 & 0,002125 & 0,011404 \\
\hline 2786,750000 & 464,725600 & 86,842030 & 0,002152 & 0,011515 \\
\hline 3126,785000 & 458,619800 & 86,197920 & 0,002180 & 0,011601 \\
\hline 3508,311000 & 452,801600 & 85,676970 & 0,002208 & 0,011672 \\
\hline 3936,389000 & 446,790400 & 85,347130 & 0,002238 & 0,011717 \\
\hline 4416,701000 & 440,781800 & 84,996600 & 0,002269 & 0,011765 \\
\hline 4955,620000 & 434,926000 & 84,654590 & 0,002299 & 0,011813 \\
\hline 5560,297000 & 429,057900 & 84,367260 & 0,002331 & 0,011853 \\
\hline 6238,757000 & 423,298700 & 84,309070 & 0,002362 & 0,011861 \\
\hline 7000,000000 & 417,527600 & 84,296100 & 0,002395 & 0,011863 \\
\hline 7854,129000 & 411,744800 & 84,325330 & 0,002429 & 0,011859 \\
\hline 8812,479000 & 405,950400 & 84,393780 & 0,002463 & 0,011849 \\
\hline 9887,763000 & 400,115100 & 84,638000 & 0,002499 & 0,011815 \\
\hline 11094,250000 & 394,268200 & 84,911340 & 0,002536 & 0,011777 \\
\hline 12447,960000 & 388,395100 & 85,278500 & 0,002575 & 0,011726 \\
\hline 13966,840000 & 382,495500 & 85,733370 & 0,002614 & 0,011664 \\
\hline 15671,050000 & 376,554400 & 86,335500 & 0,002656 & 0,011583 \\
\hline 17583,200000 & 370,601400 & 86,946320 & 0,002698 & 0,011501 \\
\hline 19728,680000 & 364,606200 & 87,689970 & 0,002743 & 0,011404 \\
\hline 22135,940000 & 358,583600 & 88,494570 & 0,002789 & 0,011300 \\
\hline 24836,940000 & 352,369500 & 89,377720 & 0,002838 & 0,011188 \\
\hline 27867,500000 & 346,259800 & 90,404210 & 0,002888 & 0,011061 \\
\hline 31267,850000 & 340,105400 & 91,527840 & 0,002940 & 0,010926 \\
\hline 35083,110000 & 333,741900 & 92,756360 & 0,002996 & 0,010781 \\
\hline 39363,890000 & 327,463000 & 94,157470 & 0,003054 & 0,010621 \\
\hline 44167,020000 & 320,809300 & 95,647030 & 0,003117 & 0,010455 \\
\hline 49556,210000 & 314,090500 & 97,234910 & 0,003184 & 0,010284 \\
\hline 55602,980000 & 307,433700 & 99,007800 & 0,003253 & 0,010100 \\
\hline 62387,570000 & 300,364600 & 100,912200 & 0,003329 & 0,009910 \\
\hline 70000,000000 & 293,189500 & 102,961900 & 0,003411 & 0,009712 \\
\hline 78541,290000 & 285,743400 & 105,131700 & 0,003500 & 0,009512 \\
\hline 88124,780000 & 278,025100 & 107,388500 & 0,003597 & 0,009312 \\
\hline 98877,620000 & 270,053000 & 109,650500 & 0,003703 & 0,009120 \\
\hline 110942,500000 & 261,629600 & 111,963000 & 0,003822 & 0,008932 \\
\hline 124479,600000 & 252,957300 & 114,202300 & 0,003953 & 0,008756 \\
\hline 139668,400000 & 243,842100 & 116,395800 & 0,004101 & 0,008591 \\
\hline
\end{tabular}




\begin{tabular}{|c|c|c|c|c|}
\hline $\begin{array}{c}\text { frequency } \\
{[\mathbf{H z}]}\end{array}$ & $\begin{array}{c}\text { real impedance } \\
\text { at } \mathbf{1 0 0} \text { bar } \\
\mathbf{Z}^{\prime}[\mathbf{\Omega} \mathbf{~ m}]\end{array}$ & $\begin{array}{c}\text { imaginary } \\
\text { impedance } \\
\text { at } \mathbf{1 0 0} \text { bar } \\
\mathbf{Z}^{\prime \prime}[\mathbf{\Omega} \mathbf{~ m}]\end{array}$ & $\begin{array}{c}\text { real admittance } \\
\text { at 100 bar } \\
\mathbf{Y}^{\prime} \text { [S m-1] }\end{array}$ & $\begin{array}{c}\text { imaginary } \\
\text { admittance } \\
\text { at 100 bar } \\
\mathbf{Y}^{\prime \prime} \text { [S m-1] }\end{array}$ \\
\hline 156710,500000 & 234,333400 & 118,403200 & 0,004267 & 0,008446 \\
\hline 175832,000000 & 224,558700 & 120,284800 & 0,004453 & 0,008314 \\
\hline 197286,800000 & 214,438200 & 121,842500 & 0,004663 & 0,008207 \\
\hline 221359,400000 & 203,862200 & 122,949500 & 0,004905 & 0,008133 \\
\hline 248369,400000 & 193,141800 & 123,678200 & 0,005178 & 0,008085 \\
\hline 278675,000000 & 182,256700 & 124,059900 & 0,005487 & 0,008061 \\
\hline 312678,500000 & 171,217100 & 123,812100 & 0,005841 & 0,008077 \\
\hline 350831,100000 & 159,973500 & 123,022600 & 0,006251 & 0,008129 \\
\hline 393638,900000 & 148,953000 & 121,656800 & 0,006714 & 0,008220 \\
\hline 441670,100000 & 137,998900 & 119,703900 & 0,007246 & 0,008354 \\
\hline 495562,000000 & 127,298000 & 117,213900 & 0,007856 & 0,008531 \\
\hline 556029,800000 & 116,890300 & 114,138200 & 0,008555 & 0,008761 \\
\hline 623875,700000 & 106,850500 & 110,595400 & 0,009359 & 0,009042 \\
\hline 700000,000000 & 97,309770 & 106,627000 & 0,010276 & 0,009378 \\
\hline 785412,900000 & 88,267470 & 102,277000 & 0,011329 & 0,009777 \\
\hline 881247,800000 & 79,776200 & 97,621240 & 0,012535 & 0,010244 \\
\hline 988776,300000 & 71,868450 & 92,730800 & 0,013914 & 0,010784 \\
\hline
\end{tabular}




\begin{tabular}{|c|c|c|c|c|}
\hline $\begin{array}{c}\text { frequency } \\
\qquad[\mathrm{Hz}]\end{array}$ & $\begin{array}{c}\text { real impedance } \\
\text { at } 150 \text { bar } \\
Z^{\prime}[\Omega \mathrm{m}]\end{array}$ & $\begin{array}{c}\text { imaginary } \\
\text { impedance } \\
\text { at } 150 \mathrm{bar} \\
\mathrm{Z}^{\prime \prime}[\Omega \mathrm{m}] \\
\end{array}$ & $\begin{array}{c}\text { real admittance } \\
\text { at } 150 \text { bar } \\
Y^{\prime}[S \text { m-1] }\end{array}$ & $\begin{array}{c}\text { imaginary } \\
\text { admittance } \\
\text { at } 150 \text { bar } \\
\mathrm{Y}^{\prime \prime}[\mathrm{S} \mathrm{m}-1]\end{array}$ \\
\hline 785,412900 & 524,461200 & 101,132600 & 0,001907 & 0,009888 \\
\hline 881,247800 & 517,435100 & 99,590530 & 0,001933 & 0,010041 \\
\hline 988,776300 & 510,240600 & 98,113510 & 0,001960 & 0,010192 \\
\hline 1109,425000 & 503,329200 & 96,784520 & 0,001987 & 0,010332 \\
\hline 1244,796000 & 496,384500 & 95,628730 & 0,002015 & 0,010457 \\
\hline 1396,684000 & 489,757500 & 94,440670 & 0,002042 & 0,010589 \\
\hline 1567,105000 & 482,997100 & 93,137030 & 0,002070 & 0,010737 \\
\hline 1758,320000 & 476,521100 & 91,974480 & 0,002099 & 0,010873 \\
\hline 1972,868000 & 470,029600 & 90,891650 & 0,002128 & 0,011002 \\
\hline 2213,594000 & 463,657800 & 89,995180 & 0,002157 & 0,011112 \\
\hline 2483,694000 & 457,468700 & 88,959520 & 0,002186 & 0,011241 \\
\hline 2786,750000 & 451,280400 & 87,919550 & 0,002216 & 0,011374 \\
\hline 3126,785000 & 445,347700 & 87,166980 & 0,002245 & 0,011472 \\
\hline 3508,311000 & 439,266500 & 86,374600 & 0,002277 & 0,011577 \\
\hline 3936,389000 & 433,127200 & 85,873930 & 0,002309 & 0,011645 \\
\hline 4416,701000 & 427,170300 & 85,235150 & 0,002341 & 0,011732 \\
\hline 4955,620000 & 421,486100 & 84,789260 & 0,002373 & 0,011794 \\
\hline 5560,297000 & 415,504600 & 84,264920 & 0,002407 & 0,011867 \\
\hline 6238,757000 & 409,781600 & 83,997760 & 0,002440 & 0,011905 \\
\hline 7000,000000 & 403,911700 & 83,675970 & 0,002476 & 0,011951 \\
\hline 7854,129000 & 398,315400 & 83,531660 & 0,002511 & 0,011972 \\
\hline 8812,479000 & 392,558100 & 83,397230 & 0,002547 & 0,011991 \\
\hline 9887,763000 & 386,775100 & 83,368190 & 0,002585 & 0,011995 \\
\hline 11094,250000 & 380,981300 & 83,372080 & 0,002625 & 0,011994 \\
\hline 12447,960000 & 375,297000 & 83,570000 & 0,002665 & 0,011966 \\
\hline 13966,840000 & 369,601800 & 83,791870 & 0,002706 & 0,011934 \\
\hline 15671,050000 & 363,881300 & 84,098240 & 0,002748 & 0,011891 \\
\hline 17583,200000 & 357,986300 & 84,448040 & 0,002793 & 0,011842 \\
\hline 19728,680000 & 352,348800 & 85,002120 & 0,002838 & 0,011764 \\
\hline 22135,940000 & 346,403100 & 85,488530 & 0,002887 & 0,011697 \\
\hline 24836,940000 & 340,698600 & 86,227700 & 0,002935 & 0,011597 \\
\hline 27867,500000 & 334,671300 & 86,942180 & 0,002988 & 0,011502 \\
\hline 31267,850000 & 328,883000 & 87,892720 & 0,003041 & 0,011378 \\
\hline 35083,110000 & 322,772000 & 88,798360 & 0,003098 & 0,011261 \\
\hline 39363,890000 & 316,718900 & 89,992430 & 0,003157 & 0,011112 \\
\hline 44167,020000 & 310,621100 & 91,254040 & 0,003219 & 0,010958 \\
\hline 49556,210000 & 304,299200 & 92,635440 & 0,003286 & 0,010795 \\
\hline 55602,980000 & 297,882100 & 94,214000 & 0,003357 & 0,010614 \\
\hline 62387,570000 & 291,383500 & 95,919000 & 0,003432 & 0,010425 \\
\hline 70000,000000 & 284,605400 & 97,832920 & 0,003514 & 0,010222 \\
\hline 78541,290000 & 277,614500 & 99,728380 & 0,003602 & 0,010027 \\
\hline 88124,780000 & 270,449100 & 101,922000 & 0,003698 & 0,009811 \\
\hline 98877,620000 & 262,926700 & 103,988900 & 0,003803 & 0,009616 \\
\hline 110942,500000 & 255,102300 & 106,181200 & 0,003920 & 0,009418 \\
\hline 124479,600000 & 246,993700 & 108,412300 & 0,004049 & 0,009224 \\
\hline 139668,400000 & 238,483100 & 110,535200 & 0,004193 & 0,009047 \\
\hline
\end{tabular}




\begin{tabular}{|c|c|c|c|c|}
\hline $\begin{array}{c}\text { frequency } \\
{[\mathbf{H z}]}\end{array}$ & $\begin{array}{c}\text { real impedance } \\
\text { at } \mathbf{1 5 0} \text { bar } \\
\mathbf{Z}^{\prime}[\mathbf{\Omega} \mathbf{~ m}]\end{array}$ & $\begin{array}{c}\text { imaginary } \\
\text { impedance } \\
\text { at } \mathbf{1 5 0} \text { bar } \\
\mathbf{Z}^{\prime \prime}[\mathbf{\Omega} \text { }]\end{array}$ & $\begin{array}{c}\text { real admittance } \\
\text { at 150 bar } \\
\mathbf{Y}^{\prime} \text { [S m-1] }\end{array}$ & $\begin{array}{c}\text { imaginary } \\
\text { admittance } \\
\text { at 150 bar } \\
\mathbf{Y}^{\prime \prime} \text { [S m-1] }\end{array}$ \\
\hline 156710,500000 & 229,559500 & 112,543300 & 0,004356 & 0,008885 \\
\hline 175832,000000 & 220,349500 & 114,492100 & 0,004538 & 0,008734 \\
\hline 197286,800000 & 210,808000 & 116,110100 & 0,004744 & 0,008613 \\
\hline 221359,400000 & 200,893400 & 117,461400 & 0,004978 & 0,008513 \\
\hline 248369,400000 & 190,668700 & 118,421700 & 0,005245 & 0,008444 \\
\hline 278675,000000 & 180,288600 & 119,027600 & 0,005547 & 0,008401 \\
\hline 312678,500000 & 169,693300 & 119,095300 & 0,005893 & 0,008397 \\
\hline 350831,100000 & 159,019500 & 118,708200 & 0,006289 & 0,008424 \\
\hline 393638,900000 & 148,280500 & 117,612100 & 0,006744 & 0,008503 \\
\hline 441670,100000 & 137,687800 & 116,065700 & 0,007263 & 0,008616 \\
\hline 495562,000000 & 127,325500 & 113,997300 & 0,007854 & 0,008772 \\
\hline 556029,800000 & 117,057100 & 111,230400 & 0,008543 & 0,008990 \\
\hline 623875,700000 & 107,197400 & 108,050400 & 0,009329 & 0,009255 \\
\hline 700000,000000 & 97,770840 & 104,429800 & 0,010228 & 0,009576 \\
\hline 785412,900000 & 88,817700 & 100,373900 & 0,011259 & 0,009963 \\
\hline 881247,800000 & 80,371640 & 96,036860 & 0,012442 & 0,010413 \\
\hline 988776,300000 & 72,474500 & 91,419830 & 0,013798 & 0,010939 \\
\hline
\end{tabular}




\begin{tabular}{|c|c|c|c|c|}
\hline $\begin{array}{c}\text { frequency } \\
{[\mathrm{Hz}]}\end{array}$ & $\begin{array}{c}\text { real impedance } \\
\text { at } 200 \text { bar } \\
Z^{\prime}[\Omega \mathrm{m}]\end{array}$ & $\begin{array}{c}\text { imaginary } \\
\text { impedance } \\
\text { at } 200 \mathrm{bar} \\
\mathrm{Z}^{\prime \prime}[\Omega \mathrm{m}]\end{array}$ & $\begin{array}{c}\text { real admittance } \\
\text { at } 200 \text { bar } \\
Y^{\prime}[S \text { m-1] }\end{array}$ & $\begin{array}{c}\text { imaginary } \\
\text { admittance } \\
\text { at } 200 \text { bar } \\
\mathrm{Y}^{\prime \prime}[\mathrm{S} \mathrm{m}-1]\end{array}$ \\
\hline 785,412900 & 524,054700 & 101,623300 & 0,001908 & 0,009840 \\
\hline 881,247800 & 516,879900 & 100,044800 & 0,001935 & 0,009996 \\
\hline 988,776300 & 509,837100 & 98,589400 & 0,001961 & 0,010143 \\
\hline 1109,425000 & 502,927200 & 97,253200 & 0,001988 & 0,010282 \\
\hline 1244,796000 & 495,983700 & 96,090030 & 0,002016 & 0,010407 \\
\hline 1396,684000 & 489,074200 & 94,751400 & 0,002045 & 0,010554 \\
\hline 1567,105000 & 482,448700 & 93,555140 & 0,002073 & 0,010689 \\
\hline 1758,320000 & 475,973900 & 92,385720 & 0,002101 & 0,010824 \\
\hline 1972,868000 & 469,499600 & 91,214050 & 0,002130 & 0,010963 \\
\hline 2213,594000 & 462,994200 & 90,201650 & 0,002160 & 0,011086 \\
\hline 2483,694000 & 456,790300 & 89,241160 & 0,002189 & 0,011206 \\
\hline 2786,750000 & 450,753100 & 88,224970 & 0,002219 & 0,011335 \\
\hline 3126,785000 & 444,536100 & 87,330240 & 0,002250 & 0,011451 \\
\hline 3508,311000 & 438,605700 & 86,562590 & 0,002280 & 0,011552 \\
\hline 3936,389000 & 432,481900 & 85,981250 & 0,002312 & 0,011630 \\
\hline 4416,701000 & 426,525600 & 85,338620 & 0,002345 & 0,011718 \\
\hline 4955,620000 & 420,691800 & 84,858570 & 0,002377 & 0,011784 \\
\hline 5560,297000 & 414,846000 & 84,432780 & 0,002411 & 0,011844 \\
\hline 6238,757000 & 409,002900 & 83,986840 & 0,002445 & 0,011907 \\
\hline 7000,000000 & 403,268700 & 83,762880 & 0,002480 & 0,011938 \\
\hline 7854,129000 & 397,537600 & 83,513340 & 0,002515 & 0,011974 \\
\hline 8812,479000 & 391,780700 & 83,374950 & 0,002552 & 0,011994 \\
\hline 9887,763000 & 385,998300 & 83,341680 & 0,002591 & 0,011999 \\
\hline 11094,250000 & 380,190400 & 83,407520 & 0,002630 & 0,011989 \\
\hline 12447,960000 & 374,521200 & 83,534390 & 0,002670 & 0,011971 \\
\hline 13966,840000 & 368,677400 & 83,717540 & 0,002712 & 0,011945 \\
\hline 15671,050000 & 362,957500 & 84,018170 & 0,002755 & 0,011902 \\
\hline 17583,200000 & 357,212300 & 84,397030 & 0,002799 & 0,011849 \\
\hline 19728,680000 & 351,441700 & 84,848150 & 0,002845 & 0,011786 \\
\hline 22135,940000 & 345,630600 & 85,425830 & 0,002893 & 0,011706 \\
\hline 24836,940000 & 339,793700 & 86,061740 & 0,002943 & 0,011620 \\
\hline 27867,500000 & 333,900600 & 86,866340 & 0,002995 & 0,011512 \\
\hline 31267,850000 & 327,980900 & 87,712920 & 0,003049 & 0,011401 \\
\hline 35083,110000 & 322,019000 & 88,651600 & 0,003105 & 0,011280 \\
\hline 39363,890000 & 315,967400 & 89,838460 & 0,003165 & 0,011131 \\
\hline 44167,020000 & 309,871200 & 91,092470 & 0,003227 & 0,010978 \\
\hline 49556,210000 & 303,551200 & 92,465600 & 0,003294 & 0,010815 \\
\hline 55602,980000 & 297,152700 & 93,983310 & 0,003365 & 0,010640 \\
\hline 62387,570000 & 290,640100 & 95,730500 & 0,003441 & 0,010446 \\
\hline 70000,000000 & 283,899000 & 97,534730 & 0,003522 & 0,010253 \\
\hline 78541,290000 & 276,894500 & 99,469740 & 0,003611 & 0,010053 \\
\hline 88124,780000 & 269,751000 & 101,605200 & 0,003707 & 0,009842 \\
\hline 98877,620000 & 262,233500 & 103,661800 & 0,003813 & 0,009647 \\
\hline 110942,500000 & 254,414500 & 105,842900 & 0,003931 & 0,009448 \\
\hline 124479,600000 & 246,330900 & 108,018900 & 0,004060 & 0,009258 \\
\hline 139668,400000 & 237,808200 & 110,172000 & 0,004205 & 0,009077 \\
\hline
\end{tabular}




\begin{tabular}{|c|c|c|c|c|}
\hline $\begin{array}{c}\text { frequency } \\
{[\mathbf{H z}]}\end{array}$ & $\begin{array}{c}\text { real impedance } \\
\text { at 200 bar } \\
\mathbf{Z}^{\prime}[\mathbf{\Omega} \mathbf{~ m}]\end{array}$ & $\begin{array}{c}\text { imaginary } \\
\text { impedance } \\
\text { at 200 bar } \\
\mathbf{Z}^{\prime \prime}[\mathbf{\Omega} \mathbf{~ m}]\end{array}$ & $\begin{array}{c}\text { real admittance } \\
\text { at 200 bar } \\
\mathbf{Y}^{\prime} \text { [S m-1] }\end{array}$ & $\begin{array}{c}\text { imaginary } \\
\text { admittance } \\
\text { at 200 bar } \\
\mathbf{Y}^{\prime \prime} \text { [S m-1] }\end{array}$ \\
\hline 156710,500000 & 229,049200 & 112,194000 & 0,004366 & 0,008913 \\
\hline 175832,000000 & 219,846300 & 114,133200 & 0,004549 & 0,008762 \\
\hline 197286,800000 & 210,332500 & 115,704800 & 0,004754 & 0,008643 \\
\hline 221359,400000 & 200,426400 & 117,047600 & 0,004989 & 0,008544 \\
\hline 248369,400000 & 190,361200 & 118,046700 & 0,005253 & 0,008471 \\
\hline 278675,000000 & 180,009000 & 118,617800 & 0,005555 & 0,008430 \\
\hline 312678,500000 & 169,421200 & 118,683900 & 0,005902 & 0,008426 \\
\hline 350831,100000 & 158,754900 & 118,295200 & 0,006299 & 0,008453 \\
\hline 393638,900000 & 148,163600 & 117,267000 & 0,006749 & 0,008528 \\
\hline 441670,100000 & 137,575000 & 115,724500 & 0,007269 & 0,008641 \\
\hline 495562,000000 & 127,102400 & 113,558100 & 0,007868 & 0,008806 \\
\hline 556029,800000 & 116,951400 & 110,897200 & 0,008551 & 0,009017 \\
\hline 623875,700000 & 107,156600 & 107,745900 & 0,009332 & 0,009281 \\
\hline 700000,000000 & 97,751720 & 104,154200 & 0,010230 & 0,009601 \\
\hline 785412,900000 & 88,811040 & 100,155000 & 0,011260 & 0,009985 \\
\hline 881247,800000 & 80,380680 & 95,809710 & 0,012441 & 0,010437 \\
\hline 988776,300000 & 72,484490 & 91,236110 & 0,013796 & 0,010961 \\
\hline
\end{tabular}




\begin{tabular}{|c|c|c|c|c|}
\hline $\begin{array}{c}\text { frequency } \\
{\left[\begin{array}{l}\mathrm{Hz}]\end{array}\right.}\end{array}$ & $\begin{array}{c}\text { real impedance } \\
\text { at } 200 \text { bar } \\
Z^{\prime}[\Omega \mathrm{m}]\end{array}$ & $\begin{array}{l}\text { imaginary } \\
\text { impedance } \\
\text { at } 200 \mathrm{bar} \\
\mathrm{Z}^{\prime \prime}[\Omega \mathrm{m}]\end{array}$ & $\begin{array}{c}\text { real admittance } \\
\text { at } 200 \text { bar } \\
Y^{\prime}[S \text { m-1] }\end{array}$ & $\begin{array}{c}\text { imaginary } \\
\text { admittance } \\
\text { at } 200 \text { bar } \\
\mathrm{Y}^{\prime \prime}[\mathrm{S} \mathrm{m}-1]\end{array}$ \\
\hline 785,412900 & 524,054700 & 101,623300 & 0,001908 & 0,009840 \\
\hline 881,247800 & 516,879900 & 100,044800 & 0,001935 & 0,009996 \\
\hline 988,776300 & 509,837100 & 98,589400 & 0,001961 & 0,010143 \\
\hline 1109,425000 & 502,927200 & 97,253200 & 0,001988 & 0,010282 \\
\hline 1244,796000 & 495,983700 & 96,090030 & 0,002016 & 0,010407 \\
\hline 1396,684000 & 489,074200 & 94,751400 & 0,002045 & 0,010554 \\
\hline 1567,105000 & 482,448700 & 93,555140 & 0,002073 & 0,010689 \\
\hline 1758,320000 & 475,973900 & 92,385720 & 0,002101 & 0,010824 \\
\hline 1972,868000 & 469,499600 & 91,214050 & 0,002130 & 0,010963 \\
\hline 2213,594000 & 462,994200 & 90,201650 & 0,002160 & 0,011086 \\
\hline 2483,694000 & 456,790300 & 89,241160 & 0,002189 & 0,011206 \\
\hline 2786,750000 & 450,753100 & 88,224970 & 0,002219 & 0,011335 \\
\hline 3126,785000 & 444,536100 & 87,330240 & 0,002250 & 0,011451 \\
\hline 3508,311000 & 438,605700 & 86,562590 & 0,002280 & 0,011552 \\
\hline 3936,389000 & 432,481900 & 85,981250 & 0,002312 & 0,011630 \\
\hline 4416,701000 & 426,525600 & 85,338620 & 0,002345 & 0,011718 \\
\hline 4955,620000 & 420,691800 & 84,858570 & 0,002377 & 0,011784 \\
\hline 5560,297000 & 414,846000 & 84,432780 & 0,002411 & 0,011844 \\
\hline 6238,757000 & 409,002900 & 83,986840 & 0,002445 & 0,011907 \\
\hline 7000,000000 & 403,268700 & 83,762880 & 0,002480 & 0,011938 \\
\hline 7854,129000 & 397,537600 & 83,513340 & 0,002515 & 0,011974 \\
\hline 8812,479000 & 391,780700 & 83,374950 & 0,002552 & 0,011994 \\
\hline 9887,763000 & 385,998300 & 83,341680 & 0,002591 & 0,011999 \\
\hline 11094,250000 & 380,190400 & 83,407520 & 0,002630 & 0,011989 \\
\hline 12447,960000 & 374,521200 & 83,534390 & 0,002670 & 0,011971 \\
\hline 13966,840000 & 368,677400 & 83,717540 & 0,002712 & 0,011945 \\
\hline 15671,050000 & 362,957500 & 84,018170 & 0,002755 & 0,011902 \\
\hline 17583,200000 & 357,212300 & 84,397030 & 0,002799 & 0,011849 \\
\hline 19728,680000 & 351,441700 & 84,848150 & 0,002845 & 0,011786 \\
\hline 22135,940000 & 345,630600 & 85,425830 & 0,002893 & 0,011706 \\
\hline 24836,940000 & 339,793700 & 86,061740 & 0,002943 & 0,011620 \\
\hline 27867,500000 & 333,900600 & 86,866340 & 0,002995 & 0,011512 \\
\hline 31267,850000 & 327,980900 & 87,712920 & 0,003049 & 0,011401 \\
\hline 35083,110000 & 322,019000 & 88,651600 & 0,003105 & 0,011280 \\
\hline 39363,890000 & 315,967400 & 89,838460 & 0,003165 & 0,011131 \\
\hline 44167,020000 & 309,871200 & 91,092470 & 0,003227 & 0,010978 \\
\hline 49556,210000 & 303,551200 & 92,465600 & 0,003294 & 0,010815 \\
\hline 55602,980000 & 297,152700 & 93,983310 & 0,003365 & 0,010640 \\
\hline 62387,570000 & 290,640100 & 95,730500 & 0,003441 & 0,010446 \\
\hline 70000,000000 & 283,899000 & 97,534730 & 0,003522 & 0,010253 \\
\hline 78541,290000 & 276,894500 & 99,469740 & 0,003611 & 0,010053 \\
\hline 88124,780000 & 269,751000 & 101,605200 & 0,003707 & 0,009842 \\
\hline 98877,620000 & 262,233500 & 103,661800 & 0,003813 & 0,009647 \\
\hline 110942,500000 & 254,414500 & 105,842900 & 0,003931 & 0,009448 \\
\hline 124479,600000 & 246,330900 & 108,018900 & 0,004060 & 0,009258 \\
\hline 139668,400000 & 237,808200 & 110,172000 & 0,004205 & 0,009077 \\
\hline 156710,500000 & 229,049200 & 112,194000 & 0,004366 & 0,008913 \\
\hline
\end{tabular}




\begin{tabular}{|c|c|c|c|c|}
\hline $\begin{array}{c}\text { frequency } \\
{[\mathbf{H z}]}\end{array}$ & $\begin{array}{c}\text { real impedance } \\
\text { at } \mathbf{2 0 0} \text { bar } \\
\mathbf{Z}^{\prime}[\mathbf{\Omega} \mathbf{~ m}]\end{array}$ & $\begin{array}{c}\text { imaginary } \\
\text { impedance } \\
\text { at } \mathbf{2 0 0} \text { bar } \\
\mathbf{Z}^{\prime \prime}[\mathbf{\Omega} \mathbf{~ m}]\end{array}$ & $\begin{array}{c}\text { real admittance } \\
\text { at 200 bar } \\
\mathbf{Y}^{\prime}[\mathbf{S} \text { m-1] }\end{array}$ & $\begin{array}{c}\text { imaginary } \\
\text { admittance } \\
\text { at 200 bar } \\
\mathbf{Y}^{\prime \prime} \text { [S m-1] }\end{array}$ \\
\hline 175832,000000 & 219,846300 & 114,133200 & 0,004549 & 0,008762 \\
\hline 197286,800000 & 210,332500 & 115,704800 & 0,004754 & 0,008643 \\
\hline 221359,400000 & 200,426400 & 117,047600 & 0,004989 & 0,008544 \\
\hline 248369,400000 & 190,361200 & 118,046700 & 0,005253 & 0,008471 \\
\hline 278675,000000 & 180,009000 & 118,617800 & 0,005555 & 0,008430 \\
\hline 312678,500000 & 169,421200 & 118,683900 & 0,005902 & 0,008426 \\
\hline 350831,100000 & 158,754900 & 118,295200 & 0,006299 & 0,008453 \\
\hline 393638,900000 & 148,163600 & 117,267000 & 0,006749 & 0,008528 \\
\hline 441670,100000 & 137,575000 & 115,724500 & 0,007269 & 0,008641 \\
\hline 495562,000000 & 127,102400 & 113,558100 & 0,007868 & 0,008806 \\
\hline 556029,800000 & 116,951400 & 110,897200 & 0,008551 & 0,009017 \\
\hline 623875,700000 & 107,156600 & 107,745900 & 0,009332 & 0,009281 \\
\hline 700000,000000 & 97,751720 & 104,154200 & 0,010230 & 0,009601 \\
\hline 785412,900000 & 88,811040 & 100,155000 & 0,011260 & 0,009985 \\
\hline 881247,800000 & 80,380680 & 95,809710 & 0,012441 & 0,010437 \\
\hline 988776,300000 & 72,484490 & 91,236110 & 0,013796 & 0,010961 \\
\hline
\end{tabular}




\begin{tabular}{|c|c|c|c|c|}
\hline $\begin{array}{c}\text { frequency } \\
{\left[\begin{array}{l}\mathrm{Hz}]\end{array}\right.}\end{array}$ & $\begin{array}{c}\text { real impedance } \\
\text { at } 250 \text { bar } \\
Z^{\prime}[\Omega \mathrm{m}]\end{array}$ & $\begin{array}{c}\text { imaginary } \\
\text { impedance } \\
\text { at } 250 \mathrm{bar} \\
\mathrm{Z}^{\prime \prime}[\Omega \mathrm{m}]\end{array}$ & $\begin{array}{c}\text { real admittance } \\
\text { at } 250 \text { bar } \\
Y^{\prime}[S \text { m-1] }\end{array}$ & $\begin{array}{c}\text { imaginary } \\
\text { admittance } \\
\text { at } 250 \text { bar } \\
\mathrm{Y}^{\prime \prime}[\mathrm{S} \mathrm{m}-1]\end{array}$ \\
\hline 785,412900 & 522,764500 & 101,846200 & 0,001913 & 0,009819 \\
\hline 881,247800 & 515,440800 & 100,232800 & 0,001940 & 0,009977 \\
\hline 988,776300 & 508,399300 & 98,771450 & 0,001967 & 0,010124 \\
\hline 1109,425000 & 501,323200 & 97,487480 & 0,001995 & 0,010258 \\
\hline 1244,796000 & 494,414600 & 96,144040 & 0,002023 & 0,010401 \\
\hline 1396,684000 & 487,623100 & 94,999920 & 0,002051 & 0,010526 \\
\hline 1567,105000 & 481,015400 & 93,712590 & 0,002079 & 0,010671 \\
\hline 1758,320000 & 474,391400 & 92,508000 & 0,002108 & 0,010810 \\
\hline 1972,868000 & 467,918200 & 91,330410 & 0,002137 & 0,010949 \\
\hline 2213,594000 & 461,413800 & 90,311570 & 0,002167 & 0,011073 \\
\hline 2483,694000 & 455,226500 & 89,265460 & 0,002197 & 0,011203 \\
\hline 2786,750000 & 449,040000 & 88,215040 & 0,002227 & 0,011336 \\
\hline 3126,785000 & 442,808600 & 87,392070 & 0,002258 & 0,011443 \\
\hline 3508,311000 & 436,894200 & 86,541600 & 0,002289 & 0,011555 \\
\hline 3936,389000 & 430,906400 & 86,058790 & 0,002321 & 0,011620 \\
\hline 4416,701000 & 424,965900 & 85,335010 & 0,002353 & 0,011719 \\
\hline 4955,620000 & 418,983000 & 84,818160 & 0,002387 & 0,011790 \\
\hline 5560,297000 & 413,152700 & 84,313350 & 0,002420 & 0,011861 \\
\hline 6238,757000 & 407,460300 & 83,892330 & 0,002454 & 0,011920 \\
\hline 7000,000000 & 401,577100 & 83,630780 & 0,002490 & 0,011957 \\
\hline 7854,129000 & 395,847000 & 83,374510 & 0,002526 & 0,011994 \\
\hline 8812,479000 & 390,091100 & 83,228800 & 0,002564 & 0,012015 \\
\hline 9887,763000 & 384,473700 & 83,152920 & 0,002601 & 0,012026 \\
\hline 11094,250000 & 378,666900 & 83,211760 & 0,002641 & 0,012018 \\
\hline 12447,960000 & 373,013400 & 83,266390 & 0,002681 & 0,012010 \\
\hline 13966,840000 & 367,185300 & 83,378740 & 0,002723 & 0,011993 \\
\hline 15671,050000 & 361,616000 & 83,707630 & 0,002765 & 0,011946 \\
\hline 17583,200000 & 355,723300 & 84,045230 & 0,002811 & 0,011898 \\
\hline 19728,680000 & 349,954400 & 84,489080 & 0,002858 & 0,011836 \\
\hline 22135,940000 & 344,308600 & 85,035380 & 0,002904 & 0,011760 \\
\hline 24836,940000 & 338,473800 & 85,664610 & 0,002954 & 0,011673 \\
\hline 27867,500000 & 332,598100 & 86,403600 & 0,003007 & 0,011574 \\
\hline 31267,850000 & 326,696300 & 87,186210 & 0,003061 & 0,011470 \\
\hline 35083,110000 & 320,869700 & 88,214780 & 0,003117 & 0,011336 \\
\hline 39363,890000 & 314,836900 & 89,338960 & 0,003176 & 0,011193 \\
\hline 44167,020000 & 308,597500 & 90,542630 & 0,003240 & 0,011045 \\
\hline 49556,210000 & 302,428500 & 91,950670 & 0,003307 & 0,010875 \\
\hline 55602,980000 & 296,034600 & 93,459300 & 0,003378 & 0,010700 \\
\hline 62387,570000 & 289,544000 & 95,145570 & 0,003454 & 0,010510 \\
\hline 70000,000000 & 282,953800 & 96,989330 & 0,003534 & 0,010310 \\
\hline 78541,290000 & 275,955700 & 98,915120 & 0,003624 & 0,010110 \\
\hline 88124,780000 & 268,836900 & 100,993200 & 0,003720 & 0,009902 \\
\hline 98877,620000 & 261,469800 & 103,096300 & 0,003825 & 0,009700 \\
\hline 110942,500000 & 253,658900 & 105,269000 & 0,003942 & 0,009499 \\
\hline 124479,600000 & 245,584000 & 107,436100 & 0,004072 & 0,009308 \\
\hline 139668,400000 & 237,248100 & 109,560800 & 0,004215 & 0,009127 \\
\hline 156710,500000 & 228,498600 & 111,578500 & 0,004376 & 0,008962 \\
\hline
\end{tabular}




\begin{tabular}{|c|c|c|c|c|}
\hline $\begin{array}{c}\text { frequency } \\
{[\mathrm{Hz}]}\end{array}$ & $\begin{array}{c}\text { real impedance } \\
\text { at } 250 \text { bar } \\
Z^{\prime}[\Omega \mathrm{m}]\end{array}$ & $\begin{array}{c}\text { imaginary } \\
\text { impedance } \\
\text { at } 250 \text { bar } \\
\mathrm{Z}^{\prime \prime}[\Omega \mathrm{m}]\end{array}$ & $\begin{array}{c}\text { real admittance } \\
\text { at } 250 \text { bar } \\
Y^{\prime}[S \text { m-1] }\end{array}$ & $\begin{array}{c}\text { imaginary } \\
\text { admittance } \\
\text { at } 250 \text { bar } \\
Y^{\prime \prime}[S \mathrm{~m}-1]\end{array}$ \\
\hline 175832,000000 & 219,306100 & 113,513000 & 0,004560 & 0,008810 \\
\hline 197286,800000 & 209,802900 & 115,080000 & 0,004766 & 0,008690 \\
\hline 221359,400000 & 200,060600 & 116,459900 & 0,004998 & 0,008587 \\
\hline 248369,400000 & 189,984700 & 117,492300 & 0,005264 & 0,008511 \\
\hline 278675,000000 & 179,662900 & 118,030400 & 0,005566 & 0,008472 \\
\hline 312678,500000 & 169,210300 & 118,184500 & 0,005910 & 0,008461 \\
\hline 350831,100000 & 158,572000 & 117,772100 & 0,006306 & 0,008491 \\
\hline 393638,900000 & 147,966800 & 116,775700 & 0,006758 & 0,008563 \\
\hline 441670,100000 & 137,404800 & 115,213500 & 0,007278 & 0,008680 \\
\hline 495562,000000 & 127,052000 & 113,154900 & 0,007871 & 0,008837 \\
\hline 556029,800000 & 116,914100 & 110,513800 & 0,008553 & 0,009049 \\
\hline 623875,700000 & 107,123200 & 107,412100 & 0,009335 & 0,009310 \\
\hline 700000,000000 & 97,739690 & 103,850500 & 0,010231 & 0,009629 \\
\hline 785412,900000 & 88,818550 & 99,882300 & 0,011259 & 0,010012 \\
\hline 881247,800000 & 80,415810 & 95,580370 & 0,012435 & 0,010462 \\
\hline 988776,300000 & 72,525930 & 91,027170 & 0,013788 & 0,010986 \\
\hline
\end{tabular}




\begin{tabular}{|c|c|c|c|c|}
\hline $\begin{array}{c}\text { frequency } \\
{\left[\begin{array}{l}\mathrm{Hz}]\end{array}\right.}\end{array}$ & $\begin{array}{c}\text { real impedance } \\
\text { at } 300 \text { bar } \\
Z^{\prime}[\Omega \mathrm{m}]\end{array}$ & $\begin{array}{l}\text { imaginary } \\
\text { impedance } \\
\text { at } 300 \text { bar } \\
Z^{\prime \prime}[\Omega \mathrm{m}]\end{array}$ & $\begin{array}{c}\text { real admittance } \\
\text { at } 300 \text { bar } \\
Y^{\prime}[S \text { m-1] }\end{array}$ & $\begin{array}{c}\text { imaginary } \\
\text { admittance } \\
\text { at } 300 \text { bar } \\
\mathrm{Y}^{\prime \prime}[\mathrm{S} \mathrm{m}-1]\end{array}$ \\
\hline 785,412900 & 521,978000 & 101,882100 & 0,001916 & 0,009815 \\
\hline 881,247800 & 514,654900 & 100,266300 & 0,001943 & 0,009973 \\
\hline 988,776300 & 507,596600 & 98,891210 & 0,001970 & 0,010112 \\
\hline 1109,425000 & 500,538300 & 97,516090 & 0,001998 & 0,010255 \\
\hline 1244,796000 & 493,613400 & 96,256350 & 0,002026 & 0,010389 \\
\hline 1396,684000 & 486,839100 & 95,023500 & 0,002054 & 0,010524 \\
\hline 1567,105000 & 480,081600 & 93,704540 & 0,002083 & 0,010672 \\
\hline 1758,320000 & 473,608400 & 92,526830 & 0,002111 & 0,010808 \\
\hline 1972,868000 & 467,119600 & 91,428340 & 0,002141 & 0,010938 \\
\hline 2213,594000 & 460,631600 & 90,325340 & 0,002171 & 0,011071 \\
\hline 2483,694000 & 454,429100 & 89,356090 & 0,002201 & 0,011191 \\
\hline 2786,750000 & 448,258600 & 88,223980 & 0,002231 & 0,011335 \\
\hline 3126,785000 & 442,177700 & 87,427850 & 0,002262 & 0,011438 \\
\hline 3508,311000 & 436,113600 & 86,545100 & 0,002293 & 0,011555 \\
\hline 3936,389000 & 430,141200 & 85,984010 & 0,002325 & 0,011630 \\
\hline 4416,701000 & 424,036100 & 85,302220 & 0,002358 & 0,011723 \\
\hline 4955,620000 & 418,203600 & 84,812290 & 0,002391 & 0,011791 \\
\hline 5560,297000 & 412,373800 & 84,304260 & 0,002425 & 0,011862 \\
\hline 6238,757000 & 406,531900 & 83,849040 & 0,002460 & 0,011926 \\
\hline 7000,000000 & 400,813600 & 83,544730 & 0,002495 & 0,011970 \\
\hline 7854,129000 & 395,083900 & 83,285770 & 0,002531 & 0,012007 \\
\hline 8812,479000 & 389,328400 & 83,137080 & 0,002569 & 0,012028 \\
\hline 9887,763000 & 383,711500 & 83,058140 & 0,002606 & 0,012040 \\
\hline 11094,250000 & 377,919800 & 83,047580 & 0,002646 & 0,012041 \\
\hline 12447,960000 & 372,252200 & 83,164660 & 0,002686 & 0,012024 \\
\hline 13966,840000 & 366,574000 & 83,307170 & 0,002728 & 0,012004 \\
\hline 15671,050000 & 360,707100 & 83,563530 & 0,002772 & 0,011967 \\
\hline 17583,200000 & 354,978800 & 83,869330 & 0,002817 & 0,011923 \\
\hline 19728,680000 & 349,210800 & 84,309550 & 0,002864 & 0,011861 \\
\hline 22135,940000 & 343,551100 & 84,911890 & 0,002911 & 0,011777 \\
\hline 24836,940000 & 337,732200 & 85,476910 & 0,002961 & 0,011699 \\
\hline 27867,500000 & 331,872700 & 86,153370 & 0,003013 & 0,011607 \\
\hline 31267,850000 & 326,089800 & 87,085300 & 0,003067 & 0,011483 \\
\hline 35083,110000 & 320,132000 & 88,011990 & 0,003124 & 0,011362 \\
\hline 39363,890000 & 314,100900 & 89,130130 & 0,003184 & 0,011220 \\
\hline 44167,020000 & 308,010300 & 90,370330 & 0,003247 & 0,011066 \\
\hline 49556,210000 & 301,859000 & 91,719990 & 0,003313 & 0,010903 \\
\hline 55602,980000 & 295,467300 & 93,223510 & 0,003384 & 0,010727 \\
\hline 62387,570000 & 288,979200 & 94,904100 & 0,003460 & 0,010537 \\
\hline 70000,000000 & 282,391800 & 96,741620 & 0,003541 & 0,010337 \\
\hline 78541,290000 & 275,396800 & 98,660580 & 0,003631 & 0,010136 \\
\hline 88124,780000 & 268,281600 & 100,731200 & 0,003727 & 0,009927 \\
\hline 98877,620000 & 260,918400 & 102,826300 & 0,003833 & 0,009725 \\
\hline 110942,500000 & 253,130200 & 104,946200 & 0,003951 & 0,009529 \\
\hline 124479,600000 & 245,200900 & 107,166500 & 0,004078 & 0,009331 \\
\hline 139668,400000 & 236,730600 & 109,221700 & 0,004224 & 0,009156 \\
\hline 156710,500000 & 227,987500 & 111,230500 & 0,004386 & 0,008990 \\
\hline
\end{tabular}




\begin{tabular}{|c|c|c|c|c|}
\hline $\begin{array}{c}\text { frequency } \\
{[\mathbf{H z}]}\end{array}$ & $\begin{array}{c}\text { real impedance } \\
\text { at 300 bar } \\
\mathbf{Z}^{\prime}[\mathbf{\Omega} \mathbf{~ m}]\end{array}$ & $\begin{array}{c}\text { imaginary } \\
\text { impedance } \\
\text { at 300 bar } \\
\mathbf{Z}^{\prime \prime}[\mathbf{\Omega} \mathbf{~ m}]\end{array}$ & $\begin{array}{c}\text { real admittance } \\
\text { at 300 bar } \\
\mathbf{Y}^{\prime}[\mathbf{S} \text { m-1] }\end{array}$ & $\begin{array}{c}\text { imaginary } \\
\text { admittance } \\
\text { at 300 bar } \\
\mathbf{Y}^{\prime \prime} \mathbf{~} \mathbf{S} \text { m-1] }\end{array}$ \\
\hline 175832,000000 & 218,957700 & 113,187400 & 0,004567 & 0,008835 \\
\hline 197286,800000 & 209,460500 & 114,749600 & 0,004774 & 0,008715 \\
\hline 221359,400000 & 199,724700 & 116,124500 & 0,005007 & 0,008611 \\
\hline 248369,400000 & 189,676100 & 117,118500 & 0,005272 & 0,008538 \\
\hline 278675,000000 & 179,340900 & 117,684600 & 0,005576 & 0,008497 \\
\hline 312678,500000 & 168,916300 & 117,803900 & 0,005920 & 0,008489 \\
\hline 350831,100000 & 158,408300 & 117,479100 & 0,006313 & 0,008512 \\
\hline 393638,900000 & 147,807900 & 116,483100 & 0,006766 & 0,008585 \\
\hline 441670,100000 & 137,367900 & 115,019300 & 0,007280 & 0,008694 \\
\hline 495562,000000 & 127,016600 & 112,964600 & 0,007873 & 0,008852 \\
\hline 556029,800000 & 116,843600 & 110,254300 & 0,008558 & 0,009070 \\
\hline 623875,700000 & 107,076300 & 107,177900 & 0,009339 & 0,009330 \\
\hline 700000,000000 & 97,704280 & 103,631700 & 0,010235 & 0,009650 \\
\hline 785412,900000 & 88,803860 & 99,690540 & 0,011261 & 0,010031 \\
\hline 881247,800000 & 80,410400 & 95,404900 & 0,012436 & 0,010482 \\
\hline 988776,300000 & 72,528950 & 90,868220 & 0,013788 & 0,011005 \\
\hline
\end{tabular}




\begin{tabular}{|c|c|c|c|c|}
\hline $\begin{array}{c}\text { frequency } \\
\qquad[\mathrm{Hz}]\end{array}$ & $\begin{array}{c}\text { real impedance } \\
\text { at } 400 \text { bar } \\
Z^{\prime}[\Omega \mathrm{m}]\end{array}$ & $\begin{array}{l}\text { imaginary } \\
\text { impedance } \\
\text { at } 400 \text { bar } \\
Z^{\prime \prime}[\Omega \mathrm{m}] \\
\end{array}$ & $\begin{array}{c}\text { real admittance } \\
\text { at } 400 \text { bar } \\
Y^{\prime}[S \text { m-1] }\end{array}$ & $\begin{array}{c}\text { imaginary } \\
\text { admittance } \\
\text { at } 400 \text { bar } \\
Y^{\prime \prime}[S \mathrm{~m}-1]\end{array}$ \\
\hline 785,412900 & 519,654700 & 101,805100 & 0,001924355 & 0,009822691 \\
\hline 881,247800 & 512,332500 & 100,185000 & 0,001951857 & 0,009981534 \\
\hline 988,776300 & 505,275100 & 98,804920 & 0,00197912 & 0,010120953 \\
\hline 1109,425000 & 498,401900 & 97,280380 & 0,002006413 & 0,010279565 \\
\hline 1244,796000 & 491,293900 & 96,159920 & 0,002035442 & 0,010399343 \\
\hline 1396,684000 & 484,520500 & 94,921950 & 0,002063896 & 0,010534971 \\
\hline 1567,105000 & 477,930400 & 93,544330 & 0,002092355 & 0,010690119 \\
\hline 1758,320000 & 471,307700 & 92,333440 & 0,002121756 & 0,010830312 \\
\hline 1972,868000 & 464,819600 & 91,230770 & 0,002151372 & 0,010961214 \\
\hline 2213,594000 & 458,498200 & 90,073130 & 0,002181034 & 0,01110209 \\
\hline 2483,694000 & 452,280800 & 89,179510 & 0,002211016 & 0,011213338 \\
\hline 2786,750000 & 446,110900 & 88,043820 & 0,002241595 & 0,011357981 \\
\hline 3126,785000 & 439,880700 & 87,212920 & 0,002273344 & 0,011466191 \\
\hline 3508,311000 & 433,967500 & 86,355300 & 0,00230432 & 0,011580065 \\
\hline 3936,389000 & 427,860800 & 85,683420 & 0,002337209 & 0,011670869 \\
\hline 4416,701000 & 421,906500 & 85,026990 & 0,002370193 & 0,011760971 \\
\hline 4955,620000 & 416,074900 & 84,531730 & 0,002403413 & 0,011829877 \\
\hline 5560,297000 & 410,260500 & 83,946790 & 0,002437476 & 0,011912308 \\
\hline 6238,757000 & 404,554800 & 83,588420 & 0,002471853 & 0,01196338 \\
\hline 7000,000000 & 398,837400 & 83,278020 & 0,002507287 & 0,01200797 \\
\hline 7854,129000 & 393,123200 & 82,944080 & 0,002543732 & 0,012056316 \\
\hline 8812,479000 & 387,368800 & 82,789290 & 0,002581519 & 0,012078857 \\
\hline 9887,763000 & 381,603600 & 82,671550 & 0,00262052 & 0,01209606 \\
\hline 11094,250000 & 375,962700 & 82,686270 & 0,002659838 & 0,012093906 \\
\hline 12447,960000 & 370,311100 & 82,731000 & 0,002700432 & 0,012087367 \\
\hline 13966,840000 & 364,634500 & 82,866390 & 0,002742472 & 0,012067619 \\
\hline 15671,050000 & 358,933000 & 83,086560 & 0,002786035 & 0,012035641 \\
\hline 17583,200000 & 353,206500 & 83,385550 & 0,002831205 & 0,011992486 \\
\hline 19728,680000 & 347,589400 & 83,853930 & 0,002876958 & 0,011925499 \\
\hline 22135,940000 & 341,798200 & 84,352110 & 0,002925703 & 0,011855068 \\
\hline 24836,940000 & 336,130300 & 84,946720 & 0,002975037 & 0,011772085 \\
\hline 27867,500000 & 330,273600 & 85,615260 & 0,003027793 & 0,011680161 \\
\hline 31267,850000 & 324,509100 & 86,481250 & 0,003081578 & 0,0115632 \\
\hline 35083,110000 & 318,702600 & 87,439630 & 0,003137721 & 0,011436462 \\
\hline 39363,890000 & 312,690800 & 88,494290 & 0,003198047 & 0,011300164 \\
\hline 44167,020000 & 306,604900 & 89,725660 & 0,003261526 & 0,011145084 \\
\hline 49556,210000 & 300,458700 & 91,065550 & 0,003328244 & 0,010981101 \\
\hline 55602,980000 & 294,218800 & 92,603900 & 0,003398831 & 0,010798681 \\
\hline 62387,570000 & 287,736700 & 94,273690 & 0,0034754 & 0,010607413 \\
\hline 70000,000000 & 281,172900 & 96,050160 & 0,003556531 & 0,010411227 \\
\hline 78541,290000 & 274,330100 & 98,008540 & 0,003645243 & 0,010203192 \\
\hline 88124,780000 & 267,223100 & 100,067900 & 0,003742191 & 0,009993215 \\
\hline 98877,620000 & 259,886600 & 102,105600 & 0,003847832 & 0,009793782 \\
\hline 110942,500000 & 252,250200 & 104,272000 & 0,003964318 & 0,009590302 \\
\hline 124479,600000 & 244,331100 & 106,482000 & 0,004092807 & 0,009391259 \\
\hline 139668,400000 & 236,010800 & 108,590100 & 0,004237094 & 0,009208943 \\
\hline 156710,500000 & 227,278300 & 110,590100 & 0,004399892 & 0,009042401 \\
\hline
\end{tabular}




\begin{tabular}{|c|c|c|c|c|}
\hline $\begin{array}{c}\text { frequency } \\
{[\mathrm{Hz}]}\end{array}$ & $\begin{array}{c}\text { real impedance } \\
\text { at } 400 \text { bar } \\
Z^{\prime}[\Omega \mathrm{m}]\end{array}$ & $\begin{array}{c}\text { imaginary } \\
\text { impedance } \\
\text { at } 400 \mathrm{bar} \\
\mathrm{Z}^{\prime \prime}[\Omega \mathrm{m}] \\
\end{array}$ & $\begin{array}{c}\text { real admittance } \\
\text { at } 400 \text { bar } \\
Y^{\prime}[S \text { m-1] }\end{array}$ & $\begin{array}{c}\text { imaginary } \\
\text { admittance } \\
\text { at } 400 \text { bar } \\
\mathrm{Y}^{\prime \prime}[\mathrm{S} \mathrm{m-1]}\end{array}$ \\
\hline 175832,000000 & 218,260100 & 112,537500 & 0,004581689 & 0,008885927 \\
\hline 197286,800000 & 208,929100 & 114,127100 & 0,004786313 & 0,008762161 \\
\hline 221359,400000 & 199,204600 & 115,496800 & 0,005019964 & 0,008658249 \\
\hline 248369,400000 & 189,167600 & 116,485700 & 0,005286318 & 0,008584745 \\
\hline 278675,000000 & 178,992800 & 117,099100 & 0,005586817 & 0,008539775 \\
\hline 312678,500000 & 168,578000 & 117,218700 & 0,005931972 & 0,008531062 \\
\hline 350831,100000 & 158,080000 & 116,894100 & 0,006325911 & 0,008554752 \\
\hline 393638,900000 & 147,629700 & 115,967300 & 0,006773705 & 0,00862312 \\
\hline 441670,100000 & 137,175900 & 114,533400 & 0,00728991 & 0,008731078 \\
\hline 495562,000000 & 126,850400 & 112,460500 & 0,007883302 & 0,008892011 \\
\hline 556029,800000 & 116,741000 & 109,849900 & 0,008565971 & 0,009103331 \\
\hline 623875,700000 & 106,987500 & 106,790600 & 0,009346886 & 0,00936412 \\
\hline 700000,000000 & 97,659600 & 103,295100 & 0,010239649 & 0,009681001 \\
\hline 785412,900000 & 88,779820 & 99,383960 & 0,011263821 & 0,010061986 \\
\hline 881247,800000 & 80,398480 & 95,154710 & 0,012438046 & 0,010509201 \\
\hline 988776,300000 & 72,525140 & 90,636230 & 0,013788322 & 0,011033116 \\
\hline
\end{tabular}


III.5 CALCUlations OF BULK CONDUCTIVITIES OF SAMPLE 17/08/00/11 CORE 1:

\begin{tabular}{|c|c|c|c|c|}
\hline $\begin{array}{c}\text { pressure } \\
\text { measurement 1 } \\
{[\text { bar] }}\end{array}$ & $\begin{array}{c}\text { resistivity R1-1of } \\
\text { elem. cell 1 } \\
{[\mathbf{\Omega}]}\end{array}$ & $\begin{array}{c}\text { capacity C1-1 * } \mathbf{E}^{-7} \\
\text { of elem. cell 1 } \\
{[\mathbf{F}]}\end{array}$ & $\begin{array}{c}\text { resistivity R2-1 of } \\
\text { elem. cell 2 } \\
{[\mathbf{\Omega}]}\end{array}$ & $\begin{array}{c}\text { capacity C2-1 * } \mathbf{E}^{-9} \\
\text { of elem. cell 2 } \\
{[\mathbf{F}]}\end{array}$ \\
\hline 0 & 174 & 2,49 & 209 & 7,72 \\
\hline 75 & 177 & 2,61 & 196 & 8,54 \\
\hline 100 & 181 & 2,7 & 184 & 9,39 \\
\hline 150 & 184 & 2,86 & 173 & 10,4 \\
\hline 200 & 185 & 2,87 & 173 & 10,5 \\
\hline 250 & 185 & 2,88 & 172 & 10,6 \\
\hline 300 & 185 & 2,89 & 171 & 10,6 \\
\hline 400 & 185 & 2,91 & 170 & 10,7 \\
\hline
\end{tabular}

\begin{tabular}{|c|c|c|}
\hline $\begin{array}{c}\text { resistivity R3-1 of } \\
\text { elem. cell 3 } \\
{[\mathbf{\Omega}]}\end{array}$ & $\begin{array}{c}\text { capacity C3-1 }{ }^{*} \mathbf{E}^{-\mathbf{9}} \\
\text { of elem. cell 3 } \\
{[\mathbf{F}]}\end{array}$ & $\mathbf{X 2 - 1}$ \\
\hline 186 & 1,22 & 0,142 \\
\hline 186 & 1,2 & 0,147 \\
\hline 182 & 1,19 & 0,156 \\
\hline 183 & 1,18 & 0,161 \\
\hline 182 & 1,18 & 0,161 \\
\hline 182 & 1,18 & 0,162 \\
\hline 182 & 1,18 & 0,162 \\
\hline 181 & 1,18 & 0,162 \\
\hline surface resistivity & surface resistivity & \\
\hline
\end{tabular}




\section{III.6 COMPLEX IMPEDANCE DATA FOR SAMPLE 17/08/00/11 CORE 2:}

\begin{tabular}{|c|c|c|c|c|}
\hline $\begin{array}{c}\text { frequency } \\
{[\mathrm{Hz}]}\end{array}$ & $\begin{array}{c}\text { real impedance } \\
\text { at } 0 \text { bar } \\
Z^{\prime}[\Omega \mathrm{m}]\end{array}$ & $\begin{array}{c}\text { imaginary } \\
\text { impedance } \\
\text { at } 0 \text { bar } \\
\mathbf{Z}^{\prime \prime}[\Omega \mathrm{m}] \\
\end{array}$ & $\begin{array}{c}\text { real admittance } \\
\text { at } 0 \text { bar } \\
Y^{\prime}[S \text { m1] }\end{array}$ & $\begin{array}{c}\text { imaginary } \\
\text { admittance } \\
\text { at } 0 \text { bar } \\
\mathrm{Y}^{\prime \prime}[\mathrm{S} \mathrm{m} 1] \\
\end{array}$ \\
\hline 785,412900 & 407,578400 & 40,158380 & 0,002454 & 0,024901 \\
\hline 881,247700 & 404,739100 & 39,807340 & 0,002471 & 0,025121 \\
\hline 988,776200 & 401,892900 & 39,527400 & 0,002488 & 0,025299 \\
\hline 1109,425000 & 399,338000 & 39,205800 & 0,002504 & 0,025506 \\
\hline 1244,796000 & 396,478100 & 39,064690 & 0,002522 & 0,025599 \\
\hline 1396,684000 & 393,909800 & 38,881010 & 0,002539 & 0,025719 \\
\hline 1567,105000 & 391,043300 & 38,804730 & 0,002557 & 0,025770 \\
\hline 1758,320000 & 388,488600 & 38,482780 & 0,002574 & 0,025986 \\
\hline 1972,868000 & 385,907100 & 38,431010 & 0,002591 & 0,026021 \\
\hline 2213,594000 & 383,332500 & 38,309680 & 0,002609 & 0,026103 \\
\hline 2483,694000 & 380,744600 & 38,319390 & 0,002626 & 0,026096 \\
\hline 2786,750000 & 378,156800 & 38,325520 & 0,002644 & 0,026092 \\
\hline 3126,785000 & 375,847100 & 38,422650 & 0,002661 & 0,026026 \\
\hline 3508,311000 & 373,246300 & 38,551560 & 0,002679 & 0,025939 \\
\hline 3936,389000 & 370,632200 & 38,804380 & 0,002698 & 0,025770 \\
\hline 4416,701000 & 368,024800 & 38,985790 & 0,002717 & 0,025650 \\
\hline 4955,620000 & 365,403900 & 39,288360 & 0,002737 & 0,025453 \\
\hline 5560,297000 & 362,776100 & 39,646100 & 0,002757 & 0,025223 \\
\hline 6238,757000 & 359,863800 & 39,963210 & 0,002779 & 0,025023 \\
\hline 7000,000000 & 357,498800 & 40,521510 & 0,002797 & 0,024678 \\
\hline 7854,129000 & 355,140300 & 41,007340 & 0,002816 & 0,024386 \\
\hline 8812,479000 & 352,482800 & 41,573050 & 0,002837 & 0,024054 \\
\hline 9887,763000 & 349,518700 & 42,274760 & 0,002861 & 0,023655 \\
\hline 11094,250000 & 346,837300 & 42,994430 & 0,002883 & 0,023259 \\
\hline 12447,960000 & 343,855800 & 43,782530 & 0,002908 & 0,022840 \\
\hline 13966,840000 & 341,125400 & 44,826440 & 0,002931 & 0,022308 \\
\hline 15671,050000 & 338,116600 & 45,751770 & 0,002958 & 0,021857 \\
\hline 17583,210000 & 335,356500 & 46,928120 & 0,002982 & 0,021309 \\
\hline 19728,680000 & 332,301200 & 48,097430 & 0,003009 & 0,020791 \\
\hline 22135,950000 & 329,225300 & 49,353880 & 0,003037 & 0,020262 \\
\hline 24836,940000 & 326,118700 & 50,751130 & 0,003066 & 0,019704 \\
\hline 27867,500000 & 322,952000 & 52,453290 & 0,003096 & 0,019065 \\
\hline 31267,850000 & 319,514200 & 53,956540 & 0,003130 & 0,018533 \\
\hline 35083,110000 & 316,002400 & 55,804610 & 0,003165 & 0,017920 \\
\hline 39363,890000 & 312,460900 & 57,712070 & 0,003200 & 0,017327 \\
\hline 44167,020000 & 308,471200 & 59,873730 & 0,003242 & 0,016702 \\
\hline 49556,210000 & 304,334600 & 62,051190 & 0,003286 & 0,016116 \\
\hline 55602,980000 & 300,212400 & 64,435890 & 0,003331 & 0,015519 \\
\hline 62387,570000 & 295,983000 & 66,884740 & 0,003379 & 0,014951 \\
\hline 70000,000000 & 291,388800 & 69,596980 & 0,003432 & 0,014368 \\
\hline 78541,290000 & 286,667200 & 72,393230 & 0,003488 & 0,013813 \\
\hline 88124,780000 & 281,568900 & 75,406110 & 0,003552 & 0,013262 \\
\hline 98877,630000 & 276,265100 & 78,289600 & 0,003620 & 0,012773 \\
\hline
\end{tabular}




\begin{tabular}{|c|c|c|c|c|}
\hline $\begin{array}{c}\text { frequency } \\
\qquad[\mathrm{Hz}]\end{array}$ & $\begin{array}{c}\text { real impedance } \\
\text { at } 0 \text { bar } \\
Z^{\prime}[\Omega \mathrm{m}]\end{array}$ & $\begin{array}{c}\text { imaginary } \\
\text { impedance } \\
\text { at } 0 \mathrm{bar} \\
\mathrm{Z}^{\prime \prime}[\Omega \mathrm{m}]\end{array}$ & $\begin{array}{c}\text { real admittance } \\
\text { at } 0 \text { bar } \\
Y^{\prime}[S \text { m1] }\end{array}$ & $\begin{array}{c}\text { imaginary } \\
\text { admittance } \\
\text { at } 0 \text { bar } \\
\mathrm{Y}^{\prime \prime}[\mathrm{S} \mathrm{m} 1]\end{array}$ \\
\hline 110942,500000 & 270,508900 & 81,370230 & 0,003697 & 0,012290 \\
\hline 124479,600000 & 264,444900 & 84,603680 & 0,003782 & 0,011820 \\
\hline 139668,400000 & 258,043200 & 87,847400 & 0,003875 & 0,011383 \\
\hline 156710,500000 & 251,228700 & 91,190960 & 0,003980 & 0,010966 \\
\hline 175832,000000 & 243,897200 & 94,450990 & 0,004100 & 0,010588 \\
\hline 197286,800000 & 236,280600 & 97,477740 & 0,004232 & 0,010259 \\
\hline 221359,400000 & 228,135200 & 100,467200 & 0,004383 & 0,009953 \\
\hline 248369,400000 & 219,642200 & 103,249200 & 0,004553 & 0,009685 \\
\hline 278675,000000 & 210,692500 & 105,766900 & 0,004746 & 0,009455 \\
\hline 312678,500000 & 201,341500 & 107,893700 & 0,004967 & 0,009268 \\
\hline 350831,100000 & 191,599800 & 109,663100 & 0,005219 & 0,009119 \\
\hline 393638,900000 & 181,662900 & 110,905200 & 0,005505 & 0,009017 \\
\hline 441670,100000 & 171,501100 & 111,686000 & 0,005831 & 0,008954 \\
\hline 495562,000000 & 161,187700 & 111,954700 & 0,006204 & 0,008932 \\
\hline 556029,800000 & 150,804500 & 111,758500 & 0,006631 & 0,008948 \\
\hline 623875,700000 & 140,419200 & 110,699900 & 0,007122 & 0,009033 \\
\hline 700000,000000 & 130,117300 & 109,180300 & 0,007685 & 0,009159 \\
\hline 785412,900000 & 120,040200 & 107,060300 & 0,008331 & 0,009341 \\
\hline 881247,800000 & 110,252100 & 104,398700 & 0,009070 & 0,009579 \\
\hline 988776,300000 & 100,808500 & 101,221400 & 0,009920 & 0,009879 \\
\hline
\end{tabular}




\begin{tabular}{|c|c|c|c|c|}
\hline $\begin{array}{c}\text { frequency } \\
{[\mathrm{Hz}]}\end{array}$ & $\begin{array}{c}\text { real impedance } \\
\text { at } 25 \text { bar } \\
Z^{\prime}[\Omega \mathrm{m}]\end{array}$ & $\begin{array}{c}\text { imaginary } \\
\text { impedance } \\
\text { at } 25 \mathrm{bar} \\
\mathrm{Z}^{\prime \prime}[\Omega \mathrm{m}]\end{array}$ & $\begin{array}{c}\text { real admittance } \\
\text { at } 25 \text { bar } \\
Y^{\prime}[S \text { m1] }\end{array}$ & $\begin{array}{c}\text { imaginary } \\
\text { admittance } \\
\text { at } 25 \text { bar } \\
\mathrm{Y}^{\prime \prime}[\mathrm{S} \mathrm{m1}]\end{array}$ \\
\hline 785,412900 & 435,246100 & 59,513350 & 0,002298 & 0,016803 \\
\hline 881,247700 & 430,702000 & 58,968550 & 0,002322 & 0,016958 \\
\hline 988,776200 & 426,451600 & 58,386620 & 0,002345 & 0,017127 \\
\hline 1109,425000 & 422,768000 & 57,882290 & 0,002365 & 0,017276 \\
\hline 1244,796000 & 418,791000 & 57,412210 & 0,002388 & 0,017418 \\
\hline 1396,684000 & 414,530800 & 56,901860 & 0,002412 & 0,017574 \\
\hline 1567,105000 & 410,573900 & 56,285720 & 0,002436 & 0,017766 \\
\hline 1758,320000 & 406,568000 & 56,025600 & 0,002460 & 0,017849 \\
\hline 1972,868000 & 402,591800 & 55,549240 & 0,002484 & 0,018002 \\
\hline 2213,594000 & 398,899000 & 55,110640 & 0,002507 & 0,018145 \\
\hline 2483,694000 & 395,480300 & 54,778930 & 0,002529 & 0,018255 \\
\hline 2786,750000 & 391,759400 & 54,542180 & 0,002553 & 0,018334 \\
\hline 3126,785000 & 388,048600 & 54,232580 & 0,002577 & 0,018439 \\
\hline 3508,311000 & 384,310000 & 54,120240 & 0,002602 & 0,018477 \\
\hline 3936,389000 & 380,590800 & 53,867360 & 0,002627 & 0,018564 \\
\hline 4416,701000 & 376,853500 & 53,740780 & 0,002654 & 0,018608 \\
\hline 4955,620000 & 373,126100 & 53,541360 & 0,002680 & 0,018677 \\
\hline 5560,297000 & 369,352800 & 53,657660 & 0,002707 & 0,018637 \\
\hline 6238,757000 & 365,872500 & 53,738740 & 0,002733 & 0,018609 \\
\hline 7000,000000 & 362,374100 & 53,935460 & 0,002760 & 0,018541 \\
\hline 7854,129000 & 358,866900 & 54,181550 & 0,002787 & 0,018456 \\
\hline 8812,479000 & 355,077500 & 54,369920 & 0,002816 & 0,018393 \\
\hline 9887,763000 & 350,996800 & 54,560010 & 0,002849 & 0,018328 \\
\hline 11094,250000 & 347,745500 & 54,986910 & 0,002876 & 0,018186 \\
\hline 12447,960000 & 343,919900 & 55,366340 & 0,002908 & 0,018062 \\
\hline 13966,840000 & 340,338200 & 56,008610 & 0,002938 & 0,017854 \\
\hline 15671,050000 & 336,202000 & 56,473050 & 0,002974 & 0,017708 \\
\hline 17583,210000 & 332,590500 & 57,239420 & 0,003007 & 0,017470 \\
\hline 19728,680000 & 328,404800 & 57,935740 & 0,003045 & 0,017261 \\
\hline 22135,950000 & 324,761600 & 58,813060 & 0,003079 & 0,017003 \\
\hline 24836,940000 & 320,804800 & 59,774190 & 0,003117 & 0,016730 \\
\hline 27867,500000 & 317,106400 & 60,861260 & 0,003154 & 0,016431 \\
\hline 31267,850000 & 313,092700 & 62,018540 & 0,003194 & 0,016124 \\
\hline 35083,110000 & 308,752200 & 63,288590 & 0,003239 & 0,015801 \\
\hline 39363,890000 & 304,145400 & 64,780720 & 0,003288 & 0,015437 \\
\hline 44167,020000 & 299,813400 & 66,267650 & 0,003335 & 0,015090 \\
\hline 49556,210000 & 295,540800 & 67,923720 & 0,003384 & 0,014722 \\
\hline 55602,980000 & 291,077700 & 69,791100 & 0,003436 & 0,014328 \\
\hline 62387,570000 & 286,235500 & 71,647140 & 0,003494 & 0,013957 \\
\hline 70000,000000 & 281,487400 & 73,702770 & 0,003553 & 0,013568 \\
\hline 78541,290000 & 276,317400 & 75,758950 & 0,003619 & 0,013200 \\
\hline 88124,780000 & 270,836900 & 78,028900 & 0,003692 & 0,012816 \\
\hline 98877,630000 & 265,179600 & 80,221370 & 0,003771 & 0,012466 \\
\hline 110942,500000 & 259,368800 & 82,730380 & 0,003856 & 0,012087 \\
\hline 124479,600000 & 253,173400 & 85,106740 & 0,003950 & 0,011750 \\
\hline 139668,400000 & 246,672600 & 87,545620 & 0,004054 & 0,011423 \\
\hline 156710,500000 & 239,849500 & 90,055850 & 0,004169 & 0,011104 \\
\hline
\end{tabular}




\begin{tabular}{|c|c|c|c|c|}
\hline $\begin{array}{c}\text { frequency } \\
{[\mathbf{H z}]}\end{array}$ & $\begin{array}{c}\text { real impedance } \\
\text { at 25 bar } \\
\mathbf{Z}^{\prime}[\mathbf{\Omega} \mathbf{~ m}]\end{array}$ & $\begin{array}{c}\text { imaginary } \\
\text { impedance } \\
\text { at } \mathbf{2 5} \text { bar } \\
\mathbf{Z}^{\prime \prime}[\mathbf{\Omega} \mathbf{~ m}]\end{array}$ & $\begin{array}{c}\text { real admittance } \\
\text { at 25 bar } \\
\mathbf{Y}^{\prime}[\mathbf{S} \mathbf{~ m 1}]\end{array}$ & $\begin{array}{c}\text { imaginary } \\
\text { admittance } \\
\text { at 25 bar } \\
\mathbf{Y}^{\prime \prime}[\mathbf{S} \text { m1] }\end{array}$ \\
\hline 175832,000000 & 232,926100 & 92,734860 & 0,004293 & 0,010783 \\
\hline 197286,800000 & 225,491400 & 94,965810 & 0,004435 & 0,010530 \\
\hline 221359,400000 & 217,683100 & 97,227550 & 0,004594 & 0,010285 \\
\hline 248369,400000 & 209,754400 & 99,406510 & 0,004767 & 0,010060 \\
\hline 278675,000000 & 201,410400 & 101,371400 & 0,004965 & 0,009865 \\
\hline 312678,500000 & 192,702000 & 103,004500 & 0,005189 & 0,009708 \\
\hline 350831,100000 & 183,599600 & 104,235500 & 0,005447 & 0,009594 \\
\hline 393638,900000 & 174,373800 & 104,999200 & 0,005735 & 0,009524 \\
\hline 441670,100000 & 165,086700 & 105,591400 & 0,006057 & 0,009470 \\
\hline 495562,000000 & 155,578800 & 105,622800 & 0,006428 & 0,009468 \\
\hline 556029,800000 & 145,993900 & 105,107800 & 0,006850 & 0,009514 \\
\hline 623875,700000 & 136,487800 & 104,243600 & 0,007327 & 0,009593 \\
\hline 700000,000000 & 127,049800 & 102,920400 & 0,007871 & 0,009716 \\
\hline 785412,900000 & 117,731200 & 101,012700 & 0,008494 & 0,009900 \\
\hline 881247,800000 & 108,627000 & 98,664830 & 0,009206 & 0,010135 \\
\hline 988776,300000 & 99,832940 & 95,929710 & 0,010017 & 0,010424 \\
\hline
\end{tabular}




\begin{tabular}{|c|c|c|c|c|}
\hline $\begin{array}{c}\text { frequency } \\
{[\mathrm{Hz}]}\end{array}$ & $\begin{array}{c}\text { real impedance } \\
\text { at } 50 \text { bar } \\
Z^{\prime}[\Omega \mathrm{m}]\end{array}$ & $\begin{array}{c}\text { imaginary } \\
\text { impedance } \\
\text { at } 50 \text { bar } \\
Z^{\prime \prime}[\Omega \mathrm{m}] \\
\end{array}$ & $\begin{array}{c}\text { real admittance } \\
\text { at } 50 \text { bar } \\
Y^{\prime}[S \text { m1] }\end{array}$ & $\begin{array}{c}\text { imaginary } \\
\text { admittance } \\
\text { at } 50 \text { bar } \\
\mathrm{Y}^{\prime \prime}[\mathrm{S} \mathrm{m1}] \\
\end{array}$ \\
\hline 785,412900 & 444,491500 & 71,238820 & 0,002250 & 0,014037 \\
\hline 881,247700 & 439,113600 & 70,455490 & 0,002277 & 0,014193 \\
\hline 988,776200 & 434,006300 & 69,791350 & 0,002304 & 0,014328 \\
\hline 1109,425000 & 429,169800 & 69,244030 & 0,002330 & 0,014442 \\
\hline 1244,796000 & 424,063700 & 68,571980 & 0,002358 & 0,014583 \\
\hline 1396,684000 & 419,240300 & 67,942150 & 0,002385 & 0,014718 \\
\hline 1567,105000 & 414,123400 & 67,335340 & 0,002415 & 0,014851 \\
\hline 1758,320000 & 409,312900 & 66,626440 & 0,002443 & 0,015009 \\
\hline 1972,868000 & 404,479500 & 66,056990 & 0,002472 & 0,015138 \\
\hline 2213,594000 & 399,658300 & 65,412790 & 0,002502 & 0,015288 \\
\hline 2483,694000 & 395,390700 & 64,926780 & 0,002529 & 0,015402 \\
\hline 2786,750000 & 390,570500 & 64,275220 & 0,002560 & 0,015558 \\
\hline 3126,785000 & 386,010800 & 63,801510 & 0,002591 & 0,015674 \\
\hline 3508,311000 & 381,452000 & 63,321480 & 0,002622 & 0,015792 \\
\hline 3936,389000 & 377,165100 & 62,947940 & 0,002651 & 0,015886 \\
\hline 4416,701000 & 372,586300 & 62,584650 & 0,002684 & 0,015978 \\
\hline 4955,620000 & 368,041100 & 62,019240 & 0,002717 & 0,016124 \\
\hline 5560,297000 & 363,735600 & 61,750580 & 0,002749 & 0,016194 \\
\hline 6238,757000 & 359,431300 & 61,471500 & 0,002782 & 0,016268 \\
\hline 7000,000000 & 355,096200 & 61,367850 & 0,002816 & 0,016295 \\
\hline 7854,129000 & 350,762600 & 61,249260 & 0,002851 & 0,016327 \\
\hline 8812,479000 & 346,722700 & 61,104940 & 0,002884 & 0,016365 \\
\hline 9887,763000 & 342,381400 & 61,017470 & 0,002921 & 0,016389 \\
\hline 11094,250000 & 338,301800 & 61,082310 & 0,002956 & 0,016371 \\
\hline 12447,960000 & 333,931800 & 61,135770 & 0,002995 & 0,016357 \\
\hline 13966,840000 & 329,823300 & 61,335520 & 0,003032 & 0,016304 \\
\hline 15671,050000 & 325,424700 & 61,516510 & 0,003073 & 0,016256 \\
\hline 17583,210000 & 321,276300 & 61,894060 & 0,003113 & 0,016157 \\
\hline 19728,680000 & 317,129400 & 62,243420 & 0,003153 & 0,016066 \\
\hline 22135,950000 & 312,962200 & 62,673810 & 0,003195 & 0,015956 \\
\hline 24836,940000 & 308,763300 & 63,234720 & 0,003239 & 0,015814 \\
\hline 27867,500000 & 304,062000 & 63,931690 & 0,003289 & 0,015642 \\
\hline 31267,850000 & 299,736700 & 64,607330 & 0,003336 & 0,015478 \\
\hline 35083,110000 & 295,299600 & 65,486120 & 0,003386 & 0,015270 \\
\hline 39363,890000 & 290,990600 & 66,397130 & 0,003437 & 0,015061 \\
\hline 44167,020000 & 286,495400 & 67,425270 & 0,003490 & 0,014831 \\
\hline 49556,210000 & 281,936300 & 68,536170 & 0,003547 & 0,014591 \\
\hline 55602,980000 & 277,216900 & 69,749550 & 0,003607 & 0,014337 \\
\hline 62387,570000 & 272,336300 & 71,052930 & 0,003672 & 0,014074 \\
\hline 70000,000000 & 267,433700 & 72,571420 & 0,003739 & 0,013780 \\
\hline 78541,290000 & 262,419500 & 74,069310 & 0,003811 & 0,013501 \\
\hline 88124,780000 & 257,146800 & 75,836860 & 0,003889 & 0,013186 \\
\hline 98877,630000 & 251,773300 & 77,413890 & 0,003972 & 0,012918 \\
\hline 110942,500000 & 246,219300 & 79,341330 & 0,004061 & 0,012604 \\
\hline 124479,600000 & 240,359100 & 81,219300 & 0,004160 & 0,012312 \\
\hline 139668,400000 & 234,328200 & 83,164520 & 0,004268 & 0,012024 \\
\hline 156710,500000 & 228,018100 & 85,114680 & 0,004386 & 0,011749 \\
\hline
\end{tabular}




\begin{tabular}{|c|c|c|c|c|}
\hline $\begin{array}{c}\text { frequency } \\
{\left[\begin{array}{l}\mathrm{Hz}]\end{array}\right.}\end{array}$ & $\begin{array}{c}\text { real impedance } \\
\text { at } 50 \text { bar } \\
Z^{\prime}[\Omega \mathrm{m}]\end{array}$ & $\begin{array}{c}\text { imaginary } \\
\text { impedance } \\
\text { at } 50 \text { bar } \\
Z^{\prime \prime}[\Omega \mathrm{m}]\end{array}$ & $\begin{array}{c}\text { real admittance } \\
\text { at } 50 \text { bar } \\
Y^{\prime}[S \text { m1] }\end{array}$ & $\begin{array}{c}\text { imaginary } \\
\text { admittance } \\
\text { at } 50 \text { bar } \\
Y^{\prime \prime}[\mathrm{S} \mathrm{m1}] \\
\end{array}$ \\
\hline 175832,000000 & 221,411300 & 87,167580 & 0,004516 & 0,011472 \\
\hline 197286,800000 & 214,642100 & 89,033120 & 0,004659 & 0,011232 \\
\hline 221359,400000 & 207,638000 & 90,878880 & 0,004816 & 0,011004 \\
\hline 248369,400000 & 200,178000 & 92,611470 & 0,004996 & 0,010798 \\
\hline 278675,000000 & 192,527500 & 94,179840 & 0,005194 & 0,010618 \\
\hline 312678,500000 & 184,618800 & 95,517990 & 0,005417 & 0,010469 \\
\hline 350831,100000 & 176,406500 & 96,641500 & 0,005669 & 0,010348 \\
\hline 393638,900000 & 168,138300 & 97,563330 & 0,005947 & 0,010250 \\
\hline 441670,100000 & 159,552400 & 97,942440 & 0,006268 & 0,010210 \\
\hline 495562,000000 & 150,924500 & 98,211020 & 0,006626 & 0,010182 \\
\hline 556029,800000 & 142,204800 & 98,072880 & 0,007032 & 0,010196 \\
\hline 623875,700000 & 133,427000 & 97,445380 & 0,007495 & 0,010262 \\
\hline 700000,000000 & 124,695900 & 96,448510 & 0,008020 & 0,010368 \\
\hline 785412,900000 & 115,998400 & 94,977600 & 0,008621 & 0,010529 \\
\hline 881247,800000 & 107,456000 & 93,046030 & 0,009306 & 0,010747 \\
\hline 988776,300000 & 99,133700 & 90,738960 & 0,010087 & 0,011021 \\
\hline
\end{tabular}




\begin{tabular}{|c|c|c|c|c|}
\hline $\begin{array}{c}\text { frequency } \\
{[\mathrm{Hz}]}\end{array}$ & $\begin{array}{c}\text { real impedance } \\
\text { at } 75 \text { bar } \\
Z^{\prime}[\Omega \mathrm{m}]\end{array}$ & $\begin{array}{c}\text { imaginary } \\
\text { impedance } \\
\text { at } 75 \mathrm{bar} \\
\mathrm{Z}^{\prime \prime}[\Omega \mathrm{m}]\end{array}$ & $\begin{array}{c}\text { real admittance } \\
\text { at } 75 \text { bar } \\
Y^{\prime}[S \text { m1] }\end{array}$ & $\begin{array}{c}\text { imaginary } \\
\text { admittance } \\
\text { at } 75 \text { bar } \\
Y^{\prime \prime}[\mathrm{S} \mathrm{m1}]\end{array}$ \\
\hline 785,412900 & 443,535400 & 73,547900 & 0,002255 & 0,013597 \\
\hline 881,247700 & 438,418700 & 72,935200 & 0,002281 & 0,013711 \\
\hline 988,776200 & 433,045800 & 72,119000 & 0,002309 & 0,013866 \\
\hline 1109,425000 & 427,673100 & 71,300930 & 0,002338 & 0,014025 \\
\hline 1244,796000 & 422,570600 & 70,601780 & 0,002366 & 0,014164 \\
\hline 1396,684000 & 417,468700 & 69,899090 & 0,002395 & 0,014306 \\
\hline 1567,105000 & 412,355100 & 69,264770 & 0,002425 & 0,014437 \\
\hline 1758,320000 & 407,524400 & 68,672640 & 0,002454 & 0,014562 \\
\hline 1972,868000 & 402,718200 & 67,934980 & 0,002483 & 0,014720 \\
\hline 2213,594000 & 397,900500 & 67,265060 & 0,002513 & 0,014867 \\
\hline 2483,694000 & 393,060000 & 66,728930 & 0,002544 & 0,014986 \\
\hline 2786,750000 & 388,243600 & 66,050610 & 0,002576 & 0,015140 \\
\hline 3126,785000 & 383,686800 & 65,550890 & 0,002606 & 0,015255 \\
\hline 3508,311000 & 379,130800 & 65,044830 & 0,002638 & 0,015374 \\
\hline 3936,389000 & 374,575800 & 64,532450 & 0,002670 & 0,015496 \\
\hline 4416,701000 & 369,999300 & 64,142840 & 0,002703 & 0,015590 \\
\hline 4955,620000 & 365,446200 & 63,616210 & 0,002736 & 0,015719 \\
\hline 5560,297000 & 360,861000 & 63,272130 & 0,002771 & 0,015805 \\
\hline 6238,757000 & 356,558900 & 62,966690 & 0,002805 & 0,015881 \\
\hline 7000,000000 & 352,236100 & 62,773730 & 0,002839 & 0,015930 \\
\hline 7854,129000 & 347,644300 & 62,456080 & 0,002877 & 0,016011 \\
\hline 8812,479000 & 343,606000 & 62,287620 & 0,002910 & 0,016055 \\
\hline 9887,763000 & 339,266100 & 62,173540 & 0,002948 & 0,016084 \\
\hline 11094,250000 & 334,917100 & 62,101470 & 0,002986 & 0,016103 \\
\hline 12447,960000 & 330,559100 & 62,069130 & 0,003025 & 0,016111 \\
\hline 13966,840000 & 326,473100 & 62,127710 & 0,003063 & 0,016096 \\
\hline 15671,050000 & 322,086300 & 62,224930 & 0,003105 & 0,016071 \\
\hline 17583,210000 & 317,949600 & 62,519600 & 0,003145 & 0,015995 \\
\hline 19728,680000 & 313,814400 & 62,787540 & 0,003187 & 0,015927 \\
\hline 22135,950000 & 309,389800 & 63,025690 & 0,003232 & 0,015867 \\
\hline 24836,940000 & 304,777900 & 63,582730 & 0,003281 & 0,015728 \\
\hline 27867,500000 & 300,542600 & 64,122970 & 0,003327 & 0,015595 \\
\hline 31267,850000 & 296,258100 & 64,723370 & 0,003375 & 0,015450 \\
\hline 35083,110000 & 291,885100 & 65,423770 & 0,003426 & 0,015285 \\
\hline 39363,890000 & 287,506600 & 66,235650 & 0,003478 & 0,015098 \\
\hline 44167,020000 & 283,026300 & 67,182260 & 0,003533 & 0,014885 \\
\hline 49556,210000 & 278,506600 & 68,114230 & 0,003591 & 0,014681 \\
\hline 55602,980000 & 273,887500 & 69,267570 & 0,003651 & 0,014437 \\
\hline 62387,570000 & 269,107800 & 70,511540 & 0,003716 & 0,014182 \\
\hline 70000,000000 & 264,304700 & 71,870860 & 0,003784 & 0,013914 \\
\hline 78541,290000 & 259,351500 & 73,252190 & 0,003856 & 0,013651 \\
\hline 88124,780000 & 254,266400 & 74,987400 & 0,003933 & 0,013336 \\
\hline 98877,630000 & 248,916300 & 76,487910 & 0,004017 & 0,013074 \\
\hline 110942,500000 & 243,470100 & 78,267960 & 0,004107 & 0,012777 \\
\hline 124479,600000 & 237,773800 & 80,114690 & 0,004206 & 0,012482 \\
\hline 139668,400000 & 231,882200 & 81,932240 & 0,004313 & 0,012205 \\
\hline 156710,500000 & 225,711700 & 83,850250 & 0,004430 & 0,011926 \\
\hline
\end{tabular}




\begin{tabular}{|c|c|c|c|c|}
\hline $\begin{array}{c}\text { frequency } \\
{[\mathrm{Hz}]}\end{array}$ & $\begin{array}{c}\text { real impedance } \\
\text { at } 75 \text { bar } \\
Z^{\prime}[\Omega \mathrm{m}]\end{array}$ & $\begin{array}{c}\text { imaginary } \\
\text { impedance } \\
\text { at } 75 \mathrm{bar} \\
\mathrm{Z}^{\prime \prime}[\Omega \mathrm{m}]\end{array}$ & $\begin{array}{c}\text { real admittance } \\
\text { at } 75 \text { bar } \\
Y^{\prime}[S \text { m1] }\end{array}$ & $\begin{array}{c}\text { imaginary } \\
\text { admittance } \\
\text { at } 75 \text { bar } \\
\mathrm{Y}^{\prime \prime}[\mathrm{S} \mathrm{m1}]\end{array}$ \\
\hline 175832,000000 & 219,287800 & 85,845940 & 0,004560 & 0,011649 \\
\hline 197286,800000 & 212,664100 & 87,604490 & 0,004702 & 0,011415 \\
\hline 221359,400000 & 205,708600 & 89,436440 & 0,004861 & 0,011181 \\
\hline 248369,400000 & 198,512500 & 91,169100 & 0,005037 & 0,010969 \\
\hline 278675,000000 & 191,046800 & 92,713260 & 0,005234 & 0,010786 \\
\hline 312678,500000 & 183,289400 & 94,101810 & 0,005456 & 0,010627 \\
\hline 350831,100000 & 175,302000 & 95,322030 & 0,005704 & 0,010491 \\
\hline 393638,900000 & 167,156000 & 96,176520 & 0,005982 & 0,010398 \\
\hline 441670,100000 & 158,801500 & 96,758290 & 0,006297 & 0,010335 \\
\hline 495562,000000 & 150,250100 & 96,990500 & 0,006656 & 0,010310 \\
\hline 556029,800000 & 141,652800 & 96,855830 & 0,007060 & 0,010325 \\
\hline 623875,700000 & 133,006200 & 96,322140 & 0,007518 & 0,010382 \\
\hline 700000,000000 & 124,355700 & 95,390390 & 0,008041 & 0,010483 \\
\hline 785412,900000 & 115,795500 & 94,004430 & 0,008636 & 0,010638 \\
\hline 881247,800000 & 107,351100 & 92,204190 & 0,009315 & 0,010845 \\
\hline 988776,300000 & 99,094670 & 89,975200 & 0,010091 & 0,011114 \\
\hline
\end{tabular}




\begin{tabular}{|c|c|c|c|c|}
\hline $\begin{array}{c}\text { frequency } \\
{[\mathrm{Hz}]}\end{array}$ & $\begin{array}{c}\text { real impedance } \\
\text { at } 100 \text { bar } \\
Z^{\prime}[\Omega \mathrm{m}]\end{array}$ & $\begin{array}{c}\text { imaginary } \\
\text { impedance } \\
\text { at } 100 \text { bar } \\
Z^{\prime \prime}[\Omega \mathrm{m}]\end{array}$ & $\begin{array}{c}\text { real admittance } \\
\text { at } 100 \text { bar } \\
Y^{\prime}[S \text { m1] }\end{array}$ & $\begin{array}{c}\text { imaginary } \\
\text { admittance } \\
\text { at } 100 \text { bar } \\
Y^{\prime \prime}[\mathrm{S} \mathrm{m1}]\end{array}$ \\
\hline 785,412900 & 443,662500 & 74,523580 & 0,002254 & 0,013419 \\
\hline 881,247700 & 438,265000 & 73,852790 & 0,002282 & 0,013540 \\
\hline 988,776200 & 432,893900 & 73,025350 & 0,002310 & 0,013694 \\
\hline 1109,425000 & 427,779700 & 72,392910 & 0,002338 & 0,013814 \\
\hline 1244,796000 & 422,409500 & 71,559900 & 0,002367 & 0,013974 \\
\hline 1396,684000 & 417,309100 & 70,845630 & 0,002396 & 0,014115 \\
\hline 1567,105000 & 412,197000 & 70,199710 & 0,002426 & 0,014245 \\
\hline 1758,320000 & 407,097900 & 69,477460 & 0,002456 & 0,014393 \\
\hline 1972,868000 & 402,269200 & 68,870050 & 0,002486 & 0,014520 \\
\hline 2213,594000 & 397,183100 & 68,070610 & 0,002518 & 0,014691 \\
\hline 2483,694000 & 392,344000 & 67,523110 & 0,002549 & 0,014810 \\
\hline 2786,750000 & 387,517500 & 66,901280 & 0,002581 & 0,014947 \\
\hline 3126,785000 & 382,691700 & 66,274420 & 0,002613 & 0,015089 \\
\hline 3508,311000 & 378,137000 & 65,757450 & 0,002645 & 0,015207 \\
\hline 3936,389000 & 373,583300 & 65,234150 & 0,002677 & 0,015329 \\
\hline 4416,701000 & 368,737400 & 64,719460 & 0,002712 & 0,015451 \\
\hline 4955,620000 & 364,456100 & 64,295750 & 0,002744 & 0,015553 \\
\hline 5560,297000 & 359,883000 & 63,877450 & 0,002779 & 0,015655 \\
\hline 6238,757000 & 355,300100 & 63,511650 & 0,002815 & 0,015745 \\
\hline 7000,000000 & 350,989100 & 63,246640 & 0,002849 & 0,015811 \\
\hline 7854,129000 & 346,679600 & 62,969720 & 0,002885 & 0,015881 \\
\hline 8812,479000 & 342,360600 & 62,740630 & 0,002921 & 0,015939 \\
\hline 9887,763000 & 337,751000 & 62,505050 & 0,002961 & 0,015999 \\
\hline 11094,250000 & 333,683800 & 62,475080 & 0,002997 & 0,016006 \\
\hline 12447,960000 & 329,326400 & 62,432590 & 0,003037 & 0,016017 \\
\hline 13966,840000 & 324,959900 & 62,427350 & 0,003077 & 0,016019 \\
\hline 15671,050000 & 320,865400 & 62,511770 & 0,003117 & 0,015997 \\
\hline 17583,210000 & 316,459300 & 62,685400 & 0,003160 & 0,015953 \\
\hline 19728,680000 & 312,324700 & 62,942920 & 0,003202 & 0,015887 \\
\hline 22135,950000 & 307,604400 & 63,332930 & 0,003251 & 0,015790 \\
\hline 24836,940000 & 303,328600 & 63,666980 & 0,003297 & 0,015707 \\
\hline 27867,500000 & 299,049100 & 64,131650 & 0,003344 & 0,015593 \\
\hline 31267,850000 & 294,737700 & 64,714490 & 0,003393 & 0,015452 \\
\hline 35083,110000 & 290,349200 & 65,345500 & 0,003444 & 0,015303 \\
\hline 39363,890000 & 285,999800 & 66,151250 & 0,003497 & 0,015117 \\
\hline 44167,020000 & 281,504800 & 67,028620 & 0,003552 & 0,014919 \\
\hline 49556,210000 & 277,014700 & 67,954230 & 0,003610 & 0,014716 \\
\hline 55602,980000 & 272,409600 & 69,045500 & 0,003671 & 0,014483 \\
\hline 62387,570000 & 267,672400 & 70,235230 & 0,003736 & 0,014238 \\
\hline 70000,000000 & 262,927500 & 71,594870 & 0,003803 & 0,013967 \\
\hline 78541,290000 & 257,962600 & 72,908510 & 0,003877 & 0,013716 \\
\hline 88124,780000 & 252,867400 & 74,574800 & 0,003955 & 0,013409 \\
\hline 98877,630000 & 247,617300 & 76,041480 & 0,004038 & 0,013151 \\
\hline 110942,500000 & 242,190300 & 77,763330 & 0,004129 & 0,012860 \\
\hline 124479,600000 & 236,568700 & 79,570820 & 0,004227 & 0,012567 \\
\hline 139668,400000 & 230,684400 & 81,373240 & 0,004335 & 0,012289 \\
\hline 156710,500000 & 224,604800 & 83,216160 & 0,004452 & 0,012017 \\
\hline
\end{tabular}




\begin{tabular}{|c|c|c|c|c|}
\hline $\begin{array}{c}\text { frequency } \\
{\left[\begin{array}{l}\mathrm{Hz}]\end{array}\right.}\end{array}$ & $\begin{array}{c}\text { real impedance } \\
\text { at } 100 \text { bar } \\
Z^{\prime}[\Omega \mathrm{m}]\end{array}$ & $\begin{array}{c}\text { imaginary } \\
\text { impedance } \\
\text { at } 100 \text { bar } \\
Z^{\prime \prime}[\Omega \mathrm{m}]\end{array}$ & $\begin{array}{c}\text { real admittance } \\
\text { at } 100 \text { bar } \\
Y^{\prime}[S \text { m1] }\end{array}$ & $\begin{array}{c}\text { imaginary } \\
\text { admittance } \\
\text { at } 100 \text { bar } \\
\mathrm{Y}^{\prime \prime}[\mathrm{S} \mathrm{m} 1]\end{array}$ \\
\hline 175832,000000 & 218,258500 & 85,179660 & 0,004582 & 0,011740 \\
\hline 197286,800000 & 211,671000 & 86,936370 & 0,004724 & 0,011503 \\
\hline 221359,400000 & 204,809600 & 88,705960 & 0,004883 & 0,011273 \\
\hline 248369,400000 & 197,719000 & 90,429080 & 0,005058 & 0,011058 \\
\hline 278675,000000 & 190,332700 & 91,956830 & 0,005254 & 0,010875 \\
\hline 312678,500000 & 182,680200 & 93,346510 & 0,005474 & 0,010713 \\
\hline 350831,100000 & 174,755200 & 94,590650 & 0,005722 & 0,010572 \\
\hline 393638,900000 & 166,703500 & 95,413610 & 0,005999 & 0,010481 \\
\hline 441670,100000 & 158,433500 & 96,042100 & 0,006312 & 0,010412 \\
\hline 495562,000000 & 149,964300 & 96,324940 & 0,006668 & 0,010382 \\
\hline 556029,800000 & 141,463200 & 96,220060 & 0,007069 & 0,010393 \\
\hline 623875,700000 & 132,846600 & 95,712800 & 0,007527 & 0,010448 \\
\hline 700000,000000 & 124,270000 & 94,843480 & 0,008047 & 0,010544 \\
\hline 785412,900000 & 115,757700 & 93,505680 & 0,008639 & 0,010695 \\
\hline 881247,800000 & 107,320800 & 91,756050 & 0,009318 & 0,010898 \\
\hline 988776,300000 & 99,070650 & 89,575740 & 0,010094 & 0,011164 \\
\hline
\end{tabular}




\begin{tabular}{|c|c|c|c|c|}
\hline $\begin{array}{c}\text { frequency } \\
{[\mathrm{Hz}]}\end{array}$ & $\begin{array}{c}\text { real impedance } \\
\text { at } 150 \text { bar } \\
Z^{\prime}[\Omega \mathrm{m}]\end{array}$ & $\begin{array}{c}\text { imaginary } \\
\text { impedance } \\
\text { at } 150 \mathrm{bar} \\
\mathrm{Z}^{\prime \prime}[\Omega \mathrm{m}] \\
\end{array}$ & $\begin{array}{c}\text { real admittance } \\
\text { at } 150 \text { bar } \\
Y^{\prime}[S \text { m1] }\end{array}$ & $\begin{array}{c}\text { imaginary } \\
\text { admittance } \\
\text { at } 150 \text { bar } \\
Y^{\prime \prime}[\mathrm{S} \mathrm{m1}]\end{array}$ \\
\hline 785,412900 & 443,892500 & 74,880710 & 0,002253 & 0,013355 \\
\hline 881,247700 & 438,226300 & 74,082150 & 0,002282 & 0,013499 \\
\hline 988,776200 & 433,124800 & 73,375170 & 0,002309 & 0,013629 \\
\hline 1109,425000 & 427,754500 & 72,542150 & 0,002338 & 0,013785 \\
\hline 1244,796000 & 422,359400 & 71,854630 & 0,002368 & 0,013917 \\
\hline 1396,684000 & 417,271900 & 71,064010 & 0,002397 & 0,014072 \\
\hline 1567,105000 & 412,160200 & 70,415420 & 0,002426 & 0,014201 \\
\hline 1758,320000 & 407,049300 & 69,761510 & 0,002457 & 0,014335 \\
\hline 1972,868000 & 401,951200 & 69,032150 & 0,002488 & 0,014486 \\
\hline 2213,594000 & 397,123600 & 68,417020 & 0,002518 & 0,014616 \\
\hline 2483,694000 & 392,308600 & 67,728420 & 0,002549 & 0,014765 \\
\hline 2786,750000 & 387,470700 & 67,171670 & 0,002581 & 0,014887 \\
\hline 3126,785000 & 382,657000 & 66,474690 & 0,002613 & 0,015043 \\
\hline 3508,311000 & 378,102600 & 65,955330 & 0,002645 & 0,015162 \\
\hline 3936,389000 & 373,256000 & 65,445420 & 0,002679 & 0,015280 \\
\hline 4416,701000 & 368,692200 & 64,976740 & 0,002712 & 0,015390 \\
\hline 4955,620000 & 364,140700 & 64,436630 & 0,002746 & 0,015519 \\
\hline 5560,297000 & 359,567900 & 64,015660 & 0,002781 & 0,015621 \\
\hline 6238,757000 & 355,266800 & 63,697580 & 0,002815 & 0,015699 \\
\hline 7000,000000 & 350,674500 & 63,379440 & 0,002852 & 0,015778 \\
\hline 7854,129000 & 346,365200 & 63,099890 & 0,002887 & 0,015848 \\
\hline 8812,479000 & 342,046400 & 62,868090 & 0,002924 & 0,015906 \\
\hline 9887,763000 & 337,718300 & 62,681800 & 0,002961 & 0,015954 \\
\hline 11094,250000 & 333,370000 & 62,596930 & 0,003000 & 0,015975 \\
\hline 12447,960000 & 329,012700 & 62,551520 & 0,003039 & 0,015987 \\
\hline 13966,840000 & 324,646400 & 62,543300 & 0,003080 & 0,015989 \\
\hline 15671,050000 & 320,541000 & 62,680760 & 0,003120 & 0,015954 \\
\hline 17583,210000 & 316,145900 & 62,795280 & 0,003163 & 0,015925 \\
\hline 19728,680000 & 311,731000 & 62,993060 & 0,003208 & 0,015875 \\
\hline 22135,950000 & 307,274200 & 63,376690 & 0,003254 & 0,015779 \\
\hline 24836,940000 & 302,987300 & 63,760930 & 0,003300 & 0,015684 \\
\hline 27867,500000 & 298,707900 & 64,222020 & 0,003348 & 0,015571 \\
\hline 31267,850000 & 294,424400 & 64,807240 & 0,003396 & 0,015430 \\
\hline 35083,110000 & 290,008000 & 65,428220 & 0,003448 & 0,015284 \\
\hline 39363,890000 & 285,602900 & 66,216940 & 0,003501 & 0,015102 \\
\hline 44167,020000 & 281,191500 & 67,109550 & 0,003556 & 0,014901 \\
\hline 49556,210000 & 276,685400 & 67,975810 & 0,003614 & 0,014711 \\
\hline 55602,980000 & 272,025100 & 69,049060 & 0,003676 & 0,014482 \\
\hline 62387,570000 & 267,343600 & 70,248660 & 0,003741 & 0,014235 \\
\hline 70000,000000 & 262,516200 & 71,581270 & 0,003809 & 0,013970 \\
\hline 78541,290000 & 257,579400 & 72,897260 & 0,003882 & 0,013718 \\
\hline 88124,780000 & 252,512200 & 74,565810 & 0,003960 & 0,013411 \\
\hline 98877,630000 & 247,235400 & 76,018590 & 0,004045 & 0,013155 \\
\hline 110942,500000 & 241,836400 & 77,742790 & 0,004135 & 0,012863 \\
\hline 124479,600000 & 236,148200 & 79,475250 & 0,004235 & 0,012583 \\
\hline 139668,400000 & 230,292600 & 81,280220 & 0,004342 & 0,012303 \\
\hline 156710,500000 & 224,214800 & 83,116170 & 0,004460 & 0,012031 \\
\hline
\end{tabular}




\begin{tabular}{|c|c|c|c|c|}
\hline $\begin{array}{c}\text { frequency } \\
{\left[\begin{array}{l}\mathrm{Hz}]\end{array}\right.}\end{array}$ & $\begin{array}{c}\text { real impedance } \\
\text { at } 150 \mathrm{bar} \\
\mathrm{Z}^{\prime}[\Omega \mathrm{m}]\end{array}$ & $\begin{array}{c}\text { imaginary } \\
\text { impedance } \\
\text { at } 150 \mathrm{bar} \\
\mathrm{Z}^{\prime \prime}[\Omega \mathrm{m}]\end{array}$ & $\begin{array}{c}\text { real admittance } \\
\text { at } 150 \text { bar } \\
Y^{\prime}[S \text { m1] }\end{array}$ & $\begin{array}{c}\text { imaginary } \\
\text { admittance } \\
\text { at } 150 \text { bar } \\
\mathrm{Y}^{\prime \prime}[\mathrm{S} \mathrm{m} 1]\end{array}$ \\
\hline 175832,000000 & 217,870700 & 85,072090 & 0,004590 & 0,011755 \\
\hline 197286,800000 & 211,327000 & 86,795120 & 0,004732 & 0,011521 \\
\hline 221359,400000 & 204,494700 & 88,569560 & 0,004890 & 0,011291 \\
\hline 248369,400000 & 197,370700 & 90,228120 & 0,005067 & 0,011083 \\
\hline 278675,000000 & 190,014000 & 91,761960 & 0,005263 & 0,010898 \\
\hline 312678,500000 & 182,390800 & 93,158530 & 0,005483 & 0,010734 \\
\hline 350831,100000 & 174,511500 & 94,380020 & 0,005730 & 0,010595 \\
\hline 393638,900000 & 166,447000 & 95,228300 & 0,006008 & 0,010501 \\
\hline 441670,100000 & 158,198000 & 95,823840 & 0,006321 & 0,010436 \\
\hline 495562,000000 & 149,774000 & 96,092000 & 0,006677 & 0,010407 \\
\hline 556029,800000 & 141,260300 & 96,009930 & 0,007079 & 0,010416 \\
\hline 623875,700000 & 132,694300 & 95,532760 & 0,007536 & 0,010468 \\
\hline 700000,000000 & 124,137700 & 94,639720 & 0,008056 & 0,010566 \\
\hline 785412,900000 & 115,628600 & 93,301400 & 0,008648 & 0,010718 \\
\hline 881247,800000 & 107,254300 & 91,569720 & 0,009324 & 0,010921 \\
\hline 988776,300000 & 99,027000 & 89,410770 & 0,010098 & 0,011184 \\
\hline
\end{tabular}




\begin{tabular}{|c|c|c|c|c|}
\hline $\begin{array}{c}\text { frequency } \\
{[\mathrm{Hz}]}\end{array}$ & $\begin{array}{c}\text { real impedance } \\
\text { at } 200 \text { bar } \\
Z^{\prime}[\Omega \mathrm{m}]\end{array}$ & $\begin{array}{l}\text { imaginary } \\
\text { impedance } \\
\text { at } 200 \text { bar } \\
\mathrm{Z}^{\prime \prime}[\Omega \mathrm{m}]\end{array}$ & $\begin{array}{c}\text { real admittance } \\
\text { at } 200 \text { bar } \\
Y^{\prime}[S \text { m1] }\end{array}$ & $\begin{array}{l}\text { imaginary } \\
\text { admittance } \\
\text { at } 200 \text { bar } \\
\mathrm{Y}^{\prime \prime}[\mathrm{S} \mathrm{m1}] \\
\end{array}$ \\
\hline 785,412900 & 443,020300 & 74,892570 & 0,002257 & 0,013352 \\
\hline 881,247700 & 437,649400 & 74,063150 & 0,002285 & 0,013502 \\
\hline 988,776200 & 432,535200 & 73,430540 & 0,002312 & 0,013618 \\
\hline 1109,425000 & 426,883200 & 72,547630 & 0,002343 & 0,013784 \\
\hline 1244,796000 & 421,783000 & 71,832270 & 0,002371 & 0,013921 \\
\hline 1396,684000 & 416,670900 & 71,186040 & 0,002400 & 0,014048 \\
\hline 1567,105000 & 411,559500 & 70,534480 & 0,002430 & 0,014177 \\
\hline 1758,320000 & 406,473300 & 69,735790 & 0,002460 & 0,014340 \\
\hline 1972,868000 & 401,363400 & 69,075370 & 0,002492 & 0,014477 \\
\hline 2213,594000 & 396,548000 & 68,389080 & 0,002522 & 0,014622 \\
\hline 2483,694000 & 391,439500 & 67,719030 & 0,002555 & 0,014767 \\
\hline 2786,750000 & 386,907100 & 67,073970 & 0,002585 & 0,014909 \\
\hline 3126,785000 & 382,058600 & 66,576740 & 0,002617 & 0,015020 \\
\hline 3508,311000 & 377,527600 & 65,922890 & 0,002649 & 0,015169 \\
\hline 3936,389000 & 372,681100 & 65,411640 & 0,002683 & 0,015288 \\
\hline 4416,701000 & 368,117500 & 64,941690 & 0,002717 & 0,015398 \\
\hline 4955,620000 & 363,555000 & 64,463810 & 0,002751 & 0,015513 \\
\hline 5560,297000 & 358,982500 & 64,040640 & 0,002786 & 0,015615 \\
\hline 6238,757000 & 354,681600 & 63,720380 & 0,002819 & 0,015694 \\
\hline 7000,000000 & 350,100600 & 63,338790 & 0,002856 & 0,015788 \\
\hline 7854,129000 & 345,780500 & 63,118010 & 0,002892 & 0,015843 \\
\hline 8812,479000 & 341,461900 & 62,883820 & 0,002929 & 0,015902 \\
\hline 9887,763000 & 337,134000 & 62,695050 & 0,002966 & 0,015950 \\
\hline 11094,250000 & 332,786000 & 62,607470 & 0,003005 & 0,015973 \\
\hline 12447,960000 & 328,429000 & 62,559270 & 0,003045 & 0,015985 \\
\hline 13966,840000 & 324,062900 & 62,548160 & 0,003086 & 0,015988 \\
\hline 15671,050000 & 319,688000 & 62,571870 & 0,003128 & 0,015982 \\
\hline 17583,210000 & 315,573900 & 62,738900 & 0,003169 & 0,015939 \\
\hline 19728,680000 & 311,439700 & 62,990740 & 0,003211 & 0,015875 \\
\hline 22135,950000 & 306,731000 & 63,320430 & 0,003260 & 0,015793 \\
\hline 24836,940000 & 302,388500 & 63,690000 & 0,003307 & 0,015701 \\
\hline 27867,500000 & 298,137500 & 64,153790 & 0,003354 & 0,015588 \\
\hline 31267,850000 & 293,798700 & 64,723240 & 0,003404 & 0,015450 \\
\hline 35083,110000 & 289,438700 & 65,352820 & 0,003455 & 0,015302 \\
\hline 39363,890000 & 285,006300 & 66,131010 & 0,003509 & 0,015121 \\
\hline 44167,020000 & 280,579500 & 66,963480 & 0,003564 & 0,014934 \\
\hline 49556,210000 & 276,007000 & 67,860200 & 0,003623 & 0,014736 \\
\hline 55602,980000 & 271,403200 & 68,941610 & 0,003685 & 0,014505 \\
\hline 62387,570000 & 266,750500 & 70,142560 & 0,003749 & 0,014257 \\
\hline 70000,000000 & 261,936700 & 71,423260 & 0,003818 & 0,014001 \\
\hline 78541,290000 & 257,001500 & 72,733710 & 0,003891 & 0,013749 \\
\hline 88124,780000 & 251,936200 & 74,395720 & 0,003969 & 0,013442 \\
\hline 98877,630000 & 246,688700 & 75,850490 & 0,004054 & 0,013184 \\
\hline 110942,500000 & 241,278200 & 77,516890 & 0,004145 & 0,012900 \\
\hline 124479,600000 & 235,606100 & 79,292800 & 0,004244 & 0,012611 \\
\hline 139668,400000 & 229,821300 & 81,068790 & 0,004351 & 0,012335 \\
\hline 156710,500000 & 223,732100 & 82,937230 & 0,004470 & 0,012057 \\
\hline
\end{tabular}




\begin{tabular}{|c|c|c|c|c|}
\hline $\begin{array}{c}\text { frequency } \\
{[\mathrm{Hz}]}\end{array}$ & $\begin{array}{c}\text { real impedance } \\
\text { at } 200 \text { bar } \\
Z^{\prime}[\Omega \mathrm{m}]\end{array}$ & $\begin{array}{l}\text { imaginary } \\
\text { impedance } \\
\text { at } 200 \text { bar } \\
Z^{\prime \prime}[\Omega \mathrm{m}]\end{array}$ & $\begin{array}{c}\text { real admittance } \\
\text { at } 200 \text { bar } \\
Y^{\prime}[S \text { m1] }\end{array}$ & $\begin{array}{c}\text { imaginary } \\
\text { admittance } \\
\text { at } 200 \text { bar } \\
Y^{\prime \prime}[\mathrm{S} \mathrm{m1}]\end{array}$ \\
\hline 175832,000000 & 217,432600 & 84,857330 & 0,004599 & 0,011784 \\
\hline 197286,800000 & 210,839500 & 86,551890 & 0,004743 & 0,011554 \\
\hline 221359,400000 & 204,079400 & 88,305140 & 0,004900 & 0,011324 \\
\hline 248369,400000 & 196,985900 & 89,969140 & 0,005077 & 0,011115 \\
\hline 278675,000000 & 189,617900 & 91,529880 & 0,005274 & 0,010925 \\
\hline 312678,500000 & 182,015700 & 92,886890 & 0,005494 & 0,010766 \\
\hline 350831,100000 & 174,183400 & 94,084790 & 0,005741 & 0,010629 \\
\hline 393638,900000 & 166,149200 & 94,942530 & 0,006019 & 0,010533 \\
\hline 441670,100000 & 157,930000 & 95,548570 & 0,006332 & 0,010466 \\
\hline 495562,000000 & 149,535300 & 95,828430 & 0,006687 & 0,010435 \\
\hline 556029,800000 & 141,066900 & 95,734680 & 0,007089 & 0,010446 \\
\hline 623875,700000 & 132,528700 & 95,273210 & 0,007546 & 0,010496 \\
\hline 700000,000000 & 123,998900 & 94,397160 & 0,008065 & 0,010594 \\
\hline 785412,900000 & 115,515500 & 93,077160 & 0,008657 & 0,010744 \\
\hline 881247,800000 & 107,138200 & 91,309170 & 0,009334 & 0,010952 \\
\hline 988776,300000 & 98,956220 & 89,190320 & 0,010105 & 0,011212 \\
\hline
\end{tabular}




\begin{tabular}{|c|c|c|c|c|}
\hline $\begin{array}{c}\text { frequency } \\
{\left[\begin{array}{l}\mathrm{Hz}]\end{array}\right.}\end{array}$ & $\begin{array}{c}\text { real impedance } \\
\text { at } 250 \text { bar } \\
Z^{\prime}[\Omega \mathrm{m}]\end{array}$ & $\begin{array}{l}\text { imaginary } \\
\text { impedance } \\
\text { at } 250 \mathrm{bar} \\
\mathrm{Z}^{\prime \prime}[\Omega \mathrm{m}]\end{array}$ & $\begin{array}{c}\text { real admittance } \\
\text { at } 250 \text { bar } \\
Y^{\prime}[S \text { m1] }\end{array}$ & $\begin{array}{c}\text { imaginary } \\
\text { admittance } \\
\text { at } 250 \text { bar } \\
Y^{\prime \prime}[\mathrm{S} \mathrm{m1}]\end{array}$ \\
\hline 785,412900 & 442,148200 & 74,903820 & 0,002262 & 0,013350 \\
\hline 881,247700 & 436,790500 & 73,996180 & 0,002289 & 0,013514 \\
\hline 988,776200 & 431,394600 & 73,314320 & 0,002318 & 0,013640 \\
\hline 1109,425000 & 426,012100 & 72,552510 & 0,002347 & 0,013783 \\
\hline 1244,796000 & 420,630200 & 71,786960 & 0,002377 & 0,013930 \\
\hline 1396,684000 & 415,812700 & 71,114090 & 0,002405 & 0,014062 \\
\hline 1567,105000 & 410,419700 & 70,412830 & 0,002437 & 0,014202 \\
\hline 1758,320000 & 405,333700 & 69,613060 & 0,002467 & 0,014365 \\
\hline 1972,868000 & 400,517800 & 68,929840 & 0,002497 & 0,014508 \\
\hline 2213,594000 & 395,678600 & 68,381320 & 0,002527 & 0,014624 \\
\hline 2483,694000 & 390,594100 & 67,572770 & 0,002560 & 0,014799 \\
\hline 2786,750000 & 386,038300 & 67,062100 & 0,002590 & 0,014912 \\
\hline 3126,785000 & 381,213300 & 66,429450 & 0,002623 & 0,015054 \\
\hline 3508,311000 & 376,377700 & 65,857430 & 0,002657 & 0,015184 \\
\hline 3936,389000 & 371,824600 & 65,328190 & 0,002689 & 0,015307 \\
\hline 4416,701000 & 367,261300 & 64,856690 & 0,002723 & 0,015419 \\
\hline 4955,620000 & 362,406200 & 64,390520 & 0,002759 & 0,015530 \\
\hline 5560,297000 & 358,126600 & 63,952430 & 0,002792 & 0,015637 \\
\hline 6238,757000 & 353,544600 & 63,579770 & 0,002828 & 0,015728 \\
\hline 7000,000000 & 349,245300 & 63,246970 & 0,002863 & 0,015811 \\
\hline 7854,129000 & 344,925400 & 63,024110 & 0,002899 & 0,015867 \\
\hline 8812,479000 & 340,325900 & 62,736000 & 0,002938 & 0,015940 \\
\hline 9887,763000 & 335,998400 & 62,544500 & 0,002976 & 0,015989 \\
\hline 11094,250000 & 331,650800 & 62,453820 & 0,003015 & 0,016012 \\
\hline 12447,960000 & 327,575200 & 62,455870 & 0,003053 & 0,016011 \\
\hline 13966,840000 & 323,209600 & 62,441940 & 0,003094 & 0,016015 \\
\hline 15671,050000 & 318,835100 & 62,462680 & 0,003136 & 0,016010 \\
\hline 17583,210000 & 314,721500 & 62,626500 & 0,003177 & 0,015968 \\
\hline 19728,680000 & 310,307400 & 62,818080 & 0,003223 & 0,015919 \\
\hline 22135,950000 & 305,823600 & 63,188760 & 0,003270 & 0,015826 \\
\hline 24836,940000 & 301,576900 & 63,519050 & 0,003316 & 0,015743 \\
\hline 27867,500000 & 297,298700 & 63,973300 & 0,003364 & 0,015632 \\
\hline 31267,850000 & 292,988700 & 64,544800 & 0,003413 & 0,015493 \\
\hline 35083,110000 & 288,629600 & 65,170140 & 0,003465 & 0,015344 \\
\hline 39363,890000 & 284,237700 & 65,900420 & 0,003518 & 0,015174 \\
\hline 44167,020000 & 279,816700 & 66,833040 & 0,003574 & 0,014963 \\
\hline 49556,210000 & 275,312700 & 67,689490 & 0,003632 & 0,014773 \\
\hline 55602,980000 & 270,710200 & 68,765570 & 0,003694 & 0,014542 \\
\hline 62387,570000 & 266,031300 & 69,953450 & 0,003759 & 0,014295 \\
\hline 70000,000000 & 261,219300 & 71,227650 & 0,003828 & 0,014039 \\
\hline 78541,290000 & 256,313500 & 72,539010 & 0,003901 & 0,013786 \\
\hline 88124,780000 & 251,235900 & 74,141310 & 0,003980 & 0,013488 \\
\hline 98877,630000 & 246,005200 & 75,640350 & 0,004065 & 0,013220 \\
\hline 110942,500000 & 240,597400 & 77,298190 & 0,004156 & 0,012937 \\
\hline 124479,600000 & 235,010200 & 79,000990 & 0,004255 & 0,012658 \\
\hline 139668,400000 & 229,202200 & 80,760500 & 0,004363 & 0,012382 \\
\hline 156710,500000 & 223,158600 & 82,591800 & 0,004481 & 0,012108 \\
\hline
\end{tabular}




\begin{tabular}{|c|c|c|c|c|}
\hline $\begin{array}{c}\text { frequency } \\
{\left[\begin{array}{l}\mathrm{Hz}]\end{array}\right.}\end{array}$ & $\begin{array}{c}\text { real impedance } \\
\text { at } 250 \text { bar } \\
Z^{\prime}[\Omega \mathrm{m}]\end{array}$ & $\begin{array}{c}\text { imaginary } \\
\text { impedance } \\
\text { at } 250 \text { bar } \\
Z^{\prime \prime}[\Omega \mathrm{m}]\end{array}$ & $\begin{array}{c}\text { real admittance } \\
\text { at } 250 \text { bar } \\
Y^{\prime}[S \text { m1] }\end{array}$ & $\begin{array}{c}\text { imaginary } \\
\text { admittance } \\
\text { at } 250 \text { bar } \\
\mathrm{Y}^{\prime \prime}[\mathrm{S} \mathrm{m} 1]\end{array}$ \\
\hline 175832,000000 & 216,849300 & 84,542520 & 0,004611 & 0,011828 \\
\hline 197286,800000 & 210,340400 & 86,261280 & 0,004754 & 0,011593 \\
\hline 221359,400000 & 203,532600 & 87,984260 & 0,004913 & 0,011366 \\
\hline 248369,400000 & 196,486500 & 89,616810 & 0,005089 & 0,011159 \\
\hline 278675,000000 & 189,192100 & 91,161660 & 0,005286 & 0,010970 \\
\hline 312678,500000 & 181,621800 & 92,526180 & 0,005506 & 0,010808 \\
\hline 350831,100000 & 173,795900 & 93,718890 & 0,005754 & 0,010670 \\
\hline 393638,900000 & 165,793100 & 94,585650 & 0,006032 & 0,010572 \\
\hline 441670,100000 & 157,604900 & 95,201740 & 0,006345 & 0,010504 \\
\hline 495562,000000 & 149,281500 & 95,482220 & 0,006699 & 0,010473 \\
\hline 556029,800000 & 140,818800 & 95,387050 & 0,007101 & 0,010484 \\
\hline 623875,700000 & 132,326200 & 94,917730 & 0,007557 & 0,010535 \\
\hline 700000,000000 & 123,824300 & 94,059730 & 0,008076 & 0,010632 \\
\hline 785412,900000 & 115,390000 & 92,776980 & 0,008666 & 0,010779 \\
\hline 881247,800000 & 107,043600 & 91,067440 & 0,009342 & 0,010981 \\
\hline 988776,300000 & 98,879410 & 88,933710 & 0,010113 & 0,011244 \\
\hline
\end{tabular}




\begin{tabular}{|c|c|c|c|c|}
\hline $\begin{array}{c}\text { frequency } \\
{[\mathrm{Hz}]}\end{array}$ & $\begin{array}{c}\text { real impedance } \\
\text { at } 300 \text { bar } \\
Z^{\prime}[\Omega \mathrm{m}]\end{array}$ & $\begin{array}{c}\text { imaginary } \\
\text { impedance } \\
\text { at } 300 \text { bar } \\
Z^{\prime \prime}[\Omega \mathrm{m}]\end{array}$ & $\begin{array}{c}\text { real admittance } \\
\text { at } 300 \text { bar } \\
Y^{\prime}[S \text { m1] }\end{array}$ & $\begin{array}{c}\text { imaginary } \\
\text { admittance } \\
\text { at } 300 \text { bar } \\
Y^{\prime \prime}[\mathrm{S} \mathrm{m1}]\end{array}$ \\
\hline 785,412900 & 441,597300 & 74,731240 & 0,002265 & 0,013381 \\
\hline 881,247700 & 435,918800 & 74,004970 & 0,002294 & 0,013513 \\
\hline 988,776200 & 430,805100 & 73,368790 & 0,002321 & 0,013630 \\
\hline 1109,425000 & 425,435600 & 72,530700 & 0,002351 & 0,013787 \\
\hline 1244,796000 & 420,348300 & 71,738850 & 0,002379 & 0,013939 \\
\hline 1396,684000 & 414,967000 & 70,969450 & 0,002410 & 0,014091 \\
\hline 1567,105000 & 409,855900 & 70,316120 & 0,002440 & 0,014221 \\
\hline 1758,320000 & 405,051800 & 69,564650 & 0,002469 & 0,014375 \\
\hline 1972,868000 & 399,660200 & 68,854030 & 0,002502 & 0,014523 \\
\hline 2213,594000 & 394,833200 & 68,235210 & 0,002533 & 0,014655 \\
\hline 2483,694000 & 390,006900 & 67,611340 & 0,002564 & 0,014790 \\
\hline 2786,750000 & 385,181300 & 66,982440 & 0,002596 & 0,014929 \\
\hline 3126,785000 & 380,356500 & 66,348500 & 0,002629 & 0,015072 \\
\hline 3508,311000 & 375,814200 & 65,758830 & 0,002661 & 0,015207 \\
\hline 3936,389000 & 371,249900 & 65,293960 & 0,002694 & 0,015315 \\
\hline 4416,701000 & 366,686700 & 64,821170 & 0,002727 & 0,015427 \\
\hline 4955,620000 & 361,843000 & 64,290460 & 0,002764 & 0,015554 \\
\hline 5560,297000 & 357,552400 & 63,914260 & 0,002797 & 0,015646 \\
\hline 6238,757000 & 352,970500 & 63,540100 & 0,002833 & 0,015738 \\
\hline 7000,000000 & 348,671400 & 63,205860 & 0,002868 & 0,015821 \\
\hline 7854,129000 & 344,070500 & 62,929920 & 0,002906 & 0,015891 \\
\hline 8812,479000 & 339,752400 & 62,691570 & 0,002943 & 0,015951 \\
\hline 9887,763000 & 335,436000 & 62,439830 & 0,002981 & 0,016015 \\
\hline 11094,250000 & 331,077800 & 62,405720 & 0,003020 & 0,016024 \\
\hline 12447,960000 & 327,013300 & 62,348740 & 0,003058 & 0,016039 \\
\hline 13966,840000 & 322,648000 & 62,333440 & 0,003099 & 0,016043 \\
\hline 15671,050000 & 318,273800 & 62,352710 & 0,003142 & 0,016038 \\
\hline 17583,210000 & 314,160500 & 62,514870 & 0,003183 & 0,015996 \\
\hline 19728,680000 & 309,746800 & 62,704590 & 0,003228 & 0,015948 \\
\hline 22135,950000 & 305,235500 & 63,067230 & 0,003276 & 0,015856 \\
\hline 24836,940000 & 301,006100 & 63,453680 & 0,003322 & 0,015760 \\
\hline 27867,500000 & 296,711600 & 63,846950 & 0,003370 & 0,015662 \\
\hline 31267,850000 & 292,441300 & 64,370720 & 0,003419 & 0,015535 \\
\hline 35083,110000 & 288,083000 & 64,993910 & 0,003471 & 0,015386 \\
\hline 39363,890000 & 283,724700 & 65,833640 & 0,003525 & 0,015190 \\
\hline 44167,020000 & 279,299800 & 66,658080 & 0,003580 & 0,015002 \\
\hline 49556,210000 & 274,796800 & 67,511820 & 0,003639 & 0,014812 \\
\hline 55602,980000 & 270,223200 & 68,591690 & 0,003701 & 0,014579 \\
\hline 62387,570000 & 265,545600 & 69,776210 & 0,003766 & 0,014332 \\
\hline 70000,000000 & 260,735000 & 71,046740 & 0,003835 & 0,014075 \\
\hline 78541,290000 & 255,830800 & 72,354190 & 0,003909 & 0,013821 \\
\hline 88124,780000 & 250,755100 & 73,951860 & 0,003988 & 0,013522 \\
\hline 98877,630000 & 245,539500 & 75,403390 & 0,004073 & 0,013262 \\
\hline 110942,500000 & 240,161500 & 77,065690 & 0,004164 & 0,012976 \\
\hline 124479,600000 & 234,590200 & 78,814270 & 0,004263 & 0,012688 \\
\hline 139668,400000 & 228,784700 & 80,568510 & 0,004371 & 0,012412 \\
\hline 156710,500000 & 222,758200 & 82,355260 & 0,004489 & 0,012143 \\
\hline
\end{tabular}




\begin{tabular}{|c|c|c|c|c|}
\hline $\begin{array}{c}\text { frequency } \\
{\left[\begin{array}{l}\mathrm{Hz}]\end{array}\right.}\end{array}$ & $\begin{array}{c}\text { real impedance } \\
\text { at } 300 \text { bar } \\
Z^{\prime}[\Omega \mathrm{m}]\end{array}$ & $\begin{array}{c}\text { imaginary } \\
\text { impedance } \\
\text { at } 300 \text { bar } \\
Z^{\prime \prime}[\Omega \mathrm{m}]\end{array}$ & $\begin{array}{c}\text { real admittance } \\
\text { at } 300 \text { bar } \\
Y^{\prime}[S \text { m1] }\end{array}$ & $\begin{array}{c}\text { imaginary } \\
\text { admittance } \\
\text { at } 300 \text { bar } \\
\mathrm{Y}^{\prime \prime}[\mathrm{S} \mathrm{m} 1]\end{array}$ \\
\hline 175832,000000 & 216,493800 & 84,273410 & 0,004619 & 0,011866 \\
\hline 197286,800000 & 210,015000 & 85,999470 & 0,004762 & 0,011628 \\
\hline 221359,400000 & 203,237300 & 87,730390 & 0,004920 & 0,011399 \\
\hline 248369,400000 & 196,179400 & 89,394090 & 0,005097 & 0,011186 \\
\hline 278675,000000 & 188,930600 & 90,913820 & 0,005293 & 0,010999 \\
\hline 312678,500000 & 181,364300 & 92,275480 & 0,005514 & 0,010837 \\
\hline 350831,100000 & 173,567900 & 93,478750 & 0,005761 & 0,010698 \\
\hline 393638,900000 & 165,610500 & 94,328380 & 0,006038 & 0,010601 \\
\hline 441670,100000 & 157,426400 & 94,944050 & 0,006352 & 0,010533 \\
\hline 495562,000000 & 149,107100 & 95,224100 & 0,006707 & 0,010502 \\
\hline 556029,800000 & 140,695800 & 95,160570 & 0,007108 & 0,010509 \\
\hline 623875,700000 & 132,206400 & 94,692150 & 0,007564 & 0,010561 \\
\hline 700000,000000 & 123,753200 & 93,869600 & 0,008081 & 0,010653 \\
\hline 785412,900000 & 115,298600 & 92,571110 & 0,008673 & 0,010803 \\
\hline 881247,800000 & 106,992200 & 90,862970 & 0,009346 & 0,011006 \\
\hline 988776,300000 & 98,813770 & 88,750020 & 0,010120 & 0,011268 \\
\hline
\end{tabular}




\begin{tabular}{|c|c|c|c|c|}
\hline $\begin{array}{c}\text { frequency } \\
{[\mathrm{Hz}]}\end{array}$ & $\begin{array}{c}\text { real impedance } \\
\text { at } 400 \text { bar } \\
Z^{\prime}[\Omega \mathrm{m}]\end{array}$ & $\begin{array}{c}\text { imaginary } \\
\text { impedance } \\
\text { at } 400 \mathrm{bar} \\
\mathrm{Z}^{\prime \prime}[\Omega \mathrm{m}]\end{array}$ & $\begin{array}{c}\text { real admittance } \\
\text { at } 400 \text { bar } \\
Y^{\prime}[S \text { m1] }\end{array}$ & $\begin{array}{c}\text { imaginary } \\
\text { admittance } \\
\text { at } 400 \text { bar } \\
Y^{\prime \prime}[\mathrm{S} \mathrm{m} 1]\end{array}$ \\
\hline 785,412900 & 439,007400 & 74,608100 & 0,002278 & 0,013403 \\
\hline 881,247700 & 433,932200 & 73,745570 & 0,002305 & 0,013560 \\
\hline 988,776200 & 428,549600 & 72,984660 & 0,002333 & 0,013702 \\
\hline 1109,425000 & 423,449400 & 72,268100 & 0,002362 & 0,013837 \\
\hline 1244,796000 & 418,055500 & 71,572720 & 0,002392 & 0,013972 \\
\hline 1396,684000 & 412,956600 & 70,848080 & 0,002422 & 0,014115 \\
\hline 1567,105000 & 407,858300 & 70,119900 & 0,002452 & 0,014261 \\
\hline 1758,320000 & 402,760600 & 69,388160 & 0,002483 & 0,014412 \\
\hline 1972,868000 & 397,651400 & 68,722250 & 0,002515 & 0,014551 \\
\hline 2213,594000 & 392,836700 & 68,031330 & 0,002546 & 0,014699 \\
\hline 2483,694000 & 388,022500 & 67,337060 & 0,002577 & 0,014851 \\
\hline 2786,750000 & 383,197300 & 66,706280 & 0,002610 & 0,014991 \\
\hline 3126,785000 & 378,654500 & 66,119670 & 0,002641 & 0,015124 \\
\hline 3508,311000 & 373,819400 & 65,544200 & 0,002675 & 0,015257 \\
\hline 3936,389000 & 369,266800 & 65,011600 & 0,002708 & 0,015382 \\
\hline 4416,701000 & 364,704000 & 64,536300 & 0,002742 & 0,015495 \\
\hline 4955,620000 & 360,142300 & 64,053090 & 0,002777 & 0,015612 \\
\hline 5560,297000 & 355,570600 & 63,624000 & 0,002812 & 0,015717 \\
\hline 6238,757000 & 351,270600 & 63,297360 & 0,002847 & 0,015798 \\
\hline 7000,000000 & 346,690600 & 62,909240 & 0,002884 & 0,015896 \\
\hline 7854,129000 & 342,371500 & 62,680910 & 0,002921 & 0,015954 \\
\hline 8812,479000 & 338,054000 & 62,439160 & 0,002958 & 0,016016 \\
\hline 9887,763000 & 333,727300 & 62,242230 & 0,002996 & 0,016066 \\
\hline 11094,250000 & 329,391500 & 62,087860 & 0,003036 & 0,016106 \\
\hline 12447,960000 & 325,316800 & 62,084110 & 0,003074 & 0,016107 \\
\hline 13966,840000 & 320,963100 & 62,007940 & 0,003116 & 0,016127 \\
\hline 15671,050000 & 316,578900 & 62,078030 & 0,003159 & 0,016109 \\
\hline 17583,210000 & 312,466600 & 62,234480 & 0,003200 & 0,016068 \\
\hline 19728,680000 & 307,892100 & 62,496880 & 0,003248 & 0,016001 \\
\hline 22135,950000 & 303,695000 & 62,748940 & 0,003293 & 0,015937 \\
\hline 24836,940000 & 299,466900 & 63,129210 & 0,003339 & 0,015841 \\
\hline 27867,500000 & 295,229700 & 63,528080 & 0,003387 & 0,015741 \\
\hline 31267,850000 & 290,933000 & 64,038730 & 0,003437 & 0,015616 \\
\hline 35083,110000 & 286,604400 & 64,660320 & 0,003489 & 0,015465 \\
\hline 39363,890000 & 282,271000 & 65,392560 & 0,003543 & 0,015292 \\
\hline 44167,020000 & 277,864800 & 66,264370 & 0,003599 & 0,015091 \\
\hline 49556,210000 & 273,392000 & 67,116130 & 0,003658 & 0,014900 \\
\hline 55602,980000 & 268,837200 & 68,239870 & 0,003720 & 0,014654 \\
\hline 62387,570000 & 264,186800 & 69,320630 & 0,003785 & 0,014426 \\
\hline 70000,000000 & 259,450500 & 70,648090 & 0,003854 & 0,014155 \\
\hline 78541,290000 & 254,562400 & 71,899550 & 0,003928 & 0,013908 \\
\hline 88124,780000 & 249,546300 & 73,500740 & 0,004007 & 0,013605 \\
\hline 98877,630000 & 244,348400 & 74,897710 & 0,004093 & 0,013352 \\
\hline 110942,500000 & 238,990000 & 76,597820 & 0,004184 & 0,013055 \\
\hline 124479,600000 & 233,438400 & 78,291340 & 0,004284 & 0,012773 \\
\hline 139668,400000 & 227,707500 & 80,010650 & 0,004392 & 0,012498 \\
\hline 156710,500000 & 221,715400 & 81,793960 & 0,004510 & 0,012226 \\
\hline
\end{tabular}




\begin{tabular}{|c|c|c|c|c|}
\hline $\begin{array}{c}\text { frequency } \\
{\left[\begin{array}{l}\mathrm{Hz}]\end{array}\right.}\end{array}$ & $\begin{array}{c}\text { real impedance } \\
\text { at } 400 \text { bar } \\
Z^{\prime}[\Omega \mathrm{m}]\end{array}$ & $\begin{array}{c}\text { imaginary } \\
\text { impedance } \\
\text { at } 400 \text { bar } \\
Z^{\prime \prime}[\Omega \mathrm{m}]\end{array}$ & $\begin{array}{c}\text { real admittance } \\
\text { at } 400 \text { bar } \\
Y^{\prime}[S \text { m1] }\end{array}$ & $\begin{array}{c}\text { imaginary } \\
\text { admittance } \\
\text { at } 400 \text { bar } \\
\mathrm{Y}^{\prime \prime}[\mathrm{S} \mathrm{m} 1]\end{array}$ \\
\hline 175832,000000 & 215,486200 & 83,708080 & 0,004641 & 0,011946 \\
\hline 197286,800000 & 209,057400 & 85,394470 & 0,004783 & 0,011710 \\
\hline 221359,400000 & 202,341800 & 87,134540 & 0,004942 & 0,011477 \\
\hline 248369,400000 & 195,361600 & 88,774590 & 0,005119 & 0,011264 \\
\hline 278675,000000 & 188,149000 & 90,295300 & 0,005315 & 0,011075 \\
\hline 312678,500000 & 180,660600 & 91,639940 & 0,005535 & 0,010912 \\
\hline 350831,100000 & 172,926000 & 92,860820 & 0,005783 & 0,010769 \\
\hline 393638,900000 & 165,045600 & 93,701810 & 0,006059 & 0,010672 \\
\hline 441670,100000 & 156,921400 & 94,341120 & 0,006373 & 0,010600 \\
\hline 495562,000000 & 148,660800 & 94,647280 & 0,006727 & 0,010566 \\
\hline 556029,800000 & 140,283100 & 94,596360 & 0,007128 & 0,010571 \\
\hline 623875,700000 & 131,866100 & 94,135510 & 0,007583 & 0,010623 \\
\hline 700000,000000 & 123,460400 & 93,308680 & 0,008100 & 0,010717 \\
\hline 785412,900000 & 115,080200 & 92,066100 & 0,008690 & 0,010862 \\
\hline 881247,800000 & 106,785400 & 90,399200 & 0,009365 & 0,011062 \\
\hline 988776,300000 & 98,670090 & 88,310470 & 0,010135 & 0,011324 \\
\hline
\end{tabular}




\begin{tabular}{|c|c|c|c|c|}
\hline $\begin{array}{c}\text { frequency } \\
{[\mathrm{Hz}]}\end{array}$ & $\begin{array}{c}\text { real impedance } \\
\text { at } 500 \text { bar } \\
Z^{\prime}[\Omega \mathrm{m}]\end{array}$ & $\begin{array}{l}\text { imaginary } \\
\text { impedance } \\
\text { at } 500 \text { bar } \\
Z^{\prime \prime}[\Omega \mathrm{m}]\end{array}$ & $\begin{array}{c}\text { real admittance } \\
\text { at } 500 \text { bar } \\
Y^{\prime}[S \text { m1] }\end{array}$ & $\begin{array}{c}\text { imaginary } \\
\text { admittance } \\
\text { at } 500 \text { bar } \\
Y^{\prime \prime}[\mathrm{S} \mathrm{m1}]\end{array}$ \\
\hline 785,412900 & 437,315700 & 74,320590 & 0,002287 & 0,013455 \\
\hline 881,247700 & 432,201900 & 73,684270 & 0,002314 & 0,013571 \\
\hline 988,776200 & 426,550600 & 72,797360 & 0,002344 & 0,013737 \\
\hline 1109,425000 & 421,168900 & 72,030140 & 0,002374 & 0,013883 \\
\hline 1244,796000 & 416,069800 & 71,307460 & 0,002403 & 0,014024 \\
\hline 1396,684000 & 410,958800 & 70,652950 & 0,002433 & 0,014154 \\
\hline 1567,105000 & 406,142700 & 69,970850 & 0,002462 & 0,014292 \\
\hline 1758,320000 & 401,057400 & 69,166790 & 0,002493 & 0,014458 \\
\hline 1972,868000 & 395,960400 & 68,430020 & 0,002526 & 0,014613 \\
\hline 2213,594000 & 391,134000 & 67,806740 & 0,002557 & 0,014748 \\
\hline 2483,694000 & 386,308400 & 67,178430 & 0,002589 & 0,014886 \\
\hline 2786,750000 & 381,471900 & 66,611640 & 0,002621 & 0,015012 \\
\hline 3126,785000 & 376,941100 & 65,956020 & 0,002653 & 0,015162 \\
\hline 3508,311000 & 372,106400 & 65,377680 & 0,002687 & 0,015296 \\
\hline 3936,389000 & 367,554200 & 64,842310 & 0,002721 & 0,015422 \\
\hline 4416,701000 & 363,003000 & 64,300620 & 0,002755 & 0,015552 \\
\hline 4955,620000 & 358,441700 & 63,815140 & 0,002790 & 0,015670 \\
\hline 5560,297000 & 353,859300 & 63,445210 & 0,002826 & 0,015762 \\
\hline 6238,757000 & 349,559800 & 63,115010 & 0,002861 & 0,015844 \\
\hline 7000,000000 & 345,261700 & 62,774400 & 0,002896 & 0,015930 \\
\hline 7854,129000 & 340,683600 & 62,371880 & 0,002935 & 0,016033 \\
\hline 8812,479000 & 336,355700 & 62,186160 & 0,002973 & 0,016081 \\
\hline 9887,763000 & 332,310800 & 62,038020 & 0,003009 & 0,016119 \\
\hline 11094,250000 & 327,975500 & 61,880200 & 0,003049 & 0,016160 \\
\hline 12447,960000 & 323,631300 & 61,762420 & 0,003090 & 0,016191 \\
\hline 13966,840000 & 319,267500 & 61,738130 & 0,003132 & 0,016197 \\
\hline 15671,050000 & 315,175700 & 61,802870 & 0,003173 & 0,016180 \\
\hline 17583,210000 & 311,075000 & 61,900880 & 0,003215 & 0,016155 \\
\hline 19728,680000 & 306,462700 & 62,206730 & 0,003263 & 0,016075 \\
\hline 22135,950000 & 302,238600 & 62,448010 & 0,003309 & 0,016013 \\
\hline 24836,940000 & 298,050700 & 62,776350 & 0,003355 & 0,015930 \\
\hline 27867,500000 & 293,831700 & 63,227250 & 0,003403 & 0,015816 \\
\hline 31267,850000 & 289,564400 & 63,737460 & 0,003453 & 0,015689 \\
\hline 35083,110000 & 285,293100 & 64,364490 & 0,003505 & 0,015537 \\
\hline 39363,890000 & 280,961500 & 65,089190 & 0,003559 & 0,015364 \\
\hline 44167,020000 & 276,596600 & 65,910930 & 0,003615 & 0,015172 \\
\hline 49556,210000 & 272,114300 & 66,802470 & 0,003675 & 0,014970 \\
\hline 55602,980000 & 267,597600 & 67,776160 & 0,003737 & 0,014754 \\
\hline 62387,570000 & 263,009300 & 68,962630 & 0,003802 & 0,014501 \\
\hline 70000,000000 & 258,300600 & 70,189810 & 0,003871 & 0,014247 \\
\hline 78541,290000 & 253,433900 & 71,580830 & 0,003946 & 0,013970 \\
\hline 88124,780000 & 248,419600 & 73,074690 & 0,004025 & 0,013685 \\
\hline 98877,630000 & 243,280700 & 74,477580 & 0,004110 & 0,013427 \\
\hline 110942,500000 & 237,980900 & 76,091340 & 0,004202 & 0,013142 \\
\hline 124479,600000 & 232,462300 & 77,783580 & 0,004302 & 0,012856 \\
\hline 139668,400000 & 226,737700 & 79,492180 & 0,004410 & 0,012580 \\
\hline 156710,500000 & 220,859900 & 81,303310 & 0,004528 & 0,012300 \\
\hline
\end{tabular}




\begin{tabular}{|c|c|c|c|c|}
\hline $\begin{array}{c}\text { frequency } \\
{\left[\begin{array}{l}\mathrm{Hz}]\end{array}\right.}\end{array}$ & $\begin{array}{c}\text { real impedance } \\
\text { at } 500 \text { bar } \\
Z^{\prime}[\Omega \mathrm{m}]\end{array}$ & $\begin{array}{c}\text { imaginary } \\
\text { impedance } \\
\text { at } 500 \text { bar } \\
Z^{\prime \prime}[\Omega \mathrm{m}]\end{array}$ & $\begin{array}{c}\text { real admittance } \\
\text { at } 500 \text { bar } \\
Y^{\prime}[S \text { m1] }\end{array}$ & $\begin{array}{c}\text { imaginary } \\
\text { admittance } \\
\text { at } 500 \text { bar } \\
\mathrm{Y}^{\prime \prime}[\mathrm{S} \mathrm{m} 1]\end{array}$ \\
\hline 175832,000000 & 214,637900 & 83,206220 & 0,004659 & 0,012018 \\
\hline 197286,800000 & 208,204800 & 84,834380 & 0,004803 & 0,011788 \\
\hline 221359,400000 & 201,576900 & 86,596790 & 0,004961 & 0,011548 \\
\hline 248369,400000 & 194,672900 & 88,215920 & 0,005137 & 0,011336 \\
\hline 278675,000000 & 187,469400 & 89,727900 & 0,005334 & 0,011145 \\
\hline 312678,500000 & 180,041900 & 91,089270 & 0,005554 & 0,010978 \\
\hline 350831,100000 & 172,399800 & 92,268510 & 0,005800 & 0,010838 \\
\hline 393638,900000 & 164,562600 & 93,162040 & 0,006077 & 0,010734 \\
\hline 441670,100000 & 156,488700 & 93,783880 & 0,006390 & 0,010663 \\
\hline 495562,000000 & 148,285700 & 94,117870 & 0,006744 & 0,010625 \\
\hline 556029,800000 & 139,980500 & 94,072910 & 0,007144 & 0,010630 \\
\hline 623875,700000 & 131,594200 & 93,629920 & 0,007599 & 0,010680 \\
\hline 700000,000000 & 123,225100 & 92,860900 & 0,008115 & 0,010769 \\
\hline 785412,900000 & 114,895600 & 91,655720 & 0,008704 & 0,010910 \\
\hline 881247,800000 & 106,643100 & 89,991660 & 0,009377 & 0,011112 \\
\hline 988776,300000 & 98,558520 & 87,963200 & 0,010146 & 0,011368 \\
\hline
\end{tabular}




\begin{tabular}{|c|c|c|c|c|}
\hline $\begin{array}{c}\text { frequency } \\
{\left[\begin{array}{l}\mathrm{Hz}]\end{array}\right.}\end{array}$ & $\begin{array}{c}\text { real impedance } \\
\text { at } 600 \mathrm{bar} \\
Z^{\prime}[\Omega \mathrm{m}]\end{array}$ & $\begin{array}{l}\text { imaginary } \\
\text { impedance } \\
\text { at } 600 \mathrm{bar} \\
\mathrm{Z}^{\prime \prime}[\Omega \mathrm{m}]\end{array}$ & $\begin{array}{c}\text { real admittance } \\
\text { at } 600 \text { bar } \\
Y^{\prime}[S \text { m1] }\end{array}$ & $\begin{array}{c}\text { imaginary } \\
\text { admittance } \\
\text { at } 600 \text { bar } \\
\mathrm{Y}^{\prime \prime}[\mathrm{S} \mathrm{m1}]\end{array}$ \\
\hline 785,412900 & 435,060000 & 73,937250 & 0,002299 & 0,013525 \\
\hline 881,247700 & 429,946500 & 73,299740 & 0,002326 & 0,013643 \\
\hline 988,776200 & 424,564500 & 72,534630 & 0,002355 & 0,013787 \\
\hline 1109,425000 & 419,465000 & 71,814030 & 0,002384 & 0,013925 \\
\hline 1244,796000 & 414,071700 & 71,113760 & 0,002415 & 0,014062 \\
\hline 1396,684000 & 408,985700 & 70,313740 & 0,002445 & 0,014222 \\
\hline 1567,105000 & 403,888000 & 69,582390 & 0,002476 & 0,014371 \\
\hline 1758,320000 & 398,790700 & 68,847500 & 0,002508 & 0,014525 \\
\hline 1972,868000 & 393,963900 & 68,226540 & 0,002538 & 0,014657 \\
\hline 2213,594000 & 388,867900 & 67,483760 & 0,002572 & 0,014818 \\
\hline 2483,694000 & 384,324300 & 66,902480 & 0,002602 & 0,014947 \\
\hline 2786,750000 & 379,511300 & 66,201070 & 0,002635 & 0,015105 \\
\hline 3126,785000 & 374,969100 & 65,610950 & 0,002667 & 0,015241 \\
\hline 3508,311000 & 370,416200 & 65,080730 & 0,002700 & 0,015366 \\
\hline 3936,389000 & 365,582700 & 64,494500 & 0,002735 & 0,015505 \\
\hline 4416,701000 & 361,009400 & 64,077380 & 0,002770 & 0,015606 \\
\hline 4955,620000 & 356,459600 & 63,526410 & 0,002805 & 0,015741 \\
\hline 5560,297000 & 352,181200 & 63,080940 & 0,002839 & 0,015853 \\
\hline 6238,757000 & 347,589700 & 62,759280 & 0,002877 & 0,015934 \\
\hline 7000,000000 & 343,292000 & 62,416280 & 0,002913 & 0,016021 \\
\hline 7854,129000 & 338,984800 & 62,121990 & 0,002950 & 0,016097 \\
\hline 8812,479000 & 334,679100 & 61,815810 & 0,002988 & 0,016177 \\
\hline 9887,763000 & 330,353500 & 61,612990 & 0,003027 & 0,016230 \\
\hline 11094,250000 & 326,289200 & 61,562050 & 0,003065 & 0,016244 \\
\hline 12447,960000 & 321,945700 & 61,440750 & 0,003106 & 0,016276 \\
\hline 13966,840000 & 317,863500 & 61,466640 & 0,003146 & 0,016269 \\
\hline 15671,050000 & 313,502500 & 61,417980 & 0,003190 & 0,016282 \\
\hline 17583,210000 & 309,392000 & 61,565980 & 0,003232 & 0,016243 \\
\hline 19728,680000 & 304,942700 & 61,787430 & 0,003279 & 0,016185 \\
\hline 22135,950000 & 300,765000 & 62,088840 & 0,003325 & 0,016106 \\
\hline 24836,940000 & 296,578300 & 62,412200 & 0,003372 & 0,016023 \\
\hline 27867,500000 & 292,399700 & 62,812390 & 0,003420 & 0,015920 \\
\hline 31267,850000 & 288,150900 & 63,373650 & 0,003470 & 0,015779 \\
\hline 35083,110000 & 283,937300 & 64,006550 & 0,003522 & 0,015623 \\
\hline 39363,890000 & 279,551800 & 64,711230 & 0,003577 & 0,015453 \\
\hline 44167,020000 & 275,284100 & 65,496680 & 0,003633 & 0,015268 \\
\hline 49556,210000 & 270,859900 & 66,394310 & 0,003692 & 0,015062 \\
\hline 55602,980000 & 266,334000 & 67,406680 & 0,003755 & 0,014835 \\
\hline 62387,570000 & 261,732900 & 68,530380 & 0,003821 & 0,014592 \\
\hline 70000,000000 & 257,027900 & 69,747660 & 0,003891 & 0,014337 \\
\hline 78541,290000 & 252,232700 & 71,099020 & 0,003965 & 0,014065 \\
\hline 88124,780000 & 247,305200 & 72,606300 & 0,004044 & 0,013773 \\
\hline 98877,630000 & 242,170800 & 73,999220 & 0,004129 & 0,013514 \\
\hline 110942,500000 & 236,903700 & 75,610250 & 0,004221 & 0,013226 \\
\hline 124479,600000 & 231,445000 & 77,308530 & 0,004321 & 0,012935 \\
\hline 139668,400000 & 225,726500 & 79,005030 & 0,004430 & 0,012657 \\
\hline 156710,500000 & 219,869600 & 80,764580 & 0,004548 & 0,012382 \\
\hline
\end{tabular}




\begin{tabular}{|c|c|c|c|c|}
\hline $\begin{array}{c}\text { frequency } \\
{\left[\begin{array}{l}\mathrm{Hz}]\end{array}\right.}\end{array}$ & $\begin{array}{c}\text { real impedance } \\
\text { at } 600 \mathrm{bar} \\
\mathrm{Z}^{\prime}[\Omega \mathrm{m}]\end{array}$ & $\begin{array}{c}\text { imaginary } \\
\text { impedance } \\
\text { at } 600 \mathrm{bar} \\
\mathrm{Z}^{\prime \prime}[\Omega \mathrm{m}]\end{array}$ & $\begin{array}{c}\text { real admittance } \\
\text { at } 600 \text { bar } \\
Y^{\prime}[S \text { m1] }\end{array}$ & $\begin{array}{c}\text { imaginary } \\
\text { admittance } \\
\text { at } 600 \text { bar } \\
\mathrm{Y}^{\prime \prime}[\mathrm{S} \mathrm{m} 1]\end{array}$ \\
\hline 175832,000000 & 213,723400 & 82,637350 & 0,004679 & 0,012101 \\
\hline 197286,800000 & 207,389600 & 84,333550 & 0,004822 & 0,011858 \\
\hline 221359,400000 & 200,799800 & 86,014130 & 0,004980 & 0,011626 \\
\hline 248369,400000 & 193,931100 & 87,635240 & 0,005156 & 0,011411 \\
\hline 278675,000000 & 186,788900 & 89,162030 & 0,005354 & 0,011216 \\
\hline 312678,500000 & 179,438000 & 90,508710 & 0,005573 & 0,011049 \\
\hline 350831,100000 & 171,799100 & 91,754330 & 0,005821 & 0,010899 \\
\hline 393638,900000 & 164,028700 & 92,595480 & 0,006096 & 0,010800 \\
\hline 441670,100000 & 156,013900 & 93,240590 & 0,006410 & 0,010725 \\
\hline 495562,000000 & 147,861000 & 93,559110 & 0,006763 & 0,010688 \\
\hline 556029,800000 & 139,612700 & 93,543150 & 0,007163 & 0,010690 \\
\hline 623875,700000 & 131,281600 & 93,131770 & 0,007617 & 0,010737 \\
\hline 700000,000000 & 122,959100 & 92,358150 & 0,008133 & 0,010827 \\
\hline 785412,900000 & 114,658800 & 91,172610 & 0,008722 & 0,010968 \\
\hline 881247,800000 & 106,477800 & 89,566380 & 0,009392 & 0,011165 \\
\hline 988776,300000 & 98,418720 & 87,561230 & 0,010161 & 0,011421 \\
\hline
\end{tabular}




\begin{tabular}{|c|c|c|c|c|}
\hline $\begin{array}{c}\text { frequency } \\
{[\mathrm{Hz}]}\end{array}$ & $\begin{array}{c}\text { real impedance } \\
\text { at } 700 \text { bar } \\
Z^{\prime}[\Omega \mathrm{m}]\end{array}$ & $\begin{array}{c}\text { imaginary } \\
\text { impedance } \\
\text { at } 700 \text { bar } \\
\mathrm{Z}^{\prime \prime}[\Omega \mathrm{m}]\end{array}$ & $\begin{array}{c}\text { real admittance } \\
\text { at } 700 \text { bar } \\
Y^{\prime}[S \text { m1] }\end{array}$ & $\begin{array}{c}\text { imaginary } \\
\text { admittance } \\
\text { at } 700 \text { bar } \\
\mathrm{Y}^{\prime \prime}[\mathrm{S} \mathrm{m1}] \\
\end{array}$ \\
\hline 785,412900 & 433,342500 & 73,800930 & 0,002308 & 0,013550 \\
\hline 881,247700 & 427,960200 & 73,037940 & 0,002337 & 0,013692 \\
\hline 988,776200 & 422,847700 & 72,393180 & 0,002365 & 0,013813 \\
\hline 1109,425000 & 417,479200 & 71,549030 & 0,002395 & 0,013976 \\
\hline 1244,796000 & 412,380500 & 70,823300 & 0,002425 & 0,014120 \\
\hline 1396,684000 & 407,282300 & 70,094030 & 0,002455 & 0,014267 \\
\hline 1567,105000 & 402,184800 & 69,361210 & 0,002486 & 0,014417 \\
\hline 1758,320000 & 397,357600 & 68,742860 & 0,002517 & 0,014547 \\
\hline 1972,868000 & 392,261200 & 68,002150 & 0,002549 & 0,014705 \\
\hline 2213,594000 & 387,435500 & 67,374440 & 0,002581 & 0,014842 \\
\hline 2483,694000 & 382,903900 & 66,724040 & 0,002612 & 0,014987 \\
\hline 2786,750000 & 378,079500 & 66,087240 & 0,002645 & 0,015132 \\
\hline 3126,785000 & 373,256000 & 65,445420 & 0,002679 & 0,015280 \\
\hline 3508,311000 & 368,714800 & 64,848100 & 0,002712 & 0,015421 \\
\hline 3936,389000 & 364,152000 & 64,373100 & 0,002746 & 0,015534 \\
\hline 4416,701000 & 359,590200 & 63,890200 & 0,002781 & 0,015652 \\
\hline 4955,620000 & 355,040700 & 63,337450 & 0,002817 & 0,015788 \\
\hline 5560,297000 & 350,751700 & 62,951190 & 0,002851 & 0,015885 \\
\hline 6238,757000 & 346,182400 & 62,505200 & 0,002889 & 0,015999 \\
\hline 7000,000000 & 341,874200 & 62,220100 & 0,002925 & 0,016072 \\
\hline 7854,129000 & 337,567400 & 61,923110 & 0,002962 & 0,016149 \\
\hline 8812,479000 & 333,251400 & 61,672350 & 0,003001 & 0,016215 \\
\hline 9887,763000 & 328,937000 & 61,408190 & 0,003040 & 0,016284 \\
\hline 11094,250000 & 324,873300 & 61,353600 & 0,003078 & 0,016299 \\
\hline 12447,960000 & 320,530400 & 61,228590 & 0,003120 & 0,016332 \\
\hline 13966,840000 & 316,459500 & 61,195140 & 0,003160 & 0,016341 \\
\hline 15671,050000 & 312,088500 & 61,197500 & 0,003204 & 0,016341 \\
\hline 17583,210000 & 307,687600 & 61,338430 & 0,003250 & 0,016303 \\
\hline 19728,680000 & 303,586500 & 61,567780 & 0,003294 & 0,016242 \\
\hline 22135,950000 & 299,420500 & 61,811290 & 0,003340 & 0,016178 \\
\hline 24836,940000 & 295,234900 & 62,129510 & 0,003387 & 0,016095 \\
\hline 27867,500000 & 291,074600 & 62,580850 & 0,003436 & 0,015979 \\
\hline 31267,850000 & 286,849100 & 63,034870 & 0,003486 & 0,015864 \\
\hline 35083,110000 & 282,648200 & 63,612340 & 0,003538 & 0,015720 \\
\hline 39363,890000 & 278,364900 & 64,385320 & 0,003592 & 0,015531 \\
\hline 44167,020000 & 274,059800 & 65,205400 & 0,003649 & 0,015336 \\
\hline 49556,210000 & 269,677000 & 66,054490 & 0,003708 & 0,015139 \\
\hline 55602,980000 & 265,181200 & 67,065700 & 0,003771 & 0,014911 \\
\hline 62387,570000 & 260,594700 & 68,135210 & 0,003837 & 0,014677 \\
\hline 70000,000000 & 255,920400 & 69,351290 & 0,003907 & 0,014419 \\
\hline 78541,290000 & 251,128700 & 70,693280 & 0,003982 & 0,014146 \\
\hline 88124,780000 & 246,205300 & 72,190080 & 0,004062 & 0,013852 \\
\hline 98877,630000 & 241,157200 & 73,597500 & 0,004147 & 0,013587 \\
\hline 110942,500000 & 235,907700 & 75,156360 & 0,004239 & 0,013306 \\
\hline 124479,600000 & 230,481600 & 76,852670 & 0,004339 & 0,013012 \\
\hline 139668,400000 & 224,863600 & 78,526910 & 0,004447 & 0,012734 \\
\hline 156710,500000 & 218,986100 & 80,266650 & 0,004566 & 0,012458 \\
\hline
\end{tabular}




\begin{tabular}{|c|c|c|c|c|}
\hline $\begin{array}{c}\text { frequency } \\
{\left[\begin{array}{l}\mathrm{Hz}]\end{array}\right.}\end{array}$ & $\begin{array}{c}\text { real impedance } \\
\text { at } 700 \text { bar } \\
Z^{\prime}[\Omega \mathrm{m}]\end{array}$ & $\begin{array}{c}\text { imaginary } \\
\text { impedance } \\
\text { at } 700 \text { bar } \\
Z^{\prime \prime}[\Omega \mathrm{m}]\end{array}$ & $\begin{array}{c}\text { real admittance } \\
\text { at } 700 \text { bar } \\
Y^{\prime}[S \text { m1] }\end{array}$ & $\begin{array}{c}\text { imaginary } \\
\text { admittance } \\
\text { at } 700 \text { bar } \\
\mathrm{Y}^{\prime \prime}[\mathrm{S} \mathrm{m1}] \\
\end{array}$ \\
\hline 175832,000000 & 212,900400 & 82,148450 & 0,004697 & 0,012173 \\
\hline 197286,800000 & 206,603100 & 83,761830 & 0,004840 & 0,011939 \\
\hline 221359,400000 & 200,033200 & 85,479320 & 0,004999 & 0,011699 \\
\hline 248369,400000 & 193,199200 & 87,101660 & 0,005176 & 0,011481 \\
\hline 278675,000000 & 186,159100 & 88,622250 & 0,005372 & 0,011284 \\
\hline 312678,500000 & 178,817300 & 89,960950 & 0,005592 & 0,011116 \\
\hline 350831,100000 & 171,254700 & 91,194950 & 0,005839 & 0,010966 \\
\hline 393638,900000 & 163,518600 & 92,044390 & 0,006116 & 0,010864 \\
\hline 441670,100000 & 155,603300 & 92,700740 & 0,006427 & 0,010787 \\
\hline 495562,000000 & 147,507600 & 93,047500 & 0,006779 & 0,010747 \\
\hline 556029,800000 & 139,291200 & 93,046380 & 0,007179 & 0,010747 \\
\hline 623875,700000 & 131,037800 & 92,684230 & 0,007631 & 0,010789 \\
\hline 700000,000000 & 122,721600 & 91,912150 & 0,008149 & 0,010880 \\
\hline 785412,900000 & 114,472200 & 90,763760 & 0,008736 & 0,011018 \\
\hline 881247,800000 & 106,317800 & 89,178760 & 0,009406 & 0,011213 \\
\hline 988776,300000 & 98,320710 & 87,197870 & 0,010171 & 0,011468 \\
\hline
\end{tabular}




\begin{tabular}{|c|c|c|c|c|}
\hline $\begin{array}{c}\text { frequency } \\
{[\mathrm{Hz}]}\end{array}$ & $\begin{array}{c}\text { real impedance } \\
\text { at } 800 \text { bar } \\
Z^{\prime}[\Omega \mathrm{m}]\end{array}$ & $\begin{array}{l}\text { imaginary } \\
\text { impedance } \\
\text { at } 800 \text { bar } \\
\mathrm{Z}^{\prime \prime}[\Omega \mathrm{m}]\end{array}$ & $\begin{array}{c}\text { real admittance } \\
\text { at } 800 \text { bar } \\
Y^{\prime}[S \text { m1] }\end{array}$ & $\begin{array}{l}\text { imaginary } \\
\text { admittance } \\
\text { at } 800 \text { bar } \\
\mathrm{Y}^{\prime \prime}[\mathrm{S} \mathrm{m1}]\end{array}$ \\
\hline 785,412900 & 453,628800 & 77,337240 & 0,002204 & 0,012930 \\
\hline 881,247700 & 447,976800 & 76,454080 & 0,002232 & 0,013080 \\
\hline 988,776200 & 442,607100 & 75,617110 & 0,002259 & 0,013225 \\
\hline 1109,425000 & 437,224600 & 74,854550 & 0,002287 & 0,013359 \\
\hline 1244,796000 & 431,829800 & 74,163570 & 0,002316 & 0,013484 \\
\hline 1396,684000 & 426,461400 & 73,318180 & 0,002345 & 0,013639 \\
\hline 1567,105000 & 421,387800 & 72,445920 & 0,002373 & 0,013803 \\
\hline 1758,320000 & 416,276700 & 71,791530 & 0,002402 & 0,013929 \\
\hline 1972,868000 & 411,216000 & 70,844870 & 0,002432 & 0,014115 \\
\hline 2213,594000 & 406,081500 & 70,325070 & 0,002463 & 0,014220 \\
\hline 2483,694000 & 401,009100 & 69,446620 & 0,002494 & 0,014400 \\
\hline 2786,750000 & 396,194400 & 68,755200 & 0,002524 & 0,014544 \\
\hline 3126,785000 & 391,380300 & 68,060420 & 0,002555 & 0,014693 \\
\hline 3508,311000 & 386,566700 & 67,362310 & 0,002587 & 0,014845 \\
\hline 3936,389000 & 381,742000 & 66,727430 & 0,002620 & 0,014986 \\
\hline 4416,701000 & 377,188200 & 66,202710 & 0,002651 & 0,015105 \\
\hline 4955,620000 & 372,365200 & 65,557080 & 0,002686 & 0,015254 \\
\hline 5560,297000 & 367,813200 & 65,020320 & 0,002719 & 0,015380 \\
\hline 6238,757000 & 363,262200 & 64,477240 & 0,002753 & 0,015509 \\
\hline 7000,000000 & 358,689800 & 64,052990 & 0,002788 & 0,015612 \\
\hline 7854,129000 & 354,129700 & 63,557460 & 0,002824 & 0,015734 \\
\hline 8812,479000 & 349,852300 & 63,104790 & 0,002858 & 0,015847 \\
\hline 9887,763000 & 345,543100 & 62,825560 & 0,002894 & 0,015917 \\
\hline 11094,250000 & 341,235300 & 62,534420 & 0,002931 & 0,015991 \\
\hline 12447,960000 & 336,669500 & 62,061980 & 0,002970 & 0,016113 \\
\hline 13966,840000 & 332,624400 & 61,916450 & 0,003006 & 0,016151 \\
\hline 15671,050000 & 328,299700 & 61,704160 & 0,003046 & 0,016206 \\
\hline 17583,210000 & 323,966000 & 61,533490 & 0,003087 & 0,016251 \\
\hline 19728,680000 & 319,623500 & 61,402180 & 0,003129 & 0,016286 \\
\hline 22135,950000 & 315,542400 & 61,417600 & 0,003169 & 0,016282 \\
\hline 24836,940000 & 311,182500 & 61,358130 & 0,003214 & 0,016298 \\
\hline 27867,500000 & 306,753900 & 61,486200 & 0,003260 & 0,016264 \\
\hline 31267,850000 & 302,562600 & 61,579960 & 0,003305 & 0,016239 \\
\hline 35083,110000 & 298,295900 & 61,741900 & 0,003352 & 0,016196 \\
\hline 39363,890000 & 294,037900 & 61,984730 & 0,003401 & 0,016133 \\
\hline 44167,020000 & 289,721600 & 62,342830 & 0,003452 & 0,016040 \\
\hline 49556,210000 & 285,390500 & 62,609980 & 0,003504 & 0,015972 \\
\hline 55602,980000 & 281,018100 & 63,039490 & 0,003558 & 0,015863 \\
\hline 62387,570000 & 276,581300 & 63,464690 & 0,003616 & 0,015757 \\
\hline 70000,000000 & 272,085800 & 63,983940 & 0,003675 & 0,015629 \\
\hline 78541,290000 & 267,542600 & 64,543100 & 0,003738 & 0,015494 \\
\hline 88124,780000 & 262,817800 & 65,201170 & 0,003805 & 0,015337 \\
\hline 98877,630000 & 258,068200 & 65,793940 & 0,003875 & 0,015199 \\
\hline 110942,500000 & 253,232000 & 66,446180 & 0,003949 & 0,015050 \\
\hline 124479,600000 & 248,316600 & 67,011840 & 0,004027 & 0,014923 \\
\hline 139668,400000 & 243,276100 & 67,659050 & 0,004111 & 0,014780 \\
\hline 156710,500000 & 238,079200 & 68,276580 & 0,004200 & 0,014646 \\
\hline
\end{tabular}




\begin{tabular}{|c|c|c|c|c|}
\hline $\begin{array}{c}\text { frequency } \\
{\left[\begin{array}{l}\mathrm{Hz}]\end{array}\right.}\end{array}$ & $\begin{array}{c}\text { real impedance } \\
\text { at } 800 \text { bar } \\
Z^{\prime}[\Omega \mathrm{m}]\end{array}$ & $\begin{array}{c}\text { imaginary } \\
\text { impedance } \\
\text { at } 800 \text { bar } \\
Z^{\prime \prime}[\Omega \mathrm{m}]\end{array}$ & $\begin{array}{c}\text { real admittance } \\
\text { at } 800 \text { bar } \\
Y^{\prime}[S \text { m1] }\end{array}$ & $\begin{array}{c}\text { imaginary } \\
\text { admittance } \\
\text { at } 800 \text { bar } \\
\mathrm{Y}^{\prime \prime}[\mathrm{S} \mathrm{m1}] \\
\end{array}$ \\
\hline 175832,000000 & 232,785500 & 68,961400 & 0,004296 & 0,014501 \\
\hline 197286,800000 & 227,370500 & 69,390010 & 0,004398 & 0,014411 \\
\hline 221359,400000 & 221,806700 & 69,855080 & 0,004508 & 0,014315 \\
\hline 248369,400000 & 216,131900 & 70,229130 & 0,004627 & 0,014239 \\
\hline 278675,000000 & 210,279100 & 70,442500 & 0,004756 & 0,014196 \\
\hline 312678,500000 & 204,305500 & 70,508980 & 0,004895 & 0,014183 \\
\hline 350831,100000 & 198,189100 & 70,494310 & 0,005046 & 0,014186 \\
\hline 393638,900000 & 192,040700 & 70,010390 & 0,005207 & 0,014284 \\
\hline 441670,100000 & 185,766300 & 69,416770 & 0,005383 & 0,014406 \\
\hline 495562,000000 & 179,452400 & 68,488200 & 0,005573 & 0,014601 \\
\hline 556029,800000 & 173,178000 & 67,273000 & 0,005774 & 0,014865 \\
\hline 623875,700000 & 166,933000 & 65,652700 & 0,005990 & 0,015232 \\
\hline 700000,000000 & 160,951300 & 63,592140 & 0,006213 & 0,015725 \\
\hline 785412,900000 & 155,126100 & 61,290590 & 0,006446 & 0,016316 \\
\hline 881247,800000 & 149,477300 & 58,697290 & 0,006690 & 0,017037 \\
\hline 988776,300000 & 144,054400 & 55,757240 & 0,006942 & 0,017935 \\
\hline
\end{tabular}


III.7 CALCUlations OF BULK CONDUCTIVITIES OF SAMPLE 17/08/00/11 CORE 2:

\begin{tabular}{|c|c|c|c|c|}
\hline $\begin{array}{c}\text { pressure } \\
\text { measurement 2 } \\
{[\mathbf{b a r}]}\end{array}$ & $\begin{array}{c}\text { resistivity R1-1of } \\
\text { elem. cell 1 } \\
{[\mathbf{\Omega}]}\end{array}$ & $\begin{array}{c}\text { capacity C1-1 * } \mathbf{E}^{-7} \\
\text { of elem. cell 1 } \\
{[\mathbf{F}]}\end{array}$ & $\begin{array}{c}\text { resistivity R2-1 of } \\
\text { elem. cell 2 } \\
{[\mathbf{\Omega}]}\end{array}$ & $\begin{array}{c}\text { capacity C2-1 * } \mathbf{E}^{-\mathbf{9}} \\
\text { of elem. cell 2 } \\
{[\mathbf{F}]}\end{array}$ \\
\hline 0 & 82 & 3,58 & 142 & 0,699 \\
\hline 25 & 115 & 3,59 & 141 & 0,917 \\
\hline 50 & 136 & 3,44 & 135 & 1,11 \\
\hline 75 & 139 & 3,45 & 132 & 1,17 \\
\hline 100 & 141 & 3,46 & 131 & 1,2 \\
\hline 150 & 142 & 3,46 & 131 & 1,2 \\
\hline 200 & 142 & 3,46 & 131 & 1,21 \\
\hline 250 & 141 & 3,47 & 130 & 1,21 \\
\hline 300 & 141 & 3,47 & 130 & 1,22 \\
\hline 400 & 141 & 3,5 & 129 & 1,3 \\
\hline 500 & 140 & 3,52 & 129 & 1,24 \\
\hline 600 & 140 & 3,55 & 128 & 1,25 \\
\hline 700 & 140 & 3,55 & 128 & 1,25 \\
\hline 800 & 148 & 2,66 & 138 & 0,772 \\
\hline
\end{tabular}

\begin{tabular}{|c|c|c|}
\hline $\begin{array}{c}\text { resistivity R3-1 of } \\
\text { elem. cell 3 } \\
{[\mathbf{\Omega}]}\end{array}$ & $\begin{array}{c}\text { capacity C3-1 * } \mathbf{E}^{-\mathbf{9}} \\
\text { of elem. cell 3 } \\
{[\mathbf{F}]}\end{array}$ & $\mathbf{X 2 - 2}$ \\
\hline 178 & 8,15 & 0,0644 \\
\hline 182 & 8,24 & 0,104 \\
\hline 180 & 8,27 & 0,124 \\
\hline 180 & 8,28 & 0,128 \\
\hline 180 & 8,29 & 0,13 \\
\hline 179 & 8,29 & 0,13 \\
\hline 179 & 8,3 & 0,131 \\
\hline 179 & 8,3 & 0,13 \\
\hline 178 & 8,3 & 0,13 \\
\hline 178 & 8,31 & 0,131 \\
\hline 177 & 8,31 & 0,131 \\
\hline 176 & 8,32 & 0,13 \\
\hline 176 & 8,32 & 0,13 \\
\hline 176 & 2,84 & 0,176 \\
\hline surface resisitivity & surface resisitivity & \\
\hline
\end{tabular}




\section{III.8 STATISTICAL ANALYSIS OF BULK CONDUCTIVITIES OF SAMPLE 17/08/00/11}

\begin{tabular}{|c|c|c|c|}
\hline $\begin{array}{c}\text { pressure } \\
\text { core 1 } \\
\text { [bar] }\end{array}$ & $\begin{array}{c}\text { bulk resistivity } \\
\text { core 1 } \\
\mathbf{R}[\mathbf{\Omega} \text { m] }\end{array}$ & $\begin{array}{c}\text { pressure } \\
\text { core 2 } \\
\text { [bar] }\end{array}$ & $\begin{array}{c}\text { bulk resistivity } \\
\text { core 2 } \\
\mathbf{R}[\mathbf{\Omega} \text { m] }\end{array}$ \\
\hline 0,000000 & 542,691000 & 25,000000 & 400,972000 \\
\hline 75,000000 & 537,642000 & 50,000000 & 425,854000 \\
\hline 100,000000 & 523,260000 & 75,000000 & 433,862000 \\
\hline 150,000000 & 511,326000 & 100,000000 & 433,004000 \\
\hline 200,000000 & 511,020000 & 150,000000 & 432,718000 \\
\hline 250,000000 & 509,490000 & 200,000000 & 433,290000 \\
\hline 300,000000 & 508,878000 & 250,000000 & 432,432000 \\
\hline 400,000000 & 506,430000 & 300,000000 & 431,288000 \\
\hline & & 400,000000 & 430,716000 \\
\hline & & 500,000000 & 428,714000 \\
\hline & & 600,000000 & 426,712000 \\
\hline & & 700,000000 & 424,424000 \\
\hline & & 800,000000 & 422,994000 \\
\hline & & & 413,842000 \\
\hline
\end{tabular}

\begin{tabular}{|l|c|l|c|}
\hline \multicolumn{2}{|c|}{ measurement 1 } & \multicolumn{1}{c|}{ measurement 2 } \\
\hline sum of bulk resistivity $[\Omega \mathrm{m}]$ & 4150,737000 & sum of bulk resistivity $[\Omega \mathrm{m}]$ & 5970,822000 \\
\hline av. bulk resistivity $[\Omega \mathrm{m}]$ & 518,842125 & av. bulk resistivity $[\Omega \mathrm{m}]$ & 426,4872857 \\
\hline av. bulk conductivity $\left[\mathrm{S} \mathrm{m}^{-1}\right]$ & 0,001927369 & av. bulk conductivity $\left[\mathrm{S} \mathrm{m}^{-1}\right]$ & 0,002344736 \\
\hline
\end{tabular}




\section{Stable Isotope Analysis}

\section{IV.1 OWN MEASUREMENT DATA AND CALCULATIONS:}

\begin{tabular}{|c|c|c|c|c|}
\hline samples & $\begin{array}{c}\mathbf{d}^{13} \mathbf{C}_{\text {calcite }} \mathrm{V} \text {-PDB } \\
\text { mean } \\
{[\% \text { ] }]} \\
\end{array}$ & $\begin{array}{c}\mathbf{d}^{18} \mathbf{O}_{\text {calcite }} \mathrm{V}-\mathrm{PDB} \\
\text { mean } \\
{[\%,]} \\
\%\end{array}$ & $\underset{[\% 0]}{d^{13} C_{\text {graphite }} \text { V-PDB }}$ & $\begin{array}{c}\Delta(\text { cal-gr }) \\
{[\% o]}\end{array}$ \\
\hline $12 / 08 / 00 / 02$ & 9,64 & $-13,99$ & 6,46 & 3,19 \\
\hline $12 / 08 / 00 / 03$ & 9,53 & $-13,55$ & & \\
\hline $15 / 08 / 00 / 01$ & 10,34 & $-12,20$ & 6,85 & 3,49 \\
\hline $17 / 08 / 00 / 04$ & 7,80 & $-11,45$ & 4,84 & 2,96 \\
\hline $17 / 08 / 00 / 05$ & 10,43 & $-10,10$ & 7,27 & 3,16 \\
\hline $17 / 08 / 00 / 10$ & 9,87 & $-11,20$ & 6,68 & 3,19 \\
\hline $17 / 08 / 00 / 11$ & 11,20 & $-10,23$ & 8,03 & 3,16 \\
\hline $17 / 08 / 00 / 12$ & 9,97 & $-11,02$ & 6,76 & 3,20 \\
\hline $17 / 08 / 00 / 13$ & 10,50 & $-8,49$ & 7,20 & 3,31 \\
\hline $17 / 08 / 00 / 15$ & 11,07 & $-10,89$ & 7,92 & 3,16 \\
\hline $17 / 08 / 00 / 16$ & 6,61 & $-9,80$ & 3,86 & 2,75 \\
\hline $17 / 08 / 00 / 17$ & 10,34 & $-10,34$ & 7,58 & 2,76 \\
\hline $17 / 08 / 00 / 19$ & 9,82 & $-12,51$ & 6,98 & 2,84 \\
\hline $17 / 08 / 00 / 20$ & 10,77 & $-7,15$ & 7,52 & 3,25 \\
\hline $17 / 08 / 00 / 21$ & 9,94 & $-11,65$ & 7,26 & 2,67 \\
\hline $18 / 08 / 00 / 02$ & 9,61 & $-11,70$ & 6,01 & 3,60 \\
\hline $3 / 10 / 01 / 3$ & 9,30 & $-11,38$ & 6,60 & 2,70 \\
\hline $3 / 10 / 01 / 4$ & 6,95 & $-11,68$ & 4,42 & 2,53 \\
\hline $3 / 10 / 01 / 5$ & 7,26 & $-9,46$ & 4,77 & 2,49 \\
\hline Elim Am & 11,08 & $-7,46$ & 7,06 & 4,02 \\
\hline Omaruru $\mathrm{Sp}$ & 8,63 & $-7,51$ & 6,18 & 2,45 \\
\hline $17 / 08 / 00 / 16 \mathrm{II}$ & & & 3,72 & \\
\hline 17/08/00/21 II & & & 7,29 & \\
\hline sum & 200,66 & $-223,74$ & 141,26 & 60,87 \\
\hline mean & 9,555047619 & $-10,65447619$ & 6,421045455 & 3,04355 \\
\hline $\min$ & 6,61 & $-13,99$ & 3,72 & 2,45 \\
\hline $\max$ & 11,2 & $-7,15$ & 8,03 & 4,02 \\
\hline standard deviation & 1,358982725 & 1,870403796 & 1,284107527 & 0,402266012 \\
\hline
\end{tabular}




\begin{tabular}{|c|c|c|c|c|}
\hline samples & ${ }^{13} \mathrm{C} /{ }^{12} \mathrm{C}$ calcite & ${ }^{13} \mathrm{C} /{ }^{12} \mathrm{C}$ graphite & $\alpha_{\text {cc-gr }}$ & $10^{3} \times \ln \alpha$ \\
\hline $12 / 08 / 00 / 02$ & 0,0113455491 & 0,0113097586 & 1,0031645664 & 3,159569693 \\
\hline \multicolumn{5}{|l|}{$12 / 08 / 00 / 03$} \\
\hline $15 / 08 / 00 / 01$ & 0,0113534151 & 0,0113141748 & 1,0034682425 & 3,462242056 \\
\hline $17 / 08 / 00 / 04$ & 0,0113248052 & 0,0112915880 & 1,0029417619 & 2,937443358 \\
\hline $17 / 08 / 00 / 05$ & 0,0113544377 & 0,0113189282 & 1,0031371833 & 3,132272574 \\
\hline $17 / 08 / 00 / 10$ & 0,0113481449 & 0,0113122645 & 1,0031718123 & 3,166792709 \\
\hline $17 / 08 / 00 / 11$ & 0,0113630005 & 0,0113274684 & 1,0031368021 & 3,131892569 \\
\hline $17 / 08 / 00 / 12$ & 0,0113491787 & 0,0113131972 & 1,0031804903 & 3,175443283 \\
\hline $17 / 08 / 00 / 13$ & 0,0113552243 & 0,0113180854 & 1,0032813806 & 3,276008641 \\
\hline $17 / 08 / 00 / 15$ & 0,0113616295 & 0,0113261761 & 1,0031302150 & 3,125326035 \\
\hline $17 / 08 / 00 / 16$ & 0,0113114779 & 0,0112805756 & 1,0027394258 & 2,735680428 \\
\hline $17 / 08 / 00 / 17$ & 0,0113533814 & 0,0113223780 & 1,0027382441 & 2,734501949 \\
\hline $17 / 08 / 00 / 19$ & 0,0113475830 & 0,0113156357 & 1,0028232934 & 2,819315405 \\
\hline $17 / 08 / 00 / 20$ & 0,0113582022 & 0,0113216925 & 1,0032247531 & 3,219564716 \\
\hline $17 / 08 / 00 / 21$ & 0,0113488641 & 0,0113188270 & 1,0026537234 & 2,650208447 \\
\hline $18 / 08 / 00 / 02$ & 0,0113452344 & 0,0113047356 & 1,0035824694 & 3,576067601 \\
\hline $3 / 10 / 01 / 3$ & 0,0113416947 & 0,0113113768 & 1,0026803073 & 2,676721674 \\
\hline $3 / 10 / 01 / 4$ & 0,0113152536 & 0,0112868235 & 1,0025188766 & 2,515709588 \\
\hline $3 / 10 / 01 / 5$ & 0,0113188158 & 0,0112908014 & 1,0024811648 & 2,478091836 \\
\hline Elim Am & 0,0113616632 & 0,0113165346 & 1,0039878458 & 3,979915428 \\
\hline Omaruru Sp & 0,0113341321 & 0,0113066459 & 1,0024309766 & 2,428026521 \\
\hline \multicolumn{5}{|l|}{$17 / 08 / 00 / 16 \mathrm{II}$} \\
\hline \multicolumn{5}{|l|}{ 17/08/00/21 II } \\
\hline sum & & & & 60,38079451 \\
\hline mean & & & & 3,019039726 \\
\hline $\min$ & & & & 2,428026521 \\
\hline $\max$ & & & & 3,979915428 \\
\hline standard deviation & & & & 0,397055763 \\
\hline
\end{tabular}




\section{IV.2 TYPES OF GRAPHITE-BEARING MARBLES:}

\begin{tabular}{|c|c|c|}
\hline Type I & Type II & Type III \\
\hline Elim Am & $12 / 08 / 00 / 02$ & $3 / 10 / 01 / 4$ \\
\hline Omaruru Sp & $12 / 08 / 00 / 03$ & $3 / 10 / 01 / 5$ \\
\hline & $15 / 08 / 00 / 01$ & \\
\hline & $17 / 08 / 00 / 04$ & \\
\hline & $17 / 08 / 00 / 05$ & \\
\hline & $17 / 08 / 00 / 10$ & \\
\hline & $17 / 08 / 00 / 11$ & \\
\hline & $17 / 08 / 00 / 12$ & \\
\hline & $17 / 08 / 00 / 13$ & \\
\hline & $17 / 08 / 00 / 15$ & \\
\hline & $17 / 08 / 00 / 16$ & \\
\hline & $17 / 08 / 00 / 17$ & \\
\hline & $17 / 08 / 00 / 19$ & \\
\hline & $17 / 08 / 00 / 20$ & \\
\hline & $17 / 08 / 00 / 21$ & \\
\hline & $18 / 08 / 00 / 02$ & \\
\hline & $3 / 10 / 01 / 3$ & \\
\hline
\end{tabular}




\section{IV.3 GEOTHERMOMETRY CALCULATIONS:}

\begin{tabular}{|c|c|c|c|c|}
\hline$\Delta$ (cc-gr) & $10^{3} x \ln \alpha$ & $\begin{array}{c}\text { Thermometer } \\
\text { Valley \& } \mathrm{O}^{\prime} \text { Neil } \\
1981\left[^{\circ} \mathrm{C}\right] \\
\left(610-760^{\circ} \mathrm{C}\right)\end{array}$ & $\begin{array}{c}\text { Thermometer } \\
\text { Wada \& Suzuki } \\
1982\left[^{\circ} \mathrm{C}\right] \\
\left(400-680^{\circ} \mathrm{C}\right)\end{array}$ & $\begin{array}{c}\text { Thermometer } \\
\text { Morikiyo } \\
1984\left[^{\circ} \mathrm{C}\right] \\
\left(270-650^{\circ} \mathrm{C}\right) \\
\end{array}$ \\
\hline 1 & 1 & 1027 & 1153 & 775 \\
\hline 1,1 & 1,1 & 1013 & 1129 & 769 \\
\hline 1,2 & 1,2 & 1000 & 1106 & 762 \\
\hline 1,3 & 1,3 & 987 & 1085 & 756 \\
\hline 1,4 & 1,4 & 973 & 1064 & 750 \\
\hline 1,5 & 1,5 & 960 & 1044 & 744 \\
\hline 1,6 & 1,6 & 947 & 1025 & 738 \\
\hline 1,7 & 1,7 & 933 & 1007 & 733 \\
\hline 1,8 & 1,8 & 920 & 990 & 727 \\
\hline 1,9 & 1,9 & 906 & 973 & 721 \\
\hline 2 & 2 & 893 & 957 & 716 \\
\hline 2,1 & 2,1 & 880 & 941 & 710 \\
\hline 2,2 & 2,2 & 866 & 926 & 705 \\
\hline 2,3 & 2,3 & 853 & 912 & 700 \\
\hline 2,4 & 2,4 & 840 & 898 & 695 \\
\hline 2,5 & 2,5 & 826 & 885 & 690 \\
\hline 2,6 & 2,6 & 813 & 872 & 685 \\
\hline 2,7 & 2,7 & 799 & 859 & 680 \\
\hline 2,8 & 2,8 & 786 & 847 & 675 \\
\hline 2,9 & 2,9 & 773 & 836 & 670 \\
\hline 3 & 3 & 759 & 824 & 666 \\
\hline 3,1 & 3,1 & 746 & 813 & 661 \\
\hline 3,2 & 3,2 & 733 & 802 & 656 \\
\hline 3,3 & 3,3 & 719 & 792 & 652 \\
\hline 3,4 & 3,4 & 706 & 782 & 648 \\
\hline 3,5 & 3,5 & 693 & 772 & 643 \\
\hline 3,6 & 3,6 & 679 & 763 & 639 \\
\hline 3,7 & 3,7 & 666 & 753 & 635 \\
\hline 3,8 & 3,8 & 652 & 744 & 630 \\
\hline 3,9 & 3,9 & 639 & 735 & 626 \\
\hline 4 & 4 & 626 & 727 & 622 \\
\hline 4,1 & 4,1 & 612 & 718 & 618 \\
\hline 4,2 & 4,2 & 599 & 710 & 614 \\
\hline 4,3 & 4,3 & 586 & 702 & 610 \\
\hline 4,4 & 4,4 & & 695 & 607 \\
\hline 4,5 & 4,5 & & 687 & 603 \\
\hline 4,6 & 4,6 & & 680 & 599 \\
\hline 4,7 & 4,7 & & 672 & 595 \\
\hline 4,8 & 4,8 & & 665 & 592 \\
\hline 4,9 & 4,9 & & 658 & 588 \\
\hline 5 & 5 & & 652 & 584 \\
\hline 5,1 & 5,1 & & 645 & 581 \\
\hline 5,2 & 5,2 & & 638 & 577 \\
\hline 5,3 & 5,3 & & 632 & 574 \\
\hline 5,4 & 5,4 & & 626 & 571 \\
\hline
\end{tabular}




\begin{tabular}{|c|c|c|c|c|}
\hline$\Delta$ (cc-gr) & $10^{3} x \ln \alpha$ & $\begin{array}{c}\text { Thermometer } \\
\text { Valley \& } \mathrm{O}^{\prime} \text { Neil } \\
1981\left[^{\circ} \mathrm{C}\right] \\
\left(610-760^{\circ} \mathrm{C}\right)\end{array}$ & $\begin{array}{c}\text { Thermometer } \\
\text { Wada \& Suzuki } \\
1982\left[{ }^{\circ} \mathrm{C}\right] \\
\left(400-680^{\circ} \mathrm{C}\right)\end{array}$ & $\begin{array}{c}\text { Thermometer } \\
\text { Morikiyo } \\
1984\left[^{\circ} \mathrm{C}\right] \\
\left(270-650^{\circ} \mathrm{C}\right)\end{array}$ \\
\hline 5,5 & 5,5 & & 620 & 567 \\
\hline 5,6 & 5,6 & & 614 & 564 \\
\hline 5,7 & 5,7 & & 608 & 561 \\
\hline 5,8 & 5,8 & & 602 & 557 \\
\hline 5,9 & 5,9 & & 597 & 554 \\
\hline 6 & 6 & & 591 & 551 \\
\hline 6,1 & 6,1 & & 586 & 548 \\
\hline 6,2 & 6,2 & & 580 & 545 \\
\hline 6,3 & 6,3 & & 575 & 542 \\
\hline 6,4 & 6,4 & & 570 & 539 \\
\hline 6,5 & 6,5 & & 565 & 536 \\
\hline 6,6 & 6,6 & & 560 & 533 \\
\hline 6,7 & 6,7 & & 555 & 530 \\
\hline 6,8 & 6,8 & & 550 & 527 \\
\hline 6,9 & 6,9 & & 546 & 524 \\
\hline 7 & 7 & & 541 & 521 \\
\hline 7,1 & 7,1 & & 537 & 519 \\
\hline 7,2 & 7,2 & & 532 & 516 \\
\hline 7,3 & 7,3 & & 528 & 513 \\
\hline 7,4 & 7,4 & & 523 & 510 \\
\hline 7,5 & 7,5 & & 519 & 508 \\
\hline 7,6 & 7,6 & & 515 & 505 \\
\hline 7,7 & 7,7 & & 511 & 502 \\
\hline 7,8 & 7,8 & & 507 & 500 \\
\hline 7,9 & 7,9 & & 503 & 497 \\
\hline 8 & 8 & & 499 & 495 \\
\hline 8,1 & 8,1 & & 495 & 492 \\
\hline 8,2 & 8,2 & & 491 & 490 \\
\hline 8,3 & 8,3 & & 487 & 487 \\
\hline 8,4 & 8,4 & & 484 & 485 \\
\hline 8,5 & 8,5 & & 480 & 482 \\
\hline 8,6 & 8,6 & & 476 & 480 \\
\hline 8,7 & 8,7 & & 473 & 477 \\
\hline 8,8 & 8,8 & & 469 & 475 \\
\hline 8,9 & 8,9 & & 466 & 473 \\
\hline 9 & 9 & & 463 & 470 \\
\hline 9,1 & 9,1 & & 459 & 468 \\
\hline 9,2 & 9,2 & & 456 & 466 \\
\hline 9,3 & 9,3 & & 453 & 464 \\
\hline 9,4 & 9,4 & & 449 & 461 \\
\hline 9,5 & 9,5 & & 446 & 459 \\
\hline 9,6 & 9,6 & & 443 & 457 \\
\hline 9,7 & 9,7 & & 440 & 455 \\
\hline 9,8 & 9,8 & & 437 & 453 \\
\hline 9,9 & 9,9 & & 434 & 450 \\
\hline 10 & 10 & & 431 & 448 \\
\hline 10,1 & 10,1 & & 428 & 446 \\
\hline
\end{tabular}




\begin{tabular}{|c|c|c|c|c|}
\hline$\Delta$ (cc-gr) & $10^{3} x \ln \alpha$ & $\begin{array}{c}\text { Thermometer } \\
\text { Valley \& } \mathrm{O}^{\prime} \text { Neil } \\
1981\left[^{\circ} \mathrm{C}\right] \\
\left(610-760^{\circ} \mathrm{C}\right) \\
\end{array}$ & $\begin{array}{c}\text { Thermometer } \\
\text { Wada \& Suzuki } \\
1982\left[{ }^{\circ} \mathrm{C}\right] \\
\left(400-680^{\circ} \mathrm{C}\right) \\
\end{array}$ & $\begin{array}{c}\text { Thermometer } \\
\text { Morikiyo } \\
1984\left[^{\circ} \mathrm{C}\right] \\
\left(270-650^{\circ} \mathrm{C}\right) \\
\end{array}$ \\
\hline 10,2 & 10,2 & & 425 & 444 \\
\hline 10,3 & 10,3 & & 422 & 442 \\
\hline 10,4 & 10,4 & & 419 & 440 \\
\hline 10,5 & 10,5 & & 417 & 438 \\
\hline 10,6 & 10,6 & & 414 & 436 \\
\hline 10,7 & 10,7 & & 411 & 434 \\
\hline 10,8 & 10,8 & & 408 & 432 \\
\hline 10,9 & 10,9 & & 406 & 430 \\
\hline 11 & 11 & & 403 & 428 \\
\hline 11,1 & 11,1 & & 401 & 426 \\
\hline 11,2 & 11,2 & & & 424 \\
\hline 11,3 & 11,3 & & & 422 \\
\hline 11,4 & 11,4 & & & 420 \\
\hline 11,5 & 11,5 & & & 419 \\
\hline 11,6 & 11,6 & & & 417 \\
\hline 11,7 & 11,7 & & & 415 \\
\hline 11,8 & 11,8 & & & 413 \\
\hline 11,9 & 11,9 & & & 411 \\
\hline 12 & 12 & & & 409 \\
\hline 12,1 & 12,1 & & & 408 \\
\hline 12,2 & 12,2 & & & 406 \\
\hline 12,3 & 12,3 & & & 404 \\
\hline 12,4 & 12,4 & & & 402 \\
\hline 12,5 & 12,5 & & & 401 \\
\hline 12,6 & 12,6 & & & 399 \\
\hline 12,7 & 12,7 & & & 397 \\
\hline 12,8 & 12,8 & & & 396 \\
\hline 12,9 & 12,9 & & & 394 \\
\hline 13 & 13 & & & 392 \\
\hline 13,1 & 13,1 & & & 391 \\
\hline 13,2 & 13,2 & & & 389 \\
\hline 13,3 & 13,3 & & & 387 \\
\hline 13,4 & 13,4 & & & 386 \\
\hline 13,5 & 13,5 & & & 384 \\
\hline 13,6 & 13,6 & & & 383 \\
\hline 13,7 & 13,7 & & & 381 \\
\hline 13,8 & 13,8 & & & 379 \\
\hline 13,9 & 13,9 & & & 378 \\
\hline 14 & 14 & & & 376 \\
\hline 14,1 & 14,1 & & & 375 \\
\hline 14,2 & 14,2 & & & 373 \\
\hline 14,3 & 14,3 & & & 372 \\
\hline 14,4 & 14,4 & & & 370 \\
\hline 14,5 & 14,5 & & & 369 \\
\hline 14,6 & 14,6 & & & 367 \\
\hline 14,7 & 14,7 & & & 366 \\
\hline 14,8 & 14,8 & & & 364 \\
\hline
\end{tabular}




\begin{tabular}{|c|c|c|c|c|}
\hline$\Delta$ (cc-gr) & $10^{3} x \ln \alpha$ & $\begin{array}{c}\text { Thermometer } \\
\text { Dunn \& Valley } \\
1992\left[\left[^{\circ} \mathrm{C}\right]\right. \\
\left(400-800^{\circ} \mathrm{C}\right) \\
\end{array}$ & $\begin{array}{c}\text { Thermometer } \\
\text { Kitchen \& Valley } \\
1995\left[{ }^{\circ} \mathrm{C}\right] \\
\left(650-850^{\circ} \mathrm{C}\right)\end{array}$ & $\begin{array}{l}\text { Thermometer } \\
\text { Chako et al } \\
1991\left[^{\circ} \mathrm{C}\right] \\
\left(400-700^{\circ} \mathrm{C}\right)\end{array}$ \\
\hline 1 & 1 & 995 & & 1710 \\
\hline 1,1 & 1,1 & 978 & & 1616 \\
\hline 1,2 & 1,2 & 962 & & 1533 \\
\hline 1,3 & 1,3 & 946 & & 1461 \\
\hline 1,4 & 1,4 & 931 & & 1396 \\
\hline 1,5 & 1,5 & 916 & & 1338 \\
\hline 1,6 & 1,6 & 902 & & 1285 \\
\hline 1,7 & 1,7 & 888 & & 1237 \\
\hline 1,8 & 1,8 & 875 & & 1193 \\
\hline 1,9 & 1,9 & 862 & & 1153 \\
\hline 2 & 2 & 849 & & 1115 \\
\hline 2,1 & 2,1 & 838 & & 1080 \\
\hline 2,2 & 2,2 & 826 & & 1048 \\
\hline 2,3 & 2,3 & 815 & & 1018 \\
\hline 2,4 & 2,4 & 804 & 945 & 989 \\
\hline 2,5 & 2,5 & 793 & 920 & 962 \\
\hline 2,6 & 2,6 & 783 & 897 & 937 \\
\hline 2,7 & 2,7 & 773 & 875 & 913 \\
\hline 2,8 & 2,8 & 763 & 854 & 891 \\
\hline 2,9 & 2,9 & 754 & 835 & 869 \\
\hline 3 & 3 & 745 & 816 & 849 \\
\hline 3,1 & 3,1 & 736 & 798 & 829 \\
\hline 3,2 & 3,2 & 727 & 782 & 811 \\
\hline 3,3 & 3,3 & 718 & 765 & 793 \\
\hline 3,4 & 3,4 & 710 & 750 & 776 \\
\hline 3,5 & 3,5 & 702 & 735 & 760 \\
\hline 3,6 & 3,6 & 694 & 721 & 745 \\
\hline 3,7 & 3,7 & 686 & 708 & 730 \\
\hline 3,8 & 3,8 & 679 & 695 & 715 \\
\hline 3,9 & 3,9 & 672 & 682 & 702 \\
\hline 4 & 4 & 664 & 670 & 688 \\
\hline 4,1 & 4,1 & 657 & 659 & 675 \\
\hline 4,2 & 4,2 & 651 & 648 & 663 \\
\hline 4,3 & 4,3 & 644 & 637 & 651 \\
\hline 4,4 & 4,4 & 637 & 626 & 640 \\
\hline 4,5 & 4,5 & 631 & 616 & 628 \\
\hline 4,6 & 4,6 & 625 & 607 & 618 \\
\hline 4,7 & 4,7 & 618 & 597 & 607 \\
\hline 4,8 & 4,8 & 612 & 588 & 597 \\
\hline 4,9 & 4,9 & 606 & 579 & 587 \\
\hline 5 & 5 & 601 & 571 & 577 \\
\hline 5,1 & 5,1 & 595 & 562 & 568 \\
\hline 5,2 & 5,2 & 589 & 554 & 559 \\
\hline 5,3 & 5,3 & 584 & 546 & 550 \\
\hline 5,4 & 5,4 & 579 & 539 & 542 \\
\hline 5,5 & 5,5 & 573 & 531 & 533 \\
\hline 5,6 & 5,6 & 568 & 524 & 525 \\
\hline
\end{tabular}




\begin{tabular}{|c|c|c|c|c|}
\hline$\Delta$ (cc-gr) & $10^{3} x \ln \alpha$ & $\begin{array}{c}\text { Thermometer } \\
\text { Dunn \& Valley } \\
1992\left[^{\circ} \mathrm{C}\right] \\
\left(400-800^{\circ} \mathrm{C}\right) \\
\end{array}$ & $\begin{array}{c}\text { Thermometer } \\
\text { Kitchen \& Valley } \\
1995\left[^{\circ} \mathrm{C}\right] \\
\left(650-850^{\circ} \mathrm{C}\right) \\
\end{array}$ & $\begin{array}{c}\text { Thermometer } \\
\text { Chako et al } \\
1991\left[^{\circ} \mathrm{C}\right] \\
\left(400-700^{\circ} \mathrm{C}\right)\end{array}$ \\
\hline 5,7 & 5,7 & 563 & 517 & 517 \\
\hline 5,8 & 5,8 & 558 & 510 & 509 \\
\hline 5,9 & 5,9 & 553 & 504 & 502 \\
\hline 6 & 6 & 548 & 497 & 495 \\
\hline 6,1 & 6,1 & 544 & 491 & 487 \\
\hline 6,2 & 6,2 & 539 & 485 & 480 \\
\hline 6,3 & 6,3 & 534 & 479 & 473 \\
\hline 6,4 & 6,4 & 530 & 473 & 467 \\
\hline 6,5 & 6,5 & 525 & 467 & 460 \\
\hline 6,6 & 6,6 & 521 & 461 & 454 \\
\hline 6,7 & 6,7 & 517 & 456 & 447 \\
\hline 6,8 & 6,8 & 513 & 450 & 441 \\
\hline 6,9 & 6,9 & 508 & 445 & 435 \\
\hline 7 & 7 & 504 & 440 & 429 \\
\hline 7,1 & 7,1 & 500 & 435 & 424 \\
\hline 7,2 & 7,2 & 496 & 430 & 418 \\
\hline 7,3 & 7,3 & 493 & 425 & 412 \\
\hline 7,4 & 7,4 & 489 & 420 & 407 \\
\hline 7,5 & 7,5 & 485 & 416 & 401 \\
\hline 7,6 & 7,6 & 481 & 411 & 396 \\
\hline 7,7 & 7,7 & 478 & 407 & 391 \\
\hline 7,8 & 7,8 & 474 & 402 & 386 \\
\hline 7,9 & 7,9 & 470 & 398 & 381 \\
\hline 8 & 8 & 467 & & 376 \\
\hline 8,1 & 8,1 & 463 & & 371 \\
\hline 8,2 & 8,2 & 460 & & 366 \\
\hline 8,3 & 8,3 & 457 & & 362 \\
\hline 8,4 & 8,4 & 453 & & 357 \\
\hline 8,5 & 8,5 & 450 & & 353 \\
\hline 8,6 & 8,6 & 447 & & 348 \\
\hline 8,7 & 8,7 & 444 & & 344 \\
\hline 8,8 & 8,8 & 440 & & 340 \\
\hline 8,9 & 8,9 & 437 & & 335 \\
\hline 9 & 9 & 434 & & 331 \\
\hline 9,1 & 9,1 & 431 & & 327 \\
\hline 9,2 & 9,2 & 428 & & 323 \\
\hline 9,3 & 9,3 & 425 & & 319 \\
\hline 9,4 & 9,4 & 422 & & 315 \\
\hline 9,5 & 9,5 & 420 & & 311 \\
\hline 9,6 & 9,6 & 417 & & 307 \\
\hline 9,7 & 9,7 & 414 & & 304 \\
\hline 9,8 & 9,8 & 411 & & 300 \\
\hline 9,9 & 9,9 & 408 & & 296 \\
\hline 10 & 10 & 406 & & 293 \\
\hline 10,1 & 10,1 & 403 & & 289 \\
\hline 10,2 & 10,2 & 400 & & 286 \\
\hline 10,3 & 10,3 & 398 & & 282 \\
\hline
\end{tabular}




\begin{tabular}{|c|c|c|c|c|}
\hline$\Delta$ (cc-gr) & $10^{3} x \ln \alpha$ & $\begin{array}{c}\text { Thermometer } \\
\text { Dunn \& Valley } \\
1992\left[^{\circ} \mathrm{C}\right] \\
\left(400-800^{\circ} \mathrm{C}\right. \\
\end{array}$ & $\begin{array}{c}\text { Thermometer } \\
\text { Kitchen \& Valley } \\
1995\left[^{\circ} \mathrm{C}\right] \\
\left(650-850^{\circ} \mathrm{C}\right. \\
\end{array}$ & $\begin{array}{c}\text { Thermometer } \\
\text { Chako et al } \\
1991\left[^{\circ} \mathrm{C}\right] \\
\left(400-700^{\circ} \mathrm{C}\right.\end{array}$ \\
\hline 10,4 & 10,4 & 395 & & 279 \\
\hline 10,5 & 10,5 & 393 & & 275 \\
\hline 10,6 & 10,6 & 390 & & 272 \\
\hline 10,7 & 10,7 & 388 & & 269 \\
\hline 10,8 & 10,8 & 385 & & 266 \\
\hline 10,9 & 10,9 & 383 & & 262 \\
\hline 11 & 11 & 380 & & 259 \\
\hline
\end{tabular}




\begin{tabular}{|c|c|c|c|}
\hline$\Delta$ (cc-gr) & $10^{3} x \ln \alpha$ & $\begin{array}{l}\text { Thermometer } \\
\text { Polyakov \& } \\
\text { Kharlashina } \\
1995\left[^{\circ} \mathrm{C}\right] \\
\left(600-1200^{\circ} \mathrm{C}\right)\end{array}$ & $\begin{array}{c}\text { Thermometer } \\
\text { Scheele \& Hoefs } \\
1992\left[{ }^{\circ} \mathrm{C}\right] \\
\left(600-1200^{\circ} \mathrm{C}\right)\end{array}$ \\
\hline 1 & 1 & 1625 & \\
\hline 1,1 & 1,1 & 1536 & \\
\hline 1,2 & 1,2 & 1457 & \\
\hline 1,3 & 1,3 & 1388 & \\
\hline 1,4 & 1,4 & 1326 & \\
\hline 1,5 & 1,5 & 1271 & \\
\hline 1,6 & 1,6 & 1220 & \\
\hline 1,7 & 1,7 & 1175 & \\
\hline 1,8 & 1,8 & 1133 & \\
\hline 1,9 & 1,9 & 1094 & \\
\hline 2 & 2 & 1058 & \\
\hline 2,1 & 2,1 & 1025 & \\
\hline 2,2 & 2,2 & 994 & \\
\hline 2,3 & 2,3 & 965 & \\
\hline 2,4 & 2,4 & 938 & \\
\hline 2,5 & 2,5 & 913 & \\
\hline 2,6 & 2,6 & 889 & \\
\hline 2,7 & 2,7 & 866 & \\
\hline 2,8 & 2,8 & 845 & \\
\hline 2,9 & 2,9 & 824 & \\
\hline 3 & 3 & 805 & 1120 \\
\hline 3,1 & 3,1 & 787 & 1039 \\
\hline 3,2 & 3,2 & 769 & 982 \\
\hline 3,3 & 3,3 & 752 & 937 \\
\hline 3,4 & 3,4 & 736 & 900 \\
\hline 3,5 & 3,5 & 721 & 868 \\
\hline 3,6 & 3,6 & 706 & 841 \\
\hline 3,7 & 3,7 & 692 & 816 \\
\hline 3,8 & 3,8 & 679 & 794 \\
\hline 3,9 & 3,9 & 666 & 774 \\
\hline 4 & 4 & 653 & 755 \\
\hline 4,1 & 4,1 & 641 & 738 \\
\hline 4,2 & 4,2 & 629 & 722 \\
\hline 4,3 & 4,3 & 618 & 707 \\
\hline 4,4 & 4,4 & 607 & 694 \\
\hline 4,5 & 4,5 & 597 & 680 \\
\hline 4,6 & 4,6 & 586 & 668 \\
\hline 4,7 & 4,7 & 577 & 657 \\
\hline 4,8 & 4,8 & 567 & 645 \\
\hline 4,9 & 4,9 & 558 & 635 \\
\hline 5 & 5 & 549 & 625 \\
\hline 5,1 & 5,1 & 540 & 615 \\
\hline 5,2 & 5,2 & 531 & 606 \\
\hline 5,3 & 5,3 & 523 & \\
\hline 5,4 & 5,4 & 515 & \\
\hline 5,5 & 5,5 & 507 & \\
\hline
\end{tabular}




\begin{tabular}{|c|c|c|c|}
\hline$\Delta$ (cc-gr) & $10^{3} \times \ln \alpha$ & $\begin{array}{l}\text { Thermometer } \\
\text { Polyakov \& } \\
\text { Kharlashina } \\
1995\left[^{\circ} \mathrm{C}\right] \\
\left(600-1200^{\circ} \mathrm{C}\right)\end{array}$ & $\begin{array}{c}\text { Thermometer } \\
\text { Scheele \& Hoefs } \\
1992\left[{ }^{\circ} \mathrm{C}\right] \\
\left(600-1200^{\circ} \mathrm{C}\right)\end{array}$ \\
\hline 5,6 & 5,6 & 500 & \\
\hline 5,7 & 5,7 & 492 & \\
\hline 5,8 & 5,8 & 485 & \\
\hline 5,9 & 5,9 & 478 & \\
\hline 6 & 6 & 471 & \\
\hline 6,1 & 6,1 & 464 & \\
\hline 6,2 & 6,2 & 458 & \\
\hline 6,3 & 6,3 & 451 & \\
\hline 6,4 & 6,4 & 445 & \\
\hline 6,5 & 6,5 & 439 & \\
\hline 6,6 & 6,6 & 433 & \\
\hline 6,7 & 6,7 & 427 & \\
\hline 6,8 & 6,8 & 422 & \\
\hline 6,9 & 6,9 & 416 & \\
\hline 7 & 7 & 411 & \\
\hline 7,1 & 7,1 & 405 & \\
\hline 7,2 & 7,2 & 400 & \\
\hline 7,3 & 7,3 & 395 & \\
\hline 7,4 & 7,4 & 390 & \\
\hline 7,5 & 7,5 & 385 & \\
\hline 7,6 & 7,6 & 380 & \\
\hline 7,7 & 7,7 & 375 & \\
\hline 7,8 & 7,8 & 371 & \\
\hline 7,9 & 7,9 & 366 & \\
\hline 8 & 8 & 361 & \\
\hline 8,1 & 8,1 & 357 & \\
\hline 8,2 & 8,2 & 353 & \\
\hline 8,3 & 8,3 & 348 & \\
\hline 8,4 & 8,4 & 344 & \\
\hline 8,5 & 8,5 & 340 & \\
\hline 8,6 & 8,6 & 336 & \\
\hline 8,7 & 8,7 & 332 & \\
\hline 8,8 & 8,8 & 328 & \\
\hline 8,9 & 8,9 & 324 & \\
\hline 9 & 9 & 321 & \\
\hline 9,1 & 9,1 & 317 & \\
\hline 9,2 & 9,2 & 313 & \\
\hline 9,3 & 9,3 & 310 & \\
\hline 9,4 & 9,4 & 306 & \\
\hline 9,5 & 9,5 & 303 & \\
\hline 9,6 & 9,6 & 299 & \\
\hline 9,7 & 9,7 & 296 & \\
\hline 9,8 & 9,8 & 293 & \\
\hline 9,9 & 9,9 & 289 & \\
\hline 10 & 10 & 286 & \\
\hline 10,1 & 10,1 & 283 & \\
\hline
\end{tabular}




\begin{tabular}{|c|c|c|c|}
\hline$\Delta$ (cc-gr) & $\mathbf{1 0}^{\mathbf{3}} \mathbf{x} \mathbf{l n} \boldsymbol{\alpha}$ & $\begin{array}{c}\text { Thermometer } \\
\text { Polyakov \& } \\
\text { Kharlashina } \\
\mathbf{1 9 9 5}\left[^{\circ} \mathbf{C}\right] \\
\left(\mathbf{6 0 0 - 1 2 0 0 ^ { \circ }} \mathbf{C}\right)\end{array}$ & $\begin{array}{c}\text { Thermometer } \\
\text { Scheele \& Hoefs } \\
\left.\mathbf{1 9 9 2}]^{\circ} \mathbf{C}\right] \\
\left(\mathbf{6 0 0 - 1 2 0 0} \mathbf{C}^{\circ} \mathbf{C}\right)\end{array}$ \\
\hline 10,2 & 10,2 & 280 & \\
\hline 10,3 & 10,3 & 277 & \\
\hline 10,4 & 10,4 & 274 & \\
\hline 10,5 & 10,5 & 271 & \\
\hline 10,6 & 10,6 & 268 & \\
\hline 10,7 & 10,7 & 265 & \\
\hline 10,8 & 10,8 & 262 & \\
\hline 10,9 & 10,9 & 259 & \\
\hline 11 & 11 & 256 & \\
\hline
\end{tabular}




\section{LEBENSLAUF:}

Am 16. Mai 1972 wurde ich als Sohn von Hiltrut Walter geb. Gellert und Joachim Walter in Windhoek, Namibia geboren. Dort wurde ich Anfang 1979 in die Deutsche Höhere Privatschule Windhoek eingeschult, an der ich dann zunächst 1990 den südafrikanischen Schulabschluss, das Matriculation Certificate, absolvierte. Im November des folgenden Jahres schloss ich dann ebenfalls an der Deutschen Höheren Privatschule Windhoek mit der deutschen Allgemeinen Hochschulreife ab.

Zum WS 1992/93 begann ich an der Georg-August-Universität Göttingen das Studium der Geologie/Paläontologie, an der ich am 18. April 1997 die Diplomvorprüfung absolvierte. Nach der Diplomkartierung und -arbeit am Institut für Geologie und Dynamik der Lithosphäre (IGDL der Universität Göttingen schloss ich das Studium am 28.10.1999 mit dem Diplom ab.

Im September 2000 begann ich mit der Promotion ebenfalls am IGDL in der ich vom 01. September 2000 bis 31. August 2001 und vom 01. September 2002 bis 31 . August 2003 über das DFG Projekt WE 488/50-1 am IGDL und am Geowissenschaftlichen Zentrum der Universität Göttingen angestellt war. Im Juni 2000 heiratete ich meine Frau Maria Elisabeth Walter geborene Rogge. Im Juli 2002 wurde unsere Tochter Franziska Leoni Walter geboren. 